This Health Hazard Evaluation (HHE) report and any recommendations made herein are for the specific facility evaluated and may not be universally applicable. Any recommendations made are not to be considered as final statements of NIOSH policy or of any agency or individual involved. Additional HHE reports are available at http://www.cdc.gov/niosh/hhe/

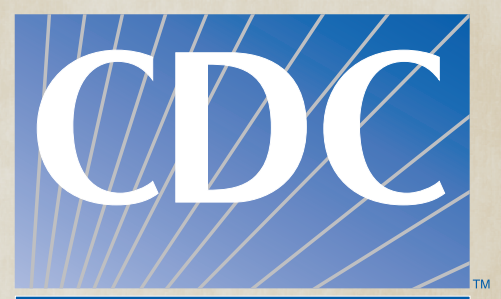

Workplace

Safety and Health

\title{
Evaluation of dampness-
} associated respiratory symptoms with relocation of staff during remediation of an elementary school

\author{
Rachel Bailey, DO, MPH \\ Ju-Hyeong Park, ScD, MPH, CIH \\ Rena Saito, $P h D$ \\ Kathleen Kreiss, $M D$ \\ Jean Cox-Ganser, PhD
}

Health Hazard Evaluation Report

HETA 2009-0172-3124

North Carolina

March 2011

DEPARTMENT OF HEALTH AND HUMAN SERVICES

Centers for Disease Control and Prevention

Safety and Health 
The employer shall post a copy of this report for a period of 30 calendar days at or near the workplace(s) of affected employees. The employer shall take steps to insure that the posted determinations are not altered, defaced, or covered by other material during such period. [37 FR 23640, November 7, 1972, as amended at 45 FR 2653, January 14, 1980]. 


\section{CONTENTS}

REPORT

Abbreviations .............................................................. iv

Highlights of the NIOSH Health Hazard Evaluation..............

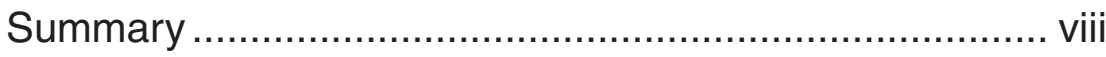

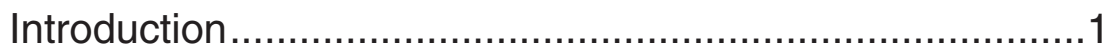

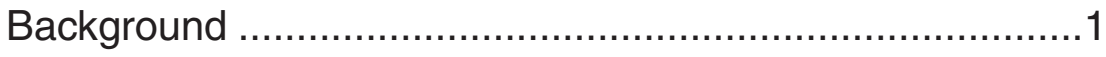

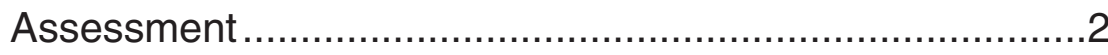

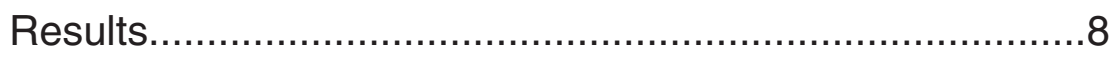

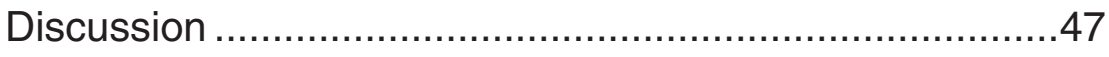

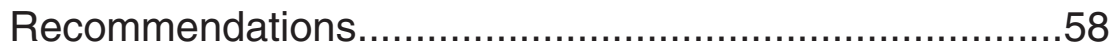

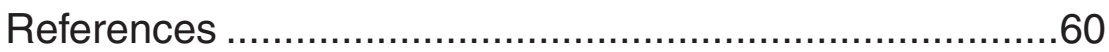

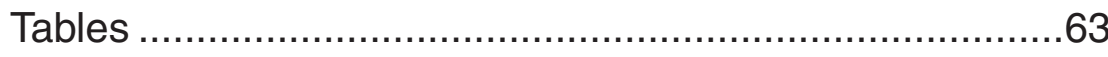

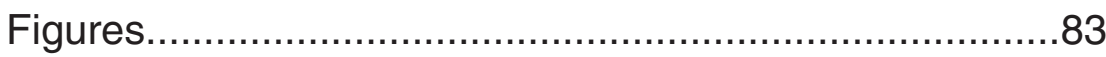

APPENDIX A County Department of Public Health Faculty/Staff

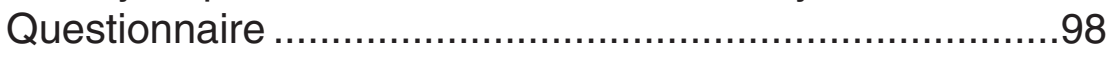

APPENDIX B County Department of Public Health Parent Questionnaire

APPENDIX C NIOSH March 2010 questionnaire

108

APPENDIX D County Department of Public Health Statement Dated 25

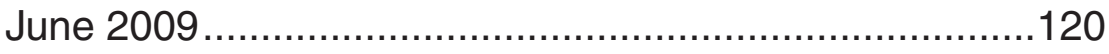

APPENDIX E NIOSH Email dated 22 July: HETA 2009-0172-Requested Summary of Findings Discussed during the Breakfast

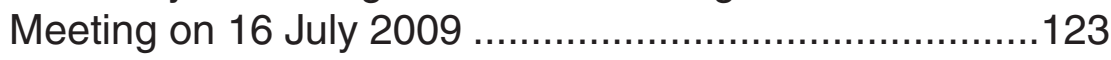


County Department of Public Health Report on Faculty/Staff

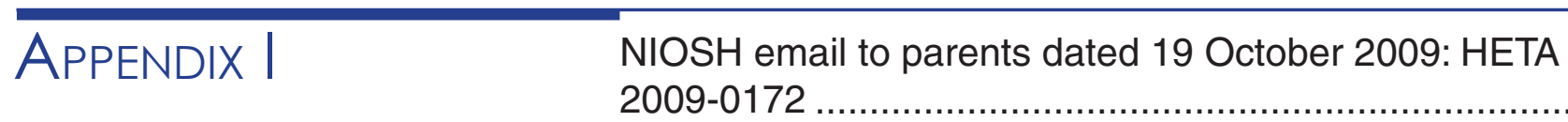


CONTENTS (Continued)

ApPENDIX Q

NIOSH Draft Mold \& Dampness Assessment Sheet........299

ACKNOWLEDGMENTS

Acknowledgements and Availability of Report...............311 


\begin{tabular}{ll}
\hline ABBREVIATIONS \\
$2 / 3$ wing & First and second grade wing \\
$4 / 5$ wing & Fourth and fifth grade wing \\
ASHRAE & American Society of Heating, Refrigeration and Air-Conditioning Engineers \\
BAS & Building automation system \\
BASE & Building Assessment Survey and Evaluation \\
BRFSS & Behavioral Risk Factor Surveillance System \\
CDC & Centers for Disease Control and Prevention \\
$\mathrm{CO}_{2}$ & Carbon dioxide \\
cfu/g & Colony forming units per gram \\
EPA & Environmental Protection Agency \\
${ }^{\circ}$ & Degrees Fahrenheit \\
GM & Geometric mean \\
$\mathrm{HEPA}$ & High-efficiency particulate air \\
$\mathrm{HP}$ & Heat pump \\
$\mathrm{HVAC}$ & Heating, ventilation, and air conditioning \\
$\mathrm{k} / 1$ wing & Kindergarten and first grade wing \\
$\mathrm{mg} / \mathrm{m}^{3}$ & Milligrams per cubic meter \\
$\mathrm{m}^{2}$ & Square meter \\
$\mathrm{m}^{3}$ & Cubic meter \\
$\mathrm{NHANES}$ III & Third National Health and Nutrition Examination Survey \\
$\mathrm{NIOSH}$ & National Institute for Occupational Safety and Health \\
$\mathrm{ppm}$ & Parts per million \\
$\mathrm{RH}$ & Relative Humidity \\
VOC & Volatile organic compounds \\
$\mathrm{WHO}$ & World Health Organization \\
&
\end{tabular}




\section{HIGHLIGHTS OF THE \\ NIOSH HEALTH \\ HazARD Evaluation}

\section{What NIOSH Did:}

- NIOSH investigators visually assessed the school in conjunction with a building science contractor hired by the school district.

- We evaluated moisture content of some floors and exterior walls.

- We monitored indoor air quality parameters (carbon dioxide, relative humidity, and temperature).

- We collected floor dust samples in some rooms to analyze for microorganisms.

- We met with staff from the county health department to review their questionnaire data.

- We analyzed de-identified questionnaire data provided by the county health department in 2009.

- We interviewed school staff members by phone.

- We reviewed and summarized historical environmental reports.

- We administered a health questionnaire to school staff when the school reopened after remediation in 2010.

\section{What NIOSH Found:}

- The school had a history of dampness, high humidity, interior mold growth, ventilation problems, biocide use on carpet and in the ventilation systems, and building-related symptoms among staff and students.

- We smelled musty, moldy odors in the basement, crawl space, and occupied space of the media center wing.

- Surface drainage problems allowed drainage of rainwater toward the concrete walkways with puddling of water adjacent to the classroom wings.

- A storm drain outside the media center was blocked.

- The foam roof on the old section of the school was damaged.

- There was excessive moisture in an exterior concrete block wall of the gym.

- Flashing at the juncture between old and new buildings was inadequate. 
HIGHLIGHTS OF THE NIOSH HEALTH HAZARD EVALUTION (CONTINUED)
- Airflow between rooms was problematic.

- Open soffits in classroom wings allowed unconditioned outdoor air into the building.

- Levels of fungi in floor dust were lower than previous sampling before floor cleaning and biocide use.

- Two staff members reported building-related symptoms suggestive of hypersensitivity pneumonitis.

- A health department questionnaire survey found that staff (and students via their parents) reported building-related health symptoms, including difficulty breathing/asthma attacks, eye symptoms, headaches, and fatigue when in the school building during the 2008/2009 school year.

- Building-related symptoms reported by elementary school staff decreased after relocation of the staff to other schools.

\section{What The School District Administration Has Done since the NIOSH Site Visit in 2009:}

- Removed carpet and replaced with hard floors (after testing slab for moisture content).

- Installed a vapor barrier and depressurized the basement and crawl space under the media center.

- Repaired the roof and inadequate flashing and sealed classroom wing soffits.

- Recommissioned the heating, ventilation, and airconditioning system.

- Relocated staff and students to alternate sites during remediation and repair at the school.

- Reopened the school in February 2010 after remediation of the school.

- Implemented the Environmental Protection Agency's Indoor Air Quality Tools for Schools Program.

\section{What The School District Administration Can Do:}

- Modify surface drainage around the school building to prevent puddling and drainage of water towards the building. 
HIGHLIGHTS OF THE NIOSH HEALTH HAZARD EVALUTION (CONTINUED)
- Follow the manufacturer's recommended maintenance schedules for maintaining the heating, ventilation, and air conditioning system.

- Routinely monitor the school in a standardized way for possible re-occurrence of dampness.

- Walk through the entire school and check for water incursion during and after heavy rains.

- Correct the cause of any identified dampness problems and clean or remove water-damaged materials.

- Use proper containment methods to prevent worker exposures and contamination of unaffected sections of the building during any remediation.

- Record the date and location of water-damaged materials and remediation steps taken.

- Avoid routine use of biocides.

- Facilitate confidential reporting of dampness problems and building-related health effects by employees.

- Monitor for possible reoccurrence of building-related symptoms, if needed, with occupant health questionnaires.

- Encourage building occupants (employees and students) to see a healthcare provider if they develop or have developed persistent or worsening respiratory or other health symptoms while in the school building.

\section{What the Elementary School Faculty and Staff Can Do:}

- Participate in health questionnaires.

- Inform the school administration of any water leaks, dampness, musty or moldy odors, or ventilation problems in the school building.

- See a healthcare provider if you develop or have developed recurring or worsening respiratory symptoms or other health symptoms while working in the school building.

- Let your supervisor know if your healthcare provider recommends relocation to another work area to prevent exposure to mold or dampness-related contaminants that may be causing or exacerbating your symptoms. 


\section{NIOSH considers dampness in occupied buildings a public health problem that requires remediation. NIOSH investigators found a history of dampness, mold growth, high indoor relative humidity, ventilation problems, biocide use, and respiratory symptoms with a building-related pattern related to the elementary school. The school closed for remediation after the 2008/2009 school year and reopened on February 22, 2010. The prevalence of building- related symptoms among staff decreased after relocation, while the school building was closed for remediation.}

In June 2009, school district management requested a health hazard evaluation to investigate concerns about indoor environmental quality at an elementary school. Since August 2005, after renovations and the addition of three classroom wings, there have been many issues regarding indoor environmental quality, including building-related symptoms and a history of dampness, mold growth, high humidity, and ventilation problems. Since 2005, the school district has had many consultants evaluate the school; we briefly summarize their findings in this report.

In July 2009, a NIOSH team visited at the elementary school. We worked in conjunction with the Turner Building Science and Design Group, hired by the school district. We found (1) a musty, moldy odor in the basement, crawl space, and occupied space in the media center; (2) surface drainage issues around the school; (3) a blocked storm drain with standing water; (4) a damaged roof; (5) inadequate flashing; (6) moisture in the gym's exterior concrete block wall; (7) pressure airflow issues between conditioned and unconditioned spaces; and (8) outdoor humid air entering through the classroom wing soffits. Except for the musty odor in the basement and crawl space under the media center, these findings were consistent with previous findings by consultants.

During the site visit, we met with the medical director and other staff of the county health department and reviewed results of a questionnaire they had offered to the staff and parents of the school during May 2009. The health department shared de-identified data with us; our analysis indicated that, during the 2008/2009 school year while in the school building (1) $31 \%$ of staff and $13 \%$ of students had difficulty breathing/asthma attacks; (2) $52 \%$ of staff and $28 \%$ of students had one or more upper respiratory symptoms; (3) $75 \%$ of staff and $28 \%$ of students had eye symptoms; (4) $92 \%$ of staff and $49 \%$ of students had headaches; (5) $44 \%$ of staff and $6 \%$ of students had fatigue; and (6) $25 \%$ of staff and $20 \%$ of students had nosebleeds. Some school staff members who were interviewed over the telephone by a NIOSH medical officer reported building-related symptoms that were suggestive of hypersensitivity pneumonitis, an uncommon and potentially chronic condition known to be related to damp buildings.

In August 2009, the school remained closed for remediation after summer break. The school district worked with the Turner Group and other consultants to address issues identified during the July 2009 site visit. The school reopened on February 22, 2010. During 
March 2-4, 2010, NIOSH investigators administered a health questionnaire to the elementary school staff. Compared with results of the previous health department survey, the NIOSH survey found that fewer staff reported headaches, sneezing, throat symptoms, eye symptoms, cough attacks, fatigue, nosebleeds, and difficulties remembering things or concentrating, consistent with a resolution of many employees' symptoms while out of the school building. The prevalence of nasal symptoms and skin symptoms were higher in the NIOSH questionnaire than the health department survey, but fewer than $25 \%$ of those with these symptoms in the NIOSH survey reported they were work-related. Of staff that participated in the health department survey and worked at the school during the 2008/2009 school year, 74\% reported they had symptoms that went away after leaving the school building, consistent with a work-related pattern. The 3\%-7\% prevalences of lower respiratory symptoms (i.e., shortness of breath, chest tightness, wheezing, and cough attacks) noted in the NIOSH questionnaire were much lower than prevalences of the two lower respiratory symptoms (breathing difficulty/asthma attacks (31\%) and cough (15\%)) in the health department survey.

At the time of the March 2010 NIOSH questionnaire, the overall prevalences of ever and current physician-diagnosed asthma among participating school staff in the NIOSH survey were not significantly different than what would be expected in North Carolina when compared to the BRFSS survey of the North Carolina adult population. Most staff reporting asthma had experienced asthma prior to beginning employment at the elementary school. Compared to the U.S. adult population in the NHANES III, a nationally representative survey, the elementary school employees had significantly lower than expected rates of lifetime and current asthma, chronic bronchitis, shortness of breath on exertion, and nasal allergies, including hay fever. When we compared the elementary school employees who participated in the NIOSH survey to U.S. office workers in the BASE study, we found no excess of work-related upper and lower respiratory symptoms, headache, unusual tiredness and fatigue, or dry itchy skin among the school employees.

The NIOSH health survey showed apparent resolution of many health symptoms likely associated with indoor environmental quality issues in the school building before remediation. If there are no future concerns, there may be no reason to resurvey the employees who have reoccupied the school. However, if concerns 
do arise in the future, another questionnaire can be administered to employees and compared to the NIOSH assessment to determine if increases in building-related symptoms have occurred. No specific environmental measurements are known to predict health outcomes for individuals, so assessing health is the only option for determining the adequacy of building remediation in improving indoor environmental quality.

Keywords: NAICS 611110 (Elementary and Secondary Schools), indoor environmental quality, asthma, hypersensitivity pneumonitis, respiratory symptoms, engineering controls, relative humidity, carbon dioxide, ventilation. 
The National Institute for Occupational Safety and Health (NIOSH) received a Health Hazard Evaluation request from the management of a North Carolina school district on June 16, 2009, to investigate concerns about indoor environmental quality at an elementary school. The request listed concerns about mold, high levels of carbon dioxide $\left(\mathrm{CO}_{2}\right)$, and problems with the heating, ventilation, and air-conditioning (HVAC) system. Health concerns included headaches, fatigue, insomnia, dizziness, bronchitis, pneumonia, sinus problems, sore throat, rashes, nausea, vision problems, and others.

The school was built in the 1930s, with additions in the 1970s. The original building is currently used for the media center, library, offices, health center, teachers' lounge, and testing room. In 2003, construction began on three new classroom wings and a cafeteria. The addition was attached to the gym, which was built in the 1970s and remodeled during the construction process. In May 2005, before the 2004/2005 school year ended, the kindergarten/ first grade $(\mathrm{k} / 1)$ wing and administrative wing were opened and occupied. The second/third grade (2/3) and fourth/fifth grade (4/5) wings (Figure 1) opened in August 2005, a few days before the start of school. Classroom wings are on the west side of the school, the gym is on the east side, and the old original building is on the north side. The playgrounds and ball fields are the highest points on the site. Except for the media center, which has a clerestory above it and a basement and crawl space beneath it, the school is single-story construction on a concrete slab.

During the 2008/2009 school year, the school had 88 faculty and staff and 738 students. Since the additions to the school in 2005, there have many issues regarding indoor environmental quality. These are summarized in the Results section of this report under "Chronological History of Environmental Findings and Remediation at the Elementary School." 


\section{July 2009 Site Visit}

A NIOSH team of two industrial hygienists, an epidemiologist, and a medical officer completed a site visit at the elementary school from July 14-16, 2009. Consultants from the Turner Building Science and Design Group, hired by the school district, also participated in the site visit, and the NIOSH team conferred with them during and after the site visit.

On the first morning, we conducted an opening meeting with school staff, parents, and news media to discuss the Health Hazard Evaluation program and the purpose of our visit. We then met with the principal, teachers, and parents for approximately one hour to discuss their concerns. This was followed by a NIOSH initial walkthrough of the school accompanied by school staff, parents, and news media. During the rest of the site visit, members of the local Parent Teacher Association often accompanied us as we evaluated the school.

In addition to visual assessment techniques, we used moisture meters (Tramex ${ }^{\mathrm{TM}}$ Concrete Encounter CME4 and Tramex Moisture Encounter, Tramex Limited, Co., Dublin, Ireland) and an infrared camera (IR-Insight ${ }^{\mathrm{TM}}$, Fluke Corporation, Everett, Washington, United States) to check for moisture in walls. Additionally, we used smoke tubes to visualize airflow and observe pressure differentials between rooms and the corridors inside the school building. Turner Group staff and NIOSH staff used digital pressure gauges (The Energy Conservatory, Minneapolis, Minnesota, United States) to measure pressure differentials at exterior doors; the results were documented on diagrams of the school and given to Turner Group staff for further analysis.

We selected 21 rooms (Table 1) to collect environmental samples (6 fully carpeted rooms, 11 carpeted rooms with 10\%-25\% tiled floor, and 4 rooms with entirely tiled or wooden floors). These rooms were selected either because occupants had reported health or environmental issues, or previous environmental consultants' reports documented elevated mold concentrations in air or floor dust. We collected floor dust samples into polyethylene filter socks using a L'il Hummer ${ }^{\mathrm{TM}}$ backpack vacuum sampler (Pro-Team Inc, Boise, Idaho, United States) (flow rate $=100$ cubic feet per minute). To collect enough dust for microbial analyses, we vacuumed either a 2 square meter $\left(\mathrm{m}^{2}\right)$ area of the floor around the teacher's workstation or a $1 \mathrm{~m}^{2}$ area of floor around the teacher's work station plus a $0.8 \mathrm{~m}^{2}$ area around the edges of the room (a total of $1.8 \mathrm{~m}^{2}$ ) for a 
total of five minutes. Room 108 was excluded because we could not collect enough dust for analysis. In the media center, which was larger than classrooms and offices, we collected two separate dust samples; one from a $2 \mathrm{~m}^{2}$ area of a rug in the room and another from the edge of the room. We also measured wet bulb and dry bulb temperatures on the floor surface to compute water activity (the amount of free or available water for microorganisms) and air temperature and relative humidity (RH) at the end of dust sampling in each room.

We sealed the vacuumed dust samples in plastic bags and transported them to the NIOSH laboratory. At the NIOSH laboratory, we emptied the dust collected in each sample into 50 milliliter pyrogen-free conical tubes and homogenized by rotation on a 360-degree rotary arm shaker at 65 revolutions per minute for 2 hours. We removed hair, fluff, and other large objects from the sample before homogenization. We weighted, partitioned, and sent the dust samples to an environmental microbiology laboratory accredited by the American Industrial Hygiene Association (EMLab $P \& K$, San Bruno, California, United States). We requested analysis of the dust samples for culturable fungi with full speciation, Gramnegative bacteria, and Actinomycetes because these microbes have been associated with dampness in buildings. We also requested analysis for Gram-positive bacteria because humans are often a source of this type of bacteria. Dust samples were cultured for Actinomycetes at three different temperatures - room temperature, 35 degrees Celsius, and 55 degrees Celsius. The laboratory reported culturable fungi, bacteria, and Actinomycetes as colony forming units per gram $(\mathrm{cfu} / \mathrm{g})$ of dust. We categorized fungal species into hydrophilic, mesophilic, and "other fungi" groups. Hydrophilic fungi (or water-loving fungi) are defined as fungal species requiring high water content for survival and growth in building materials (water activity >0.9). Mesophilic fungi are fungal species that require water activity between 0.8 and 0.9 . In damp conditions, hydrophilic fungi will overgrow mesophilic fungi. Fungal species not categorized as either hydrophilic or mesophilic were included in the "other fungi" group. We used three sources [WHO 2009; Hung et al. 2005; Flannigan and Miller 2001] to determine the water activity requirement of each fungal species and to categorize them into one of three groups; hydrophilic, mesophilic, "other" fungi. Among the three sources [WHO 2009; Hung et al. 2005; Flannigan and Miller 2001], we choose the classification with the highest water activity. We computed fractions of the hydrophilic and mesophilic fungi for each dust sample by dividing the concentration of each group of 
fungi by the total concentration.

We visited the county health department; the medical director shared de-identified data from a self-administered paper questionnaire that they had offered to the faculty/staff (Appendix A) and parents of students (Appendix B) at the elementary school in May and June 2009. We interviewed with three staff members by phone regarding health concerns they believed were related to the school building.

On the last day of the site visit, we had a breakfast meeting with representatives of the school district about our findings and recommendations. This was followed by a brief closing meeting at the elementary school with school staff, parents, local health department staff, and the news media. Prior to, during, and after the site visit, we obtained documentation from the school district and the health department regarding indoor environmental quality issues at the school. We used this documentation to summarize the historical environmental problems and actions that had occurred at the school during the past five years.

\section{Health Department Questionnaire Data}

In May 2009, the county health department surveyed elementary school employees and parents/guardians of students at the elementary school with self-administered paper questionnaires (Appendices A and B) that focused on the 2008/2009 and 2007/2008 school years. Throughout this report, we refer to faculty and staff as "staff" or "staff members" and parents or guardians as "parents." The health department chose the 2008/2009 and 2007/2008 time frame to determine if there were changes in building-related symptoms after the installation of a dehumidification system in the classroom section of the school during the summer of 2008.

The staff and parent questionnaires included questions on primary office or classroom, symptoms, health conditions, medication, smoking status, and home exposures. Participants were first asked if they experienced any of the following symptoms while in the school building during the 2008/2009 school year: (1) headaches; (2) dry eyes; (3) unusual sneezing episodes; (4) difficulty breathing/asthma attacks; (5) nosebleeds; or (6) any other symptoms (participant asked to write in other symptoms). Participants who answered "no" or did not write-in a symptom they experienced during the $2008 / 2009$ school year were asked to stop the questionnaire at that point. For these participants, no information was collected on symptoms during the 2007/2008 school year, health conditions, 
medication, smoking status, or home exposures. Participants who did report experiencing symptoms in 2008/2009 were asked about whether they experienced any of the same list of symptoms during the 2007/2008 school year. They were also asked if they had the following conditions: (1) chronic respiratory disease; (2) undergoing chemotherapy or radiation therapy; (3) immune system stressed by disease or other cause; (4) allergic rhinitis; or (5) asthma. Those participants were further asked to report rooms or areas in the building where their symptoms were worst and if their symptoms/ problems went away after leaving the building. Participants also had the opportunity to write in additional information at the end of the questionnaire. The questionnaire did not seek information about physician diagnosis or date of onset of health conditions. The questionnaires did not include standardized symptom or asthma questions, which would have allowed us to compare staff rates to U.S. or state population rates.

We received de-identified questionnaire data from the health department in Microsoft Excel ${ }^{\circledR}$ (Microsoft, Redmond, Washington, United States) format and imported it into SAS® (version 9.2, SAS Institute Inc., Cary, North Carolina, United States) for analysis. The Excel database included variables created for the specific health conditions and symptoms asked in the questionnaire, as well as variables for write-in symptoms. The write-in variables included rash, nausea, sinus problems, insomnia, dizziness, fatigue, sore throat, and vision problems. We created additional variables for other symptoms written in by participants for each school. These included cough, allergies, metallic taste, memory loss, eye symptoms (burning, itching, red, dry, or tearing), skin problems (eczema, rash, dry skin, itchy skin, or tingling skin), nasal symptoms (stuffy, itchy, burning, runny, or dry), throat symptoms (dry, scratchy, or sore), and voice symptoms (hoarse voice or laryngitis). Additionally, we grouped symptoms into upper respiratory symptoms (sinus problems, nasal symptoms, sneezing, throat symptoms, and/or voice symptoms), lower respiratory symptoms (breathing problem/asthma attack and/or cough), and mucous membrane irritation symptoms (eye, nasal, throat, and/or voice symptoms).

We grouped school building areas into six categories (gym/cafeteria wing, administrative wing, media center wing, $\mathrm{k} / 1$ wing, $2 / 3$ wing, and $4 / 5$ wing). Staff was assigned to a work wing based on their primary classrooms or offices. We assigned students to a classroom wing based on their primary classrooms/homerooms. If a student did not have a primary classroom/homeroom listed in the database, we assigned the student to a classroom wing based on his or her age 
(if it was available). Students 5 or 6 years old were assigned to the k/ 1 wing; students 8 years old to the $2 / 3$ wing; and students 10,11 , or 12 years old to the $4 / 5$ wing. We did not assign students aged 7 or 9 years old because their wing assignment would differ based on whether they started kindergarten at 5 or 6 years-old. Therefore in 2008/2009, we did not assign wings for three 7-year-olds and four 9 -year olds.

\section{March 2010 Site Visit}

During March 2-4, 2010, we returned and administered an interview-based health questionnaire (Appendix C) with questions about respiratory symptoms and other symptoms, chest illnesses, medications, work environment, work history at the elementary school, and smoking habits. Some questions were adapted from two national surveys (1) the Building Assessment Survey and Evaluation (BASE) study [Womble et al. 1996], a 1994-1998 Environmental Protection Agency (EPA) survey of office employees at office buildings with no known or perceived problems in large U.S. cities; and (2) the Third National Health and Nutrition Examination Survey (NHANES III) [CDC 1996], a 1988-1994 survey of the U.S. civilian, noninstitutionalized population. We asked about symptoms during the four-week period (January 24 through February 21, 2010) prior to the reopening of the elementary school on February 22, 2010. Some participants had re-entered the school approximately two to six weeks before February 22, 2010 to get their work areas ready. For these participants, we had them answer the four-week symptom questions relating to the four weeks prior to re-entering the school building. For example, if they started coming to the elementary school on February 15, we had them answer the four week symptoms questions for the period of January 17 through February 14, 2010.

We asked if symptoms stayed the same, worsened, or improved when away from the school building. If symptoms improved away from the building, we considered them work-related. We grouped symptoms into upper respiratory symptoms (stuffy, itchy, or runny nose; sneezing; sore or dry throat; or sinusitis or sinus problems), lower respiratory symptoms (chest wheezing; chest tightness; shortness of breath attacks; coughing attacks; awakened by breathing difficulty; shortness of breath on exertion; or cough with phlegm), mucous membrane symptoms (stuffy, itchy, or runny nose; watery, itchy eyes; or sore or dry throat), and constitutional symptoms (fever and chills; flu-like achiness or achy joints; tiredness, fatigue, or drowsiness; difficulty remembering or concentrating; or dizziness or 
lightheadedness; headache). All school employees were invited to participate.

\section{Statistical Analysis}

We used SAS software for data analyses. When analyzing the health department survey, we calculated prevalence of reported symptoms experienced by staff and by parents (for their children who attend the elementary school) during the 2008/2009 and/or 2007/2008 school years. We used McNemar's test to determine if there were statistically significant differences in symptom prevalences during the 2008/2009 and 2007/2008 school years among staff and students who worked or attended during the 2008/2009 and $2007 / 2008$ school years and who had answered "yes" or wrote in a response to one of the six symptom questions on the first page of the health department questionnaire (Appendices A and B). We used the likelihood ratio chi-square test to test for significant differences when comparing 2008/2009 symptom prevalences among classroom wings. When analyzing data from the NIOSH survey, we used Pearson's exact test to test for significant differences in symptom prevalences between different work areas at the elementary school. McNemar's test was used to compare symptom prevalences among employees who had worked at the elementary school during the $2008 / 2009$ school year and returned to the school for the opening in February 2010. A probability level of $\mathrm{p}<0.05$ was considered statistically significant. A probability level of $0.05 \leq p<0.10$ was considered marginally significant. We calculated prevalence ratios of diagnoses and respiratory symptoms in comparison with U.S. adult population expected prevalences from NHANES III data and BASE data, and with 2009 expected values for North Carolina from the Behavioral Risk Factor Surveillance System (BRFSS) [NCCDPHP 2009]. 


\section{Chronology of Environmental Findings and Remediation at the Elementary School}

\section{August 2005}

Prior to school opening in August 2005, library books were shrinkwrapped on rolling homemade presswood carts and moved from the library in the media center wing of the original building to the pre-kindergarten classroom (room 613) in the new k/1 wing (Figure 1 ), where they were stored for two months. In August 2005, when the $2 / 3$ and $4 / 5$ wings were completed, the books were moved to a temporary media center (classroom 500) in the 4/5 wing; at this time, the books and carts in the pre-kindergarten classroom were covered in a gray-green mold and an overwhelming moldy smell was noted in the room. A contractor cleaned the books before they were moved to classroom 500 and stored on wooden bookcases.

In August 2005, visible mold was also found on carpet in classrooms 427 and 625 (Figure 2), upholstered chairs in the records room (room 109) (Figure 3), and on picture frames in the storage room (room 111) (Figure 4). Later, after the school year began, mold reappeared on some of the books in the temporary media center in classroom 500. Many books were thrown out while others were again cleaned. By the end of August 2005, visible mold had been identified in the $\mathrm{k} / 1$ wing (classroom 613, 625), 2/3 wing (classroom 427), 4/5 wing (classroom 500), and the administrative wing (rooms 109 and 111).

During this time frame, the school was having problems with high $\mathrm{RH}$ and the new HVAC system was being commissioned to assure that it would perform as intended. Additionally, parents had begun notifying the school that their children were complaining of headaches and respiratory symptoms. On August 25, 2005, the county health department received two complaints about mold on library books. The health department inspector who visited the school reported that the school was humid, and that this issue needed to be addressed to control mold growth. The inspector returned on August 31, 2005 and noted that the humidity issues had improved; however, staff reported headaches and irritation of the eyes, throat, and ears.

\section{September 2005}

During September 2005, school maintenance staff continued to adjust the HVAC system; mold and RH were still a concern. A consultant found that air filters in the heat pump (HP) units were 
very dusty and poorly fitting; they were replaced. The unit (HP-42) that served classroom 500 had some visible dry buildup on the coil front; a sample of the material grew mold (Penicillium). RH in sampled rooms exceeded $60 \%$ (range: $61-72 \%$ ). Because mold was found in carpet samples, new carpet was put in classroom 625 and vinyl composition tile in the health center (room 101).

In September 2005, the North Carolina Division of Occupational Safety and Health received a complaint regarding (1) elevated carbon dioxide $\left(\mathrm{CO}_{2}\right)$ levels (2000 parts per million (ppm) and $5000 \mathrm{ppm}$ ) in classrooms 502 and 508 in the 4/5 wing; (2) exposure to mold in the school (including the media center, which was closed due to mold on books, the health center, classroom 520, and around the gym); and (3) respiratory symptoms among employees. Parents continued to report that their children were experiencing respiratory symptoms, headaches, and fatigue.

The school hired an industrial hygiene consultant to test and monitor indoor air quality. He reported that a malfunctioning HP and damper were the cause of the elevated $\mathrm{CO}_{2}$ levels in classrooms 502 and 508. The HP was replaced, and the damper was fixed. The consultant also noted that an outside wall in classroom 400 was damaged by water from an exterior leak during construction, and this was to be removed by a mold remediation firm.

\section{October 2005}

In October 2005, the North Carolina Division of Occupational Safety and Health closed their case at the school on the grounds that the alleged hazardous conditions had been corrected or no longer existed. However, during October 2005, the county health department notified the school district that the health department continued to receive complaints from parents regarding indoor environmental quality issues such as mold and RH at the elementary school. There were also concerns about the water supply quality and radon levels at the school, which were later found to be within normal ranges.

\section{November 2005}

In November 2005, $\mathrm{CO}_{2}$ levels continued to be elevated in some classrooms, including classroom 503 (1534 ppm fully occupied), classroom 505 (1484 ppm fully occupied), classroom 623 (1250 ppm fully occupied), and classroom 625 (1370 ppm fully occupied). 
Return air dampers were installed in some classrooms to increase outdoor air supply to these rooms. However, during this same month, the water boiler and several HPs had "tripped out" for unknown reasons. The HP fans may have been off during $\mathrm{CO}_{2}$ testing, and this may have resulted in the elevated $\mathrm{CO}_{2}$ levels. In a further attempt to improve ventilation, the $\mathrm{CO}_{2}$ setpoints for the two HPs serving the four classrooms with elevated $\mathrm{CO}_{2}$ levels (HP-28 for classrooms 623 and 625 and HP-41 for classrooms 503 and 505) were reduced to $900 \mathrm{ppm}$. However, the school continued to have problems with elevated $\mathrm{CO}_{2}$ levels as well as high $\mathrm{RH}$.

\section{February 2006}

In February 2006, mold was identified from surface samples taken from the top of a dusty mobile cart and from a small canvas bag that sat on a dusty bookcase in the Testing Room (room 115). The canvas bag was discarded. The industrial hygiene consultant recommended that the surfaces in this room be professionally cleaned, which was done.

\section{May-August 2006}

In May 2006, many teachers reported "stuffy" rooms and left their windows open for end-of-grade testing to help prevent symptoms in their students during the testing. In early June 2006, the architect notified the construction company that the $\mathrm{CO}_{2}$ controllers did not meet the specifications in the contract documents. Additionally, he notified them of a broken storm drainage line and problems with sewage lines (also identified in a February 2006 inspection).

In mid-June 2006, a Trane Tracer ${ }^{\mathrm{TM}}$ MP581 programmable controller with temperature, $\mathrm{CO}_{2}$, and humidity sensors was installed at the school. A two-day test run was done with the new unit controlling classroom 618 (HP-26) in the k/1 wing. This was compared to classroom 627 (HP-25) across the hall, which did not have the new sequence installed. During the first day of testing, it was rainy with an outside temperature of 65 degrees Fahrenheit $\left({ }^{\circ} \mathrm{F}\right)$ and an RH of $90 \%$; during the second day, it was sunny with an outside temperature and $\mathrm{RH}$ ranging from $73^{\circ} \mathrm{F}-80^{\circ} \mathrm{F}$ and $32 \%-49 \%$, respectively. The Trane $\operatorname{Tracer}^{\mathrm{TM}}$ programmable unit sequence of operation called for the unit to be placed in dehumidification mode when the zone humidity reached $65 \%$ RH and off when the zone humidity reached 55\% RH. However, during the two days, the compressor did not turn off due to the zone humidity levels ranging between approximately $64 \%$ and $70 \%$ and never reaching 
the $55 \%$ level. The temperature stayed between approximately $71^{\circ} \mathrm{F}$ and $73^{\circ} \mathrm{F}$. The Trane consultants determined that since there was no type of reheat available, the only way to keep the room from over-cooling during the dehumidification mode was to switch the unit from cooling to heating. Once this occurred, the air entering the space was no longer dehumidified, and the humidity rapidly rose. However, once the discharge air temperature was low enough to dehumidify the air, the room again became over cooled, and the unit had to be changed back to the heating mode. The Trane consultants had concerns about the wear and tear on the equipment. The reversing valve changed from cooling to heating or from heating to cooling over 25 times during three hours of operation. During normal operation, the reversing valve should only change once or twice in a given day because it is usually heating or cooling, not switching back and forth between the two. The consultants thought that most of the humidity problems were associated with the introduction of outdoor air. They recommended that the outdoor air be pre-treated with dedicated units to allow the existing HPs to control the zone temperature.

With continued oversight by Trane personnel, the test control system was adjusted to better handle humidity with minimal reversing of the unit from cooling to heating and heating to cooling. In July 2006, the temperature and RH in classroom 520, a room with complaints, was monitored for five days. During the day, the temperature ranged from approximately $70^{\circ} \mathrm{F}-77^{\circ} \mathrm{F}$, and $\mathrm{RH}$ ranged from $50 \%-60 \%$; however, during other times, the conditions in the room exceeded $80^{\circ} \mathrm{F}$ and $65 \% \mathrm{RH}$.

On July 22, 2006, several roof leaks occurred after a heavy rain. Water penetrated rooms in the $\mathrm{k} / 1$ wing $(603,606,608,610,616$, 619, corridor between 603 and 608), 2/3 wing (401, 407, 418, 421, 427), 4/5 wing (500, 505), administrative wing (104, 106, 108), and gym/cafeteria wing (201). In August 2006, visible mold was discovered on carpet in classrooms 406, 424, 427, and 623. The indoor $\mathrm{RH}$ was reported to be high during this time. The carpets in these rooms were cleaned and treated with an antimicrobial agent (or biocide). This same month, a leak occurred in a water-line joint, which was subsequently fixed.

\section{February 2007}

In February 2007, the North Carolina Department of Environmental and Natural Resources reported several ceiling leaks during a school 
inspection.

\section{October 2007}

In October 2007, fungal spore concentrations were measured in the air outdoor and inside the school. By spore trap sampling, the outdoor total spore count was 14,302 spores per cubic meter (spores $/ \mathrm{m}^{3}$ ); the top four outside fungal genera identified were Cladosporium, Ascospores, Basidiospores, and Aspergillus/Penicillium. Eleven indoor locations (416, 503, 505, 514, 517, 519, 521, 618, 625, 627 , and the main office) were tested. The five rooms with the highest total spore counts were classroom $521\left(7,680\right.$ spores $\left./ \mathrm{m}^{3}\right)$, the main office $\left(5,055\right.$ spores $\left./ \mathrm{m}^{3}\right)$, classroom $517\left(3,370\right.$ spores $\left./ \mathrm{m}^{3}\right)$, classroom 505 (2,586 spores/m³), and classroom 503 (2,429 spores/ $\left.\mathrm{m}^{3}\right)$. Although the four predominant fungal genera found outside were also found in many of the classrooms, a number of other fungal genera were more predominant in indoor locations. Compared with outdoor air, Alternaria counts were higher in the main office and in classrooms 503, 505, 514, 517, and 521; Curvularia counts were higher in the main office and classrooms 503, 514, 517, and 521; Drechslera/Bipolaris counts were higher in the main office and classrooms 505, 517, and 521; Pithomyces counts were higher in the main office and classroom 521, and Epicoccum counts were higher in classroom 521. Myxomycete/Smut-like was not found outside but was found in the main office and classrooms 503, 505, 514, 517, 521, 625, and 627. Nigrospora was not found outside but was found in the main office and classrooms 517 and 521.

(NOTE: NIOSH indoor air scientists do not currently recommend routine air sampling for mold in indoor environmental quality evaluations because there are no established health-based standards for acceptable levels of mold and limited short-term air sampling may be misleading.)

Later in October 2007, consultants tested for airborne $\mathrm{CO}_{2}$ levels and analyzed air, surface, and carpet samples for fungal materials. $\mathrm{CO}_{2}$ levels were above the level recommended by the American Society of Heating, Refrigeration and Air-Conditioning Engineers (ASHRAE) in 10 (416, 503, 514, 517, 519, 520, 521, 618, 625, 627) of 13 rooms tested. Three (classrooms 416, 618, 625) of the 10 rooms with elevated $\mathrm{CO}_{2}$ had levels that exceeded 1,500 ppm. Spore trap sampling inside and outside the school revealed outdoor total counts of 2,083 spores $/ \mathrm{m}^{3}$ (outside the front of the school) and 2,983 spores $/ \mathrm{m}^{3}$ (outside classroom 405) and indoor total spore 
counts ranging from 66 to 359 spores $/ \mathrm{m}^{3}$ (classrooms 405, 416, $503,514,517,519,521,608,625$, and office lobby). The five rooms with the highest total spore counts were classroom 503 (359 spores/ $\left.\mathrm{m}^{3}\right)$, classroom $517\left(346 \mathrm{spores} / \mathrm{m}^{3}\right)$, classroom 521 (293 spores $\left./ \mathrm{m}^{3}\right)$, classroom $625\left(213\right.$ spores $\left./ \mathrm{m}^{3}\right)$, and classroom $608\left(212\right.$ spores $\left./ \mathrm{m}^{3}\right)$. The top four fungal genera identified both outside and indoors were Basidiospores, Cladosporium, Penicillium/Aspergillus, and Ascospores.

Surface samples were collected from insulation in the supply air plenums of nine air handling units. Six of the nine samples grew Cladosporium. Samples from three of these air handlers (air handlers 37, 41, and 49) had trace to minimal fungal growth; one (air handler 26) had low fungal growth, one (air handler 12) had moderate fungal growth, and one (air handler 14) had high fungal growth. The consultants recommended that the insulation inside these six air handlers be cleaned or replaced. In December 2007, a cleaning company cleaned air handlers 12, 14, 26, and 49.

Carpet dust samples from classrooms 423, 517, 519, 521, and the office lobby showed viable fungal spore concentrations ranging from $132,600 \mathrm{cfu} / \mathrm{g}$ in classroom 517 to $1,176,000 \mathrm{cfu} / \mathrm{g}$ in classroom 423. The consultants recommended (1) cleaning carpets with a commercial-grade high efficiency particulate air (HEPA) vacuum cleaner; (2) use of mats to capture particulate at the outdoor entryway to each affected room or area; and (3) possibly routing students returning from the outside through the main entrance to the school. The school district subsequently reported (1) delivery of three new HEPA-filtered back-pack vacuum cleaners and two new HEPA-filtered upright vacuum cleaners; (2) securing doorway mats; and (3) addressing student reentry into the school through the main entrance.

\section{January 2008}

In January 2008, an inspector from the North Carolina Department of Environmental and Natural Resources reported a bad leak in the central receiving room ceiling during a school inspection.

\section{September 2008}

In September 2008, consultants measured temperature, humidity, and $\mathrm{CO}_{2}$ and sampled carpet dust for fungal spores and indoor air for total volatile organic compound (VOC). Indoor temperatures (range: $71.3^{\circ} \mathrm{F}-72.4^{\circ} \mathrm{F}$ ) and $\mathrm{RH}$ (range: $48.9 \%-53.0 \%$ ) were within the ASHRAE recommended levels. $\mathrm{CO}_{2}$ measurements 
(range: 657-1,189 ppm) were elevated in classroom $514(1,189$ ppm) and classroom 519 (1,086 ppm). The consultants reported that the air handling unit serving classroom 519 might not have been functioning properly at the time of testing. Additionally, the consultant noted the window in classroom 521 (651 ppm) may have been open during testing in that room. These rooms were retested, and the $\mathrm{CO}_{2}$ levels in classroom $519(954 \mathrm{ppm})$ and classroom $521(898 \mathrm{ppm})$ were below the ASHRAE recommended limit. Classroom 514 had an elevated $\mathrm{CO}_{2}$ measurement (1,158 $\mathrm{ppm})$. The school district reported making adjustments to the air handler for room 514 to decrease the $\mathrm{CO}_{2}$ level. In classroom 407, carpet sampling for fungal spores revealed $1,300,000 \mathrm{cfu} / \mathrm{g}$; the top three fungal genera identified were Alternaria, Aspergillus, and Cladosporium. Fungal spore trap samples of air were analyzed from two locations outside of the school building (total counts of 10,736 spores $/ \mathrm{m}^{3}$ and 6,896 spores $/ \mathrm{m}^{3}$ ) and 11 indoor locations - an office and classrooms 405, 407, 414, 501, 514, 519, 520, 521, 618, and 627 (total count range: $<78-392$ spores $/ \mathrm{m}^{3}$ ). The three rooms with the highest total spore counts were classroom $514\left(392 \mathrm{spores} / \mathrm{m}^{3}\right)$, classroom $618\left(313\right.$ spores $\left./ \mathrm{m}^{3}\right)$, and classroom 521 (with window open) (313 spores $\left./ \mathrm{m}^{3}\right)$. All three total VOCs were below $0.5 \mathrm{mg} /$ $\mathrm{m}^{3}$. The specific locations of VOC sampling were not mentioned in the report.

\section{October 2008}

In October 2008, carpet in the main lobby, the secretary/treasurer's office, and classrooms 407, 501, 503 was cleaned and treated with Sporicidin ${ }^{\circledR}$. HVAC units 12 (front office), 48 (classroom 501), and 50 (classroom 407) were cleaned by contact vacuuming with a HEPA vacuum. Fan blowers and coils were HEPA-vacuumed, and the HVACs were sanitized with Sporicidin ${ }^{\circledR}$. The rooms were wiped down and HEPA-vacuumed following the cleaning process.

\section{December 2008}

In December 2008, an off-white substance was observed coming from the ductwork in the main office area and in classroom 618 after some air handling units had been cleaned. There was concern that the white substance was from the cleaning agent used or possibly the mineral content in the water. An industrial hygiene consultant observed an off-white powdery substance in three air handler units inspected and informed a school official. The school official contacted the cleaning contractor and was told that no white substances were used in the cleaning of the coils. The coil 
cleaner contained butyl cellosolve (ethylene glycol monobutyl ether or 2-butoxyethanol), a type of glycol ether, which is water soluble and often used as a solvent. An industrial hygiene consultant sampled the air for total particulates, butyl cellosolve, and fungal materials and sampled surfaces for fungal materials in the office area and room 618. In the three areas (office lobby and rooms 113 and 618) where the white substance was observed coming out of the ductwork, total particulate concentrations (range: $0.20-0.53 \mathrm{mg} /$ $\mathrm{m}^{3}$ ) did not differ substantially from total particulate concentration in the break room $\left(0.34 \mathrm{mg} / \mathrm{m}^{3}\right)$, which had not had coil cleaning. All three air sampling results (office lobby and rooms 113 and 618) for butyl cellosolve were below the detection limit $<0.72$ ppm) for the analytical method; thus, they were below the NIOSH recommended exposure limit (5 ppm) and the Occupational Safety and Health Administration permissible exposure limit (50 ppm) at the time of testing. Two outdoor fungal spore trap samples revealed total counts of 1,746 spores $/ \mathrm{m}^{3}$ and 653 spores $/ \mathrm{m}^{3}$ compared to total spore counts of 53 structures $/ \mathrm{m}^{3}$ in room 618,93 spores $/ \mathrm{m}^{3}$ in the office lobby, and 653 spores $/ \mathrm{m}^{3}$ in room 113. The laboratory detected an Aspergillus conidiophore in the air sample taken in room 113. The consultants conducted surface sampling on horizontal surfaces in three locations (office conference room table, lobby login book, and plastic lid in classroom 618) where the white substance was observed. Very few miscellaneous spores were present; no mold grew from these samples.

\section{April 2009}

In early April 2009, an industrial hygiene consultant from the Occupational and Environmental Epidemiology Branch of the North Carolina Department of Health and Human Services and a building systems engineer for the public schools of North Carolina visited the school to evaluate the indoor environment. During the site visit, the industrial hygiene consultant reported a moldy odor in the northwest corner of the assistant principal's office (room 108) where carpet had been pulled up. He noted that the northwest wall was common to the new and old buildings. He also noted a history of recurrent water damage to the ceiling tiles above the ramp connecting the new and old buildings. Additionally, there was evidence of termites swarming in that area. In classroom 520 in the $4 / 5$ wing, he reported a musty, earthy odor or an odor similar to citrus-based cleaning products when a carpet square was lifted. He also reported a microbial odor outside the bathrooms in the 4/5 hall and a history of roof leaks in the music room (room 407) and 
the Spanish room (room 501), which shared a common wall located where a north-south roof intersected an east-west roof. In classroom 427 , he reported a history of recurring problems with mold growth in the carpet. He recommended the school district:

1. remove the carpet (using proper procedures) and replace with vinyl composition tile (after measurement of concrete floor moisture content) in rooms 108, 520, and the music room;

2. identify and correct the sources of water movement through the exterior wall in room 108;

3. repair flashing on the parapet where the old and new building were joined;

4. investigate the source of roof leaks above the music and Spanish room;

5. encourage students to use the main doors to enter and exit the building to reduce the amount of soils, dust, debris and allergens brought into the building;

6. install barrier mats at each entrance long enough that a person would take at least five full steps on the mat;

7. vacuum carpet daily;

8. identify and correct odor sources at the bathroom in the $4 / 5$ hall;

9. investigate sources of moisture under the concrete slab;

10. integrate pest management techniques to control the termite infestation where the new and old buildings meet;

11. analyze absentee data or other data to look for patterns where problems may exist.

During the same site visit, the building systems engineer for the public schools of North Carolina reported ceiling tile stains throughout the school, especially in the area where the old and new buildings were joined. He noted a tendency for maintenance staff to replace stained ceiling tiles without first repairing the leaks. He recommended fixing leaks before replacing the ceiling tiles. He noted that foam roof sections in the older section of the school were showing signs of deterioration and would need to be replaced, especially if the number of leaks increased. He noted that flashing in some areas over the media center did not cover enough of the wall to prevent rain penetration, and that some exterior bricks on the wall had deteriorated, which may have contributed to the 
leaks into the assistant principal's office. He recommended that the metal cap flashing on the north side be extended down to cover gaps between the existing flashing and the brick wall. The engineer also reported that surface water drainage toward the building kept the slab wet. To help keep surface water away from the building slab, he recommended installing a French drain between the school and the higher ground of the playground area to drain groundwater toward the streets. He recommended construction of swales around the building on the playground side and improvement of drainage around the courtyard surface areas to better drain water away from the buildings. He noted that some of the existing drop inlets might need to be lowered. He reported $\mathrm{RH}$ variations indicating moisture-related issues in the classrooms closest to the playground. He recommended keeping the RH between $30 \%$ and $60 \%$ with installation of room dehumidifiers in classrooms where the HVAC system could not maintain an RH of $60 \%$ or below. (The school district reported that they tested the $\mathrm{RH}$ in the school, and on average, the $\mathrm{RH}$ readings were at or below $60 \%$.) Lastly, the engineer reported a significant amount of visible mold under the carpet in the assistant principal's office. He noted that several classrooms had odors which were masked by carpet cleaning compounds. He recommended removal of carpet and replacement with floor tile in carpeted rooms where odors and/or excessive moisture were detected.

Later in April 2009, another industrial hygiene consultant from Workplace Group monitored temperature, humidity, and $\mathrm{CO}_{2}$ in classrooms 516 and 520. He also sampled adhesive from beneath the carpet in classroom 501 and the underside of carpet in classroom 520 and the assistant principal's office for fungal materials. The adhesive beneath the carpet in classroom 501 was negative for fungal growth or spores. In the assistant principal's office and classroom 520, Aspergillus and Penicillium were detected on the carpet backing. Mold growth, scored from 1 to 4 with 4 denoting the highest concentration, was scored 3 in classroom 520 and 4 in the assistant principal's office. In classroom 516, the temperature ranged from $66.7^{\circ} \mathrm{F}-75.1^{\circ} \mathrm{F}$ (average $70.8^{\circ} \mathrm{F}$, median $70.2^{\circ} \mathrm{F}$ ), the RH ranged from $22.7 \%-30.9 \%$ (average: $25.9 \%$, median: $26.1 \%$ ), and $\mathrm{CO}_{2}$ from $350-750 \mathrm{ppm}$ (average: $500 \mathrm{ppm}$, median: $460 \mathrm{ppm}$ ). In room 520 , the temperature ranged from $67.4^{\circ} \mathrm{F}-75.3^{\circ} \mathrm{F}$ (average: $73.0^{\circ} \mathrm{F}$, median: $73.5^{\circ} \mathrm{F}$ ), the $\mathrm{RH}$ from $20.6 \%-51.0 \%$ (average: $33.3 \%$, median $33.0 \%$ ), and $\mathrm{CO}_{2}$ from $330-1710 \mathrm{ppm}$ (average: 700 ppm, median: $540 \mathrm{ppm}$ ), exceeding the recommended limit of 1100 ppm at one point during testing. 
In mid-April 2009, floor consultants performed moisture testing of interior floor slabs in the assistant principal's office and classrooms $427,516,518,520$, and 618 . In addition, a consultant engineer reviewed floor and wall conditions. In his report, he noted that interior floor slabs were being tested for moisture by a flooring company, and the surface preparation was suspect, as evidenced by adhesive residue and curing compounds under the testing chamber. He mentioned that the school was reported to have had high levels of humidity for an extended period of time when the new addition was opened in 2005, and adjustments had been made to the HVAC system. Monitors for temperature and humidity were removed, and relative humidity was no longer monitored on the interior of the school. Surface water draining from the elevated ball fields was ponding adjacent to concrete walkways instead of being directed across the gravel roadway and away from the school. He also noted significant roof leaks on the flat roof over the utility/storage room at the southeast corner of the school just west of the gym. He reported over 18 ceiling tiles were damaged. In the main hallway where the old and the new buildings interface, he reported damaged ceiling tiles from water intrusion which appeared to be from a roof leak.

In mid-April, the carpet in the assistant principal's office was replaced with vinyl composition tile.

\section{May 2009}

During the second week in May 2009, a senior environmental health specialist from the county health department visited the site and noted a water-stained ceiling tile in room 619. She noted in her report that a previous site visit by state and local agencies on April 3, 2009 outlined a variety of issues and recommendations.

Also during the second week of May 2009, an industrial hygiene consultant from Workplace Hygiene sampled carpet, surface, and air for fungal materials (in rooms 115, 206, 420, 421, 422, 423, $425,427,500,503,514,515,516,610,616,618,627)$ and air for VOC. The indoor fungal air sampling results ranged from no spores detected in classroom 627 to 940 spores $/ \mathrm{m}^{3}$ in classroom 420. The six rooms with the highest total spores were classroom $420\left(940\right.$ spores $\left./ \mathrm{m}^{3}\right)$, classroom $206\left(705\right.$ spores $\left./ \mathrm{m}^{3}\right)$, classroom $427\left(549\right.$ spores $\left./ \mathrm{m}^{3}\right)$, classroom $514\left(549\right.$ spores $\left./ \mathrm{m}^{3}\right)$, classroom 115 $\left(470\right.$ spores $\left./ \mathrm{m}^{3}\right)$, and classroom $425\left(470\right.$ spores $\left./ \mathrm{m}^{3}\right)$. The outdoor samples (outside the $2 / 3$ and $\mathrm{k} / 1$ wings) ranged from 18,103 to 28,134 spores $/ \mathrm{m}^{3}$; the top three outside fungi species identified were 
Ascospores, Basidiospores, and Cladosporium. The outdoor counts were higher than the indoor counts except for Myxomycete/Smutlike, which was higher in room 420 than outside. Surfaces beneath the carpeting were sampled in the same 17 rooms, in a paper tray in room 115, and on a water-damaged wall in room 500 . Because the carpet was glued to the floor, only one location was selected from each room. With the exception of the $\mathrm{k} / 1$ wing (where carpet did not extend to exterior doors) and room 206 (which had no exterior door), the samples were collected near the exterior doors of each classroom because the consultants thought this area would more likely have excessive moisture. Spores were not detected on the carpet backing in rooms $425,503,515$, and 616 . With the exception of room 421, spore concentrations were characterized as "trace to light." The carpet backing in room 421 had a surface concentration of mold spores identified as Aspergillus/Penicillium characterized as "loaded" and concentrations of Basidiospores and Cladosporium characterized as "trace" to "light."

Carpet sampling results for mold ranged from $30,000 \mathrm{cfu} / \mathrm{g}$ in room 115 to $>2,000,000 \mathrm{cfu} / \mathrm{g}$ in classroom 516. Six of the 16 results exceeded 1,000,000 cfu/g (room 206, > 1,333,333; classroom 514, > 1,600,000; classroom 515, > 1,600,000; classroom 516, > 2,000,000; classroom 618, > 1,000,000; classroom 627, 1,460,000). Total VOC results ranged from 0.18 milligrams per cubic meter $\left(\mathrm{mg} / \mathrm{m}^{3}\right)$ for a sample collected outdoors to $1.6 \mathrm{mg} / \mathrm{m}^{3}$ in classroom 425 . The consultants noted that the Green Building Council established a recommended limit for VOCs of $0.5 \mathrm{mg} / \mathrm{m}^{3}$ for new buildings, and that Washington State established a limit of $0.5 \mathrm{mg} / \mathrm{m}^{3}$ in buildings. Six (rooms 420, 423, 425, 500, 501, 514) of the 20 areas sampled exceeded the $0.5 \mathrm{mg} / \mathrm{m}^{3}$ limit. The consultant reported cleaning activities were going on during the sampling, and this may have affected the results. The cleaning products used were not listed. The industrial hygiene consultant recommended the school district (1) test for aldehydes to determine whether their presence contributed to reports of bloody noses; (2) test (by smoke or pressure) the drain lines to check for leaks; (3) inspect and (if necessary) clean the air handlers, and replace any contaminated insulation found; and (4) forward the consultant's report to the county health department.

Later in May 2009, an engineer consultant assessed the school and found "slight humidity in the administration office", but thought the air quality in the school seemed acceptable. He evaluated the mechanical systems in the $2 / 3$ and $4 / 5$ wings and thought the system was well designed. However, in an outdoor air unit, 
he found standing water in the condensate drain pan and noted the filters for these units needed to be replaced. The engineer relieved the pressure on the unit and drained the pan completely. He noted that the units were designed to be free of standing water and recommended that drainage of these units be evaluated to determine the problem. Another contractor checked the drain pans and found that the drain piping did not appear to allow complete drainage in the drain pan. However, he did not note carryover of moisture to the fan compartment or ductwork and did not see any dirt or other problems that would cause a mold issue in the unit or drain pan. The two consultants agreed that an adjustment in the trays should solve the problem. A school staff member was reported to have immediately started the adjustment.

The engineer consultant also found that $\mathrm{CO}_{2}$ sensors in the ventilation system had been electrically jumped and thereby disabled, and that the outdoor air units had been set at 100\% fresh air to get as much air into the building as possible. However, outdoor air did not appear to be getting into the building. $\mathrm{CO}_{2}$ concentrations inside the building were around $500 \mathrm{ppm}$ when the building was unoccupied for three days. Additionally, the building was noted to be in a neutral pressure condition when it should have been in a positive pressure condition. The consultant noted that the HVAC system serving the school was designed properly but was not operating correctly. He recommended the system be recommissioned and the outdoor air system be checked to see if it was sized correctly. A third consultant noted that the $\mathrm{CO}_{2}$ sensors were part of a previous attempt to control fresh air intake and were not intended to be part of the current ventilation control. However, when the system was most recently balanced, the dampers were bypassed in the open position to allow the scheduled fresh air to be delivered from the new air handling unit to the classroom units to ensure the right amount of air reached each unit. The prior summer, when the new system was installed, fans and dampers were set up and a balance contractor verified flow to each unit and all were apparently $95 \%$ or better for scheduled air supply. The school planned to have the ventilation air calculations reviewed to determine if any further re-commissioning was necessary. The engineer consultant found a lot of construction dust in the return ductwork and recommended the duct system be cleaned. He noted the filters were being maintained and the ductwork on the other side of the filter was clean.

During May 2009, the carpet in classrooms 407, 501, 508, 518, 
and 625 was replaced with vinyl composition tile. During the last weekend in May 2009, a carpet cleaning company cleaned all the remaining carpet in the school with an antimicrobial agent.

During the last week in May 2009, the county health department distributed questionnaires for staff and parents at the elementary school.

\section{June 2009}

In mid-June 2009, the medical director and others from the county health department toured the school. In a media briefing on the following day (June 16, 2009), he reported that when they toured the school a month earlier they could find no mold or dampness; however, during the June tour, they did observe moisture in a wall and a small patch of mildew behind a banner in the gymnasium. The county health department reported in a document dated June 25, 2009 that approximately 800 questionnaire surveys were distributed and about half were returned within the one-week deadline (Appendix D). The document stated that the summary of the responses showed that the "driving" symptoms were headache and fatigue that occurred within the school and resolved when fresh air was obtained by opening windows and doors to the classrooms, by going outside, or by going home. The document noted that other symptoms, particularly nosebleeds, were also reported, though without a clear pattern of occurrence to suggest they were buildingrelated.

\section{July 2009}

In mid-July 2009, NIOSH investigators and Turner Group consultants visited the elementary school on three consecutive days.

During this visit, we noted a strong musty, moldy odor in the basement (Figure 5) and crawl space under the media center. Lime was observed on the crawl space dirt floor. We also noted a musty smell in the corridor around the media center, particularly near the school museum. Surface water from playgrounds drained towards the classroom wings; it appeared water could puddle near concrete walkways adjacent to classroom wings, especially near the $2 / 3$ and $4 / 5$ wings (Figures 6,7 , and 8 ). A storm drain outside the media center was blocked, and there was standing water in it (Figure 9). There were multiple locations on the foam roof surface where the roof membrane had been compromised; there were signs of softness and bubbling of the membrane (Figures 10 and 11). There did not 
appear to be adequate flashing at the junction of the parapet wall (above room 206) and the gym (Figure 12). Room 206 had stained carpet, wavy baseboards, and walls which appeared to have been newly painted. Inside the school, we noted excessive moisture (with an infrared camera) in the gym's exterior concrete block wall where a leak had been repaired. We also noted that multiple classrooms were under negative pressure instead of positive pressure relative to the corridor. Multiple restrooms were also under positive pressure instead of negative pressure. During the day, the plenum above the classroom ceilings was generally under positive pressure in relation to the exterior of the building. However at night, when the dehumidifier (which served the classroom wings) was turned off, this sometimes resulted in the plenum being under negative pressure in relation to the outside of the building. This allowed outside humid air to enter the plenum through the soffits in the classroom wings. From the mezzanine area, where the dehumidifier was located, we observed dust on classroom ceilings and ducts (Figures 13 and 14). Some of the coils for the HVAC air handlers had residue that may have been mold contamination (Figure 15).

Water activity on floor materials from the 23 areas (21 rooms) sampled ranged from 0.52 to 0.81 (median $=0.69$ ) on the date of investigation. Average water activity on the floor in the administration wing $(0.76)$ and media center wing $(0.73)$ were above the median, while average water activity in the $\mathrm{k} / 1$ wing was lowest (0.57) (Table 2). RH in the building ranged from 34\% to $69 \%$. Average RH was higher in the administrative wing (59\%) than the other wings of the building and lowest in $\mathrm{k} / 1$ wing $(37 \%)$, and 2/3 wing $(39 \%)$. Air temperature did not show a wide range within the building (range $\left.=70.5^{\circ} \mathrm{F}-74 \cdot 1^{\circ} \mathrm{F}\right)$.

From the 22 floor dust samples, we recovered 20 fungal species, yeasts, and unidentifiable non-sporulating fungi (Figure 16). Identified hydrophilic (water-loving) fungi from the dust samples included Alternaria alternata, Epicoccum nigrum, Trichoderma konigii, Aspergillus fumigatus, Mucor plumbeus, Rhizopus stolonifer, and yeasts. Identified mesophilic fungi included Cladosporium cladosporioides, Aureobasidium pullulans, Fusarium oxysporum, Aspergillus ochraceus, Aspergillus niger, Aspergillus versicolor, Paecilomyces varioti, Penicillium aurantiogriseum. Among the species recovered, Cladosporium cladosporioides occurred most frequently (recovered from all 22 samples). Eighty-six percent of the samples contained Epicoccum nigrum and 64\% contained Alternaria alternate, both hydrophilic fungi. Bipolaris australiensis, which was not classified as either 
hydrophilic or mesophilic, was recovered from $64 \%$ of samples.

The overall geometric mean (GM) concentration of total culturable fungi measured in the dust samples was $6,000 \mathrm{cfu} / \mathrm{g}$ dust (Table 3). Overall sample GM concentration of culturable hydrophilic fungi was $1,200 \mathrm{cfu} / \mathrm{g}$. Analysis of the fractions of hydrophilic and mesophilic fungi showed that, $83 \%$ of the total culturable fungi were either mesophilic (61\%) or hydrophilic (22\%). Among fungi found in more than $40 \%$ of samples, Cladosporium cladosporioides $(\mathrm{GM}=2,852 \mathrm{cfu} / \mathrm{g})$, Epicoccum nigrum $(\mathrm{GM}=1,130 \mathrm{cfu} / \mathrm{g})$, and Aureobasidium pullulans $(\mathrm{GM}=733 \mathrm{cfu} / \mathrm{g}$ ) were recovered at higher concentrations than others. The overall GM concentration of Gram-positive bacteria measured in the building was 396,000 cfu/g where as the concentration of Gram-negative bacteria was 8,500 $\mathrm{cfu} / \mathrm{g}$. None of the 22 samples grew Actinomycetes (fungus-like bacteria).

Levels of total fungi, Gram-positive, and Gram-negative bacteria by building wing are summarized in Table 4 . The gym/cafeteria wing $(\mathrm{N}=1 ; 24,000 \mathrm{cfu} / \mathrm{g})$ and the $2 / 3$ wing $(\mathrm{N}=4 ; 23,500$ $\mathrm{cfu} / \mathrm{g}$ ) had the highest level of total culturable fungi in floor dust, approximately eight-times higher than those of the media center $(\mathrm{N}$ $=3 ; 2,900 \mathrm{cfu} / \mathrm{g})$ and the $4 / 5$ wing $(\mathrm{N}=6 ; 2,900 \mathrm{cfu} / \mathrm{g})$. Similarly, concentrations of hydrophilic fungi in dust were several times higher in the gym/cafeteria and $2 / 3$ wing than in other areas. The proportions of total fungi represented by hydrophilic fungi were similar among wings and ranged between 20\% (administration wing) and 29\% ( $\mathrm{k} / 1$ wing). Except for the $\mathrm{k} / 1$ wing, the pattern of Gram-negative bacteria concentrations by building wing was similar to those observed for culturable fungi. The level of Gram-negative bacteria was more than an order of magnitude higher in the $2 / 3$ wing $(52,000 \mathrm{cfu} / \mathrm{g})$ than in the media center $(2,500 \mathrm{cfu} / \mathrm{g})$ and the $4 / 5$ wing $(3,900 \mathrm{cfu} / \mathrm{g})$. The levels of Gram-positive bacteria were highest in the $\mathrm{k} / 1$ wing $(855,600 \mathrm{cfu} / \mathrm{g})$, followed by the $2 / 3$ wing $(660,100 \mathrm{cfu} / \mathrm{g})$, and the media center $(631,600 \mathrm{cfu} / \mathrm{g})$.

We identified 10 rooms where measurements were made by both NIOSH investigators and previous consultants: two rooms in the administration area, one in the gym area, two in the $2 / 3$ wing, three in the 4/5 wing, and two in the $\mathrm{k} / 1$ wing. Eight of the 10 rooms had measurements in May 2009 and July 2009 (Table 5). Room 100 (lobby in the administration area) had measurements in October 2007 and July 2009 (Table 6). Room 407 had measurements in September and October 2008 and July 2009 (Table 6). Based on 
the comparisons, we found that the fungal levels measured during the 2009 NIOSH investigation were 4 to 1,000-fold lower than those measured previously by consultants in all rooms except for room 115 in the administration wing, which showed similar levels at the two sampling times. Measurements from three rooms in the 4/5 wing showed dramatically decreased (90 to 1000-fold lower) fungal levels in the NIOSH investigation compared to those measured about two months before (May 2009).

On the last day of the July 2009 site visit, we communicated preliminary findings with the concurrence of the Turner Group at a breakfast meeting with officials from the school district. The following week, we e-mailed school district officials a brief summary of the findings discussed during the meeting (Appendix E). Additionally in July 2009, we notified school district officials via teleconference that some elementary school employees interviewed over the telephone reported building-related symptoms suggestive of hypersensitivity pneumonitis. We also communicated that there appeared to be a high prevalence of respiratory symptoms at the school based on the summary of the county health department questionnaire survey results. (Appendices F and G).

The Turner Group summarized their preliminary findings in a document dated July 21, 2009 (Appendix H). Their findings and recommendations included:

1. Finding: dirt-floor crawl space under the media center is a possible source of mold. Recommendation: install flexible vapor barrier and depressurize air space under the vapor barrier in the crawl space under the media center.

2. Finding: inadequate flashing at junction between the parapet wall and gym wall above room 206 causing water intrusion leading to mold growth in the carpet. Recommendation: install through-wall-flashing at edge of parapet.

3. Finding: discolored drywall on inside of exterior wall in classroom 400, at the site of an earlier leak that led to occupant reports of mold under the cove base. Recommendation: repair roof junction joints to provide adequate flashing.

4. Finding: dehumidification system operation suspect, evidenced by high humidity $(70 \%+)$ in the $4 / 5$ wing. Recommendation: provide retro-commissioning of HVAC system to verify proper system capacity and sequence of 
operation.

5. Finding: possible HP refrigerant leaks indicated by $64^{\circ} \mathrm{F}$ discharge at HP serving classroom 520 and humidity levels monitored at $70 \%-80 \%$ in room. Alternatively, quantity of outdoor air being handled exceeded coil capacity. Recommendation: provide retro-commissioning of HVAC system to verify proper system capacity and sequence of operation;

6. Finding: classroom wings not sealed at soffit, allowing excess air leakage in and out (depending on mechanical system operation). Recommendation: seal soffit to prevent air infiltration and ensure means for exhaust/relief.

7. Finding: HP design conducive to growing mold due to proximity of filter and cooling coil. Recommendation: provide new filter boxes upstream to create separation between filter and coil.

8. Finding: concerns about odors from drains, particularly HP drains, when the traps are allowed to dry out.

Recommendation: review maintenance tasks and schedules; on-going maintenance to assure that drain traps do not dry out.

9. Finding: bathrooms not consistently at negative pressure relative to hall. Recommendation: balance air flows as part of retro-commissioning process, possibly increase supply air to hall;

10. Finding: mechanical room in office area under significant negative pressure. Recommendation: seal duct joints on all return air and possibly supply air and verify air flows on retro-commissioning.

11. Finding: unknown issues regarding storm runoff at the end of the $2 / 3$ and $4 / 5$ wings with possibility of water getting under slab if it pools against the exterior walls. Recommendation: verify adequate site drainage, clean site drainage system as necessary, and provide means to limit runoff of sand and other surface material.

12. Finding: original design of classroom wings modulated between 3.5 cubic feet per minute per person and 7.5 cubic feet per minute per person from $1000 \mathrm{ppm} \mathrm{CO}_{2}$ to 1800 ppm; when dehumidification systems installed, $\mathrm{CO}_{2}$ control eliminated and all areas receive 7.5 cubic feet per minute per person. Recommendation: balance outdoor air supply and 
mark dampers as part of retro-commissioning project.

13. Finding: numerous open bubbles on the foam roof with compromise of the aluminum paint. Recommendation: obtain estimate to repair/replace the foam roof.

14. Finding: possible leak from sewer vent and/or roof flashing of vent through roof in classroom 500. Recommendation: replace or repair flashing.

15. Finding: building not observed during the occupied mode and some operational conditions were not observed. Recommendation: provide monitoring of conditions during occupancy to evaluate operation under occupancy (after retro-commissioning of systems).

\section{August 2009}

In August 2009, the elementary school remained closed for remediation after the summer break. Students attended three alternate sites. Kindergartners and first-graders attended another elementary school; second and third graders attended a nearby military academy; and fourth and fifth graders attended a middle school.

\section{October 2009}

In October 2009, NIOSH sent an e-mail to parents who requested a summary of our findings from the site visit (Appendix I).

\section{November 2009}

In November 2009, EMC Engineers, Inc. was contracted by the school district to retro-commission the HVAC systems at the school (Appendix J). Regarding the water-source HPs, EMC personnel encountered the following issues:

1. The building automation system (BAS) was not scheduled to vary any building equipment function based on building occupancy; there was 24/7/365 operation. EMC personnel decided to leave it as found until the new schedule was implemented. The new recommended schedule was (a) office and media center areas, occupied $5 \mathrm{am}-10 \mathrm{pm}$; (b) classroom wings and gym/cafeteria, occupied $6 \mathrm{am}-10 \mathrm{pm}$; and (c) data closets, occupied continuously.

2. Setpoint control was found to be from the BAS for all HPs.

3. A significant number of HP discharge air temperature 
sensors were found to be out of range. EMC personnel reported that this point was not critical to unit operation but should read accurately to correctly convey HP operation.

4. Various graphics errors were found on the Trane workstation in the boiler room.

5. Low air flows were observed in several HPs.

6. All space temperature setpoints for HPs were independently controlled in the BAS and did not follow any unified area control. EMC and Turner Group personnel discussed this, and a determination was made that global setpoints should be applied to the HPs, with local temperature sensor control.

7. HP-11 (which serves offices in the physical education area and a small corridor) was found to be incapable of supplying the designed amount of air to the offices it served. To bring values to within $10 \%$ of design values, SCI personnel installed larger supply diffusers and Phoenix T\&B personnel proportioned the supply.

8. Sheaves for the fans of HP-48 and HP-55 (which serve areas in the $2 / 3$ wing) were found to be adjusted incorrectly, and setscrews were damaged. Sheaves were replaced; Phoenix $T \& B$ personnel adjusted them to the correct settings, which were then verified by EMC personnel.

9. HP-43 (serving areas in the $4 / 5$ wing) was not accessible on the $4 / 5$ wing graphic. This was corrected by SCI personnel.

10. All HPs were set up as members of a variable air volume system in the Tracer Summit ${ }^{\mathrm{TM}}$ BAS, which was incorrect. This was corrected. EMC personnel verified the corrections.

11. A foreign object, measuring approximately 2' $\times 2$ ' was found inside one of the supply ducts in the $4 / 5$ wing. EMC reported that the object, which appeared to be a camping shower, was removed by Phoenix T\&B personnel.

12. EMC personnel found that the majority of HPs had air flows within $+/-10 \%$ of design values. However, a small number of units exhibited air flow slightly beyond this threshold. The outdoor air units were providing air to the associated wings of the building, at volumes consistent with those specified by the designer. Based on the final Test and Balance Report, the units were operating generally within the $+/-10 \%$ of design. EMC personnel recommended: 
1. Setpoints of $70^{\circ} \mathrm{F}$ during the heating season and $74^{\circ} \mathrm{F}$ during the cooling season.

2. That all outside doors and operable windows remain closed at all times to aid in maintaining acceptable indoor conditions.

3. Regular air filter inspections of all units, including the makeup air units, at least every three months per manufacturer's recommendations, with filters changed when warranted by visual inspection.

4. Consistent space temperature setpoints within logical zones of the building (i.e., classroom wings; office and media center areas; gym/cafeteria areas; and data closets) to help reduce the potential for wide temperature fluctuations among each zone's spaces.

5. Use of global setpoint values, referenced in each HP controller, in the Trane BAS.

6. Unoccupied setpoint temperature offsets of $5^{\circ} \mathrm{F}$ from occupied setpoints (i.e., unoccupied cooling setpoints $5^{\circ} \mathrm{F}$ higher than occupied cooling setpoints; unoccupied heating setpoints $5 \circ \mathrm{F}$ lower than occupied heating setpoints).

EMC personnel evaluated the tempered water system and found the following issues, which were corrected:

1. No programming was in place to rotate the building loop pumps upon failure of a pump. SCI personnel corrected this programming.

2. The outside air temperature sensor was not calibrated, and the outside air humidity sensor was incorrectly terminated. SCI personnel corrected both of these issues.

3. All pumps in the main mechanical room were operating in manual mode instead of being automatically controlled from the BAS.

After functional testing on the tempered water system, EMC personnel were confident that the system would be able to maintain the loop water temperature, so that the water source HPs throughout the building would be able to maintain space temperature effectively. $T \& B$ consultants verified the loop water balance.

EMC personnel evaluated the makeup air units and found the following issues, which were corrected:

1. The makeup air units were found to trip when outside air 
temperatures fell below a low temperature limit of $29^{\circ} \mathrm{F}$. Trends were set up in the BAS to capture system parameters at the time of trip.

2. The location of the outdoor air sensor was incorrect, resulting in readings not reflective of actual outdoor air conditions. The sensor was relocated to a proper location on the north wall of the main mechanical room and verified by EMC personnel.

EMC personnel evaluated the fans and systems and found the following issues, which were corrected:

1. Several exhaust fans (ER-1, EF-3, EF-4, EF-5, EF-6, and EF-10) did not respond (start/stop) when the associated HPs were occupied / unoccupied.

2. The ceiling grill in the janitor's closet outside the multipurpose room was not connected to an exhaust duct, which resulted in no exhaust from the space, and likely contributed to the objectionable odors noted in that vicinity.

3. Exhaust fan EF-9 was located above the fire-rated ceiling in the 4/5 wing and was not accessible. Access was cut in beneath the fan.

4. A substantial number of dampers located above fire-rated ceilings were not accessible due to the absence of access doors or panels. This was corrected by cutting in access.

EMC personnel planned to be onsite the first week of May 2010 to confirm proper cooling-season operation of the makeup air units, HPs, and associated systems.

The school district administration continued to consult with the Turner Group to address issues found during the initial site visit in July 2009 and during further testing. Additional findings by the Turner Group (Appendices K and L) included visible mold on some small portions of gypsum board removed at floor level and water-damaged walls in the clerestory area above the media center that were suspected to have supported mold growth in the past. The walls were removed or cleaned. Dried leaves and other dried organic material found trapped in the structure of the media center were removed. Some of the clerestory windows were resealed and existing roof scuppers (i.e., roof drains) were lowered. An old unused ventilation system in the clerestory space was removed, and the ventilation openings sealed because they were defeating 
the building's thermal envelope. Blower-door tests and infrared imaging indicated that the soffits in the classroom wings were a source of many air leaks. Other heat and air leak areas identified on the thermal imaging included doors, windows, and the base of the building wall. Turner Group consultants reviewed photos that showed some plumbing waste line vents in the classroom wings that should have been connected to the waste line roof vents were instead open to the space above the ceilings. This was corrected. Additionally, some of the waste line roof vents were located less than 10 feet from roof-mounted air inlets. They were apparently relocated to meet the minimum 10 feet distance between roof vents and air inlets specified by construction drawings. Slab testing results reported in the Turner Group's final report noted that the concrete flooring in some rooms $(106,110,400,425,502,514,608,610$, $614,616,617,618$, and 627) had more moisture penetration (over 5.0 pounds per 1000 square feet per 24 hours) than other rooms. They also noted that some classrooms $(505,516,608$, and 614) had rusted sill plates, possibly due to water damage. In addition to the recommendations in their preliminary report, the Turner Group also recommended:

1. new floor maintenance systems and schedules because the school no longer had textile floor covers but either hard vinyl or terracotta floor surfaces.

2. limiting noise by fitting feet of desks, chairs, and other objects with felt pads or with rolling castor wheels designed for hard floors.

3. avoiding use of textile floor coverings of any size except track-off mats.

4. using track-off mats at all main entries and avoiding use of classroom fire-exits for routine entry and exit.

5. setting occupied temperature setpoint of $70^{\circ} \mathrm{F}$ heating and $74^{\circ} \mathrm{F}$ cooling in accordance with recommendations made by the commissioning agent.

6. operating the school in occupied and unoccupied cycles.

7. continuing additional monitoring of space conditions throughout the remaining heating season and the upcoming cooling season to verify proper operation of the HVAC system and controls.

8. additional evaluation and possible corrective work to further limit heat loss and air leaks from doors, windows, and base of the walls. 


\section{February 2010}

In February 2010, NIOSH investigators released an interim report (Appendix M), which summarized the environmental problems at the school, our walkthrough observations and environmental sample results, our analysis of the health department questionnaire data, and our recommendations, which had been previously shared with school district officials.

\section{March 2010}

During the NIOSH walkthrough inspection in March 2010, after the school had reopened, we found the school to be clean and organized. We did not observe any dampness or stained ceiling tiles. The carpet in the school building had been replaced with hard floors (Figures 17 and 18). In the corridors, doormats were noted at the exterior doors (Figures 19-22). In the library/media center, an exterior door was being installed (Figure 23).

\section{May 2010}

In May 2010, EMC Engineers, Inc. performed seasonal testing of the HVAC systems (Appendix N). Overall, EMC personnel found the building systems to be operating well within the guidelines and recommendations established in the Turner Group report and the EMC retro-commissioning report. Regarding the water-source HPs, EMC personnel observed the following:

1. The BAS was found to control all HPs on the following schedules: (a) Office and Media Center areas, occupied 5 a.m. - 10 p.m.; (b) classroom wings and gym/cafeteria, occupied 6 a.m. - 10 p.m.; (c) data closets, occupied continuously.

2. Space temperature measurements immediately following transition from unoccupied to occupied operation revealed that the HPs overshot the occupied setpoints by up to 2.5 F. Classroom HPs took anywhere from 30 minutes to 1 hour to resolve the overshoot condition. When overshoot was occurring, there were instances of teachers arriving to prepare for class while room temperatures were either too warm or too cold. This resulted in trouble calls to school district maintenance department. EMC personnel were informed by operations staff of the school district that generally there was no occupancy of the school after 9 p.m. As a result, EMC personnel recommended that the BAS occupancy schedule be adjusted. 


$\begin{array}{ll}\frac{\text { HP }}{40} & \text { Room } \\ 47 & 422 \\ 1 & \text { Central Receiving } \\ 6 & \text { Gym hallway } \\ 7 & \text { Kitchen } \\ 37 & 517 \\ 39 & 515 \\ 53 & 406 \\ 55 & 401 \\ 58 & \text { Office hallway }\end{array}$

3. Setpoint control was found to be from the BAS for all HPs, with setpoints as follows: (a) office and media center occupied cooling at $74 \circ \mathrm{F}$ and unoccupied cooling at $79 \circ \mathrm{F}$, (b) classroom wings and gym/cafeteria - occupied cooling at $72^{\circ} \mathrm{F}$ and unoccupied cooling at $77^{\circ} \mathrm{F}$.

4. A review of trouble calls generated since reoccupying the school revealed that a significant number of calls originated from staff working in the office and media center. Testing showed that the HPs controlling these spaces were able to satisfactorily maintain a lower setpoint consistent with the classroom wings. EMC personnel recommended lowering the cooling setpoints, while remaining within the guidelines of the Turner Group report.

5. Space temperature sensor calibrations for the HPs were found to generally be within $+/-0.5^{\circ} \mathrm{F}$, with the following exceptions:

\begin{tabular}{ccc} 
Tracer Summit Temp. & Measured Temp. & Cal. Error \\
\cline { 2 - 3 } 71.2 & 69.7 & -1.5 \\
72.2 & 71.0 & -1.1 \\
74.4 & 76 & +1.6 \\
72.8 & 75.1 & +2.3 \\
74.9 & 73.1 & -1.8 \\
72.8 & 73.6 & +0.8 \\
71.9 & 72.7 & +0.8 \\
72.1 & 70.4 & -1.7 \\
71.4 & 69.9 & -1.5 \\
74 & 75.9 & +1.9
\end{tabular}

6. EMC consultants observed the position of outside doors and windows and found the exterior door and windows open in one classroom on the 4/5 wing. They also observed rocks or other objects (i.e., microphone stand base and bricks) outside of exterior doors to classrooms and corridors, apparently used to prop the doors open at times. They noted the exterior door and door frame in classroom 521 was damaged, allowing conditioned air to escape to the outside. Additionally, they noted it was difficult to pull the door shut to latch it, allowing potential unauthorized access to the school if the door was left unlatched. 
7. The outdoor air humidity sensor connected to the BAS was found to be reading incorrectly. It gave an implausible reading of $122.9 \%$ while a calibrated test instrument gave a reading of $74 \%$.

8. On the BAS graphic screen, classroom 501 did not have a link to HP-48, which serves that area.

9. A heat-generating cathode ray tube computer monitor was found to have been placed directly beneath the thermostat of HP-55 in classroom 401. The heat generated by this monitor was influencing the thermostat and keeping the room much colder than the BAS setpoint.

10. The cooling setpoint of HP-34 (serving areas in the $\mathrm{k} / 1$ wing) was found to be $74^{\circ} \mathrm{F}$, slightly higher than the intended setpoint of $72^{\circ} \mathrm{F}$.

11. The space temperature sensor installed in classroom 501 was found to be wired to an unknown point. EMC personnel were unable to determine the termination point for this sensor due to inability to access above the hard ceiling in the hallway.

12. HP-48 serves classroom 421 and classroom 501, where the single thermostat controlling this HP is located, but control of space temperatures of both these classrooms was found to be acceptable and consistent.

13. Air filters for HPs showed expected amounts of build-up consistent with the approximately 90 days of use they had experienced.

14. No issues with the BAS were found and communication to all HPs was present.

15. HP-24 was continually producing cold air. Although temporarily altering the setpoints caused the HP controller to switch to heating mode, discharge air temperature remained at levels consistent with cooling operation. The reversing valve was tested and found to operate properly, but the HP controller was operating inappropriately constantly energizing the reversing valve.

16. Review of the Tracer Summit alarm log revealed three instances when the Building Control Unit communications ceased during the week of May 3, 2010. The alarm log contained no indication of how long communications were down or why communications ceased. There was no impact 
on HP operation or indoor conditions; however, EMC personnel recommended that the alarm log be periodically reviewed to determine if communication losses increase in frequency.

17. The low refrigerant pressure switch of HP-9 circuit \#1 was found to be in the off position. Subsequent investigation by school district personnel revealed that this circuit's refrigerant had escaped.

18. The controller for HP-26 had been replaced with the same type of controller used for all other similar HPs in the school. The operation of this HP was now consistent with the others, consistent with a recommendation in the original EMC report.

19. The supply fan motor for HP-12 stopped working while EMC personnel were conducting seasonal testing. Maintenance personnel from the school district made repairs to correct this.

20. The rigid copper piping associated with the water-source HP building loop, located in the mezzanines above the classroom wings, lacked sufficient structural support in numerous locations. Unnecessary strain was being placed on soldered pipe joints, which could lead to failure of the joints and a potential for catastrophic flooding inside the school.

EMC personnel made the following recommendations:

1. Revise the BAS occupancy schedule to help settle space temperatures before employees arrive in the mornings. The revised schedules were as follows: (a) office and media center areas, occupied 4 a.m-9 p.m.; (b) classroom wings and gym/cafeteria, occupied 5 a.m.- 9 p.m.; and (c) data closets, occupied continuously. These changes were implemented on May 7, 2010.

2. Revise the temperature setpoints to provide acceptable space temperatures and alleviate trouble calls to school district maintenance department; the revised temperature setpoints were as follows: (a) office and media center, $72^{\circ} \mathrm{F}$ for occupied cooling and $77^{\circ} \mathrm{F}$ for unoccupied cooling; (b) classroom wings and gym/cafeteria, no changes; and (c) data closets, no changes. These changes were implemented by May 7, 2010. EMC personnel recommended that all unoccupied off-sets remain at $+/-5.0^{\circ} \mathrm{F}$. 
3. Continue regular air filter inspections on all units, including the makeup air units, at least every 3 months per manufacturer's recommendations, changing filters when warranted by visual inspection.

4. Use trained personnel with the proper calibrated equipment to assess air flow to help prevent unnecessary trouble calls stemming from inaccurate observations or assumptions.

5. Calibrate the thermostats in the kitchen, central receiving, the gym hallway, office hallway, and rooms 401, 406, 422, $514,515,517$, and 522 to within factory tolerances $\left(+/-0.5^{\circ}\right.$ F). If calibration is not possible, replace the thermostat and verify the new thermostat calibration with a calibrated test instrument.

6. Keep windows and outside doors closed to aid in maintaining acceptable indoor conditions. Additionally, custodial staff should regularly clear the areas around all exterior doors of rocks or objects that may deliberately or inadvertently impede full closing of those doors.

7. Replace or repair the outside doorframe and correct the door alignment for room 521.

8. Calibrate the outdoor air humidity transmitter for the BAS and verify the scaling of the device input in the BAS.

9. Add a link to HP-48 in the $4 / 5$ wing graphic of the BAS to facilitate easy access to the HP controller from the $2 / 3$ wing graphic.

10. Investigate the HP-24 controller to determine why the reversing valve was always energized, and repair or replace the controller.

11. Set the setpoints for HP-34 to the same profile as for the other classroom HPs.

12. Continue to follow unit manufacturer's recommendations on frequency for changing air filters, but observe filters for increased build-up and increase filter-change frequency as necessary, especially during periods of high traffic (i.e., move-in and move-out at beginning and end of school year).

13. Review the Tracer Summit alarm log weekly and look for building control unit communication failures. If these failures start to appear more frequently than weekly, consider building control unit replacement.

14. Investigate the cause of the refrigerant leak out of HP-9 
circuit \#1 and repair as necessary. Once repairs have been made, HP-9 performance should be reviewed daily for 1 week to determine effectiveness of repairs.

15. Install pipe supports with dielectric insulation properties, where missing, on all HP loop piping in the building. Straight, plumb, and properly sized saddle-bracket supports should be installed every six feet to evenly distribute the weight of the piping.

No issues were discovered with respect to the tempered water system (i.e., the operation of the cooling tower, heat exchanger, or pumps in cooling-season operation), makeup air units, or the building exhaust fans. Fans controlled by HPs and thermostatically-controlled fans were operational.

Appendix $\mathrm{O}$ summarizes the remediation activities completed at the elementary school since the NIOSH site visit in July 2009.

\section{Health Findings}

\section{Telephone interviews with three staff members}

\section{Staff Member A}

During the early part of the 2008/2009 school year, this staff member, with no prior history of respiratory illness, developed headaches, scratchy throat, laryngitis, sinus infection, and deep cough. He/She noted that the carpet in the classroom sometimes felt damp, and the room smelled like "a wet dog," or musty, especially when it rained, or when the power was off and there was no ventilation. He/She was diagnosed with bronchitis and then pneumonia. While he/she had pneumonia, he/she had profound fatigue and night sweats but no fever or chills. He/She did not respond to antibiotics and was eventually given corticosteroid shots. His/Her symptoms improved when he/she was restricted from work for a short period of time, but then returned when he/ she went back to work. He/She improved until the carpet was pulled up, at which time he/she experienced the same progression of symptoms, diagnoses, antibiotics with no effect, and finally corticosteroid (prednisone) treatment, which made him/her feel better. When the ventilation ducts were cleaned, he/she developed wheezing and was treated with an inhaler (corticosteroid with longacting bronchodilator) and oral corticosteroids. Additionally, he/ 
she reported that fish in one of the classrooms died, and the class was relocated for a few days because of the strong disinfectant smell. In the summer of 2009, while out of the school, he/she had no wheezing, cough, fatigue, headaches, or medication requirement.

\section{Staff Member B}

Since the opening of the new school building in 2005, this staff member developed recurrent sinus infections. He/She had postnasal drip, difficulty breathing, chest tightness, a metallic taste in his/her mouth, lungs that "hurt," and "clogged" ears. During the 2008/2009 school year, he/she experienced three episodes of "dizziness" and "lightheadedness" with a sense that he/she might "pass out" in the school. Other symptoms included a deep cough during exercise and some dyspnea on exertion, as if he/she "couldn't take a deep breath" when he/she walked. He/She noted his/her cough more outside of the building than inside, and it was sometimes dry and other times productive. Other than cough, he/ she reported no symptoms outside of the school building. His/Her symptoms worsened progressively over the course of the work week. During weekends and holiday breaks, the symptoms improved, but he/she would get sick upon returning to the building. During May and June 2009, his/her symptoms got much worse after the carpet was torn up at the school.

Being in the media room made him/her nauseous and feel like he/ she was getting the flu and had excessive fatigue. Sometimes he/she had night sweats and felt feverish and occasionally chilly. During the last two months of school during the 2008/2009 school year, he/she noted difficulty concentrating, remembering, and recalling. $\mathrm{He} / \mathrm{She}$ also experienced frequent wheezing and sneezing attacks, itchy/burning eyes, and headaches. By the end of each week, he/ she felt he/she was getting sick, but then felt better on Saturdays. $\mathrm{He} /$ She normally is an active person but gave up exercise during the second half of the 2008/2009 school year due to excessive fatigue. $\mathrm{His} / \mathrm{Her}$ fatigue resolved within two weeks following the end of the 2008/2009 school year.

$\mathrm{He} / \mathrm{She}$ reported no allergies on testing, except to cats, which was apparently minimal, and he/she does not have a cat. Though some symptoms during the last couple months of the 2008/2009 school year were treated with alternating antibiotics and corticosteroids, medications did not helped his/her symptoms. However, within a couple of weeks of school ending, he/she felt well again, with no 
need for medications.

$\mathrm{He} /$ She provided personal observations: the $4 / 5$ wing had leaks from bathroom pipes; when it rained, the ceiling tiles got stained and were changed; the gym had mold on the walls; the carpet in the physical education teacher's room separated from a damp wall; the administration offices smelled musty, like an old basement; a dehumidifier was placed in the administration area, but it did not seem to serve all the offices; and there were two termite swarms in the corridor near the library.

\section{Staff Member C}

This staff member, with no prior history of respiratory illness, developed respiratory and sinus infections, itchy/watery eyes, rashes, decreased appetite, fatigue, and difficulty concentrating and focusing during the 2008/2009 school year. He/she did not note any cough, wheezing, fever, or chills, but sometimes "gasped for air." Since the end of the 2008/2009 school year, his/her symptoms have improved, and he/she feels less fatigued and more alert.

$\mathrm{He} /$ She provided personal observations: there were many ceiling leaks at the school; water was sometimes evident in ceiling light fixtures in a classroom wing; stained ceiling tiles were frequently replaced; and there was mold growth and condensation on the gym walls.

\section{Health Department Questionnaire results}

\section{Participation and Demographics}

Fifty-two of 88 (59\%) staff members participated in the county health department questionnaire in May 2009. All 52 participants worked at the elementary school during the 2008/2009 school year; 45 of the 52 participants also worked at the school during the $2007 / 2008$ school year. Two of the staff participants who worked at the school during both school years stopped the questionnaire after the first page, as directed by the questionnaire instructions; they had answered "no" to experiencing any of the following symptoms while in the school building during the 2008/2009 school year (1) headaches; (2) dry eyes; (3) unusual sneezing episodes; (4) difficulty breathing/asthma attacks; (5) nosebleeds; or (6) other symptoms. Therefore, these two participants who worked at the school during both school years did not provide information on symptoms in the school building during the 2007/2008 school year, or on health 
conditions, medication, smoking status, or home exposures.

Seven hundred and thirty-eight (738) students attended the elementary school in 2008/2009 and 734 in 2007/2008. Parents of 388 students completed the questionnaire. We excluded data from one questionnaire because the student did not attend the school during the 2008/2009 or 2007/2008 school year. Of the remaining 387 student questionnaires, 385 attended during the $2008 / 2009$ school year, representing about $52 \%$ of the 738 students who attended that year; 308 attended during the 2007/2008 school year; and 306 attended during both school years.

Of the 387 students, 122 reported not having the symptoms on the first page of the questionnaire while in the school building during the 2008/2009 school year. Despite instructions to stop the questionnaire at that point, questionnaires were completed for 11 of these 122. Therefore, 276 (71\%) of the 387 participants completed the entire questionnaire, representing less than $40 \%$ of the student body.

Of the 48 staff who reported their 2008/2009 primary classroom or office, $40(83 \%)$ worked primarily in classroom wings and eight (17\%) in other wings (Table 7). Of the 385 participating students who attended the school during the 2008/2009 school year, we were able to determine the primary classroom/homeroom wing for 376 students; 111 of these 376 (30\%) were located in the $\mathrm{k} / 1$ wing, 136 (36\%) in the $2 / 3$ wing, and $129(34 \%)$ in the $4 / 5$ wing (Table 7 ).

Five of $50(10 \%)$ staff members reported smoking cigarettes, pipes, or cigars or having a smoker in their household. Nineteen of 276 (7\%) parents reported a smoker in the household.

Six of $50(12 \%)$ participating staff members reported one or more of the following in their homes: a history of water leaks; water stains on the wall and ceiling; or mold or mildew problems. Of the participating student households, 23 of $276(8 \%)$ reported one or more water leaks; water stains on the wall or ceiling; or mold or mildew.

\section{Six Staff with Daily Breathing Problems/Asthma Attacks}

As an indication of the presence of chronic lower respiratory problems possibly associated with the school building, we 
summarized responses from the questionnaires of six staff members who reported having "difficulty breathing/asthma attacks" daily while at work in the school building during the 2008/2009 school year. None of them reported smoking or having smokers in their households. Two of the six staff members reported having asthma and one reported allergic rhinitis. We do not have information on dates of diagnosis of these conditions, but one person reported an onset of 2007/2008 or earlier and the other reported an onset in $2008 / 2009$. Only the two staff members with asthma reported being on prescription breathing medication.

These six staff members worked in the $2 / 3,4 / 5$, or administrative wings. They reported symptoms associated with their own primary work areas and also with the staff lounge and media center. Four of the six staff members reported that their symptoms resolved hours to several days after leaving the school.

All six employees reported a variety of symptoms related to being in the school building. As a group, their symptoms included chesttightness, shortness of breath, coughing, difficulty breathing on awakening, fatigue, sore throat, headache, eye problems, nasal and sinus problems, skin problems, nosebleeds, nausea, vomiting, and a metallic taste in the mouth. Five of these six staff members had visited a doctor multiple times (from two to more than 30 times) during the 2008/2009 school year for the health problems reported on the questionnaire.

\section{Health Conditions}

Of participating staff, 34\% reported seasonal allergies; $8 \%$ asthma; and $8 \%$ allergic rhinitis. For students, $48 \%$ had seasonal allergies; $16 \%$ asthma; and $12 \%$ allergic rhinitis (Table 8 ).

\section{Building-related Symptoms During the 2008/2009 School Year}

Seventy-four percent (74\%) (37 of 50) of staff and 53\% (145 of 274) of students had at least one symptom/problem that resolved after leaving the building during the 2008/2009 school year.

\section{Lower respiratory symptoms}

Thirty-eight percent (38\%) of staff and 19\% of students experienced at least one lower respiratory symptom while in the school; difficulty breathing/asthma attacks was the most commonly experienced lower respiratory symptom occurring while in the school for both staff (31\%) and students (13\%) (Tables 9 and 10$)$. Of the 16 staff 
who reported difficulty breathing/asthma attacks while in the school, 38\% (6 of 16) reported them daily, 56\% (9 of 16) sometimes, and $6 \%$ ( 1 of 16) rarely. Of the 42 (of 49) students with available frequency data; $5 \%$ (2 of 42 ) were reported to have had difficulty breathing/asthma attacks daily, 79\% (33 of 42) sometimes, and $17 \%$ ( 7 of 42 ) rarely. Fifteen percent of staff and $7 \%$ of students had a cough while in the school.

\section{Upper respiratory symptoms}

Fifty-two percent $(52 \%)$ of staff and $28 \%$ of students experienced at least one upper respiratory symptom while in the school; unusual sneezing was the most commonly experienced upper respiratory symptom for both staff (31\%) and students (23\%) (Tables 9 and 10 ). Other reported upper respiratory symptoms while in the school included: sinus problems ( $25 \%$ of staff and $5 \%$ of students); voice or throat symptoms (27\% of staff and $5 \%$ of students); and nasal symptoms (10\% of staff and 5\% of students) (Tables 9 and 10 ).

\section{Mucous membrane irritation}

Eighty-seven percent (87\%) of staff and 32\% of students experienced at least one mucous membrane symptom while in the school; eye symptoms were the most commonly experienced mucous membrane symptoms for both staff (75\%) and students (28\%) (Tables 9 and 10).

\section{Headache}

Headache was the most common symptom experienced while in the school for both staff (92\%) and students (49\%) (Tables 9 and 10). Of the 47 (out of 48) staff for whom headache frequency data were available, $55 \%$ (26 of 47 ) reported experiencing headaches daily while in the school, $43 \%$ (20 of 47) sometimes, and $2 \%$ (1 of 47) rarely. Of the 182 (out of 187) students for whom headache frequency data were available, $25 \%$ (45 of 182) were reported to have experienced headaches daily at school, $57 \%$ (104 of 182) sometimes, and $18 \%$ (33 of 182) rarely.

\section{Fatigue}

Fatigue while in the school was reported for $44 \%$ of staff and $6 \%$ of students.

\section{Nosebleed}

Nosebleeds while in the school were also commonly reported for both staff (25\%) and students (20\%). Of the 10 (out of 13) staff for whom nosebleed frequency data were available, $10 \%$ (1 of 10$)$ 
reported them daily, $70 \%$ (7 of 10) sometimes, and 20\% ( 2 of 10) rarely. Of the 72 (out of 77) students for whom nosebleed frequency data were available, $11 \%$ (8 of 72) were reported to have had them daily, $60 \%$ (43 of 72) sometimes, and 29\% (21 of 72) rarely.

\section{Symptoms by Classroom Wing}

The health department questionnaire asked the participants to provide room numbers or descriptions of areas in the school where they experienced more symptoms or discomfort. Of the 52 participating staff members who worked at the school during the 2008/2009 school year, 27 indicated one or more rooms or areas where they experienced more symptoms or discomfort in the school. Of these 27 staff members, 12 reported the media room, 12 reported areas in the administrative wing, 10 reported areas in the $4 / 5$ wing, eight reported areas in the $2 / 3$ wing, four reported areas in the $\mathrm{k} / 1$ wing, and three reported areas in the gym/cafeteria wing.

Of the 385 students who attended the school during the 2008/2009 school year, parents of 86 of these students indicated one or more rooms or areas where their child experienced more symptoms or discomfort in the school. Fifty-four of the 86 parents reported areas in the $4 / 5$ wing, 43 reported areas in the $2 / 3$ wing, 18 reported areas in the $\mathrm{k} / 1$ wing, and two reported the gym. The music room (in the $2 / 3$ wing) was most commonly mentioned, with 16 writeins.

Table 9 shows the prevalences of reported symptoms among participating staff by primary work wing during the 2008/2009 school year. Upper respiratory symptoms were significantly more prevalent $(\mathrm{p}<0.05)$ among staff who worked in the $2 / 3$ or $4 / 5$ wings compared to staff who worked in the $\mathrm{k} / 1$ wing (Figure 24). Lower respiratory symptoms were marginally more prevalent $(\mathrm{p}<$ 0.10 ) among staff who worked in the $2 / 3$ or $4 / 5$ wings compared to staff who worked in the $\mathrm{k} / 1$ wing.

Table 10 shows the prevalence of reported symptoms by homeroom wing of students during the 2008/2009 school year. Headaches and nosebleeds were significantly more prevalent $(\mathrm{p}<0.05)$ among students whose primary/homeroom classrooms were in the $2 / 3$ or $4 / 5$ wing compared to students whose primary/homeroom classrooms were in the $\mathrm{k} / 1$ wing (Figure 25). Dry eyes, unusual sneezing, breathing difficulties/asthma attacks, and upper 
respiratory symptoms were marginally more prevalent $(\mathrm{p}<0.10)$ among students whose primary/homeroom classrooms were in the $2 / 3$ or $4 / 5$ wing compared to students whose primary/homeroom classroom were in the $\mathrm{k} / 1$ wing.

\section{Symptoms During the 2008/2009 and 2007/2008 School Years}

Staff (Figure 26) and students (Figure 27) who reported symptoms in the 2008/2009 school year and who worked or attended the elementary school during 2007/2008 and 2008/2009 school years had headache, dry eyes, unusual sneezing, breathing difficulties/ asthma attacks, nosebleeds, and upper and lower respiratory symptoms were more likely to report experiencing these symptoms during the 2008/2009 school year than during the 2007/2008 school year; the results were statistically significant $(\mathrm{p}<0.05)$.

\section{NIOSH Questionnaire Results}

\section{Demographics}

At the elementary school, 70 of 89 (79\%) current staff members participated in the March 2010 questionnaire. Demographics and the primary work areas of the participants are summarized in Tables 11 and 12 . Table 13 shows the schools that the participants worked at while the elementary school was closed for remediation. Of the 70 participants, 66 had worked at the elementary school during the 2008/2009 school year. The majority of staff members were nonsmokers (94\%).

\section{Physician-diagnosed health conditions}

Self-reported physician-diagnosed health conditions are summarized in Table 14. Physician-diagnosed sinusitis or sinus infections were reported by $64 \%(45 / 70)$. Of these, 29 reported they were first diagnosed with sinusitis or sinus problems before they starting work at the elementary school, five reported they were diagnosed after starting work at the elementary school but before the school reopened in 2005, and seven reported they were first diagnosed after it reopened in 2005. Four did not report a date of diagnosis.

Thirteen participants reported sinusitis or sinus problems during the four weeks before returning to the elementary school following its remediation; 11 of these reported their sinusitis or sinus problems were diagnosed by a physician. 
Physician-diagnosed hay fever or nasal allergies were reported by $34 \%$ (24 of 70 ); however, $41 \%$ (29 of 70 ) reported having nasal allergies, including hay fever. Of the 24 with a reported physician diagnosis of hay fever or nasal allergies, 17 reported they were first diagnosed before they started working at the elementary school, five reported they were first diagnosed after starting work at the elementary school but before it reopened in 2005, and one reported being initially diagnosed after it reopened in 2005. One participant did not report a date of diagnosis.

Prevalence of ever having been diagnosed with asthma was 10\% (7 of 70), and prevalence of current physician-diagnosed asthma was $7 \%$ (5 of 70). Six of the seven participants with a history of asthma reported their asthma was diagnosed prior to employment at the elementary school. One participant reported diagnosis of asthma after the school reopened in 2005.

\section{Health Symptoms During the Four Weeks Before Returning to the School in $\mathbf{2 0 1 0}$}

Tables 15 and 16 show prevalences of symptoms during the previous four weeks prior to starting back at the elementary following its remediation. In these tables, work-related symptoms were those reported to be better while away from the school (i.e., military academy, other elementary school, or middle school) that they worked at while the elementary school was closed for remediation. Stuffy, itchy, or runny nose (24\%) was the most commonly reported symptom, followed by dry or itchy skin (21\%), headache (19\%), sneezing $(16 \%)$, sore or dry throat (14\%), cough with phlegm (14\%), and watery, itchy eyes (13\%). Thirty-one percent $(37 \%)$ of staff reported using medications for nasal-sinus or eye problems during this four-week period.

Table 17 shows the prevalence of various health conditions reported one or more times per week by school staff during the four-week period prior to reporting to the elementary school in February 2010. When we excluded staff members who had not worked at the elementary school during the 2008/2009 school year, we observed similar prevalences.

Compared with participants in the BASE study, which asked U.S. office workers about symptoms that occurred one or more days per week in the last four weeks and that got better away from work, the elementary school employees did not have statistically significant excesses of sneeze, sore/dry throat, unusual tiredness, chest 
wheezing, shortness of breath, cough, headache, or dry itchy skin (Table 18).

When we compared questionnaire responses of the elementary school staff with those of NHANES III examinees, school employees had a statistically significant excess of nasal allergies compared to the U.S. adult population (Table 19). There were no statistically significant differences between the school staff and the U.S. adult population with respect to prevalences of shortness of breath on exertion, asthma, or chronic bronchitis.

The overall prevalences of lifetime and current physician-diagnosed asthma among participating school staff were not significantly different from expected compared to BRFSS survey data from the North Carolina adult population (Table 20).

\section{Health Symptoms During the 2008/2009 School Year at the Elementary School}

Table 21 shows prevalences of symptoms by type of wing (classroom vs. non-classroom). Compared to non-classroom wings, classroom wings were associated with statistically higher prevalences of unusual tiredness or fatigue; watery, itchy eyes; and upper respiratory symptoms. Table 22 shows the prevalence of symptoms by primary work area. There were no statistically significant differences in the distribution of symptoms among the $\mathrm{k} / 1,2 / 3$, and $4 / 5$ wings.

\section{Health Symptoms Following Remediation at the Elementary School}

Of the 70 school staff, 16 (23\%) reported having health symptoms that they considered possibly related to working in the elementary school building since its reopening in February 2010. Of the 16, six reported eye symptoms (four-dry eyes, one-allergy symptoms, and one-drippy eyes); five reported fatigue, tiredness, or feeling worn-out; five reported dry, scratchy, or sore throat symptoms; four reported headaches; four reported dry, flaky, or eczematous skin; three reported cough; two reported hoarseness; two reported nasal symptoms; one reported dehydration; one reported sneezing attacks; one reported clogged ears, and one reported sinus problems. 
Comparison of health symptom prevalences during the 2008/2009 school year and during the fourweek period prior to returning to the elementary school in February 2010

Table 23 shows prevalences of symptoms and the percentage of staff who reported their symptoms were work-related during the $2008 / 2009$ school year and during the four week period prior to returning to the elementary school following remediation. The prevalences of headaches; unusual tiredness or fatigue; upper and lower respiratory symptoms; watery, itchy eyes; difficulty remembering things or concentrating; and fever, chills, or flu-like symptoms were significantly greater during the 2008/2009 school year than during the four weeks prior to returning to the school. Less than 10 staff members reported nosebleeds during either time period. Work-relatedness for these 2008/2009 symptoms ranged from $73 \%-90 \%$. Because of the differences in time periods, we did not present statistical testing for data in Table 23.

\section{Comparison of NIOSH and Health Department Questionnaire Responses Concerning Symptoms During the 2008/2009 School Year at the Elementary School}

Headache, eye symptoms, and fatigue or tiredness were three of the most commonly reported symptoms during the 2008/2009 school year in both surveys. Many more participants in the NIOSH survey reported difficulty with memory or concentration during the 2008/2009 school year (44\%) than in the health department survey $(13 \%)$. Substantially more staff reported nosebleeds in the health department survey (25\%) than in the NIOSH survey (11\%). Similar percentages of upper and lower respiratory symptoms were reported in both surveys. 
Following a request from the school district to investigate respiratory illness and dampness at the elementary school, NIOSH assisted the school district and county health department in the evaluation of the school, motivated the hiring of building consultants, provided recommendations, and supported the decision to close the school for remediation at the end of the 2008/2009 school year. During the remediation process, school management acted on many of the $\mathrm{NIOSH}$ and building consultant recommendations, including (1) repairing the roof and inadequate flashing; (2) removing the carpet in the school and replacing it with hard floors (after testing slab for moisture content); (3) installing a vapor barrier and depressurizing the basement and crawl space under the media center; (4) sealing classroom wing soffits; (5) recommissioning the HVAC system; and (6) implementing the EPA's Indoor Air Quality Tools for Schools Program. The school reopened on February 22, 2010. In Appendix $\mathrm{O}$, there is a list with pictures compiled by the school district of remediation activities completed at the school.

The decision to close the elementary school during the remediation to address the underlying issues of water intrusion, humidity, and ventilation was a prudent one. The remediation we recommended is easier to do when a building is not occupied; health effects associated with dampness commonly are made worse when remediation occurs in occupied buildings. To help prevent worker exposures and contamination of unaffected sections of the building during remediation, proper containment is always warranted during remediation, whether the building being remediated is occupied or unoccupied.

As documented in this report (see Results section and Appendices $\mathrm{J}$ and $\mathrm{N}$ ), there have been problems with the HVAC system at the elementary school after renovations and the addition of the classroom wings. In addition, there have been widespread dampness and mold growth at the school since 2005. Visible mold was found recurrently on books, carpet, upholstered furniture, picture frames, and walls. The significance of the visible mold is that it reflects chronic dampness and potential health hazards for staff and students. Musty odors indicate that mold is actively growing. Mold cannot grow without moisture and a source of organic nutrients; high relative humidity is sufficient to support mold growth on organic substrates in indoor environments, including paper, adhesives, wood, and textiles.

There were several sources of dampness in the school. Consultants 
reported $\mathrm{RH}$ measurements above $70 \%$, exceeding recommended ranges of $30 \%-60 \%$ for most indoor environments. Problems with the building's HVAC system and with air flow in parts of the building allowed the outdoor, unconditioned air to enter the school through areas such as the crawl space under the media center, clerestory above the media center, and soffits in the classroom wings. There was potential for the formation of condensation. Additionally, the air flow and ventilation problems at the school may have allowed the transport of mold spores from reservoirs such as the crawl space and basement under the media center, where a strong musty odor were detected.

Inadequate flashing was also a source of dampness at the school. During the July 2009 site visit, NIOSH investigators and Turner Group consultants found inadequate flashing at the junction between the parapet wall above room 206 and the gym. In room 206, the carpet was stained and the baseboards were warped, likely from water damage. A few months earlier, a consulting engineer visited the school and reported that inadequate flashing over areas of the media center had caused deterioration of exterior bricks, which likely allowed penetration of rain water and contributed to leaks in the assistant principal's office. During the same site visit, the consultant also reported finding a substantial amount of visible mold under the carpet in that office. In 2005, an exterior wall in classroom 400 was damaged by water from an exterior leak during construction. In July 2009, the Turner Group reported that the drywall in this room was discolored, and that there had been occupant reports of mold found under the cove base. Although the wall flashing had been repaired, the Turner Group recommended repair of the associated roof junction joints to provide adequate flashing.

Consultants over the years have also reported ceiling and roof leaks. Following a heavy rain in July 2006, roof leaks occurred in all three classroom wings, the administrative wing, and one of the offices near the gym. In February 2007, an official of the North Carolina Department of Environmental and Natural Resources reported several ceiling leaks. The following year during a similar inspection, a bad leak was reported in the ceiling over the central receiving room. In April 2009, an engineer consultant reported significant roof leaks over the utility/storage room near the gym. Also during April 2009, more than one consultant noted stained ceiling tiles throughout the school, especially where the old and new buildings were structurally joined. There was also a history 
of roof leaks where the north-south roof intersects with the eastwest roof above the music and Spanish rooms, which shared a common wall. Consultants and building occupants have reported that water-stained ceiling tiles were often replaced before the leaks were repaired. Although we observed no stained ceiling tiles during our July 2009 visit, we did find defects in the flat roof over the old section of the school, suggesting the likelihood of water leaks.

Consultants have reported that landscaping around the school tends to result in the drainage of water toward the school building, allowing pooling of water adjacent to the school. Slab test results reported in the Turner Group's final report demonstrated that the concrete flooring in some rooms had more moisture (over 5.0 pounds per 1000 square feet per 24 hours) than other rooms. Turner Group consultants also noted that some classrooms had rusted sill plates possibly due to water damage.

Since the school was remodeled in 2005, various methods have been tried to correct the ventilation problems, excessive humidity, and water leakage through the building envelope. However, additional indoor environmental quality issues may have resulted from use of cleaning agents, such as biocides, in the HVAC system and on the carpet. The school had tried to keep the carpets clean with HEPAfiltered vacuuming; the carpeting had also had repeated moisture infusion from steam cleaning and chemical treatments with biocides intended to control mold growth. Carpet can act like a sponge to absorb and often hold these fluids and odors (as was noted by some consultants). Carpeted areas are frequently associated with increased building-related symptoms. Carpets may release volatile organic compounds, especially when new or after treatments with chemicals, including biocides intended to control mold growth. Even with routine cleaning, carpets can accumulate dust and harbor dust mites that can cause allergic symptoms. In damp conditions, accumulated dusts may contain fungi and bacteria, as was the case at the elementary school.

Air, swab, and vacuum sampling documented the presence of microorganisms within the school. Microorganisms can be found in samples collected from any indoor environment, and usually the indoor fungi reflect what has come in with outdoor air. Repeated air sampling for fungi often reveals that levels of fungi in the air are highly variable at different times during the same day or at different locations in the same room. At present, no environmental standards exist with which to predict health risk based on air sampling. For 
that reason, NIOSH scientists do not recommend the common consultant practice of conducting room air sampling, nor have we found room air sampling helpful for detecting reservoirs of hidden mold within walls or under carpets. Although counts of fungi in the air, whether as spores or colony forming units, are difficult to interpret, air sampling is sometimes helpful. For example, when the qualitative or quantitative distribution of airborne fungal types indoors differs from outdoors, an indoor source of fungal growth may exist. The consultant sampling documented some indoor fungal species that were higher in indoor samples than outdoor samples or did not occur in outdoor samples. This suggests that there were indoor sources of mold growth, as was evident by visual inspection and by smelling moldy odors at the school. The predominance of hydrophilic fungi documented in the elementary school suggests indoor sources related to chronic dampness.

In July 2009, we collected floor dust samples at the elementary school and found that mesophilic and hydrophilic fungi together accounted for more than $80 \%$ of the total fungal concentration. According to the American Industrial Hygiene Association guidelines [Hung et al. 2005], samples dominated by the presence of hydrophilic fungi indicate chronic dampness, while the predominance of a species mix of mesophilic fungi indicates alternating wet and dry cycles.

Our sampling results showed that the $2 / 3$ wing had the highest average fungal concentrations (of both hydrophilic and total fungi) in floor dust, while the $4 / 5$ wing and the media center had the lowest. These findings suggest chronic dampness in the $2 / 3$ wing. We also found a high concentration of gram-negative bacteria in rooms of the 2/3 wing, which is also consistent with chronic dampness in that wing because, as with hydrophilic fungi, gram-negative bacteria require relatively high moisture for proliferation [Burge et al. 1994]. The rooms in the $\mathrm{k} / 1$ wing had the lowest average water activity on floor materials compared to the other wings. This finding may imply that the carpets or tiles in the $\mathrm{k} / 1$ wing were relatively drier than those in other wings.

The concentrations of culturable fungi in the floor dust samples we collected during July 2009 were consistently and substantially lower than those in samples collected earlier by environmental consultants. During the last weekend in May 2009, the school had cleaned all the carpets in the school with an antimicrobial (biocide) agent. Additionally in May 2009, the carpet in room 407 had been replaced with vinyl composite tile. Cleaning with biocide 
and replacement of carpet with tiles may account for the apparent decrease in levels of fungi in floor carpet dust; however, microbial growth could recur if dampness problems are not sufficiently resolved at present or adequately controlled in the future.

Upon review of environmental reports of activities at the elementary school, we determined that the biocide Sporicidin ${ }^{\circledR}$ (The Sporicidin Company, Rockville, Maryland) had been applied in the HVAC system and on carpets. Biocides, including fungicides and disinfectants, are chemical agents that destroy or harm living organisms [EPA 1997; Cole and Foarde 1999; Prezant et al. 2008]. Any biocide must be registered for use by the EPA under the Federal Insecticide, Fungicide, and Rodenticide Act, and EPA biocide registration is granted only for specific biocide uses. Thus, only biocides specifically approved for use in an HVAC system can be applied in a HVAC system [EPA 1997]. Sporicidin Disinfectant Solution and Sporicidin Brand Disinfectant Spray are approved by the EPA for cleaning air ducts (EPA Registration \# 8383-3 and 8383-4, respectively). The product label (Appendix P) indicates that Sporicidin ${ }^{\circledR}$ must be only applied to hard, non-porous surfaces of air ducts. This product is not approved for use in internally insulated air duct systems. According to the EPA, biocides registered for use in HVAC systems have low toxicity levels and the lowest level of efficacy [Lantz 2009]; however, the EPA notes that risk assessment for use of most biocides in HVAC systems has not been completed [EPA 2002]. The product label (Appendix P) indicates that Sporicidin ${ }^{\circledR}$ is approved for use on carpets; however, formal risk assessment for Sporicidin ${ }^{\circledR}$ use on carpets has not been done [EPA 2009]. There is potential for exposure to biocide residues in treated carpet especially since the product label claims that this product has residual anti-fungal properties lasting for over six months.

Use of biocides in HVAC systems is controversial. Although biocide use may help reduce mold and bacterial growth, all biocides have some level of toxicity and unnecessary exposures should be avoided. The growth of mold and bacteria in indoor environments is due to excess moisture; thus, application of biocide as a protective action is unnecessary if moisture is properly controlled. Applying biocides directly into HVAC ducts may result in dissemination of these chemicals throughout occupied office areas and classrooms, resulting in possible occupant exposures and health effects. Exposure to airborne mold can cause respiratory health effects even when the mold is not viable (alive), so mold exposures can continue even after biocide treatment, further detracting from biocide use in 
HVAC ducts [Cole and Foarde 1999; NYCDHMH 2008; Prezant et al. 2008].

The New York City Guidelines [NYCDHMH 2008] and the American Conference of Governmental Industrial Hygienists [Cole and Foarde 1999] do not recommend biocide use in HVAC systems as a routine cleaning practice due to the questionable effectiveness of some of these chemicals to prevent microbiological growth and also due to uncertainties regarding health outcomes from associated exposures of building occupants to biocides. Biocides should not be applied in an occupied building or in an operating HVAC system [Sesline et al. 1994]. Given current uncertainty about the safety of biocide use in HVAC system ducts, any biocide use in HVAC systems or ductwork, even EPA-approved biocides such as Sporicidin ${ }^{\circledR}$, should be minimized, carefully monitored in terms of occupant health and air quality, and guided by professional judgment [Prezant et al. 2008].

An environmental sampling report we reviewed stated that the coil cleaner used in December 2008 at the elementary school contained butyl cellosolve (2-butoxyethanol). The Agency for Toxic Substances and Disease Registry stated that exposure to 2-butoxyethanol may result in irritation of the nose and eyes, headache, a metallic taste, and vomiting [ATSDR 1999]. High exposure levels may cause breathing problems [ATSDR 1999]. Air sampling for 2-butoxyethanol after the coil cleaning showed levels were below both the Occupational Safety and Health Administration permissible exposure limit and the NIOSH recommended exposure limit; however, we have no information on air levels of 2-butoxyethanol during the cleaning, when levels are likely to have been higher.

Excessive indoor dampness is a recognized public health problem. In 2004, the Institute of Medicine of the National Academies published an extensive review of past scientific studies on the health effects of damp buildings [IOM 2004]. In 2009, the World Health Organization (WHO) published guidelines for dampness and mold based on an updated review [WHO 2009]. The WHO report states that "excess moisture on almost all indoor materials leads to growth of microbes, such as mould, fungi, and bacteria, which subsequently emit spores, cells, fragments and volatile organic compounds into indoor air. Moreover, dampness initiates chemical or biological degradation of materials, which also pollutes indoor air." With respect to health effects, the WHO report points out that (1) clinical evidence is sufficient to conclude that exposure to 
mold and other dampness-related microbial agents in the indoor environment increases the risk of hypersensitivity pneumonitis, chronic rhinosinusitis, and allergic fungal sinusitis; (2) epidemiological evidence is sufficient to conclude that occupants of damp or moldy buildings are at increased risk of respiratory symptoms (specifically upper respiratory tract symptoms, cough, wheeze, and shortness of breath), respiratory infections, asthma development, current asthma, and asthma exacerbation; and (3) epidemiological evidence suggests that occupants of damp or moldy buildings are also at increased risk of bronchitis and allergic rhinitis. Importantly, the WHO guidelines note that although atopy and allergy increases susceptibility to dampness-related health effects, such health effects are also found in non-atopic building occupants.

The WHO guidelines relevant to occupational environments are as follows [WHO 2009]:

- Persistent dampness and microbial growth on interior surfaces and in building structures should be avoided or minimized, as they may lead to adverse health effects.

- Indicators of dampness and microbial growth include the presence of condensation on surfaces or in structures, visible mold, perceived moldy odor and a history of water damage, leakage or penetration. Thorough inspection and, if necessary, appropriate measurements can be used to confirm indoor moisture and microbial growth.

- As the relations between dampness, microbial exposure and health effects cannot be quantified precisely, no quantitative healthbased guideline values or thresholds can be recommended for acceptable levels of contamination with microorganisms. Instead, it is recommended that dampness and mold-related problems be prevented. When they occur, they should be remediated because they increase the risk of hazardous exposure to microbes and chemicals.

- Well-designed, well-constructed, well-maintained building envelopes are critical to the prevention and control of excess moisture and microbial growth, as they prevent thermal bridges and the entry of liquid or vapor-phase water. Management of moisture requires proper control of temperatures and ventilation to avoid excess humidity, condensation on surfaces and excess moisture in materials. Ventilation should be distributed effectively throughout spaces, and stagnant air zones should be avoided.

- Building owners are responsible for providing a healthy workplace or living environment free of excess moisture and mold, by ensuring proper building construction and maintenance. The occupants are 
responsible for managing the use of water, heating, ventilation and appliances in a manner that does not lead to dampness and mold growth.

From our limited telephone interviews and from the health department questionnaire responses, we found evidence of buildingrelated lower respiratory problems among building occupants, and we suspect at least two employees had hypersensitivity pneumonitis caused by microbial exposures in the school. Hypersensitivity pneumonitis is a serious lung disease in which an individual's immune system reacts to repeated exposure to organic dusts (materials from living things such as plants, animals, bacteria, or fungi) or other sensitizing agents [Patel et al. 2001]. Symptoms can include cough, shortness of breath, muscle aches, fever, chills, and fatigue. In work-related hypersensitivity pneumonitis cases, the first sign that the illness is due to exposures at work may be that the worker's symptoms and medical test abnormalities (e.g., pulmonary function tests) improve during periods of time away from work and worsen on return to work. Continued exposure can lead to lung scarring and permanent shortness of breath. The two staff members with symptoms suggestive of hypersensitivity pneumonitis both reported that their symptoms (e.g., fatigue, fever, chills, cough, and shortness of breath) resolved with time away from the school and recurred upon return to the school building. Physicians often fail to consider a diagnosis of hypersensitivity pneumonitis. As a result, hypersensitivity pneumonitis is under-recognized, and even a single case related to a building should be considered a sentinel event requiring investigation and remediation [Weltermann et al. 1998]. Hypersensitivity pneumonitis has been documented in workers in buildings with contaminated air-conditioners, ductwork, and HVAC filters [Bernstein et al. 1983; Scully et al. 1979; Fink et al. 1976; Banaszak et al. 1970; Arnow et al. 1978]. Hypersensitivity pneumonitis has also occurred employees in waterdamaged buildings with roof leaks, plumbing leaks, poorly draining condensation pans, or high indoor relative humidity [Seuri et al. 2000; Thörn et al. 1996 Weltermann et al. 1998; Hoffman et al. 1993].

The health department survey done in May 2009 showed that elementary school staff had high rates of lower respiratory symptoms, mucous membrane irritation, and headaches during the 2008/2009 school year. Even if we adjusted for the response rate of $59 \%$, these symptom rates would still be high. Staff and students who attended the school during the 2007/2008 and 2008/2009 school years had more upper and lower respiratory symptoms, as 
well as headaches, dry eyes, and nosebleeds during the 2008/2009 school year than during the 2007/2008 school year, suggesting the problems at the school were getting worse. Based on the NIOSH and health department surveys, headaches, eye symptoms, and fatigue or tiredness were commonly experienced by school staff during the 2008/2009 school year. In the NIOSH survey, staff also commonly reported difficulty remembering things or concentrating during the 2008/2009 school year.

There were some limitations in our evaluation of the health department survey data. The health department questionnaire contained some open-ended questions in which the participants were asked to answer in their own words. These types of answers are often difficult to categorize for statistical analysis. Additionally, the questionnaire did not ask specifically about cough, wheeze, chest tightness, itchy or runny nose, or other symptoms; some participants wrote in these and other symptoms on the questionnaire. While the questionnaire did ask about improvement of symptoms after leaving the school building, we could not determine which specific symptoms improved away from the school building. Additionally, the response rate (that is, the number of questionnaires that were returned to the health department) was low, and the results may not be representative of the whole school population. Parents filled out the questionnaires for their children. Such surrogate or proxy reporting is often used for surveys of children, but may be less accurate than direct reporting. Thus, with respect to our analyses of health data relating to the elementary school, survey data for the staff may be more reliable than that for the students. Lastly, the health department questionnaire did not ask about physiciandiagnosed asthma and did not contain other standardized symptom questions needed to compare with results from national or state surveys to determine if the school staff had elevated symptom rates. Nevertheless, the questionnaire represented an attempt to systemically collect data to determine policy and prioritize interventions. Priority had to be given to the entire school because of the prevalence of reported symptoms and the environmental findings throughout the school. Additionally, the school buildingrelatedness of the symptoms reported by staff and parents were consistent with building-related exposures causing symptoms. Also, case reports obtained by non-standardized telephone interviews indicated that some illness that may have been caused by exposures in the school building was severe enough to have required medical consultation and treatment. Most worrisome were symptoms suggestive of building-related hypersensitivity pneumonitis. This, 
along with the high rates of asthma/breathing difficulties reported by building occupants during the 2008/2009 school year, warranted action, taken by the school, to relocate the staff and students during remediation.

After remediation for indoor dampness and mold problems, building managers, building occupants, and their employers often wish to know if the building is "safe." Building consultants often recommend and perform "clearance" air sampling after remediation in an attempt to demonstrate that the building is safe for occupants. However, there is no scientific basis for the use of air sampling for this purpose. Once remediation is completed (sources of excessive dampness corrected; moldy and water-damaged materials removed; musty odors no longer evident), the best evidence that the building is safe may be that employees no longer experience building-related symptoms.

The NIOSH questionnaire administered to school staff a week after they reoccupied the elementary school building addressed health symptoms experienced during the four weeks prior to the reopening of the school in February 2010 and during the 2008/2009 school year at the elementary school. Upper and lower respiratory symptoms (with the exception of nasal symptoms), as well as mucous membrane symptoms and constitutional/nonspecific type symptoms, were reported much less commonly in the four weeks prior to returning to the elementary school than during the 2008/2009 school year, suggesting that building-related symptoms resolved in most staff while they were temporarily out of the damp school building. We did not observe lower prevalences of skin or nasal symptoms. This may have been because of the winter season and dry air. In any case, fewer than $25 \%$ of staff members with stuffy, itchy, or runny noses or dry or itchy skin reported it was work-related.

The overall prevalences of lifetime and current physician-diagnosed asthma among the elementary school staff who participated in the NIOSH survey were not significantly different than what would be expected in North Carolina based on the BRFSS survey of the North Carolina adult population. Most staff with asthma reported that onset of their asthma occurred before they began working at the school. Compared to the U.S. adult population surveyed in NHANES III, elementary school employees had significantly lower than expected rates of lifetime and current asthma, chronic bronchitis, shortness of breath on exertion, and nasal allergies, 
including hay fever. Compared to U.S. office workers who participated in BASE, elementary school employees did not have excess rates of work-related upper and lower respiratory symptoms, headache, unusual tiredness and fatigue, or dry itchy skin.

Findings from the NIOSH health survey after remediation can serve as a baseline to help assess whether the apparently improved health is maintained among the employees who have reoccupied the elementary school. Of the school staff who participated in the NIOSH survey, 16 (23\%) reported having health symptoms that they considered possibly related to working in the school building after its reopening in February 2010. Fatigue, headaches, and eye and throat symptoms (often described as dry, scratchy, or itchy) were the most commonly reported symptoms among these 16 staff members. Some of these recent eye and throat symptoms may have been due to dry air; during winter months, indoor relative humidity tends to be low because the heating system is on more of the time. Additionally, improved ventilation resulting from the retro-commissioning of the HVAC system may have enhanced a dry sensation among some building occupants.

Controlling water activity is the most effective approach for preventing fungal growth in indoor settings. This is accomplished by fixing water leaks, controlling relative humidity, and keeping surface temperatures high enough to prevent condensation; dry buildings do not become moldy. When water activity is high, mold can be limited by reducing the availability of nutrients that support fungal growth. One good way to help limit fungal growth in indoor settings is to replace carpeted flooring with hard flooring, as the school has done. Hard floors provide little inherent nutrients and can be cleaned to prevent dust accumulation.

Our specific recommendations for the elementary school are presented in the following section. 
1. Assess surface drainage around the school building and make modifications to prevent puddling and drainage of water towards the building.

2. Maintain the HVAC system by following the manufacturer's recommended maintenance schedules, including replacing air filters at least every three months, checking drip pans, ensuring thermostats are in working order, and checking/ cleaning ventilation system dampers to ensure proper functioning.

3. Make all the repairs recommended in the EMC Engineers, Inc. report dated May 2010 (Appendix N), which are summarized in the Results section of this report.

4. Do not use biocides in ductwork or on floors as a routine practice.

5. Continue to routinely assess the school for water intrusion and damage and high relative humidity and correct these upon discovery. During and after heavy rains, walk through the entire school and check for water incursion. When sources of moisture are identified, repairs should be made to prevent further water entry into the building. Mold and moisture-damaged materials should be promptly removed or cleaned with appropriate containment to minimize exposure of building occupants.

6. Keep a record of when and where water-damaged materials are discovered and what has been done to fix the underlying problem leading to the water damage. Areas with moldy or earthy odors or visible mold should likewise be identified, remediated, and documented.

7. Use a dampness/mold check-sheet (Appendix Q) during routine inspections of the school to record current problem areas and prioritize responsive action.

8. If and when any remediation is required, use proper containment methods to minimize exposures for remediation workers, building occupants, and unaffected sections of the building.

9. Encourage employees to report dampness and buildingrelated health problems and designate a contact person for this purpose. Allow confidential reports with an appropriately located locked drop box or an appropriately designed web-based electronic reporting system. 
10. If needed, use occupant health questionnaires to collect information on building-related symptoms and compare symptom prevalences with the NIOSH questionnaire done in March 2010 to determine if symptom prevalences have changed and appear to be work-related.

11. Continue to implement the EPA's Indoor Air Quality Tools for Schools Program.

12. Any member of the staff or student body who experiences worsening, persistent, or recurrent respiratory or other health symptoms that may be associated with being in the school building should see a healthcare provider. 
ATSDR (Agency for Toxic Substances and Disease Registry) [1999]. ToxFAQs for 2-Butoxyethanol and 2-Butoxyethanol Acetate. [http://www.atsdr.cdc.gov/toxfaqs/tf.asp?id=346\&tid=61] Date accessed: March 2011.

Arnow PM, Fink JN, Schlueter DP, Barboriak JJ, Mallison G, Said SI, Martin S, Unger GF, Scanlon G, Kurup VP [1978]. Early detection of hypersensitivity pneumonitis in office workers. Am J Med 64:236-242.

Banaszak EF, Thiede WH, Fink JN [1970]. Hypersensitivity pneumonitis due to contamination of an air conditioner. N Engl J Med 283:271-276.

Bernstein RS, Sorenson WG, Garabrant D, Reaux C, Treitman RD [1983]. Exposures to respirable, airborne Penicillium from a contaminated ventilation system: clinical, environmental and epidemiological aspects. Am Ind Hyg Assoc J 44:161-169.

Burge HA, Su HJ, Spengler JD [1994]. Moisture, organisms, and health effects. In: Trechsel HR, ed. Moisture control in buildings: (MNL 18). ASTM International, pp. 84-90.

CDC (Centers for Disease Control and Prevention) [1996]. Third National Health and Nutrition Examination Survey, 1988-1994, NHANES III Examination Data File [CDROM]. Hyattsville, Maryland: U.S. Department of Health and Human Services, Centers for Disease Control and Prevention. (Public use data file documentation No. 76300.)

Cole EC and Foarde KK [1999]. Biocides and antimicrobial agents. In: Macher J, ed. Bioaerosols: assessment and control. Cincinnati, $\mathrm{OH}$ : American Conference of Governmental Industrial Hygienists, pp. 16-1 - 16-9.

EPA (Environmental Protection Agency) [1997]. Should you have the air ducts in your home cleaned? [http://www.epa.gov/iaq/pdfs/airducts.pdf]. Date accessed: March 2011.

EPA (Environmental Protection Agency) [2002]. Use of disinfectants and sanitizers in heating, ventilation, air conditioning, and refrigeration systems. [http://www.epa.gov/oppad001/hvac.htm]. Date accessed: March 2011.

EPA (Environmental Protection Agency) [2009]. Reregistration eligibility decision for phenol \& salts. Washington, DC: U.S. Environmental Protection Agency, Prevention, Pesticides and Toxic Substances (7510P), EPA 739-R-08-010.

Flannigan B, Miller JD [2001]. Microbial growth in indoor environments. In: Flannigan B, Samson RA, Miller JD, eds. Microorganisms in home and indoor work environments: diversity, health impacts, investigation and control. $1^{\text {st }}$ ed. New York: Taylor \& Francis, Inc., pp.35-67.

Fink JN, Banaszak EF, Baroriak JJ, Hensley GT, Kurup VP, Scanlon GT, Schlueter DP, Sosman AJ, Thiede WH, Unger GF [1976]. Interstitial lung disease due to contamination of forced air systems. Ann Intern Med 84:406-413. 
REFERENCES (CONTINUED)

Hoffman RE, Wood RC, Kreiss K [1993]. Building-related asthma in Denver office workers. Am J Public Health 83:89-93.

Hung LL, Miller JD, Dillon HK, eds. [2005]. Field guide for the determination of biological contaminants in environmental samples. $2^{\text {nd }}$ ed. Fairfax, VA: American Industrial Hygiene Association, pp. 29-38.

IOM (Institute of Medicine) [2004]. Damp Indoor Spaces and Health. Washington, DC: National Academy of Sciences.

Lantz T (Lantz.Tracy@epamail.epa.gov) [2009]. Biocide use in HVAC. Private e-mail message to Rena Saito (hve5@cdc.gov), December 8.

NCCDPHP (National Center for Chronic Disease Prevention and Health Promotion) [2009]. Behavioral Risk Factor Surveillance System (BRFSS). Prevalence Data. [http://apps.nccd.cdc.gov/BRFSS/] Date accessed: October 2010.

NYCDHMH (New York City Department of Health and Mental Hygiene) [2008]. Guidelines on assessment and remediation of fungi in indoor environments. [http://www.nyc.gov/html/doh/ downloads/pdf/epi/epi-mold-guidelines.pdf]. Date accessed: March 2011.

Patel A, Ryu J, Reed C [2001]. Hypersensitivity pneumonitis: current concepts and future questions. J Allergy Clin Immunol 108:661-670.

Prezant B, Weekes DM, Miller JD, ed. [2008]. Recognition, evaluation, and control of indoor mold. Fairfax, VA: American Industrial Hygiene Association, p. 207.

Scully RF, Galdabini JJ, McNeely BU [1979]. Case records of the Massachusetts General Hospital: Weekly clinicopathological exercises. Case 47-1979. N Engl J Med 301:1168-1174.

SeslineD, Ames RG, Howd RA [1994]. Irritative and systemic symptoms following exposure to Microban disinfectant through a school ventilation system. Arch Environ Health 49:439-444.

Seuri M, Husman K, Kinnunen H, Reiman M, Kreus R, Kuronen P, Lehtomäki K, Paananen M [2000]. An outbreak of respiratory diseases among workers at a water-damaged building - a case report. Indoor Air $10: 138-145$.

Thörn A, Lewné M, Belin L [1996]. Allergic alveolitis in a school environment. Scan J Work Environ Health 22:311-314.

Weltermann BM, Hodgson M, Storey E, DeGraff AC, Bracker A, Groseclose S, Cole SR, Cartter M, Phillips D [1998]. Hypersensitivity pneumonitis: a sentinel event investigation in a wet building. Am J Ind Med 34:499-505. 
Womble SE, Ronca EL, Girman JR, Brightman HS [1996]. Developing baseline information on buildings and indoor air quality. In: IAQ 96 Paths to Better Building Environments/Health Symptoms in Building Occupants. Atlanta, GA: ASHRAE (American Society of Heating, Refrigeration and Air-Conditioning Engineers), pp. 109-117.

WHO (World Health Organization) [2009]. WHO guidelines for indoor air quality: dampness and mould. WHO Regional Office for Europe. 


\section{TABLES}

Table 1. Microbial agents in floor dust samples collected by NIOSH, July 2009

\begin{tabular}{|c|c|c|c|c|}
\hline \multirow{2}{*}{ Room* } & \multicolumn{2}{|l|}{ Fungi } & \multicolumn{2}{|l|}{ Bacteria } \\
\hline & Species & $\mathrm{cfu} / \mathrm{g} \dagger$ & & $\mathrm{cfu} / \mathrm{g}_{\dagger}^{\dagger}$ \\
\hline $100^{\mathrm{a}}$ & $\begin{array}{l}\text { Alternaria alternata } \\
\text { Cladosporium cladosporioides } \\
\text { Epicoccum nigrum } \\
\text { Fusarium oxysporum } \\
\text { Total fungi }\end{array}$ & $\begin{array}{l}2,000 \\
4,900 \\
2,000 \\
980 \\
9,800\end{array}$ & $\begin{array}{l}\text { Gram positive } \\
\text { Gram negative, non-fermentative } \\
\text { Broad spectrum }\end{array}$ & $\begin{array}{l}610,000 \\
7,300 \\
380,000\end{array}$ \\
\hline $113^{a}$ & $\begin{array}{l}\text { Aureobasidium pullulans } \\
\text { Biopolaris australiensis } \\
\text { Cladosporium cladosporioides } \\
\text { Mucor plumbeus } \\
\text { Nonsporulating fungi } \\
\text { Penicillium lividium } \\
\text { Trichoderma koningii } \\
\text { Total fungi }\end{array}$ & $\begin{array}{l}640 \\
91 \\
1,500 \\
91 \\
180 \\
180 \\
91 \\
2,800\end{array}$ & $\begin{array}{l}\text { Gram positive } \\
\text { Gram negative, non-fermentative } \\
\text { Broad spectrum }\end{array}$ & $\begin{array}{l}280,000 \\
5,600 \\
450,000\end{array}$ \\
\hline $115^{\mathrm{a}}$ & $\begin{array}{l}\text { Aspergillus versicolor } \\
\text { Aureobasidium pullulans } \\
\text { Biopolaris australiensis } \\
\text { Cladosporium cladosporioides } \\
\text { Epicoccum nigrum } \\
\text { Nonsporulating fungi } \\
\text { Penicillium lividium } \\
\text { Total fungi }\end{array}$ & $\begin{array}{l}960 \\
960 \\
1,900 \\
13,000 \\
2,900 \\
1,900 \\
960 \\
23,000\end{array}$ & $\begin{array}{l}\text { Gram positive } \\
\text { Gram negative, non-fermentative } \\
\text { Broad spectrum }\end{array}$ & $\begin{array}{l}67,000 \\
6,100 \\
95,000\end{array}$ \\
\hline $116^{\mathrm{a}}$ & $\begin{array}{l}\text { Cladosporium cladosporioides } \\
\text { Epicoccum nigrum } \\
\text { Nonsporulating fungi } \\
\text { Penicillium aurantiogriseum } \\
\text { Total fungi }\end{array}$ & $\begin{array}{l}1,600 \\
540 \\
270 \\
89 \\
2,500\end{array}$ & $\begin{array}{l}\text { Gram positive } \\
\text { Gram negative, non-fermentative } \\
\text { Broad spectrum }\end{array}$ & $\begin{array}{l}450,000 \\
4,600 \\
63,000\end{array}$ \\
\hline $206^{a}$ & $\begin{array}{l}\text { Alternaria alternata } \\
\text { Aspergillus glaucus } \\
\text { Aspergillus niger } \\
\text { Aspergillus versicolor } \\
\text { Biopolaris australiensis } \\
\text { Cladosporium cladosporioides } \\
\text { Epicoccum nigrum } \\
\text { Fusarium oxysporum } \\
\text { Rhizopus stolonifer } \\
\text { Total fungi }\end{array}$ & $\begin{array}{l}1,000 \\
2,000 \\
1,000 \\
1,000 \\
1,000 \\
14,000 \\
2,000 \\
1,000 \\
1,000 \\
24,000\end{array}$ & $\begin{array}{l}\text { Gram positive } \\
\text { Gram negative, non-fermentative } \\
\text { Broad spectrum }\end{array}$ & $\begin{array}{l}170,000 \\
7,500 \\
210,000\end{array}$ \\
\hline $407^{b}$ & $\begin{array}{l}\text { Alternaria alternata } \\
\text { Aspergillus glaucus } \\
\text { Aspergillus niger }\end{array}$ & $\begin{array}{l}1,900 \\
2,800 \\
940\end{array}$ & $\begin{array}{l}\text { Gram positive } \\
\text { Gram negative, non-fermentative } \\
\text { Broad spectrum }\end{array}$ & $\begin{array}{l}94,000 \\
6,300 \\
1,200,000\end{array}$ \\
\hline
\end{tabular}




\begin{tabular}{|c|c|c|c|c|}
\hline$T A B$ & $\begin{array}{l}\text { (CONTINUED) } \\
\text { Aspergillus ochraceus } \\
\text { Cladosporium cladosporioides } \\
\text { Epicoccum nigrum } \\
\text { Nonsporulating fungi } \\
\text { Total fungi }\end{array}$ & $\begin{array}{l}940 \\
7,500 \\
1,900 \\
1,9001 \\
8,000\end{array}$ & & \\
\hline $418^{c}$ & $\begin{array}{l}\text { Biopolaris australiensis } \\
\text { Cladosporium cladosporioides } \\
\text { Epicoccum nigrum } \\
\text { Mucor plumbeus } \\
\text { Total fungi }\end{array}$ & $\begin{array}{l}1,900 \\
19,000 \\
1,900 \\
950 \\
24,000\end{array}$ & $\begin{array}{l}\text { Gram positive } \\
\text { Gram negative, non-fermentative } \\
\text { Broad spectrum }\end{array}$ & $\begin{array}{l}1,700,000 \\
3,800,000 \\
1,800,000\end{array}$ \\
\hline $421^{\mathrm{c}}$ & $\begin{array}{l}\text { Alternaria alternata } \\
\text { Aureobasidium pullulans } \\
\text { Biopolaris australiensis } \\
\text { Cladosporium cladosporioides } \\
\text { Epicoccum nigrum } \\
\text { Nonsporulating fungi } \\
\text { Total fungi }\end{array}$ & $\begin{array}{l}920 \\
4,600 \\
1,800 \\
4,600 \\
5,500 \\
2,800 \\
20,000 \\
\end{array}$ & $\begin{array}{l}\text { Gram positive } \\
\text { Gram negative, non-fermentative } \\
\text { Broad spectrum }\end{array}$ & $\begin{array}{l}540,000 \\
10,000 \\
220,000\end{array}$ \\
\hline $424^{\circ}$ & $\begin{array}{l}\text { Alternaria alternata } \\
\text { Aureobasidium pullulans } \\
\text { Biopolaris australiensis } \\
\text { Cladosporium cladosporioides } \\
\text { Curvularia lunata } \\
\text { Epicoccum nigrum } \\
\text { Fusarium oxysporum } \\
\text { Nonsporulating fungi } \\
\text { Yeasts other } \\
\text { Total fungi }\end{array}$ & $\begin{array}{l}1,000 \\
2,000 \\
1,000 \\
21,000 \\
1,000 \\
4,000 \\
1,000 \\
2,000 \\
2,000 \\
35,000\end{array}$ & $\begin{array}{l}\text { Gram positive } \\
\text { Gram negative, non-fermentative } \\
\text { Broad spectrum }\end{array}$ & $\begin{array}{l}2,200,000 \\
19,000 \\
8,900,000\end{array}$ \\
\hline $502^{\mathrm{a}}$ & $\begin{array}{l}\text { Alternaria alternata } \\
\text { Aureobasidium pullulans } \\
\text { Biopolaris australiensis } \\
\text { Cladosporium cladosporioides } \\
\text { Epicoccum nigrum } \\
\text { Nonsporulating fungi } \\
\text { Yeasts other } \\
\text { Total fungi }\end{array}$ & $\begin{array}{l}93 \\
370 \\
190 \\
1,900 \\
470 \\
280 \\
280 \\
3,600\end{array}$ & $\begin{array}{l}\text { Gram positive } \\
\text { Gram negative, non-fermentative } \\
\text { Broad spectrum }\end{array}$ & $\begin{array}{l}380,000 \\
1,900 \\
2,700,000\end{array}$ \\
\hline $503^{c}$ & $\begin{array}{l}\text { Alternaria alternata } \\
\text { Aureobasidium pullulans } \\
\text { Biopolaris australiensis } \\
\text { Cladosporium cladosporioides } \\
\text { Epicoccum nigrum } \\
\text { Total fungi }\end{array}$ & $\begin{array}{l}88 \\
350 \\
88 \\
1,600 \\
530 \\
2,700\end{array}$ & $\begin{array}{l}\text { Gram positive } \\
\text { Broad spectrum }\end{array}$ & $\begin{array}{l}650,000 \\
560,000\end{array}$ \\
\hline $505^{\mathrm{c}}$ & $\begin{array}{l}\text { Alternaria alternata } \\
\text { Aspergillus fumigates } \\
\text { Biopolaris australiensis }\end{array}$ & $\begin{array}{l}880 \\
880 \\
880\end{array}$ & $\begin{array}{l}\text { Gram positive } \\
\text { Gram negative, non-fermentative } \\
\text { Broad spectrum }\end{array}$ & $\begin{array}{l}120,000 \\
3,800 \\
210,000\end{array}$ \\
\hline
\end{tabular}




\begin{tabular}{|c|c|c|c|c|}
\hline$T_{A B}$ & $\begin{array}{l}\text { (CONTINUED) } \\
\text { Cladosporium cladosporioides } \\
\text { Epicoccum nigrum } \\
\text { Nigrospora sphaerica } \\
\text { Total fungi }\end{array}$ & $\begin{array}{l}3,500 \\
2,600 \\
880 \\
9,600\end{array}$ & & \\
\hline $515^{\mathrm{c}}$ & $\begin{array}{l}\text { Alternaria alternata } \\
\text { Aspergillus niger } \\
\text { Biopolaris australiensis } \\
\text { Cladosporium cladosporioides } \\
\text { Epicoccum nigrum } \\
\text { Fusarium oxysporum } \\
\text { Nigrospora sphaerica } \\
\text { Total fungi }\end{array}$ & $\begin{array}{l}88 \\
270 \\
180 \\
710 \\
180 \\
88 \\
88 \\
1,600\end{array}$ & $\begin{array}{l}\text { Gram positive } \\
\text { Gram negative, non-fermentative } \\
\text { Broad spectrum }\end{array}$ & $\begin{array}{l}360,000 \\
3,800 \\
380,000\end{array}$ \\
\hline $516^{c}$ & $\begin{array}{l}\text { Alternaria alternata } \\
\text { Aspergillus niger } \\
\text { Cladosporium cladosporioides } \\
\text { Nonsporulating fungi } \\
\text { Total fungi }\end{array}$ & $\begin{array}{l}200 \\
300 \\
1,600 \\
400 \\
2,500\end{array}$ & $\begin{array}{l}\text { Gram positive } \\
\text { Gram negative, non-fermentative } \\
\text { Broad spectrum }\end{array}$ & $\begin{array}{l}1,000,000 \\
8,700 \\
360,000\end{array}$ \\
\hline $520^{b}$ & $\begin{array}{l}\text { Aspergillus versicolor } \\
\text { Cladosporium cladosporioides } \\
\text { Curvularia lunata } \\
\text { Epicoccum nigrum } \\
\text { Nigrospora sphaerica } \\
\text { Nonsporulating fungi } \\
\text { Total fungi }\end{array}$ & $\begin{array}{l}390 \\
490 \\
98 \\
290 \\
98 \\
200 \\
1,600\end{array}$ & $\begin{array}{l}\text { Gram positive } \\
\text { Gram negative, non-fermentative } \\
\text { Broad spectrum }\end{array}$ & $\begin{array}{l}5,900 \\
2,600 \\
19,000\end{array}$ \\
\hline $610^{\mathrm{c}}$ & $\begin{array}{l}\text { Alternaria alternata } \\
\text { Aureobasidium pullulans } \\
\text { Biopolaris australiensis } \\
\text { Cladosporium cladosporioides } \\
\text { Epicoccum nigrum } \\
\text { Nonsporulating fungi } \\
\text { Trichoderma koningii } \\
\text { Total fungi }\end{array}$ & $\begin{array}{l}960 \\
960 \\
960 \\
5,800 \\
2,900 \\
1,900 \\
960 \\
14,000\end{array}$ & $\begin{array}{l}\text { Gram positive } \\
\text { Gram negative, non-fermentative } \\
\text { Broad spectrum }\end{array}$ & $\begin{array}{l}290,000 \\
2,000 \\
4,000,000\end{array}$ \\
\hline $612^{\mathrm{c}}$ & $\begin{array}{l}\text { Biopolaris australiensis } \\
\text { Cladosporium cladosporioides } \\
\text { Epicoccum nigrum } \\
\text { Mucor plumbeus } \\
\text { Total fungi }\end{array}$ & $\begin{array}{l}980 \\
4,900 \\
2,000 \\
980 \\
8,800\end{array}$ & $\begin{array}{l}\text { Gram positive } \\
\text { Gram negative, non-fermentative } \\
\text { Broad spectrum }\end{array}$ & $\begin{array}{l}12,000,000 \\
5,200 \\
810,000\end{array}$ \\
\hline $618^{c}$ & $\begin{array}{l}\text { Alternaria alternata } \\
\text { Aspergillus fumigates } \\
\text { Aureobasidium pullulans } \\
\text { Biopolaris australiensis } \\
\text { Cladosporium cladosporioides } \\
\text { Epicoccum nigrum } \\
\text { Nonsporulating fungi }\end{array}$ & $\begin{array}{l}93 \\
93 \\
190 \\
93 \\
1,300 \\
470 \\
93\end{array}$ & $\begin{array}{l}\text { Gram positive } \\
\text { Gram negative, non-fermentative } \\
\text { Broad spectrum }\end{array}$ & $\begin{array}{l}550,000 \\
170,000 \\
46,000,000\end{array}$ \\
\hline
\end{tabular}




\begin{tabular}{|c|c|c|c|c|}
\hline TABLE & $\begin{array}{l}\text { (CONTINUED) } \\
\text { Trichoderma koningii } \\
\text { Total fungi }\end{array}$ & $\begin{array}{l}93 \\
2,400\end{array}$ & & \\
\hline $623^{c}$ & $\begin{array}{l}\text { Aureobasidium pullulans } \\
\text { Biopolaris australiensis } \\
\text { Cladosporium cladosporioides } \\
\text { Epicoccum nigrum } \\
\text { Total fungi }\end{array}$ & $\begin{array}{l}460 \\
92 \\
1,500 \\
460 \\
2,500\end{array}$ & $\begin{array}{l}\text { Gram positive } \\
\text { Gram negative, non-fermentative } \\
\text { Broad spectrum }\end{array}$ & $\begin{array}{l}280,000 \\
32,000 \\
220,000\end{array}$ \\
\hline MR-1 $A^{d}$ & $\begin{array}{l}\text { Alternaria alternata } \\
\text { Aspergillus niger } \\
\text { Aspergillus ochraceus } \\
\text { Aspergillus versicolor } \\
\text { Cladosporium cladosporioides } \\
\text { Fusarium oxysporum } \\
\text { Paecilomyces variotii } \\
\text { Penicillium crustosum } \\
\text { Total fungi }\end{array}$ & $\begin{array}{l}86 \\
86 \\
170 \\
340 \\
170 \\
170 \\
86 \\
260 \\
1,400\end{array}$ & $\begin{array}{l}\text { Gram positive } \\
\text { Gram negative, non-fermentative } \\
\text { Broad spectrum }\end{array}$ & $\begin{array}{l}6,000 \\
86 \\
26,000\end{array}$ \\
\hline MR-1B & $\begin{array}{l}\text { Aspergillus niger } \\
\text { Cladosporium cladosporioides } \\
\text { Epicoccum nigru } \\
\text { Trichoderma koningii } \\
\text { Total fungi }\end{array}$ & $\begin{array}{l}93 \\
370 \\
93 \\
93 \\
650\end{array}$ & $\begin{array}{l}\text { Gram positive } \\
\text { Gram negative, non-fermentative } \\
\text { Broad spectrum }\end{array}$ & $\begin{array}{l}21,000,000 \\
1,600 \\
350,000\end{array}$ \\
\hline$M R-2^{e}$ & $\begin{array}{l}\text { Alternaria alternata } \\
\text { Aspergillus versicolor } \\
\text { Cladosporium cladosporioides } \\
\text { Epicoccum nigrum } \\
\text { Fusarium oxysporum } \\
\text { Nonsporulating fungi } \\
\text { Trichoderma koningii } \\
\text { Total fungi }\end{array}$ & $\begin{array}{l}1,700 \\
860 \\
17,000 \\
5,200 \\
860 \\
860 \\
860 \\
28,000\end{array}$ & $\begin{array}{l}\text { Gram positive } \\
\text { Gram negative, non-fermentative } \\
\text { Broad spectrum }\end{array}$ & $\begin{array}{l}2,000,000 \\
110,000 \\
4,100,000\end{array}$ \\
\hline
\end{tabular}

${ }^{*}$ Room 108 was excluded because we were unable to collect enough dust for microbial analyses. $\dagger c f u / g$ : colony forming units per gram (Total fungi values have been rounded by the reporting laboratory.) Note: MR-1A, MR-1B = library - samples collected from wood floor edge; MR-2 = library - samples collected from rugs; ${ }^{\text {a }}$ carpet; ${ }^{\mathrm{b}}$ tile; ${ }^{\mathrm{c}}$ carpet with partial tile floor; ${ }^{\mathrm{d}}$ wood floor; ${ }^{\mathrm{e}}$ rug 
TABLES (CONTINUED)

Table 2. Arithmetic mean and standard deviation of water activity on the floor surface, and relative humidity and temperature in air by school building wing, July 2009

\begin{tabular}{|c|c|c|c|c|}
\hline Building wing & $\begin{array}{c}\text { Number of } \\
\text { samples }\end{array}$ & Water activity & $\begin{array}{c}\text { Relative humidity } \\
(\%)\end{array}$ & Temperature $\left({ }^{\circ} \mathrm{F}\right)$ \\
\hline Gym/cafeteria & 1 & $0.60(-)^{*}$ & $53.0(-)$ & $73.0(-)$ \\
\hline Administration & 5 & $0.76(0.09)$ & $59.0(9.4)$ & $71.6(0.7)$ \\
\hline Media center & 3 & $0.73(0.03)$ & $55.0(1.7)$ & $72.1(0.2)$ \\
\hline $\mathrm{k} / 1$ & 4 & $0.57(0.07)$ & $36.8(2.2)$ & $72.4(1.4)$ \\
\hline $2 / 3$ & 4 & $0.66(0.10)$ & $38.8(5.7)$ & $72.1(0.4)$ \\
\hline $4 / 5$ & 6 & $0.69(0.06)$ & $53.8(3.6)$ & $72.8(0.7)$ \\
\hline
\end{tabular}

${ }^{*}$ Values in parentheses represent standard deviations

Table 3. Overall building floor dust concentrations (cfu/g*) of culturable fungi and bacteria, July 2009

\begin{tabular}{|c|c|c|c|c|c|}
\hline $\begin{array}{c}\mathrm{N}=22 \text { dust } \\
\text { samples }\end{array}$ & Total fungi & $\begin{array}{c}\text { Hydrophilic } \\
\text { fungi }\end{array}$ & $\begin{array}{c}\text { Mesophilic or } \\
\text { hydrophilic } \\
\text { fungi }\end{array}$ & $\begin{array}{c}\text { Gram negative } \\
\text { bacteria }\end{array}$ & $\begin{array}{c}\text { Gram positive } \\
\text { bacteria }\end{array}$ \\
\hline Minimum & 650 & 86 & 650 & 86 & 5,900 \\
\hline Maximum & 35,000 & 7,800 & 31,000 & $5,600,000$ & $21,000,000$ \\
\hline $\begin{array}{c}\text { Geometric } \\
\text { mean }\end{array}$ & 6,000 & 1,200 & 5,200 & 8,500 & 396,000 \\
\hline $\begin{array}{c}\text { Geometric } \\
\text { standard } \\
\text { deviation }\end{array}$ & 3.3 & 4.2 & 3.3 & 8.1 & 7.0 \\
\hline
\end{tabular}

${ }^{*}$ Colony forming units per gram

Table 4. Floor dust concentrations (cfu/g*) of culturable fungi and bacteria by wing, July 2009

\begin{tabular}{|c|c|c|c|c|c|}
\hline Building wing & $\mathrm{N}$ & $\begin{array}{c}\text { Total } \\
\text { fungi }\end{array}$ & $\begin{array}{c}\text { Hydrophilic } \\
\text { fungi }\end{array}$ & $\begin{array}{c}\text { Gram negative } \\
\text { bacteria }\end{array}$ & $\begin{array}{c}\text { Gram positive } \\
\text { bacteria }\end{array}$ \\
\hline Gym/cafeteria & 1 & $24,000(-) \dagger$ & $4,000(-)$ & $7,700(-)$ & $170,000(-)$ \\
\hline Administration & 4 & $6,300(2.9)$ & $1,000(4.3)$ & $5,900(1.2)$ & $267,900(2.7)$ \\
\hline Media center & 3 & $2,900(7.3)$ & $500(11.1)$ & $2,500(37.7)$ & $631,600(66.7)$ \\
\hline $\mathrm{k} / 1$ & 4 & $5,200(2.4)$ & $1,500(3.0)$ & $15,800(7.1)$ & $855,600(6.0)$ \\
\hline $2 / 3$ & 4 & $23,500(1.3)$ & $4,700(1.5)$ & $52,000(23.4)$ & $660,100(4.2)$ \\
\hline $4 / 5$ & 6 & $2,900(2.0)$ & $600(3.1)$ & $3,900(1.7)$ & $199,500(6.5)$ \\
\hline
\end{tabular}

* Colony forming units per gram

†Values in parentheses represent geometric standard deviations 
TABLES (CONTINUED)

Table 5. Results of floor dust fungal sampling collected by environmental consultant, May 2009, and NIOSH, July 2009

\begin{tabular}{|c|c|c|}
\hline $\begin{array}{l}\text { Room number } \\
\text { and wing }\end{array}$ & $\begin{array}{l}\text { Consultant }-05 / 08 / 2009 \\
\left(\mathrm{cfu} / \mathrm{g}^{*}\right)\end{array}$ & $\begin{array}{l}\text { NIOSH - July } 2009 \\
(\mathrm{cfu} / \mathrm{g} *)\end{array}$ \\
\hline $\begin{array}{l}115 \\
\text { in administration wing }\end{array}$ & Total $(30,000)$ & $\begin{array}{l}\text { Aspergillus versicolor }(960) \\
\text { Aspergillus pullulans }(960) \\
\text { Biopolaris australiensis }(1,900) \\
\text { Cladosporium cladosporioides }(13,000) \\
\text { Epicoccum nigrum }(2,900) \\
\text { Nonsporulating fungi }(1,900) \\
\text { Penicillium lividium }(960) \\
\text { Total } \mathbf{( 2 3 , 0 0 0 )}\end{array}$ \\
\hline $\begin{array}{l}206 \\
\text { in gym/cafeteria wing }\end{array}$ & $\begin{array}{l}\text { Alternaria } \\
\text { Aspergillus } \\
\text { Epicoccum } \\
\text { Eurotium } \\
\text { Nonsporulating fungi } \\
\text { Penicillium } \\
\text { Yeast } \\
\text { Total (> } \mathbf{1 . 3} \text { million) }\end{array}$ & $\begin{array}{l}\text { Alternaria alternata }(1,000) \\
\text { Aspergillus glaucus }(2,000) \\
\text { Aspergillus niger }(1,000) \\
\text { Aspergillus versicolor }(1,000) \\
\text { Biopolaris australiensis }(1,000) \\
\text { Cladosporium cladosporioides }(14,000) \\
\text { Epicoccum nigrum }(2,000) \\
\text { Fusarium oxysporum }(1,000) \\
\text { Rhizopus stolonifer }(1,000) \\
\text { Total }(\mathbf{2 4 , 0 0 0 )}\end{array}$ \\
\hline $\begin{array}{l}421 \\
\text { in } 2 / 3 \text { wing }\end{array}$ & $\begin{array}{l}\text { Alternaria }(5,000) \\
\text { Aspergillus }(65,000) \\
\text { Bipolaris }(5,000) \\
\text { Cladosporium }(75,000) \\
\text { Curvularia }(5,000) \\
\text { Epicoccum }(60,000) \\
\text { Exserohilium }(5,000) \\
\text { Nonsporulating fungi }(70,000) \\
\text { Yeast }(40,000) \\
\text { Total }(\mathbf{3 3 0 , 0 0 0 )}\end{array}$ & $\begin{array}{l}\text { Alternaria alternata }(920) \\
\text { Aspergillus pullulans }(4,600) \\
\text { Biopolaris australiensis }(1,800) \\
\text { Cladosporium cladosporioides }(4,600) \\
\text { Epicoccum nigrum }(5,500) \\
\text { Nonsporulating fungi }(2,800)\end{array}$ \\
\hline $\begin{array}{l}503 \\
\text { in } 4 / 5 \text { wing }\end{array}$ & $\begin{array}{l}\text { Acremonium }(8,000) \\
\text { Alternaria }(40,000) \\
\text { Bipolaris }(4,000) \\
\text { Cladosporium }(36,000) \\
\text { Epicoccum }(48,000) \\
\text { Nonsporulating fungi }(60,000) \\
\text { Phoma }(12,000) \\
\text { Trichoderma }(12,000) \\
\text { Yeast }(24,000) \\
\text { Total }(\mathbf{2 4 4 , 0 0 0 )}\end{array}$ & $\begin{array}{l}\text { Alternaria alternata }(88) \\
\text { Aspergillus pullulans }(350) \\
\text { Biopolaris australiensis }(88) \\
\text { Cladosporium cladosporioides }(1,600) \\
\text { Epicoccum nigrum }(530)\end{array}$ \\
\hline $\begin{array}{l}515 \\
\text { in } 4 / 5 \text { wing }\end{array}$ & $\begin{array}{l}\text { Alternaria } \\
\text { Aspergillus } \\
\text { Aureobasidium }\end{array}$ & $\begin{array}{l}\text { Alternaria alternata }(88) \\
\text { Aspergillus niger }(270) \\
\text { Biopolaris australiensis }(180)\end{array}$ \\
\hline
\end{tabular}




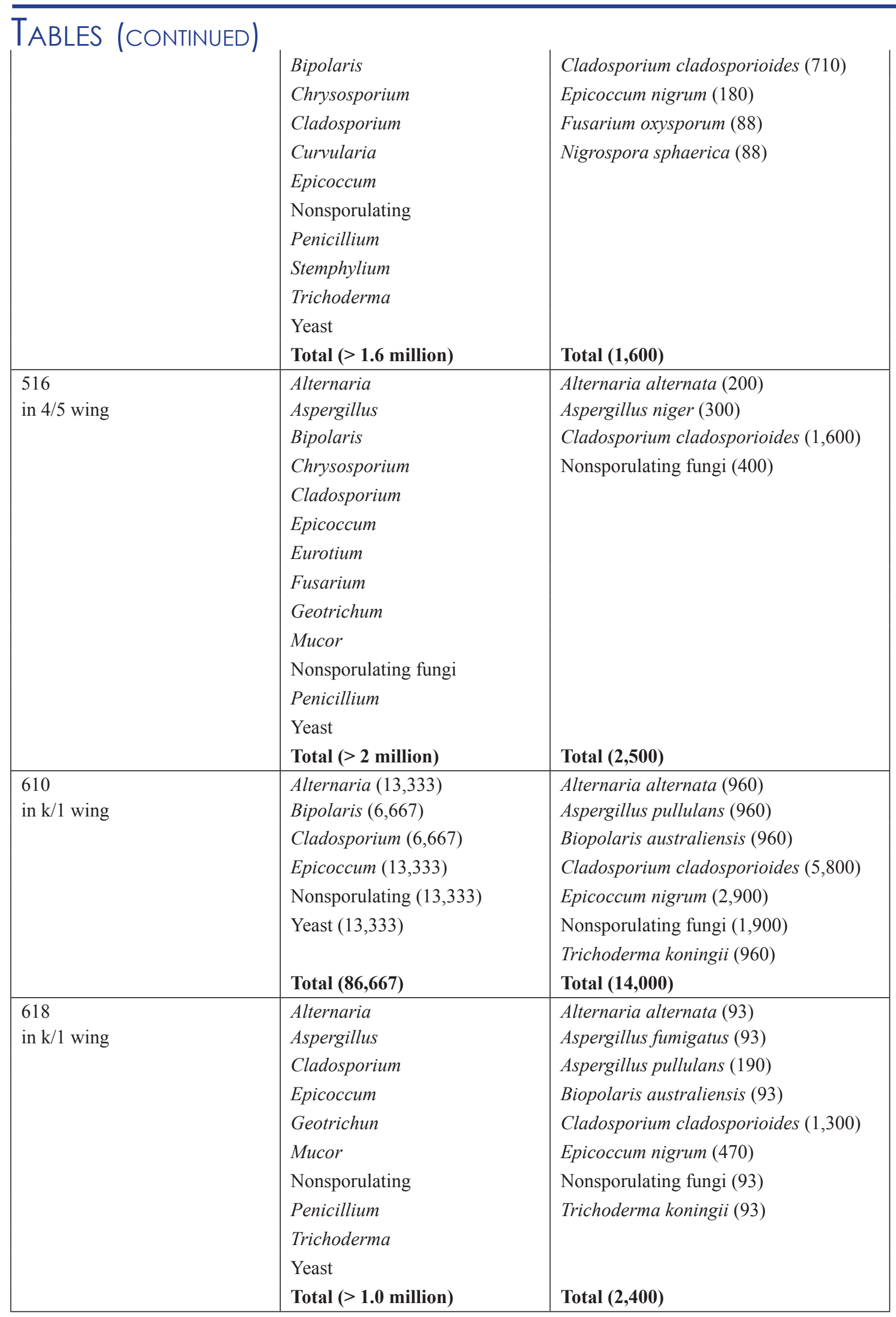

*cfu/g: colony forming units per gram (values preceded by '>' symbol were too numerous to count - no individual fungal species counts were made) 
TABLES (CONTINUED)

Table 6. Results of floor dust fungal sampling collected by environmental consultants, October 2007, September 2008, and October 2008, and by NIOSH, July 2009

\begin{tabular}{|c|c|c|c|c|}
\hline $\begin{array}{l}\text { Room } \\
\text { number }\end{array}$ & $\begin{array}{c}\text { Consultant - Oct } 2007 \\
\left(\mathrm{cfu} / \mathrm{g}^{*}\right)\end{array}$ & $\begin{array}{l}\text { Consultant - Sept } \\
2008\left(\mathrm{cfu} / \mathrm{g}^{*}\right)\end{array}$ & $\begin{array}{c}\text { Consultant - Oct } 2008 \\
\left(\mathrm{cfu} / \mathrm{g}^{*}\right)\end{array}$ & $\begin{array}{l}\text { NIOSH - July } 2009 \\
\left(\mathrm{cfu} / \mathrm{g}^{*}\right)\end{array}$ \\
\hline 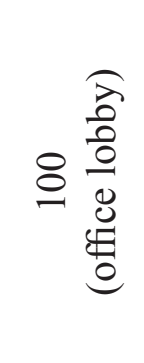 & $\begin{array}{l}\text { Acremonium }(380,000) \\
\text { Cladosporium }(290,000) \\
\text { Curvularia lunata }(48,000) \\
\text { Epicoccum nigrum }(95,000) \\
\text { Fusarium solani }(95,000) \\
\text { Total } \mathbf{( 9 0 8 , 0 0 0 )}\end{array}$ & & & $\begin{array}{l}\text { Alternaria alternata }(2,000) \\
\text { Cladosporium cladosporioides }(4,900) \\
\text { Epicoccum nigrum }(2,000) \\
\text { Fusarium oxysporum }(980) \\
\text { Total } \mathbf{( 9 , 8 0 0 )}\end{array}$ \\
\hline 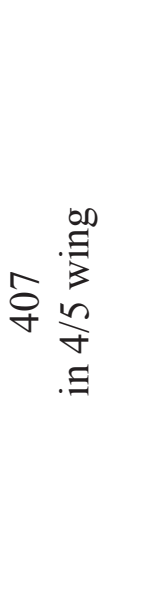 & & $\begin{array}{l}\text { Alternaria }(200,000) \\
\text { Aspergillus }(320,000) \\
\text { Cladosporium }(40,000) \\
\text { Bipolaris }(40,000) \\
\text { Epicoccum }(20,000) \\
\text { Penicillium }(20,000) \\
\text { Phoma }(600,000) \\
\text { Wallemia }(60,000) \\
\text { Total }(\mathbf{1 , 3 0 0 , 0 0 0 )}\end{array}$ & $\begin{array}{l}\text { Alternaria }(1,081) \\
\text { Aspergillus }(17,838) \\
\text { Aureobasidium }(1,081) \\
\text { Cladosporium }(4,865) \\
\text { Bipolaris }(1,622) \\
\text { Epicoccum }(6,486) \\
\text { Wallemia }(541) \\
\text { Yeast }(1,081) \\
\text { Nonsporulating fungi }(541) \\
\text { Total }(\mathbf{3 5 , 1 3 5 )}\end{array}$ & $\begin{array}{l}\text { Alternaria alternata }(1,900) \\
\text { Aspergillus glaucus }(2,800) \\
\text { Aspergillus niger }(940) \\
\text { Aspergillus ochraceus }(940) \\
\text { Cladosporium cladosporioides }(7,500) \\
\text { Epicoccum nigrum }(1,900) \\
\text { Nonsporulating fungi }(1,9001)\end{array}$ \\
\hline
\end{tabular}

${ }^{*} \mathrm{cfu} / \mathrm{g}$ : colony forming units per gram 


\section{TABLES (CONTINUED)}

Table 7. Primary work area or homeroom wing during 2008/2009 school year; health department survey, May 2009

\begin{tabular}{|c|c|c|}
\hline Wing & $\begin{array}{c}\text { Staff } \\
\mathrm{n}=48^{*}\end{array}$ & $\begin{array}{c}\text { Students } \\
\mathrm{n}=376 \dagger\end{array}$ \\
\hline $\mathrm{k} / 1$ & $18(38 \%)$ & $111(30 \%)$ \\
\hline $2 / 3$ & $13(27 \%)$ & $136(36 \%)$ \\
\hline $4 / 5$ & $9(19 \%)$ & $129(34 \%)$ \\
\hline Non-classroom $\leftarrow$ & $8(17 \%)$ & - \\
\hline
\end{tabular}

${ }^{*}$ Four of the 52 staff participants did not indicate their primary work location.

tWe could not classify the primary classroom wing for 9 of the 385 students whose parents responded to the questionnaire. $\ddagger g y m /$ cafeteria wing, administration wing, or media center wing

Table 8. Prevalence of health conditions; health department survey, May 2009

\begin{tabular}{|l|c|c|}
\hline & $\begin{array}{c}\text { Staff } \\
\mathrm{n}=50^{*}\end{array}$ & $\begin{array}{c}\text { Students } \\
\mathrm{n}=276 \dagger\end{array}$ \\
\hline Seasonal allergies & $17(34 \%)$ & $132(48 \%)$ \\
\hline Asthma & $4(8 \%)$ & $44(16 \%)$ \\
\hline Allergic rhinitis & $4(8 \%)$ & $33(12 \%)$ \\
\hline Chronic respiratory disease & 0 & $4(1 \%)$ \\
\hline Suppressed immune system & $1(2 \%)$ & $2(0.7 \%)$ \\
\hline Cystic fibrosis & $1(2 \%)$ & 0 \\
\hline Radiation or chemotherapy & 0 & 0 \\
\hline
\end{tabular}

${ }^{*}$ Data missing for 2 of the 52 staff participants.

$\nmid$ Data missing for 111 of the 387 students whose parents participated in the questionnaire. 
TABLES (CONTINUED)

Table 9. Prevalence of staff symptoms reported to occur while in the school building during the 2008/2009 school year, by primary work wing*; health department survey, May 2009

\begin{tabular}{|c|c|c|c|c|c|}
\hline & $\begin{array}{c}\text { Overall } \\
n=52\end{array}$ & $\begin{array}{c}\mathrm{k} / 1 \text { wing } \\
\mathrm{n}=18\end{array}$ & $\begin{array}{c}4 / 5 \text { wing } \\
n=9\end{array}$ & $\begin{array}{c}2 / 3 \text { wing } \\
n=13\end{array}$ & $\begin{array}{c}\text { Non- } \\
\text { classroom } \\
\text { wings } \$ \\
n=8\end{array}$ \\
\hline Headache $\dagger$ & $48(92 \%)$ & $18(100 \%)$ & $8(89 \%)$ & $12(92 \%)$ & $7(100 \%)$ \\
\hline Eye symptoms & $39(75 \%)$ & $15(83 \%)$ & $8(89 \%)$ & $9(69 \%)$ & $4(57 \%)$ \\
\hline Fatigue & $23(44 \%)$ & $9(50 \%)$ & $6(67 \%)$ & $5(38 \%)$ & $1(14 \%)$ \\
\hline Throat symptoms & $12(23 \%)$ & $5(28 \%)$ & 0 & $6(46 \%)$ & 0 \\
\hline $\begin{array}{l}\text { Breathing difficulty/ } \\
\text { asthma } \dagger\end{array}$ & $16(31 \%)$ & $4(22 \%)$ & $4(44 \%)$ & $5(38 \%)$ & $3(43 \%)$ \\
\hline Unusual sneezing $\dagger$ & $16(31 \%)$ & $4(22 \%)$ & $6(67 \%)$ & $3(23 \%)$ & $3(38 \%)$ \\
\hline Nosebleed $\dagger$ & $13(25 \%)$ & $4(22 \%)$ & $2(22 \%)$ & $3(23 \%)$ & $2(29 \%)$ \\
\hline Sinus problems & $13(25 \%)$ & $2(11 \%)$ & $2(22 \%)$ & $6(46 \%)$ & $3(43 \%)$ \\
\hline Nausea & $7(13 \%)$ & $2(11 \%)$ & $1(11 \%)$ & $3(23 \%)$ & $1(14 \%)$ \\
\hline Cough & $8(15 \%)$ & $2(11 \%)$ & $2(22 \%)$ & $3(23 \%)$ & $1(14 \%)$ \\
\hline Skin problems & $10(19 \%)$ & $1(6 \%)$ & $3(33 \%)$ & $4(31 \%)$ & $1(14 \%)$ \\
\hline Dizziness & $11(21 \%)$ & $6(33 \%)$ & $1(11 \%)$ & $3(23 \%)$ & $1(14 \%)$ \\
\hline Memory loss & $7(13 \%)$ & $1(6 \%)$ & $1(11 \%)$ & $3(23 \%)$ & $1(14 \%)$ \\
\hline Nasal symptoms & $5(10 \%)$ & 0 & 0 & $3(23 \%)$ & $1(1 \%)$ \\
\hline Metallic taste & $4(8 \%)$ & 0 & $1(11 \%)$ & $2(15 \%)$ & $1(14 \%)$ \\
\hline Voice symptoms & $5(10 \%)$ & $1(6 \%)$ & $2(22 \%)$ & $2(15 \%)$ & 0 \\
\hline Insomnia & $3(6 \%)$ & 0 & $1(11 \%)$ & $2(15 \%)$ & 0 \\
\hline Vision problems & $4(8 \%)$ & $1(6 \%)$ & $2(22 \%)$ & 0 & 0 \\
\hline Allergies & $1(2 \%)$ & 0 & 0 & 0 & $1(14 \%)$ \\
\hline $\begin{array}{l}\text { Throat or voice } \\
\text { symptoms }\end{array}$ & $14(27 \%)$ & $5(28 \%)$ & $2(22 \%)$ & $6(46 \%)$ & 0 \\
\hline $\begin{array}{l}\text { Lower respiratory } \\
\text { symptoms }\end{array}$ & $20(38 \%)$ & $5(28 \%)$ & $5(56 \%)$ & $7(54 \%)$ & $3(43 \%)$ \\
\hline $\begin{array}{l}\text { Upper respiratory } \\
\text { symptoms }\end{array}$ & $27(52 \%)$ & $7(39 \%)$ & $7(78 \%)$ & $9(69 \%)$ & $3(43 \%)$ \\
\hline $\begin{array}{l}\text { Mucous membrane } \\
\text { irritation }\end{array}$ & $45(87 \%)$ & $16(89 \%)$ & $9(100 \%)$ & $13(100 \%)$ & $4(57 \%)$ \\
\hline
\end{tabular}

${ }^{*}$ Four of the 52 staff participants did not indicate their primary work location.

†Symptoms specifically asked about in the questionnaire

$\ddagger$ Gym/cafeteria wing, administration wing, or media center wing 


\section{TABLES (CONTINUED)}

Table 10. Prevalence of student symptoms reported to occur while in the school building during the 2008/2009 school year, overall and by homeroom wing*; health department survey, May 2009

\begin{tabular}{|c|c|c|c|c|}
\hline & $\begin{array}{l}\text { Overall } \\
n=385\end{array}$ & $\begin{array}{c}\mathrm{k} / 1 \text { wing } \\
\mathrm{n}=111\end{array}$ & $\begin{array}{c}2 / 3 \text { wing } \\
n=136\end{array}$ & $\begin{array}{c}4 / 5 \text { wing } \\
n=129\end{array}$ \\
\hline Headache $\dagger$ & $187(49 \%)$ & $40(36 \%)$ & $72(53 \%)$ & $72(56 \%)$ \\
\hline Eye symptoms & $106(28 \%)$ & $22(20 \%)$ & $41(30 \%)$ & $41(32 \%)$ \\
\hline Fatigue & $23(6 \%)$ & $8(7 \%)$ & $5(4 \%)$ & $10(8 \%)$ \\
\hline Throat symptoms & $19(5 \%)$ & $4(4 \%)$ & $6(4 \%)$ & $7(5 \%)$ \\
\hline $\begin{array}{l}\text { Breathing difficulty/ } \\
\text { asthma } \dagger\end{array}$ & $49(13 \%)$ & $9(8 \%)$ & $18(13 \%)$ & $20(16 \%)$ \\
\hline Usual sneezing $\dagger$ & $88(23 \%)$ & $19(17 \%)$ & $31(23 \%)$ & $36(28 \%)$ \\
\hline Nosebleed $\dagger$ & $77(20 \%)$ & $15(14 \%)$ & $35(26 \%)$ & $25(19 \%)$ \\
\hline Sinus problems & $18(5 \%)$ & $6(5 \%)$ & $7(5 \%)$ & $5(4 \%)$ \\
\hline Nausea & $16(4 \%)$ & $2(2 \%)$ & $7(5 \%)$ & $7(5 \%)$ \\
\hline Cough & $28(7 \%)$ & $9(8 \%)$ & $12(9 \%)$ & $5(4 \%)$ \\
\hline Skin problems & $34(9 \%)$ & $10(9 \%)$ & $11(8 \%)$ & $12(9 \%)$ \\
\hline Dizziness & $8(2 \%)$ & $1(1 \%)$ & $6(4 \%)$ & $1(0.8 \%)$ \\
\hline Memory loss & $1(0.3 \%)$ & 0 & 0 & $1(0.8 \%)$ \\
\hline Nasal symptoms & $20(5 \%)$ & $5(5 \%)$ & $10(7 \%)$ & $4(3 \%)$ \\
\hline Metallic taste & 0 & 0 & 0 & 0 \\
\hline Voice symptoms & 0 & 0 & 0 & 0 \\
\hline Insomnia & $3(0.8 \%)$ & 0 & $2(1 \%)$ & $1(0.8 \%)$ \\
\hline Vision problems & $6(2 \%)$ & $1(1 \%)$ & $2(1 \%)$ & $3(2 \%)$ \\
\hline Allergies & $9(2 \%)$ & $2(2 \%)$ & $6(4 \%)$ & $1(0.8 \%)$ \\
\hline Throat or voice symptoms & $19(5 \%)$ & $4(4 \%)$ & $6(4 \%)$ & $7(5 \%)$ \\
\hline $\begin{array}{l}\text { Lower respiratory } \\
\text { symptoms }\end{array}$ & $72(19 \%)$ & $17(15 \%)$ & $26(19 \%)$ & $25(19 \%)$ \\
\hline $\begin{array}{l}\text { Upper respiratory } \\
\text { symptoms }\end{array}$ & $106(28 \%)$ & $24(22 \%)$ & $39(29 \%)$ & $40(31 \%)$ \\
\hline $\begin{array}{l}\text { Mucous membrane } \\
\text { irritation }\end{array}$ & $123(32 \%)$ & $27(24 \%)$ & $45(33 \%)$ & $48(37 \%)$ \\
\hline
\end{tabular}

* We could not classify the primary classroom wing for 9 of the 385 participants.

†Symptoms specifically asked about in the questionnaire 
TABLES (CONTINUED)

Table 11. Characteristics of staff; NIOSH survey, March 2010

\begin{tabular}{|l|c|}
\hline \multicolumn{1}{|c|}{ Variable } & $\begin{array}{c}\text { Staff } \\
\mathrm{n}=70\end{array}$ \\
\hline Mean age, years (range) & $45(21-66)$ \\
\hline Mean years employed at school (range) & $7(0.04-30)$ \\
\hline Female, n (\%) & $59(84 \%)$ \\
\hline Smoking status & $4(6 \%)$ \\
Current smoker, n (\%) & $7(10 \%)$ \\
Former smoker, n (\%) & $59(84 \%)$ \\
Never smoker, n (\%) & \\
\hline Job category & $47(67 \%)$ \\
Teachers/student teachers/teacher assistants & $7(10 \%)$ \\
Office/administration staff & $7(10 \%)$ \\
Custodians & $9(13 \%)$ \\
Other & \\
\hline
\end{tabular}

Table 12. Primary work area of staff during the 2009/2010 school year; NIOSH survey, March 2010

\begin{tabular}{|l|c|}
\hline \multicolumn{1}{|c|}{ Wing } & $\begin{array}{c}\text { Staff } \\
\mathrm{n}=70\end{array}$ \\
\hline $\mathrm{k} / 1$ & $17(24 \%)$ \\
\hline $2 / 3$ & $17(24 \%)$ \\
\hline $4 / 5$ & $13(19 \%)$ \\
\hline Administrative & $7(10 \%)$ \\
\hline Everywhere & $7(10 \%)$ \\
\hline Gym/cafeteria & $3(4 \%)$ \\
\hline Other* & $6(9 \%)$ \\
\hline
\end{tabular}

*More than one primary area but not 'everywhere'

Table 13. Work location of staff while the elementary school closed for remediation; NIOSH survey, March 2010

\begin{tabular}{|l|c|}
\hline & $\begin{array}{c}\text { Staff } \\
\mathrm{n}=70\end{array}$ \\
\hline Military academy & $25(36 \%)$ \\
\hline Other elementary school & $17(24 \%)$ \\
\hline Middle school & $18(26 \%)$ \\
\hline Other & $10(14 \%)$ \\
\hline
\end{tabular}


TABLES (CONTINUED)

Table 14. Participant reported health conditions ever-diagnosed by a physician; NIOSH survey, March 2010

\begin{tabular}{|l|c|}
\hline & $\begin{array}{c}\text { Staff } \\
\mathrm{n}=70\end{array}$ \\
\hline Sinusitis or sinus infections & $45(64 \%)$ \\
\hline Hay fever or nasal allergies & $24(34 \%)$ \\
\hline Acute bronchitis & $16(23 \%)$ \\
\hline Eczema, dermatitis, or skin allergy & $15(21 \%)$ \\
\hline Pneumonia & $9(13 \%)$ \\
\hline Ever asthma & $7(10 \%)$ \\
\hline Current asthma & $5(7 \%)$ \\
\hline Heart disease & $4(6 \%)$ \\
\hline Chronic bronchitis & $3(4 \%)$ \\
\hline
\end{tabular}




\section{TABLES (CONTINUED)}

Table 15. Prevalence of symptoms and their work-relatedness (portion of those with symptoms), health conditions, and medication usage during the four-week period prior to reporting to the elementary school for the February 2010 reopening; NIOSH survey, March 2010

\begin{tabular}{|l|c|c|}
\hline & $\begin{array}{c}\text { Staff } \\
\mathrm{n}=70\end{array}$ & $\begin{array}{c}\text { Work- } \\
\text { related }\end{array}$ \\
\hline Stuffy, itchy, or runny nose & $17(24 \%)$ & $4(24 \%)$ \\
\hline Dry or itchy skin & $15(21 \%)$ & $3(20 \%)$ \\
\hline Headache & $13(19 \%)$ & $2(15 \%)$ \\
\hline Sneezing & $11(16 \%)$ & 0 \\
\hline Sore or dry throat & $10(14 \%)$ & $2(20 \%)$ \\
\hline Cough with phlegm & $10(14 \%)$ & 0 \\
\hline Watery, itchy eyes & $9(13 \%)$ & $4(44 \%)$ \\
\hline Cough attacks & $5(7 \%)$ & $1(20 \%)$ \\
\hline Shortness of breath on level ground or up slight hill & $3(4 \%)$ & $1(33 \%)$ \\
\hline Chest tightness & $3(4 \%)$ & 0 \\
\hline Tiredness or fatigue & $3(4 \%)$ & 0 \\
\hline Nosebleed & $3(4 \%)$ & 0 \\
\hline Shortness of breath attacks & $2(3 \%)$ & 0 \\
\hline Fever and chills & $2(3 \%)$ & 0 \\
\hline Chest wheezing & $2(3 \%)$ & 0 \\
\hline Difficulty remembering things or concentrating & $2(3 \%)$ & 0 \\
\hline Dizziness or lightheadedness & $1(1 \%)$ & 0 \\
\hline Awakened by breathing difficulty & $1(1 \%)$ & 0 \\
\hline Flu-like achiness or achy joints & 0 & 0 \\
\hline Cold & $13(19 \%)$ & - \\
\hline Sinusitis or sinus problems & $13(19 \%)$ & - \\
\hline Bronchitis & $4(6 \%)$ & - \\
\hline Upper respiratory symptoms* & $27(39 \%)$ & - \\
\hline Mucous membrane irritation $\dagger$ & $23(33 \%)$ & - \\
\hline Lower respiratory symptoms & $15(21 \%)$ & - \\
\hline Constitutional / nonspecific symptoms§ & $14(20 \%)$ & - \\
\hline Used any medications for breathing problems & $5(7 \%)$ & - \\
\hline Used any inhaled beta-agonists & $4(6 \%)$ & - \\
\hline Used any medications for nasal-sinus or eye problems & $22(31 \%)$ & - \\
\hline Oner or & $22 \%)$ \\
\hline
\end{tabular}

${ }^{*}$ One or more of the following symptoms: stuffy, itchy, or runny nose; sneezing; sore or dry throat; sinusitis or sinus problems

$\dagger$ One or more of the following symptoms: stuffy, itchy, or runny nose; watery, itchy eyes; or sore or dry throat $\ddagger$ One or more of the following symptoms: chest wheezing; chest tightness; shortness of breath attacks; coughing attacks; awakened by breathing difficulty; shortness of breath on exertion (hurrying on level ground or walking up hill); cough with phlegm

§One or more of the following symptoms: headache; tiredness or fatigue; fever and chills; difficulty remembering things or concentrating; dizziness or lightheadedness; or flu-like achiness or achy joints 


\section{TABLES (CONTINUED)}

Table 16. Prevalence of symptoms and their work-relatedness (portion of those with symptoms), health conditions, and medication usage during the four-week period prior to reporting to the elementary school for its February 2010 reopening, as reported by current staff, by temporary school location; NIOSH survey, March 2010

\begin{tabular}{|c|c|c|c|c|c|c|c|c|}
\hline & $\begin{array}{c}\text { Military } \\
\text { academy } \\
\text { n=25 }\end{array}$ & $\begin{array}{l}\text { Work- } \\
\text { related } \\
\text { Military } \\
\text { academy }\end{array}$ & $\begin{array}{c}\text { Other } \\
\text { elementary } \\
\text { school }\end{array}$ & $\begin{array}{c}\frac{\text { Work- }}{\text { related }} \\
\text { Other } \\
\text { elementary } \\
\text { school }\end{array}$ & $\begin{array}{c}\text { Middle } \\
\text { school } \\
\text { n=18 }\end{array}$ & $\begin{array}{l}\frac{\text { Work- }}{\text { related }} \\
\text { Middle } \\
\text { school }\end{array}$ & $\begin{array}{c}\text { Other } \\
\text { locations }\end{array}$ & $\begin{array}{c}\frac{\text { Work- }}{\text { related }} \\
\begin{array}{c}\text { Other } \\
\text { locations }\end{array}\end{array}$ \\
\hline Stuffy, itchy, or runny nose & $9(36 \%)$ & $3(33 \%)$ & $2(12 \%)$ & 0 & $5(28 \%)$ & 0 & $1(10 \%)$ & $1(100 \%)$ \\
\hline Dry or itchy skin & $5(20 \%)$ & $1(20 \%)$ & $3(18 \%)$ & 0 & $5(28 \%)$ & $1(20 \%)$ & $2(20 \%)$ & $1(50 \%)$ \\
\hline Headache & $6(24 \%)$ & $1(17 \%)$ & $3(18 \%)$ & $1(33 \%)$ & $2(11 \%)$ & 0 & $2(20 \%)$ & 0 \\
\hline Sneezing & $4(16 \%)$ & 0 & $3(18 \%)$ & 0 & $4(22 \%)$ & 0 & 0 & 0 \\
\hline Sore or dry throat & $6(24 \%)$ & $1(17 \%)$ & $2(12 \%)$ & 0 & $2(11 \%)$ & $1(50 \%)$ & 0 & 0 \\
\hline Cough with phlegm & $2(8 \%)$ & 0 & $3(18 \%)$ & 0 & $3(17 \%)$ & 0 & $2(20 \%)$ & 0 \\
\hline Watery, itchy eyes & $3(12 \%)$ & $3(100 \%)$ & $3(18 \%)$ & 0 & $2(11 \%)$ & 0 & $1(10 \%)$ & $1(100 \%)$ \\
\hline Cough attacks & $2(8 \%)$ & $1(50 \%)$ & $1(6 \%)$ & 0 & $1(6 \%)$ & 0 & $1(10 \%)$ & 0 \\
\hline $\begin{array}{l}\text { Shortness of breath on level } \\
\text { ground or up slight hill }\end{array}$ & $1(4 \%)$ & $1(100 \%)$ & 0 & 0 & $1(6 \%)$ & 0 & $1(10 \%)$ & 0 \\
\hline Chest tightness & 0 & 0 & $1(6 \%)$ & 0 & $1(6 \%)$ & 0 & $1(10 \%)$ & 0 \\
\hline Tiredness or fatigue & 0 & 0 & $1(6 \%)$ & 0 & $1(6 \%)$ & 0 & $1(10 \%)$ & 0 \\
\hline Shortness of breath attacks & 0 & 0 & $1(6 \%)$ & 0 & 0 & 0 & $1(10 \%)$ & 0 \\
\hline Fever and chills & 0 & 0 & $1(6 \%)$ & 0 & $1(6 \%)$ & 0 & 0 & 0 \\
\hline Chest wheezing & $1(4 \%)$ & 0 & $1(6 \%)$ & 0 & 0 & 0 & 0 & 0 \\
\hline Nosebleed & $2(8 \%)$ & 0 & $1(6 \%)$ & 0 & 0 & 0 & 0 & 0 \\
\hline $\begin{array}{l}\text { Difficulty remembering things } \\
\text { or concentrating }\end{array}$ & 0 & 0 & $1(6 \%)$ & 0 & 0 & 0 & $1(10 \%)$ & 0 \\
\hline Dizziness or lightheadedness & 0 & 0 & 0 & 0 & 0 & 0 & $1(10 \%)$ & 0 \\
\hline $\begin{array}{l}\text { Awakened by breathing } \\
\text { difficulty }\end{array}$ & 0 & 0 & 0 & 0 & 0 & 0 & $1(10 \%)$ & 0 \\
\hline Flu-like achiness or achy joints & 0 & 0 & 0 & 0 & 0 & 0 & 0 & 0 \\
\hline Cold & $5(20 \%)$ & - & $4(24 \%)$ & - & $3(17 \%)$ & - & $1(10 \%)$ & - \\
\hline Sinusitis or sinus problems & $4(16 \%)$ & - & $4(24 \%)$ & - & $3(17 \%)$ & - & $2(20 \%)$ & - \\
\hline Bronchitis & $1(4 \%)$ & - & $2(12 \%)$ & - & $1(6 \%)$ & - & 0 & - \\
\hline Upper respiratory symptoms* & $12(48 \%)$ & - & $5(29 \%)$ & - & $7(39 \%)$ & - & $3(30 \%)$ & - \\
\hline Mucous membrane irritation $\dagger$ & $10(40 \%)$ & - & $4(24 \%)$ & - & $7(39 \%)$ & - & $2(20 \%)$ & - \\
\hline Lower respiratory symptoms & $4(16 \%)$ & - & $4(24 \%)$ & - & $4(22 \%)$ & - & $3(30 \%)$ & - \\
\hline $\begin{array}{l}\text { Constitutional / nonspecific } \\
\text { symptoms } \S\end{array}$ & $6(24 \%)$ & - & $4(24 \%)$ & - & $2(11 \%)$ & - & $2(20 \%)$ & - \\
\hline $\begin{array}{l}\text { Used any medications for } \\
\text { breathing problems }\end{array}$ & $1(4 \%)$ & - & $1(6 \%)$ & - & $2(11 \%)$ & - & $1(10 \%)$ & - \\
\hline Used any inhaled beta-agonists & $1(4 \%)$ & - & $1(6 \%)$ & - & $1(6 \%)$ & - & $1(10 \%)$ & - \\
\hline $\begin{array}{l}\text { Used any medications for } \\
\text { nasal-sinus or eye problems }\end{array}$ & $7(28 \%)$ & - & $8(47 \%)$ & - & $5(28 \%)$ & - & $2(20 \%)$ & - \\
\hline
\end{tabular}

*One or more of the following symptoms: stuffy, itchy, or runny nose, sneezing, sore or dry throat, sinusitis or sinus problems † One or more of the following symptoms: stuffy, itchy, or runny nose; watery, itchy eyes, or sore or dry throat ¥One or more of the following symptoms: chest wheezing, chest tightness, shortness of breath attacks, coughing attacks, awakened by breathing difficulty, shortness of breath on exertion (hurrying on level ground or walking up hill), cough with phlegm $\S$ One or more of the following symptoms: headache; tiredness or fatigue; fever and chills; difficulty remembering things or concentrating; dizziness or lightheadedness; or flu-like achiness or achy joints 


\section{TABLES (CONTINUED)}

Table 17. Prevalence of staff health symptoms occurring one or more times per week during the four-week period before returning to the elementary school for its reopening; NIOSH survey, March 2010

\begin{tabular}{|c|c|c|}
\hline & $\begin{array}{l}\text { Current elementary } \\
\text { school staff } \\
\text { n=70 }\end{array}$ & $\begin{array}{c}\text { Current elementary } \\
\text { school staff who also } \\
\text { worked at the school } \\
\text { during the } 2008 / 2009 \\
\text { school year } \\
n=66\end{array}$ \\
\hline Dry or itchy skin & $15(21 \%)$ & $14(21 \%)$ \\
\hline Stuffy, itchy, or runny nose & $14(20 \%)$ & $13(20 \%)$ \\
\hline Sneezing & $10(14 \%)$ & $10(15 \%)$ \\
\hline Headache & $10(14 \%)$ & $10(15 \%)$ \\
\hline Watery, itchy eyes & $9(13 \%)$ & $8(12 \%)$ \\
\hline Cough with phlegm & $8(11 \%)$ & $8(12 \%)$ \\
\hline Sore or dry throat & $8(11 \%)$ & $8(12 \%)$ \\
\hline Cough attacks & $5(7 \%)$ & $5(8 \%)$ \\
\hline $\begin{array}{l}\text { Shortness of breath on exertion (hurrying on } \\
\text { level ground or walking up hill) }\end{array}$ & $3(4 \%)$ & $3(5 \%)$ \\
\hline Chest wheezing & $2(3 \%)$ & $2(3 \%)$ \\
\hline Chest tightness & $2(3 \%)$ & $2(3 \%)$ \\
\hline Tiredness or fatigue & $2(3 \%)$ & $2(3 \%)$ \\
\hline Difficulty remembering things or concentrating & $2(3 \%)$ & $2(3 \%)$ \\
\hline Nosebleed & $2(3 \%)$ & $2(3 \%)$ \\
\hline Shortness of breath attacks & $1(1 \%)$ & $1(2 \%)$ \\
\hline Awakened by breathing difficulty & 0 & 0 \\
\hline Fever and chills & 0 & 0 \\
\hline Flu-like achiness or achy joints & 0 & 0 \\
\hline Dizziness or lightheadedness & 0 & 0 \\
\hline Upper respiratory symptoms* & $19(27 \%)$ & $18(28 \%)$ \\
\hline Mucous membrane irritation $\uparrow$ & $18(26 \%)$ & $17(26 \%)$ \\
\hline Lower respiratory symptoms & $13(19 \%)$ & $13(20 \%)$ \\
\hline Constitutional / nonspecific symptoms§ & $10(14 \%)$ & $10(15 \%)$ \\
\hline
\end{tabular}

${ }^{*}$ One or more of the following symptoms: stuffy, itchy, or runny nose, sneezing, sore or dry throat, sinusitis or sinus problems

†One or more of the following symptoms: stuffy, itchy, or runny nose; watery, itchy eyes, or sore or dry throat.

¥One or more of the following symptoms: chest wheezing, chest tightness, shortness of breath attacks, coughing attacks, awakened by breathing difficulty, shortness of breath on exertion (hurrying on level ground or walking up hill), cough with phlegm.

§One or more of the following symptoms: headache; tiredness or fatigue; fever and chills; difficulty remembering things or concentrating; dizziness or lightheadedness; or flu-like achiness or achy joints. 


\section{TABLES (CONTINUED)}

Table 18. Symptom prevalence ratios* based on comparison with BASE survey; NIOSH survey, March 2010

\begin{tabular}{|l|c|}
\hline & Elementary school staff \\
\hline Sneeze & $\begin{array}{c}\text { Prevalence ratio } \\
(95 \% \mathrm{CI})+\dagger\end{array}$ \\
\hline Sore/dry throat & $0(0-0.5)$ \\
\hline Unusual tiredness, fatigue & $0.2(0.04-1.2)$ \\
\hline Wheezing or whistling in the chest & $0(0-0.4)$ \\
\hline Chest tightness & $0(0-3.0)$ \\
\hline Shortness of breath & $0(0-2.5)$ \\
\hline Cough & $0(0-3.0)$ \\
\hline Headache & $0.3(0.05-1.6)$ \\
\hline Dry itchy skin & $0.2(0.05-0.7)$ \\
\hline
\end{tabular}

${ }^{*}$ Unadjusted prevalence ratios

†95\% CI: 95\% confidence interval

$\$ 95 \%$ CIs that exclude 1.0 are statistically significantly different from comparison with U.S. office workers

Table 19. Comparison of respiratory symptoms and diagnoses reported by school employees (during the four-week period prior to reporting to the elementary school for the reopening) with the U.S. adult population (NHANES III)* NIOSH survey, March 2010

\begin{tabular}{|l|l|l|l|}
\hline & Overall & Male & Female \\
\hline & $\begin{array}{l}\text { Prevalence ratio } \\
(95 \% \mathrm{CI}) \dagger+\end{array}$ & $\begin{array}{l}\text { Prevalence ratio } \\
(95 \% \mathrm{CI}) \dagger+\end{array}$ & $\begin{array}{l}\text { Prevalence ratio } \\
(95 \% \mathrm{CI}) \dagger+\end{array}$ \\
\hline $\begin{array}{l}\text { Shortness of breath on exertion } \\
\text { (hurrying on level ground or walk up a } \\
\text { slight hill) }\end{array}$ & $0.2(0.08-0.7)$ & $0.8(0.1-4.6)$ & $0.2(0.04-0.6)$ \\
\hline Nasal allergies, including hay fever§ & $2.3(1.5-3.3)$ & $3.1(1.2-7.9)$ & $2.1(1.4-3.3)$ \\
\hline $\begin{array}{l}\text { Chronic bronchitis (physician- } \\
\text { diagnosed) }\end{array}$ & $0.7(0.2-2.1)$ & $0(0-13.7)$ & $0.8(0.3-2.2)$ \\
\hline Ever asthma (physician-diagnosed) & $1.3(0.6-2.7)$ & $2.5(0.7-9.0)$ & $1.1(0.5-2.6)$ \\
\hline Current asthma (physician-diagnosed) & $1.5(0.6-3.5)$ & $2.1(0.4-11.8)$ & $1.4(0.5-3.6)$ \\
\hline
\end{tabular}

*Indirect standardization for age, gender, smoking status, and race

$\uparrow 95 \%$ CI: $95 \%$ confidence interval

$\$ 95 \%$ CIs that exclude 1.0 are statistically significantly different from comparison with U.S. adult population

${ }^{\S}$ NIOSH question included physician-diagnosed hay fever or nasal allergies; NHANES III question asked about physiciandiagnosed hay fever ("Has a doctor ever told you that you had hay fever?") 


\section{TABLES (CONTINUED)}

Table 20. Comparison of physician-diagnosed asthma among elementary school staff to the North Carolina state population prevalence (2009 BRFSS); NIOSH survey, March 2010

\begin{tabular}{|c|c|c|}
\hline & \multicolumn{2}{|c|}{ Staff } \\
\hline & \multicolumn{2}{|c|}{$\begin{array}{c}\text { Prevalence ratio } \\
(95 \% \mathrm{CI})^{* \dagger}\end{array}$} \\
\hline Ever asthma \\
\hline Male & 1.7 & $0.5-6.1$ \\
\hline Female & 0.6 & $0.2-1.3$ \\
\hline Overall & 0.7 & $0.3-1.5$ \\
\hline Current asthma & 1.6 & $0.3-9.1$ \\
\hline Male & 0.7 & $0.3-1.8$ \\
\hline Female & 0.8 & $0.3-1.8$ \\
\hline Overall &
\end{tabular}

${ }^{*} 95 \%$ CI: $95 \%$ confidence interval

$\lceil 95 \%$ CIs that exclude 1.0 are statistically significantly different from comparison with the North Carolina state population

Table 21. Prevalence of symptoms reported by elementary school staff during the 2008/2009 school year, by primary work area*; NIOSH survey, March 2010

\begin{tabular}{|l|c|c|c|}
\hline & $\begin{array}{c}\text { Classroom wings } \\
(\mathrm{n}=45)\end{array}$ & $\begin{array}{c}\text { Non-classroom wings } \\
(\mathrm{n}=13)\end{array}$ & P value \\
\hline Headaches & $34(76 \%)$ & $7(54 \%)$ & 0.17 \\
\hline Unusual tiredness or fatigue & $33(73 \%)$ & $4(31 \%)$ & 0.008 \\
\hline Upper respiratory symptoms & $30(67 \%)$ & $4(31 \%)$ & 0.03 \\
\hline $\begin{array}{l}\text { Difficulty remembering } \\
\text { things or concentrating }\end{array}$ & $25(56 \%)$ & $4(31 \%)$ & 0.21 \\
\hline Watery, itchy eyes & $25(56 \%)$ & $2(15 \%)$ & 0.01 \\
\hline Lower respiratory symptoms & $24(53 \%)$ & $6(46 \%)$ & 0.76 \\
\hline $\begin{array}{l}\text { Fever, chills, or flu-like } \\
\text { symptoms }\end{array}$ & $10(22 \%)$ & $4(31 \%)$ & 0.71 \\
\hline Nosebleeds & $3(7 \%)$ & $3(23 \%)$ & 0.12 \\
\hline
\end{tabular}

${ }^{*} 8$ of the 66 participants who worked at the elementary school during the 2008/2009 school year had more than one primary work area and could not be classified as working primarily in classroom or non-classroom wings.

†Classroom wings include the $\mathrm{k} / 1,2 / 3$, and $4 / 5$ wings.

$\ddagger$ Non-classroom wings include the administration, media center, gym, and cafeteria areas.

§Pearson Chi-square exact test; $\mathrm{p}<0.05$ - statistically significant; $0.05<\mathrm{p} \leq 0.10$ - marginally significant 


\section{TABLES (CONTINUED)}

Table 22. Prevalence of symptoms reported by elementary school staff during the 2008/2009 school year, by primary work area; NIOSH survey, March 2010

\begin{tabular}{|l|c|c|c|c|c|c|c|c|}
\hline & $\begin{array}{c}\mathrm{k} / 1 \text { wing } \\
(\mathrm{n}=17)\end{array}$ & $\begin{array}{c}2 / 3 \text { wing } \\
(\mathrm{n}=16)\end{array}$ & $\begin{array}{c}4 / 5 \text { wing } \\
(\mathrm{n}=11)\end{array}$ & $P$ value* & $\begin{array}{c}\text { Admin } \\
\text { wing } \\
(\mathrm{n}=7)\end{array}$ & $\begin{array}{c}\text { Gym/ } \\
\text { cafeteria } \\
\text { wing } \\
(\mathrm{n}=3)\end{array}$ & $\begin{array}{c}\text { Other } \\
(\mathrm{n}=7)\end{array}$ & $\begin{array}{c}\text { Everywhere } \\
(\mathrm{n}=5)\end{array}$ \\
\hline $\begin{array}{l}\text { Lower } \\
\text { respiratory } \\
\text { symptoms }\end{array}$ & $7(41 \%)$ & $11(69 \%)$ & $5(45 \%)$ & 0.26 & $4(57 \%)$ & $1(33 \%)$ & $2(29 \%)$ & 0 \\
\hline $\begin{array}{l}\text { Watery, itchy } \\
\text { eyes }\end{array}$ & $9(53 \%)$ & $9(56 \%)$ & $6(55 \%)$ & 1.0 & $1(14 \%)$ & $1(33 \%)$ & $2(29 \%)$ & $1(20 \%)$ \\
\hline $\begin{array}{l}\text { Upper } \\
\text { respiratory } \\
\text { symptoms }\end{array}$ & $9(53 \%)$ & $12(75 \%)$ & $8(73 \%)$ & 0.37 & $2(29 \%)$ & $2(67 \%)$ & $3(43 \%)$ & $1(20 \%)$ \\
\hline $\begin{array}{l}\text { Unusual } \\
\text { tiredness or } \\
\text { fatigue }\end{array}$ & $13(76 \%)$ & $12(75 \%)$ & $7(64 \%)$ & 0.83 & $4(57 \%)$ & 0 & $2(29 \%)$ & 0 \\
\hline $\begin{array}{l}\text { Fever, chills, } \\
\text { or flu-like } \\
\text { symptoms }\end{array}$ & $3(18 \%)$ & $5(31 \%)$ & $2(18 \%)$ & 0.66 & $3(43 \%)$ & $1(33 \%)$ & 0 & 0 \\
\hline $\begin{array}{l}\text { Difficulty } \\
\text { remembering } \\
\text { things or } \\
\text { concentrating }\end{array}$ & $9(53 \%)$ & $8(50 \%)$ & $7(64 \%)$ & 0.80 & $4(57 \%)$ & 0 & $1(14 \%)$ & 0 \\
\hline Headaches & $14(82 \%)$ & $11(69 \%)$ & $8(73 \%)$ & 0.68 & $5(71 \%)$ & $2(67 \%)$ & $4(57 \%)$ & $1(20 \%)$ \\
\hline Nosebleeds & $2(12 \%)$ & 0 & $1(9 \%)$ & 0.46 & $3(43 \%)$ & 0 & $1(14 \%)$ & 0 \\
\hline
\end{tabular}

${ }^{*}$ Pearson Chi-square exact test; $\mathrm{p}<0.05$ - statistically significant; $0.05 \leq \mathrm{p}<0.10$ - marginally significant; comparison was among classroom wings 


\section{TABLES (CONTINUED)}

Table 23. Prevalence of health symptoms and their work-relatedness (portion of those with symptom) reported by elementary school staff during the 2008/2009 school year and during the four-week period prior to returning to the elementary school for its reopening in February 2010; NIOSH survey, March 2010

\begin{tabular}{|c|c|c|c|c|}
\hline & $\begin{array}{c}\text { 2008/2009 school } \\
\text { year } \\
\text { n=66* }\end{array}$ & $\begin{array}{c}\text { Work-related } \\
\text { 2008/2009 school } \\
\text { year } \\
\text { n (\%) }\end{array}$ & $\begin{array}{l}\text { 4-week period } \\
\text { prior to returning } \\
\text { to the school } \\
\qquad n=66^{*}\end{array}$ & $\begin{array}{l}\text { Work-related } \\
\text { 4-week period } \\
\text { prior to returning } \\
\text { to the school } \\
\text { n }(\%)\end{array}$ \\
\hline Headaches & $45(68 \%)$ & $36(80 \%)$ & $12(18 \%)$ & $2(17 \%)$ \\
\hline $\begin{array}{l}\text { Unusual tiredness or } \\
\text { fatigue }\end{array}$ & $38(58 \%)$ & $34(89 \%)$ & $3(5 \%)$ & 0 \\
\hline $\begin{array}{l}\text { Upper respiratory } \\
\text { symptoms }\end{array}$ & $37(56 \%)$ & $27(73 \%)$ & $26(39 \%)$ & - \\
\hline $\begin{array}{l}\text { Lower respiratory } \\
\text { symptoms }\end{array}$ & $30(45 \%)$ & $25(83 \%)$ & $15(23 \%)$ & - \\
\hline Watery, itchy eyes & $29(44 \%)$ & $26(90 \%)$ & $8(12 \%)$ & $3(38 \%)$ \\
\hline $\begin{array}{l}\text { Difficulty remembering } \\
\text { things or concentrating }\end{array}$ & $29(44 \%)$ & $24(83 \%)$ & $2(3 \%)$ & 0 \\
\hline $\begin{array}{l}\text { Fever, chills, or flu-like } \\
\text { symptoms }\end{array}$ & $14(21 \%)$ & $12(86 \%)$ & $2(3 \%) \dagger$ & 0 \\
\hline Nosebleeds & $7(11 \%)$ & $6(86 \%)$ & $3(5 \%)$ & 0 \\
\hline
\end{tabular}

*Excludes participating staff who did not work at the elementary school during the 2008/2009 school year

†Includes participants who reported episodes of fever and chills and/or episodes of flu-like achiness or achy joints during the 4 weeks before returning to the elementary school. 


\section{FIGURES}

Figure 1. Diagram of the elementary school.

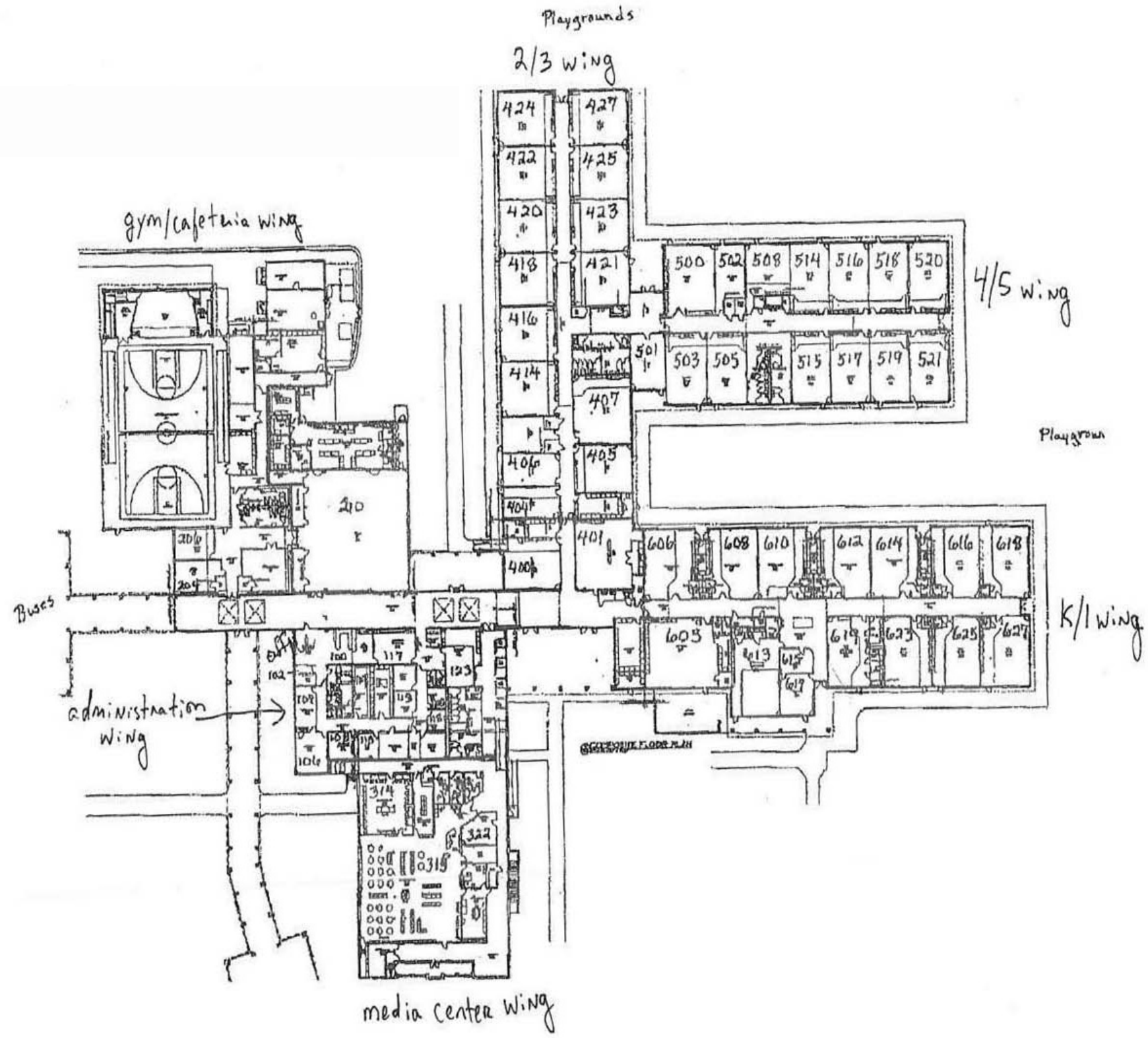




\section{FIGURES (CONTINUED)}

Figure 2. Visible mold on carpet in a classroom (room 625), August 2005 (courtesy of building occupant).

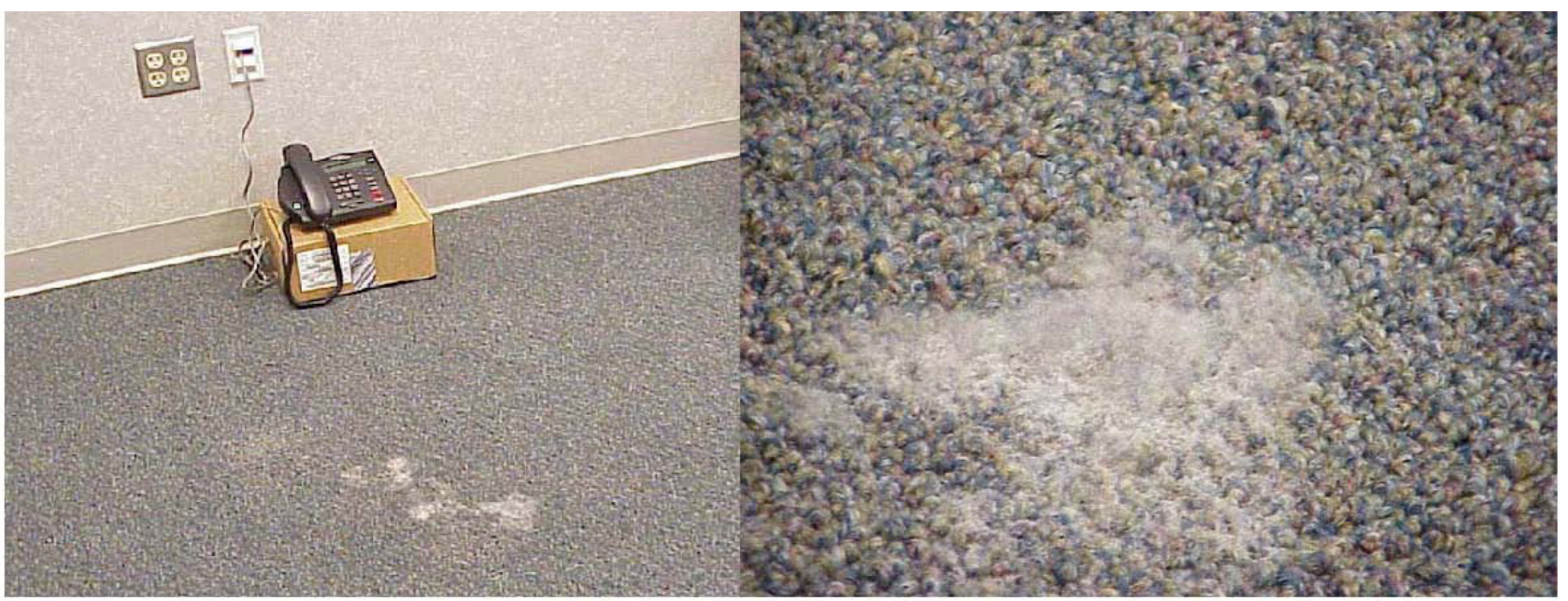

Figure 3. Visible mold on upholstered chairs in the records room (room 109), August 2005 (courtesy of building occupant).

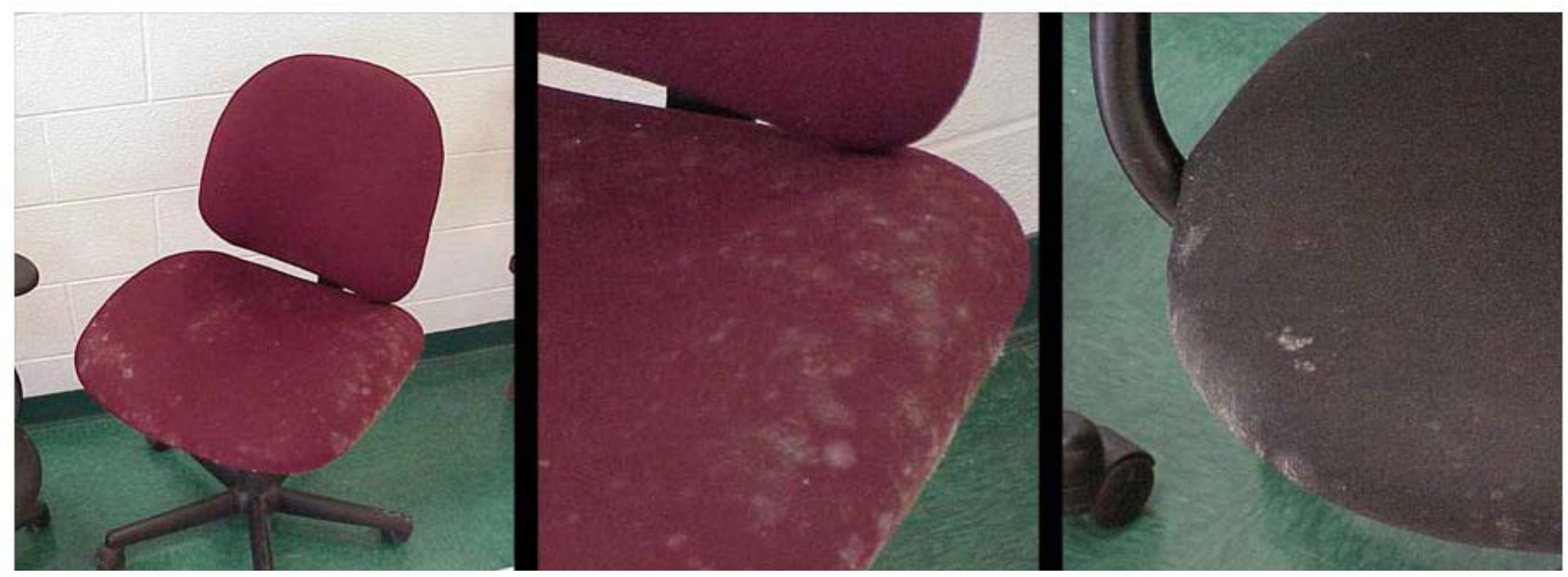




\section{FIGURES (CONTINUED)}

Figure 4. Visible mold on picture frames in the storage room (room 111), August 2005 (courtesy of building occupant).
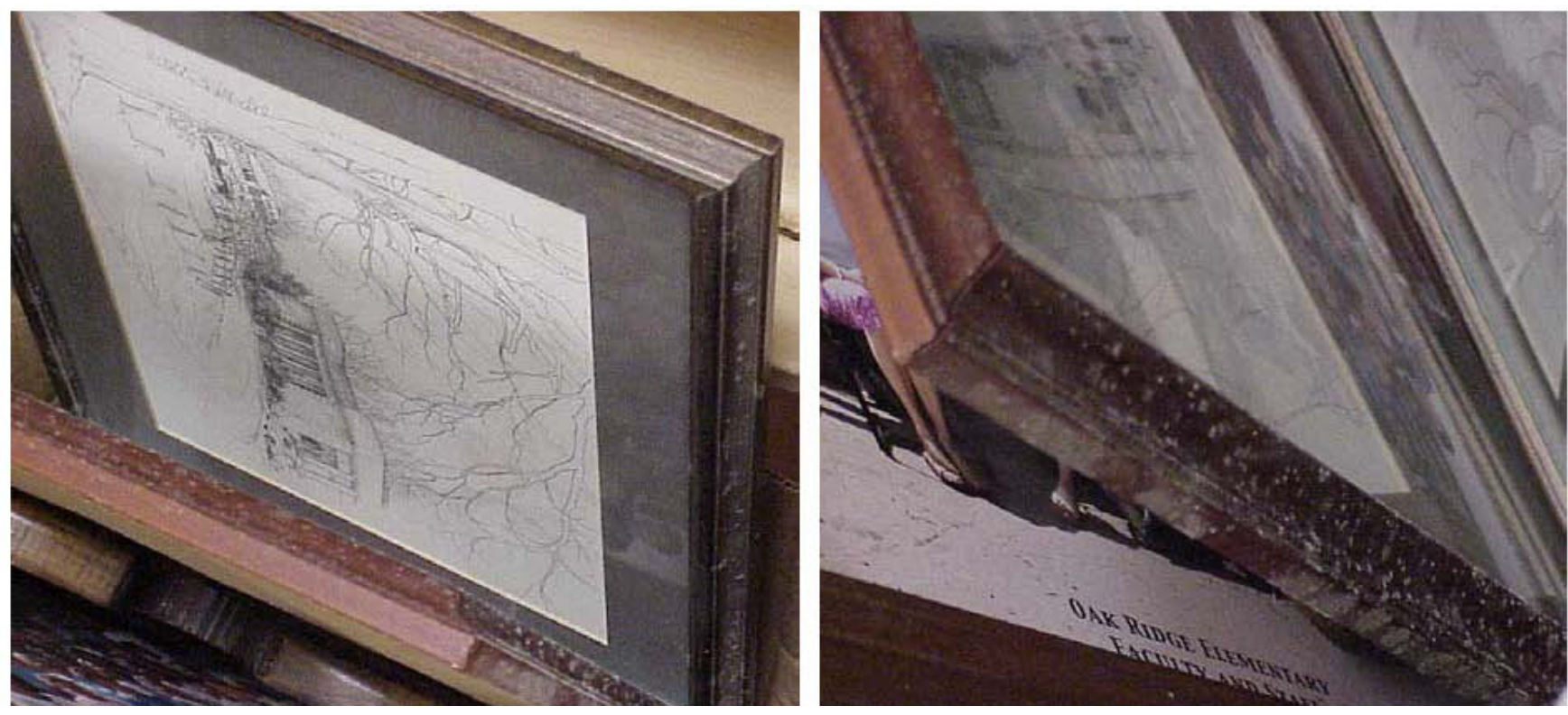

Figure 5. Basement below the media center wing, July 2009.

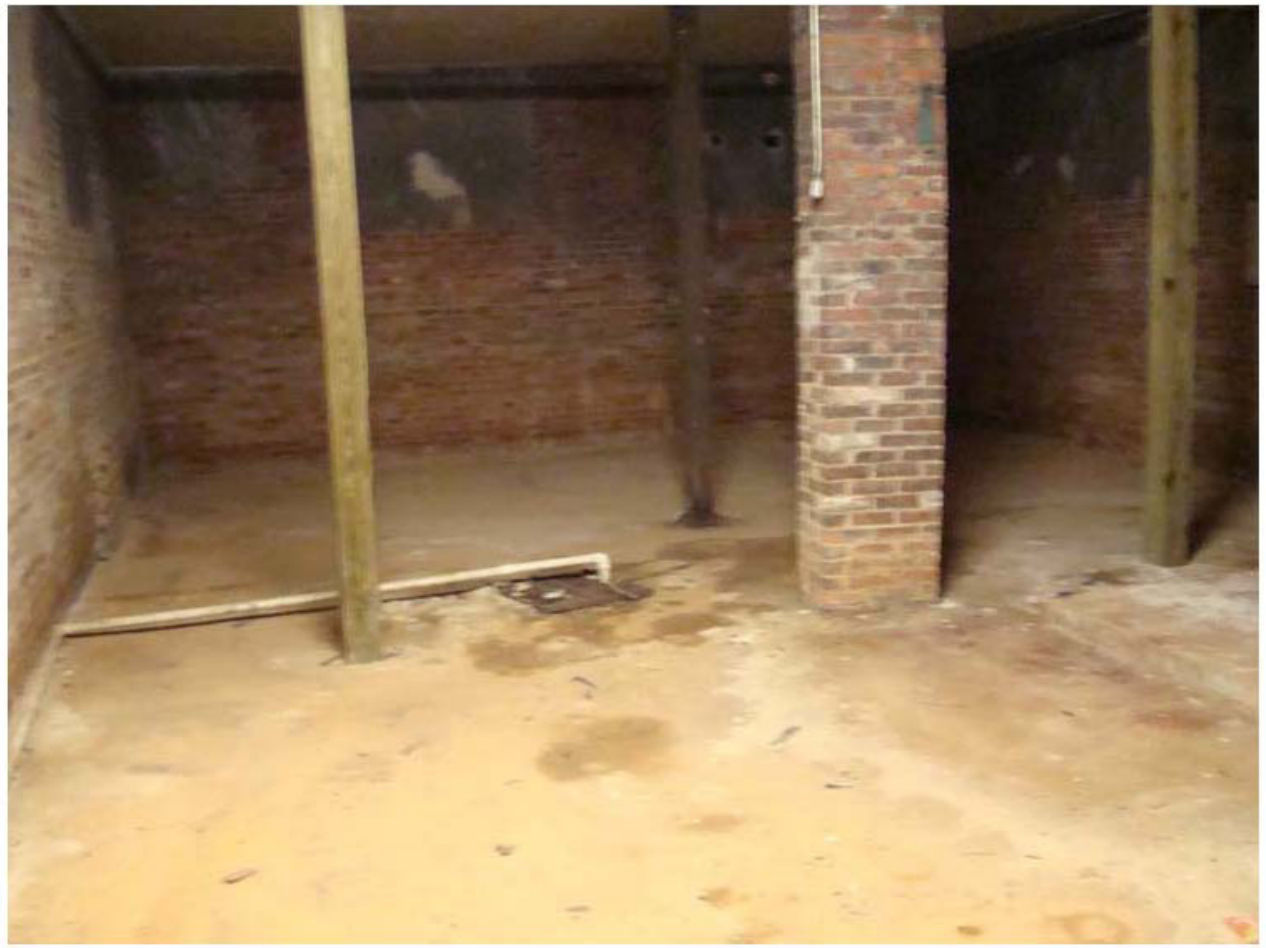




\section{FIGURES (CONTINUED)}

Figure 6. School grounds sloping downward towards classroom wings, July 2009.

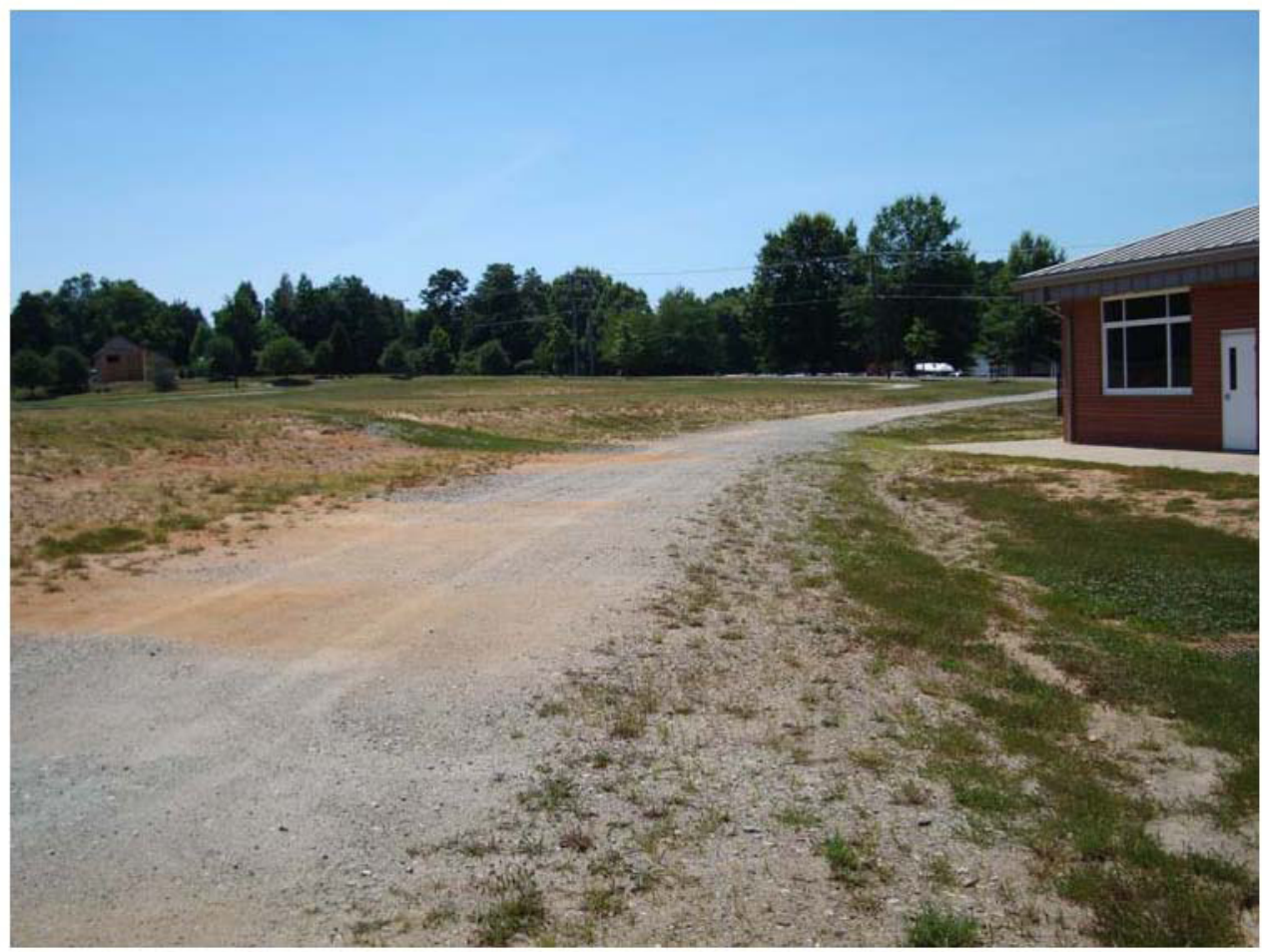

Figure 7. School grounds sloping downward towards classroom wings, July 2009.

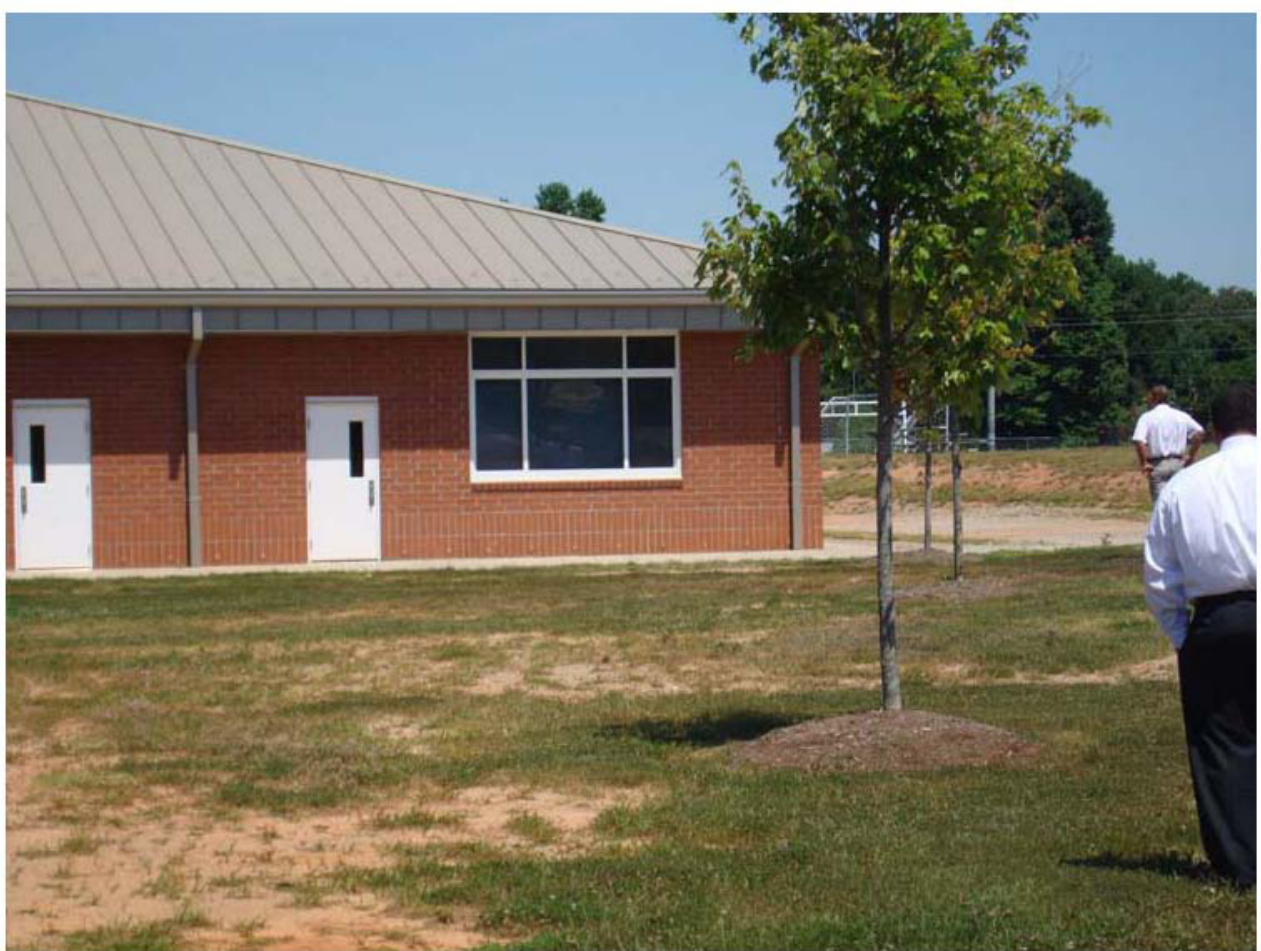


FIGURES (CONTINUED)

Figure 8. School grounds sloping downward towards classroom wings, July 2009.

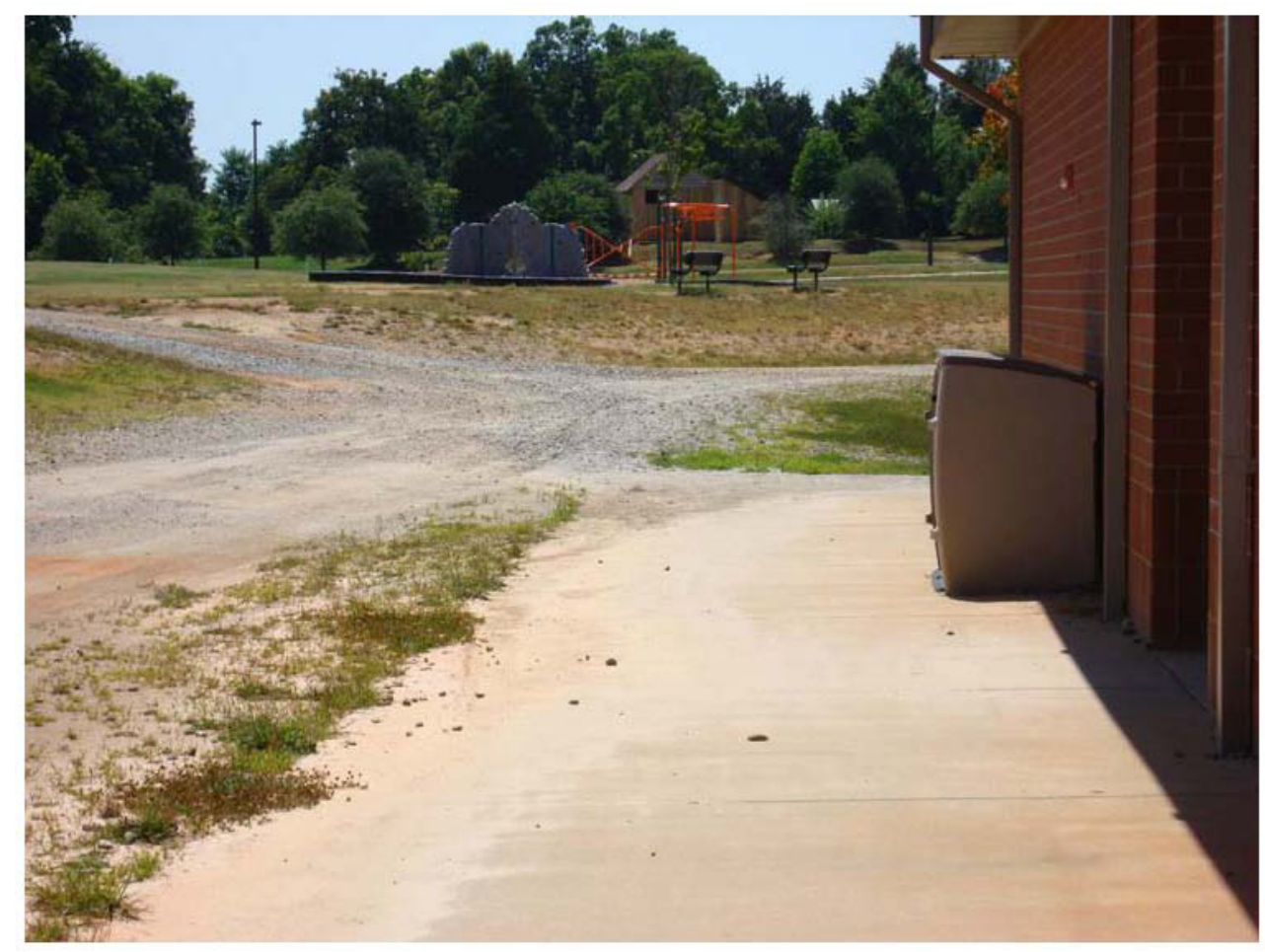

Figure 9. Blocked storm drain outside of the media center, July 2009.

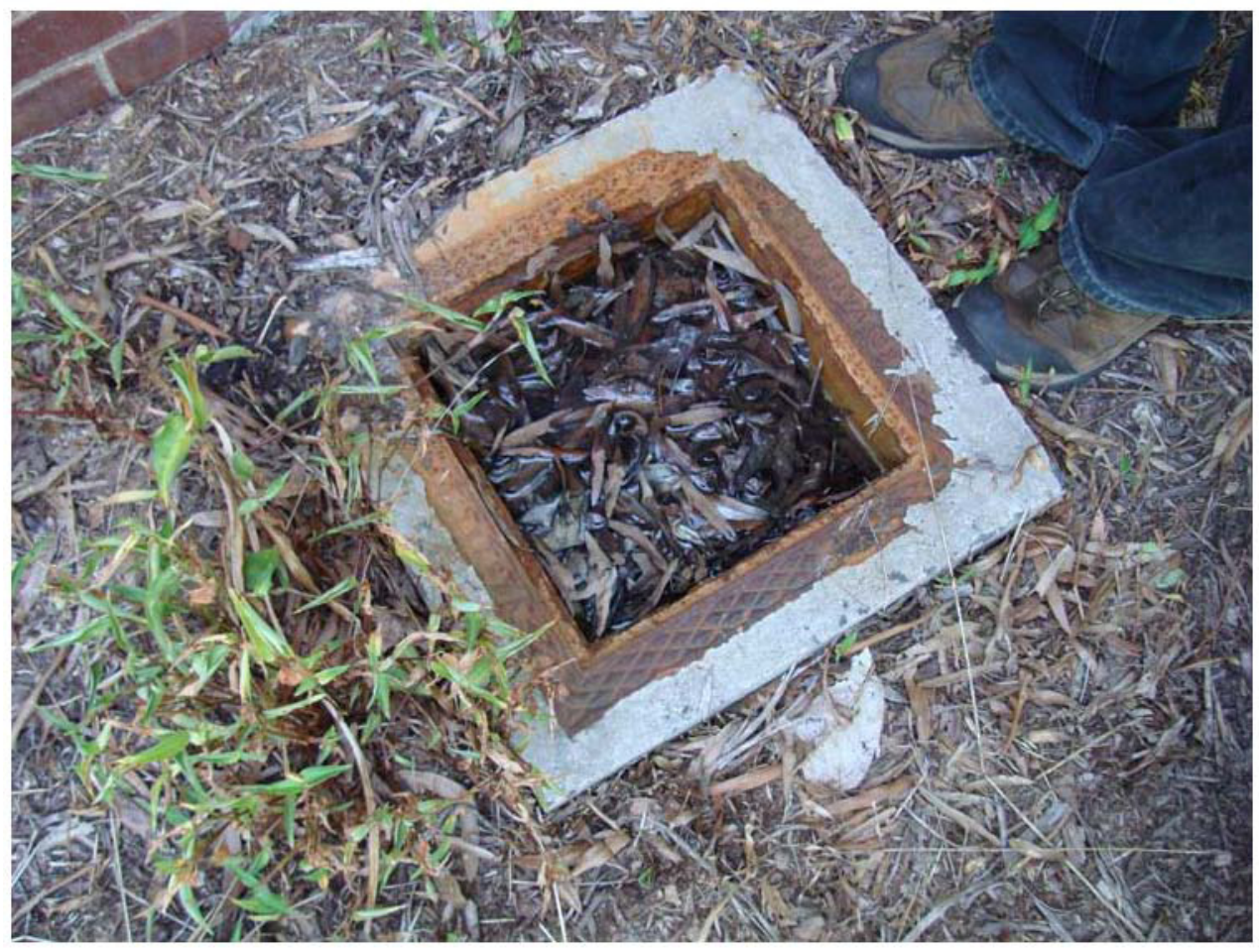




\section{FIGURES (CONTINUED)}

Figure 10. Foam roof adjacent to gym, July 2009.

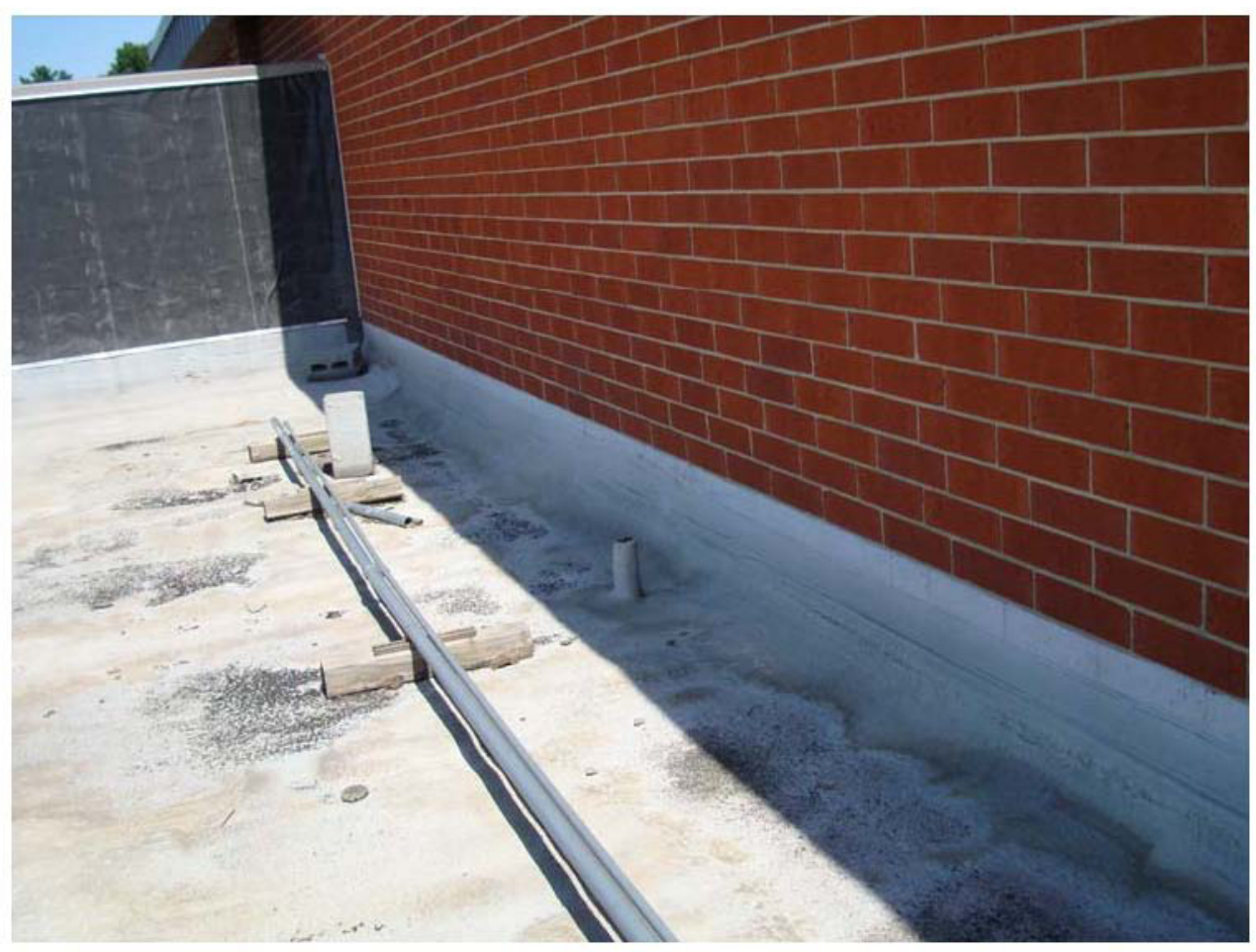

Figure 11. Foam roof over media center wing, July 2009.

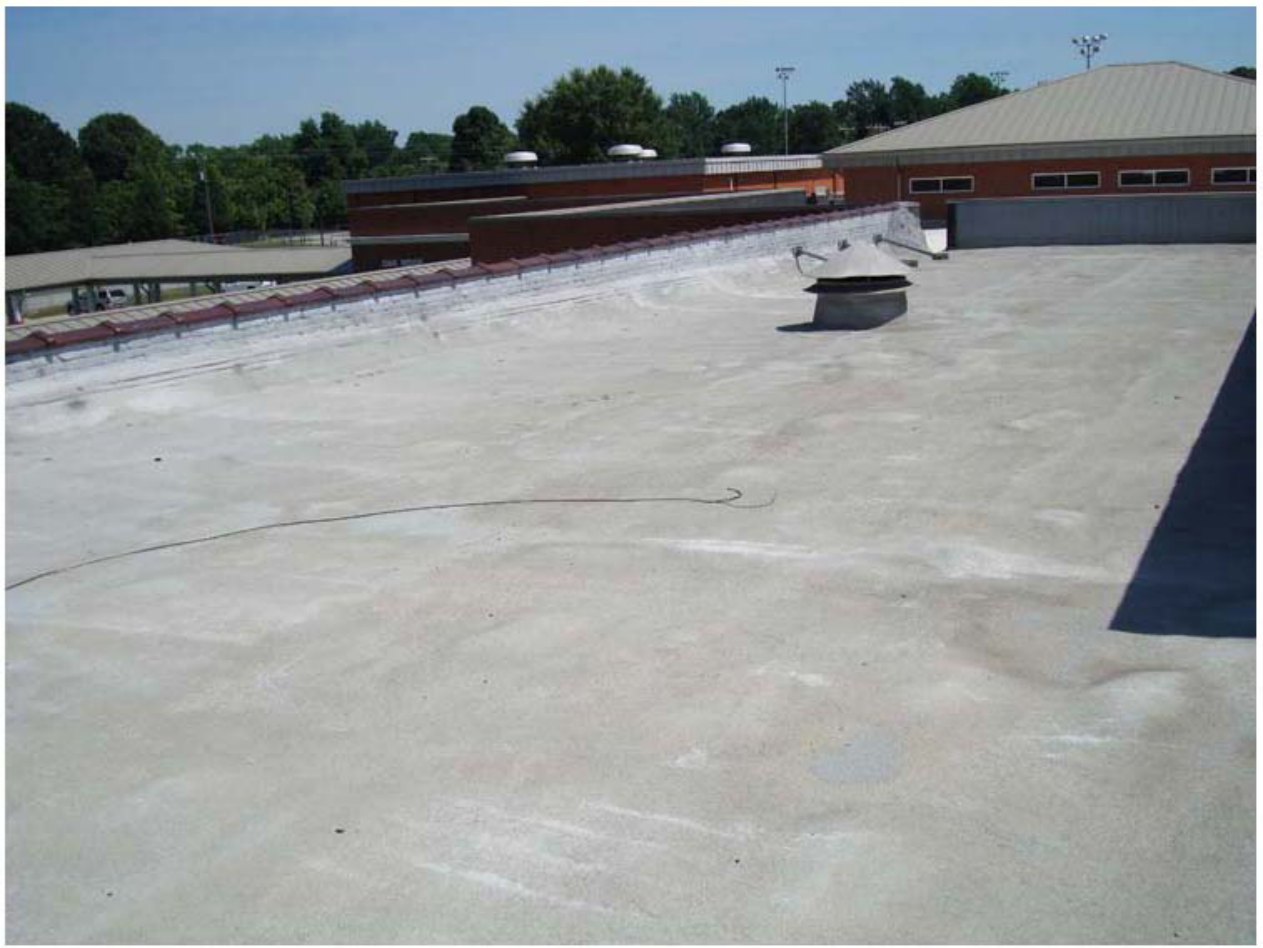




\section{FIGURES (CONTINUED)}

Figure 12. Flashing judged to be inadequate and that appeared to be mounted superficially at the junction between the parapet wall (above room 206) and the gym, July 2009.

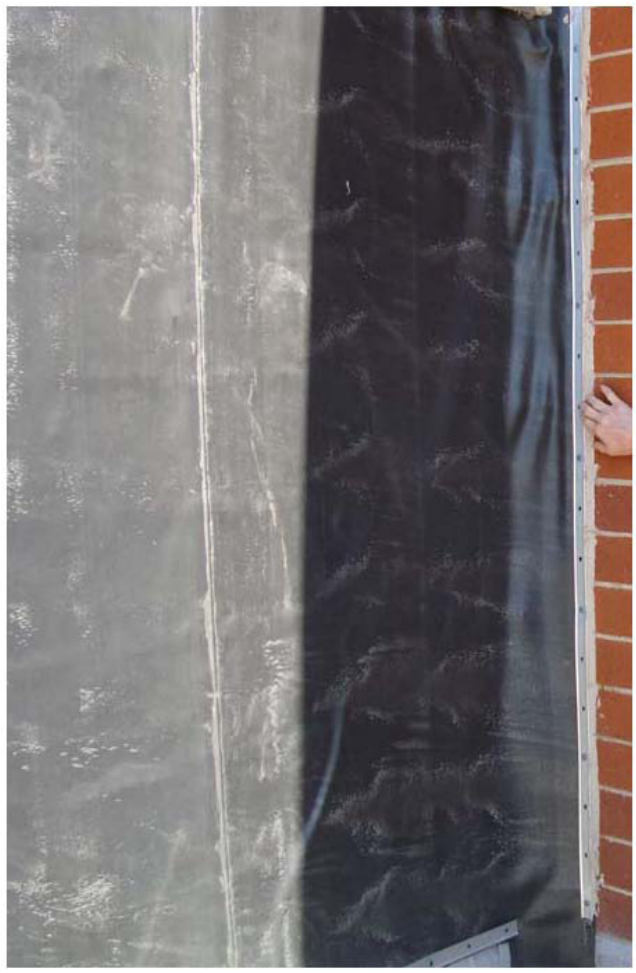

Figure 13. Plenum above classroom ceiling, showing ductwork and dust, July 2009.

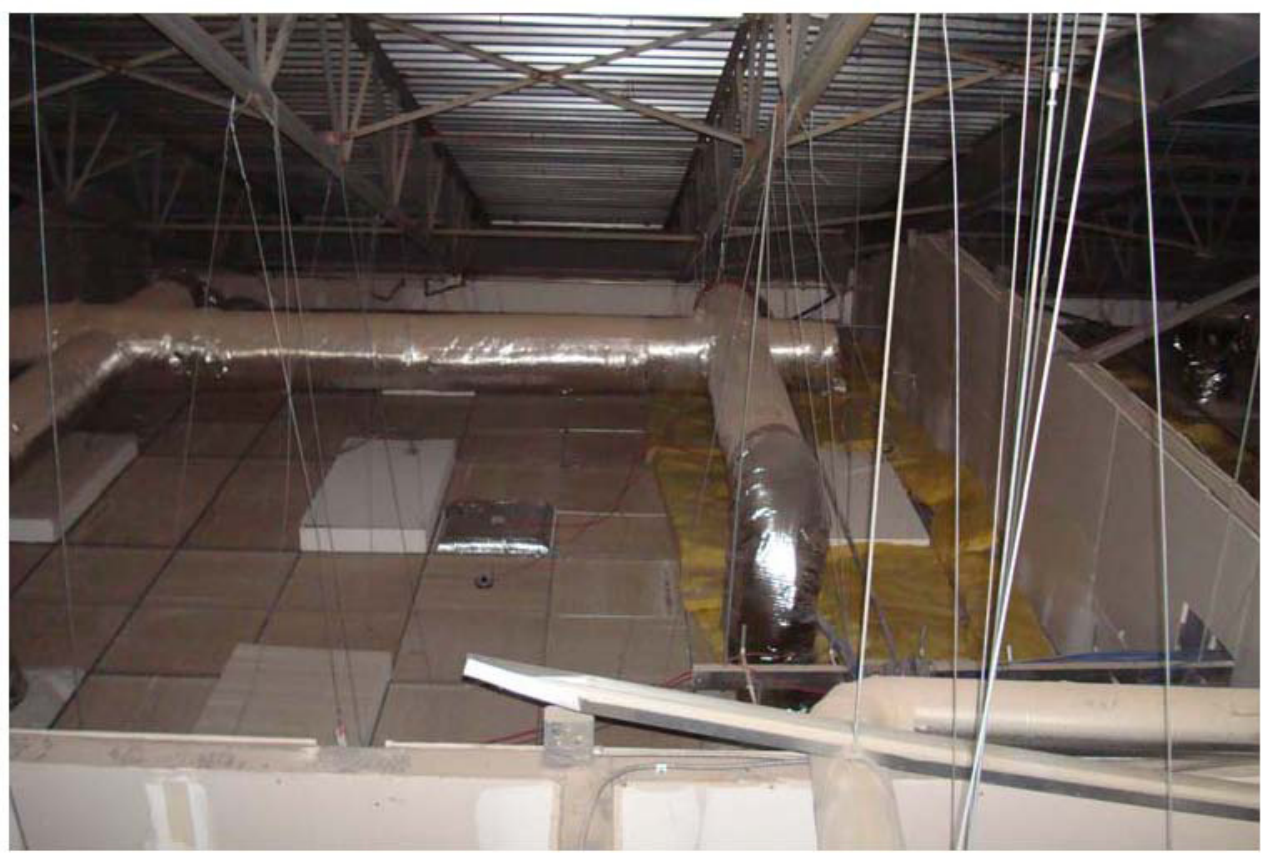




\section{FIGURES (CONTINUED)}

Figure 14. Plenum above classroom ceiling, showing ductwork and dust, July 2009.

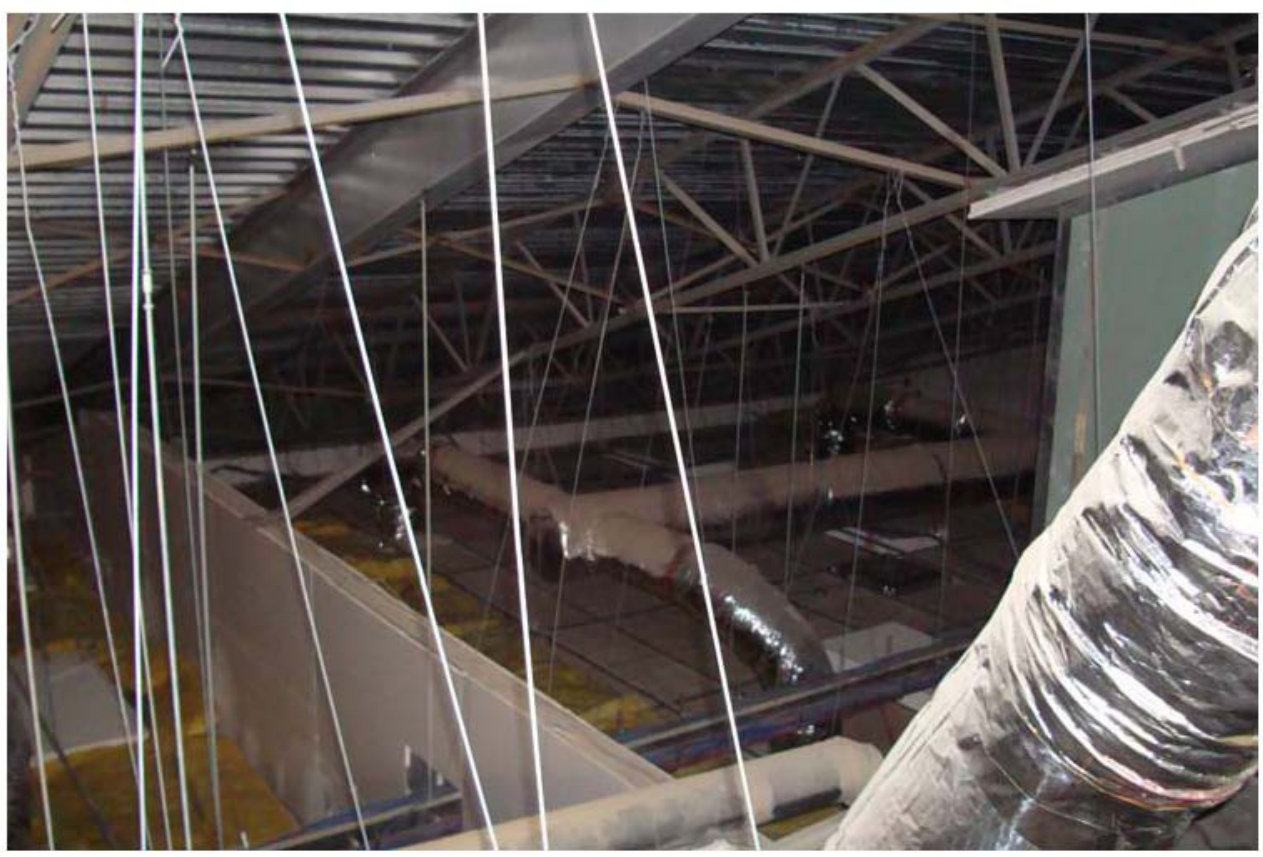

Figure 15. Coils in HVAC air handlers, showing dust and possible mold contamination, July 2009.
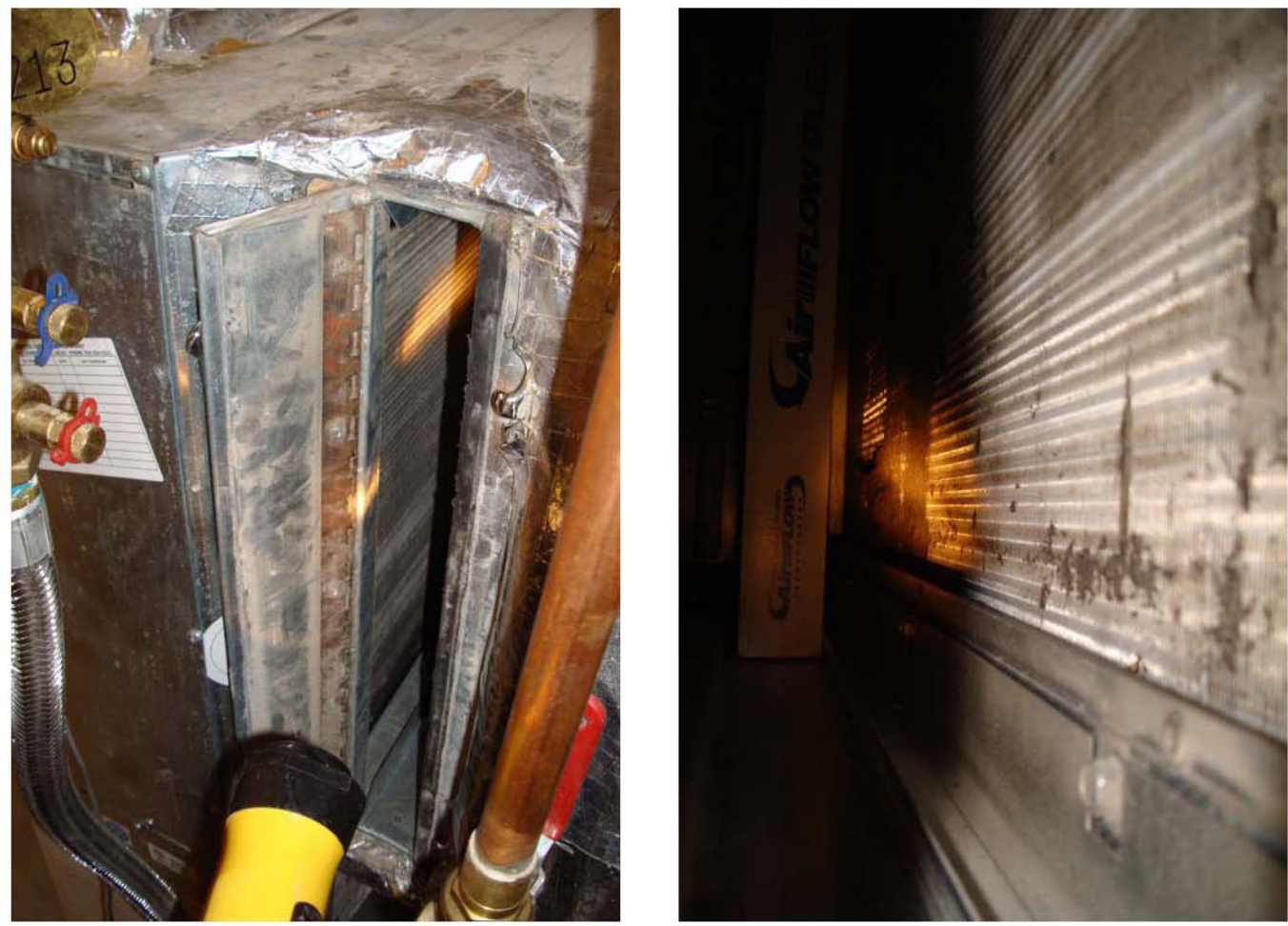
FIGURES (CONTINUED)

Figure 16. Percent frequency of occurrence individual fungal species in floor dust samples, July 2009.
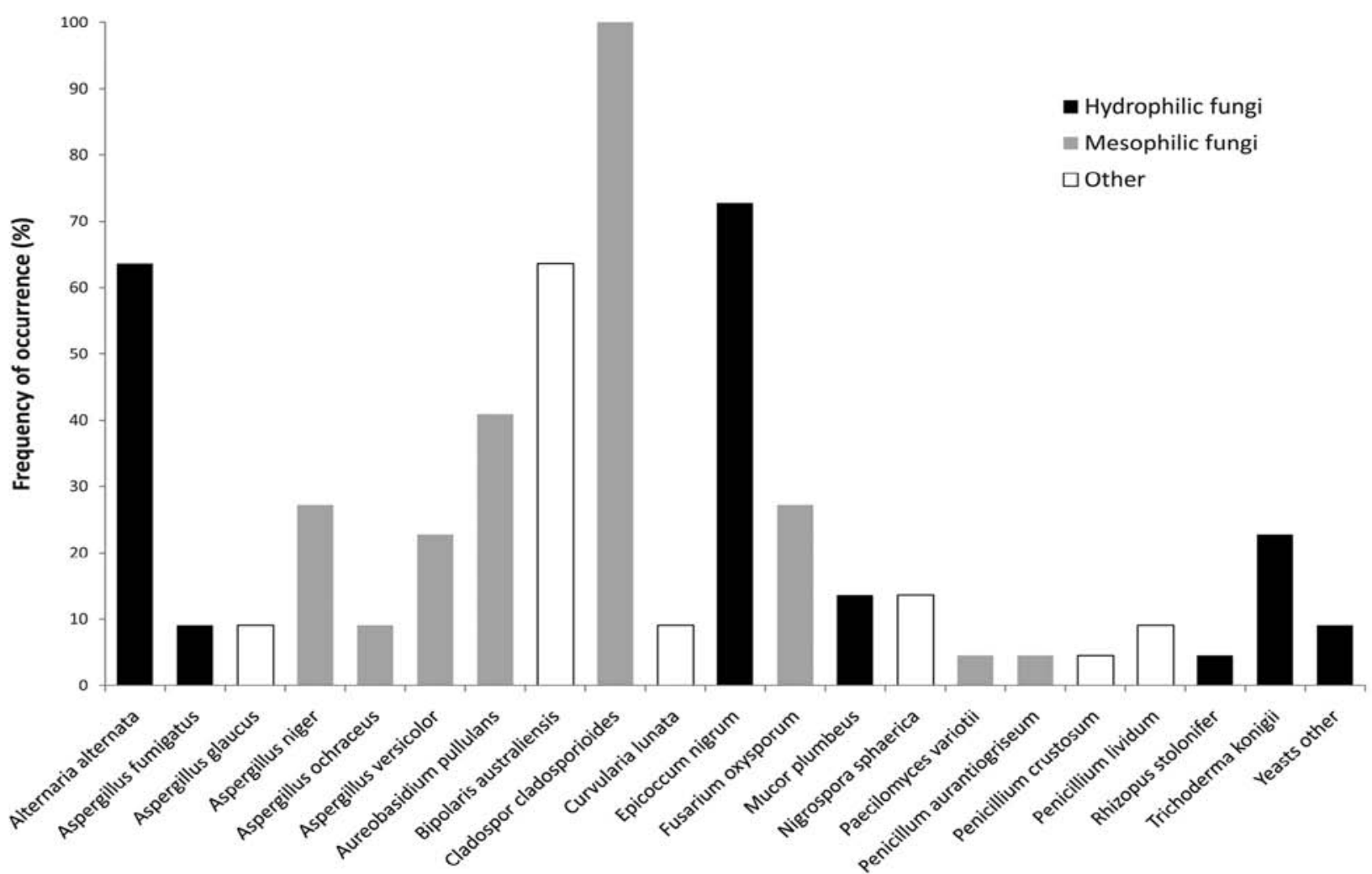
FIGURES (CONTINUED)

Figure 17. Classroom, March 2010.

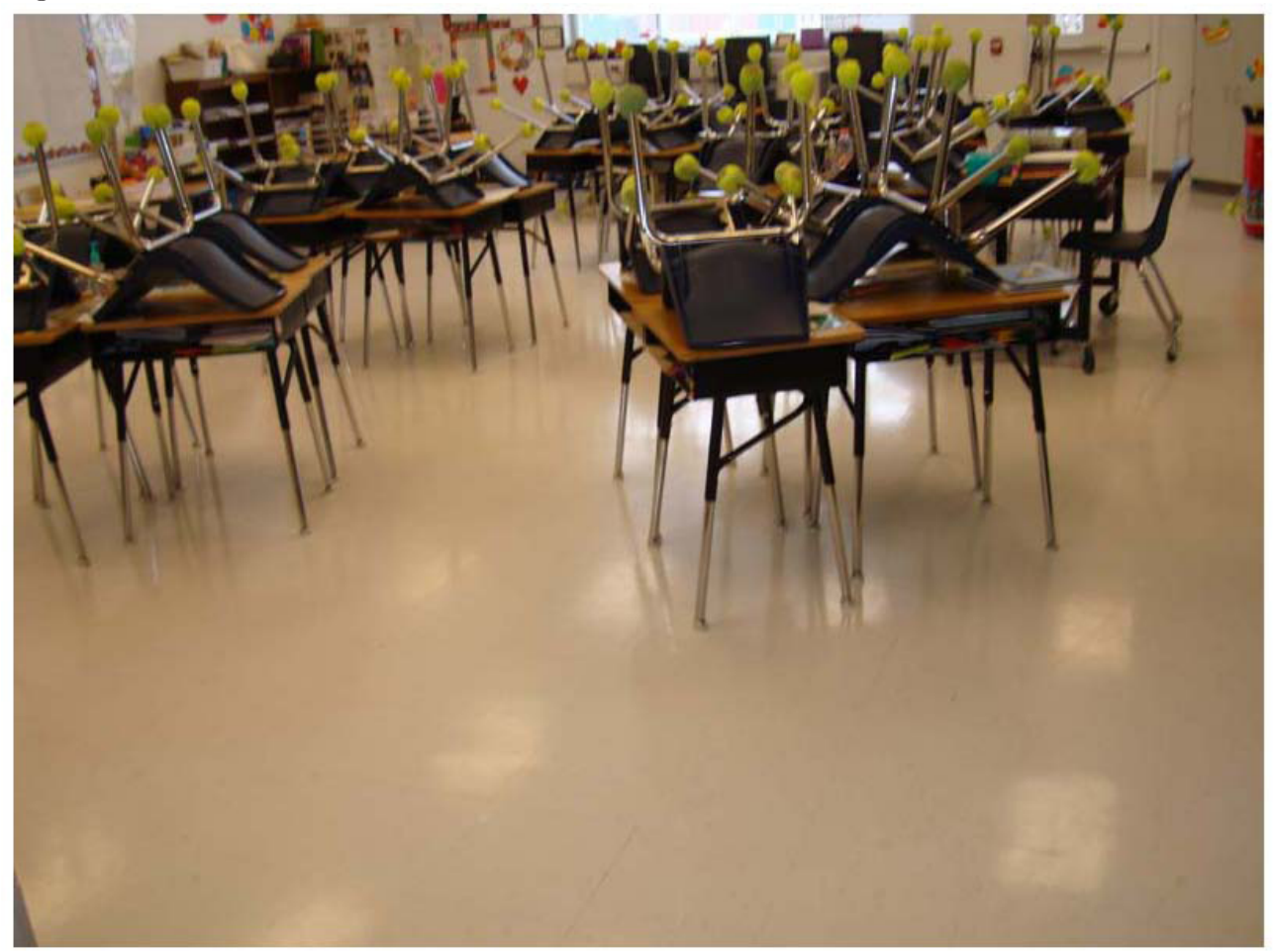

Figure 18. Classroom, March 2010.

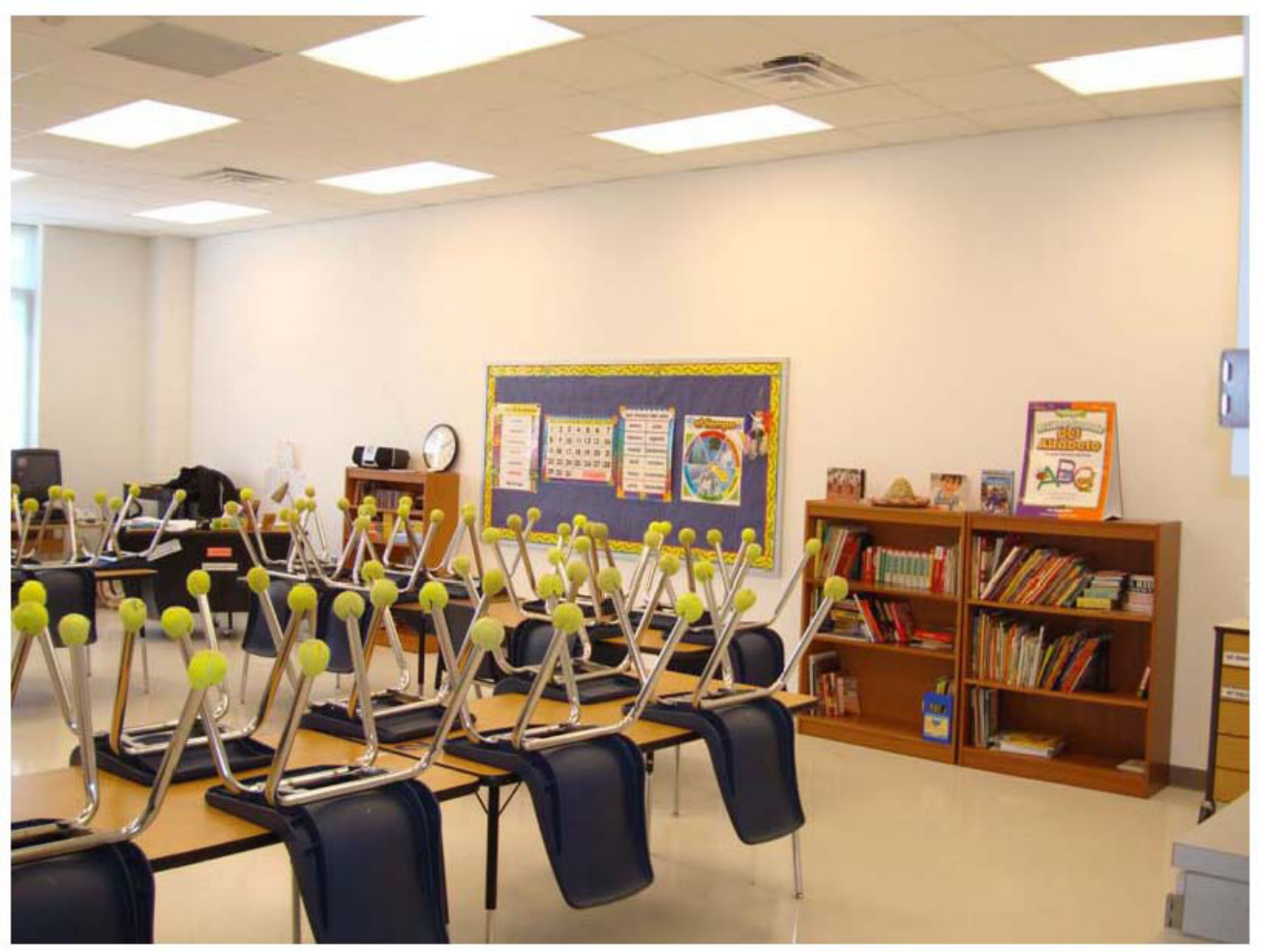


FIGURES (CONTINUED)

Figure 19. Corridor in classroom wing, March 2010.

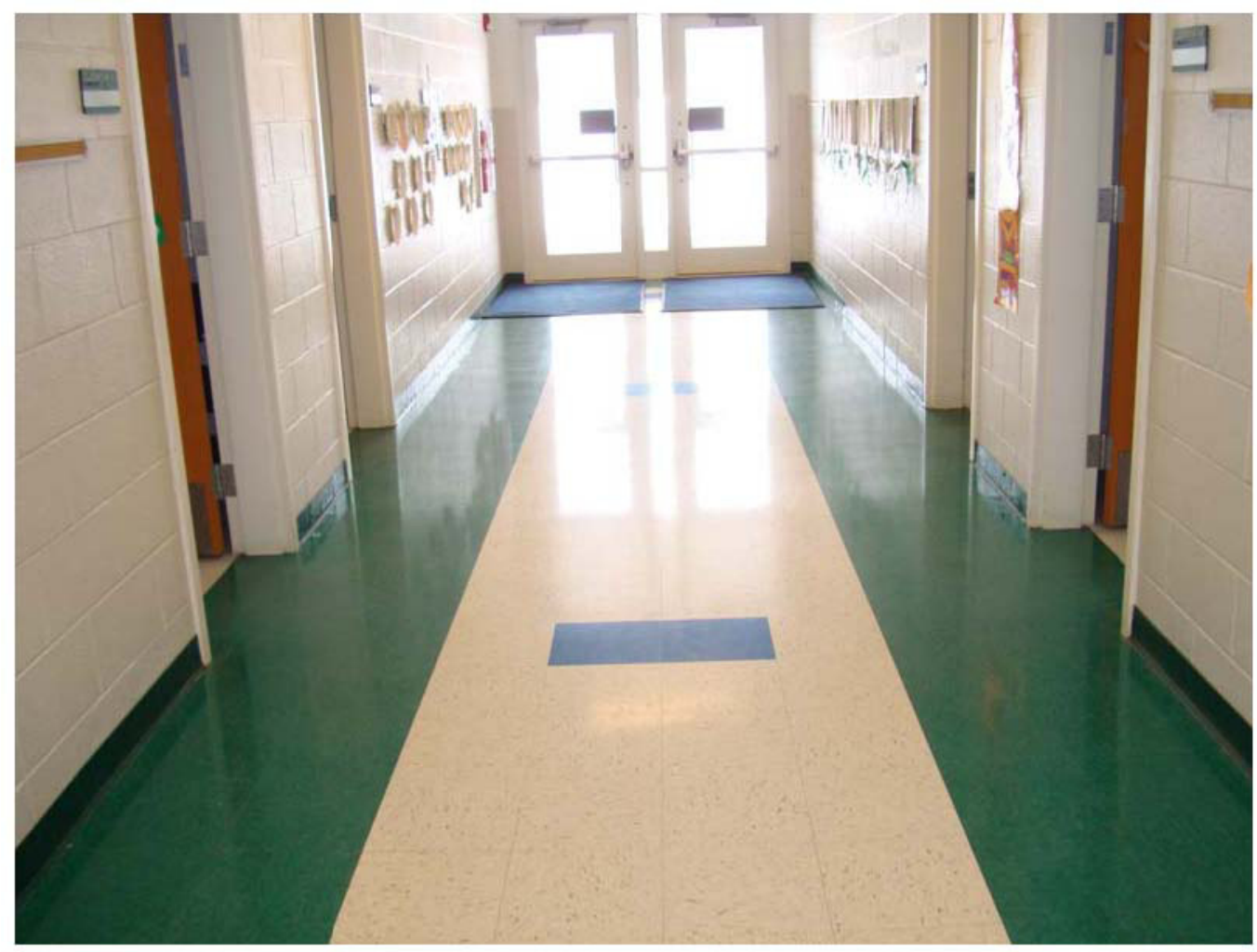

Figure 20. Main corridor, March 2010.

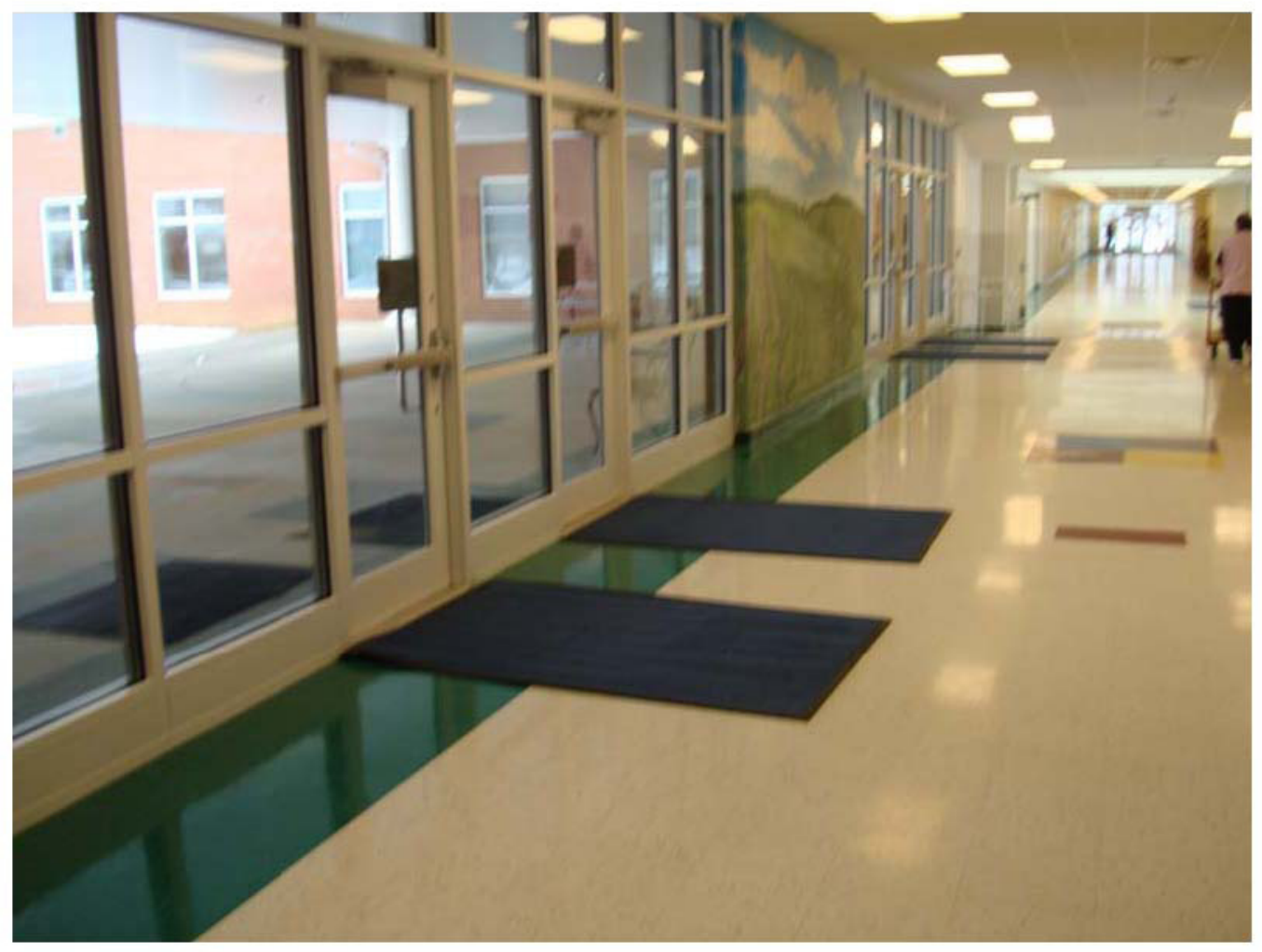




\section{FIGURES (CONTINUED)}

Figure 21. Corridor in media center wing, March 2010.

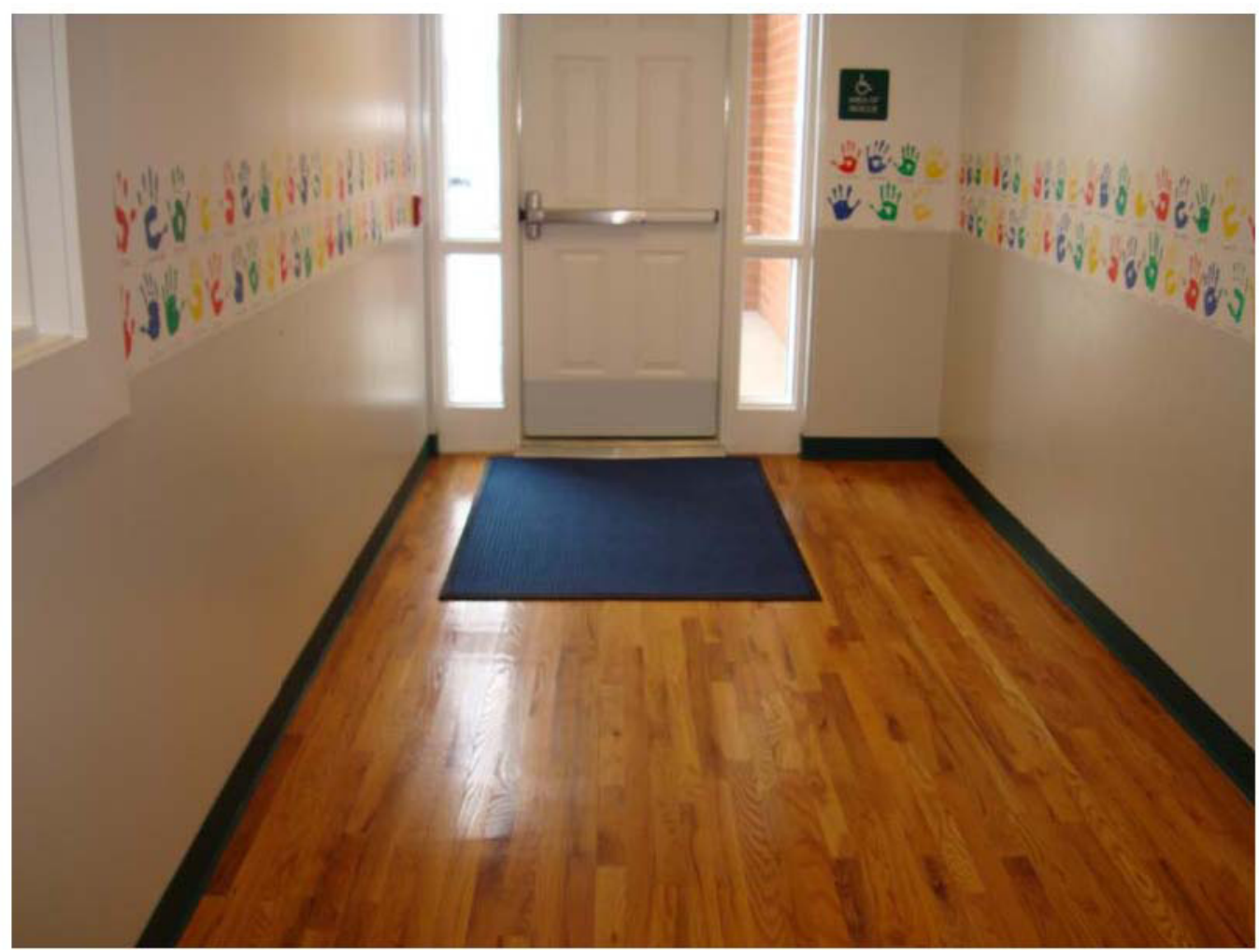

Figure 22. Corridor in media center wing, March 2010.

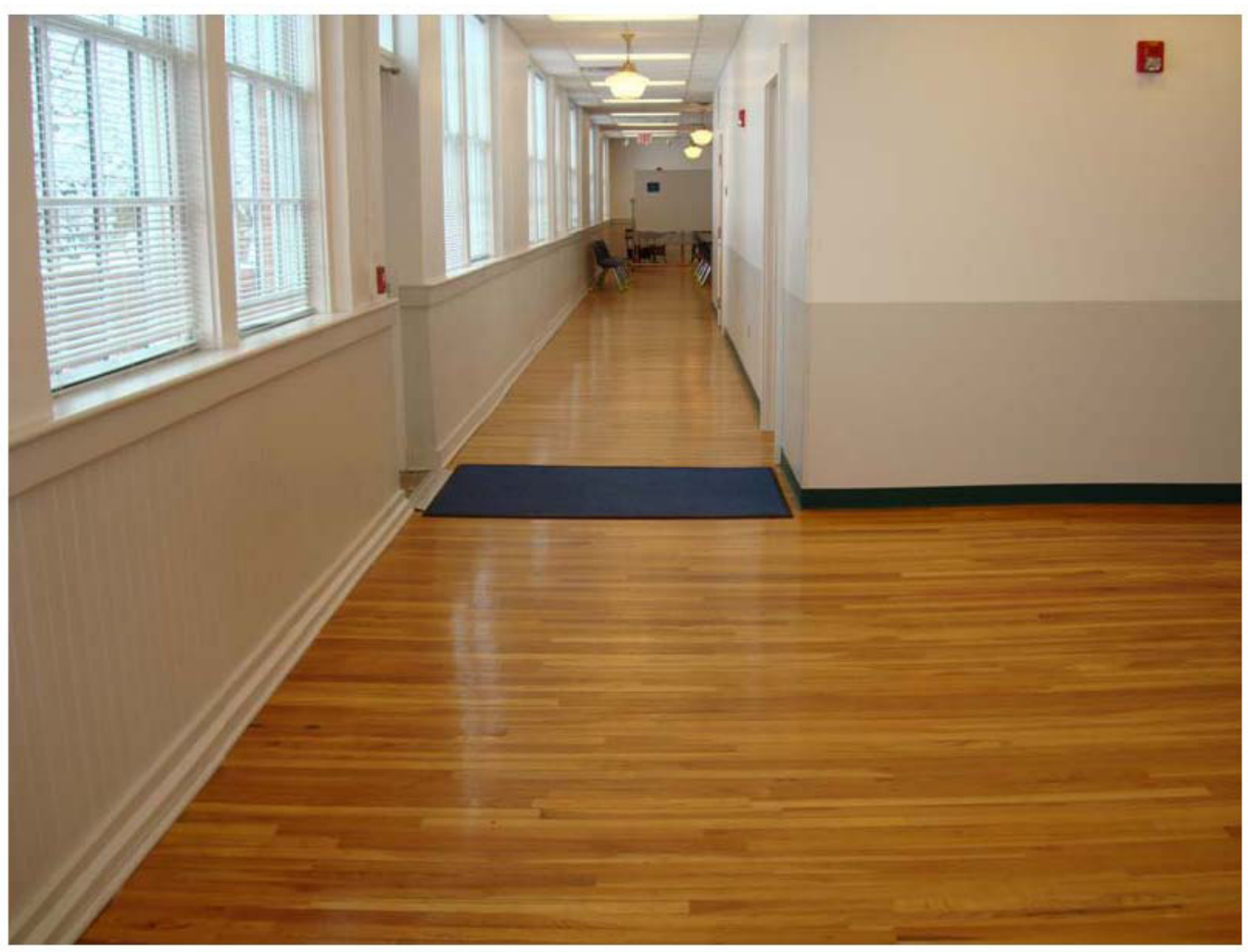




\section{FIGURES (CONTINUED)}

Figure 23. Media center, showing temporary containment barrier for installation of exterior door, March 2010.

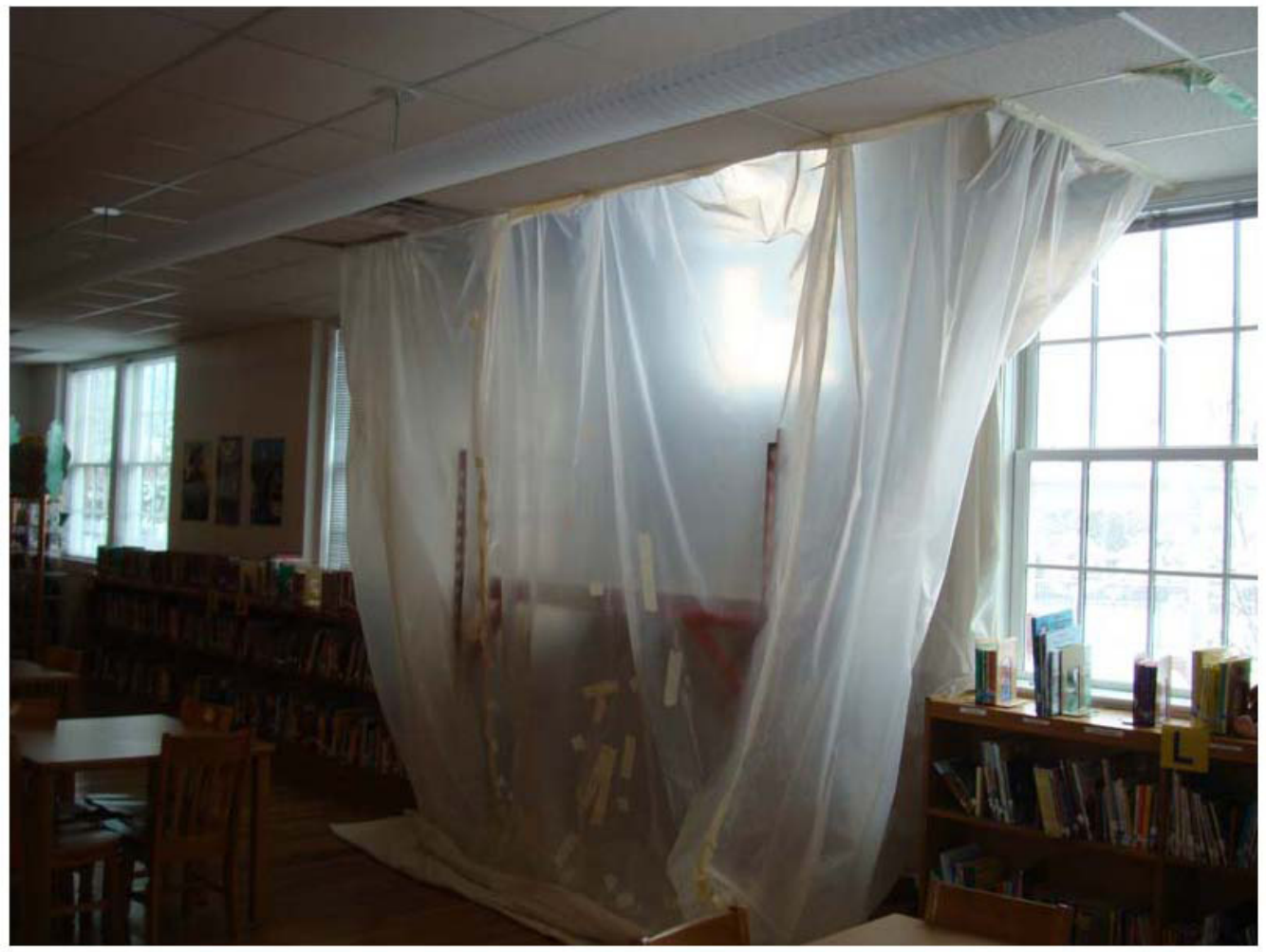

Figure 24. Comparison of symptom prevalences in 2008/2009 school year by classroom wing in 40 staff members ${ }^{1}$.

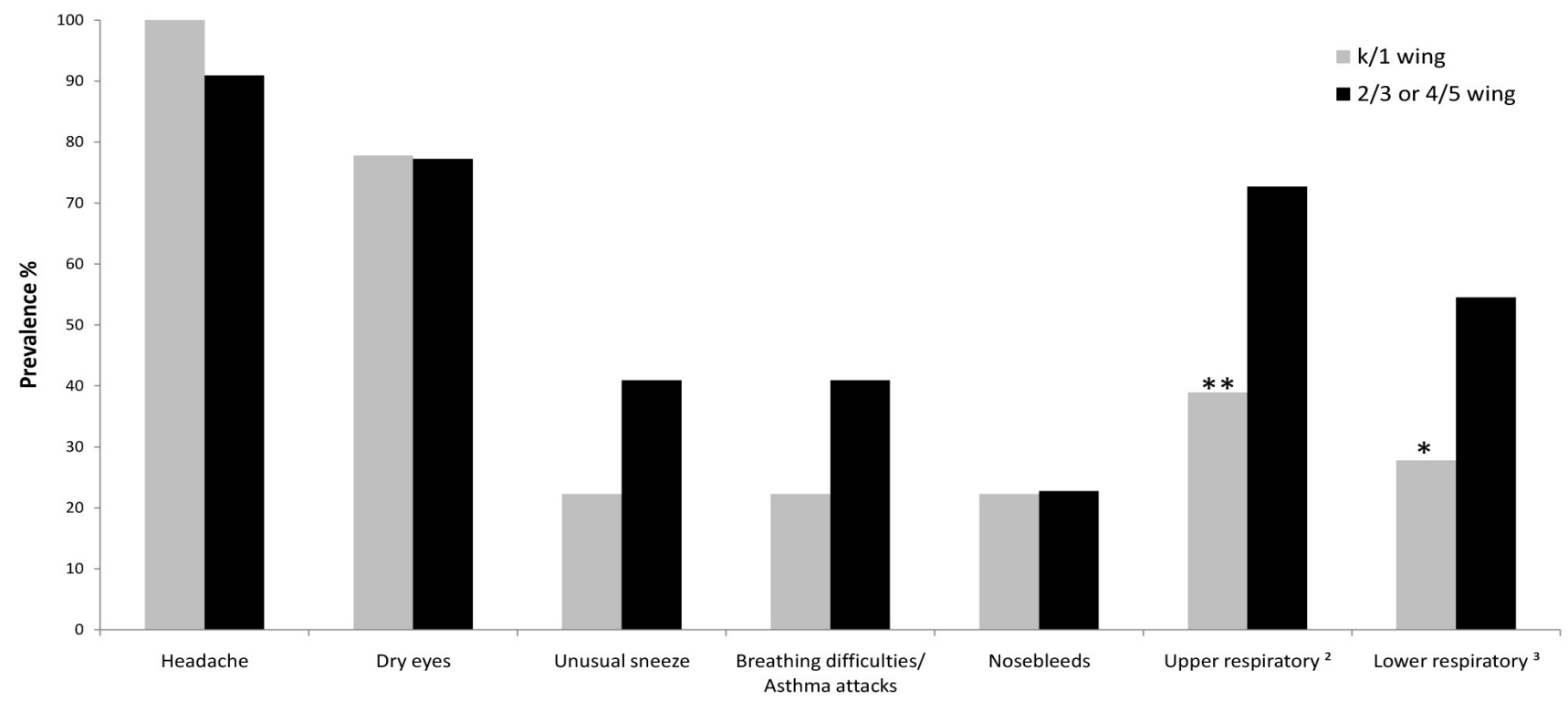

Likelihood ratio chi-square test different at $* * p<0.05$ and $* p<0.10$.

${ }^{1}$ We did not have primary classroom wing information for four of the 52 staff members. Of the 48 staff members with work area information, 40 worked primarily in classroom wings. ${ }^{2}$ Upper respiratory symptoms include one more of the following: sinus problems,
nasal symptoms, sneezing, throat symptoms, and/or voice symptoms.

${ }^{3}$ Lower respiratory symptoms include cough and/or breathing problem/asthma attack. 


\section{FIGURES (CONTINUED)}

Figure 25. Comparison of symptom prevalences in 2008/2009 school year by classroom wing in 376 students ${ }^{1}$.

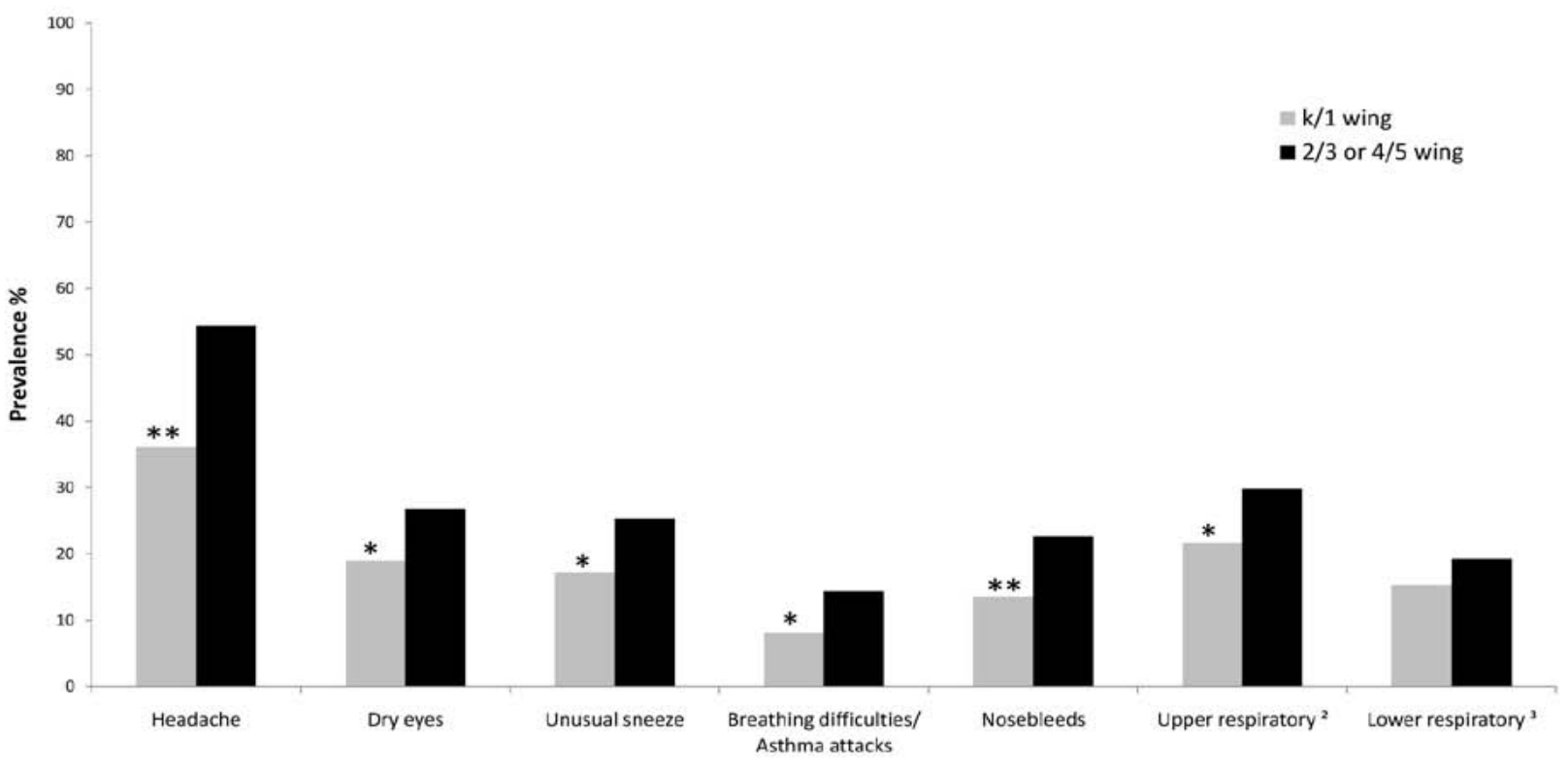

Likelihood ratio chi-square test different at $* p<0.05$ and *p<0.10.

${ }^{2}$ Upper respiratory symptoms include one more of the following: sinus problems, nasal symptoms, sneezing, throat symptoms, and/or voice symptoms.

${ }^{1}$ We did not have classroom wing information for 9 of the 385 students

Figure 26. Comparison of symptoms prevalences in 2007/2008 and 2008/2009 school years in 43 staff members ${ }^{1}$ who had symptoms during the 2008/2009 school year and worked at this school both years.

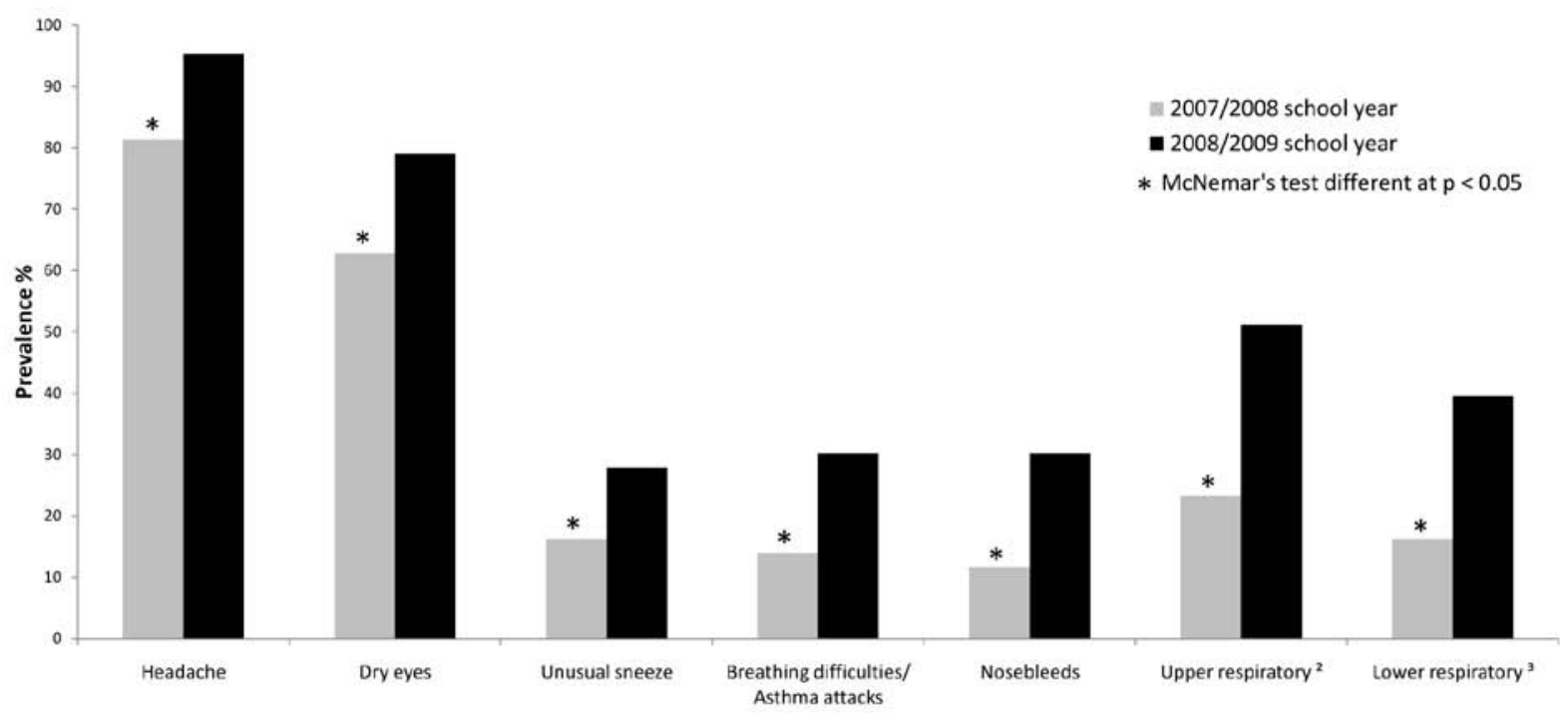

${ }^{1}$ Staff members who worked at the elementary school both years and answered "yes" or wrote in a response to one of the six symptom questions on the first page of the health department questionnaire (Appendix A).

2 Upper respiratory symptoms include one more of the following: sinus problems, nasal symptoms, sneezing, throat symptoms, and/or voice symptoms.

${ }^{3}$ Lower respiratory symptoms include cough and/or breathing problem/asthma attack. 


\section{FIGURES (CONTINUED)}

Figure 27. Comparison of symptom prevalences in 2007/2008 and 2008/2009 school years in 221 students ${ }^{1}$ who had symptoms during the 2008/2009 school year and attended this school both years.

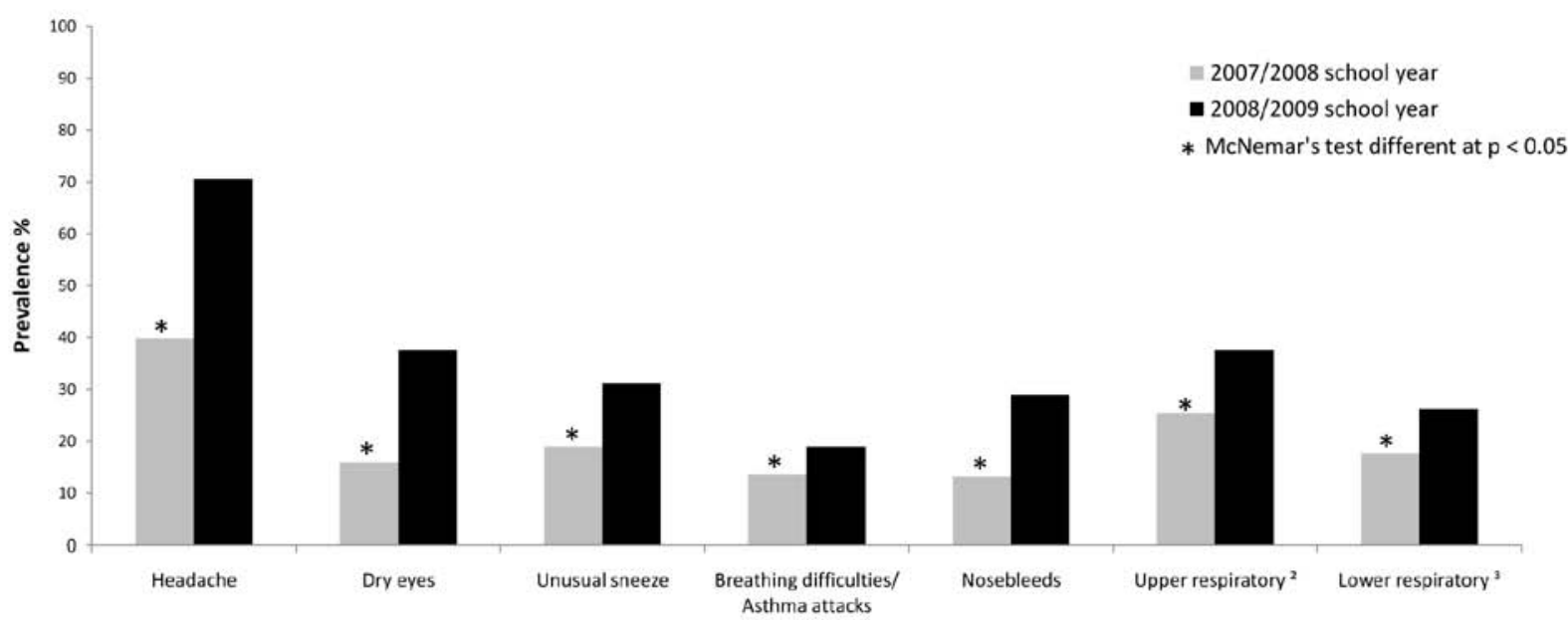

${ }^{1}$ Students who worked at the elementary school both years and answered "yes" or wrote in a response to one of the six symptom questions on the first page of the health department questionnaire (Appendix A).

${ }^{2}$ Upper respiratory symptoms include one more of the following: sinus problems, nasal symptoms, sneezing, throat symptoms, and/or voice symptoms.

${ }^{3}$ Lower respiratory symptoms include cough and/or breathing problem/asthma attack. 


\section{Appendix A: Guilford County Department of Public Health FACULTY/StAFF QUESTIONNAIRE}

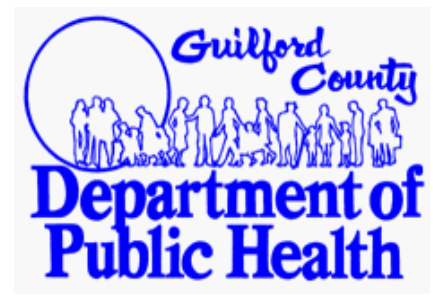

\section{HEALTH \\ QUESTIONNAIRE \\ Oak Ridge Elementary School}

May 2009

This is a survey of the Guilford County Department of Public Health. The purpose of the survey is to assess the health of faculty and staff of the Oak Ridge Elementary School. Participation is voluntary. The information you provide is confidential. Your name and address is requested in the event that we need to follow-up with you for additional information.

Name (Last/First/Middle Initial)

Home Address

$\begin{array}{lcc}\text { City } & \text { State Z Zip } \\ \text { Home Phone } & \text { Age__ }\end{array}$

When did you work at Oak Ridge Elementary School? (Check all that apply)

2008-2009 School Year (August 2008-May 2009)

2007-2008 School Year (August 2007-May 2008)

Where at Oak Ridge Elementary School is your office or classroom located currently and in previous years?

2008-2009 School Year: Primary Classroom \# 2007-2008 School Year: Primary Classroom \# or Office \# or Office \#

During the 2008-9 school year (from August 2008-May 2009) did you experience any of the following symptoms while at work in the school building? (Circle all that apply)

If YES, how frequently? (daily, sometimes, rarely)

$\begin{array}{llcl}\text { 1. Headaches } & \text { YES } & \text { NO } \\ \text { 2. } & \text { Dry eyes } & \text { YES } & \text { NO } \\ \text { 3. } & \text { Unusual sneezing episodes } & \text { YES } & \text { NO } \\ \text { 4. } & \text { Difficulty breathing, asthma attacks } & \text { YES } & \text { NO } \\ \text { 5. } & \text { Nosebleeds } & \text { YES } & \text { NO }\end{array}$

6. Other symptoms, discomfort 


\section{Appendix A: Guilford County Department of Public Health Faculty/StafF Questionnaire (COntinued)}

If you answered NO to questions 1-5 and reported no other symptoms or discomfort in question 6, you may STOP the survey at this point and return your survey to Oak Ridge Elementary School according to the cover sheet instructions.

If you answered YES to any of the symptom questions above or listed other symptoms, did you seek medical care for any of these concerns? YES NO

If you did seek medical care in 2008-9, where did you seek care? (If so, please check and in the parentheses write in how many times)

Doctor's Office ) Emergency Room ( $($ ) Hospital Admission (

During the 2007-8 school year (August 2007-May 2008) did you experience any of the following symptoms while at work in the school building? (Circle all that apply)

If YES, how frequently? (daily, sometimes, rarely)

\begin{tabular}{llll} 
1. & Headaches & YES & NO \\
2. & Dry eyes & YES & NO \\
3. & Unusual sneezing episodes & YES & NO \\
4. & Difficulty breathing, asthma attacks & YES & NO \\
5. & Nosebleeds & YES & NO \\
6. & Other symptoms, discomfort & \\
\hline
\end{tabular}

If you answered YES to any of the symptom questions above or listed other symptoms, did you seek medical care for any of these concerns? YES NO

If you did seek medical care in 2007-8, where did you seek care? (If so, please check and then in the parentheses write in how many times):

Doctor's Office ) Emergency Room ( ${ }^{\prime}$ Hospital Admission (

Is there a particular room or area in the building where you experience more symptoms or discomfort?

If YES, what is room number or description of room?

$$
\text { YES NO }
$$

Do you have any of the following health conditions? (This information will be kept confidential.)

1. Chronic respiratory disease

2. Undergoing chemotherapy or radiation therapy

3. Immune system suppressed by disease or other causes
YES NO

YES NO

YES NO 


\section{Appendix A: County Department of Public Health Faculty/StafF Questionnaire (CONtInUed)}
4. Allergic rhinitis
YES NO
5. Asthma
YES NO
6. Seasonal Allergies
YES NO
7. Cystic Fibrosis
YES NO

What prescription medications are you taking?

What over the counter medications are you taking?

What herbal therapies are you taking?

When did the symptoms/problems start?

2007-2008 school year or earlier

2008-2009 school year

Of the symptoms/problems you have been experiencing, which are the worst?

When are the symptoms/problems generally worse?

Beginning of the week End of the week Particular days

Morning

Afternoon

Particular times of day

Spring

Summer

Fall

Winter

When do symptoms/problems go away?

After leaving the building YES NO

If YES, how long does it take to go away? 


\section{Appendix A: County Department of Public Health FaCUlty/StafF QuestionNaIRE (CONTINUed)}

Do you smoke cigarettes, a pipe or cigars?

Are there any other smokers in your household?

If YES, how many total smokers in household?

Please provide us with some information about your current home:

house

duplex

mobile home

YES NO

YES NO
a. Age of building:
years
b. Type of heating:
forced hot air water/steam electric gas oil

c How many people live in your household?

d. Are there any pets in your household?

YES NO If YES, please specify.

e. Do you use a humidifier at home?

YES NO

f. Do you have wall to wall carpeting in your home?

YES NO

g. Have there been any water leaks in your home?

YES NO

If YES, describe.

h. Have you noticed visible water stains on the walls?

YES NO

i. Visible stains on ceiling or ceiling tiles?

YES

NO

j. Have you noticed mold or mildew?

YES NO

If YES, was the mold problem corrected?

YES NO

When?

Is there any additional information you feel we should know?

This concludes the survey. Please see the cover letter for instructions on returning the survey. Thank you for your participation! 


\section{Appendix A: County Department of Public Health \\ Faculty/StafF Questionnaire (continued)}

Extra page for any additional information you wish to provide. 


\section{Appendix B: County Department of Public Health Parent QUESTIONNAIRE}

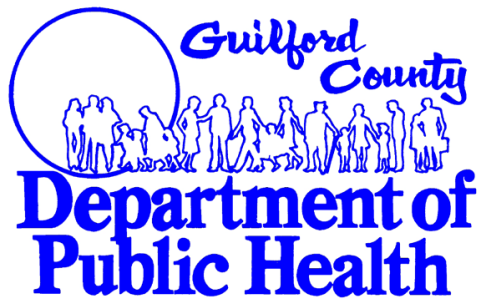

\section{Parent/Guardian}

\section{HEALTH \\ QUESTIONNAIRE \\ Oak Ridge Elementary School}

May 2009

This is a survey of the Guilford County Department of Public Health. The purpose of the survey is to assess the health of students attending the Oak Ridge Elementary School. This survey should be completed by one of the student's parents or guardian. If you have more than one child at Oak Ridge Elementary School, a separate survey should be completed for each child. Participation is voluntary. The information you provide is confidential. Your name and address is requested in the event that we need to follow-up with you for additional information.

Name of Child (Last/First/Middle Initial) Home Address

City
Home Phone
Name of Parent or Guardian who completed survey ___ Age

Which years did your child attend Oak Ridge Elementary School? (Check all that apply)

2008-2009 School Year (August 2008-May 2009)

2007-2008 School Year (August 2007-May 2008)

2006-2007 School Year (August 2006-May 2007)

2005-2006 School Year (August 2005-May 2006)

2004-2005 School Year (August 2004-May 2005)

Where at Oak Ridge Elementary School is your child's main classroom or homeroom located for the last two years?

2008-2009 School Year: Primary Classroom or Homeroom \# 2007-2008 School Year: Primary Classroom or Homeroom \#

During the 2008-9 school year (from August 2008-May 2009) did your child experience any of the following symptoms while in the school building? (Circle all that apply)

If YES, how frequently? (daily, sometimes, rarely)

\begin{tabular}{|c|c|c|}
\hline 1. & Headaches & YES \\
\hline 2 & Dry eyes & YES \\
\hline 3. & Unusual sneezing episodes & YES \\
\hline
\end{tabular}




\section{Appendix B: County Department of Public Health Parent Questionnaire (CONTINUED)}

4. Difficulty breathing, asthma attacks YES NO

5. Nosebleeds $\quad$ YES NO

6. Other symptoms, discomfort

If you answered NO to questions 1-5 and your child reported no other symptoms or discomfort in question 6, you may STOP the survey at this point and return your survey to Oak Ridge Elementary School according to the cover sheet instructions.

If you answered YES to any of the symptom questions above or listed other symptoms, did you seek medical care for your child for any of these concerns?

If you did seek medical care for your child in 2008-9, where did you seek care? (If so, please check and in the parentheses write in how many times)

Doctor's Office ___ ( ) Emergency Room ___ ( ) Hospital Admission ___ ( )

During the 2007-8 school year (August 2007-May 2008) did your child experience any of the following symptoms while in the school building? (Circle all that apply)

If YES, how frequently?

1. Headaches YES NO

(daily, sometimes, rarely)

2. Dry eyes $\quad$ YES NO

3. Unusual sneezing episodes $\quad$ YES NO

4. Difficulty breathing, asthma attacks YES NO

5. Nosebleeds $\quad$ YES NO

6. Other symptoms, discomfort

If you answered YES to any of the symptom questions above or listed other symptoms, did you seek medical care for your child for any of these concerns? $\quad$ YES NO

If you did seek medical care for your child in 2007-8, where did you seek care? (If so, please check and in the parentheses write in how many times)

Doctor's Office ___ ( ) Emergency Room ___ ( ) Hospital Admission ___ ( )

Is there a particular room or area in the building where your child experienced more symptoms or discomfort (Room number or description)? $\quad$ YES NO

If YES, what is the room number or description of area?

Does your child have any of the following health conditions? (This information will be kept confidential.)

ORES Parent/Guardian 


\section{Appendix B: County Department of Public Health Parent Questionnaire (COntinued)}

1. Chronic respiratory disease

2. Undergoing chemotherapy or radiation therapy

3. Immune system suppressed by disease or other causes

4. Allergic rhinitis

5. Asthma

6. Seasonal Allergies

7. Cystic Fibrosis
YES NO

YES NO

YES NO

YES NO

YES NO

YES NO

YES NO

What prescription medications does your child take?

What over the counter medications does your child take?

What herbal therapies does your child take?

When did the symptoms/problems start?

2007-2008 school year or earlier 2008-2009 school year

Of the symptoms/problems your child has been experiencing, which are the worst?

When are the symptoms/problems generally worse?

Beginning of the week End of the week Particular days

Morning

Afternoon

Spring

Summer

Fall

Winter 


\section{Appendix B: County Department of Public Health Parent Questionnaire (CONtInUed)}

\section{When do symptoms/problems go away?}

After leaving the building YES NO

If YES, how long does it take to go away?

\section{Are there any smokers in your household? YES NO}

If YES, how many total smokers in household?

Please provide us with some information about the home where your child lives:

house duplex mobile home other

a. Age of building: years

b. Type of heating: forced hot air water/steam electric gas oil

c How many people live in your household?

d. Are there any pets in your household?

YES NO If YES, please specify.

e. Do you use a humidifier at home?

YES NO

f. Do you have wall to wall carpeting in your home?

YES NO

g. Have there been any water leaks in your home/ apartment?

If YES, describe.

h. Have you noticed visible water stains on the walls?

YES NO

i. Visible stains on ceiling or ceiling tiles?

YES NO

j. Have you noticed mold or mildew?

YES NO

If YES, was the mold problem corrected?

YES NO

When?

Is there any additional information you feel we should know? 


\section{Appendix B: County Department of Public Health Parent Questionnalre (continued)}

This concludes the survey. Please see the cover letter for instructions on returning the survey. Thank you for your participation!

Extra space for any additional information you wish to provide. 


\title{
Appendix C: NIOSH March 2010 questionNAIRE
}

\author{
U.S. DEPARTMENT OF HEALTH AND HUMAN SERVICES \\ CENTERS FOR DISEASE CONTROL AND PREVENTION \\ NATIONAL INSTITUTE FOR OCCUPATIONAL SAFETY AND HEALTH
}

Oak Ridge Elementary School

Oak Ridge, $\mathrm{NC}$

HETA 2009-0172

The National Institute for Occupational Safety and Health (NIOSH) is a part of the United States Public Health Service and an institute within the Centers for Disease Control and Prevention (CDC) that is concerned with workplace health and safety. In 2009, we received a Health Hazard Evaluation request from officials of Guilford County Schools to evaluate health concerns that may be related to your workplace environment. The purpose of this survey is to assess the current health of employees.

This is a questionnaire about your health history and work history. All medical and other personal information that you provide NIOSH is considered confidential in accordance with the Privacy Act of 1974 (Public Law 93-579). The information you provide NIOSH will be summarized so that no individual is identified. All information is stored at NIOSH until destroyed. Management will not see your response.

If you have any questions about this survey or your participation, please contact the NIOSH project officer Dr. Rachel Bailey. She will be at Oak Ridge Elementary School from March 2-4, 2010. Additionally, you may contact her at 1(800) 232-2114 or (304) 285-5757.

\section{"BY COMPLETING THIS QUESTIONNAIRE, YOU INDICATE YOUR CONSENT TO PARTICIPATE IN THIS STUDY."}

\section{Thank you for your participation.}




\section{Appendix C: NIOSH March 2010 questionnalRe (CONtinued)}

\section{NIOSH-Administered Current Employee Health Questionnaire}

\section{Identification and Demographic Information}

1. Survey Date: $/ 2010$

2. Name:

First $\overline{\text { MI }}$

$\overline{\text { Last }}$

3. Home Telephone Number:

4. Date of Birth:

$$
\overline{\text { Month }}{ }^{\prime} \overline{\text { Day }}{ }^{\prime}-\overline{\text { Year }}-
$$

5. Gender:
1.___ Male

2.___ Female

6. $\quad$ Ethnicity (Please choose one):

1.___ Hispanic or Latino

$0 . \quad$ Not Hispanic or Latino

7. Race (Please choose all that apply):

1.___ American Indian or Alaska Native

2._Asian

3. Black or African American

4._ Native Hawaiian or Other Pacific Islander

5._White 


\section{Appendix C: NIOSH March 2010 questionNAIRE (CONTINUED)}

I am going to ask you some questions about your health in the four-week timeframe prior to Oak Ridge Elementary School re-opening on February 22, 2010; therefore from January 24, 2010 to February 21, 2010. Some questions will ask about your symptoms away from "the building". "The building" refers to the school building you worked in during January 24, 2010 to February 21, 2010. The answers to many of these questions will be "Yes" or "No." If you are in doubt about whether to answer "Yes" or "No," then please answer "No."

8.1 During the 4 weeks before returning to Oak Ridge Elementary School, did you have wheezing or whistling in your chest at any time?

\section{IF YES:}
1. Yes
0. No

8.2 Did you have wheezing or whistling in your chest one or more times per week during those 4 weeks?

1.Yes 0. No

8.3 When you were away from the building was this wheezing or whistling in your chest:

$$
\text { 1.Same }
$$
2.Worse 3.Better

8.4 In what month and year did you first have wheezing or whistling in your chest?

$$
\overline{\text { Month }}{ }^{\prime}-\overline{\text { Year }}-
$$

9.1 During the 4 weeks before returning to Oak Ridge Elementary School, did you have chest tightness?

\section{IF YES:}

9.2 Did you have chest tightness one or more times per week during those 4 weeks?

$$
\text { 1.Yes _ } 0 \text {. No }
$$

9.3 When you were away from the building was the chest tightness:

$$
\text { 1. Same }
$$

2. Worse

3. Better

9.4 In what month and year did you first have chest tightness?

$$
\overline{\text { Month }}+\frac{\text { Y }}{\mathrm{ear}}-
$$

10.1 During the 4 weeks before returning to Oak Ridge Elementary School, did you have attacks of shortness of breath?

1. Yes 0 . No

\section{IF YES:}

10.2 Did you have attacks of shortness of breath one or more times per week during those 4 weeks?

$$
\text { 1. Yes }
$$
0 . No

10.3 When you were away from the building were the attacks of shortness of breath:

1.Same 2.Worse 3.Better

10.4 In what month and year did you first have attacks of shortness of breath?

$\overline{\text { Month }} \overline{\text { Year }}-$




\section{Appendix C: NIOSH MARCh 2010 questionNAIRE (CONTINUED)}

11.1 During the 4 weeks before returning to Oak Ridge Elementary School, did you have coughing attacks?

IF YES:

11.2 Did you have coughing attacks one or more times per week during those 4 weeks?

1. Yes _ 0 . No

11.3 When you were away from the building were the coughing attacks:

1.Same _ 2.Worse _ _ 3.Better

11.4 In what month and year did you first have coughing attacks?

$\overline{\text { Month }}^{\prime}-\overline{\mathrm{Y}} \overline{\mathrm{ear}}-$

12.1 During the 4 weeks before returning to Oak Ridge Elementary School, were you awakened by an attack of breathing difficulty?

\section{IF YES:}

1. Yes _ 0 . No

12.2 Were you awakened by an attack of breathing difficulty one or more times per week during those 4 weeks?

1. Yes 0. No

12.3 When you were away from the building was the awakening by attacks of breathing difficulty:

1.Same 2.Worse 3.Better

12.4 In what month and year were you first awakened by an attack of breathing difficulty?

$\overline{\text { Month }}-\overline{\text { Year }}-$

13.1 During the 4 weeks before returning to Oak Ridge Elementary School, did you have shortness of breath when hurrying on level ground or walking up a slight hill? $\quad 1$. Yes _ 0 . No

IF YES:

13.2 Did you have shortness of breath when hurrying on level ground or walking up a slight hill one or more times per week during those 4 weeks?

1. Yes 0 . No

13.3 When you were away from the building was this shortness of breath:

$$
\text { 1.Same _ 2.Worse _ _ 3.Better }
$$

13.4 In what month and year did you first have this shortness of breath?

$$
\overline{\text { Month }}+\frac{\text { Year }}{-}
$$

14.1 During the 4 weeks before returning to Oak Ridge Elementary School, did you have a cough with phlegm?

1.Yes 0. No

\section{IF YES:}

14.2 Did you have cough with phlegm one or more times per week during those 4 weeks?

1.Yes _ 0. No

14. 3 When you were away from the building was the cough with phlegm:
1.Same 2.Worse 3.Better

14.4 In what month and year did you first have cough with phlegm?

$\overline{\text { Month }}+\frac{1}{\text { Year }}-$




\section{ApPENDIX C: NIOSH MARCH 2010 QuestiOnNAIRE (CONTINUED)}

15.1 During the 4 weeks before returning to Oak Ridge Elementary School, did you have episodes of fever and chills?

1.Yes 0. No

IF YES:

15.2 Did you have episodes of fever and chills one or more times per week during those 4 weeks?

$$
\text { 1.Yes _ } 0 . \text { No }
$$

15.3 When you were away from the building were these episodes of fever and chills:

1.Same

2.Worse

3.Better

15.4 In what month and year did you first have episodes of fever and chills?

$\overline{\text { Month }}{ }^{\prime}-\overline{\text { Year }}-$

16.1 During the 4 weeks before returning to Oak Ridge Elementary School, did you have episodes of flulike achiness or achy joints?

\section{IF YES:}

1.Yes _ 0. No

16.2 Did you have episodes of flu-like achiness or achy joints one or more times per week during those 4 weeks?

1.Yes 0. No

16. 3 When you were away from the building was the flu-like achiness or achy joints:

1.Same 2.Worse 3.Better

16.4 In what month and year did you first have episodes of flu-like achiness or achy joints?

$\overline{\text { Month }}{ }^{\prime}-\overline{\text { Year }}-$

17.1 During the 4 weeks before returning to Oak Ridge Elementary School, did you have unusual tiredness or fatigue?

IF YES:

1. Yes _ 0 . No

17.2 Did you have unusual tiredness or fatigue one or more times per week during those 4 weeks?

1. Yes 0 . No

17.3 When you were away from the building was the unusual tiredness or fatigue:

$$
\text { 1.Same _ 2. Worse _ _ 3.Better }
$$

17.4 In what month and year did you first have unusual tiredness or fatigue?

$$
\overline{\text { Month }}{ }^{\prime}-\overline{\text { Year }}-
$$




\section{Appendix C: NIOSH MARCH 2010 questionNAIRE (CONTINUED)}

18.1 During the 4 weeks before returning to Oak Ridge Elementary School, did you have difficulty remembering things or concentrating?

IF YES:

1.Yes _

18.2 Did you have difficulty remembering things or concentrating one or more times per week during those 4 weeks?

1.Yes 0. No

18.3 When you were away from the building was the difficulty remembering things or concentrating:

$$
\text { 1.Same _ 2.Worse _ _ 3.Better }
$$

18.4 In what month and year did you first have difficulty remembering things or concentrating?

$\overline{\text { Month }}{ }^{\prime}-\overline{\text { Year }}$

19.1 During the 4 weeks before returning to Oak Ridge Elementary School, did you have dizziness or lightheadedness?

1.Yes 0. No

IF YES:

19.2 Did you have dizziness or lightheadedness one or more times per week during those 4 weeks?

$$
1 . \text { Yes } \quad 0 . \text { No }
$$

19.3 When you were away from the building was the dizziness or lightheadedness:

$$
\text { 1.Same _ 2. Worse _ } 3 . \text { Better }
$$

19.4 In what month and year did you first have dizziness or lightheadedness?

$\overline{\text { Month }}{ }^{\prime}-\overline{\text { Year }}-$

20.1 During the 4 weeks before returning to Oak Ridge Elementary School, did you have headaches?

IF YES:
1.Yes
0. No

20.2 Did you have headaches one or more times per week during those 4 weeks?

1. Yes _ $0 . \mathrm{No}$

20.2 When you were away from the building were the headaches:

1.Same

2.Worse _ 3.Better

21.1 During the 4 weeks before returning to Oak Ridge Elementary School, did you have any episodes of stuffy, itchy or runny nose?

IF YES:

1.Yes _ 0. No

21.2 Did you have a stuffy, itchy or runny nose one or more times per week during those 4 weeks?

1.Yes _ 0. No

21.3 When you were away from the building was the stuffy, itchy or runny nose:

1.Same

2.Worse _ 3.Better

22.1 During the 4 weeks before returning to Oak Ridge Elementary School did you have sneezing?

\section{IF YES:}

$$
1 . \text { Yes _ } 0 . \text { No }
$$




\section{Appendix C: NIOSH MARCH 2010 questionNAIRE (CONTINUED)}

22.2 Did you have sneezing one or more times per week during those 4 weeks?

1.Yes 0. No

22.3 When you were away from the building was the sneezing:

1.Same 2.Worse 3.Better

23.1 During the 4 weeks before returning to Oak Ridge Elementary School, did you have dry or itchy skin?

1.Yes $\quad 0$. No

\section{IF YES:}

23.2 Did you have dry or itchy skin one or more times per week during those 4 weeks?

1.Yes_ 0. No

23.3 When you were away from the building was the dry or itchy skin:

1.Same _ 2.Worse _ _ 3.Better

23.4 In what month and year did you first have dry or itchy skin?

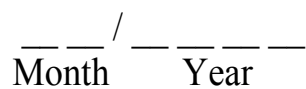

24.1 During the 4 weeks before returning to Oak Ridge Elementary School, did you have episodes of watery, itchy eyes?

\section{IF YES:}

$$
\text { 1.Yes_ } 0 . \text { No }
$$

\subsection{Did you have watery or itchy eyes one or more times per week during those 4 weeks?}

1.Yes

0. No

24.3 When you are away from the building were the watery or itchy eyes:
1.Same
2. Worse 3.Better

24.4 In what month and year did you first have watery or itchy eyes?

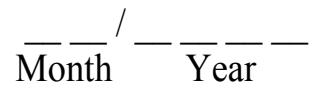

25.1 During the 4 weeks before returning to Oak Ridge Elementary School, did you have a sore or dry throat? 1. Yes _ 0. No

\section{IF YES:}

25.2 Did you have a sore or dry throat one or more times per week during those 4 weeks?

1.Yes 0. No

25.3 When you were away from the building was the sore or dry throat:

1.Same _ 2.Worse _ _ 3.Better

25.4 In what month and year did you first have a sore or dry throat?

$\overline{\text { Month }}{ }^{\prime}-\overline{\text { Year }}-$

26.1 During the 4 weeks before returning to Oak Ridge Elementary School, did you have a nosebleed?

IF YES:

1.Yes__ 0. No

26.2 Did you have nosebleeds one or more times per week during those 4 weeks?

1. Yes _ 0. No 


\section{Appendix C: NIOSH MARCH 2010 questionNAIRE (CONTINUED)}

26.3 When you were away from the building were the nosebleeds:

1.Same

2.Worse

3.Better

27. During the 4 weeks before returning to Oak Ridge Elementary School did you have a cold?

$$
1 . \text { Yes _ } 0 . \text { No }
$$

28. During the 4 weeks before returning to Oak Ridge Elementary School did you have sinusitis or sinus problems?

1.Yes 0. No

29.1 During the 4 weeks before returning to Oak Ridge Elementary School did you have bronchitis?

IF YES:

1. Yes _ 0. No

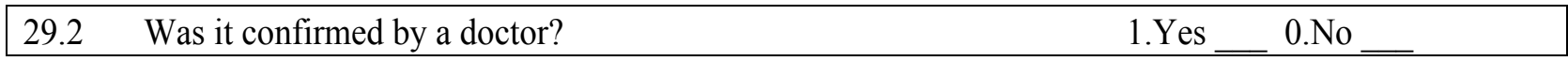

30.1 Do you have any nasal allergies, including hay fever?

1. Yes

0.No

\section{IF YES:}

30.2 How old were you when you first had hay fever or nasal allergies?

31. Have you ever had eczema or any kind of skin allergy?

1. Yes

0.No

32.1 Has a physician ever told you that you have asthma?

1. Yes

0. No

\section{IF YES:}

32.2 In what month and year were your first diagnosed with asthma?

32.3 Do you still have asthma?

$\overline{\text { Month }}{ }^{\prime}-\overline{\text { Year }}-$

1. Yes _ 0. No

32.4 During the last six months prior to returning to Oak Ridge Elementary School, how many times did you get treatment for an acute asthma attack at a doctor's office, urgent care facility, or emergency department? Times

33. During the 4 weeks before returning to Oak Ridge Elementary School, how many days did you miss work because of respiratory health problems?

Days

34. During the 4 weeks before returning to Oak Ridge Elementary School, how many days did you miss work because of health problems other than respiratory? Days

\section{Medications for Breathing Problems}

I am now going to ask you some questions about medication use in the four-week timeframe prior to Oak Ridge Elementary School re-opening on February 22, 2010; therefore from January 24, 2010 to February 21, 2010.

35.1 During the 4 weeks before returning to Oak Ridge Elementary School did you use any prescription or over-the-counter medications for breathing problems?

IF YES:

1.Yes 0.No 


\section{Appendix C: NIOSH March 2010 questionNaIRE (CONTINUEd)}

35.2 In those 4 weeks, did you use any inhaled beta-agonists (quick-relief medicine, such as Albuterol or Proventil) for breathing problems? 1 .Yes _ 0. No If yes to 35.2 :

35.3 Did you use your beta-agonist inhaler (quick-relief medicine, such as Albuterol or Proventil) on a daily basis during those 4 weeks? 1 .Yes __ 0.No

35.4 Did you use any over-the-counter inhalers or pills (e.g. Primatene) for breathing problems during those 4 weeks?

1. Yes _ 0. No

36.1 During the 4 weeks before returning to Oak Ridge Elementary School did you use any prescription or over-the-counter medications for nasal-sinus or eye problems?

1.Yes 0. No

IF YES:

36.2 Select the prescription or over-the-counter medications you used for nasal-sinus or eye problems during those 4 weeks?

$\square$ Antihistamine pills (Claritin, Zyrtec, Allegra etc)

$\square$ Decongestant pills (Sudafed, etc)

$\square$ Decongestant nasal spray (Afrin, Otrivin, etc)

$\square$ Prescription nasal spray (Flonase, Nasalcrome, Atrovent nasal spray,etc)

$\square$ Eye drops (Visine, Clear eyes, Livostin, etc)

$\square$ Other (please specify

37. Have you ever been told by a physician that you had any of the following conditions?

IF YES:

\begin{tabular}{|c|c|c|}
\hline Conditions & Told by a physician you had it? & $\begin{array}{l}\text { Month and Year of } \\
\text { first diagnosis? }\end{array}$ \\
\hline 37.1 Hay fever or nasal allergies & 1. Yes _ $0 . \mathrm{No}$ & \\
\hline 37.2 Sinusitis or sinus infections & 1.Yes__ 0.No & \\
\hline 37.3 Eczema, dermatitis, or skin allergy & 1.Yes_ $0 . \mathrm{No}$ & \\
\hline 37.4 Acute bronchitis & $1 . \mathrm{Yes}_{\text {_ }}$ 0.No & \\
\hline 37.5 Chronic bronchitis & 1.Yes__ $0 . \mathrm{No}$ & \\
\hline 37.6 Emphysema & 1.Yes__ 0.No & \\
\hline 37.7 Pneumonia & 1.Yes $\quad 0$. No & \\
\hline 37.8 Hypersensitivity pneumonitis & 1.Yes__ $0 . \mathrm{No}$ & \\
\hline 37.9 Sarcoidosis & $1 . \mathrm{Yes}_{\text {_ }} 0 . \mathrm{No}$ & \\
\hline 37.10 Heart disease & 1.Yes__ $0 . \mathrm{No}$ & \\
\hline
\end{tabular}




\section{Appendix C: NIOSH March 2010 questionNaIRE (CONTINUED)}

38. Did you work at Oak Ridge Elementary School during the 2008/2009 school year?

\section{IF YES:}

1. Yes _ 0. No

39.1. Did you have any lower respiratory symptoms such as cough, shortness of breath, chest tightness, or wheezing while at work in the Oak Ridge Elementary School building during the 2008/2009 school year?

IF YES

$$
\text { 1. Yes _ } 0 . \text { No _ _ year }
$$

39.2 When you were away from the Oak Ridge Elementary School building while it was closed for remediation were your lower respiratory symptoms: $\quad$ 1.Same __ 2.Worse _ _ 3.Better

40.1. Did you have episodes of watery, itchy eyes while at work in the Oak Ridge Elementary School building during the 2008/2009 school year?

\section{IF YES}

$$
\text { 1.Yes _ } 0 . \text { No }
$$

40.2 When you were away from the Oak Ridge Elementary School building while it was closed for remediation were your watery, itchy eyes: $\quad$ 1.Same _ 2 .Worse _ 3. Better

41.1. Did you have upper respiratory symptoms such as stuffy, itchy or runny nose; sore or dry throat; or sneezing while at work in the Oak Ridge Elementary School building during the 2008/2009 school year?

\section{IF YES}

$$
\text { 1.Yes_ } 0 . \text { No }
$$

41.2 When you were away from the Oak Ridge Elementary School building while it was closed for remediation were your upper respiratory symptoms: $\quad$ 1.Same _ _ 2.Worse _ _ 3.Better

42.1. Did you have unusual tiredness or fatigue while at work in the Oak Ridge Elementary School building during the 2008/2009 school year?

1.Yes 0. No

IF YES

42.2 When you were away from the Oak Ridge Elementary School building while it was closed for remediation was your unusual tiredness or fatigue:

1.Same 2.Worse 3.Better

43.1. Did you have fever, chills, or flu-like symptoms while at work in the Oak Ridge Elementary School building during the 2008/2009 school year?
1.Yes 0. No

IF YES

43.2 When you were away from the Oak Ridge Elementary School building while it was closed for remediation were your fever, chills, or flu-like symptoms: 1 .Same _ _ 2.Worse _ _ 3.Better 


\section{Appendix C: NIOSH MARCH 2010 questionNAIRE (CONTINUED)}
44.1. Did you have difficulty remembering things or concentrating while at work in the Oak Ridge Elementary School building during the 2008/2009 school year? 1.Yes 0. No
IF YES

44.2 When you were away from the Oak Ridge Elementary School building while it was closed for remediation was the difficulty remembering things or concentrating:

1.Same 2.Worse 3.Better

45.1. Did you have headaches while at work in the Oak Ridge Elementary School building during the 2008/2009 school year? IF YES

1.Yes _ 0. No

45.2 When you were away from Oak Ridge Elementary School while it was closed for remediation were your headaches:

1.Same 2.Worse 3.Better

46.1. Did you have nosebleeds while at work in the Oak Ridge Elementary School building during the 2008/2009 school year?

1.Yes 0. No

IF YES

46.3 When you were away from the Oak Ridge Elementary School building while it was closed for remediation were your nosebleeds:

1.Same 2.Worse 3.Better

47. Where was your primary work area during the 2008/2009 school year at Oak Ridge Elementary School?
$\mathrm{k} / 1$ wing _ $2 / 3$ wing
gym area cafeteria $4 / 5$ wing administrative wing media center wing other (please describe:

48.1 Since the Oak Ridge Elementary School re-opening in February 2010, have you had any health symptoms that you consider possibly related to working in this building? 1. Yes __ 0 . No IF YES

48.2 Please describe:

\section{Work Information}

49. Where did you work when Oak Ridge Elementary School was closed for repair and remediation from August 2009 to February 2010?

Oak Ridge Military Academy

Colfax Elementary School
Pearce Elementary School 


\section{Appendix C: NIOSH March 2010 questionNaIRE (CONTINUED)}

Northwest Middle School Campus

Other (Please specify:

50. What is your current job title:

\begin{tabular}{|c|c|}
\hline 1. & Teacher \\
\hline 2. & Teacher's Aide \\
\hline 3. & Clerical/Secretarial \\
\hline 4. & Administration \\
\hline 5. & Maintenance \\
\hline 6. & Custodian \\
\hline 7. & Librarian \\
\hline 8. & Counselor \\
\hline 9. & Nurse \\
\hline 10. & Cafeteria worker \\
\hline 11. & Other (please specify \\
\hline
\end{tabular}

51. When did you first start working at Oak Ridge Elementary School?

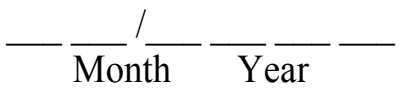

52. Where is your current primary work area at Oak Ridge Elementary School?

$\mathrm{k} / 1$ wing _ $2 / 3$ wing __ $4 / 5$ wing __ administrative wing __ media center wing

gym area

cafeteria

other (please describe :

\section{Tobacco Use Information}

I am now going to ask you a few questions about tobacco use.
53.1 Have you ever smoked cigarettes?
(NO if less than 20 packs of cigarettes
1. __ Yes 0.
in a lifetime or less than 1 cigarette a day for 1 year.)

\section{IF YES:}

53.2 How old were you when you first started smoking regularly? _ Y Years old

53.3 Over the entire time that you have smoked, what is the average number of cigarettes that you smoked per day?

Cigarettes/day

53.4 Do you still smoke cigarettes?

IF NO:

53.5 How old were you when you stopped smoking cigarettes regularly?

1. Y Yes

0.

53.5. How old were you when you stopped smoking cigarettes regularly?

54. Do you have any comments or concerns that might contribute to our investigation?

\section{Thank you for participating in this survey!}

Years old 


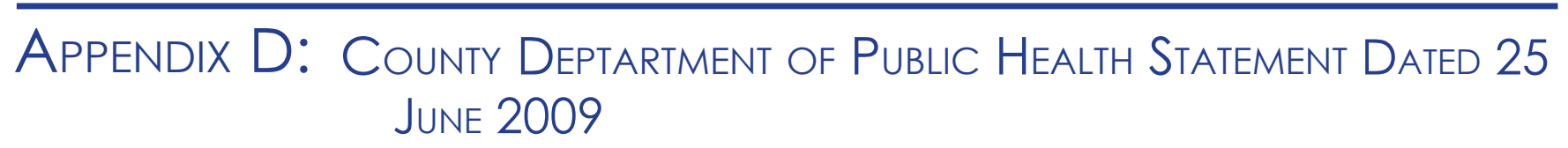

25 June 2009

In April 2009, the Guilford County Department of Public Health received a request to evaluate reports that the staff and students of Oak Ridge Elementary School (ORES) had been exposed to and had become ill from mold growth within the building. Since the request, Public Health staff has:

1. Inspected the building

2. Interviewed teachers and staff

3. Attended the open forum at the ORES

4. Reviewed environmental reports

5. Met with School officials and administration

6. Reviewed the medical literature pertaining to mold and human health

7. Acquired information from representatives of the Centers for Disease Control and Prevention

8. Acquired information from two EPA funded physicians expert on mold and human health

9. Acquired information from the North Carolina Department of Public Health

10. Constructed, administered, collated and interpreted a health survey of staff and parents/guardians of students at ORES

The following statements represent our current understanding of the origin of the ORES concerns and recommendations based on the available science and medical knowledge. As with any diagnosis, our opinions are subject to change as inspections proceed at the school over the summer.

Oak Ridge Elementary School is an amalgam of a renovated building and adjacent new construction. As many such large patchwork facilities experience, there were leaks in the roofline that allowed water to enter at various points. The indoor dampness elicited mold growth that ultimately became visible to parents and staff. Cultures were taken of the area in 2007 and yielded Aspergillus and Penicillium. The testing procedure is not standardized and there are no accepted guidelines to allow interpretation of results.)

The Guilford County School Administration has hired and listened to numerous experts on indoor air quality. They have patched rooflines, diverted drainage channels, replaced drywall, and put down tile in place of carpet. The areas of the building which have dampness and mold have either been remediated or are in the process of remediation.

The appropriate response to finding visible mold in any building is to find and to repair the leak, which allowed the moisture to enter, and to clean or to replace the surfaces where the mildew has grown. Further additional efforts are not medically indicated at this time. The detection of antibodies to molds in any person is expected and a sign of an intact and normal immune system. 


\section{Appendix D: County Deptartment of Public Health Statement Dated 25 JUNE 2009 (CONTINUED)}

The purpose of our recent survey was to catalog the symptoms of students and staff. We asked in the survey whether during the last two years individuals had had dry eyes, rashes, allergic symptoms, headaches and the like. Approximately 800 surveys were distributed. Half of the surveys returned within the week's deadline: a respectable response. A summary of the responses showed that the driving symptoms were headache and fatigue that occurred within the school and that resolved when fresh air was obtained by opening windows and doors to the classrooms, by going outside or by going home (see Appendices 1 and 2). Nosebleeds were also common. Other symptoms were tabulated, but their frequency was not as elevated; nor was there a specific pattern evident.

The building appears to be under-ventilated. A century ago, all buildings were constructed to have large windows, high ceilings, front porches, fireplaces or steam radiators. It has been only within the last decades that we've closed the windows and truly moved indoors. Conditioned air, must be humidified or dehumidified, filtered or circulated, heated or cooled in combinations. As a consequence, some building dwellers have found that the absence of a constant source of large volumes of fresh air elicits headache, fatigue, difficulty concentrating and other, equally immeasurable but real symptoms. These symptoms are related to build up of $\mathrm{CO} 2$ or other human and building castoffs. The symptoms abate with larger amounts of circulated air. The symptoms detailed in the ORES survey match this pattern. Our own inspection of the building encountered many rooms in the school that needed more air flow for comfort.

Another reported and frequent problem reported by staff and parents and found in the survey is nosebleeding (epistaxis). Nosebleeds are common in school-aged children. The two leading causes of nosebleeds are dry air and trauma (nose-picking). We know that the introduction of a dehumidifier last summer in response to the finding of mold resulted in dryer air this year compared to last. However, we found no significant difference between last year's experience and this year's with respect to nosebleeding. Regardless, rather than a sign of exposure to molds, nosebleeds are likely due to dry air. Dry air has been raised as a concern by the state's environmental review.

Beyond the survey, in direct interviews and in emails, teachers have reported an unusual clustering of students needing glasses. Twenty-five percent of children will develop refractive errors during these school years. The sudden increase in discovered refractive errors is due to the appropriate vigilance of the teachers for reading difficulties and then hyper-vigilance for others once the index cases were found. The continued surveillance of school-aged children for visual problems is encouraged, but there is no scientific or medical basis on which to blame a school's environmental for students needing eyeglasses. Our recommendations continue to be:

1. Focus on the HVAC system and recalibrate air exchanges according to the guidelines in the engineer's reports;

2. Reassess the settings on the carry our dehumidification system to assure the best settings for each season of the year.

3. Take advantage of summertime school closings to continue to research possible causes of the symptoms. Invite official governmental and research agencies on iste for testing such as National Institute of Occupational Safety and Health (NIOSH), US Dept of Health and Human Services (USDHHS), and the Centers for Disease Control (CDC). 


\section{Appendix D: County Deptartment of Public Health Statement Dated 25 JUNE 2009 (CONTINUED)}

References:

Bush, R. K., Portnoy, J. M., Saxon, A. (2006). The medical effects of mold exposure. Journal of Allergy \& Clinical Immunology 117(2), 326-333.

CDC. (2006) Mold Prevention Strategies and Possible Health Effects in the Aftermath of Hurricanes and Major Floods. MMWR 55(RR08), 1-27.

Chang, C. and Gershwin, M. E. (2005) Mold hysteria: Origin of the hoax. Clinical and Developmental Immunology 12(2), 151-158.

Coats, D.K. and Paysse, E.A. Refractive errors in children. Available at www.uptodate. com.

Geller R. (2006) Mold. Safe and Healthy School Environments. New York: Oxford University Press.

Gomzi M, Bobic J, Radosevic-Vidacek B, et al. (2007) Sick building syndrome: psychological, somatic, and environmental determinants. Arch Environ Occup Health. 62(3), 147-55.

Hansen AM, Meyer HW, and Gyntelberg F. (2008) Building-related symptoms and stress indicators. Indoor Air 18(6):440-6.

Institute of Medicine. (2004) Damp Indoor Spaces and Health. Washington: National Academies Press.

Messner, AH. Evaluation of epistaxis in children. Available at www.uptodate.com.

NIOSH HEALTH HAZARD EVALUATION REPORT. HETA \#2005-0112-2980 Taft Elementary School, Santa Ana, California. September 2005. Available at http://www.cdc.gov/niosh/hhe/reports.

Norbäck D, Nordström K. (2008) Sick building syndrome in relation to air exchange rate, CO(2), room temperature and relative air humidity in university computer classrooms: an experimental study. Int Arch Occup Environ Health. 82(1), 21-30.

Rabito, F. A., Iqbal, S., Kiernan, M.P. et al. (2008) Children's respiratory health and mold levels in New Orleans after Katrina: A preliminary look. Journal of Allergy \& Clinical Immunology. 121(3), 622-625.

Redd, S.C. 2002. State of the Science on Molds and Human Health. Statement for the Record Before the Subcommittees on Oversight and Investigations and Housing and Community Opportunity Committee on Financial Services. United States House of Representatives. Available at http://www.cdc.gov/mold/pdfs/ moldsci.pdf. 


\section{ApPendix E: NIOSH eMAIL DATEd 22 JULY: HETA 2009-0172 - ReQUESTED SUMMARY OF FINDINGS DISCUSSED DURING THE BREAKFAST MEETING ON 16 JULY 2009}

From: Bailey, Rachel L. (CDC/NIOSH/DRDS)

Sent: Wednesday, July 22, 2009 12:32 PM

To: 'Becoats, Eric J'

Cc: Bailey, Rachel L. (CDC/NIOSH/DRDS); Cox-Ganser, Jean (CDC/NIOSH/DRDS); Park, Ju-Hyeong (CDC/NIOSH/ DRDS)

Subject: HETA 2009-0172 - Requested summary of findings discussed during the breakfast meeting on July 16, 2009.

\section{Dr. Becoats,}

As requested, below is a summary of our interim findings discussed during the breakfast meeting on July $16,2009$.

We noted a strong musty, moldy odor from the basement and crawl space under the media center. Lime was observed on the crawl space floor. We also occasionally noted a musty smell in the corridor around the media center in particular near the school museum. We discussed that this problem should be mitigated such that no air from the basement and crawl space reaches the occupied space.

We noted surface drainage issues around the school mainly between the $2 / 3$ and $4 / 5$ wings. Additionally, a storm drain outside the media center was blocked with standing water in it. Such issues may lead to water infiltrating the concrete slab. We discussed that these problems be addressed.

The foam roof on the old section of the school had multiple damaged areas, and there did not appear to be adequate flashing at the junction between Room 206 and the Gym. Inside the school, we noted moisture (with the infrared camera) in the gym's exterior concrete block wall where the bleachers are located. This was an area where a leak had been repaired. We addressed the need for the roof and flashing to be repaired.

We noted pressure airflow issues between rooms and spaces. For example, the classrooms should generally have a positive pressure in regard to the corridor, and the bathrooms should have a negative pressure in regard to the outer room. This was not always the case. During the day, the attic was under positive pressure in regard to the exterior of the building. However at night when the dehumidifier (which serves the classroom wings) was turned off, this resulted in the attic being under negative pressure in regard to the outside of the building. This allowed outside humid air to enter the attic from the soffits. From the mezzanine area where the dehumidifier is located, we observed dust on classroom ceilings and ducts. We discussed that the Turner Group would be addressing solutions for these issues.

From our initial inspection, it is possible that some of the coils for the HVAC air handlers may have mold contamination. As further discussed by the Turner Group, these issues will have to be addressed.

We agreed that the classroom carpets should continued to be removed following appropriate protocols. We also discussed the need to evaluate the moisture levels of the concrete slab. 


\section{Appendix E: NIOSH emaIL DATEd 22 July: HETA 2009-0172 - ReQuested SUMMARY OF FINDINGS DISCUSSED DURING THE BREAKFAST MEETING ON 16 JULY 2009 (CONTINUED)}

We appreciate the cooperation from Guilford County Schools and the faculty, staff, and parents of Oak Ridge Elementary School. We will continue to analyze the data from your school and will be providing you with an interim report in the future. If you have any questions or concerns, please feel free to contact us.

Sincerely,

Jean Cox-Ganser, Ph.D.

Epidemiologist

Ju-Hyeong Park, Sc.D., C.I.H.

Industrial Hygienist

Rachel L. Bailey, D.O., M.P.H.

Medical Officer

Respiratory Disease Hazard Evaluation and

Technical Assistance Program

Field Studies Branch

Division of Respiratory Disease Studies 
Appendix F: County Department of Public Health Report on the Faculty/ Staff Questionnaire Results at the Elementary SchoOl

Guilford County Department of Public Health June 12, 2009

\section{Oak Ridge Elementary School}

Faculty/Staff Questionnaire Results

Survey conducted between May 29 and June 5, 2009

Completed Surveys: 50 of 88 faculty and staff

Average age of teachers and staff: 44 Age range: $25-61$

Worked at school during the 08-09 school year: $96 \%$

Worked at school during the $07-08$ school year: $92 \%$

\section{Symptoms, 08-09}

Headache: $92 \%$

Of those reporting headaches, $54.3 \%$ reported daily, $43.5 \%$ sometimes, and $2.2 \%$ rarely

Dry Eyes: $74 \%$

Of those reporting dry eyes, $70.3 \%$ reported daily, $27 \%$ sometimes

Unusual Sneezing Episodes: $30 \%$

Of those reporting sneezing, $26.7 \%$ daily, $53.3 \%$ sometimes, and $13.3 \%$ rarely

Difficulty Breathing/asthma attacks: $28 \%$

Of those reporting breathing problems, $42.9 \%$ daily, $50 \%$ sometimes, and $7.1 \%$ rarely

Nosebleeds: $26 \%$

Of those reporting nosebleeds, $7.7 \%$ daily, $53.8 \%$ sometimes, $15.4 \%$ rarely

$\begin{array}{ll}\text { Rashes: } & 10 \% \\ \text { Nausea: } & 14 \% \\ \text { Sinus problems: } & 20 \% \\ \text { Insomnia: } & 6 \% \\ \text { Dizziness: } & 22 \% \\ \text { Fatigue: } & 42 \% \\ \text { Sore throat: } & 18 \% \\ \text { Vision problems: } & 8 \%\end{array}$

Other symptoms written in:

Acid reflux: $\quad 6 \%$

Vomiting: $\quad 2 \%$

Allergy symptoms: $\quad 4 \%$

Eczema: $\quad 6 \%$

Bronchitis: $\quad 2 \%$

Pneumonia: $\quad 2 \%$

Metallic taste in mouth: $8 \%$

Cough: 
Appendix F: County Department of Public Health Report on the Faculty/ Staff Questionnaire Results at the Elementary School (continued)

Trouble concentrating: $6 \%$

Trouble remembering: $14 \%$

Losing voice/hoarse: $\quad 8 \%$

Watery eyes: $\quad 4 \%$

Dry skin: $\quad 2 \%$

Dry throat: $\quad 6 \%$

Red eyes/burning $\quad 14 \%$

Ear pressure/ache $\quad 8 \%$

Miscarriage $\quad 2 \%$

Muscle cramp $2 \%$

Body aches/pain $\quad 4 \%$

Lacerations on eyes $\quad 2 \%$

Reduced sensation of taste, smell $\quad 2 \%$

\# of Symptoms reported: For the 2008-2009 school year, $6 \%$ of faculty/staff reported 0 symptoms, $10 \%$ reported 1 symptom, $6 \%$ reported 2 symptoms, $18 \%$ reported 3 symptoms, $22 \%$ reported 4 symptoms, $16 \%$ reported 5 symptoms, $12 \%$ reported 6 symptoms, $2 \%$ reported 9 symptoms, and $2 \%$ reported 10 symptoms.

Did persons seek care for symptoms in 08-09?

$\mathbf{5 4 \%}$ of all teachers/staff respondents sought care for their symptoms at a doctor's office, ER or hospital. The most common source of care was the doctor's office, with 24 persons reporting doctor visits. Reports of doctor visits range from one visit to more than 30 visits. Three individuals went to the emergency room for care, with one reporting 4 ER visits. Two persons were hospitalized for their symptoms.

\section{Symptoms, 07-08}

Headache: $72 \%$

Of those reporting headaches, $36.1 \%$ reported daily, $58.3 \%$ sometimes, and $6.6 \%$ rarely

Dry Eyes: $56 \%$

Of those reporting dry eyes, $42.9 \%$ reported daily, $53.6 \%$ sometimes

Unusual Sneezing Episodes: 16\%

Of those reporting sneezing, $100 \%$ sometimes

Difficulty Breathing/asthma attacks: $14 \%$

Of those reporting breathing problems, $28.6 \%$ daily, $71.4 \%$ sometimes

Nosebleeds: $10 \%$

Of those reporting nosebleeds, $100 \%$ sometimes

Rashes: $\quad 0 \%$

Nausea: $\quad 4 \%$

Sinus problems: $\quad 18 \%$

Insomnia:

Dizziness:
$2 \%$

$4 \%$ 
$\overline{\text { Appendix F: County Department of Public Health Report on the Faculty/ }}$
Staff Questionnaire Results at the Elementary School (continued)

Fatigue: $\quad 20 \%$

Sore throat: $\quad 8 \%$

Vision problems: $\quad 6 \%$

Other symptoms written in:

Acid reflux: $2 \%$

Bronchitis: $\quad 2 \%$

Cough: $\quad 2 \%$

Trouble concentrating: $\quad 4 \%$

Trouble remembering: $\quad 6 \%$

Losing voice/hoarse: $\quad 2 \%$

Red eyes/burning $\quad 10 \%$

Body aches/pain $4 \%$

Reduced sensation of taste, smell $\quad 2 \%$

\# of Symptoms reported: For the 2007-2008 school year, $12 \%$ of faculty/staff reported 0 symptoms, $18 \%$ reported 1 symptom, 20\% reported 2 symptoms, 10\% reported 3 symptoms, $14 \%$ reported 4 symptoms, $4 \%$ reported 5 symptoms, $2 \%$ reported 6 symptoms, and $6 \%$ reported 7 symptoms.

Did persons seek care for symptoms in 07-08?

$34 \%$ of all faculty/staff respondents sought care for their symptoms at a doctor's office, ER or hospital. The most common source of care was the doctor's office with 14 persons reporting doctor visits. Reports of visits range from one visit to 20 visits. Three individuals went to the emergency room for care, with one person going to the ER on 5 occasions. Two persons reported being hospitalized.

Is there a particular area of the building where persons experience more symptoms? Rooms listed include 406, 407, 421, 209, 405, 501, 423, 425, 405, 500 (2), 514, 518, 520, 606, 613 , and 617 . Other areas include the media center (9 reports), lounge, main office (4 reports), Spanish room, 4/5 hallway (2 reports), testing room, and gym (2 reports)

\section{Pre-existing health conditions:}

Chronic respiratory disease: $\quad 2 \%$

Undergoing chemotherapy or radiation therapy: $\quad 0 \%$

Immune system suppressed by disease or other cause: $\quad 0 \%$

Allergic rhinitis: $\quad 8 \%$

Asthma: $\quad 8 \%$

Seasonal allergies: $\quad 32 \%$

Cystic fibrosis: $\quad 2 \%$ 
Appendix F: County Department of Public Health Report on the Faculty/ Staff Questionnaire Results at the Elementary School (continued)

When did symptoms start?

2007-2008 or earlier $\quad 58 \%$

2008-2009 $30 \%$

Missing $12 \%$

Of the symptoms being experienced, which are the worst?

$\begin{array}{ll}\text { Headaches } & 32 \% \\ \text { Dry or itchy eyes } & 18 \% \\ \text { Fatigue } & 18 \% \\ \text { Breathing } & 10 \% \\ \text { Coughing } & 6 \%\end{array}$

Other symptoms listed: sinus problems, allergy symptoms, weight loss, dizziness, eczema on legs and arms, tightness in chest, metallic taste

\section{When are symptoms generally worse?}

Beginning of the week: $\quad 22 \%$

End of week: $\quad 38 \%$

Particular days: $\quad$ All week, $28 \%$, Monday, $2 \%$, Friday, $2 \%$

Morning: $\quad 30 \%$

Afternoon: $\quad 58 \%$

Particular times of day: $\quad$ After 4:00, 2\%; All day, $8 \%$; night time, $2 \%$; random, $2 \%$

Spring: $\quad 56 \%$

Summer: $\quad 18 \%$

Fall: $\quad 44 \%$

Winter: $\quad 54 \%$

Do symptoms go away after leaving the building: $Y e s=72 \%$, No $=18 \%$

How long does it take for the symptoms to go away: no clear pattern; responses range from a few minutes to a few days.

\section{Home Characteristics}

Does respondent smoke?

Are there others in household who smoke?

House type: $\quad$ House $=90 \%$, Other $=6 \%$

Age of home: $\quad$ Mean 15 years old, range 2-40
Yes $=4 \%$, No $=92 \%$

Yes $=6 \%$, No $=90 \%$ 
Appendix F: County Department of Public Health Report on the Faculty/ Staff Questionnaire Results at the Elementary School (continued)

Type of heat: $\quad$ Electric $=36 \%$, Forced hot air $=10 \%$, Gas $=46 \%$, Oil $=2 \%$, Steam $=2 \%$

Number of persons in household: $\quad 1=10 \% ; 2=28 \% ; 3=20 \% ; 4=34 \% ; 5=4 \%$

Pets in household: $Y e s=58 \%$, No $=38 \%$

Type of pet: dogs, cats and gerbils

Humidifier: Yes $=24 \%$; No $=72 \%$

Wall to wall carpet: $\quad$ Yes $=48 \%$, No $=48 \% \quad$ Water leaks? $\quad$ Yes $=6 \%$ 
Appendix G: County Department of Public Health Report on Parent Questionnaire Results at the Elementary SchoOl

Guilford County Department of Public Health

June 12, 2009

Oak Ridge Elementary School

Parent Questionnaire Results

Survey conducted between May 29 and June 5, 2009

Completed Surveys: 377

Average age of children: 8.5 Age range: 5-12

Child attended school during the 08-09 school year: 99.2\%

Child attended school during the $07-08$ school year: $79.0 \%$

Child attended school during the $06-07$ school year: $53.6 \%$

Child attended school during the $05-06$ school year: $32.4 \%$

Child attended school during the $04-05$ school year: $14.6 \%$

\section{Symptoms, 08-09}

Headache: $\quad 47.7 \%$

Of those reporting headaches, $22.8 \% \%$ reported daily, $56.7 \%$ sometimes, and $17.8 \%$ rarely

Dry Eyes: $24.4 \% \%$

Of those reporting dry eyes, $27.2 \%$ reported daily, $54.3 \%$ sometimes, and $14.1 \%$ rarely

Unusual Sneezing Episodes: 22.8\%

Of those reporting sneezing, $27.9 \%$ daily, $51.2 \%$ sometimes, and $11.7 \%$ rarely

Difficulty Breathing/asthma attacks: $12.8 \%$

Of those reporting breathing problems, $4.3 \%$ daily, $69.4 \%$ sometimes, and $14.9 \%$ rarely

Nosebleeds: $19.6 \%$

Of those reporting nosebleeds, $10.8 \%$ daily, $55.4 \%$ sometimes, $28.4 \%$ rarely

$\begin{array}{ll}\text { Rashes: } & 6.6 \% \\ \text { Nausea: } & 4 \% \\ \text { Sinus problems: } & 3.7 \% \\ \text { Insomnia: } & 0.8 \% \\ \text { Dizziness: } & 1.9 \% \\ \text { Fatigue: } & 4.8 \% \\ \text { Sore throat: } & 4.8 \% \\ \text { Vision problems: } & 1.6 \%\end{array}$

Other symptoms written in:

Vomiting and diarrhea: $2 \%$

Allergy symptoms: $\quad 1 \%$

Eczema: $\quad 2 \%$

Bronchitis: $\quad 0.5 \%$

Pneumonia: $\quad 0.5 \%$ 


\section{Appendix G: County Department of Public Health Report on Parent Questionnaire Results at the Elementary School (continued)}

$\begin{array}{ll}\text { Cough: } & 6 \% \\ \text { Trouble concentrating: } & 0.2 \% \\ \text { Trouble remembering: } & 0.2 \% \\ \text { Eyes red, burn, watery: } & 2 \% \\ \text { Dry itchy skin: } & 2 \% \\ \text { Dry throat: } & 0.2 \% \\ \text { Ear pressure/ache } & 2 \% \\ \text { Body aches/pain } & 3 \% \\ \text { Stuffy, runny nose 4\% } & \\ \text { Irritable } & 0.5 \% \\ \text { Circles under eyes } & 0.8\end{array}$

Number of Symptoms: For the $08-09$ school year, 36.1\% of parents reported 0 symptoms, $20.2 \%$ reported 1 symptom, $18.0 \%$ reported 2 symptoms, $13.3 \%$ reported 3 symptoms, $6.9 \%$ reported 4 symptoms, $3.7 \%$ reported 5 symptoms, $1.6 \%$ reported 5 symptoms, and 1 parent reported 13 symptoms.

Did persons seek care for symptoms in 08-09? $23 \%$ of parents sought care for their child's symptoms at a doctor's office, with number of visits ranging from 1-15. Four parents sought care for their child at the emergency room and one parent had their child hospitalized

\section{Symptoms, 07-08}

Headache: $23.6 \%$

Of those reporting headaches, $13.5 \%$ reported daily, $68.5 \%$ sometimes, and $11.2 \%$ rarely

Dry Eyes: $\quad 9.8 \%$

Of those reporting dry eyes, $18.9 \%$ reported daily, $48.6 \%$ sometimes, $16.2 \%$ rarely

Unusual Sneezing Episodes: $12.2 \%$

Of those reporting sneezing, $19.6 \%$ reported daily, $58.6 \%$ sometimes, and $8.7 \%$ rarely

Difficulty Breathing/asthma attacks: $7.7 \%$

Of those reporting breathing problems, $10.3 \%$ daily, $65.5 \%$ sometimes, and $13.8 \%$ rarely

Nosebleeds: $8.0 \%$

Of those reporting nosebleeds, $16.7 \%$ reported daily, $70 \%$ sometimes and $20 \%$ rarely

Rashes:

Nausea:

Sinus problems:

Insomnia:

Dizziness:

Fatigue:

Sore throat:
$2.7 \%$

$1.3 \%$

$2.4 \%$

$0 \%$

$0.3 \%$

$1.9 \%$

$2.1 \%$ 
Appendix G: County Department of Public Health Report on Parent
Questionnalre Results at the Elementary SChool (continued)

Vision problems: $\quad 0.3 \%$

Other symptoms written in:

Vomiting and diarrhea: $\quad 2 \%$

Allergy symptoms: $\quad 1 \%$

Eczema: $\quad 2 \%$

Bronchitis: $\quad 0.5 \%$

Pneumonia: $\quad 0.5 \%$

Cough: $\quad 6 \%$

Trouble concentrating: $\quad 0.2 \%$

Trouble remembering: $\quad 0.2 \%$

Eyes red, burn, watery: $\quad 2 \%$

Dry itchy skin: $\quad 2 \%$

Dry throat: $\quad 0.2 \%$

Ear pressure/ache $\quad 2 \%$

Body aches/pain $3 \%$

Stuffy, runny nose $\quad 4 \%$

Irritable $\quad 0.5 \%$

Circles under eyes $\quad 0.8$

Number of Symptoms: For the $07-08$ school year, $64.7 \%$ of parents reported 0 symptoms, $14.9 \%$ reported 1 symptom, $9.3 \%$ reported 2 symptoms, $7.4 \%$ reported 3 symptoms, $2.4 \%$ reported 4 symptoms, $1.1 \%$ reported 5 symptoms, and $0.3 \%$ reported 6 symptoms.

Did persons seek care for symptoms in $07-08$ ?

$23 \%$ of parents sought care for a child's symptoms at a doctor's office, with number of visits ranging from 1 to $10+$. Four parents took their child to the emergency room for care and one parent reported having their child hospitalized due to his or her symptoms.

Is there a particular area of the building where persons experience more symptoms?

Nearly every room in the school was reported at least once. Rooms reported by more than one parent included $416,418,420,424,425,500,514,515,517,518,520,521,610,618,619$, and the music room.

Pre-existing health conditions:

Chronic respiratory disease:

$1.1 \%$

Undergoing chemotherapy or radiation therapy: $\quad 0 \%$

Immune system suppressed by disease or other cause: $\quad 0.5 \%$

Allergic rhinitis:

$8.2 \%$

Asthma:

$11.7 \%$

Seasonal allergies:

$33.4 \%$

Cystic fibrosis:

$0 \%$ 


\section{Appendix G: County Department of Public Health Report on Parent Questionnaire Results at the Elementary SChoOl (COntinued)}

When did symptoms start?

2007-2008 or earlier $\quad 35 \%$

2008-2009 $27 \%$

Missing $\quad 38 \%$

Of the symptoms being experienced, which are the worst?

The most common reported "worst" symptom was headaches, followed by nosebleeds, coughs and congestion, asthma and breathing problems, eye problems and rashes.

\section{When are symptoms generally worse?}

Beginning of the week: $\quad 6.4 \%$

End of week: $\quad 15.6 \%$

Particular days: $\quad$ All week, $7.5 \%$, specific days each less than $1 \%$

Morning: $\quad 10.3 \%$

Afternoon: $\quad 25.7 \%$

Particular times of day: $\quad$ No specific time of day reported by more than $1 \%$

Spring: $\quad 38 \%$

Summer: $\quad 9.8 \%$

Fall: $\quad 25.7 \%$

Winter: $\quad 28.9 \%$

Do symptoms go away after leaving the building: $Y e s=37.1 \%$, No $=22 \%$

How long does it take for the symptoms to go away: no clear pattern; responses range from improvement immediately after leaving the building to a few days.

\section{Home Characteristics}

Are there smokers in household?

Yes $=4.8 \%$

Total smokers in household Eleven parents reported one smoker, two reported two smokers and one reported four smokers in the household.

House type: $\quad$ House $=66.8 \%$, Mobile home $=0.3 \%$, Other $=0.5 \%$

Age of home: $\quad$ Mean 10 years old, range 1-80

Type of heat: $\quad$ Electric $=19.6 \%$, Forced hot air $=13.8 \%$, Gas $=30.2 \%$, Oil $=0.3 \%$, Steam $=0.5 \%$ 


\section{Appendix G: County Department of Public Health Report on Parent Questionnaire Results at the Elementary SChoOl (continued)}

Number of persons in household: $\quad$ Mean number $=4.3$, Mode $=4$; range from 2-9

Pets in household: $43.5 \%$ reported pets in the household

Type of pet: dogs and cats primarily, as well as hamsters, fish, mice, geckos, rabbits, turtles and gerbils

Humidifier: Yes $=20.4 \%$

Wall to wall carpet: $\quad$ Yes $=35.3 \% \quad$ Water leaks? Yes $=4.2 \%$

Water stains: $\quad$ Yes $=0 \% \quad$ Ceiling stains? Yes $=2.1 \%$

Mold: $\quad$ Yes $=1.6 \%$ 


\section{Appendix H: The Turner Building Science and Design preliminary REPORT DATED 21 JULY 2009}

TURNER

GROUP

\section{TURNER BUILDING SCIENCE \& DESIGN, LLC}

26 PINEWOOD LANE, HARRISON, MAINE 04040-4334 TEL: 207-583-4571 FAX: 207-583-4572

www.hlturner.com

www.turnerbuildingscience.com

\section{Oak Ridge Elementary School}

Very Preliminary Draft Indoor Air Quality Issues

- Issue: Dirt floor crawl space under the original building is a likely source of mold.

Observation: Occupant reports of mold odors, observation of conditions and odors in crawl space and basement.

Preliminary Recommendation: Provide flexible vapor barrier (fire retardant?) and depressurization of air space under vapor barrier.

- Issue: Inadequate flashing at junction between parapet wall and Gym wall above Room 206 causing water intrusion leading to mold growth in carpet.

Observation: Flashing clearly appears to be mounted on surface rather than continuing through brick Gym wall to drainage plane, occupant reports of wetted materials in Room 206, observation of Room 206 walls, floor (newly painted cove base wavy, carpet stains.

Preliminary Recommendation: Install through wall flashing at edge of parapet to connect through brick to drainage layer behind.

- Issue: Repaired flashing at wall in Room 400 was once a leak source.

Observation: Discolored drywall on exterior wall, occupant reports, mold found under cove base

Preliminary Recommendation: Repair roof junction joints to provide adequate flashing

- Issue: Dehumidification system operation suspect, evidenced by high humidity $(70 \%+)$ in $4 / 5$ wing,

Observation: Measurement of relative humidity in hall, mechanical space and Room 520 (long-term)

Preliminary Recommendation: Provide retro commissioning of HVAC system with respect to verify proper system capacity and sequence of operation, are all HP units involved with dehumidification system working

$$
\text { BUILDING SCIENTISTS • IAQ CONSULTANTS }
$$


- Issue: Possible heat pump refrigerant leaks,

Observation: Indicated by $64^{\circ} \mathrm{F}$ discharge at HP serving Room 520 and humidity levels monitored at $70 \%-80 \%$ in room, or quantity of outside air being handled exceeds that of coil capacity

Preliminary Recommendation: Provide retro commissioning of HVAC system with respect to verifying proper system capacity and sequence of operation.

- Issue: Classroom wings not sealed at soffit, allowing excess air leakage in and out (depending on mechanical system operation).

Observation: Pressure measurements indicate indoor/outdoor delta $\mathrm{P}$ only 2-4 $\mathrm{Pa}$ (low) while providing approx. 10,000 cfm excess OA. Also noted that rooms with highest number of complaints tended to be near extended overhang or have two exposures of soffit. Verified air directon at soffit openings with chemical smoke. Dust on top of ductwork in attic space.

Preliminary Recommendation: Sealing of soffit to prevent air infiltration. Note: If soffit sealed, provide means for exhaust/relief. Determine design logic for current system. Dirt/pesticide being drawn in through soffit?

- Issue: Heat pump design conducive to growing mold due to proximity of filter and cooling coil.

Observation: Observation and photos of configuration. New filters installed- no signs of current mold growth on filters.

Preliminary Recommendation: Provide new filter boxes upstream to create separation between coil and filter

- Issue: Concerns about odors from dry traps, particularly at heat pump drains, since air is distributed to occupants.

Observation: Traps at heat pumps had water when we observed, but addition of dehumidification systems will tend to dry out downstream traps.

Preliminary Recommendation: Review maintenance tasks and schedules, On-going maintenance issue.

Oak Ridge Elementary School 


\section{Appendix H: The Turner BuILding SCIEnCe and Design Preliminary REPORT DATED 21 JULY 2009 (CONTINUED)}

- Issue: Bathrooms not at negative pressure consistently with respect to hall.

Observation: Measurements, drawings, and smoke test

Preliminary Recommendation: Balance air flows as part of retro commissioning process, move some supply air to hall?

- Issue: Mechanical room in Office area under significant negative pressure.

Observation: Change in duct noise when opening door, May indicate excess leakage on the return side of ducts, insufficient outside air, etc.

Preliminary Recommendation: Provide duct joint sealant on all RA (\&SA?). Verify air flows on retro commissioning

- Issue: Unknown issues regarding storm runoff at end of 2/3 and 4/5 wings.

Observation: Drawing review seems to indicate the possibility of water getting under slab if it pools against exterior wall. Observations indicate possible plugged storm drains - downspouts could overcharge drainage.

Preliminary Recommendation: Verify adequate site drainage and clean site drainage system as necessary. Provide means to limit runoff sand and other surface material

- Issue: Original design of classroom wings modulated between $3.5 \mathrm{cfm} /$ person and $7.5 \mathrm{cfm} /$ person from $1000 \mathrm{ppm} \mathrm{CO}_{2}$ to $1800 \mathrm{ppm}$. When dehumidification systems installed, carbon dioxide control eliminated and all areas receive 7.5 $\mathrm{cfm} /$ person??

Observation: Original drawings and renovation drawings. Need confirmation of damper position (not clearly marked on damper shaft). No balancing report available.

Preliminary Recommendation: Provide balancing of OA system and marking of dampers as part of retro commissioning project

- Issue: Foam roof appears to be a concern. Numerous locations where the aluminum paint has been comprised.

Observation: Many opened bubbles of foam.

Preliminary Recommendation: Obtain roofing contractors estimate to repair/replace foam roof

Oak Ridge Elementary School 


\section{Appendix H: The Turner BuILding Science and Design preliminary REPORT DATED 21 JULY 2009 (CONTINUED)}

- Issue: Occupant photos of room 500 indication possible leak from sewer vent and/or roof flashing of VTR

Observation: Photos and observation of stained ceiling tile tees.

Preliminary Recommendation: Replace or repair flashing.

- Issue: Building not observed during occupied mode, some operational conditions not observed.

Preliminary Recommendation: Provide monitoring of conditions during occupancy to evaluate operation under occupancy (after retro commissioning of systems) 


\section{APPENDIX I: NIOSH EMAIL TO PARENTS DATED 19 OCTOBER 2009: HETA 2009-0172}

From: Bailey, Rachel L. (CDC/NIOSH/DRDS)

Sent: Monday, October 19, 2009 12:58 PM

To: 'Becoats, Eric J'

Cc: Bobadilla, Leobardo; 'Carr, Nora'

Subject: HETA 2009-0172 - Oak Ridge Elementary School

\section{Dr. Becoats,}

We have had some parents inquire about when the school will reopen and who will make the decision to open the school. Below is text from the email that was sent out to parents requesting this information.

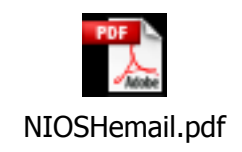

Dear Parent,

Guilford County Schools will determine when the school reopens, and NIOSH has no role to play in this decision. As you know in July 2009, we performed a three day site visit at Oak Ridge Elementary School at the request of Guilford County Schools. On the last day during a breakfast meeting, we met with representatives from Guilford County Schools and discussed our observations and recommendations. We discussed the following findings:

1. Musty, moldy odor from the basement and crawl space under the media center

2. Surface drainage/landscaping issues around the school

3. Blocked storm drain with standing water

4. Damaged roof

5. Inadequate flashing

6. Moisture in the gym's exterior concrete block wall

7. Pressure airflow issues between conditioned and unconditioned spaces

8. Outside humid air entering through the classroom wing soffits

After the site visit, we sent an email to the school administration that briefly summarized our conversation (please see attachment) and have been in contact with them since our visit. NIOSH has evaluated numerous buildings and has found that indoor environmental quality problems can generally be corrected using remediation practices that are fairly standardized. For Oak Ridge Elementary School these are the following: 1) remove carpet and replace with hard floors (after testing slab for moisture content), 2) install a vapor barrier and depressurize the crawl space under the media center, 3) repair the roof, 4) repair the flashing, 5) seal classroom wing soffits, and 6) recommission the HVAC system. 


\section{ApPENDIX I: NIOSH EMAIL tO PARENTS DATED 19 OCtOber 2009: HETA 2009-0172 (CONTINUED)}

Although the final report is not out, these specific issues and our recommendations were discussed with the school administration. We have found that remediation similar to what we recommended is easier to

do when a building is not occupied and that health effects associated with dampness commonly continue and are made worse when remediation occurs in occupied buildings. Accordingly, we think the decision to close Oak Ridge Elementary School during the remediation to address the underlying issues of water intrusion, humidity, and ventilation was a prudent one. In our report, we are summarizing the many consultant reports and timeline of remediation of which we hope will be useful. However, we do not anticipate that the decisions regarding reopening the school are dependent on receipt of the final report.

Sincerely,

Jean Cox-Ganser, Ph.D. Epidemiologist

Ju-Hyeong Park, Sc.D., C.I.H. Industrial Hygienist

Rachel L. Bailey, D.O., M.P.H. Medical Officer

Centers for Disease Control and Prevention

National Institute for Occupational Safety and Health

Division of Respiratory Disease Studies

Field Studies Branch

1095 Willowdale Road

$\mathrm{M} / \mathrm{S} \mathrm{H} 2800$

Morgantown, West Virginia 26505 
Appendix J: EMC EngIneERS, INC. HVAC Retro-COMmissioning Report dated DeCEMber 2009

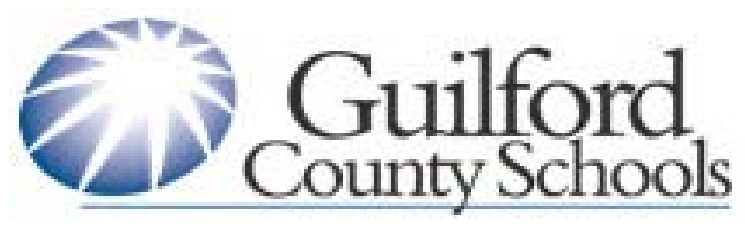

FINAL REPORT

\section{Retro-Commissioning of HVAC Systems}

Oak Ridge Elementary

Oak Ridge, NC

Prepared for:

Guilford County Schools

EMC Project No. 1C409021.000

December 2009

Prepared by:

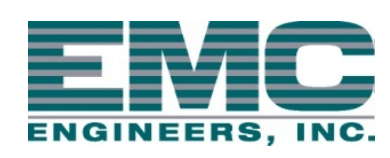

E M C Engineers, Inc.

706 Hillsborough Street, Suite 102

Raleigh, NC 27603 
APPENDIX J: EMC ENGINEERS, INC. HVAC RETRO-COMMISSIONING Report DAted DeCEMBer 2009 (CONTINUEd)

\section{INTENTIONAL BLANK PAGE}

EMC Engineers, Inc.

$1-2$

December 2009 


\section{APPENDIX J: EMC ENGINEERS, INC. HVAC RETRO-COMMISSIONING Report dated DeCeMber 2009 (continued)}

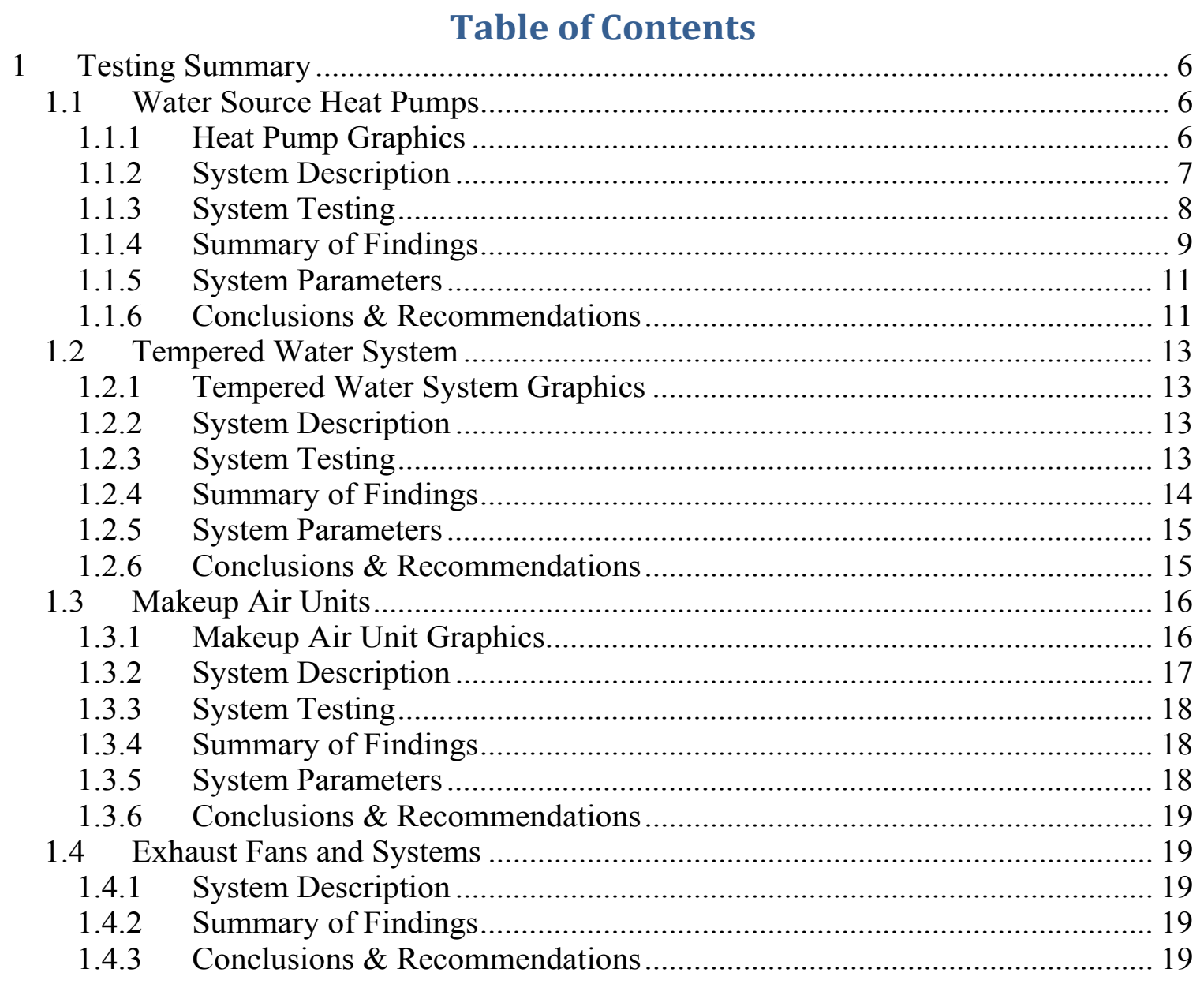

\section{List of Tables}

Table 1: Supply Air Balance Test Sample.....................................11

Table 2: Loop water test and balance data..................................... 14

Table 3: Boiler and Cooling Tower As-Found Set-points.......................... 15

Table 4: AHU-G Supply Air Balance........................................... 18

Table 5: AHU-G As-Found Set-points..........................................

\section{List of Figures}

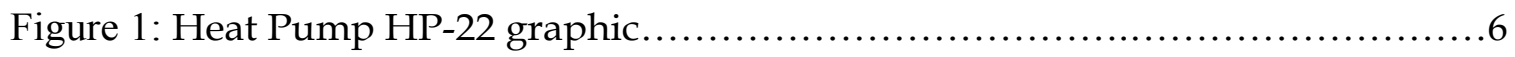

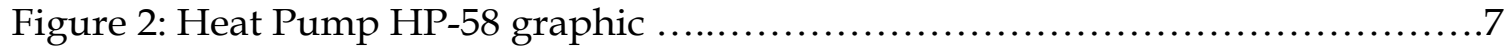

Figure 3: Tempered Water System Graphic ..................................... 13

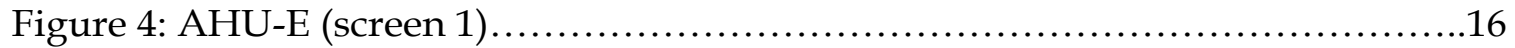

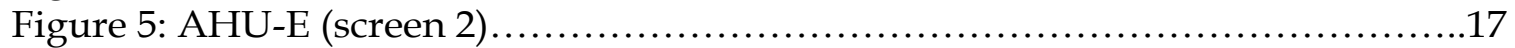

EMC Engineers, Inc. 1-3 December 2009 


\section{Appendix J: EMC EngINEeRS, InC. HVAC Retro-COMMISSIONING Report DATED DECEMBER 2009 (CONTINUED)}

\section{List of Appendices}

Appendix A - Scope of Work

Appendix B - Issues Log

Appendix C - TAB Verification

Appendix D - Activity Reports

Appendix E - Functional Tests

Appendix F - Test \& Balance Report

Appendix G - Heat Pump Start-Up Log Sheets 
Executive Summary

EMC was selected to perform retro-commissioning functional testing at Oak Ridge Elementary School, a part of the Guilford County Schools System (GCS). In addition to EMC, GCS retained the services of The Phoenix Agency to perform test and balance work on the process water and air distribution systems, and Systems Contractors, Inc. (SCI) to perform various mechanical and building automation system (BAS) repairs. Functional testing performed by EMC included point-to-point verification of field devices associated with each piece of mechanical equipment and verification of BAS graphic linkages, setpoints, and reporting.

The American Society of Heating and Refrigeration, and Air Conditioning Engineers (ASHRAE) defines building commissioning as "the process of ensuring that systems are designed, installed, functionally tested and capable of being operated and maintained to perform in conformity with design intent." The owner of a facility can further define goals for commissioning according to their desired outcomes for the selected facility. This project was a retro-commissioning project and as such, did not involve all aspects of commissioning found in new construction projects.

EMC was contracted for retro-commissioning of the building in November 2009. EMC performed a comprehensive review of the available plans, and was scoped to commission the following systems:

- Building automation systems, including linkages to remote monitoring and control sites

- Testing of building automation operator workstation graphics

- Heat Pumps, water pumps, piping, and associated equipment

- Make Up Air Unit

- Hot Water, pumps, piping, condensate, cooling towers, heat exchangers and associated equipment

- Local Boiler(s), combustion air systems, and boiler controls

- Exhaust fans, kitchen ventilation systems

Functional acceptance testing of the systems generated over 150 items in the issues log. Those issues were addressed as work progressed. The complete issues log can be found in Appendix B. 
ApPendix J: EMC EngINEeRs, INC. HVAC Retro-COMmISSIONING Report dated DeCeMber 2009 (continued)

\section{Testing Summary}

\subsection{Water Source Heat Pumps}

\subsubsection{Heat Pump Graphics}

Below are screen captures which represent the typical heat pump graphics found on the Trane Tracer Summit front-end of the building automation system.

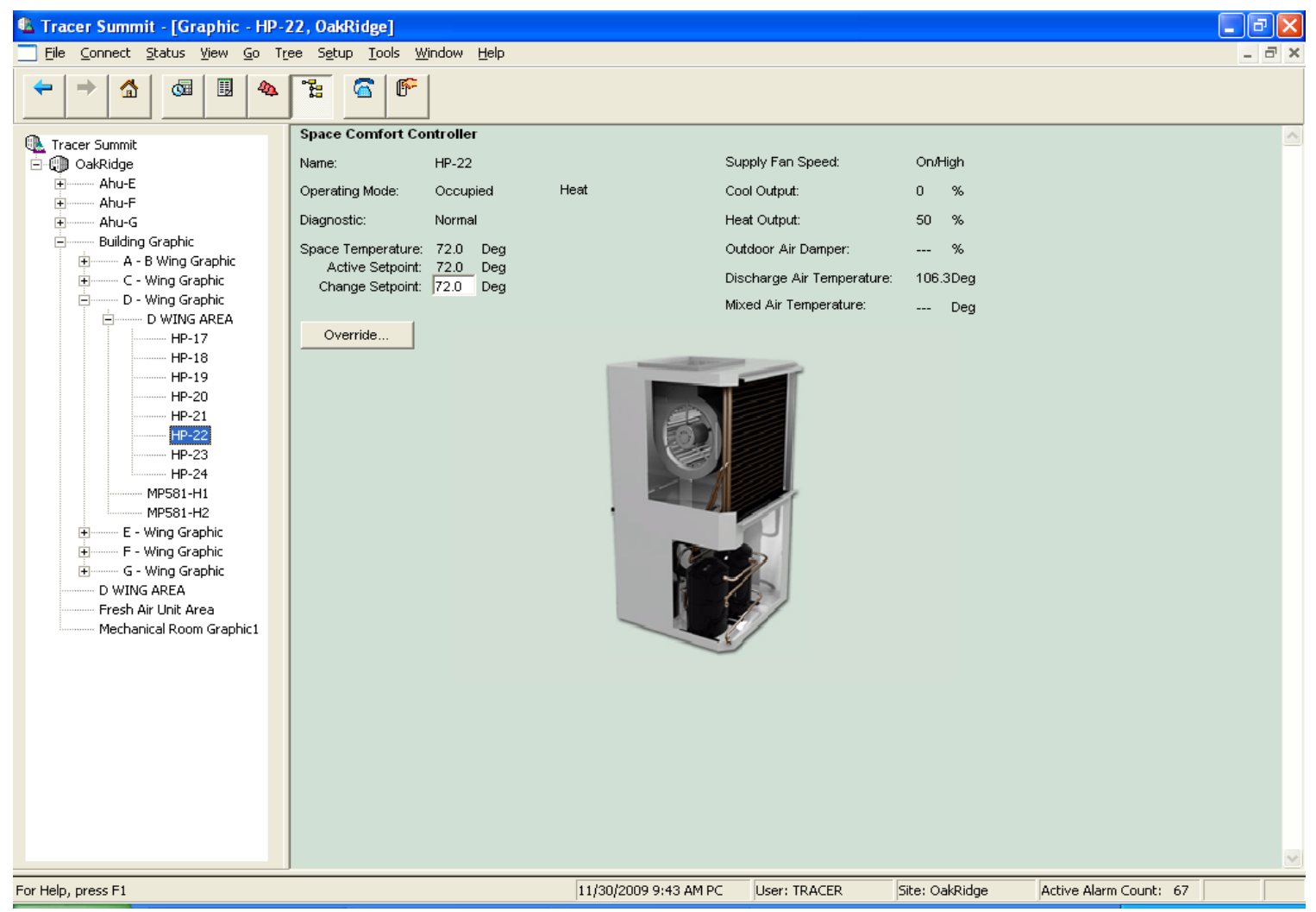

Figure 1 - Heat Pump HP-22 graphic 


\section{APPENDIX J: EMC ENGINEERS, INC. HVAC RETRO-COMMISSIONING Report DATED DECEMBER 2009 (CONTINUED)}

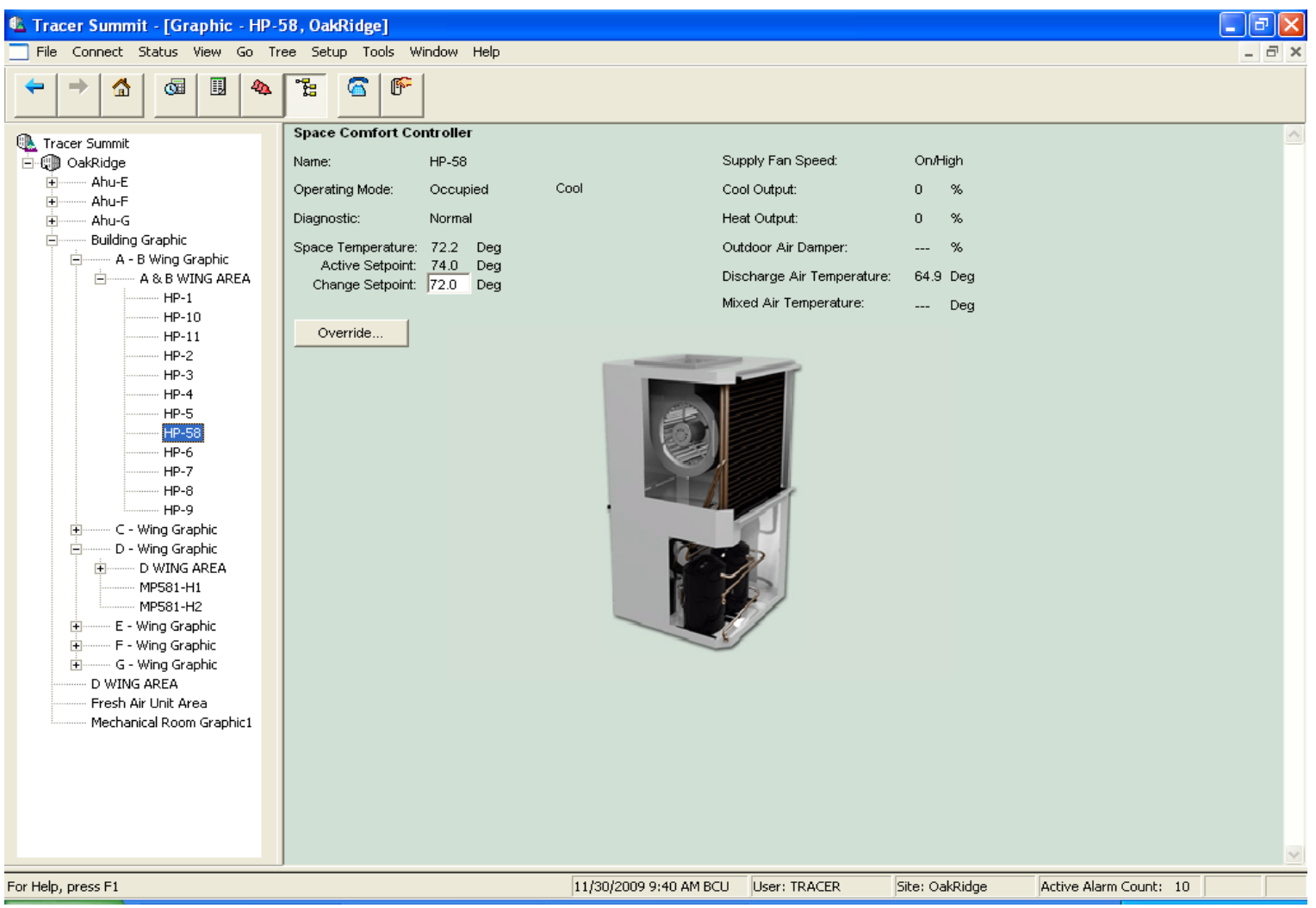

Figure 2 - Heat Pump HP-58 graphic

\subsubsection{System Description}

There are 58 water source heat pumps in the building, serving various spaces independently. The loop water condensing coils in the heat pump units are served by one hot water boiler for heating, and a cooling tower for cooling. There is also an associated plate-type heat exchanger used to transfer heat to or from the building loop. The building is divided into wings identified on plans as follows:

- A/B - Multi-Purpose and Dining/Kitchen areas

- Heat pump 1 serves the Central Receiving area

- Heat pumps 2 through 5 serve the Multi-Purpose Room and associated stage areas

- Heat pump 6 serves corridor and toilet spaces

- Heat pumps 7 through 9 serve Kitchen and Dining areas

- Heat pump 10 serves a Data Room

- Heat Pump 11 serves the two P.E. offices and a small corridor

- C/D - Offices and Media Center

- Heat pump 58 serves the main corridor between the Offices and the Multi-Purpose Room 


\section{ApPendix J: EMC EngINeERS, INC. HVAC Retro-COMMISSIONING Report dated DeCeMber 2009 (continued)}

- Heat pumps 12 through 18 serve office spaces and are connected to a common outside air intake hood

- Heat pump 19 serves a Data Room

- Heat pump 21 serves the Media Center Workroom and Speech Room. This unit has a re-heat coil, which is intended to utilize loop water for control of supply air temperature during dehumidification of the associated spaces

- Heat pumps 20 and 24 serve the corridors outside the Media Center. Each of these units has a re-heat coil, which is intended to utilize loop water for control of supply air temperature during dehumidification of the associated spaces

- Heat pumps 22 and 23 serve the Media Center. Each of these units has a re-heat coil, which is intended to utilize loop water for control of supply air temperature during dehumidification of the associated spaces

- $\quad$ E-Wing (400-series Classrooms)

- Heat pumps 44 through 56 primarily serve classroom spaces, with a small portion of capacity utilized for support spaces within the wing. Each of these heat pumps is also connected to AHU-E for the purposes of introduction of outside air. These heat pumps are located on a mezzanine above this classroom wing

- F-Wing (500-series Classrooms)

○ Heat pumps 35 through 43 primarily serve classroom spaces, with a small portion of capacity utilized for support spaces within the wing. Each of these heat pumps is also connected to AHU-F for the purposes of introduction of outside air. These heat pumps are located on a mezzanine above this classroom wing

- G-Wing (600-series Classrooms)

- Heat pumps 25 through 34 primarily serve classroom spaces, with a small portion of capacity utilized for support spaces within the wing. Each of these heat pumps is also connected to AHU-G for the purposes of introduction of outside air. These heat pumps are located on a mezzanine above this classroom wing

Compressor staging and power characteristics of the heat pumps vary. The heat pump units all control space temperature by utilizing building loop water condensing coils.

\subsubsection{System Testing}

EMC was scoped to verify the TAB effort for diffusers and outlets of heat pumps, dedicated outdoor air system, exhaust and hydronic systems by witnessing measurements made by the TAB contractor. For a given heat pump unit, each 


\section{APPENDIX J: EMC ENGINEERS, INC. HVAC RETRO-COMMISSIONING Report DATED DECEMBER 2009 (CONTINUED)}

diffuser was measured by the TAB contractor and recorded by EMC. EMC also verified outside air balance where applicable on heat pump units.

EMC was scoped to perform testing as follows:

- $100 \%$ testing of Heat Pump operation

- $100 \%$ testing on Makeup Air Units

- $100 \%$ testing of heat exchange hydronic system, including pumps, heat exchangers, cooling tower and associated equipment

- $100 \%$ testing of Supervisory Controls, Graphics, Schedules

- $100 \%$ testing of Exhaust Fans

Point-to-point testing included checking calibration of the space temperature sensor and discharge air temperature sensor for each heat pump. Verification of the calibration of humidity sensors, where applicable, was also performed.

The functional test verification involved imposing varying degrees of heating and cooling demand on a unit, and recording whether or not the unit reacted properly with its associated compressor and/or control valves.

\subsubsection{Summary of Findings}

The following issues were commonly encountered by EMC, and subsequently addressed by various personnel:

- The BAS was not scheduled to unoccupy any building equipment at any time. This $24 / 7 / 365$ operation was discussed amongst the testing team and the decision was made to leave as found until the new schedule is implemented. The new recommended schedule is as follows:

- Office and Media Center areas

- Occupied 5 a.m. - 10 p.m.

- Unoccupied 10 p.m. - 5 a.m.

- Classroom wings and Gymnasium / Cafeteria

- Occupied 6 a.m. - 10 p.m.

- Unoccupied 10 p.m. -6 a.m.

- Data closets

- Occupied continuously

- Setpoint control was found to be from the BAS for all heat pumps.

- A significant number of heat pump discharge air temperature sensors were found to be out of range. This point is not critical to unit operation, but should read accurately in order to accurately convey heat pump operation

- Various graphics errors on the Trane workstation in the boiler room

- Low air flows in several heat pumps

- All heat pump space temperature setpoints are independently controlled in the BAS, and do not follow any unified area control. EMC and Turner Building Sciences discussed this and a determination was made that global setpoints should be applied to the heat pumps, with local temperature sensor control 

Report Dated DeCeMber 2009 (continued)

The following issues were isolated instances found by EMC and corrected:

- Heat pump 11 was found to be capable of supplying less than the designed amount of air to the offices it served. SCI installed larger supply diffusers and Phoenix T\&B proportioned supply to bring values to within $10 \%$ of design values

- Sheaves for the fans of Heat pumps 48 and 55 were found to be adjusted incorrectly and setscrews damaged. Sheaves were replaced and Phoenix T\&B adjusted to correct settings, which were then verified by EMC

- Heat pump 43 was not accessible from the F-Wing graphic. SCI personnel corrected this issue

- All Heat pumps were set up as members of a VAV Air System in Tracer Summit. This was incorrect, and was later corrected. EMC verified the corrections

- A foreign object, measuring approximately $2^{\prime} \times 2^{\prime}$ was found to have been placed inside one supply duct in the F-Wing (500-series rooms). This object appears to be a camping shower. The object was removed by Phoenix T\&B personnel (see attached photograph) 


\section{APPENDIX J: EMC ENGINEERS, INC. HVAC RETRO-COMMISSIONING Report dated DeCEMber 2009 (continued)}

\subsubsection{System Parameters}

Table 1 shows a sampling of the test and balance verification performed on the Heat Pump Units:

Table 1: Supply Air Balance Test Sample

\begin{tabular}{|l|c|c|r|c|}
\hline \multicolumn{5}{|c|}{ Supply Air Balance } \\
\hline Heat Pump & Design & TAB & Actual & $\begin{array}{c}\% \\
\text { Design }\end{array}$ \\
\hline HP-58 & 2400 & 2127 & 2162 & $90 \%$ \\
\hline HP-43 & 400 & 437 & 437 & $109 \%$ \\
\hline HP-17 & 1400 & 0 & 1425 & $102 \%$ \\
\hline HP-22 & 2000 & 0 & 1853 & $93 \%$ \\
\hline HP-23 & 2000 & 0 & 1886 & $94 \%$ \\
\hline HP-32 & 2200 & 0 & 2082 & $95 \%$ \\
\hline HP-24 & 1800 & 0 & 1792 & $100 \%$ \\
\hline HP-21 & 1250 & 0 & 1325 & $106 \%$ \\
\hline HP-29 & 2700 & 0 & 2534 & $94 \%$ \\
\hline HP-31 & 2700 & 0 & 2492 & $92 \%$ \\
\hline HP-51 & 2400 & 0 & 2716 & $113 \%$ \\
\hline HP-49 & 2400 & 0 & 2651 & $110 \%$ \\
\hline HP-50 & 1800 & 0 & 1890 & $105 \%$ \\
\hline HP-48 & 2500 & 2817 & 2823 & $113 \%$ \\
\hline HP-46 & 2400 & 2666 & 2360 & $98 \%$ \\
\hline HP-33 & 1800 & 0 & 1886 & $105 \%$ \\
\hline HP-35 & 1400 & 1473 & 1485 & $106 \%$ \\
\hline HP-39 & 2000 & 0 & 2051 & $103 \%$ \\
\hline HP-28 & 2000 & 0 & 1932 & $97 \%$ \\
\hline HP-26 & 1450 & 0 & 1422 & $98 \%$ \\
\hline
\end{tabular}

\subsubsection{Conclusions \& Recommendations}

The majority of heat pumps have air flows within $+/-10 \%$ of design values.

However, a small number of units exhibited air flow slightly beyond this threshold. Appendix F details each unit's air flow values. The outside air units are providing air to the associated wings of the building, at volumes consistent with those specified by the designer. Based on the final Test and Balance Report, the units are operating generally within $+/-10 \%$ of design.

Recommended setpoints are as follows: Heating Season (70 deg F); Cooling Season (74 deg F). In order to aid in maintaining acceptable indoor conditions, all outside doors as well as all operable windows should remain closed at all times. 


\section{AppendIX J: EMC ENGINEERS, INC. HVAC RETRO-COMMISSIONING Report DATED DeCEMBer 2009 (CONTINUED)}

EMC recommends that regular air filter inspections be performed on all units, including the makeup air units, at least every 3 months per manufacturer's recommendations, with filter changes conducted when visual inspection indicates necessity.

Space temperature setpoints should be consistent within logical zones of the building, in order to help reduce the potential for wide temperature fluctuations between that zone's spaces. EMC recommends using global setpoint values in the Trane BAS, which are referenced in each heat pump controller. Logical zones with common setpoints should be as follows:

- Classroom wings - common setpoint

- Office and Media Center areas - common setpoint

- Gymnasium and Cafeteria areas - common setpoint

- Data closets - common setpoint

Recommended Trane BAS schedules for occupied and unoccupied operation of the system are as follows:

- Office and Media Center areas

○ Occupied 5 a.m. - 10 p.m.

- Unoccupied 10 p.m. - 5 a.m.

- Classroom wings and Gymnasium / Cafeteria

○ Occupied 6 a.m. -10 p.m.

○ Unoccupied 10 p.m. -6 a.m.

- Data closets

- Occupied continuously

Recommended setpoint temperature offset of 5 degrees from occupied setpoints (i.e. unoccupied cooling setpoints 5 deg F higher than occupied cooling setpoints; unoccupied heating setpoints 5 deg F lower than occupied heating setpoints). 

Report DATED DeCEMBER 2009 (CONTINUed)

\subsection{Tempered Water System}

\subsubsection{Tempered Water System Graphics}

Below is a screen capture of the tempered water system graphic found on the Trane Tracer Summit front-end of the building automation system.

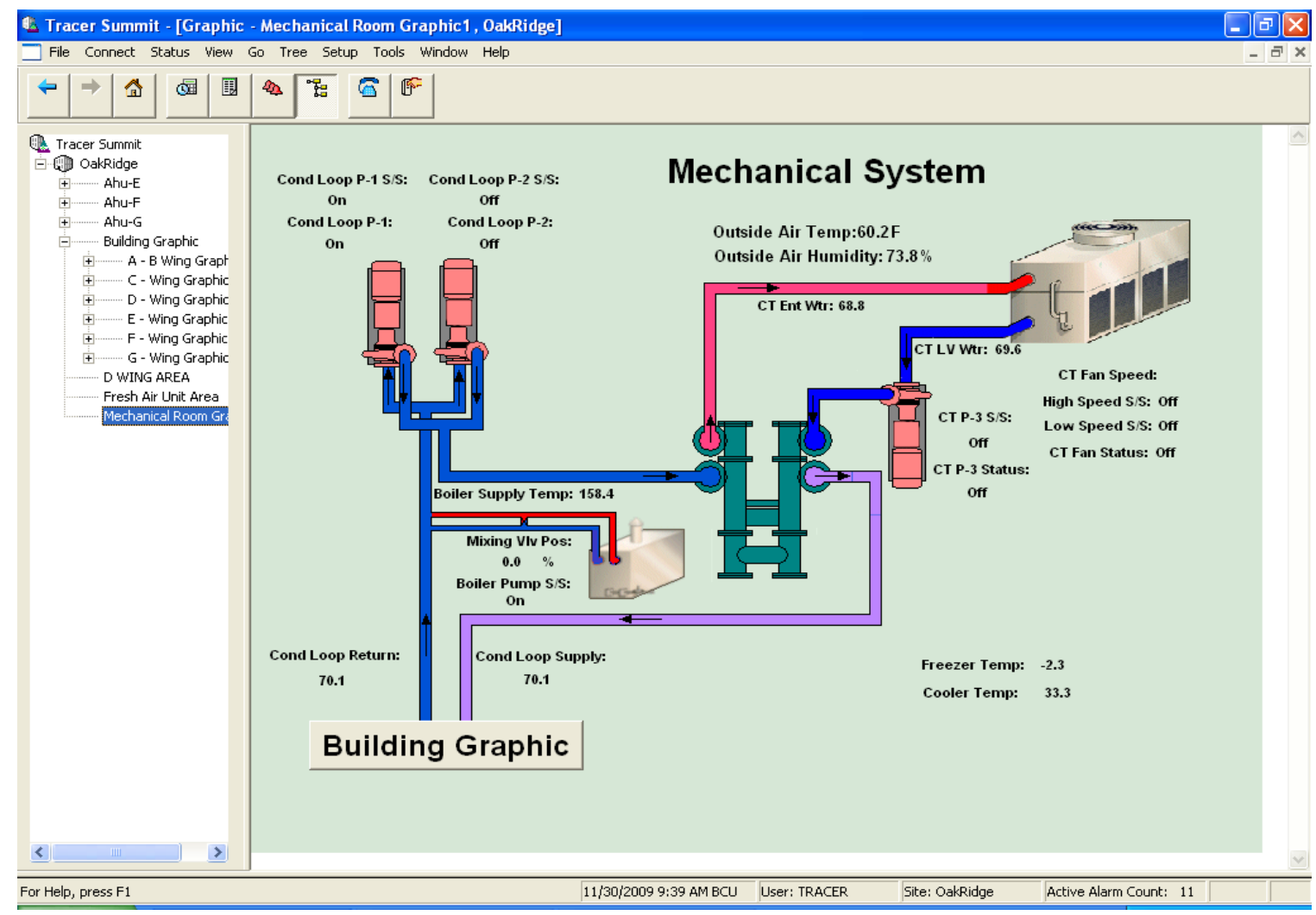

Figure 3 - Tempered Water System Graphic

\subsubsection{System Description}

The condensing coils on the water source heat pump units are served by loop water, which is heated and cooled by a heat exchanger. This heat exchanger is piped to one (1) hot water boiler, and also one cooling tower. The loop water is circulated by two constant-speed pumps (P-1 and P-2). Water is pumped through the cooling tower by one (1) pump (P-3), and water is pumped through the hot water boiler by one (1) pump (P-4). The tempered water system maintains the loop water temperature between 60 and $82^{\circ} \mathrm{F}$, all year round, so that the water source heat pumps can control space temperature effectively.

\subsubsection{System Testing}

EMC worked with the T\&B Contractor to verify the water balance on the loop water. Table 2 below lists the findings of that work. 

Report DATED DECEMBER 2009 (CONTINUED)

Table 2: Loop water test and balance data

\begin{tabular}{|c|l|r|r|r|r|r|r|r|r|r|}
\hline \# & Description & $\begin{array}{c}\text { Disch. } \\
\text { press } \\
\text { (Feet) }\end{array}$ & $\begin{array}{c}\text { Suct. } \\
\text { Press } \\
\text { (Feet) }\end{array}$ & $\begin{array}{c}\text { Disch. } \\
\text { SHUT } \\
\text { OFF } \\
\text { Head } \\
\text { (FEET) }\end{array}$ & $\begin{array}{c}\text { Suct. } \\
\text { SHUT } \\
\text { OFF } \\
\text { Head } \\
\text { (FEET) }\end{array}$ & $\begin{array}{c}\text { Design } \\
\text { Ft Head }\end{array}$ & $\begin{array}{c}\text { Total } \\
\text { Design } \\
\text { GPM }\end{array}$ & $\begin{array}{c}\text { As Found } \\
\text { Measured } \\
\text { GPM }\end{array}$ & $\begin{array}{c}\% \text { of } \\
\text { Design }\end{array}$ & $\begin{array}{c}\text { Meas. } \\
\text { Tot. ft. } \\
\text { head }\end{array}$ \\
\hline P-1 & Loop Pump & 80 & 11.74 & 86 & 15 & 145 & 875 & 800 & $91.4 \%$ & 157 \\
\hline P-2 & Loop Pump & 75.97 & 11.8 & 85 & 17.7 & 145 & 875 & 850 & $97.1 \%$ & 148 \\
\hline P-3 & $\begin{array}{l}\text { Tower } \\
\text { Pump }\end{array}$ & 28.5 & 0.58 & 40.4 & 3.2 & 60 & 1050 & 920 & $87.6 \%$ & 64.5 \\
\hline P-4 & Boiler Pump & 25.5 & 2.2 & 35.5 & 3.8 & 55 & 160 & 175 & $109 \%$ & 53.8 \\
\hline
\end{tabular}

EMC verified the control inputs and control outputs used in the operation of the Boiler, Cooling Tower, and Heat Exchanger. All control input data points corresponding to temperatures and humidity were verified for calibration within $\pm 1^{\circ} \mathrm{F}$ and $\pm 3 \% \mathrm{RH}$ respectively, with a NIST-traceable temperature and humidity measurement device provided by EMC. All digital commands, such as pump start/stop commands were visually verified for proper equipment reaction. All analog commands such as valve positions were visually verified for proper equipment reaction by sending commands of $0 \%, 50 \%$, and $100 \%$ of scale from the Building Automation System (BAS). Valves were verified to fully open and close upon command. All applicable heat pump digital points, such as compressor start/stop were verified by commanding units to run or stop. All applicable heat pump and outside air unit control input data points such as temperature sensors were verified for calibration within $\pm 1^{\circ} \mathrm{F}$ and $\pm 3 \% \mathrm{RH}$ respectively, with a NISTtraceable temperature and humidity measurement device provided by EMC.

Upon satisfactory operation and accuracy of control input and output data points, EMC worked to verify that the sequence of operations was fully and correctly implemented, and that the transient changes in operation of the units were made apparent on the Graphics for the BAS.

Any deficiencies that were found during controls verification were documented online by EMC in the WebCx database and were readily available to project team members, so that issues could be corrected, and EMC could verify the corrections.

\subsubsection{Summary of Findings}

The following were isolated instances found by EMC, and corrected:

- No programming in place to rotate the building loop pumps upon failure of a pump. SCI personnel corrected this programming

- The outside air temperature sensor was not calibrated. Also, the outside air humidity sensor was incorrectly terminated. Both of these were corrected by SCI personnel 

Report dated DeCeMber 2009 (continued)

- All pumps in the main mechanical room were found to be operating in the "Hand" position - not being controlled automatically from the BAS

\subsubsection{System Parameters}

Table 3 shows the set-points found for the Boilers and Cooling Tower.

Table 3: Boiler and Cooling Tower As-Found Set-points

\begin{tabular}{|l|l|}
\hline \multicolumn{1}{|c|}{ Set-point Name } & \multicolumn{1}{c|}{ As-Found Value } \\
\hline Condenser Water Loop Supply Temperature & $75^{\circ} \mathrm{F}$ \\
\hline Boiler Loop Supply Temperature & $145^{\circ} \mathrm{F}$ \\
\hline Boiler OA Disable & $60^{\circ} \mathrm{F}$ \\
\hline Boiler Loop Temp Low-Limit Startup & $50^{\circ} \mathrm{F}$ \\
\hline
\end{tabular}

\subsubsection{Conclusions \& Recommendations}

After functional testing on the Tempered Water System, EMC is confident that the system will be able to maintain the loop water temperature, such that the water source heat pumps throughout the building will be able to maintain space temperature effectively. 

Report dated DeCeMber 2009 (continued)

\subsection{Makeup Air Units}

\subsubsection{Makeup Air Unit Graphics}

Below are screen captures of the typical outside/makeup air unit graphics found on the Trane Tracer Summit front-end of the building automation system.

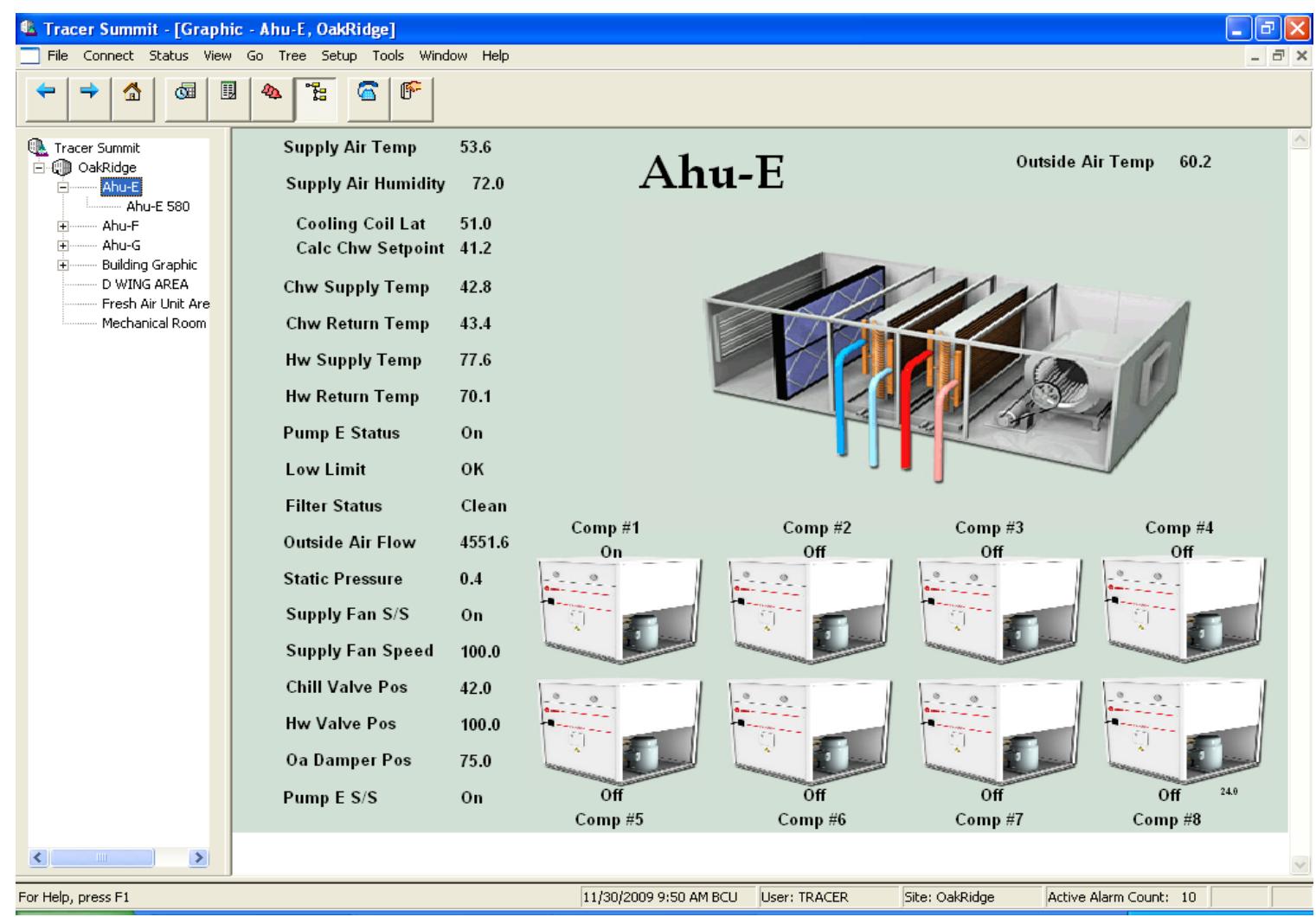

Figure 4 - AHU-E (screen 1) 


\section{APPENDIX J: EMC ENGINEERS, INC. HVAC RetRO-COMMISSIONING Report DATED DECEMBER 2009 (CONTINUED)}

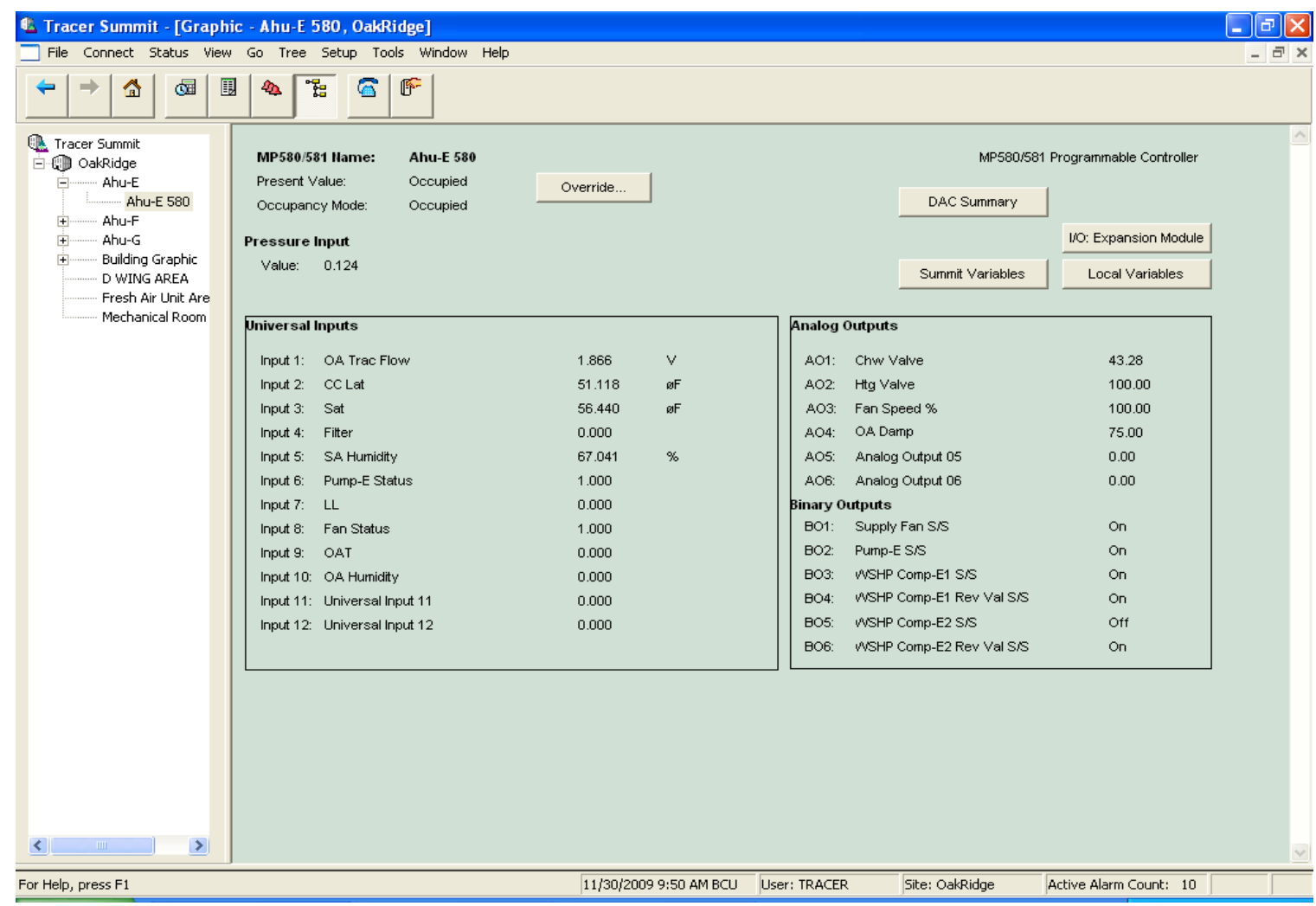

Figure 5 - AHU-E (screen 2)

\subsubsection{System Description}

- AHU-E provides conditioned outside air to the heat pumps associated with this wing of the building. The unit is connected to eight (8) water-to-water heat pump modules whose sole purpose is to condition the outside air prior to introduction into the building heat pumps

- AHU-F provides conditioned outside air to the heat pumps associated with this wing of the building. The unit is connected to seven (7) water-to-water heat pump modules whose sole purpose is to provide cooling and re-heat capability for the conditioning of outside air prior to introduction into the building heat pumps

- AHU-G provides conditioned outside air to the heat pumps associated with this wing of the building. The unit is connected to six (6) water-to-water heat pump modules whose sole purpose is to provide cooling and re-heat capability for the conditioning of outside air prior to introduction into the building heat pumps 


\subsubsection{System Testing}

EMC verified the air balance on AHU-G as a sample, by witnessing the Test and Balance contractor measure airflow on the unit under design conditions.

EMC verified the control inputs and control outputs used in the operation of the Air Handler. All control input data points corresponding to temperatures and humidity were verified for calibration within $\pm 1^{\circ} \mathrm{F}$ and $\pm 3 \% \mathrm{RH}$ respectively, with a NISTtraceable temperature and humidity measurement device provided by EMC. All digital commands, such as supply fan start/stop, were visually verified for proper equipment reaction. All analog commands such as air damper position, and hot and chilled water valve positions were visually verified for proper equipment reaction by sending commands of $0 \%, 50 \%$, and $100 \%$ of scale from the Building Automation System (BAS). Dampers were verified to fully open and close upon command.

Upon satisfactory operation and accuracy of control input and output data points, EMC verified that the sequence of operations was fully and correctly implemented, and that the transient changes in operation of the air handler were made apparent on the Graphics for the BAS.

Any deficiencies that were found during controls verification were documented and distributed by EMC, so that the Controls Contractor or Mechanical Contractor could correct the issues, and EMC could verify the corrections. Appendix B contains the list of issues discovered and resolved.

\subsubsection{Summary of Findings}

The following issues were found by EMC:

- The makeup air units were found to trip on freezestat (low temperature limit) when outside air temperatures fell below 29 degrees. Trends were set up in the BAS to capture system parameters at the time of trip.

- Outdoor air sensor location was discovered to be incorrect, resulting in readings not reflective of actual outdoor air conditions. The sensor was relocated to a proper location on the north wall of the main mechanical room and verified by EMC.

\subsubsection{System Parameters}

Table 4 shows the air balance on AHU-G, as witnessed by EMC.

Table 4: AHU-G Supply Air Balance

\begin{tabular}{|c|c|c|c|}
\hline Design (CFM) & $\begin{array}{c}\text { Measured } \\
\text { (CFM) }\end{array}$ & $\begin{array}{c}\text { Average } \\
\text { FPM }\end{array}$ & Percent of Design \\
\hline $\mathbf{3 4 9 0}$ & $\mathbf{3 4 0 2}$ & $\mathbf{9 2 2 . 7}$ & $97 \%$ \\
\hline
\end{tabular}


Table 5 shows the set-points found for AHU-G.

Table 5: AHU-G As-Found Set-points

\begin{tabular}{|l|l|}
\hline \multicolumn{1}{|c|}{ Set-point Name } & \multicolumn{2}{c|}{ As-Found Value } \\
\hline Winter Discharge Temp & $70^{\circ} \mathrm{F}$ \\
\hline Summer Discharge Temp & $70^{\circ} \mathrm{F}$ \\
\hline Dehumidification Start & $60 \%$ \\
\hline
\end{tabular}

\subsubsection{Conclusions \& Recommendations}

EMC will schedule to be onsite the first week of May, 2010 to confirm proper cooling season operation of the makeup air units, heat pumps, and associated systems.

\subsection{Exhaust Fans and Systems}

\subsubsection{System Description}

There are seventeen (17) Exhaust Fans installed, which serve various mechanical equipment spaces, kitchen spaces, janitor's closets, art classroom kiln spaces, and bathrooms. Exhaust fans serving bathrooms and janitor's closets are controlled by the BAS through the starting and stopping of the associated water source heat pump's fan. Exhaust fan 17 is controlled by a local wall thermostat in the main electrical room, behind the boiler room. Exhaust fan 16 is controlled by the BAS, and serves the boiler room. This fan runs continuously.

System Testing

EMC was scoped to verify sequence of operations on $100 \%$ of the exhaust fans, by performing a functional test. The functional test involved imposing start/stop commands on the exhaust fans from the BAS, and recording whether or not the exhaust fans reacted properly.

\subsubsection{Summary of Findings}

The following issues were isolated instances found by EMC, and corrected:

- Exhaust fans 1, 3, 4, 5, 6, and 10 would not respond (start/stop) when the associated heat pumps were occupied/unoccupied)

- The exhaust duct to the janitor's Closet outside the Multi-Purpose Room was not connected to the ceiling grill. This resulted in no exhaust from the space, and likely contributed to objectionable odors in the vicinity

- Exhaust Fan EF-9 was not accessible due to being located above the rated ceiling in the F-Wing. Necessary access was cut in beneath the fan

- A substantial number of dampers located above rated ceilings were not accessible due to the absence of access doors or panels. Access was subsequently cut in

\subsubsection{Conclusions \& Recommendations}

The exhaust fans are operating correctly. No recommendations at this time. 
Appendix K: The Turner Building SCience and Design Final Report DAted 8 January 2010

\title{
OVERVIEW OF WORK COMPLETED TO IMPROVE INDOOR AIR QUALITY AND HVAC OPERATION OAK RIDGE ELEMENTARY SCHOOL OAK RIDGe, North CAROLINA
}

\author{
JANUARY 2010
}




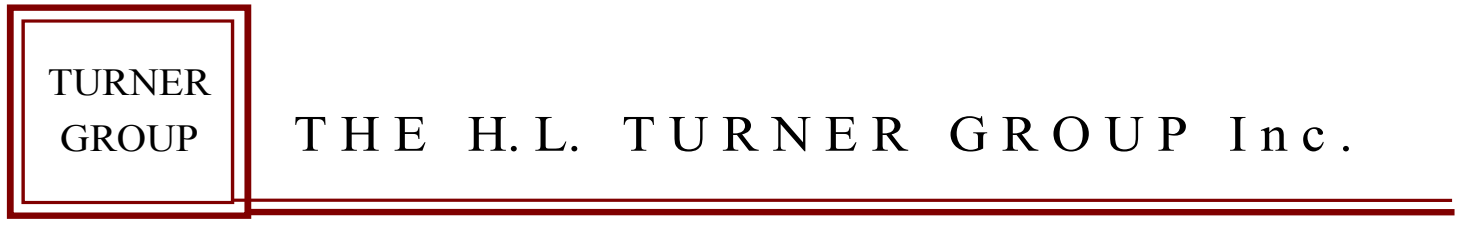

27 LOCKE ROAD, CONCORD, NH 03301-5417 TELEPHONE: 603-228-1122 FAX: 603-228-1126

January 8,2010

Mr. Leo Bobadilla

Chief Operations Officer

Via Email: bobadil@gcsnc.com

Guilford County Schools

617 West Market Street

Greensboro, NC 27401

SUBJECT: Report on Work Completed at Oakridge Elementary School

Oakridge, North Carolina

TBS Project \#S0873-01

Dear Mr. Bobadilla:

In accordance with our approved Scope of Services, we are pleased to offer the following report of our observations and analysis of the indoor air quality, building envelope, and HVAC systems at the Oakridge School in Oakridge, North Carolina. The work performed was in accordance with our approved Scope of Services dated July 10, 2009, and as amended on July 29, 2009. Our final site visit was completed on December 17, 2009.

An analysis of the adequacy of indoor air quality provided to the occupants of the school is the focus of this work effort. During our analysis, we have taken into consideration US EPA, State of North Carolina, and other professional guidance concerning indoor air quality within school facilities. Our site observations, building environmental measurements, and air sampling are the basis of all our recommendations.

The enclosed report is of a technical nature. Therefore, to evaluate the recommendations made herein properly, it is recommended that the reader have technical knowledge of the facility.

The H.L. Turner Group Inc. (TTG) has enjoyed the opportunity to serve as professional consultants to Guilford County Schools. Please contact me if you have any questions or need further clarification of any items within this report. You can reach me at our Vermont office at (802) 592-3097, Mr. William Turner in our Harrison, Maine office at (207) 583-4571, ext. 11, or Mr. Steven Caulfield at ext. 14.

Sincerely,

THE H.L. TURNER GROUP INC.

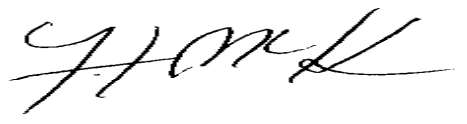

Frederick T. McKnight, P.E.

Chief Indoor Air Quality Engineer

FTM/sai

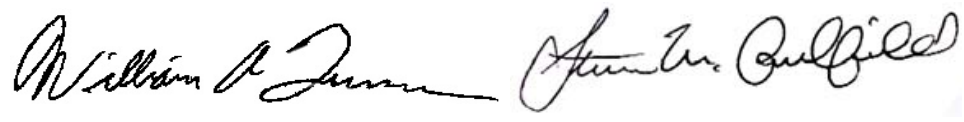

William A. Turner P.E.

Steven M. Caulfield, P.E. Senior Vice President Senior Vice President

Enclosures

cc: /James Smith, GCS/Gerald Greeson, GCS/Alex Elkan

ARCHITECTS • ENGINEERS • BUILDING SCIENTISTS • CONSTRUCTION MANAGERS 

DATED 8 January 2010 (CONTINUED)

\section{TABLE OF CONTENTS}

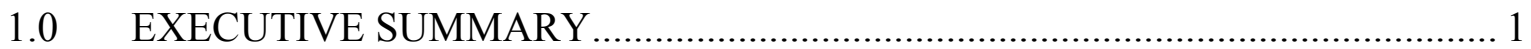

$1.1 \quad$ Summary of Recommendations …………….......................................... 3

2.0 BUILDING DESCRIPTION .................................................................... 5

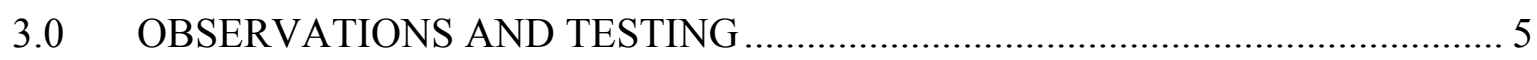

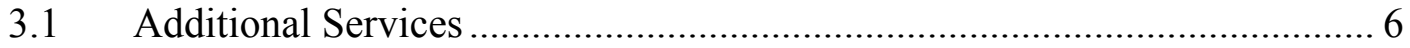

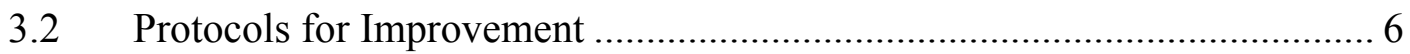

4.0 RESULTS AND DISCUSSION OF RESULTS.............................................. 7

4.1 Work Completed to Limit Potential for Mold Growth .................................. 8

4.1.1 Removal of Water Damaged Building Material ............................... 8

4.1.2 Installation of Replacement Flooring.............................................. 9

4.1.3 Modifications to Existing Heat Pumps .......................................... 9

4.2 Work Completed to Limit Water Intrusion ................................................... 10

4.2.1 Site Work to Limit Possible Liquid Water Entry ............................... 10

4.2.2 Roof and Flashing Work to Limit Liquid Water Entry...................... 10

4.3 Work Completed to Limit Water Vapor and Unplanned Air Flows............. 11

4.3.1 Crawl Space Moisture and Microbial Control ................................... 11

4.3.2 Soffit Sealing: Controlling Unplanned Air Flow and Humidity ....... 12

4.3.3 Waste Piping Vent Line Repairs ............................................................ 13

4.4 Work Completed to Meet Design Air Flows ................................................... 13

4.4.1 Retro-Commissioning, Terminal Air Balancing Controlling Unplanned Air Flows, and Maintaining Humidity Levels................. 13

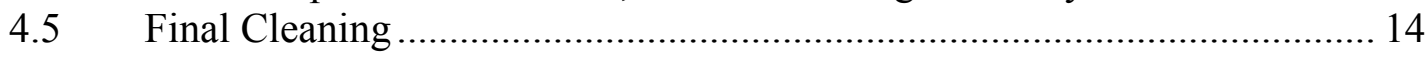

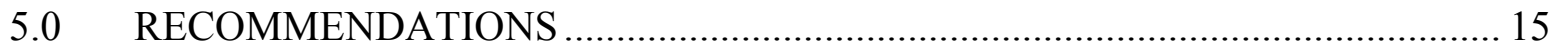

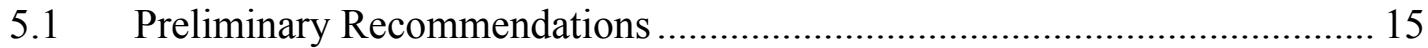

5.2 Additional Recommendations .............................................................. 15

Recommendation \#1: New Floor Maintenance Systems and Schedules ................. 15

Recommendation \#2: Limiting Noise ............................................................... 15

Recommendation \#3: Do Not Use Textile Floor Coverings.................................... 15

Recommendation \#4: Use Track-Off Mats and Limit Exiting to Control Dirt

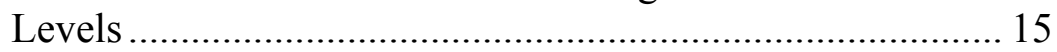

Recommendation \#5: Occupied Temperature Set Points ………………………... 16

Recommendation \#6: Occupied Cycles ............................................................... 16

Recommendation \#7: Additional Monitoring of Space Temperatures, Humidity, and Ventilation Rates ......................................................... 16

Recommendation \#8: Additional Engineering Services to Increase Energy Efficiency 16 


\section{Appendix K: The Turner Building Science and Design Final Report DATED 8 JANuary 2010 (CONTINUED)}

\section{APPENDICES}

Appendix A: Protocols for Improvement

Appendix B: Material Removal Spore Trap Clearance Tests

Appendix C: Final Cleaning Spore Trap Clearance Tests

Appendix D: Temperature and Humidity

Appendix E: Blower Door Test Results

Appendix F: Concrete Moisture Tests

Appendix G: Photos 


\subsection{EXECUTIVE SUMMARY}

At the request of Ms. Heather Larkin and Mr. Leo Bobadilla, The H.L. Turner Group Inc. (TTG) conducted an initial building evaluation in conjunction with NIOSH, and subsequently produced a series of protocol documents providing detailed recommendations for improvement. Our work also included a series of site visits to observe work completed by contractors and school personnel that was based on the written protocols. This report concludes all currently approved observations, measurements, and sampling as outlined in our approved Scope of Services dated July 10, 2009, and an addendum dated July 29, 2009. (Note: Additional site work has yet to be completed as noted below, and additional work may be required to complete the new recommendations made herein.)

These recommendations focus on changes in operation and maintenance procedures for the building. The recommendations are made because of the modifications made to the existing building envelope and HVAC systems. The building modifications were completed based on a series of recommendations that were made after our initial site visit, which was completed in conjunction with a site visit from a NIOSH team of health experts. The initial recommendations were outlined on July 21, 2009 in our report "Oak Ridge Elementary School Very Preliminary Draft Indoor Air Quality Issues".

The building was not occupied during our evaluations; therefore, direct observation of the spaces, when occupied, was not possible. However, reports completed by other consultants prior to our work that included some measurements of indoor conditions during occupancy were made available for our use. Our analysis and recommendations made in previous reports, and made herein, are based primarily on our observations, test results, available reports from other consultants, and our understanding of guidelines and standards published by ASHRAE, ACGIH, NIOSH, USEPA, and the State of North Carolina concerning the indoor environment of educational facilities.

We anticipate that the work completed to decrease unplanned airflows, increase the building ventilation rates, improve control of space humidity, remove and replace building materials, improve site drainage, as well as complete air and water balancing and commissioning the mechanical system, will improve the air quality within the facility. However, if occupants begin to acquire symptoms typical of poor air quality, then further building evaluations and assessments may be required.

The Guilford County Schools system reportedly is working to implement the USEPA Tools for Schools program for all schools in the county. When completely implemented the Tools for Schools program will provide a framework for professional staff, parents, maintenance staff, and the school administrators to identify and assign resources to make improvements to address indoor air quality concerns. 


\section{Appendix K: The Turner Building SCIence and Design Final Report Dated 8 January 2010 (CONTINUed)}

In essence, our evaluations, observations, and resulting test reports indicate the following:

- That there are no amplified microbial sources contributing spores to the indoor air of the tested spaces of the Oak Ridge School.

- Initial air balancing measurements indicated some HVAC units were not meeting the design airflow parameters.

- Modifications to the existing HVAC systems combined with balancing and retrocommissioning of the systems have verified that the building HVAC systems are now operating within the parameters of the initial design.

- Cleaning of dust accumulations on the coils and the fan casings of the heat pumps has been completed.

- Envelope repairs to the soffit, crawl space, and to the clerestory have been completed to minimize the impact of unplanned air flows.

- Building repairs to walls and roofs have eliminated known sources of moisture intrusion.

- Modifications to the existing plumbing vent piping have helped to reduce emission of odors into the occupied space.

- Removal and replacement of textile floor coverings with vinyl tiles and removal of glue-on ceiling tiles have decreased the risk of future amplified microbial sources being found within the building.

- Modifications to the existing HVAC system with respect to the location of filters and cooling coils have been completed to minimize the opportunity for the coils to wet the filter surfaces and promote mold growth.

- The building has been thoroughly cleaned as part of the remediation project. A series of spore trap samples has indicated that the indoor environment has fewer spores than the outdoor air, suggesting that the indoor spaces do not have sources of amplified microbial growth.

In addition, some site work has not yet been completed, due to seasonal climate and weather conditions. The work may not be completed until spring of 2010 . The site work is intended to minimize the amount of surface run-off that pools next to the building during torrential storm events.

Additional recommendations made herein include:

- Installing track-off mats to control the amounts of dirt and other material tracked into the building. 
- Restricting the use of classroom fire-exit doors to only those doors that have trackoff matting systems in place.

- Not reintroducing textile floor covings (throw rugs) back into the spaces with ground contact flooring.

- The hard nature of the new flooring will increase the reverberation of noise and will do little to absorb noise energy. Measures including placing felt pads on movable furniture are recommended.

- Floor maintenance and cleaning schedules will need to be adjusted for the new floor types installed.

- Temperature set points and offsets recommended by the commissioning agent are recommended, and occupied/unoccupied scheduling of HVAC equipment is also recommended.

- Additional monitoring of space temperatures, humidity, and ventilation rates to verify system operation and capacity.

- Additional engineering studies to provide options to improve building air and heat leaks found in thermal images.

\subsection{Summary of Recommendations}

See July 21, 2009 report Oak Ridge Elementary School Very Preliminary Draft Indoor Air Quality Issues for details on the preliminary recommendations. The preliminary recommendations are summarized below. Additional recommendations are also summarized below. See Section 5 for more details on the additional recommendations made herein.

\section{Preliminary Recommendations:}

1. Crawl Space: Provide flexible vapor barrier (fire retardant?) and depressurization of air space under vapor barrier.

2. Install through-wall flashing at edge of parapet to connect through brick to drainage layer behind.

3. Repair roof junction joints to provide adequate flashing.

4. Provide retro-commissioning of HVAC system with respect to verifying proper system capacity and sequence of operation. 


\section{Appendix K: The Turner Building Science and Design Final Report DATED 8 JANUARY 2010 (CONTINUED)}

5. Provide retro-commissioning of HVAC system with respect to verifying proper system capacity and sequence of operation.

6. Sealing of soffit to prevent air infiltration. (Note: If soffit is sealed, provide means for exhaust/relief.)

7. Provide new filter boxes upstream to create separation between coil and filter.

8. Review maintenance tasks, schedules, and on-going maintenance issues.

9. Balance air flows as part of retro-commissioning process; move some supply air to hall.

10. Verify air flows on retro-commissioning. Provide duct joint sealant on all RA \& SA if necessary.

11. Verify adequate site drainage and clean site drainage system as necessary. Provide means to limit run-off sand and other surface material.

12. Provide balancing of OA system and marking of dampers as part of retrocommissioning project.

13. Obtain roofing contractors estimate to repair/replace foam roof.

14. Replace or repair flashing.

15. Provide monitoring of conditions during occupancy to evaluate operation under occupancy (after retro-commissioning of systems).

\section{Additional Recommendations:}

Recommendation \#1: New Floor Maintenance Systems and Schedules

Recommendation \#2: Limiting Noise

Recommendation \#3: Do Not Use Textile Floor Coverings

Recommendation \#4: Use Track-Off Mats and Limit Exiting to Control Dirt Levels

Recommendation \#5: Occupied Temperature Set Points

Recommendation \#6: Occupied Cycles

Recommendation \#7: Monitoring of Occupied Spaces for Temperature, Humidity and Ventilation Rates

Recommendation \#8: Additional Engineering Services to Increase Energy Efficiency 


\section{$\underline{2.0}$ BUILDING DESCRIPTION}

The facility reportedly underwent a major renovation six years ago. During that renovation, the majority of the interior finishes were replaced, some materials such as the media center floor were refurbished, and new mechanical and electrical systems were installed. The school facility included a main building and remote buildings (i.e. remote from the main building, but within the current site). The renovations included building additions that connected all of the buildings on-site into a single continuous facility (under one roof). The subject areas of our evaluation were all normally occupied spaces within the Oak Ridge Elementary, and other spaces that may be affecting the occupied spaces, such as crawl spaces and utility rooms.

The walls (in general) based on observation, are composed of a brick facade. The new classroom wings (400 wing, 500 wing, and 600 wing) and the administration wing have a cavity frame wall behind the facade. The structure is light gauge steel framing with an exterior grade gypsum sheathing covered with 1 " thick rigid insulation board. The cavity space is filled with 6" fiberglass batt insulation with a Kraft-type paper backing. The interior side of the cavity is covered with paper faced interior grade gypsum board. The wall in the gym/cafeteria wing is a CMU block wall behind the exterior brick facade. The original building is a solid brick wall in lieu of a brick facade. The interior finish of the media center is a plasterboard or gypsum board on strapping attached to the brick.

Doors and windows are steel framed; doors are steel panel and glass, with a varying proportion of glass. Windows are thermo-pane-type with double-glazing. The frame types are either fixed-type, or combination fixed and operable sliding casement-type. All floors except those in the media center wing are slab-on-grade poured concrete. The media center wing has wood floors supported by wood framing above a crawl space.

Roofs are a combination of flat (low pitch) and pitched roofs. Reportedly, the pitched roof is comprised of a metal exterior roof panel mounted on a 3 " thick foam gypsum board panel that is mounted on a $1-1 / 2$ " metal roof deck. The flat roofs are either a ballasted builtup bituminous roof, rolled roofing, or a foam-in-place roof. Components that lie under these roofs were not observed, except for the media center where the roof deck and structure are wood. The finished ceiling is predominately a suspended lay-in ceiling. Some exceptions include the gym, which has no finished ceiling and utility rooms.

\subsection{OBSERVATIONS AND TESTING}

A series of tests and observations were completed in our initial site visit. The test results and the observations that were of concern to us were briefly reported in our July 21, 2009 report Oak Ridge Elementary School Very Preliminary Draft Indoor Air Quality Issue. 


\section{Appendix K: The Turner Building SCience and Design Final Report DATED 8 JanuARY 2010 (CONTINUED)}

\section{$\underline{3.1 \quad \text { Additional Services }}$}

As a result of our initial recommendations, an addendum to expand our Scope of Services was requested by the school administration. The addendum, dated July 29, 2009, included performing additional analysis and providing written protocols detailing the recommendations made in the preliminary report.

Additional testing completed after our initial site visit included:

- Measuring concrete moisture emission rates. Concrete emissions tests were conducted by others. Test results were made available to us. We completed additional real-time moisture content measurements of selected concrete floors.

- Observing wall conditions for visible mold behind selected walls finishes with vinyl wallpaper.

- Completing observations of the clerestory space above the media center ceiling for both visible mold and conditions that may result in unplanned air flows.

- The exterior sides of the building walls were scanned with a thermal imaging camera (Flir ModelB360) to locate air leaks. The scans were completed before the recommended soffit sealing was begun, and then again, after the soffit sealing was completed. The tests completed after the soffit sealing included a series of scans taken during occupied cycle, and a scan completed during unoccupied cycle.

- Blower door testing was completed in conjunction with the thermal scans to quantify the air leakage. The blower door tests were completed with the HVAC system in unoccupied cycle in order to measure the total pressure induced leakage rate.

\subsection{Protocols for Improvement}

A number of protocols were assembled based on the preliminary recommendations. The protocols are detailed recommendations for improvement of specific conditions observed. It is our understanding that the protocols were used to develop plans and specifications that the school department used to solicit bids to make the recommended improvements. Below is a summary list of protocols released under this project. The protocols are available for review in Appendix A.

- Air and water balance (with respect to retro-commissioning) $(10 / 08 / 09)$

- Automatic controls (with respect to retro-commissioning) (10/08/09)

- Coil cleaning (including heat pump casing) (10/08/09)

- Crawl space venting (including vapor barrier) (10/08/09)

Turner_final_report.doc 
- Filter boxes (and return duct modifications) (10/08/09)

- Filter returns $(10 / 08 / 09)$

- Final cleaning $(11 / 24 / 09)$

- Oak Ridge carpet removal (including removal and cleaning of all contents) $(8 / 13 / 09)$

- Oak Ridge glue-on ceiling tile removal (10/18/09)

- Oak Ridge VCT (8/13/09)

- Retro-commissioning (10/08/09)

- Roof flashing (and miscellaneous roofing repairs) (10/08/09)

- Soffit (10/20/09)

- Site work comments on submitted plan (11/20/09)

\subsection{RESULTS AND DISCUSSION OF RESULTS}

The focus of all the work completed included:

- Removal of flooring textiles and other building materials that had a high potential to promote microbial growth.

- Minimizing unplanned air flows.

- Increasing control over the indoor humidity.

- Measuring and adjusting outdoor air flow to meet the intent of the initial design (7.5 CFM/Occupant as set by building codes of the State of North Carolina).

The textile floor coverings were replaced with solid vinyl tiles. Other removed materials were replaced with new suitable materials where the proper function of the building required replacement. (Note: Some materials such as the glue-on ceiling tiles were removed without replacement, because the tiles did not affect the function or appearance of the building.)

The recommendations for improvement fall into four major groupings:

- Work needed to reduce the potential for mold growth.

- Work needed to reduce potential moisture intrusions.

- Work needed to control water vapor in air (humidity), including correction of unplanned air flows.

- Work needed to correct the building operation to verify that it meets the initial design intent with respect to air flows, system capacity, and operation. 


\section{Appendix K: The Turner Building SCience and Design Final Report DATED 8 JanuARY 2010 (CONTINUED)}

After all interior work was completed, the building was given a final thorough cleaning where all surfaces were vacuumed or damp wiped, floors were vacuumed, wet cleaned with an extractor, and reportedly waxed.

The following sections summarize work completed to improve indoor air quality and are presented by the categories outlined above.

\subsection{Work Completed to Limit Potential for Mold Growth}

\subsubsection{Removal of Water Damaged Building Material}

A series of bulk samples of carpet dust collected from the installed carpets (samples collected by others) revealed repeated incidents of amplified microbial reservoirs within the carpet. Mitigation of amplified reservoirs found in textile floor coverings is not recommended by ACGIH. Removal of the textile is the recommended procedure. (Note: this was not on our preliminary list of recommendations because the school department had informed us of their plans to remove the carpet. However, we did provide them with a protocol for removal of carpet, at their request.)

The first task undertaken to improve air quality at this facility was to remove the carpeting and other water-damaged materials found in the facility (small quantities of ceiling tiles and gypsum board). As a precaution, the carpeting and other materials were removed under conditions of isolation and containment. The removal process included removal and cleaning of all non-fixed contents (such as desks, chairs, and materials) from the building. The non-fixed contents were visually inspected for signs of mold growth; none was reportedly found. All contents not showing visible signs of mold growth were then cleaned using ACGIH recommended practices and shipped to a conditioned storage site or to other locations for use at that location. See Oak Ridge Carpet Removal Protocol and Oak Ridge Glue on Ceiling Tile Removal for details of cleaning and containment systems. (Note: Reportedly, none of the non-fixed contents were found to have visual signs of mold. In addition, the removal of glue-on tiles was not part of the preliminary list of recommendations because they were discovered after the list of recommendations was presented.)

The conditioned storage site was another building (former school) owned by the school system. The storage site and the contents, while in storage, were observed and found to be in a dry location with normal occupied temperatures and humidity. Reportedly, the storage site was observed daily by maintenance personnel who entered the building to look for water leaks or abnormal temperatures.

Removal of water damaged building materials and finishes were completed in each of the classroom wings, the administrative wing, and the media center under conditions of isolation and containment. Each wing was separately isolated and contained before removal of water damaged building materials. Isolation and containment means that the

Turner_final_report.doc 


\section{Appendix K: The Turner Building SCIence and Design Final Report Dated 8 January 2010 (CONTINued)}

space is physically isolated from the rest of the building with the installation of a physical barrier. HVAC systems were also isolated from the space by covering registers and grilles. Containment of the space was accomplished by inducing an air pressure in the isolated area that was lower than the air pressure in the surrounding building areas.

Materials removed, in addition to the textile floor coverings, included some small portions of gypsum board at floor level. These pieces of gypsum showed signs of visible mold. Glue-on ceiling tiles located above the dropped ceiling tiles in the media center wing, and wood bead board ceilings also located above the dropped ceiling in the media center wing, were also removed or cleaned. Areas of water damaged walls in the clerestory area above the dropped ceiling tile in the media center were suspected of having supported mold growth in the past. Although the walls were not currently found to be supporting growth, they were also removed or cleaned, as well as removing water damaged building materials, dried leaves, and other dried organic material found trapped in the building construction in the media center wing. In addition, selected portions of the walls in the classroom wings covered with vinyl wallpaper were observed for visible mold growth by peeling back the wallpaper. No visible mold growth was observed under the vinyl wallpaper.

Once the identified suspected water damaged materials (carpet, wallboard sections, organic materials, and ceiling materials) were removed from each wing, the wing was thoroughly cleaned as outlined in the protocol. A series of airborne spore trap samples were collected and sent to the lab for analysis. See Appendix B for lab results of spore trap sampling. The installed isolation and containment systems were removed after the spore trap sample results indicated that the spore count inside the containment area was less than the outdoor spore count.

\subsubsection{Installation of Replacement Flooring}

After removal of the containment and isolation systems, moisture emission tests were conducted on the bare concrete subfloor. See Appendix F for test results and real-time moisture meter readings. In conjunction with the vapor emission tests, visual observations were completed, and real-time moisture content readings were collected with a Protimeter (Serial \#6600-089). The emissions test results and the moisture measurements were used to determine the type of flooring and adhesive that would replace the textile floor coverings. See Oak Ridge VCT Protocol for details.

\subsubsection{Modifications to Existing Heat Pumps}

The location of the air filters in front (upstream) of the cooling/heating coils at each heat pump in the HVAC system provide an elevated risk of mold growth, especially during any period of cooling. The downstream filter surfaces were observed to be within a few inches of upstream coil surfaces. The make and model of the heat pump with a similar air filter arrangement had been observed (in a different facility) to have a high risk of developing microbial reservoirs, due to the very close proximity of organic material in the filter, and the moisture from condensation at the cooling coil. While visible mold growth was not

Turner_final_report.doc

THE H.L. TURNER GROUP Inc. 


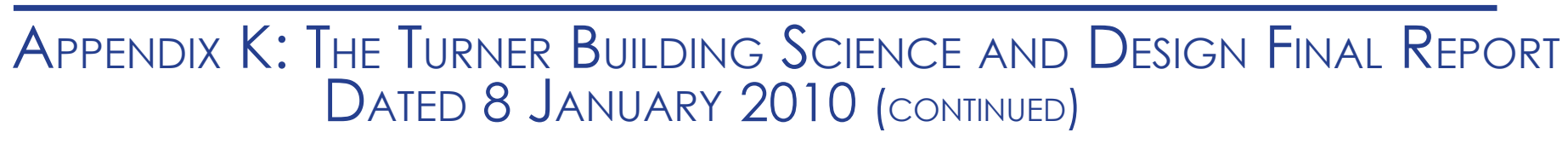

observed on the filter surfaces at the time of our site work, the arrangement of filters and coils have been known to provide an elevated risk for microbial growth. (Note: The observed filters in the heat pumps were fairly new, having been changed days before our site visit as part of the ongoing filter maintenance program.)

Based on the known risks and our recommendations, the air filters were moved further upstream from the cooling/heating coils. The filters were moved to the return air registers in heat pump systems serving the classroom wings, and in other selected locations where the arrangement of return air and outdoor air ducts restricted the relocation of the filters, and the outdoor air was filtered separately. The remaining heat pump systems had arrangements that provided sufficient room to locate the filter further upstream while not allowing unfiltered outdoor air to bypass the filter. See Filter Boxes Protocol and Filter Returns Protocol for details.

Additionally, the cooling/heating coils and the heat pump fan casing were cleaned to remove a build-up of filter material that had collected on the coil surface. See Coil Cleaning Protocol for details. (Note: We did not recommend duct cleaning because duct cleaning would have little beneficial impact due to an absence of significant deposition of dust or nutrient material in the duct system. Our observation of some portions of the return ducts in the classroom wings did not show signs of heavy accumulation of material or signs of moisture damage (staining).)

\subsection{Work Completed to Limit Water Intrusion}

\subsubsection{Site Work to Limit Possible Liquid Water Entry}

Based on observation and reports from occupants, the site around the building has finished grades that would allow water to pond against the building walls during times of torrential rainfall. Water at the base of the exterior walls may have been responsible at times for conditions that promoted microbial growth within the carpets in some classrooms. Site grade modification plans have been developed and reportedly will be implemented at the earliest opportunity after the winter rainy season ends. The implementation of the modifications in the spring is not likely to have an adverse impact on the building in the short-term because the carpets have been removed and replaced with a floor covering that is more resistant to moisture.

\subsubsection{Roof and Flashing Work to Limit Liquid Water Entry}

Our observations of the roofs and the visible flashing at the base of high walls where these walls intersected the roofs, observations of reported past leak areas, and occupants reports, suggested that the flashing may not be functioning properly. We recommended additional evaluation including destructive disassembly of some of these areas to make additional evaluations and then repairs as necessary. The disassembly revealed through-the-wall flashing that was extended up the wall approximately $1 / 4 "$. The observed extension up the

Turner_final_report.doc 
wall would fail to keep water from getting behind the flashing when winds were greater than an estimated $17 \mathrm{MPH}$.

In addition, some of the corner and cap flashing assemblies that were observed appeared not to be constructed well enough to keep water from getting behind the flashing. Once water gets behind the flashing and into the wall structure, damage to the wall may occur, and water may be found inside of the building.

Work was completed on the flashing to increase the extension and limit the potential for wind driven rain to defeat the flashing. See Roof Flashing Protocol for details. Additional work was completed to repair flashing that was identified as needing replacement.

Additional evaluation of the flashing and edge details of the roof at the clerestory was also completed and some of the clerestory windows were resealed. The existing roof scuppers were also lowered.

The clerestory space above the media center was also found to have abandoned ventilation systems that were not removed in the last renovation. The abandoned system did not serve any purpose with respect to the current use of the space. The ventilation system was defeating the building thermal envelope. The systems were removed and the openings sealed.

\subsection{Work Completed to Limit Water Vapor and Unplanned Air Flows}

\subsubsection{Crawl Space Moisture and Microbial Control}

The media center wing of the building does not have a slab-on-grade floor that is typical of the remaining building areas, but has a crawl space under a wood framed and wood deck floor. The crawl space was an earthen floor space enclosed by perimeter foundation walls. The crawl space also included a walk-in space that was the old boiler/electrical room for the original building. The heating plant and electrical distribution panels are no longer located in this space.

Depending upon the operation of the HVAC system, moist air from the crawl space would flow into the media center wing from the crawl space (i.e. unplanned air flow). In addition to moist air, the potential for the transport of mold spores (from reservoirs observed in the crawl space) into the media center wing was high during times that the HVAC system operation would allow infiltration into the media center wing.

To limit moisture entry into the crawl space and to prevent excessive moisture build-up, the crawl space was isolated from the earthen floor with a poly vapor barrier. The vapor barrier is sealed to the foundation wall. The wall is insulated with polyisocyanurate and all the existing vent openings through the foundation wall were sealed. Other openings between the crawl space and the media center above were sealed with spray foam sealant.

Turner_final_report.doc

THE H. L. TURNER GROUP Inc. 


\section{Appendix K: The Turner Building SCience and Design Final Report DATED 8 JanuARY 2010 (CONTINUED)}

The crawl space was provided with a sub-membrane sump pump to remove liquid ground water that rises to the surface beneath the poly vapor barrier. A sub-membrane vapor removal system was also installed to control water vapor levels under the membrane. A system of dehumidifiers was placed in the crawl space and the old boiler room to limit moisture (as vapor in air) to 50\% relative humidity. See Crawl Space Venting Protocol for details. (Note: The poly vapor barrier was attached to the foundation wall using an alternate method that eliminated the wood strip, which was feared to introduce an additional source of food for termites. The alternate system used was the standard attachment method used by the installing contractor.)

\subsubsection{Soffit Sealing: Controlling Unplanned Air Flow and Humidity}

The soffit area of the three classroom wings was observed and found to incorporate a number of large and small openings through the building thermal enclosure, allowing leakage of unconditioned air into and out of the building, depending on the operation of the HVAC system. The leakage sites would allow humid air into the building, especially during unoccupied times. The introduction of humid air into the building is not desirable. Humid air counteracts the dehumidification systems and adds moisture to the space that can result in condensation upon cooler surfaces (such as the floors). See Soffit Protocol for details of modifications.

Soffit sealing included adding gypsum sheathing to the large openings at the end of each classroom corridor, adding fire-stop to all cracks and openings, applying spray-on foam to seal all openings in the wall behind the soffit, installing relief vents from the classrooms up into the plenum above the classroom ceilings, and installing relief vents to the outside, fitted with weighted back draft dampers to allow air to leave the plenum freely, but restrict the flow from the outside into the plenum.

Blower door tests and infrared imaging indicated that the soffit was a source of numerous large and small air leak areas.

Initial blower door testing was completed prior to sealing the soffit. A second set of tests were completed after the soffit sealing was complete. See Appendix E for blower door test results and Appendix $\mathrm{G}$ for infrared images. The blower door results are graphed separately for each wing tested. The graphs show the total leakage from the wing prior to the soffit sealing work, and the leakage from the wing after completion of the soffit sealing work. The difference between the two tests indicates the amount of air leakage that existed at the soffit prior to sealing the soffit.

Other heat and air leak areas identified on the thermal imaging included doors, windows, and the base of the building wall.

Turner_final_report.doc 


\section{Appendix K: The Turner Building SCIence and Design Final Report Dated 8 January 2010 (CONTINUED)}

\subsubsection{Waste Piping Vent Line Repairs}

Several photos of the plumbing vent piping provided by other consultants working with us on this project were reviewed. The photos indicated some plumbing waste line vents in the classroom wings were open to the space above the ceilings. The latest as-built drawings indicated that these lines should have been connected to the waste line vents. These opened vents have reportedly been connected to the waste vent systems. In addition, some of the waste line vents through the roof were reportedly less than 10 feet from roof mounted air inlets. These through the roof vent lines were reportedly relocated to meet the minimum 10 foot distance between roof vents and air inlets as specified by the construction drawings.

\subsection{Work Completed to Meet Design Air Flows}

\subsubsection{Retro-Commissioning, Terminal Air Balancing Controlling Unplanned Air Flows, and Maintaining Humidity Limits}

Initial space temperature and humidity measurements combined with our observations and occupant provided information, pertaining to thermal comfort and air quality, lead to our recommendation for retro-commissioning and air balancing. Commissioning is a systematic process of ensuring that all building systems perform interactively according to the design intent and the Owner's operational needs. As retro-commissioning (post occupancy commissioning), the commissioning process encompasses system documentation, equipment operation, control system calibration and operation, testing and balancing, and system performance testing.

Testing and balancing included balancing the air flows (supply air, return air, exhaust air, and outdoor air) of all heat pumps and the associated outdoor air systems. Providing the proper amounts of air to each space with the correct proportion of outside air is critical for maintaining air quality, including recommended humidity levels.

Retro-commissioning services verified that the design amounts of air, including outdoor air, are currently being provided. In addition, it verified that the HVAC system is conditioning the air to the extent that the design intended. The services were provided in accordance with our protocol, Retro-Commissioning Protocol and Air and Water Balance and Automatic Controls. For protocol details, see Appendix A. The commissioning report will be released separately by the commissioning agent, EMC Engineering, Inc., Raleigh, NC.

Indoor temperature and humidity data collected at the site prior to the beginning of improvement work, and at a point where the work was approximately $95 \%$ complete, can be viewed in Appendix D. The data shows high humidity in Room 520 during July when the system was operating in an unoccupied cycle (outdoor air units were off). In addition, data was collected after the soffit sealing was complete, but prior to the reinstallation of the 


\section{Appendix K: The Turner Building SCience and Design Final Report Dated 8 January 2010 (CONTINUed)}

soffit panels. At that time, the work to modify the HAVC system was approximately $95 \%$ complete, and temperature monitoring indicated that the space temperatures were very constant (within a degree variation). The same data also depicts the different operating conditions under which the corner classroom (Room 520) operates. The temperature swings in Room 520 were greater than non-corner classrooms. Similar temperature readings were collected in July with respect to temperature variation; however, the space temperature remained within $1-1 / 2$ degrees.

Preliminary balancing indicated that the outdoor air supply to the media center was low and could not be adjusted to meet the design intent. Additional equipment was added to the media center HVAC systems to provide the quantities of OA required to meet the original design amounts. Reportedly, balancing of all the other systems in the building to meet the original design was successfully completed. Replacement parts (thermostats and other devices) were required in order to provide the intended control sequence. The retrocommissioning efforts are reported in the final commissioning report submitted by the commissioning agent. The report also includes the final air and water balancing report.

\section{$\underline{4.5} \quad$ Final Cleaning}

At the completion of all interior work to improve air quality, the building was thoroughly cleaned. Cleaning occurred prior to the reintroduction of any of the contents that were in storage or in use at other sites. A series of spore trap tests were completed that compared inside levels to outside levels. The series included 300-second sample intervals and 600second sample intervals. The sample interval is selected based on observation and other information which is used to determine the potential of overloading (or under loading) the samplers. Results from both interval lengths indicate that the spaces have lower spore counts than the outdoors.

After the final cleaning of the building materials at the off-site storage, and in use in other locations were, or will be, reintroduced into the Oak Ridge School. Materials in storage were observed for signs of visible mold growth and odors by trained industrial hygienists from S\&ME prior to reintroduction into the Oak Ridge School facility.

\section{Conclusion}

We believe the buildings HVAC system is performing in accordance with the intent of the design as reported in the commissioning agents report. Additionally testing suggests that unplanned airflows that affected humidity levels in the building, as well as the air quality have been reduced. Moisture damaged materials and materials that were found to be at an elevated risk of damage from moisture have been removed and replaced with material less susceptible to moisture damage. Based on the improved building performance as outlined above, our experience, our observations of conditions at the facility, the available spore trap test results, the commissioning report, and air leakage test results, we believe the

Turner_final_report.doc 
building can be reoccupied. Occupants should be aware of, and conform to, the intent of our additional recommendations made herein concerning the operation of the facility.

\subsection{RECOMMENDATIONS}

\subsection{Preliminary Recommendations}

Our initial observations and tests were the basis for our preliminary recommendations, and were reported in our July 21, 2009 preliminary report, Oak Ridge Elementary School Very Preliminary Draft Indoor Air Quality Issue. The additional recommendations made below are presented in recognition that the building has undergone a series of fundamental changes and will require some additional changes in maintenance and operation.

Our recommendations made below include some best practices with respect to maintenance and verification monitoring. These recommendations do not necessarily need to be completed prior to re-occupancy. Many are dependent on specific modes of operations that may not reflect actual operating conditions.

\section{$\underline{5.2 \quad \text { Additional Recommendations }}$}

\section{Recommendation \#1: New Floor Maintenance Systems and Schedules}

The school no longer has textile floor coverings. All finished floors are either hard vinyl surfaces or TerraCotta. The hard surface floors will not hide dirt and organic materials as the previous textile floor coverings did. Reportedly, the school system's Custodial Support Procedures document outlines the system's procedures and schedules for maintaining hard surface floors. These procedures should be followed with respect to maintenance of the new floors.

\section{Recommendation \#2: Limiting Noise}

Hard surface flooring will not absorb noise energy as readily as textile flooring. Desks, chairs, and other objects that are regularly moved across the floor should be fitted with felt pads on the bottom of their feet, or fitted with rolling castor wheels designed for hard surface floors.

\section{Recommendation \#3: Do Not Use Textile Floor Coverings}

We do not recommend using textile floor coverings of any size (except track-off mats) in any area of the building where the floor type is concrete slab-on-grade.

\section{Recommendation \#4: Use Track-Off Mats and Limit Exiting to Control Dirt Levels} We recommend that the floors at all main entries be covered with track-off mats. A recommended track-off mat system should be designed to remove moisture from the bottom of footwear, to remove course material, and to remove fine material from the bottom of shoes. The mats should extend beyond the entry door a sufficient distance to allow adequate moisture and debris removal. Additionally, we do not recommend that the 


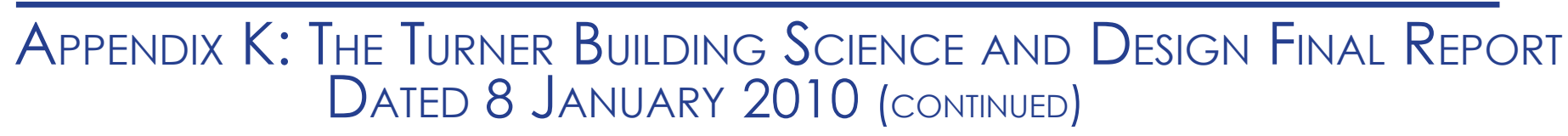

classroom fire-exits be used as normal entry doors. All entry into the building should be through main entries fitted with the proper track-off mats. Some doors in some classrooms, such as the kindergarten area, may need to be fitted with track-off mats to allow access to the gated play area.

\section{Recommendation \#5: Occupied Temperature Set Points}

The HVAC systems have been commissioned, which included calibration of all sensors and is capable of maintaining set point conditions. We recommend set points of $70^{\circ} \mathrm{F}$ heating and $74^{\circ} \mathrm{F}$ cooling in accordance with recommendations made by the commissioning agent. However, the sensors that control the systems are zoned such that two rooms are served by a single heat pump with a thermostat placed in one of the two spaces. The sensors may need to be adjusted to different values than recommended in order to maintain the desired temperatures (between 70 and 74 degrees) in the spaces. Additionally, some sensors may not properly sense the space temperature due to its location; therefore, these too may need an adjustment at the thermostat dial that is beyond the recommended range.

\section{Recommendation \#6: Occupied Cycles}

We recommend, in the interest of energy savings, that the school be operated with an occupied cycle and an unoccupied cycle. The occupied cycle should include continuous fan operation of the heat pumps and outdoor air systems, and occupied temperatures as recommended above in Recommendation \#5. The unoccupied cycle should include a 5 degree offset from the occupied temperature set points, and the heat pump fans should run only on demand for heating or cooling. The outdoor air systems should be off and the associated OA dampers closed.

\section{Recommendation \#7: Additional Monitoring of Space Temperatures, Humidity, and Ventilation Rates}

We recommend additional monitoring of space conditions throughout the remaining heating season of this year, and the upcoming cooling season, to verify that the HVAC systems and controls are operating properly, and that the systems have the capacity to maintain space conditions at the recommended set point conditions.

\section{Recommendation \#8: Additional Engineering Services to Increase Energy Efficiency The thermal images taken of the buildings perimeter walls indicated that the door, windows, and the base of the walls were additional sources of heat leaks. Collectively these leaks may account for another large portion of the total air leakage measured by the blower door tests. We recommend additional evaluation and possible corrective work to further limit the heat loss and air leaks. Limiting the air leaks may have an additional favorable impact on the unplanned air flows that occur during the unoccupied cycles. Limiting air leaks at doors may be as simple as adding commercial grade weather-stripping to the perimeter of doors.}




\section{Appendix L: The Turner Building Science and Design Final Report Pictures}

Shell Pressure Monitoring

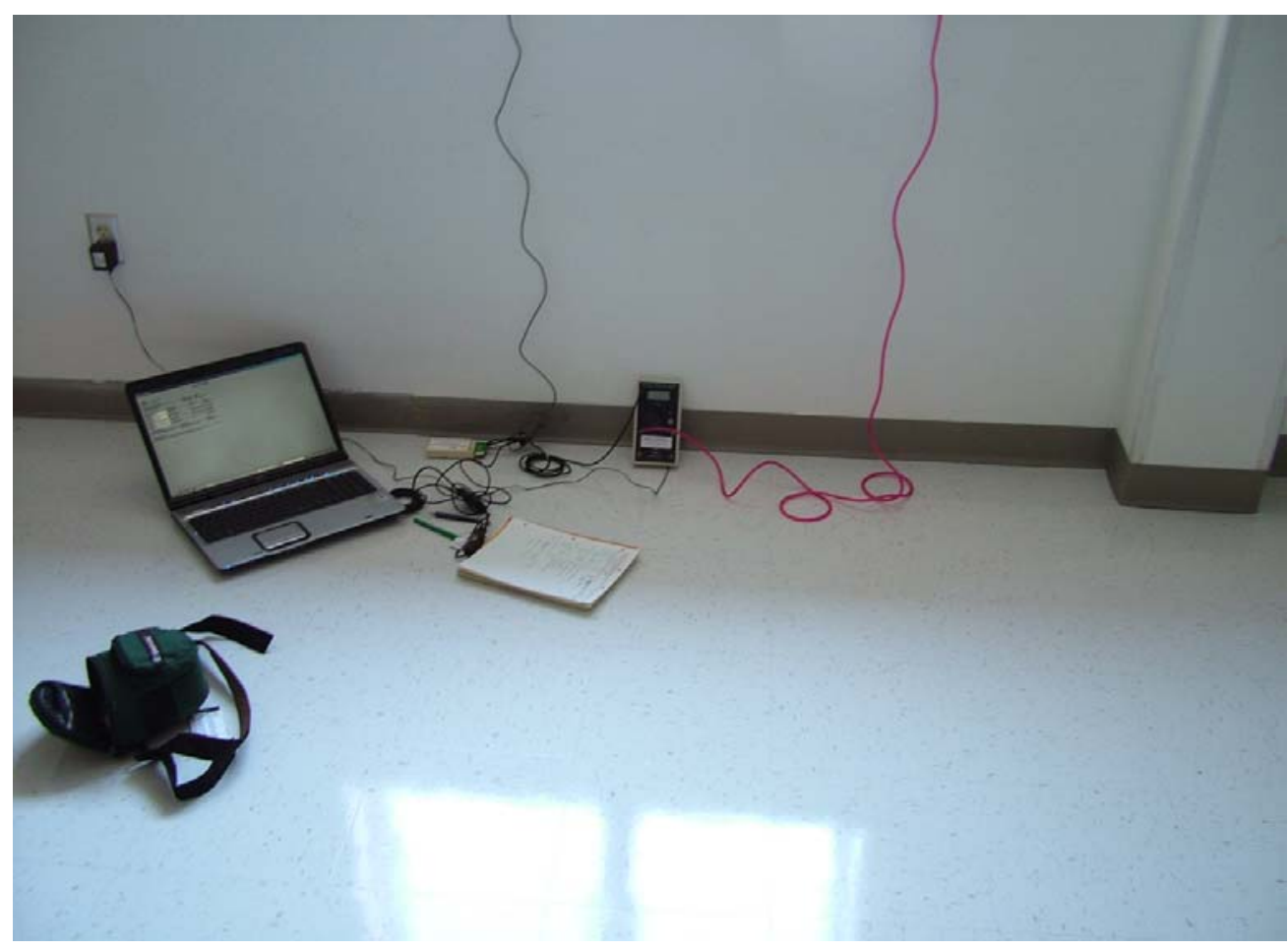

Temperature \&, Humidity Monitoring

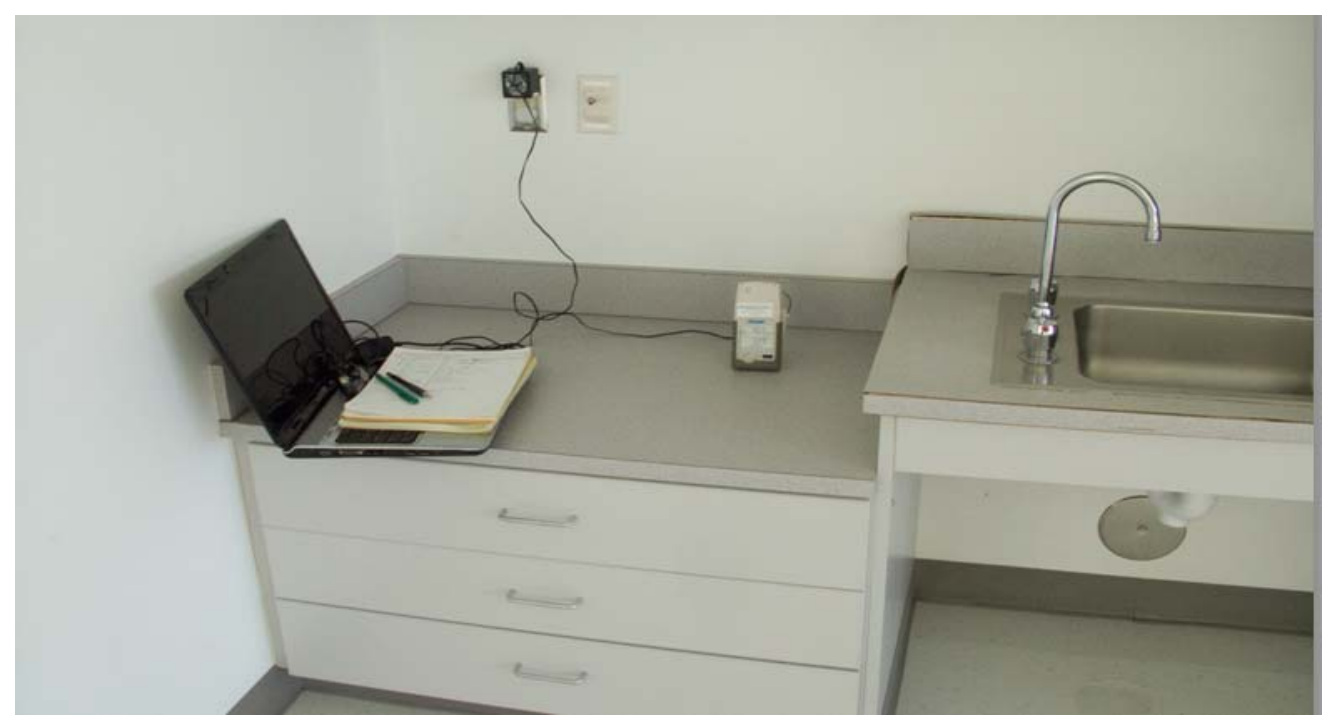




\section{Appendix L: The Turner Building Science and Design Final Report Pictures (CONTINUED)}

\section{Blower Door Testing}

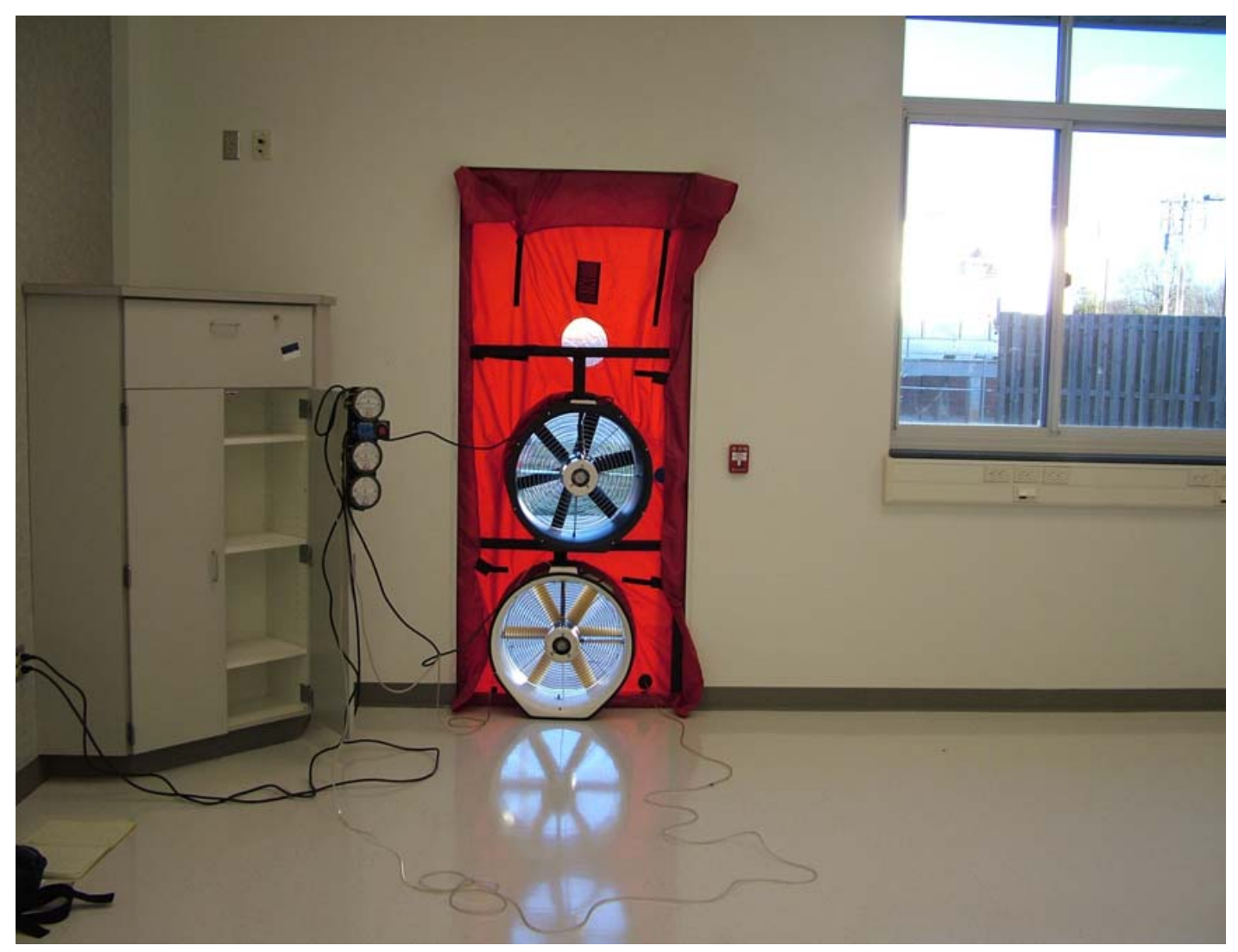

Typical Vinyl Wallpaper Observation

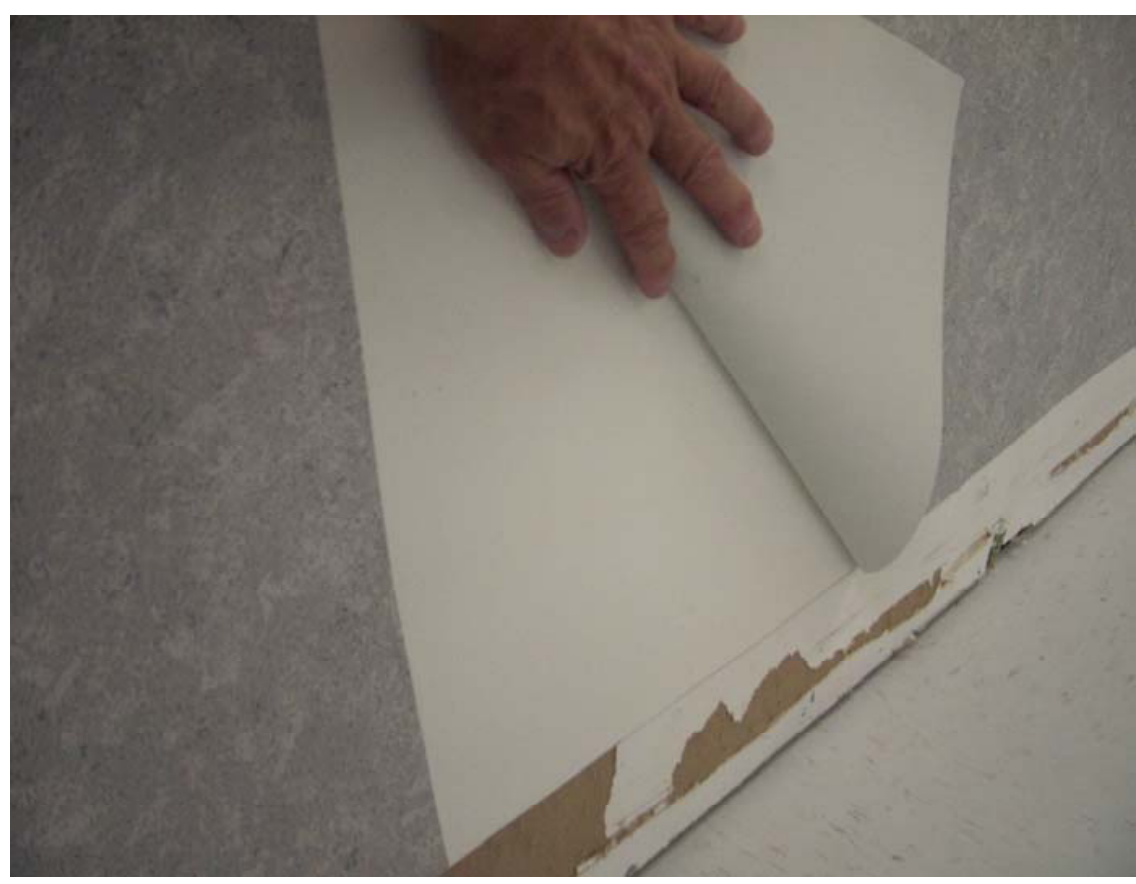




\section{Typical Wall Observation}

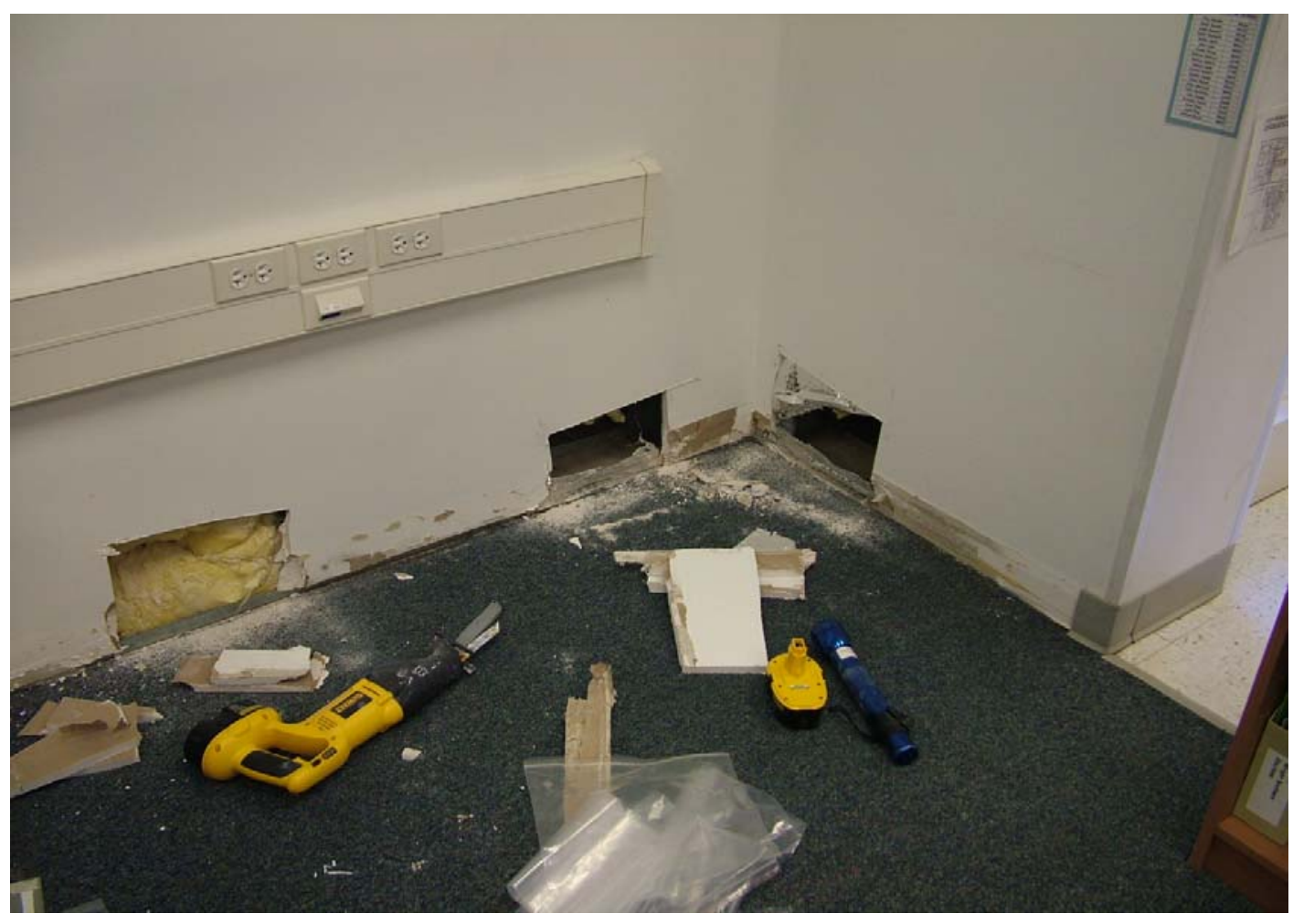

Typical Classroom Containment \& Isolation

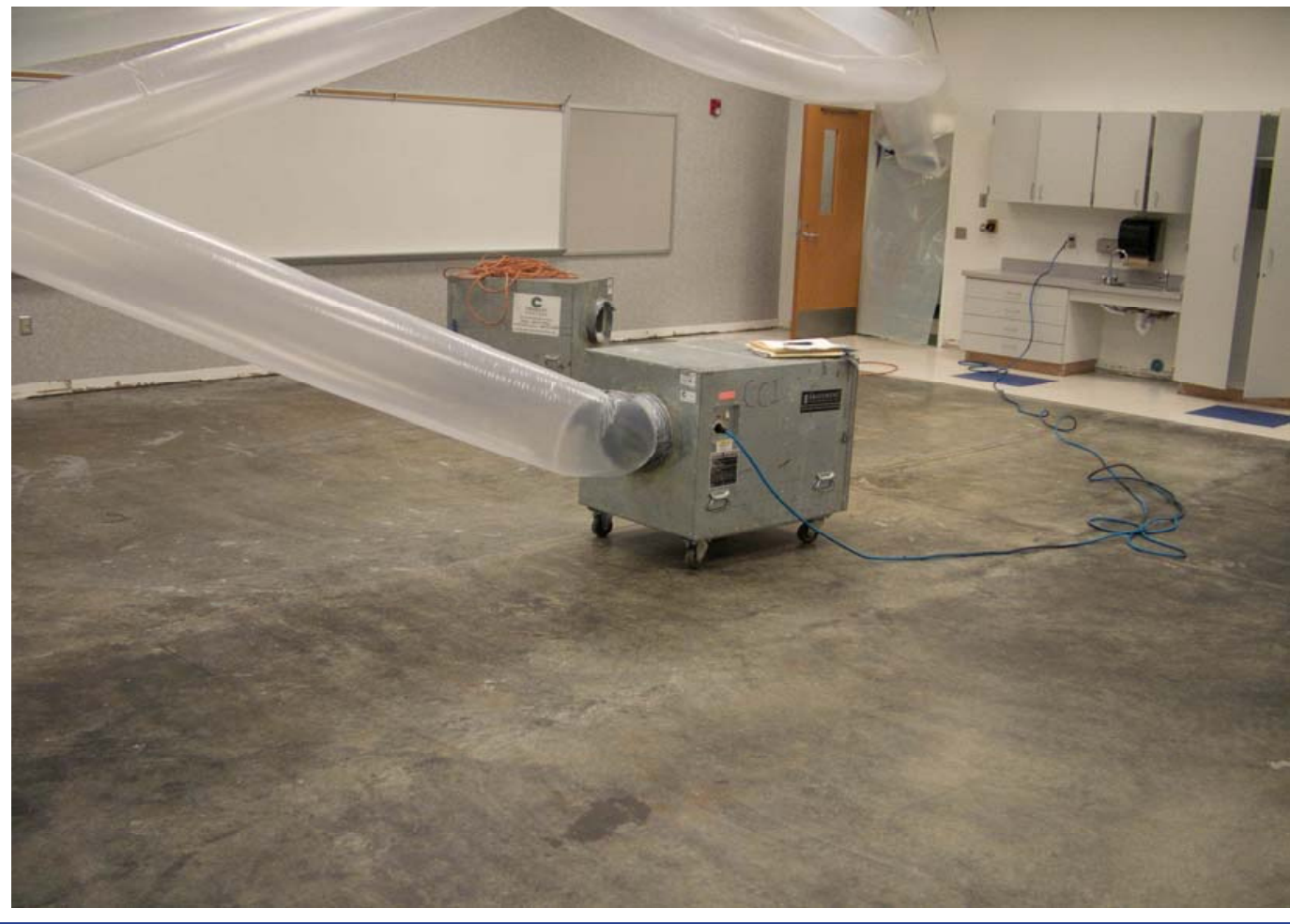




\section{Appendix L: The Turner Building Science and Design Final Report Pictures (CONTINUED)}

Typical Poor Filter Installation

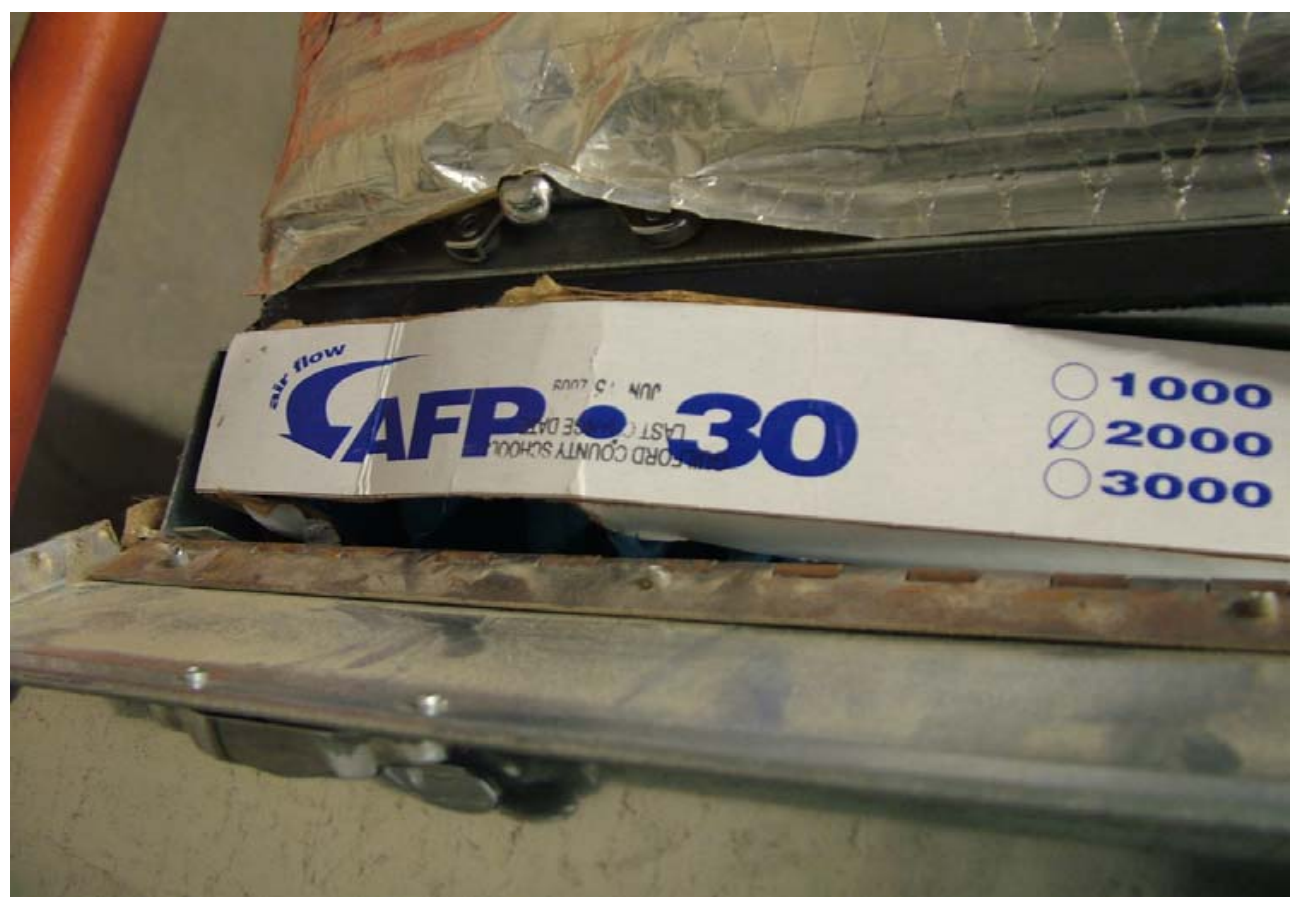

Dirty Coil

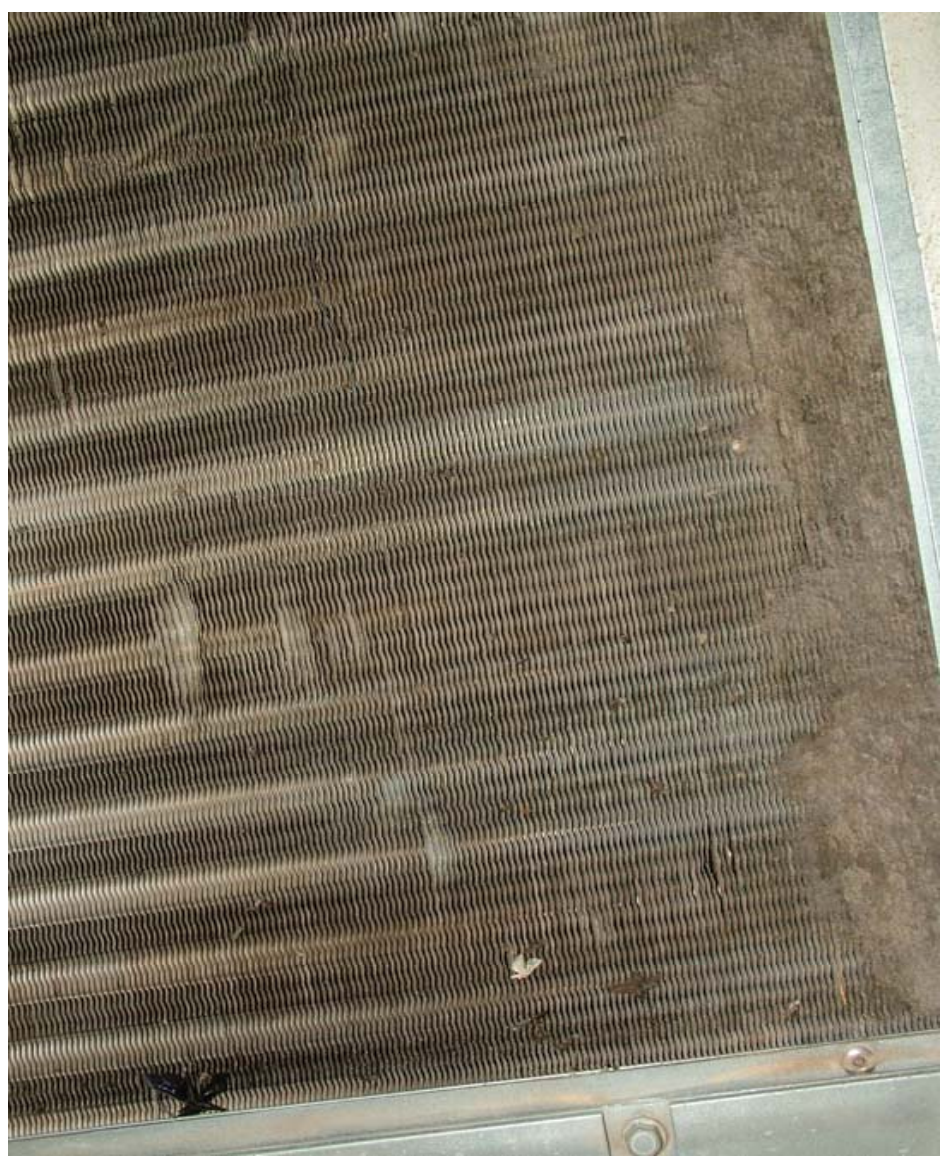


Appendix L: The Turner Building Science and Design Final Report Pictures (CONTINUED)

Typical Marked OA Balancing Damper

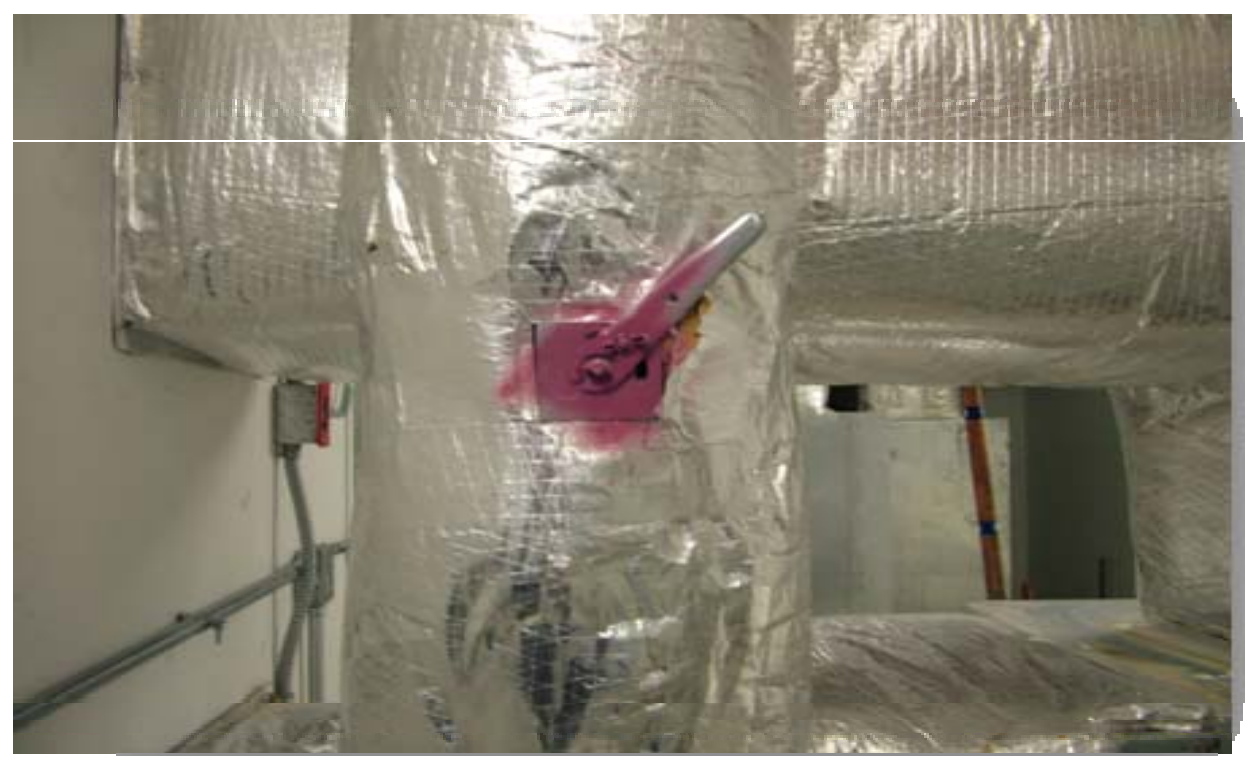

Clean Coil

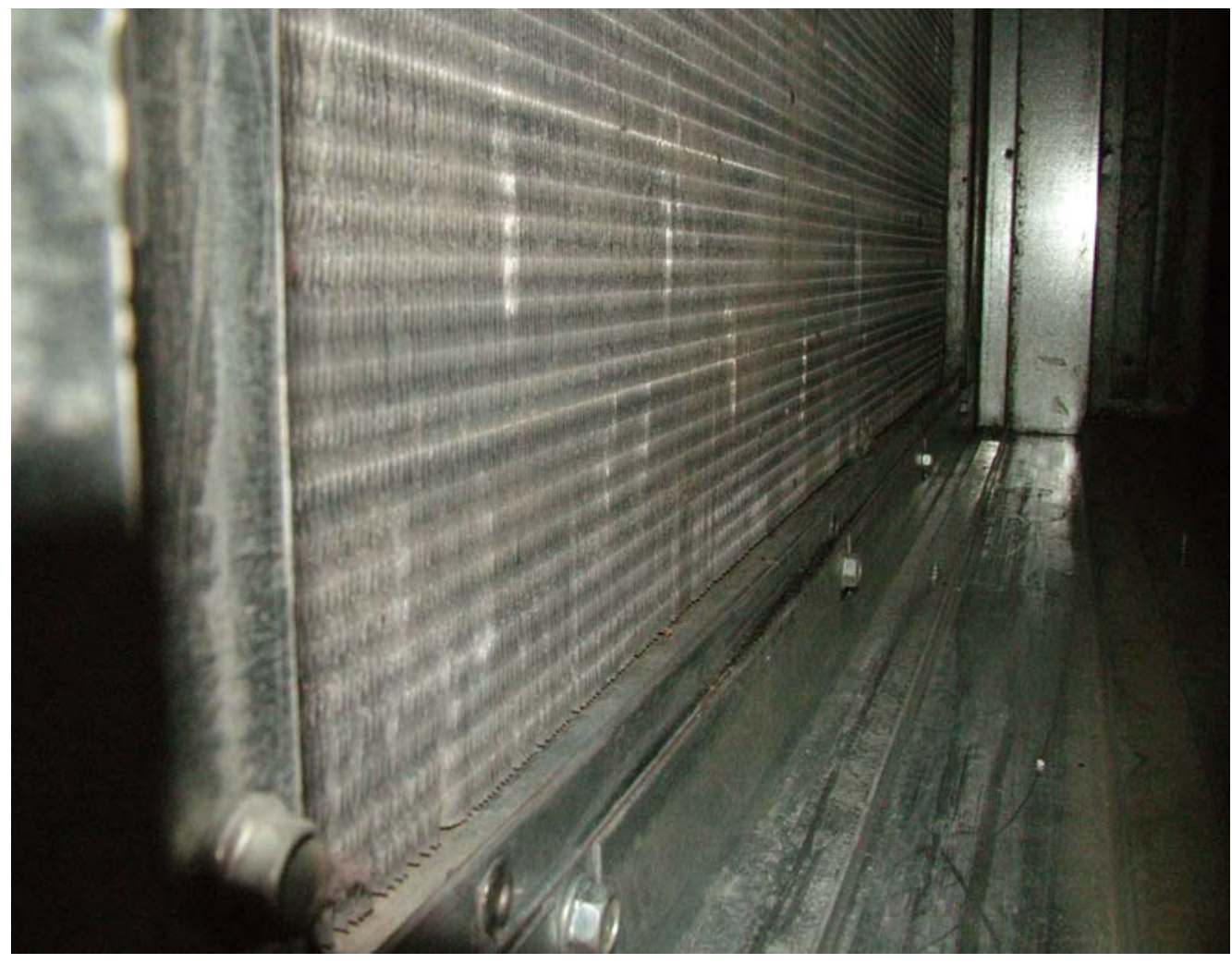




\section{Appendix L: The Turner Building Science and Design Final Report Pictures (CONTINUED)}

Media Center Clearstory

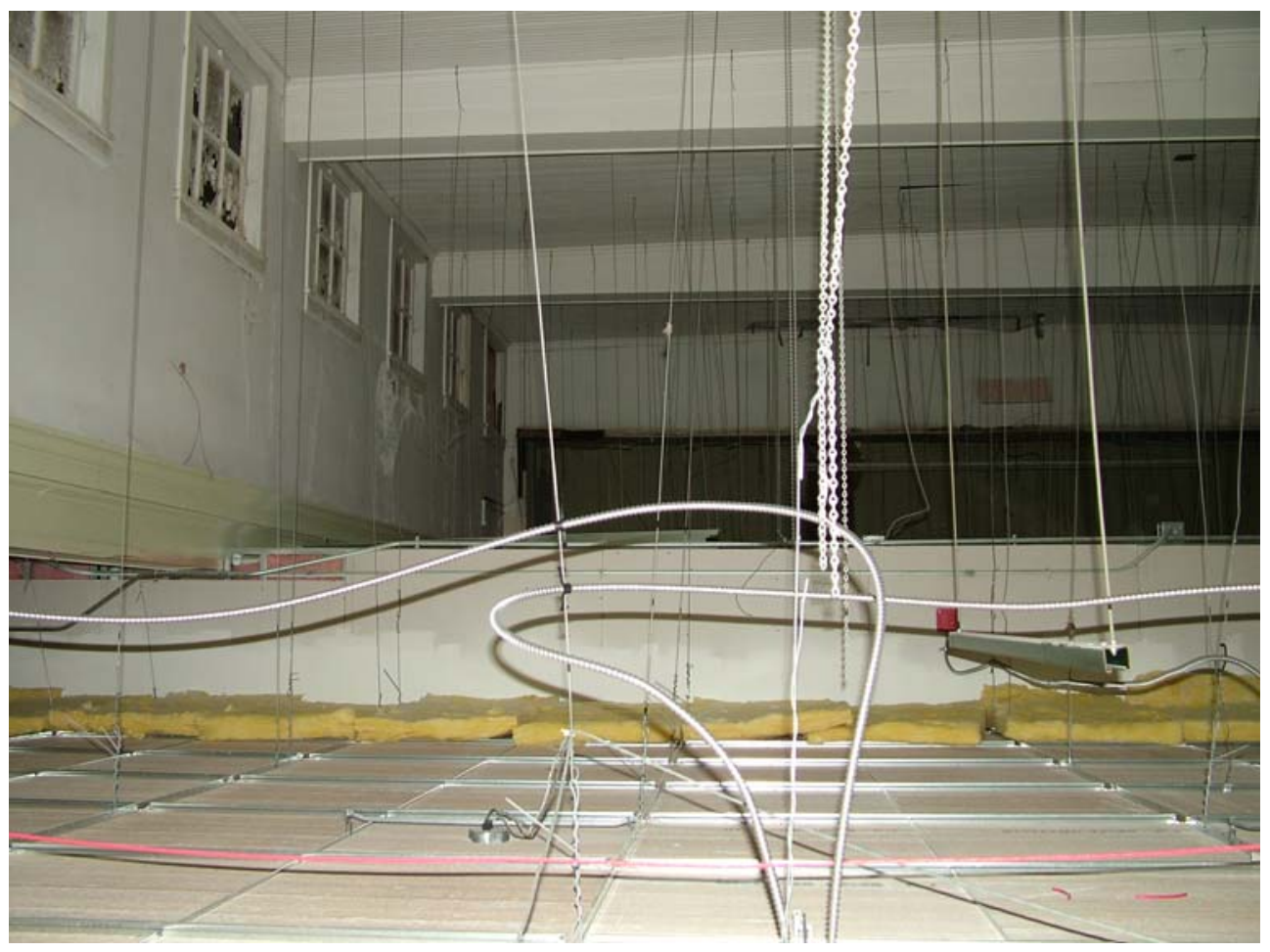

Media Center Clearstory

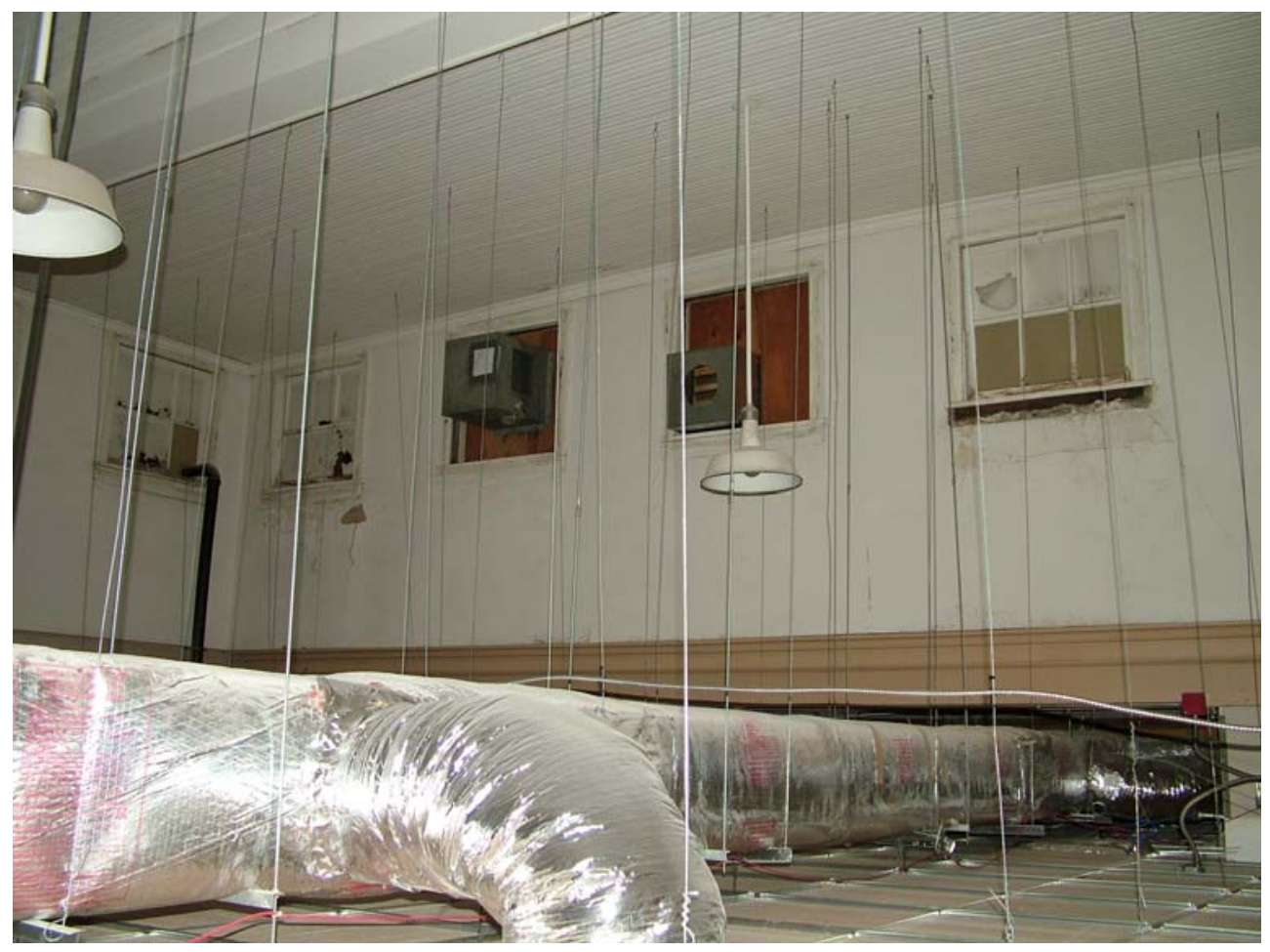


Appendix L: The Turner Building Science and Design Final Report Pictures (CONTINUED)

Media Center Clearstory

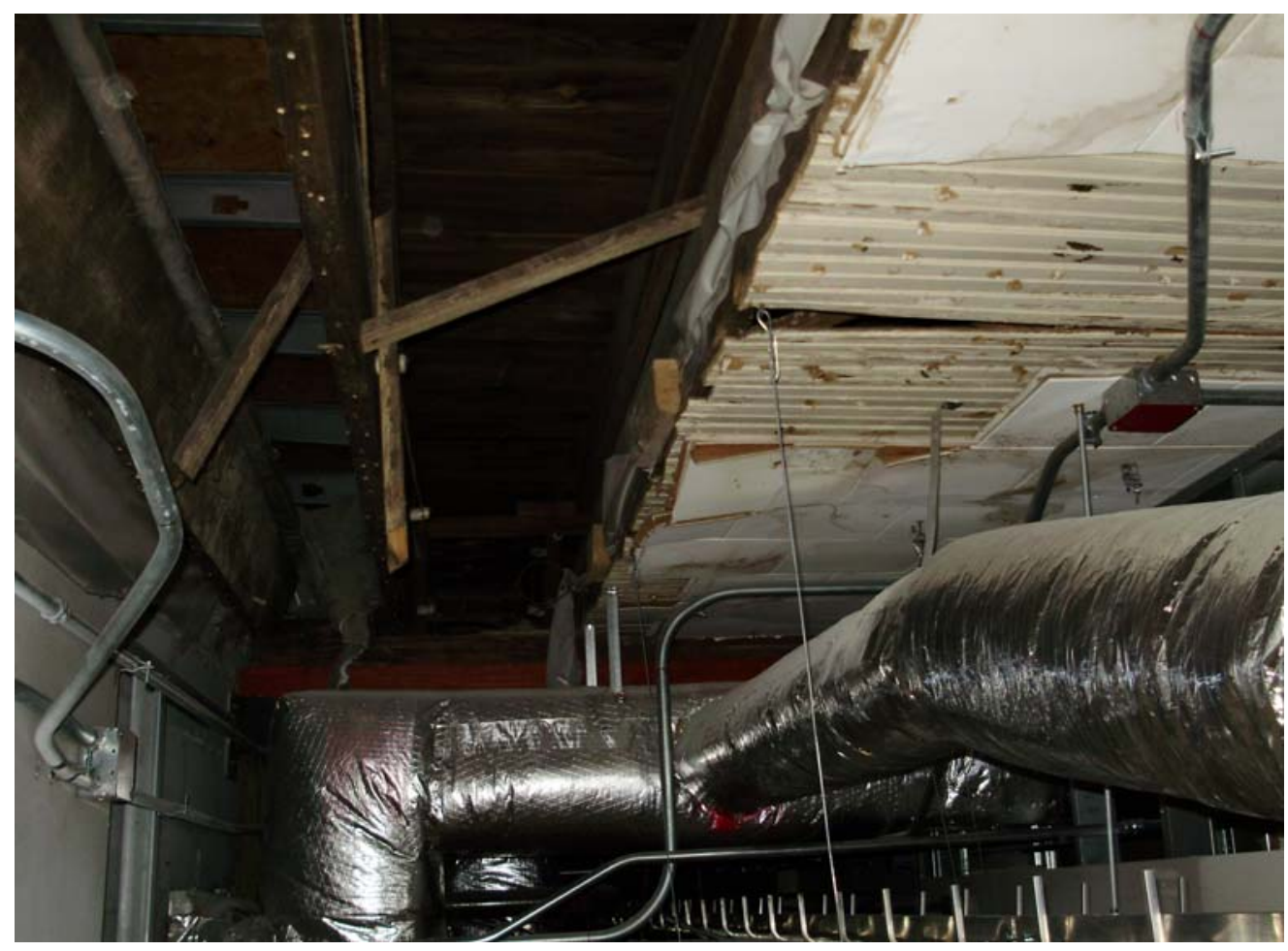

Open Plumbing Vent Lines

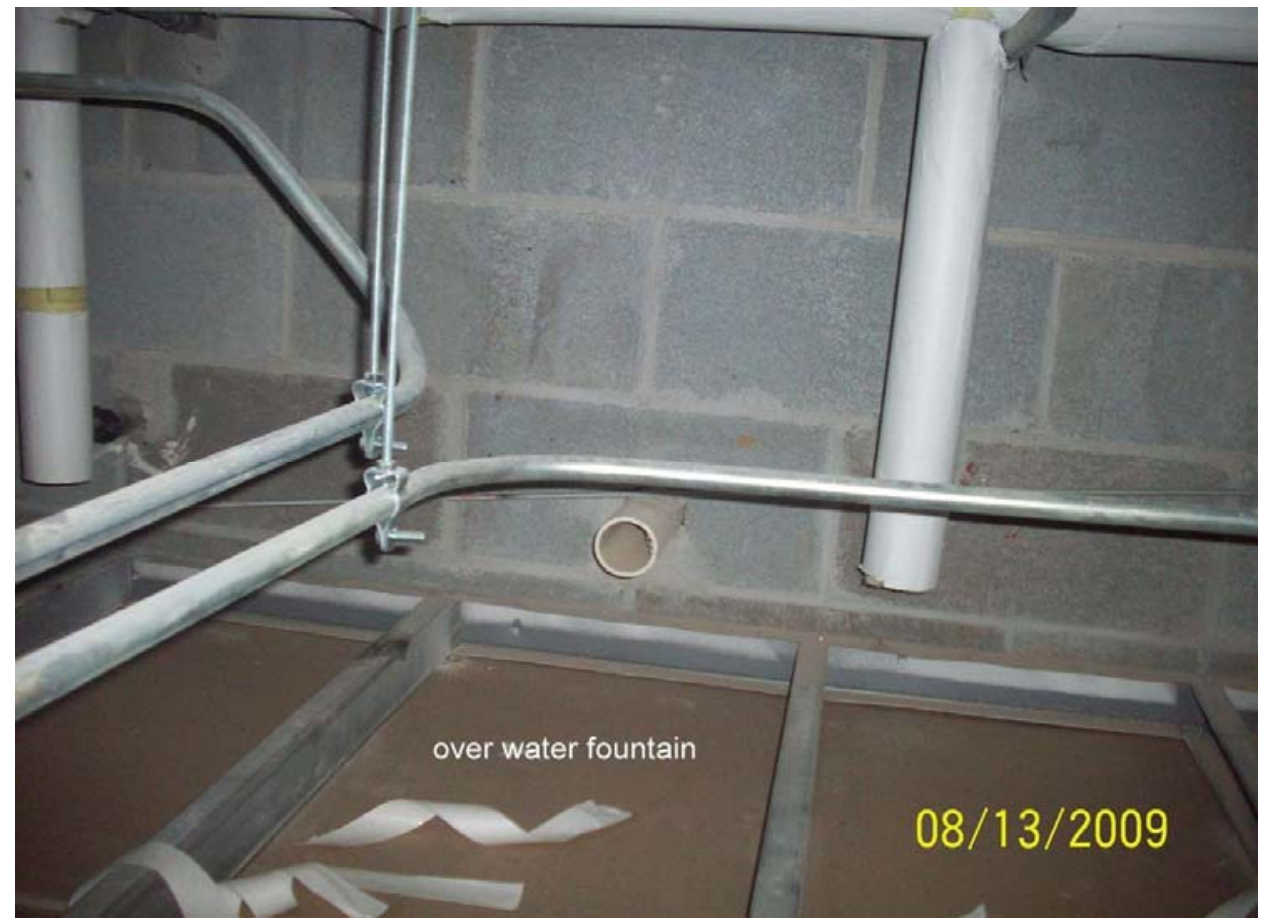




\title{
Appendix L: The Turner Building Science and Design Final Report Pictures (CONTINUED)
}

\author{
Vent Through Roof And OA Inlet
}

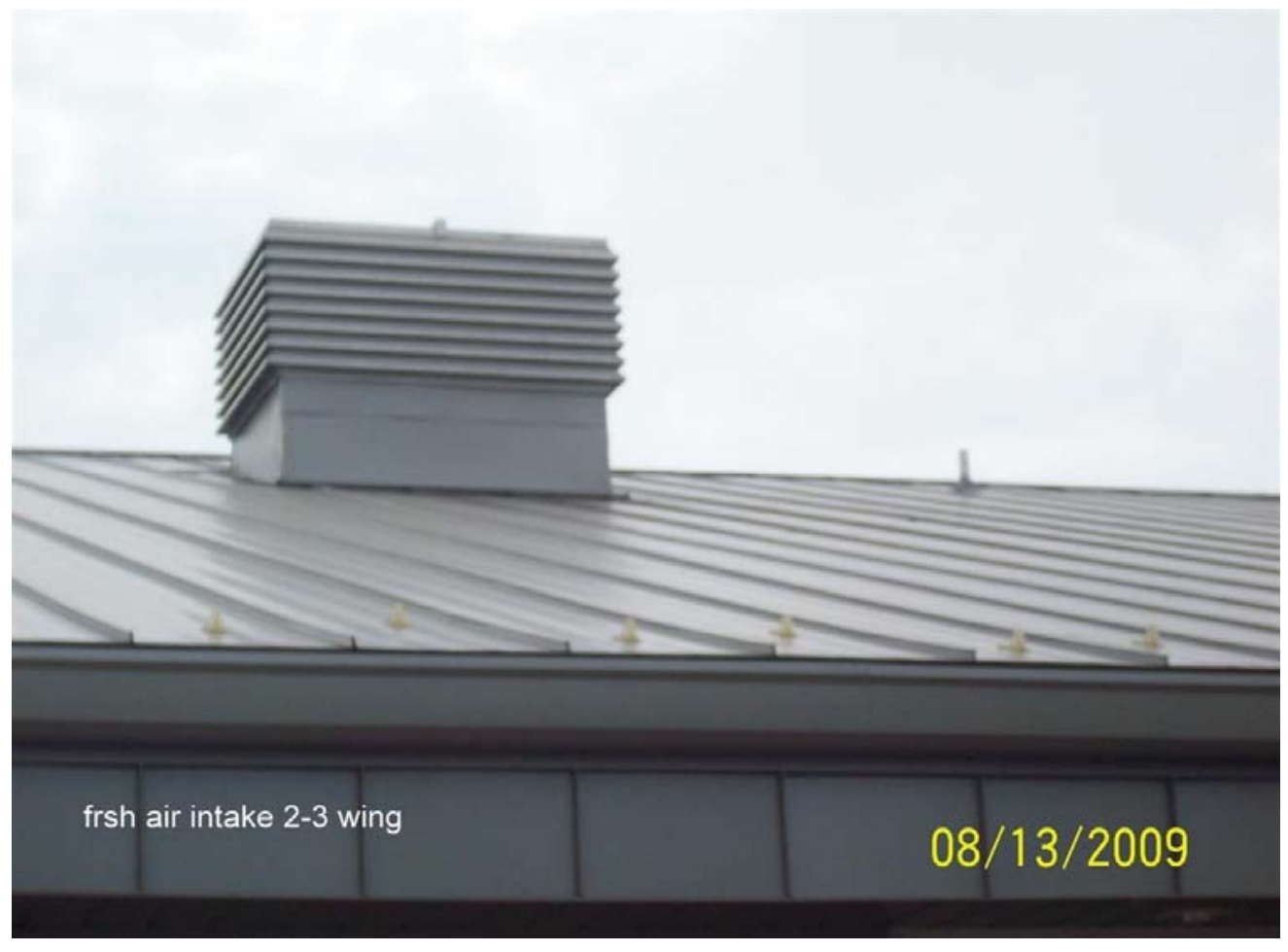

Crawl Space Vapor Barrier

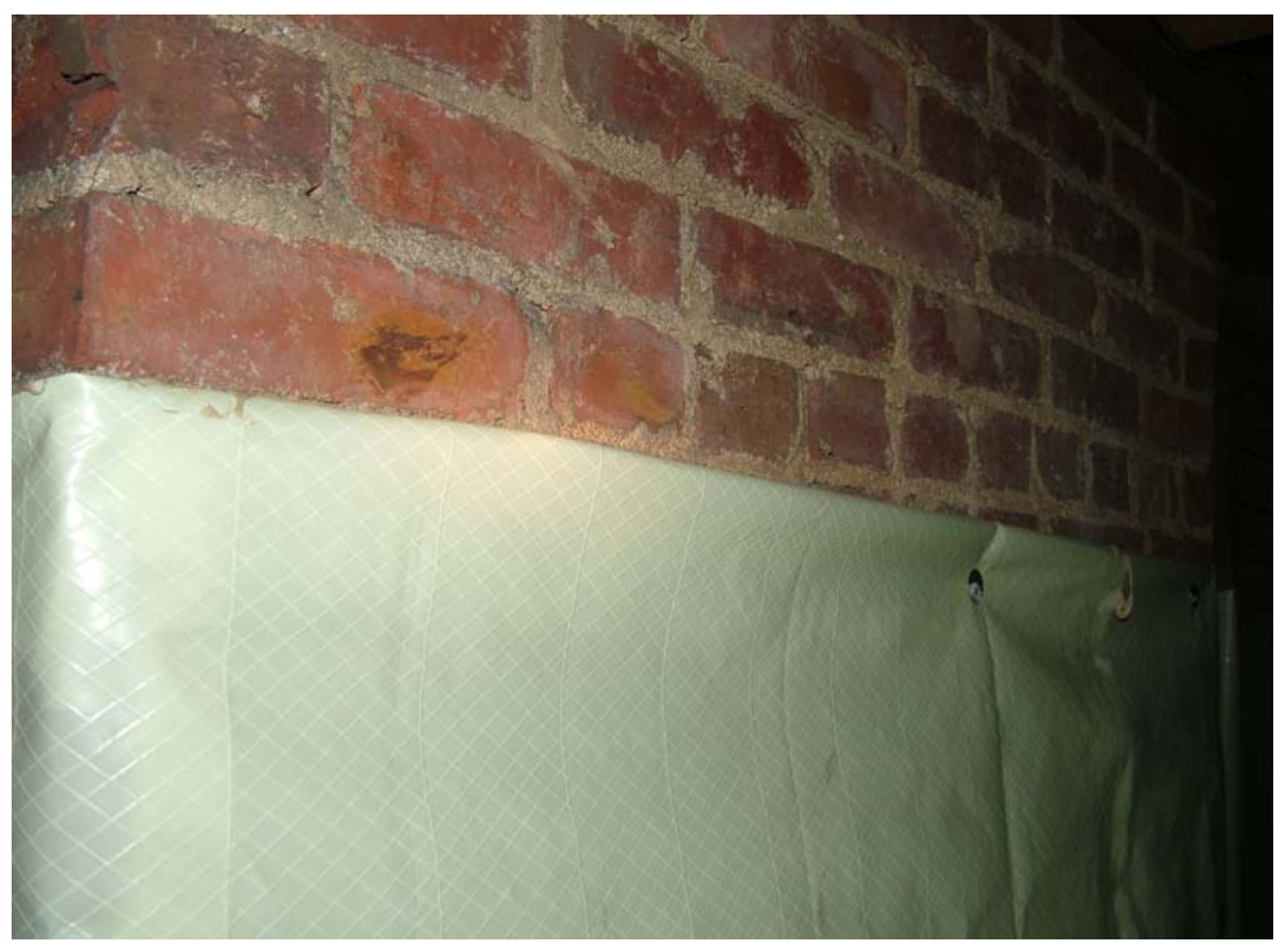




\section{Appendix L: The Turner Building Science and Design Final Report Pictures (CONTINUED)}

\section{Crawl Space Vapor Barrier}

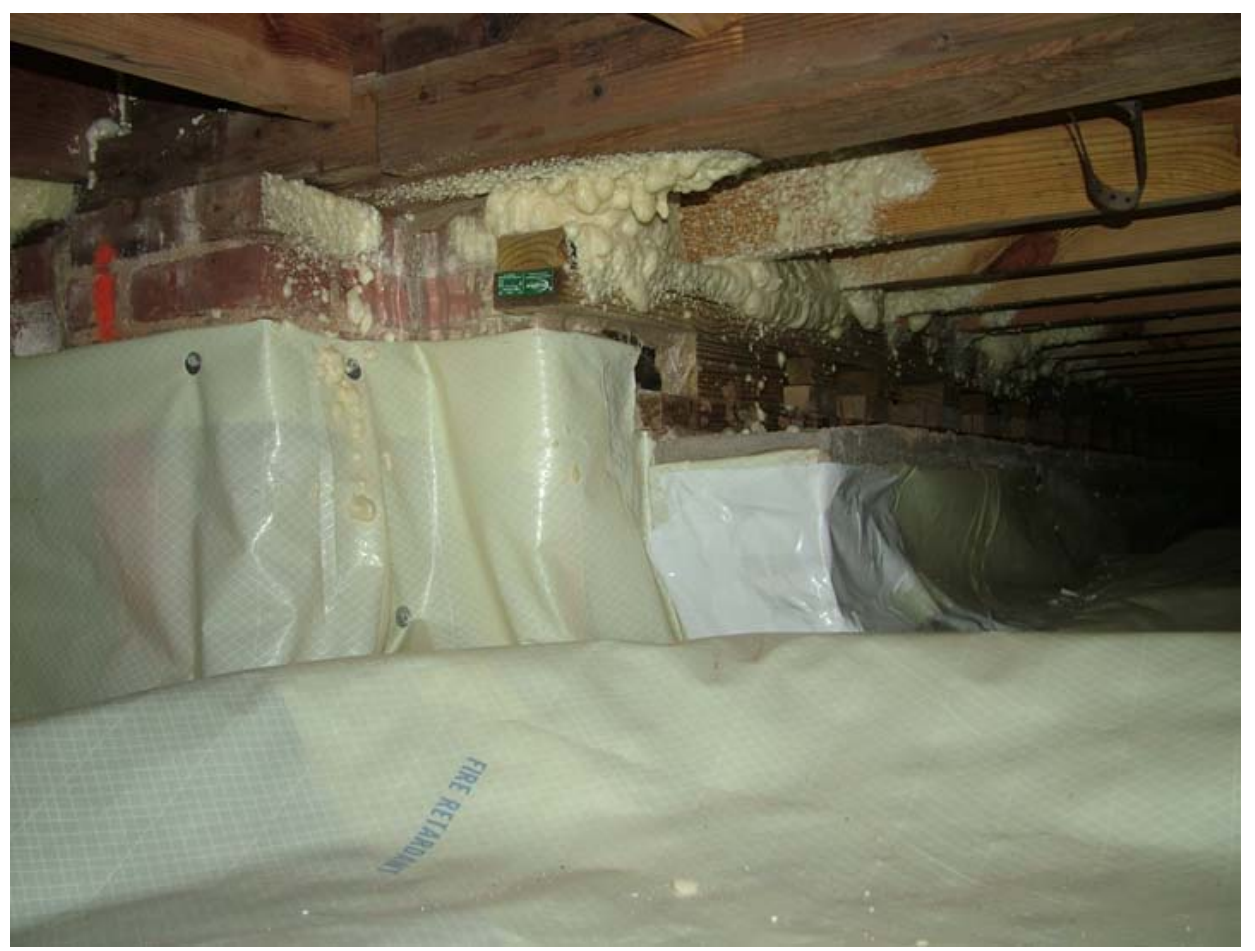

Crawl Space Vapor Barrier

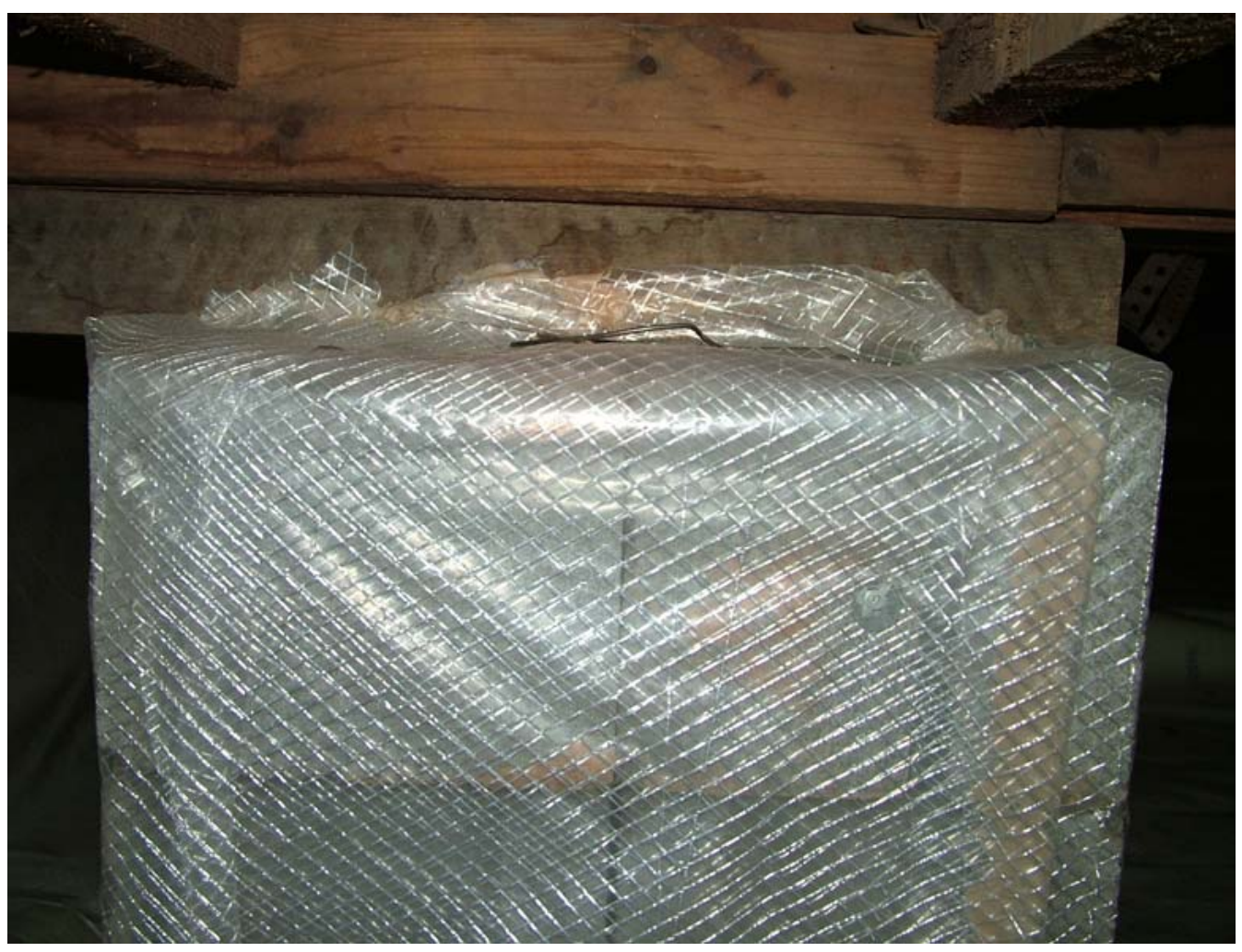




\section{Appendix L: The Turner Building Science and Design Final Report Pictures (CONTINUED)}

\section{Crawl Space Vapor Barrier}

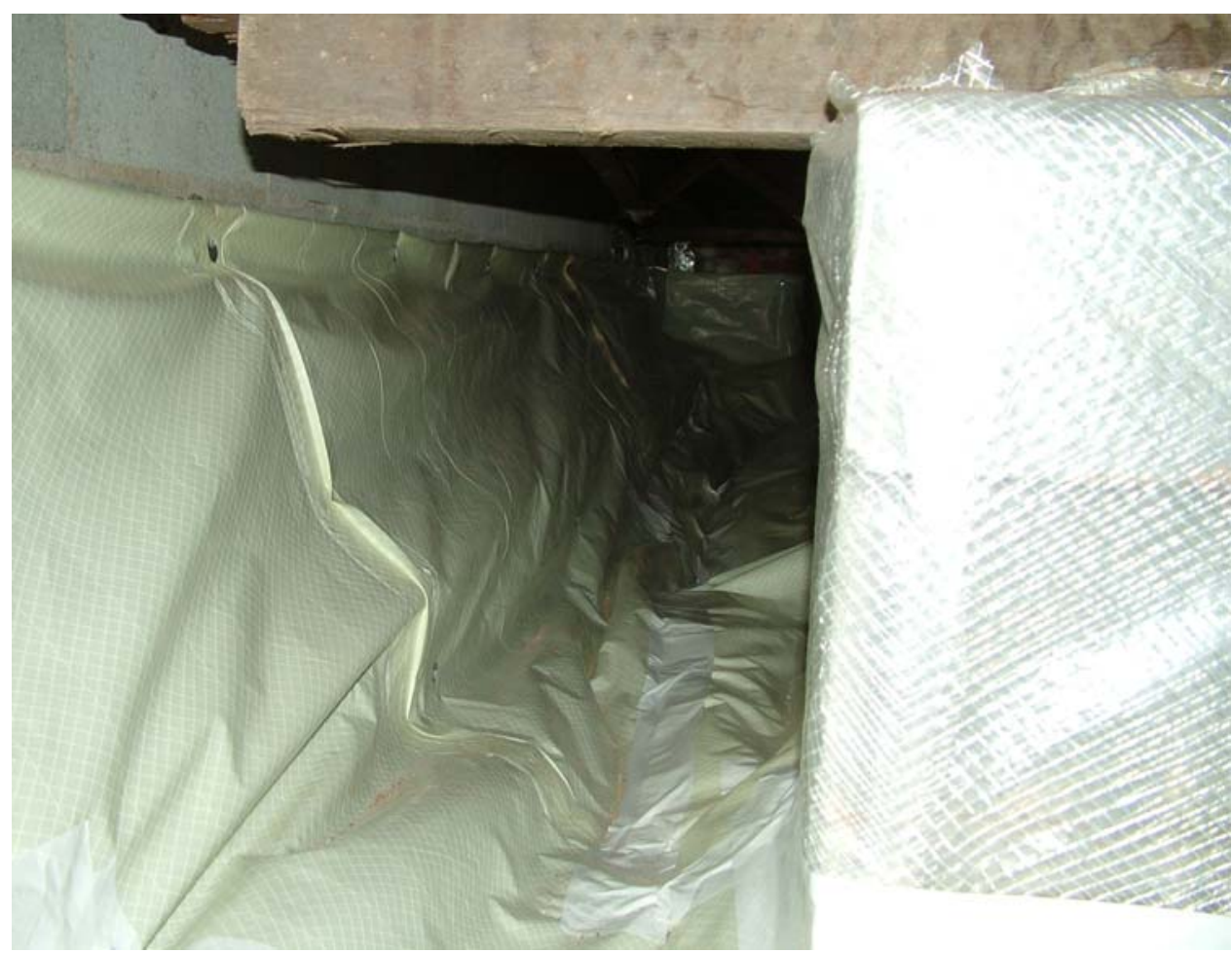

Crawl Space Vapor Barrier

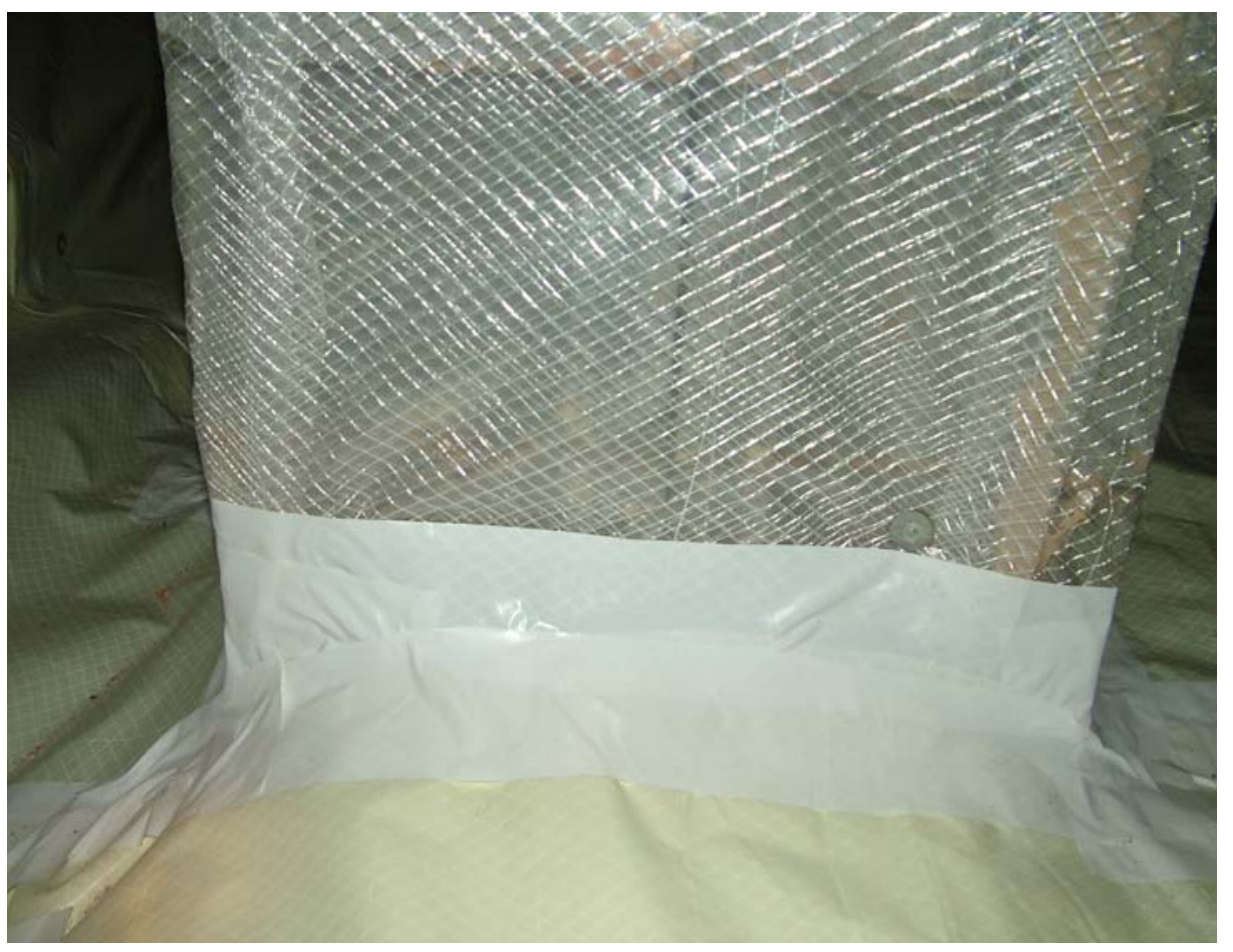


Appendix L: The Turner Building Science and Design Final Report Pictures (CONTINUED)

\section{Crawl Space Vapor Barrier}

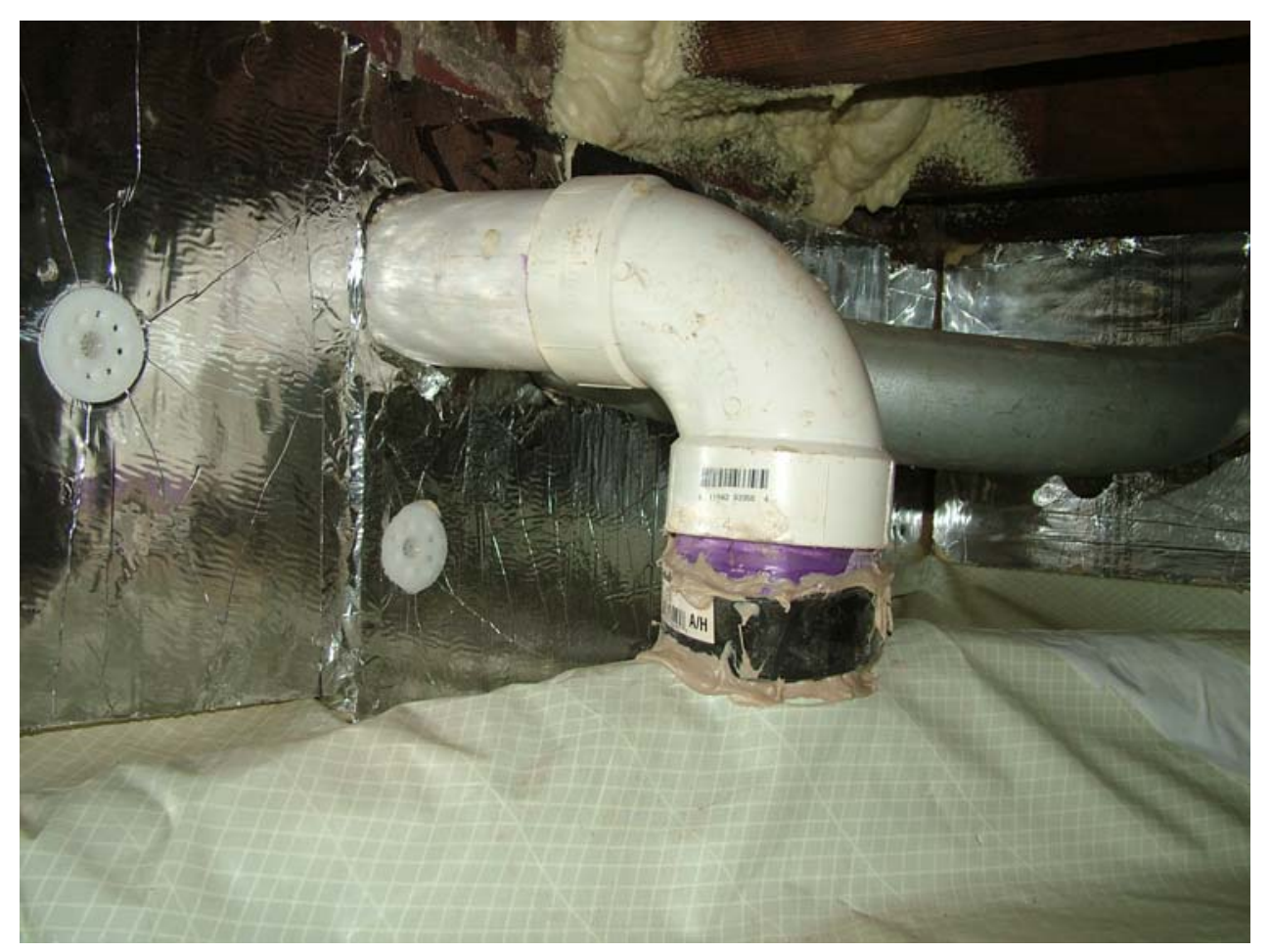

As Found Roof Flashing

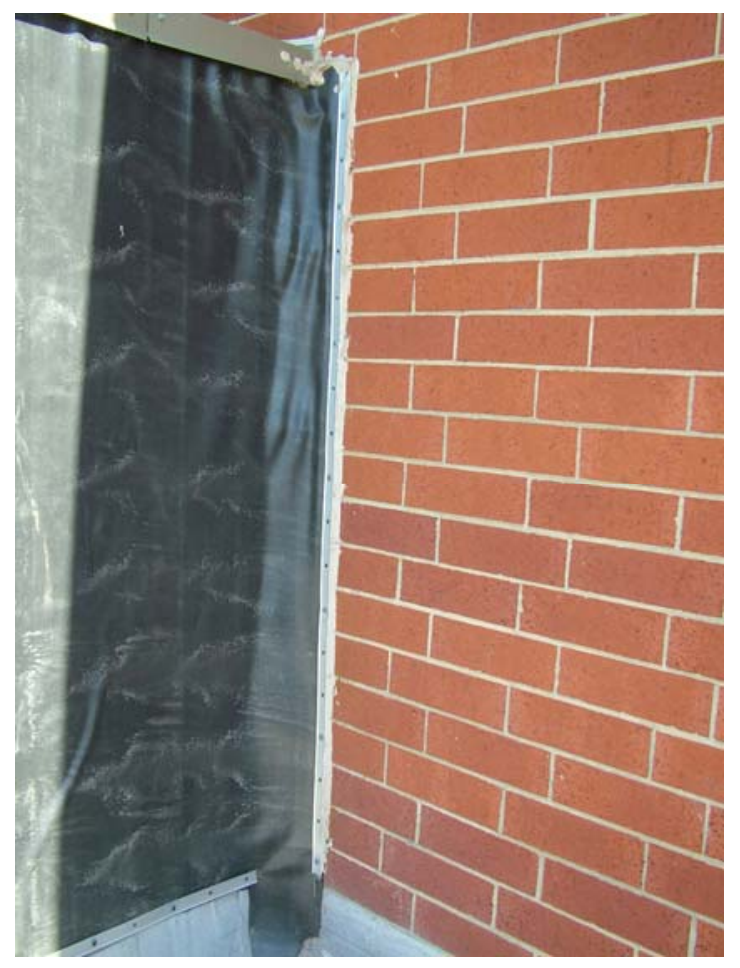




\section{Appendix L: The Turner Building Science and Design Final Report Pictures (CONTINUED)}

\section{As Found Roof Flashing}

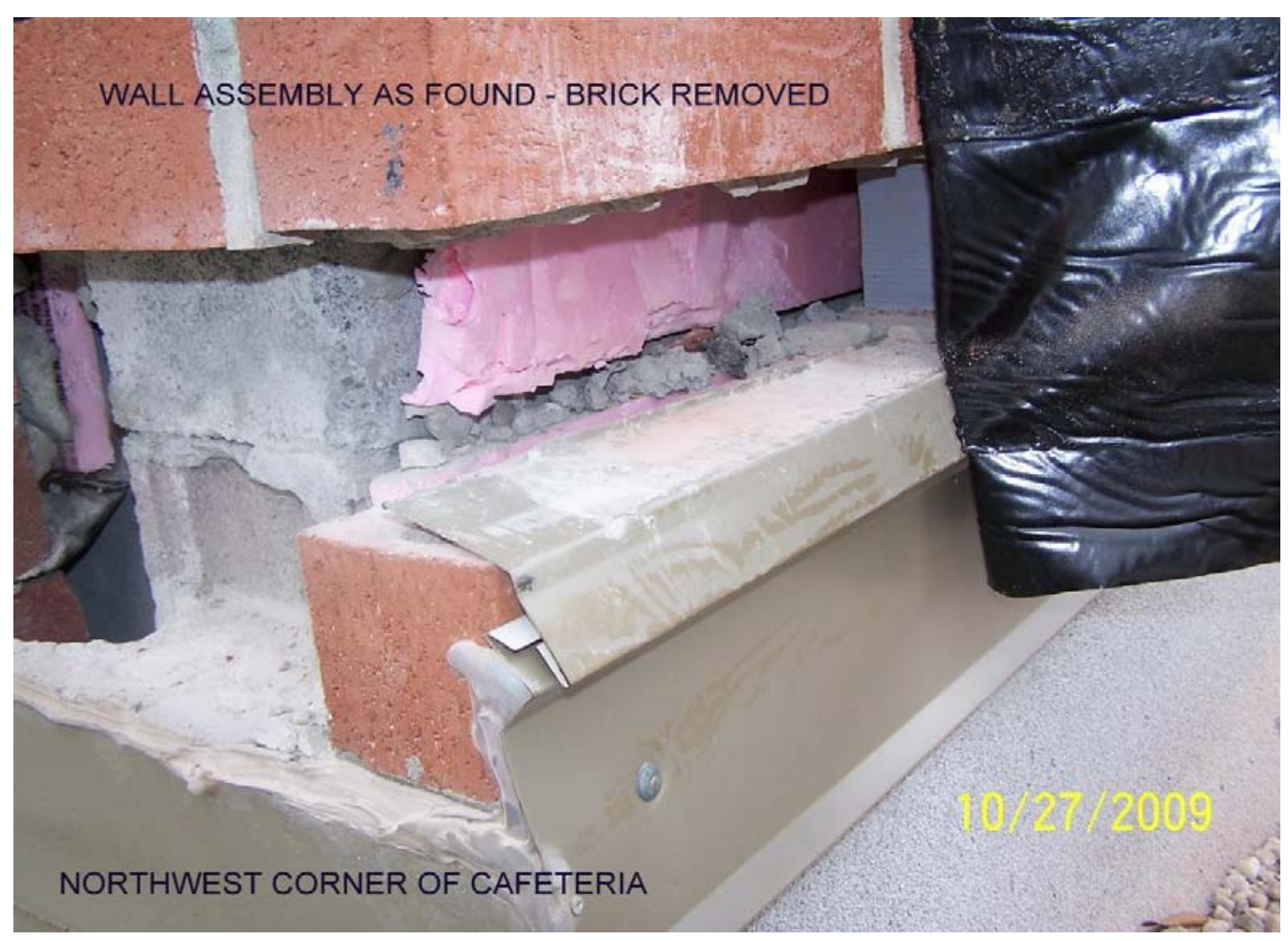

As Found Roof Flashing

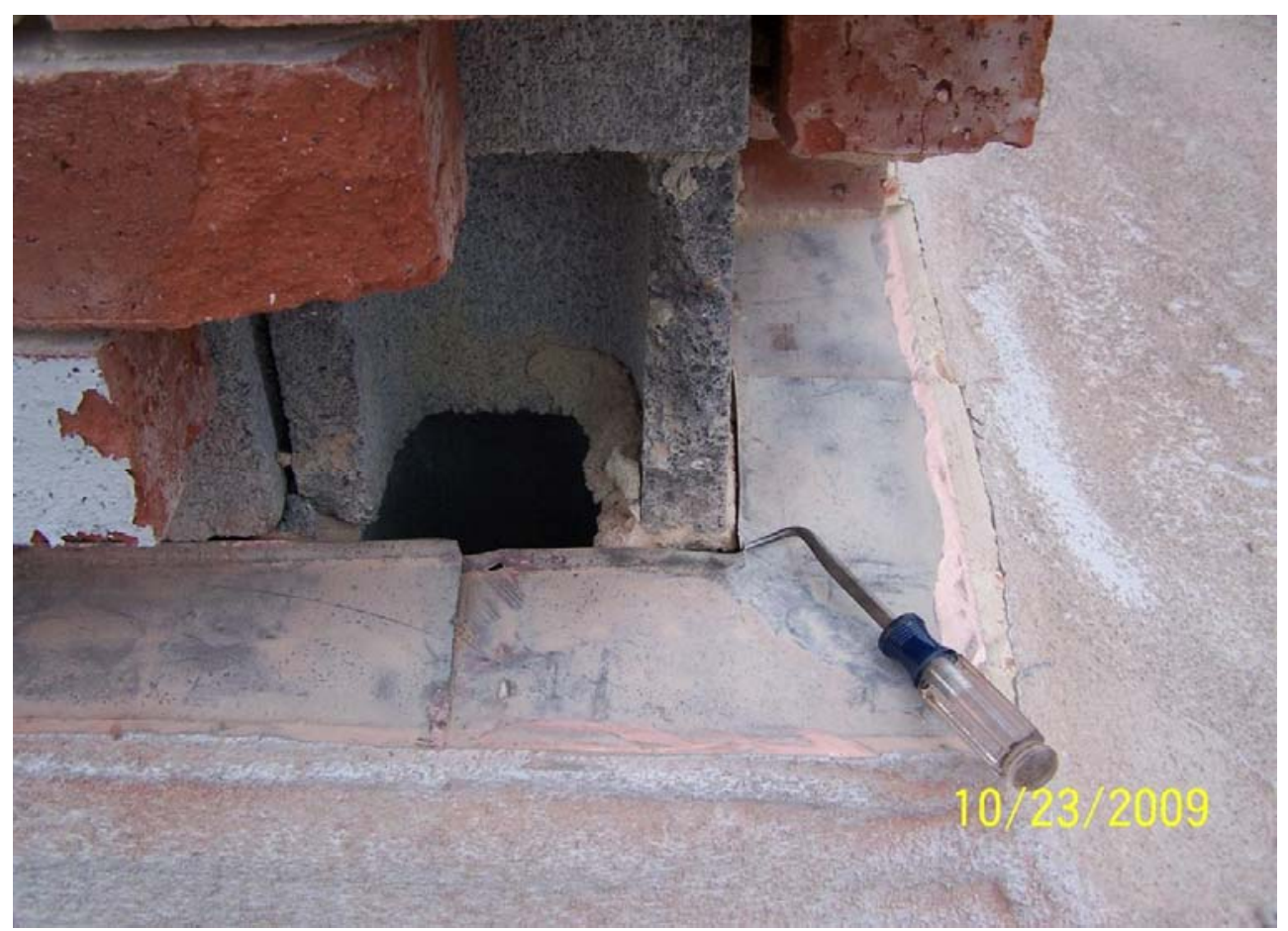




\section{Appendix L: The Turner Building Science and Design Final Report Pictures (CONTINUED)}

\section{Visual signs of Site runoff}

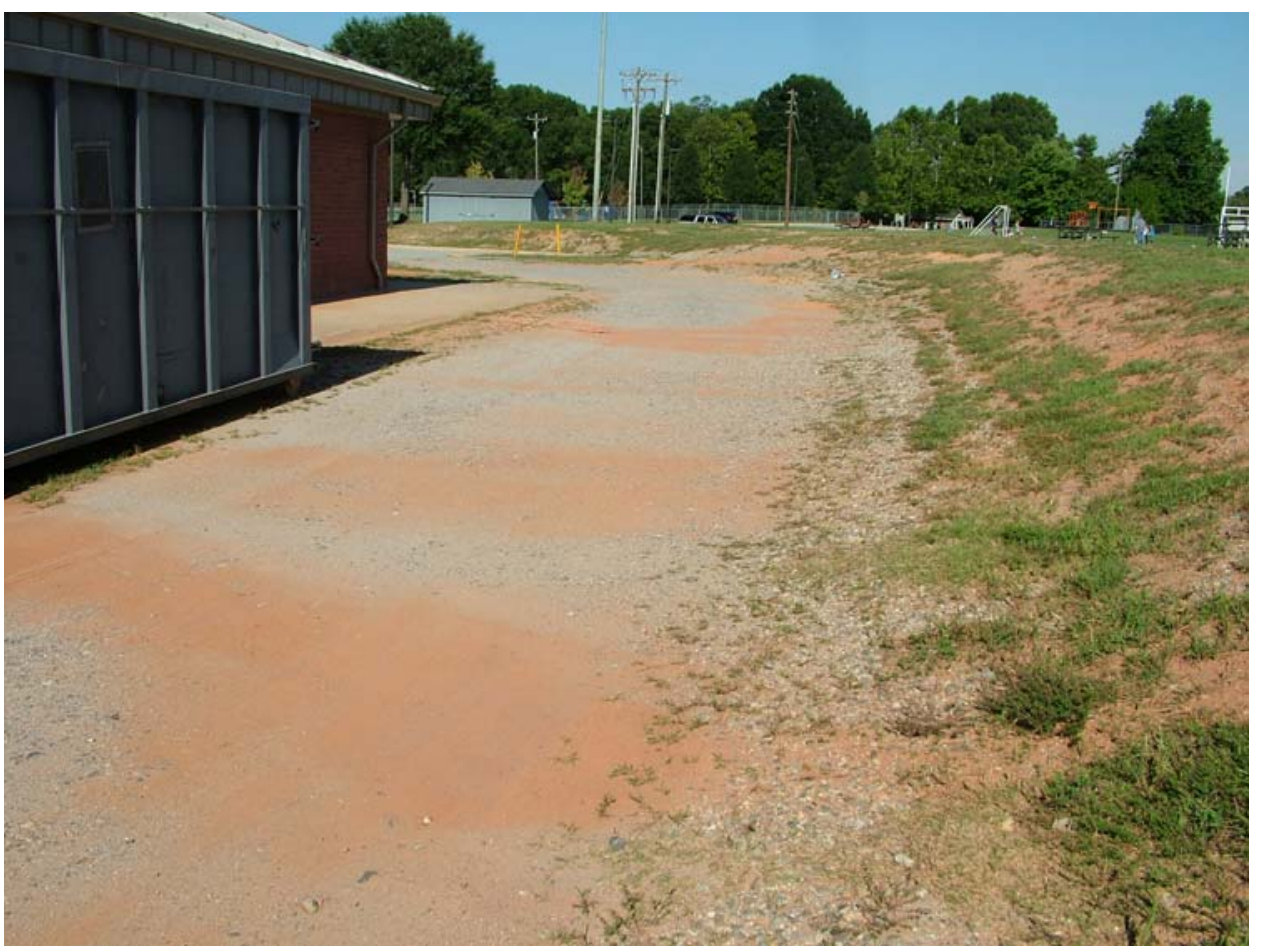

Visual signs of Site runoff

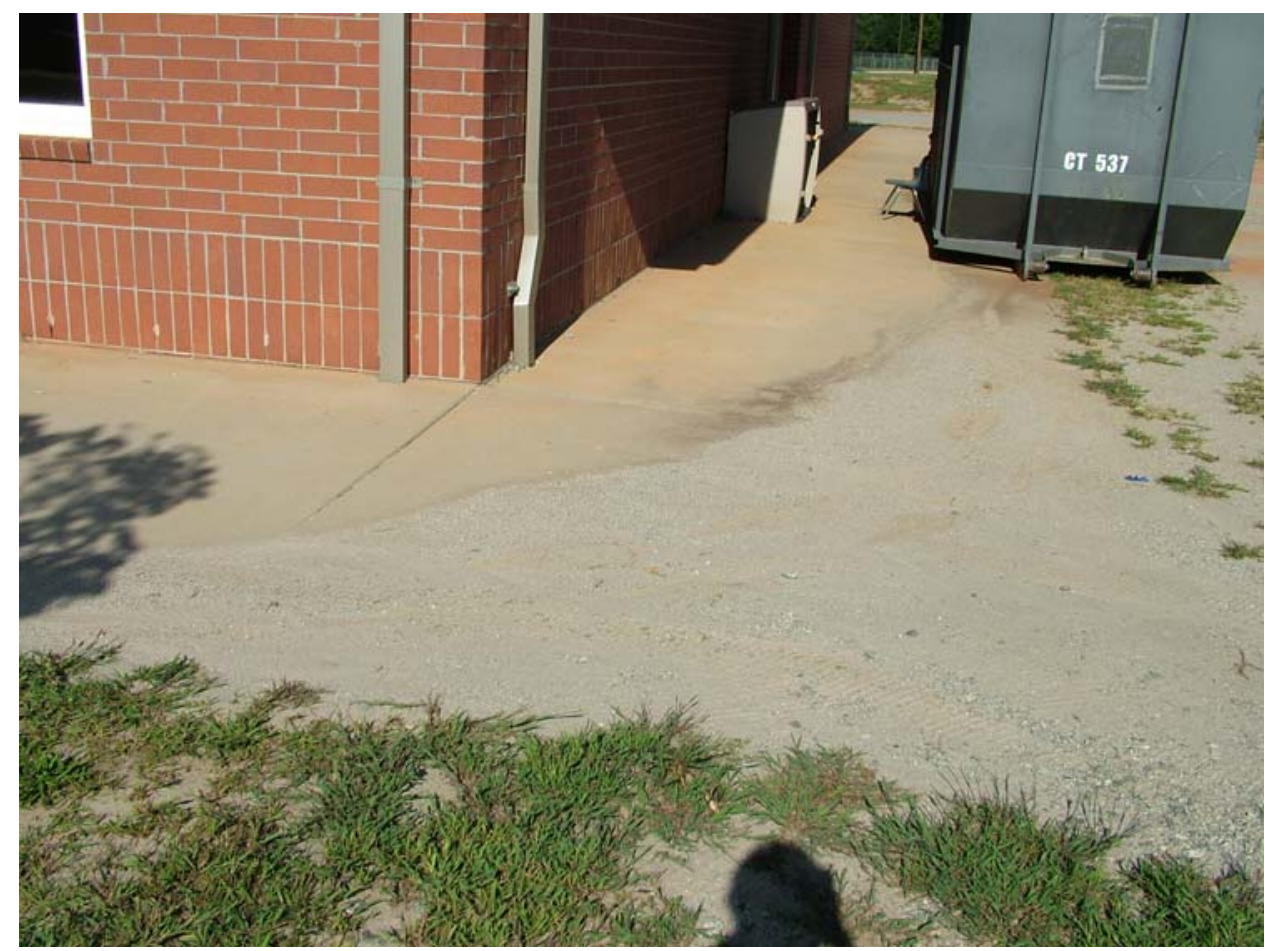




\section{Appendix L: The Turner Building Science and Design Final Report Pictures (CONTINUED)}

\section{Visual signs of Site runoff}

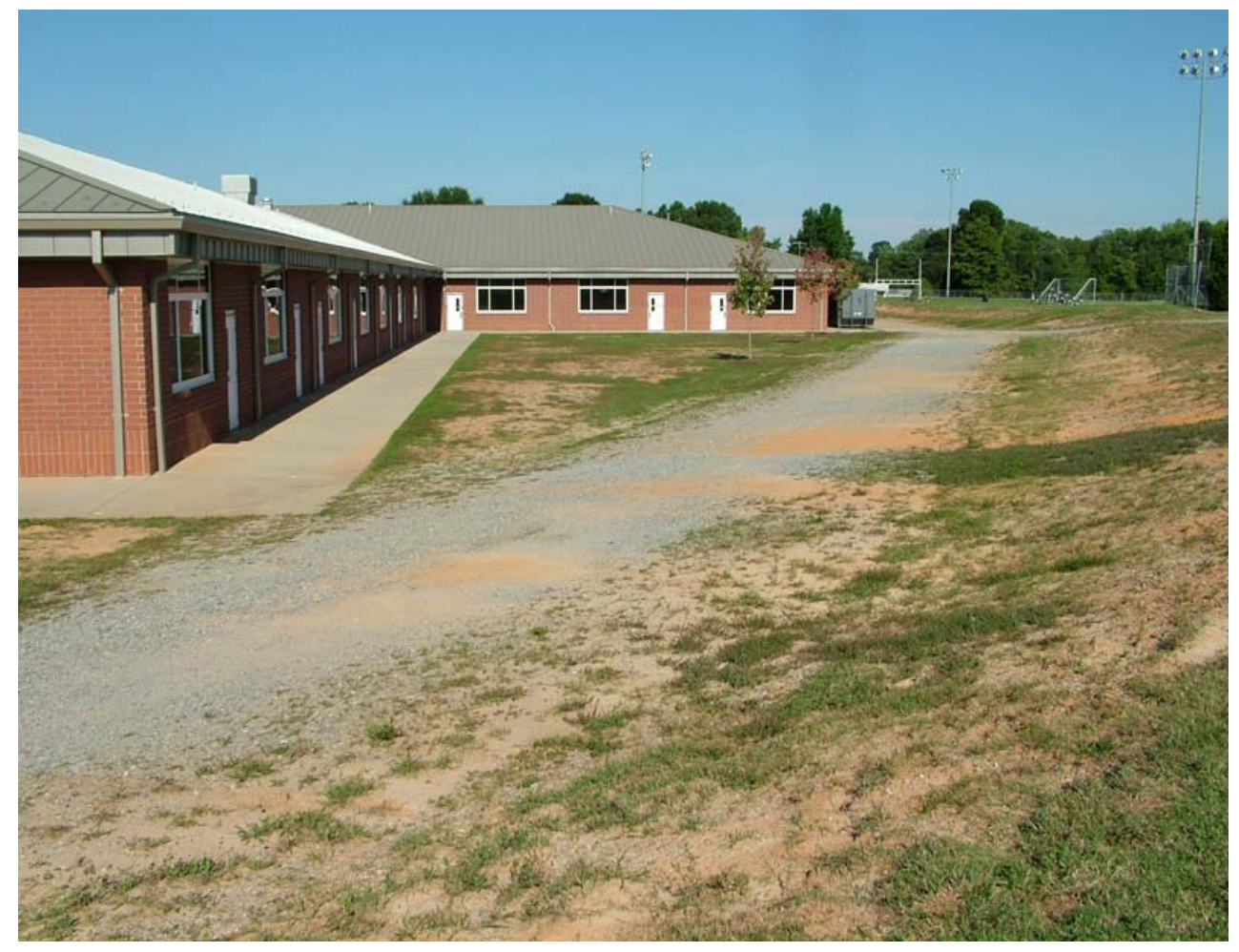

As Found Soffit Air Leaks

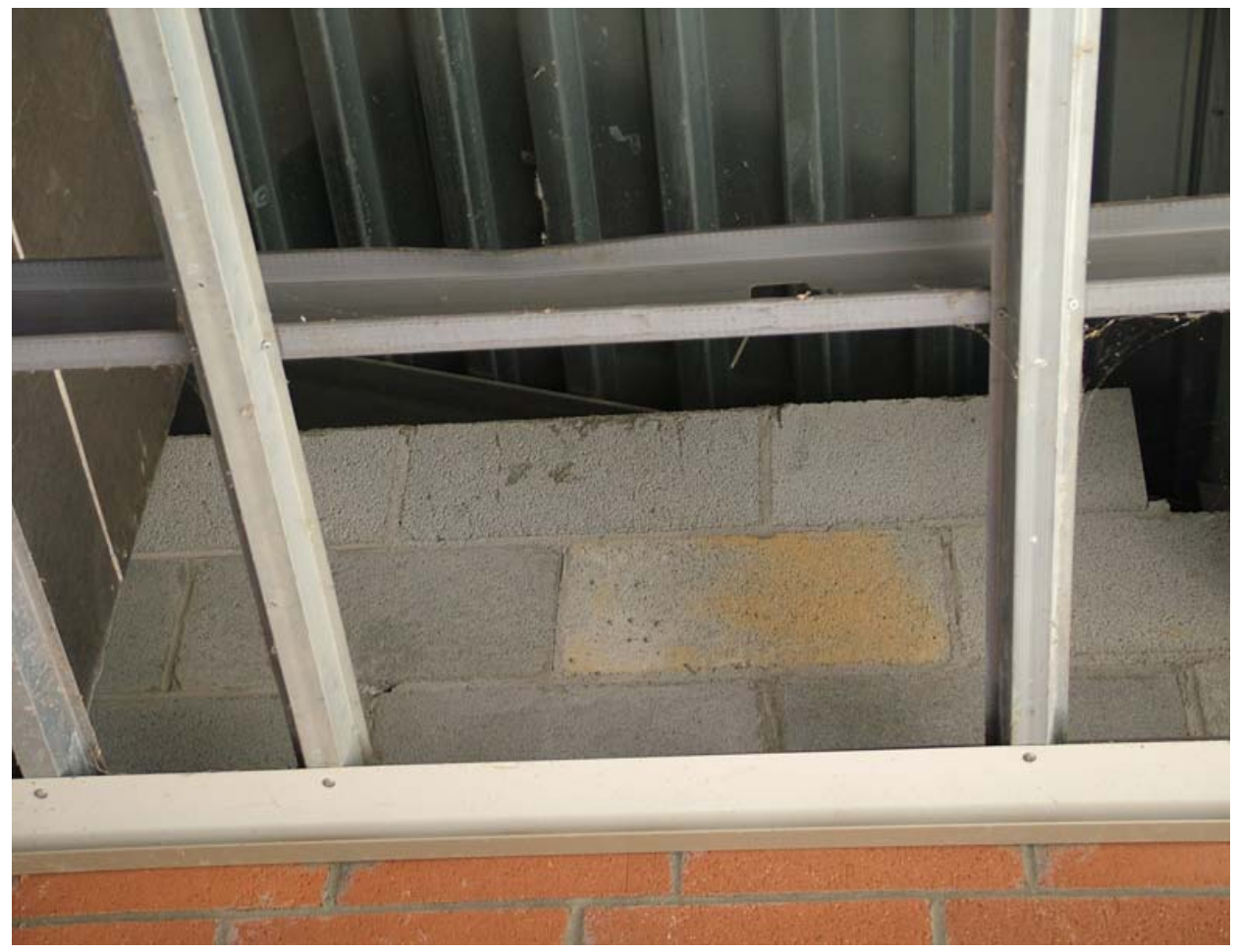


Appendix L: The Turner Building Science and Design Final Report Pictures (CONTINUED)

\section{As Found Soffit Air Leaks}

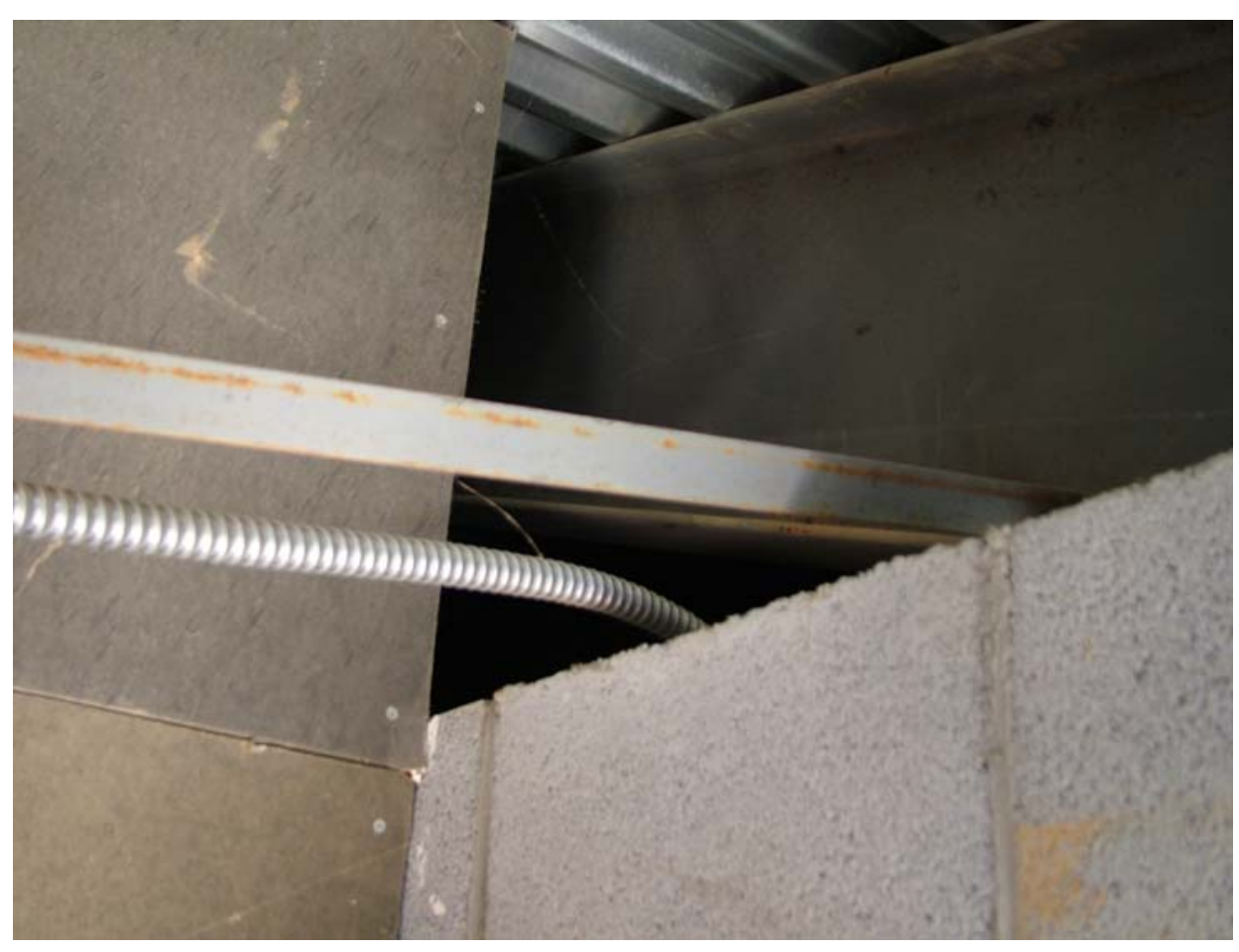

As Found Soffit Air Leaks

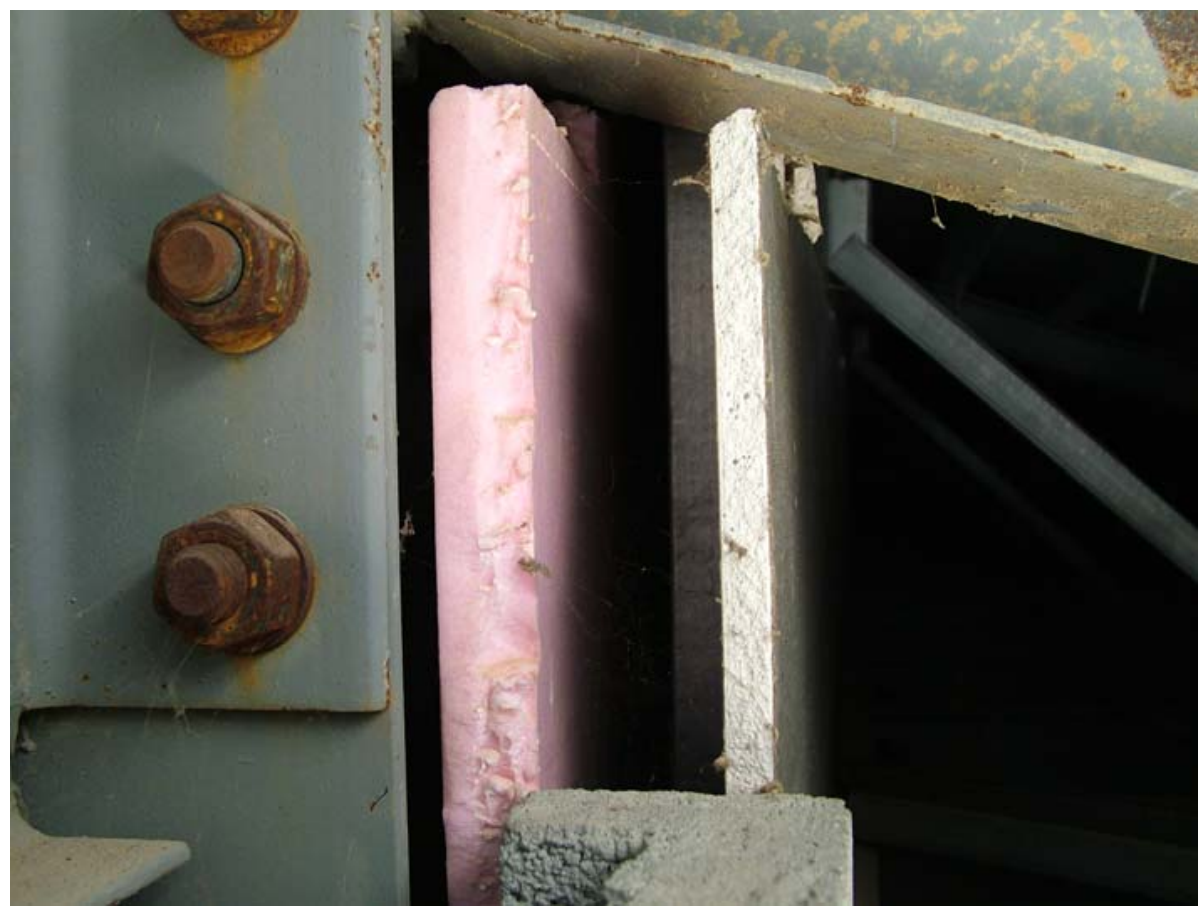




\section{Appendix L: The Turner Building Science and Design Final Report Pictures (CONTINUED)}

\section{As Found Soffit Air Leaks}

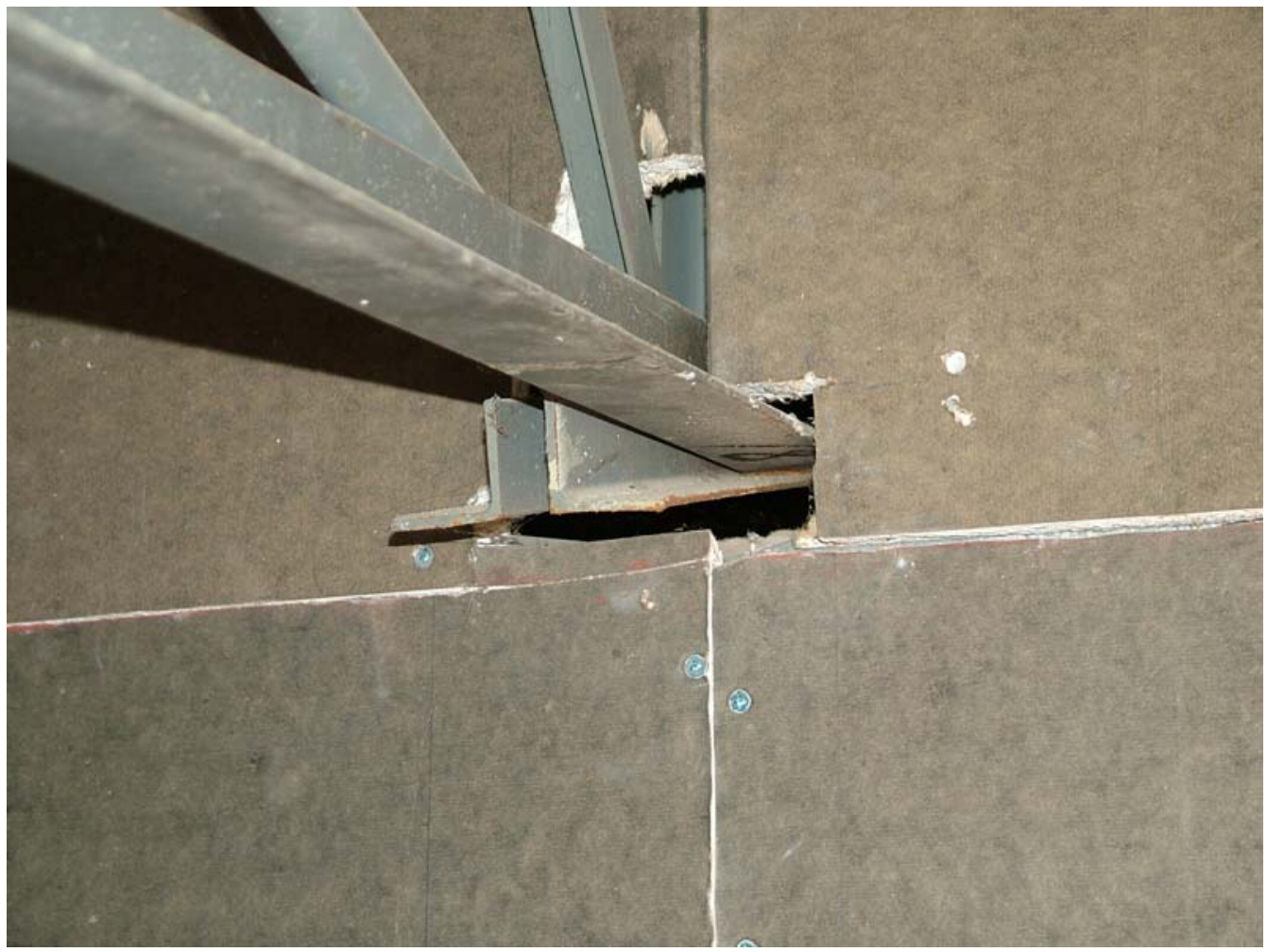

As Found Soffit Air Leaks

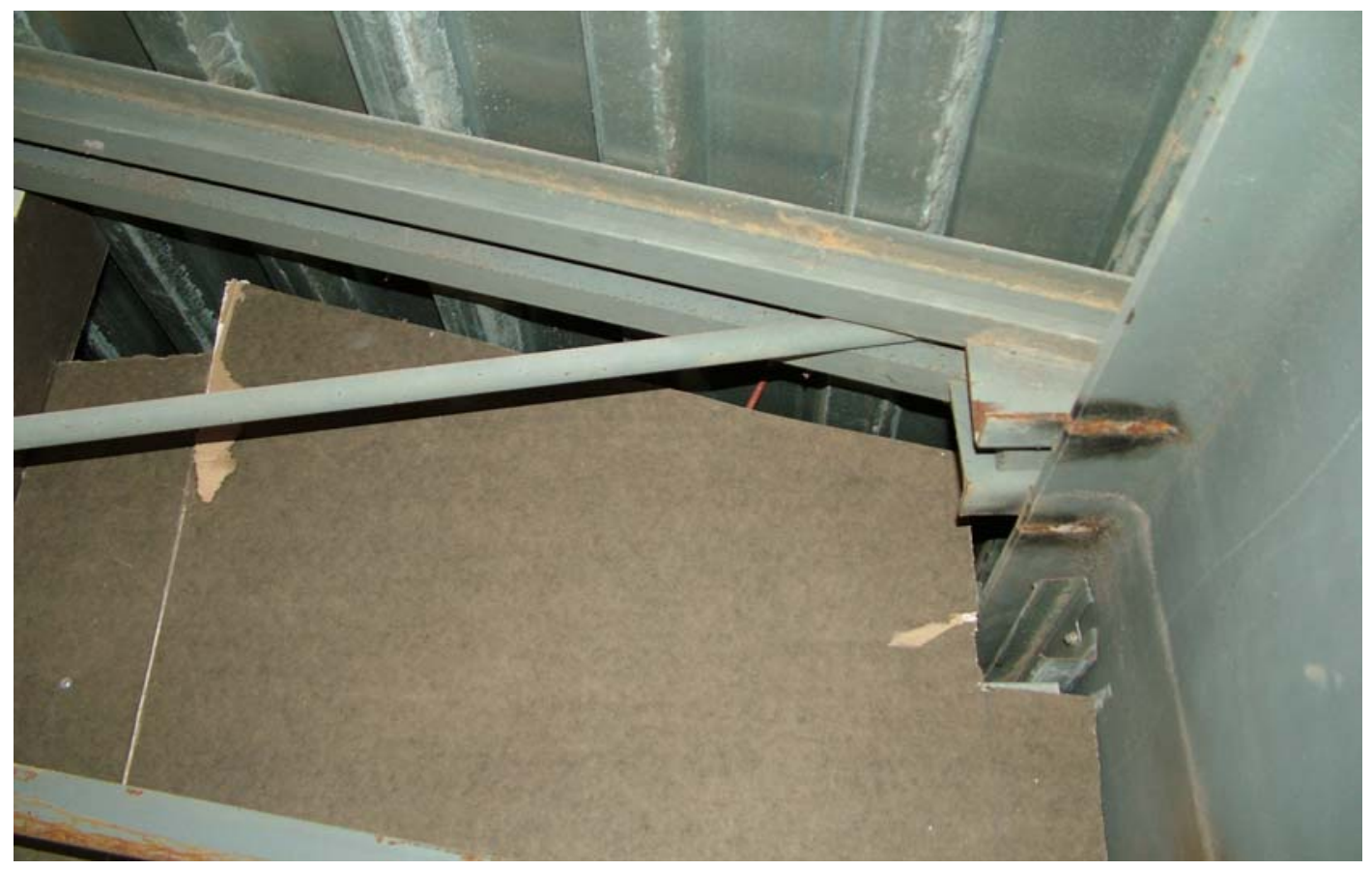


Appendix L: The Turner Building Science and Design Final Report Pictures (CONTINUED)

\section{As Found Soffit Air Leaks}

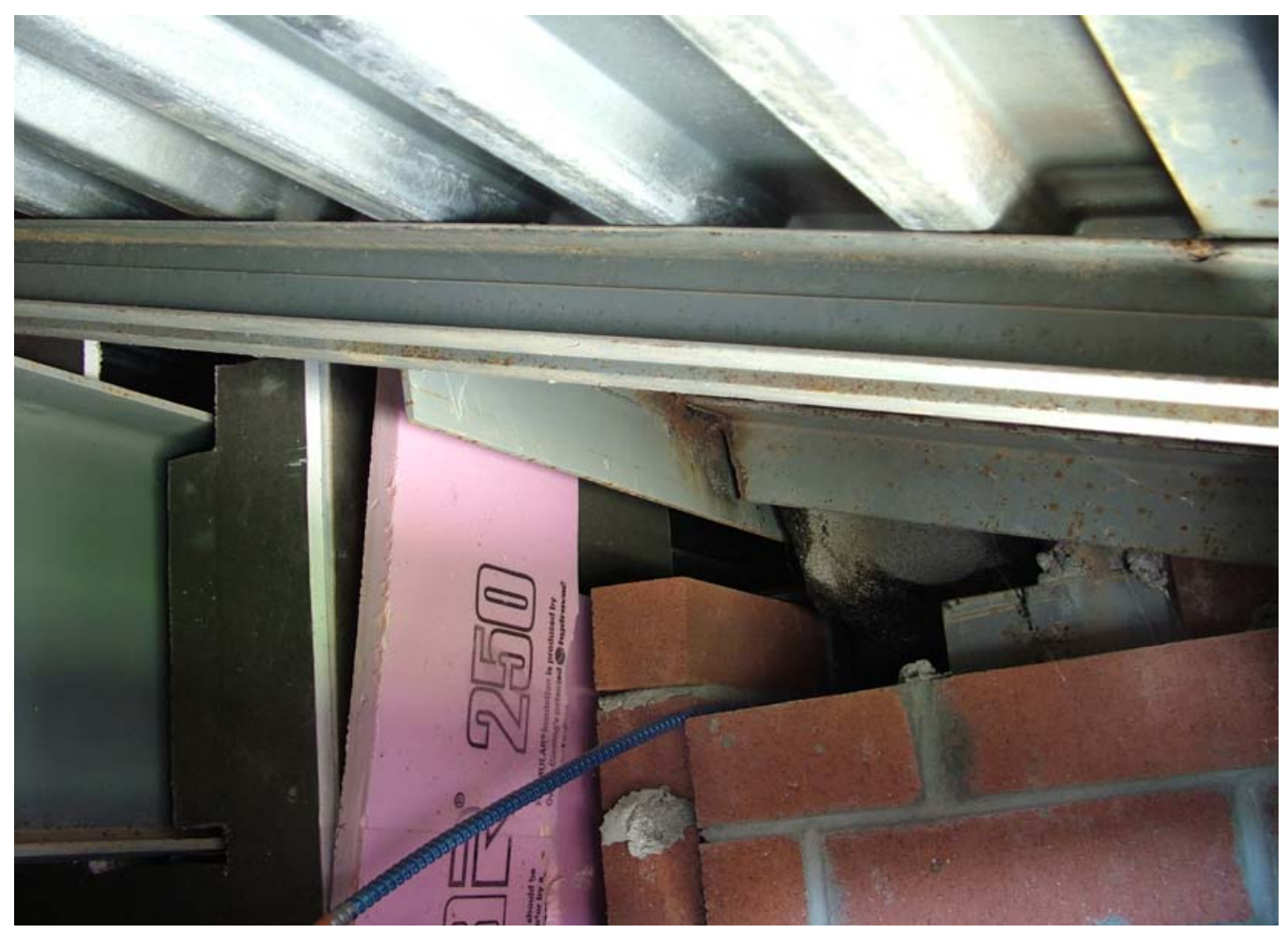

Soffit Sealing

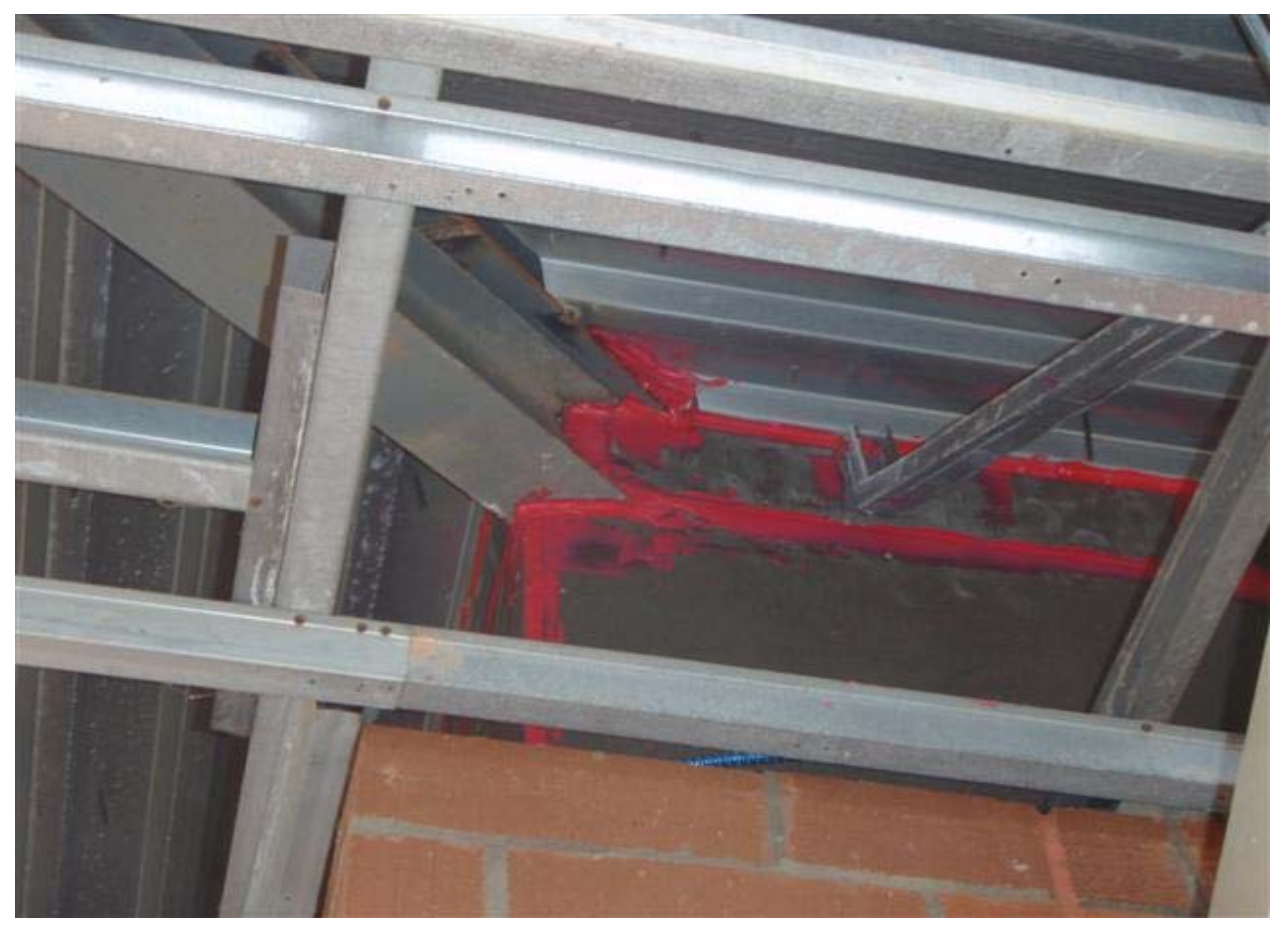




\section{Appendix L: The Turner Building Science and Design Final Report Pictures (CONTINUED)}

\section{Soffit Sealing}

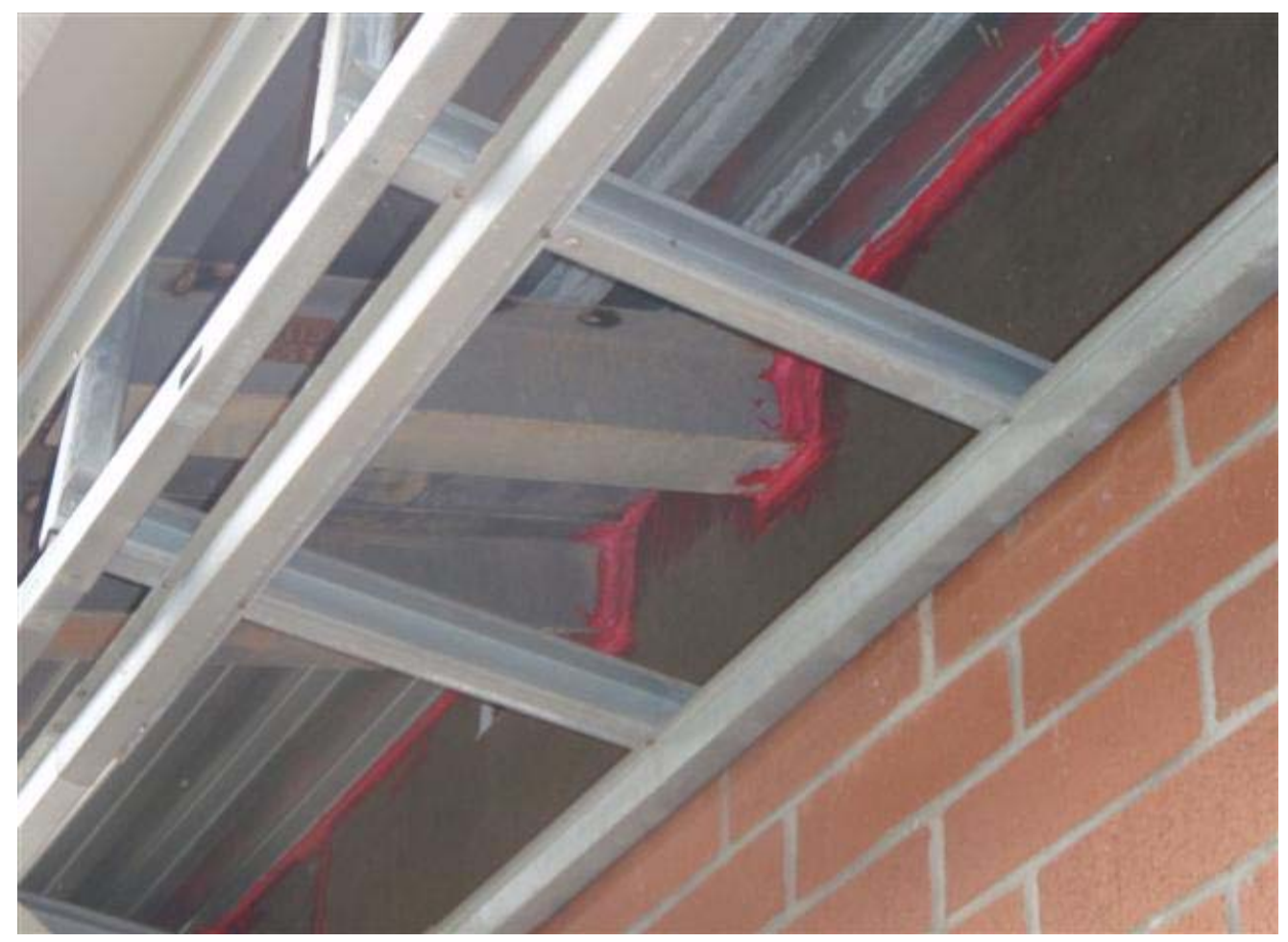

Soffit Sealing

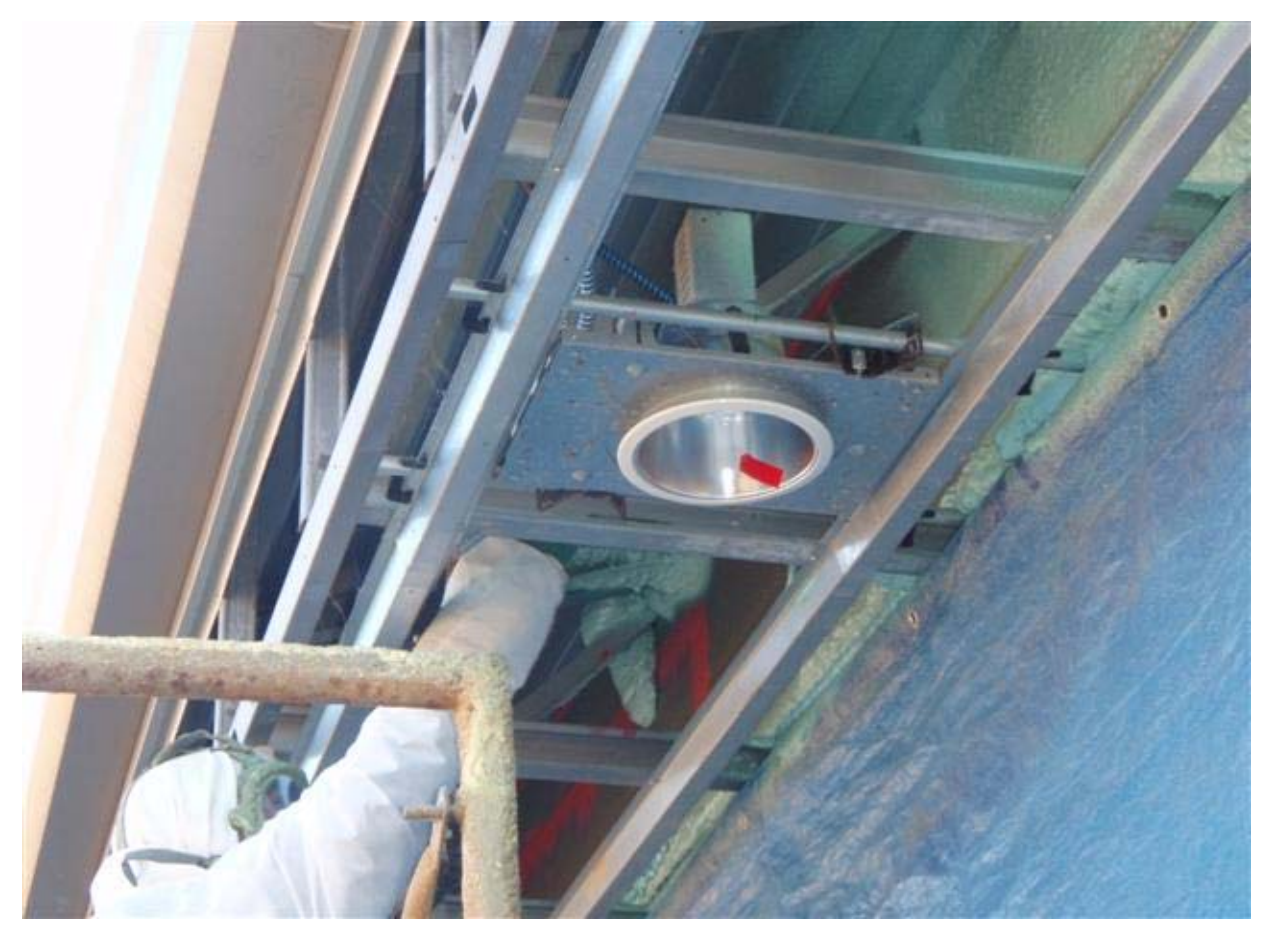


Appendix L: The Turner Building Science and Design Final Report Pictures (CONTINUED)

Soffit Sealing

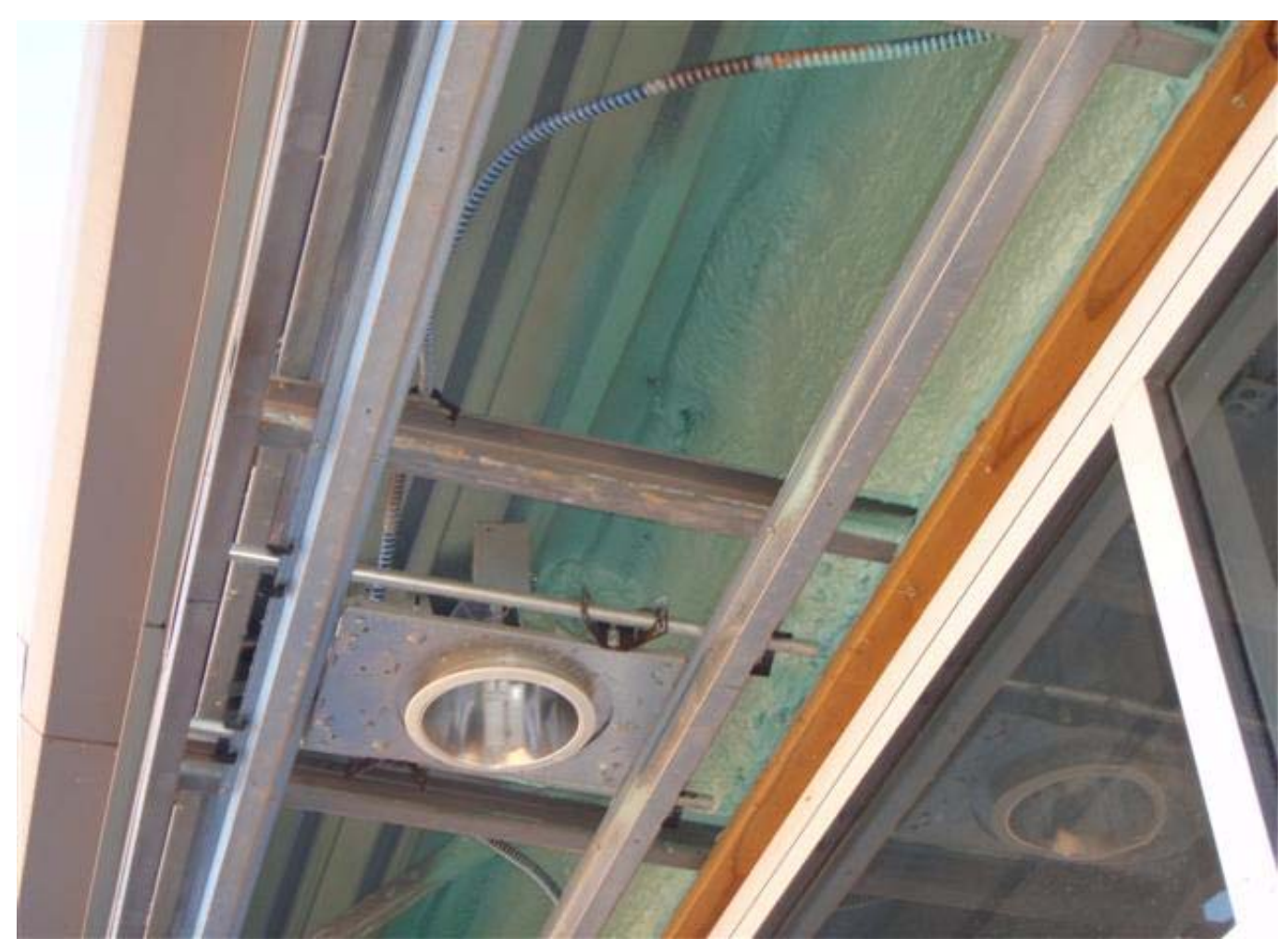

Soffit Sealing

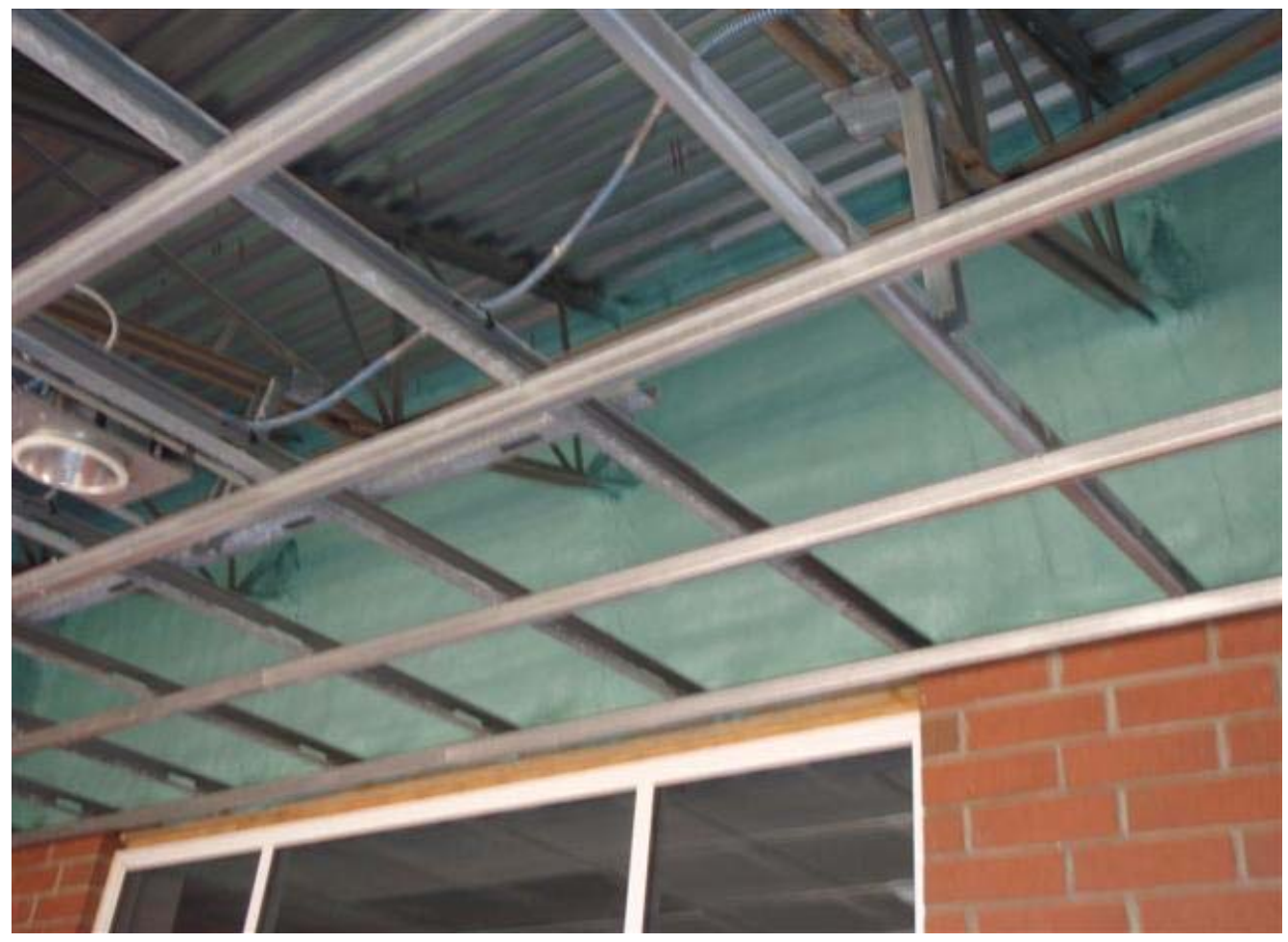




\section{Appendix L: The Turner Building Science and Design Final Report Pictures (CONTINUED)}

\section{Soffit Sealing}

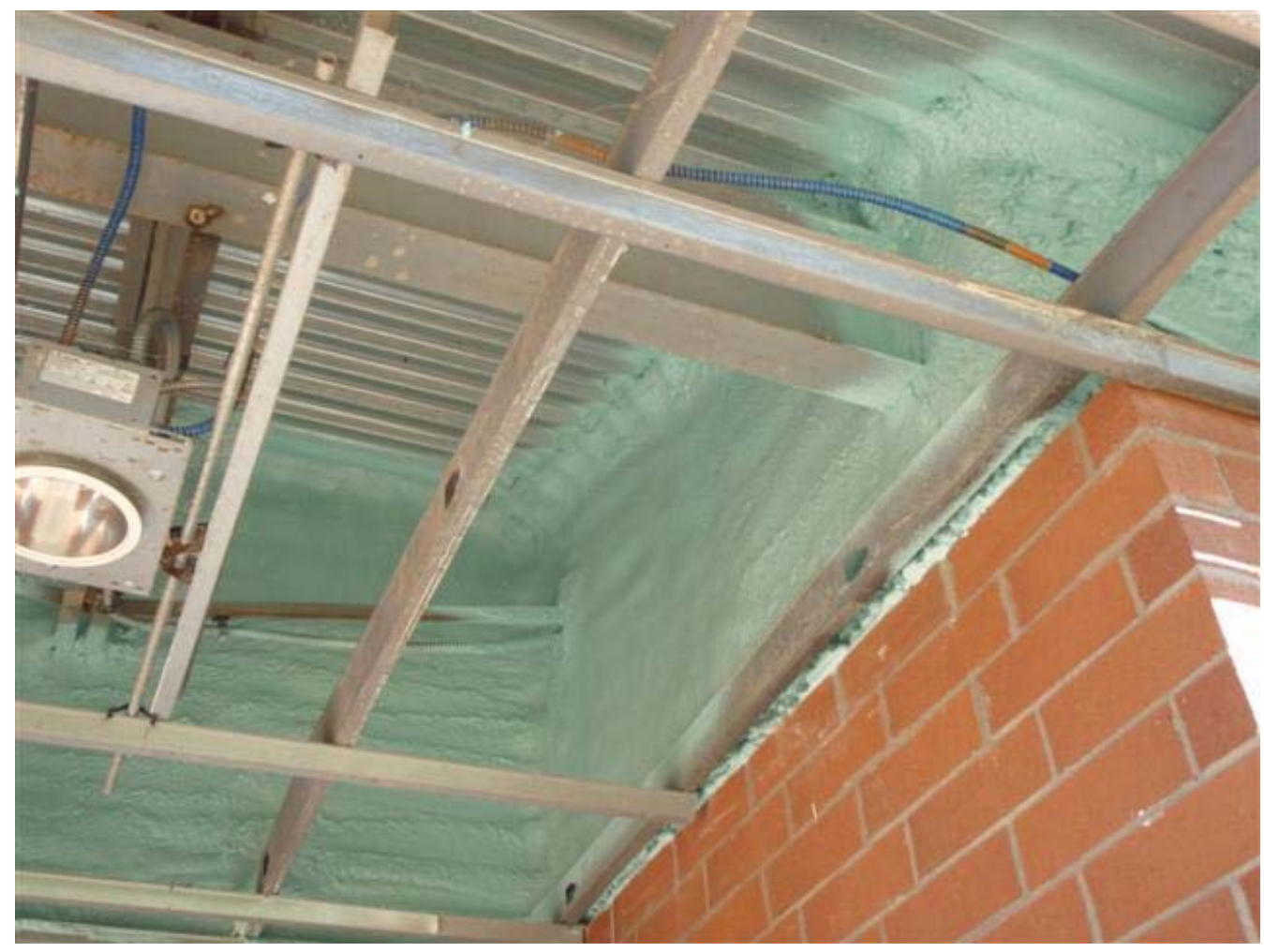

IR November 10, 2009 Soffit Leaks

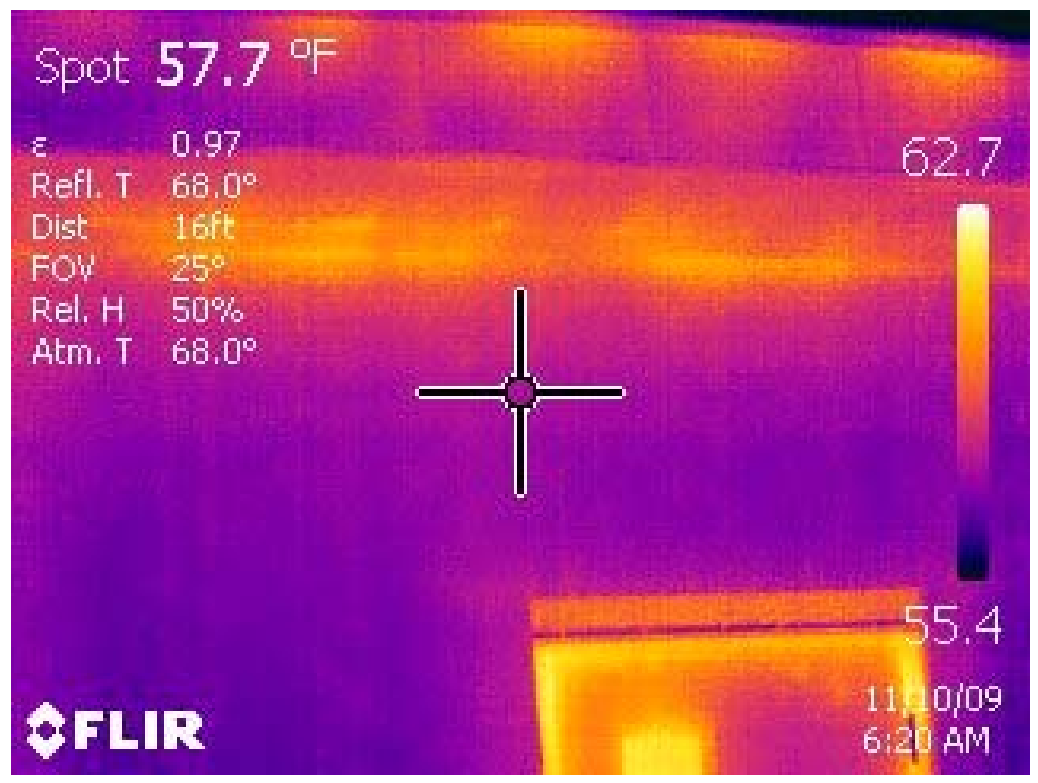


Appendix L: The Turner Building Science and Design Final Report Pictures (CONTINUED)

IR November 10, 2009 Soffit Leaks

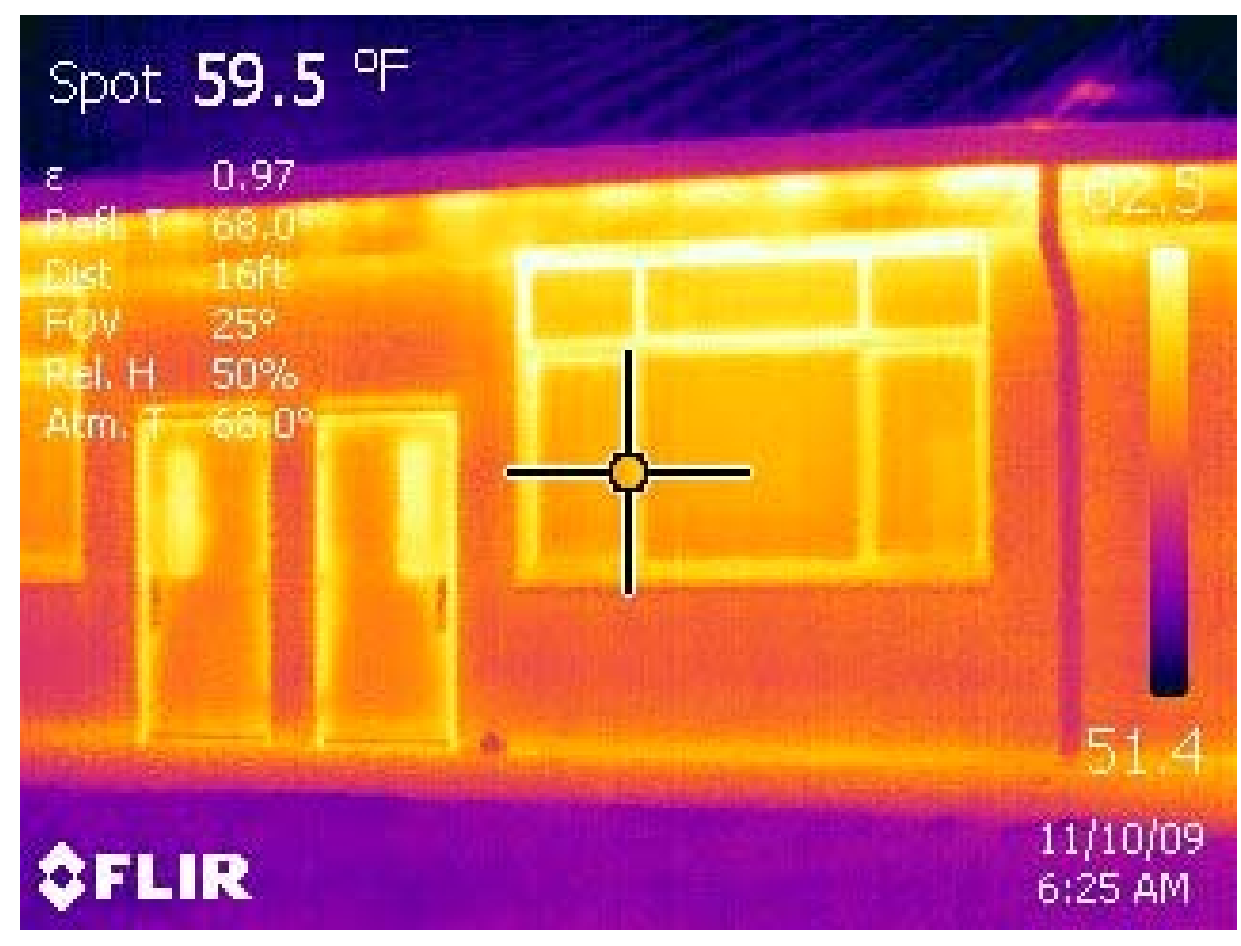

IR November 10, 2009 Soffit Leaks

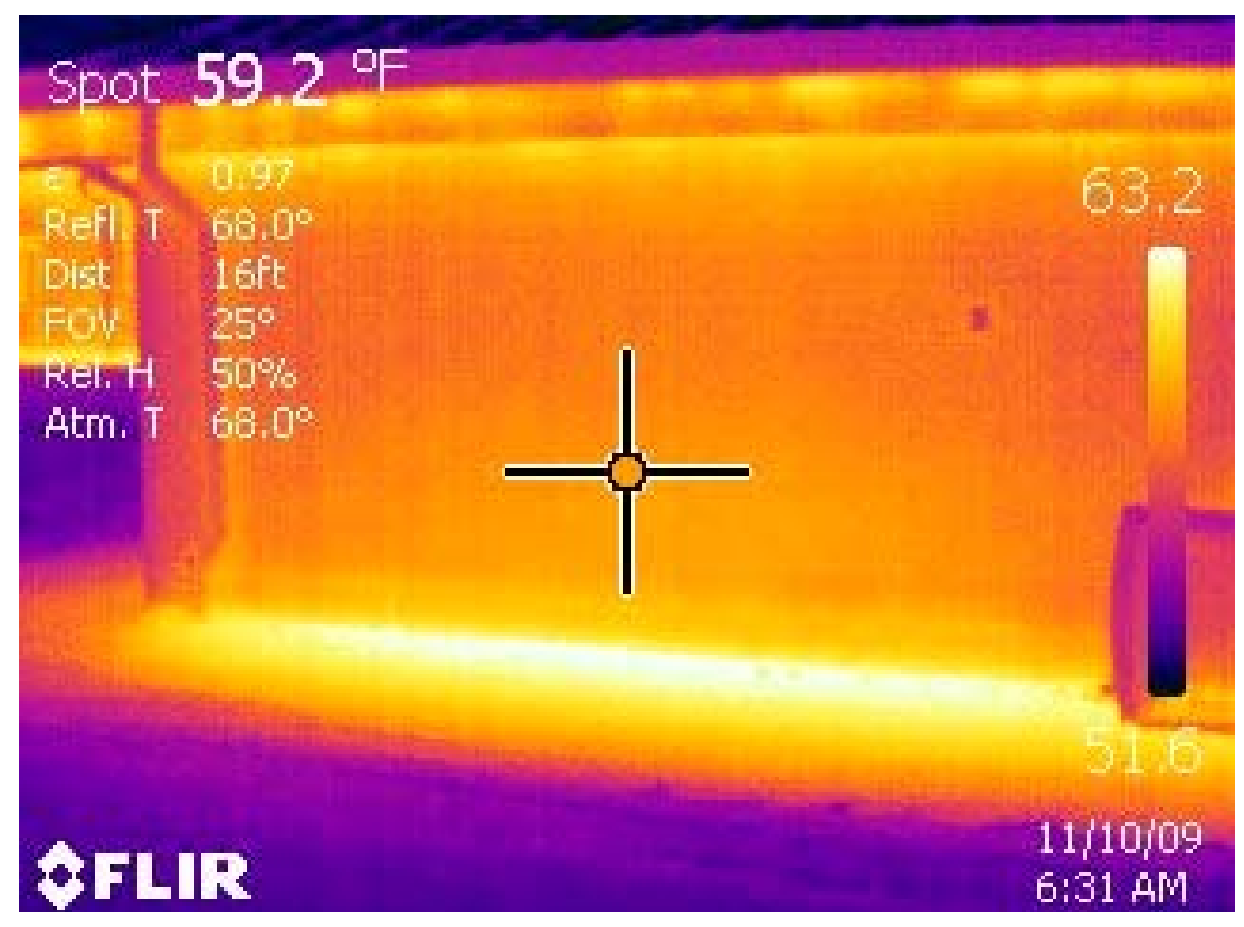


Appendix L: The Turner Building Science and Design Final Report Pictures (CONTINUED)

IR November 10, 2009 Soffit Leaks

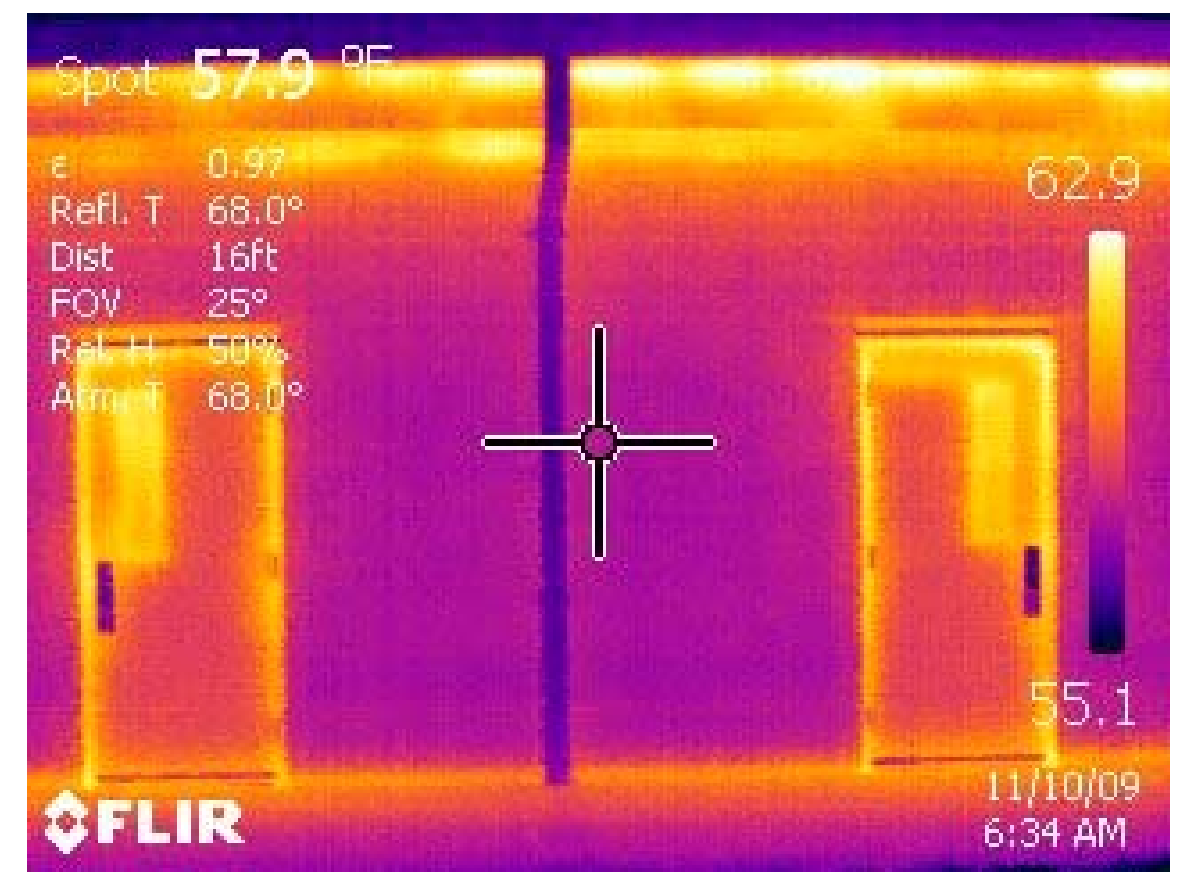

IR November 10, 2009 Soffit Leaks

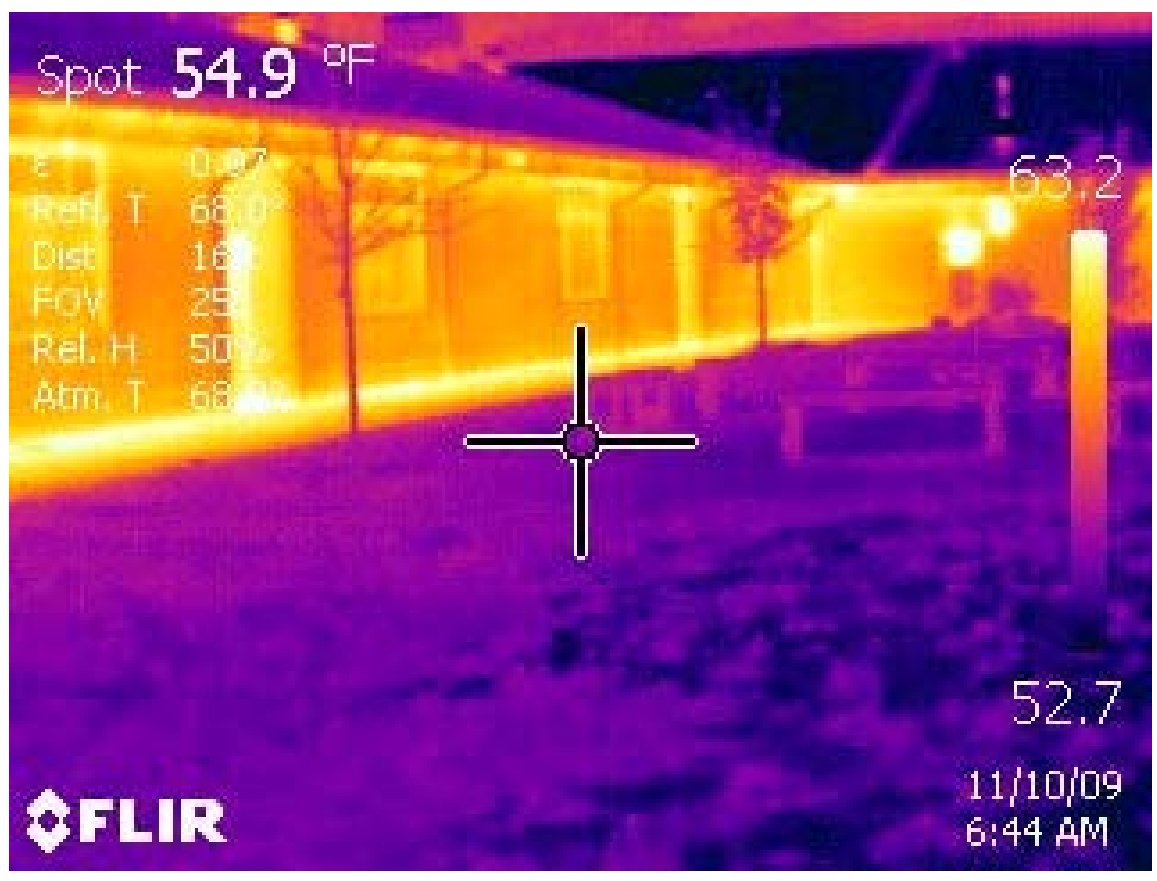


Appendix L: The Turner Building Science and Design Final Report Pictures (CONTINUED)

IR November 10, 2009 Soffit Leaks

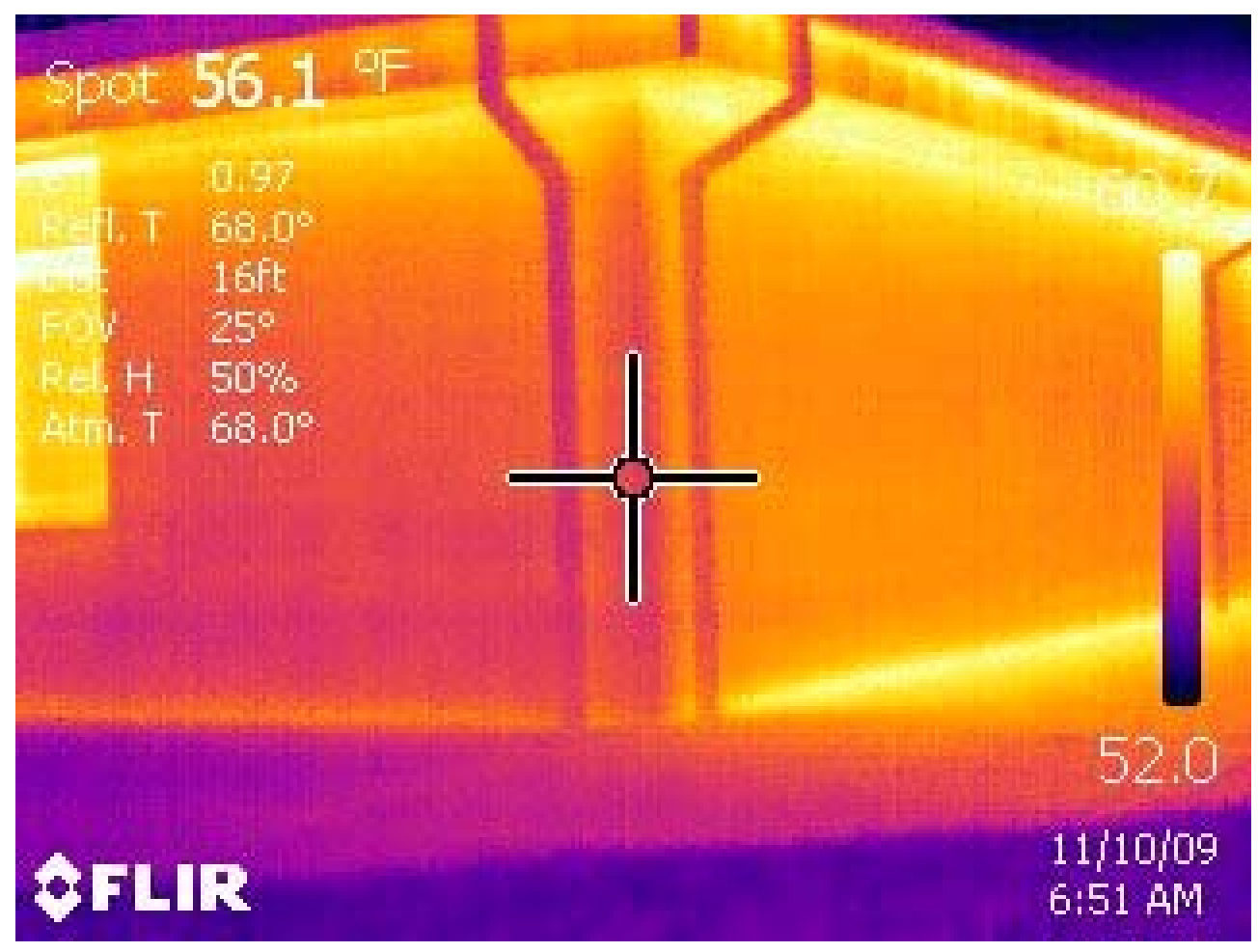

IR December 16, 2009 Soffit Sealing

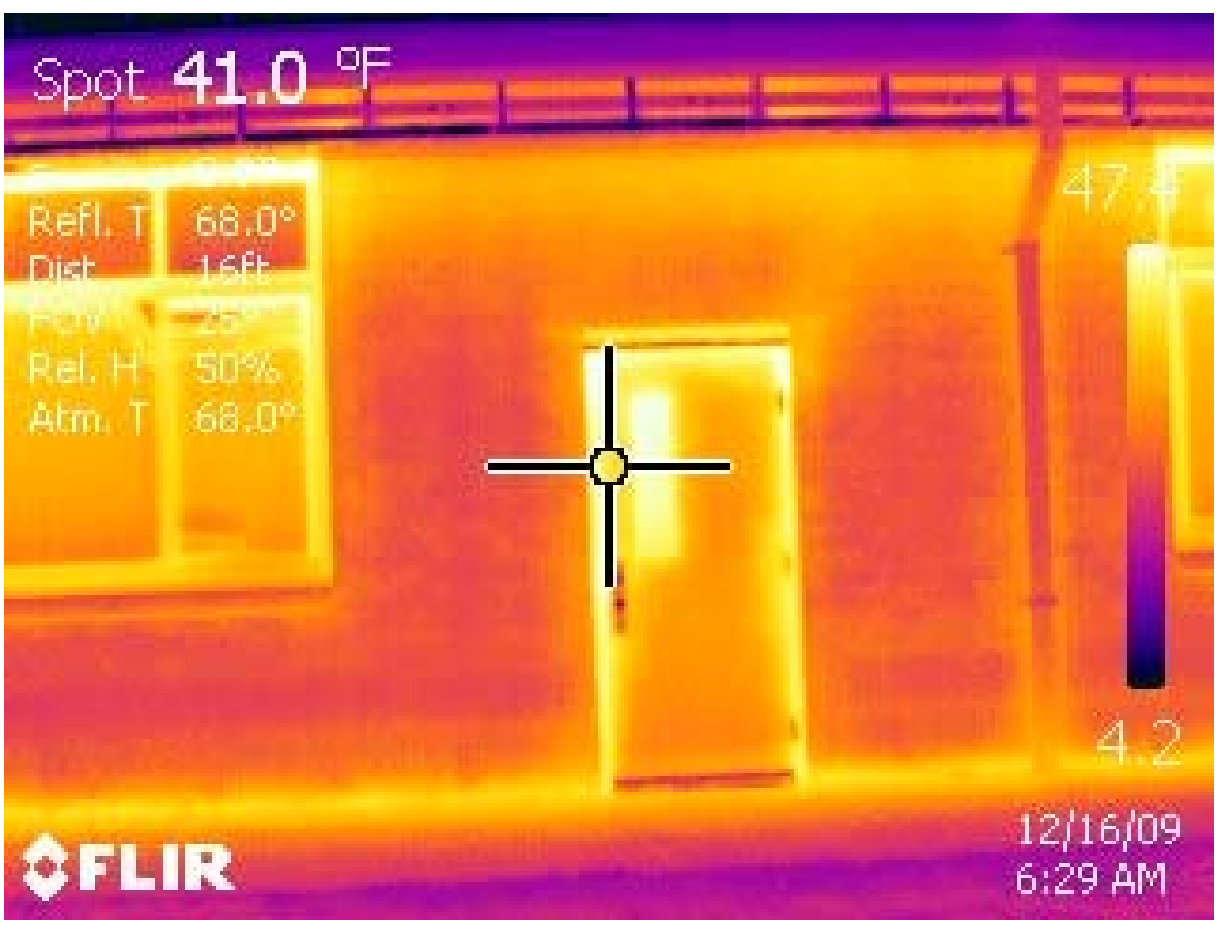




\section{Appendix L: The Turner Building Science and Design Final Report Pictures (CONTINUED)}

IR December 16, 2009 Soffit Sealing

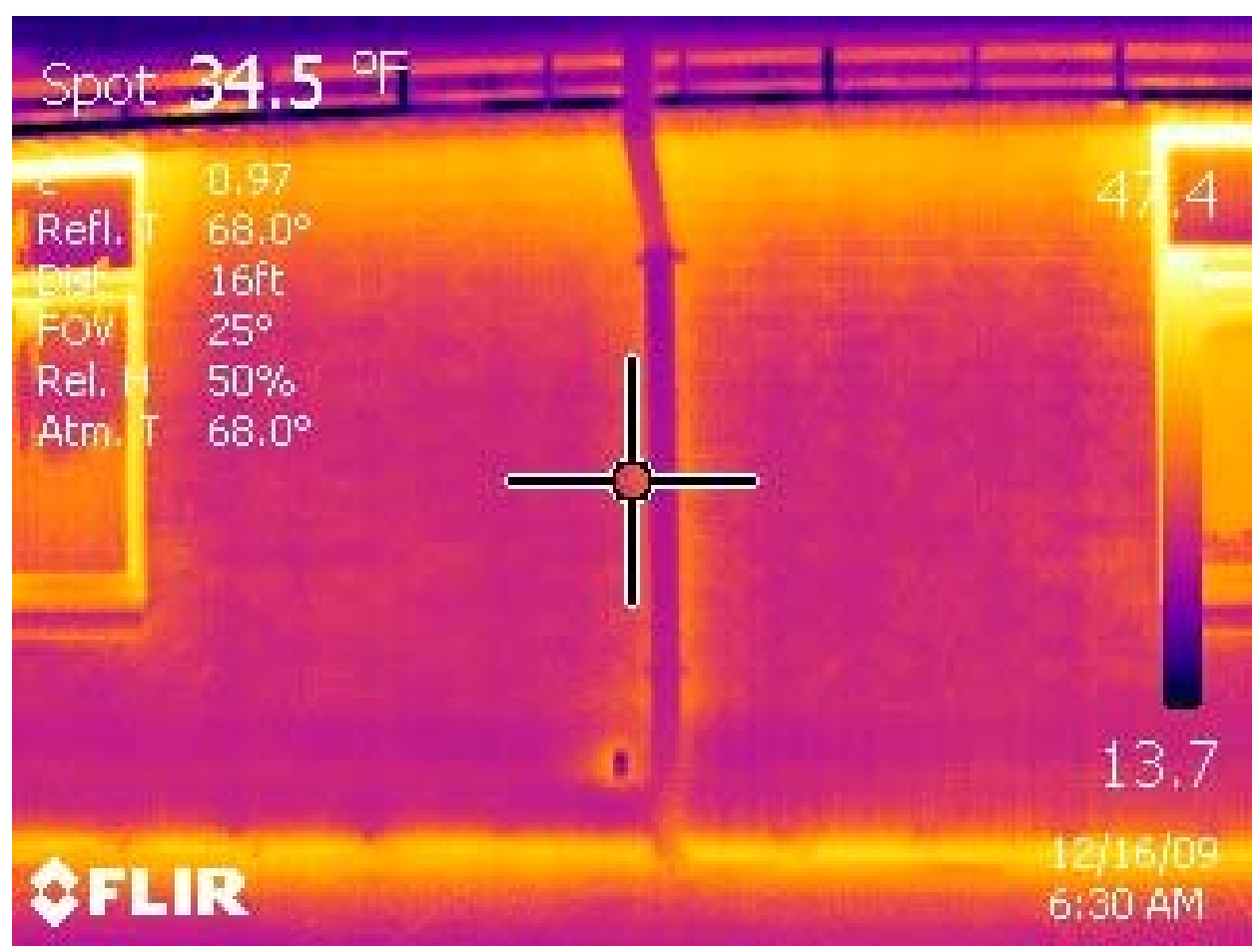

IR December 16, 2009 Soffit Sealing

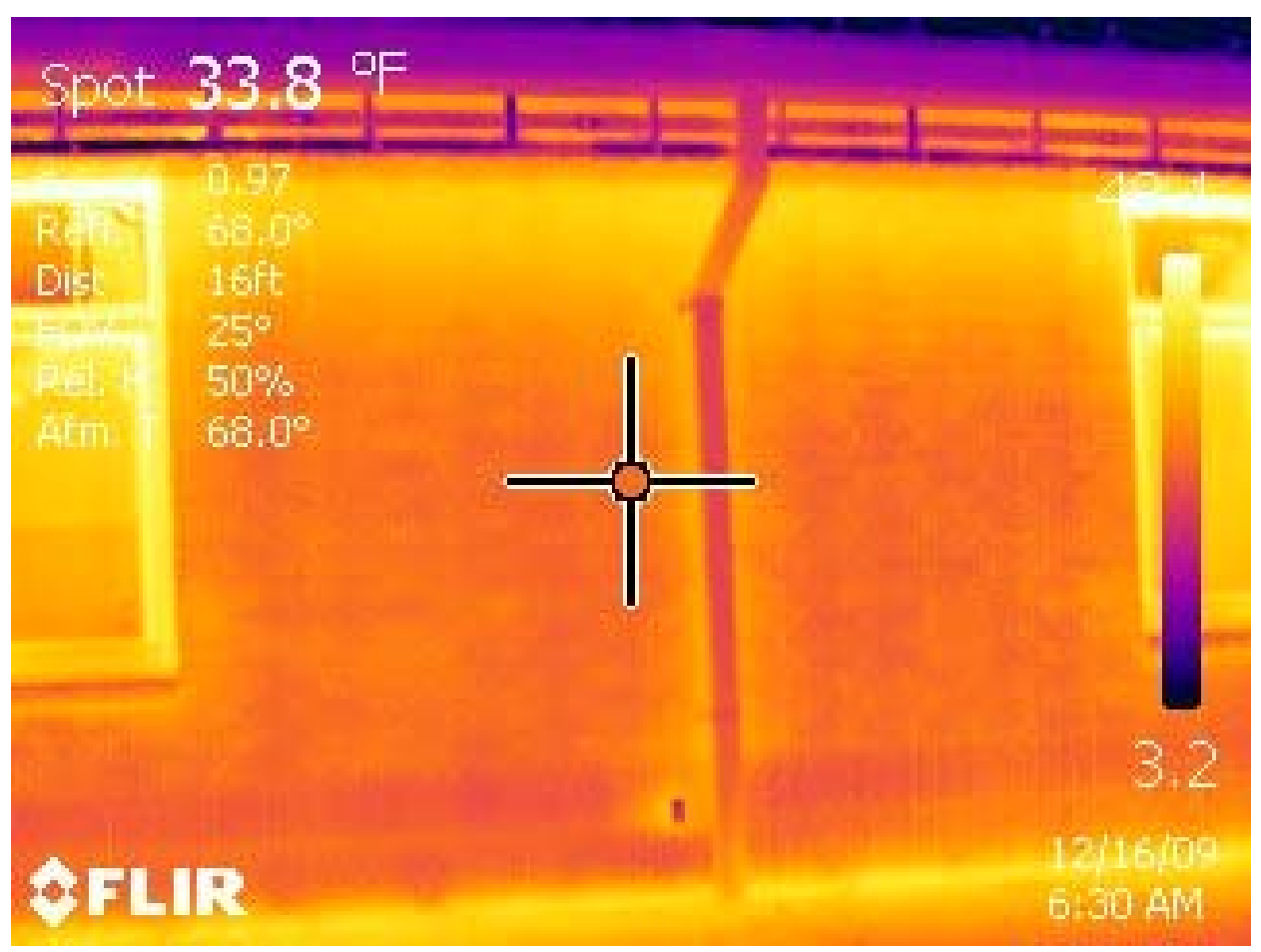


Appendix L: The Turner Building Science and Design Final Report Pictures (CONTINUED)

IR December 16, 2009 Soffit Sealing

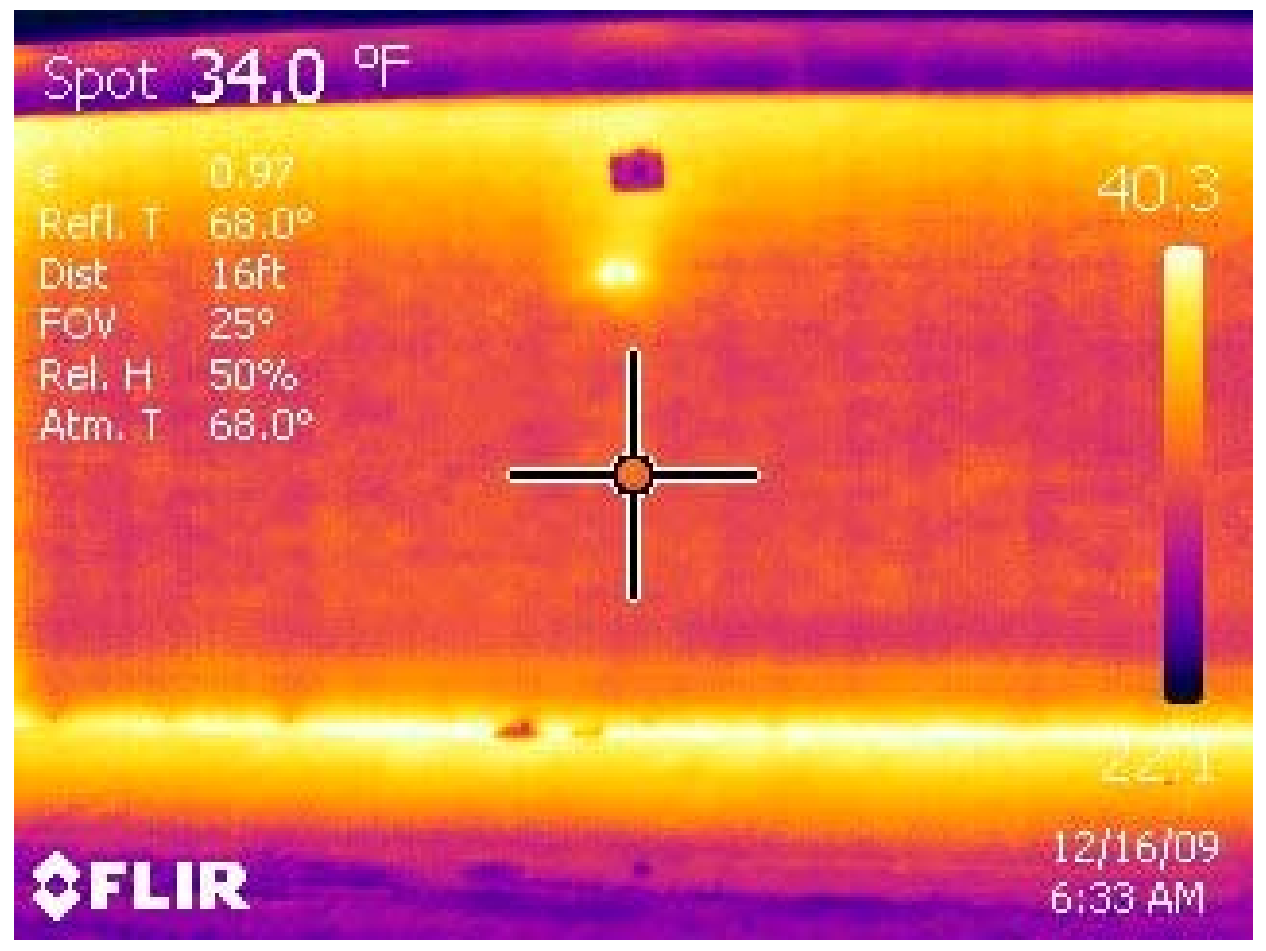

IR December 16, 2009 Soffit Sealing

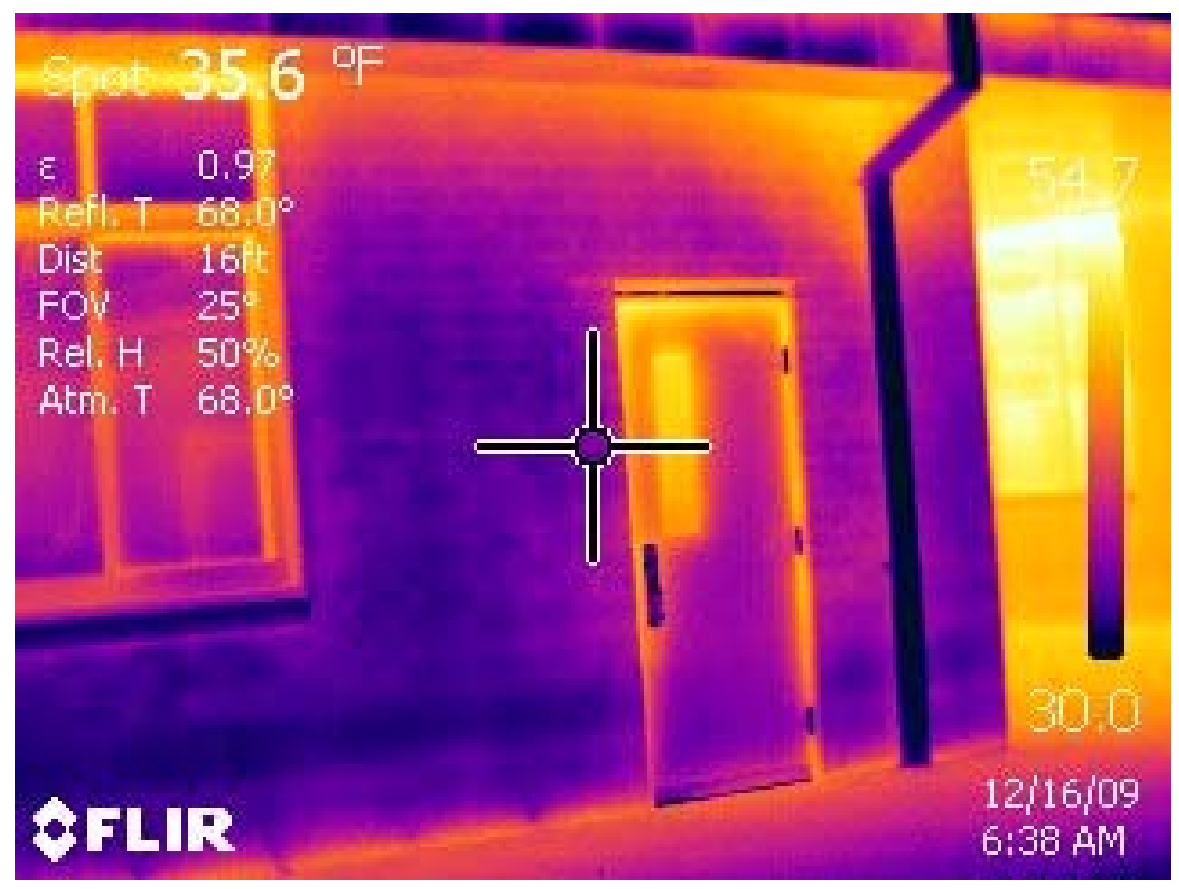




\section{Appendix L: The Turner Building Science and Design Final Report Pictures (CONTINUED)}

IR December 16, 2009 Soffit Sealing

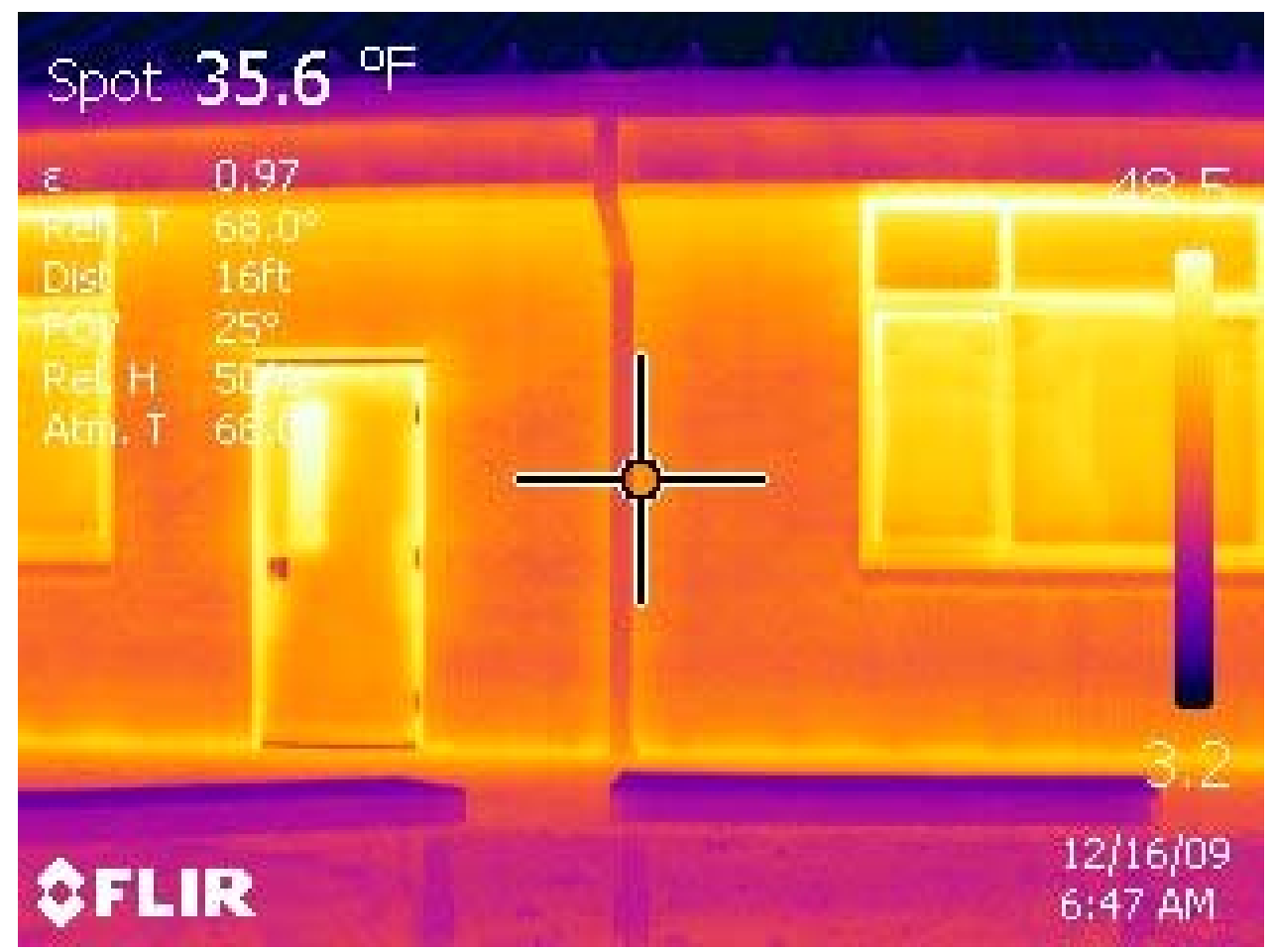

IR December 17, 2009 Soffit Sealing

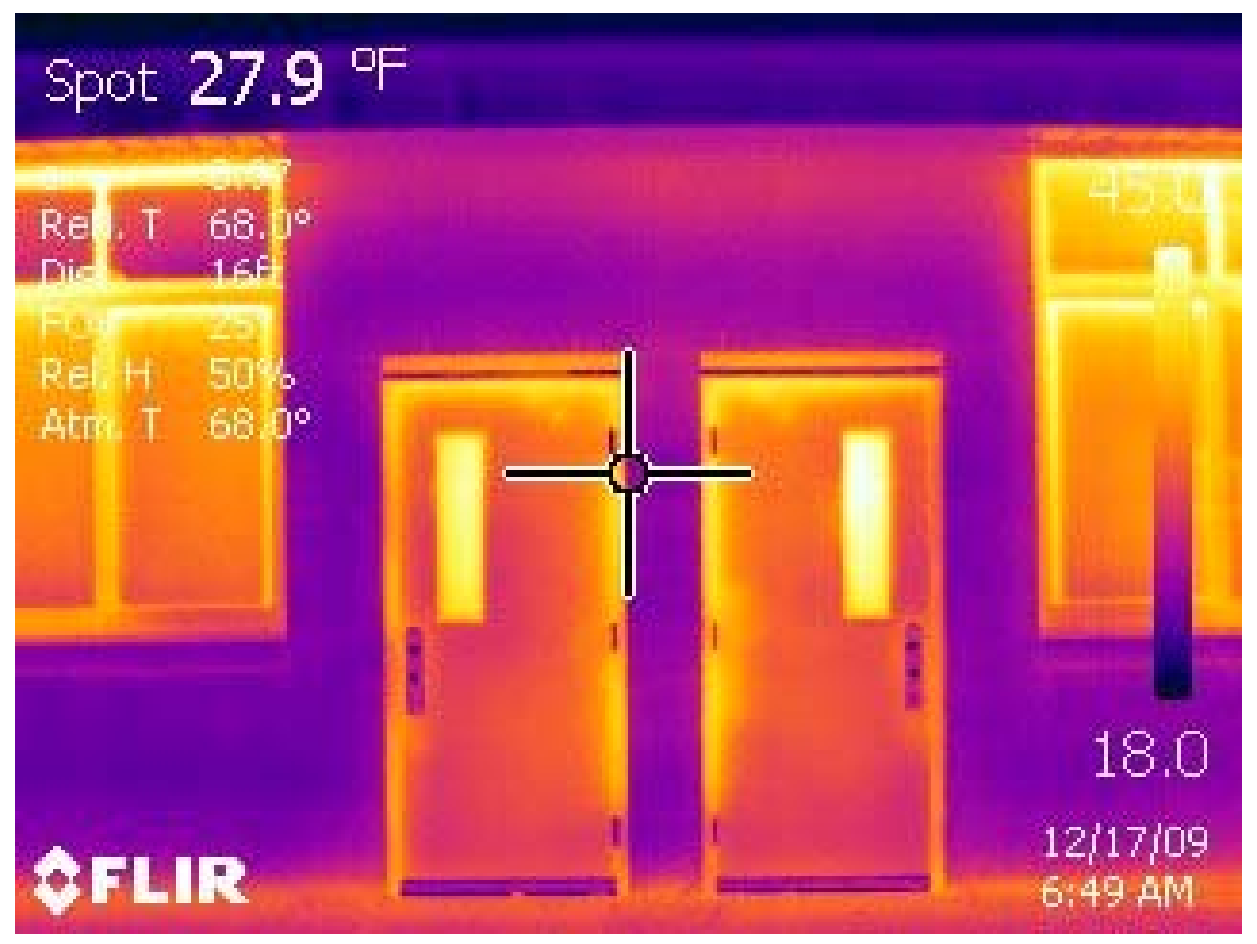


Appendix L: The Turner Building Science and Design Final Report Pictures (CONTINUED)

IR December 17, 2009 Soffit Sealing

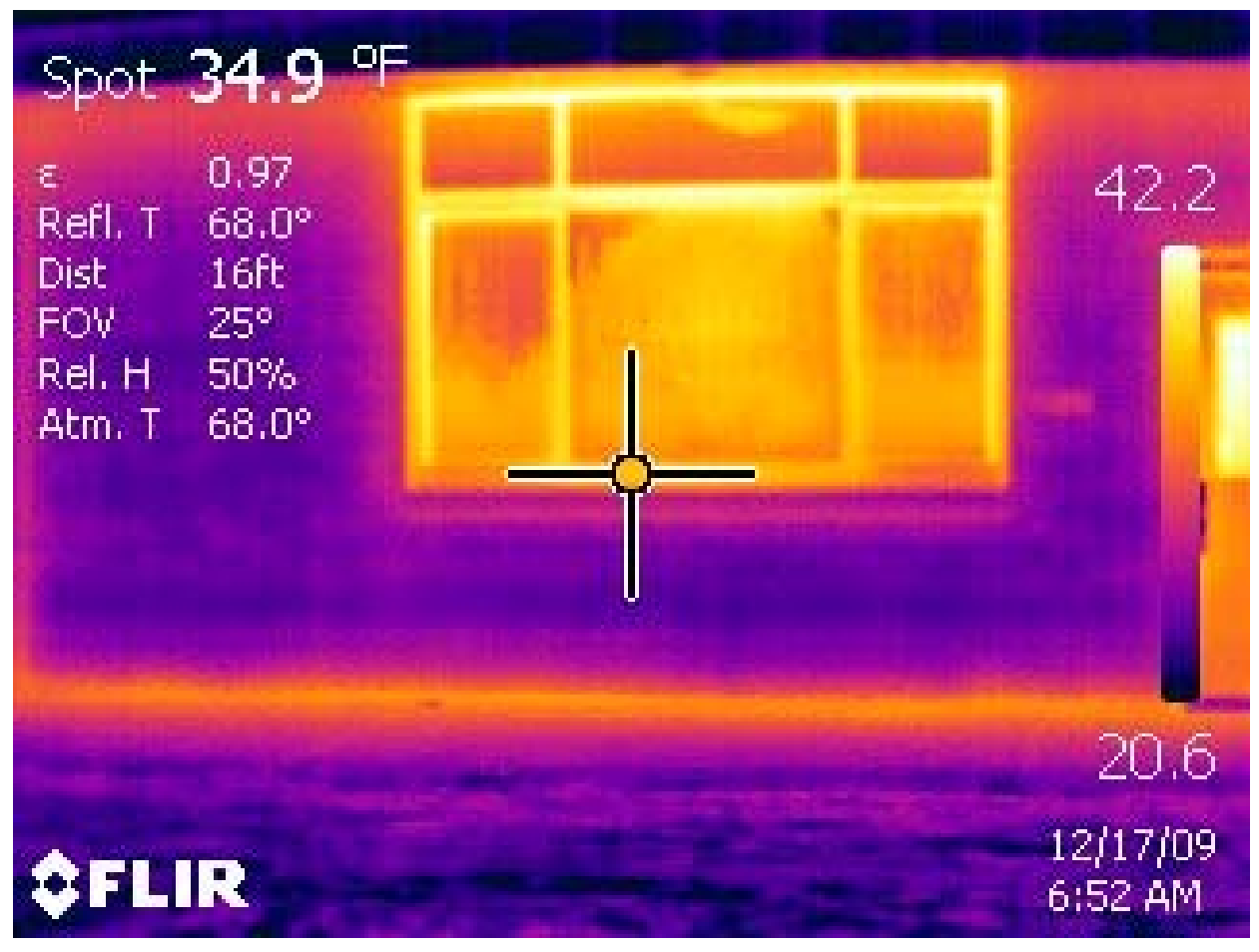

IR December 17, 2009 Soffit Sealing

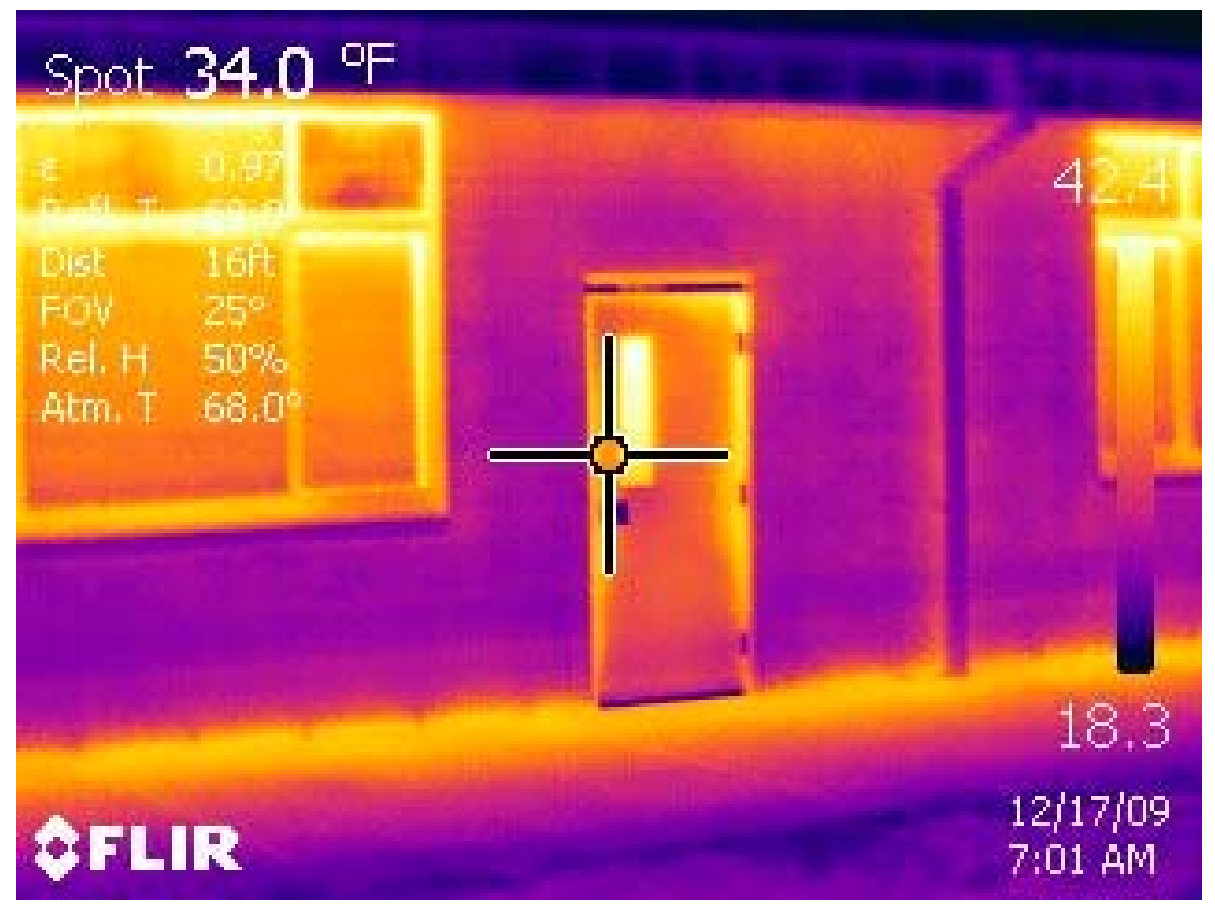




\section{Appendix L: The Turner Building Science and Design Final Report Pictures (CONTINUED)}

IR December 17, 2009 Soffit Sealing

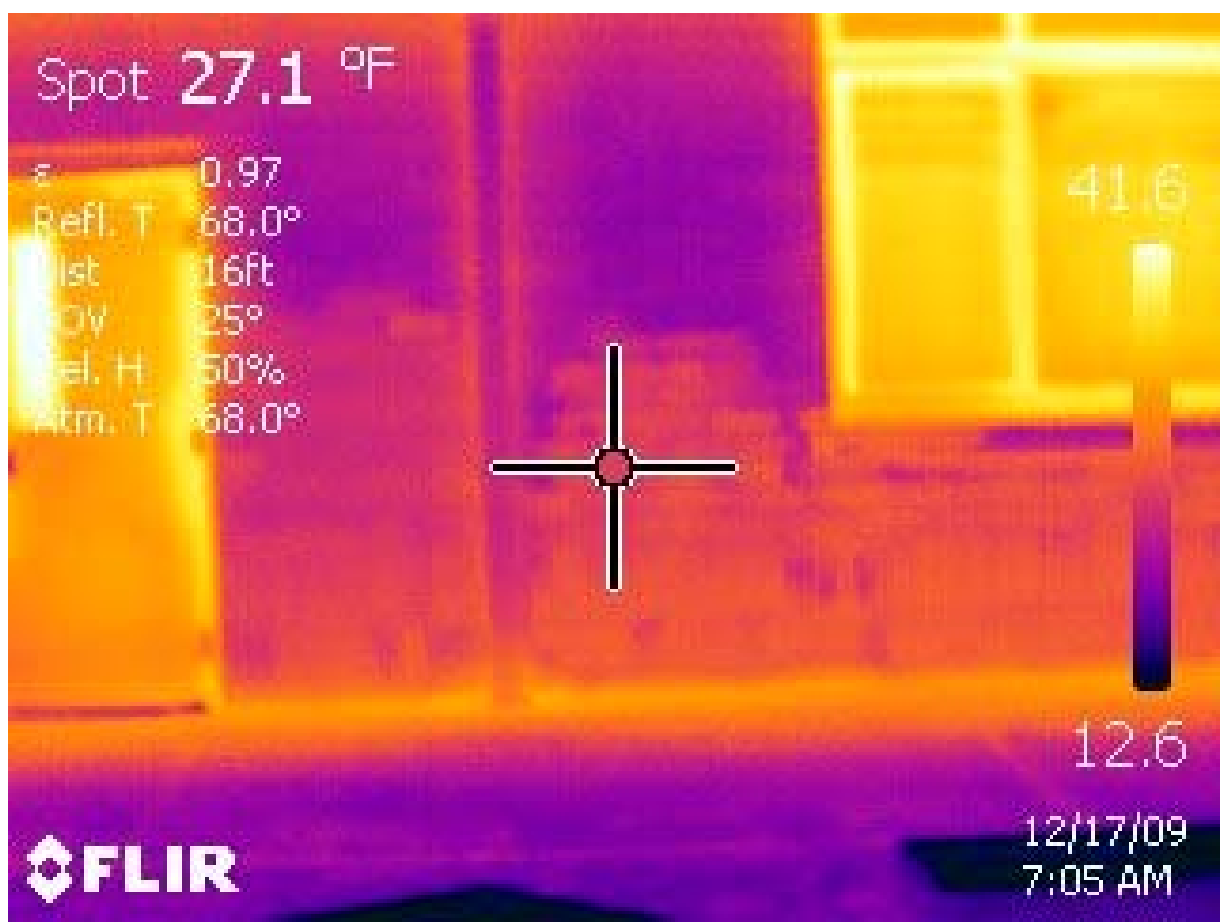

IR December 17, 2009 Soffit Sealing

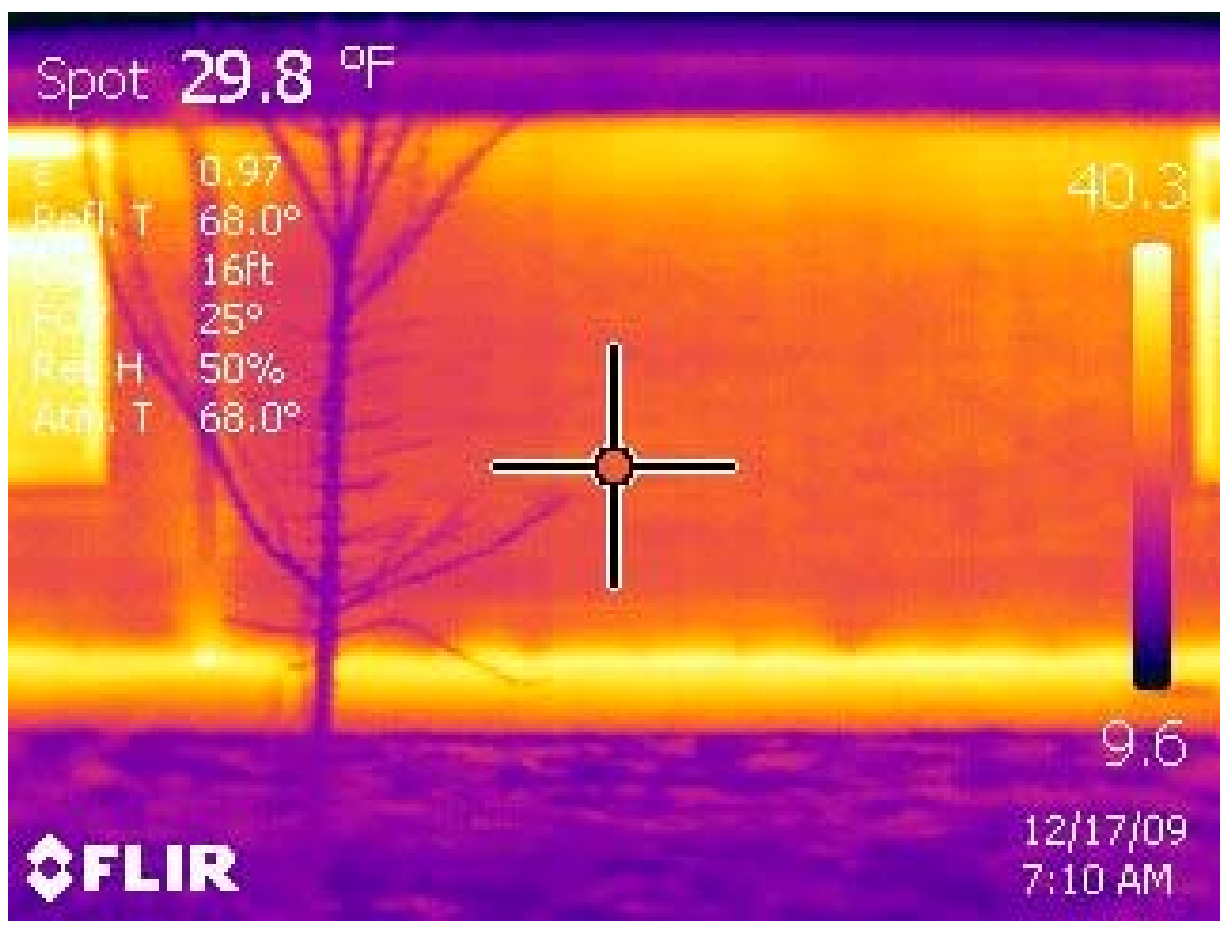


Appendix L: The Turner Building Science and Design Final Report Pictures (CONTINUED)

IR December 17, 2009 Soffit Sealing

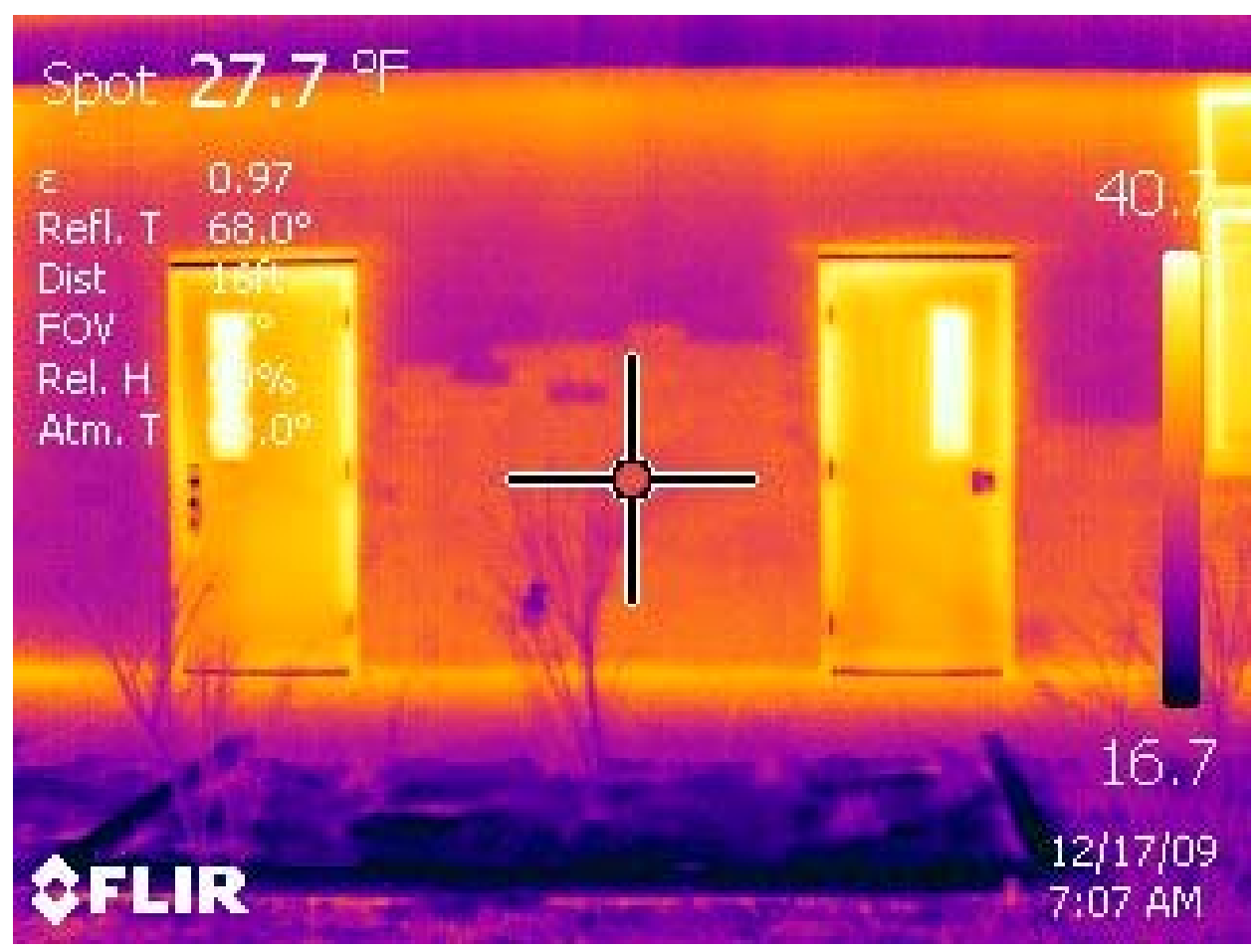




\section{Appendix M: NIOSH INTERIM RepORT, HETA 2009-0172}

\section{DEPARTMENT OF HEALTH \& HUMAN SERVICES}

Phone: (304) 285-5751

Fax: (304) 285-5820
Public Health Service

Centers for Disease Control and Prevention (CDC) National Institute for Occupational Safety and Health (NIOSH) 1095 Willowdale RoaD Morgantown, WV26505-2888

February 24, 2010

HETA 2009-0172

Interim Letter

Eric Becoats, Ed.D.

Chief Administrative Officer

Guilford County Schools

712 North Eugene Street

Greensboro, NC 27401

Dear Dr. Becoats:

The purpose of this letter is to convey a report on the findings of the National Institute for Occupational Safety and Health (NIOSH) Health Hazard Evaluation at Oak Ridge Elementary School at 2050 Oak Ridge Road, in Oak Ridge, North Carolina. A copy of the interim report is enclosed. We recommend that you post the interim report in a prominent place accessible to the employees for a period of 30 calendar days.

As you are aware, during the week of March 1, 2010, we are planning to administer a health survey questionnaire at Oak Ridge Elementary School to employees who wish to participate. Much of the questionnaire will address health symptoms experienced the four weeks prior to the re-opening of the school. We see this cross-sectional survey as part of surveillance to document the extent of resolution of a public health problem likely associated with dampness in the building before remediation. We will analyze the data from that questionnaire and provide you with the findings as soon as we can.

If you have any questions or concerns, please feel free to contact us at (800) 232-2114.

Sincerely,

Rachel L. Bailey, DO, MPH

Medical Officer

Ju-Hyeong Park, ScD, CIH

Industrial Hygienist

Rena Saito, PhD

Industrial Hygienist 


\title{
$\overline{\text { Appendix M: NIOSH INTERIM Report, HETA 2009-0172 (CONTINUEd) }}$
}

\author{
Jean Cox-Ganser, Ph.D. \\ Research Team Supervisor \\ Respiratory Disease Hazard Evaluation and \\ Technical Assistance Program \\ Field Studies Branch \\ Division of Respiratory Disease Studies
}

cc:

Mr. Maurice "Mo" Green, Superintendent

Ms. Nora Carr, Chief of Staff

Ms. Ann Kraft, Oak Ridge Elementary School Principal

Ms. Jackie Burney, Oak Ridge Elementary School Assistant Principal

Dr. Ward Robinson, Guilford County Department of Public Health

Ms. Ashley Royal, Parent Teacher Association 


\section{$\overline{\text { Appendix M: NIOSH INTERIM RePORT, HETA 2009-0172 (CONTINUED) }}$ \\ HETA 2009-0172 \\ Interim Report \\ February 24, 2010}

\section{INTRODUCTION}

The National Institute for Occupational Safety and Health (NIOSH) received a Health Hazard Evaluation (HHE) request from Guilford County Schools' management on June 16, 2009 to investigate concerns about indoor environmental quality (IEQ) at Oak Ridge Elementary School located at 2050 Oak Ridge Road, in Oak Ridge, North Carolina (NC). The request listed concerns about mold, high levels of carbon dioxide $\left(\mathrm{CO}_{2}\right)$, and problems with the heating, ventilation, and air-conditioning (HVAC). Health concerns included headaches, fatigue, insomnia, dizziness, bronchitis, pneumonia, sinus problems, sore throat, rashes, nauseas, vision problems, and others.

\section{BACKGROUND}

Oak Ridge Elementary School was built in the 1930s with additions in the 1970s. The original building is currently used for the media center, library and offices including the health center, teachers' lounge, and testing room. In 2003, construction began on three new classroom wings. The addition was attached to the gym that was built in the 1970s and remodeled during the construction process. In May 2005, before the 2004/2005 school year ended, the kindergarten/first grade (k/1) wing and administrative wing were opened and occupied. The second/ third grade (2/3) and fourth/fifth grade (4/5) wings (Figure 1) opened in August 2005, a few days before the start of school. Classroom wings are to the west side of the school, the gym is to the east side, and the old original building is to the north side (Figure 1). The playgrounds and ball fields are the highest points on the site.

During the 2008/2009 school year, the school had 88 faculty and staff and 738 students. Since the reopening of the school in 2005, there have many issues regarding IEQ which are summarized in the result section under "Brief history of environmental findings and remediation at Oak Ridge Elementary School."

\section{ASSESSMENT}

A NIOSH team of two industrial hygienists, an epidemiologist, and a medical officer completed a site visit at Oak Ridge Elementary School from July 14-16, 2009. Consultants of the H.S. Turner Group (also known as the "Turner Group"), a building science and design company, hired by Guilford County Schools also participated in the site visit, and the NIOSH team conferred with them during and since the site visit.

On the first morning, we conducted an opening meeting with school staff, parents, and news media to discuss the HHE program and the purpose of our visit. We then met with the principal, teachers, and parents for approximately one hour to discuss their concerns. This was followed by a NIOSH initial walkthrough of the school accompanied by school staff, parents, and news media. During the rest of the site visit, members of the Parent Teacher Association often accompanied us as we evaluated the school.

In addition to a visual assessment of the school, we used moisture meters and an infrared camera to check for moisture in walls and other surfaces. Additionally, we used smoke tubes to visualize airflow and observe pressure differentials between rooms and the corridors inside the school building. The Turner Group used digital pressure gauges (The Energy Conservatory, Minneapolis, MN, USA), and NIOSH staff worked with the Turner Group staff to measure pressure differentials at exterior doors; the results were documented on diagrams of the school and given to the Turner Group for further analysis.

We selected 21 rooms (Table 1) to collect environmental samples (6 fully carpeted rooms, 11 carpeted rooms with 10 percent (\%) to $25 \%$ tiled floor, 4 rooms with tiled or wooden floor). These rooms were selected either because occupants had reported health or environmental issues, or the previous environmental consultants' reports documented elevated mold concentrations either in air or floor dust. We collected floor dust samples into polyethylene filter socks using a L'il Hummer ${ }^{\mathrm{TM}}$ backpack vacuum sampler (flow rate $=100$ cubic feet per minute). To collect enough dust for microbial analyses, we vacuumed either a 2 meter squared $\left(\mathrm{m}^{2}\right)$ area on the 
floor around the teacher's work station or around the edges of the room $\left(0.8 \mathrm{~m}^{2}\right)$ and $1 \mathrm{~m}^{2}$ area of floor around the teacher's work station (a total of $1.8 \mathrm{~m}^{2}$ ) for a total of five minutes. Even using these methods, we were not able to collect enough dust from room 108 for microbial analyses, and thus, the room was excluded in data analyses. In the media center, which was larger than classrooms and offices, we collected two separate dust samples; one from a $2 \mathrm{~m}^{2}$ area on a rug in the room and another from the edge of the room. We also measured wet bulb and dry bulb temperatures on the floor surface to compute water activity (Aw: the amount of free or available water for microorganisms) and air temperature and relative humidity $(\mathrm{RH})$ at the end of the dust sampling in each room.

The vacuumed dust samples were sealed in plastic bags and transported to the NIOSH laboratory where the collected dust in the filter socks was empted into 50 milliter $(\mathrm{ml})$ pyrogen-free conical tubes and homogenized by rotation on a 360-degree rotary arm shaker at 65 revolutions per minute for 2 hours. We removed hair, fluff, and other larger objects from the sample before homogenization. The dust samples were then weighed, partitioned, and sent to the American Industrial Hygiene Association (AIHA)-accredited Environmental Microbiology Laboratory P\&K (EMLab P\&K, San Bruno, California). We analyzed dust samples for culturable fungi with full speciation, gram negative bacteria, and Actinomycetes because these microbes have been associated with dampness in buildings. We also analyzed for gram positive bacteria as humans are often a source of this type of bacteria. Dust samples were cultured for Actinomycetes at three different temperatures - room temperature, 35 degrees Celsius $\left({ }^{\circ} \mathrm{C}\right)$, and $55^{\circ} \mathrm{C}$. The laboratory reported culturable fungi, bacteria, and Actinomycetes as colony forming units (cfu) per gram (g) of dust. We categorized fungal species into hydrophilic, mesophilic, and "other fungi" groups. Hydrophilic fungi (or water-loving fungi) are defined as fungal species which require high water content in building materials $(\mathrm{Aw}>0.9)$ for survival and growth. Mesophilic fungi are fungal species that require water activity to be between 0.8 and 0.9 . In damp conditions, hydrophilic fungi will overgrow mesophilic fungi. Fungal species which were not categorized as either hydrophilic or mesophilic were included in the "other fungi" group. We used three sources [WHO 2009; Hung et al. 2005; Flannigan and Miller 2001] to determine the water activity of the fungi in order to categorize them into one of the three groups. If a fungal species was classified differently (hydrophilic vs. mesophilic vs. "other") among the three sources, we choose the classification with the highest water activity. We computed a fraction of the hydrophilic and mesophilic fungi for each collected dust sample by dividing the concentration of each group of fungi by the total concentration.

We visited the Guilford County Department of Public Health. The medical director shared de-identified data from a self-administered paper questionnaire that the health department offered to the faculty/staff (Appendix A) and parents of students (Appendix B) at Oak Ridge Elementary School in May and June 2009.

NIOSH staff conducted telephone interviews with three staff members regarding health concerns believed related to the school.

On the last day of the site visit, we had a breakfast meeting with Guilford County School representatives regarding our findings and recommendations. This was followed by a brief closing meeting at the Oak Ridge Elementary School.

Prior to, during, and after the site visit, we obtained documentation from the school regarding indoor environmental quality issues at the school. We used this documentation to summarize the historical environmental problems and actions that had occurred at the school over the past five years.

\section{Health department questionnaire data}

In May 2009, the Guilford County Department of Public Health surveyed faculty/staff and parents/guardians of students at Oak Ridge Elementary School with self-administered paper questionnaires (Appendices A and B) that focused on the 2008/2009 and 2007/2008 school years. Throughout the report, we will refer to faculty and staff as "staff" or "staff members" and "parents" or "guardians" as "parents". The health department chose the 2008/2009 and 2007/2008 time frame to determine if there were any retrospective changes in building-related symptoms 


\section{ApPendIX M: NIOSH INTERIM RePORT, HETA 2009-0172 (CONTINUED)}

after the installation of a dehumification system in the classroom section of the school during the summer of 2008 between the two school years.

The staff and parent questionnaires included questions on primary office or classroom, symptoms, health conditions, medication, smoking status, and home exposures. For each specific school year (2008/2009 then 2007/2008), the participants were asked if they experienced any of the following symptoms while in the school building: 1) headaches, 2) dry eyes, 3) unusual sneezing episodes, 4) difficulty breathing/asthma attacks, 5) nosebleeds, or 6) any other symptoms (participant asked to write-in other symptoms). However, participants who answered "no" or did not write-in a symptom on the first page of the questionnaire (that asked about symptoms during the 2008/2009 school year) were asked to stop the questionnaire at that point. For these participants, no information was collected on symptoms during the 2007/2008 school year, health conditions, medication, smoking status, and home exposures. For participants who did have symptoms in 2008/2009, they were also asked if they had the following conditions: 1) chronic respiratory disease, 2) undergoing chemotherapy or radiation therapy, 3) immune system stressed by disease or other cause, 4) allergic rhinitis, or 5) asthma. The participants were asked to report rooms or areas in the building where their symptoms were worst and if their symptoms/problems went away after leaving the building. Additionally, they had the opportunity to write in additional information at the end of the questionnaire. Information about physician diagnosis of health conditions and date of onset of the health conditions in the questionnaire was not sought in the questionnaire. The questionnaires did not include standardized symptom or asthma questions which would have allowed us to compare rates to the US or state population rates.

We received the de-identified questionnaire data from the health department in excel format and converted it into SAS databases. The excel database had variables created for the specific health conditions and symptoms asked in the questionnaire. The database also had additional variables created for write-in symptoms that corresponded to the 2008/2009 or the 2007/2008 school year. The write-in variables included rash, nausea, sinus problems, insomnia, dizziness, fatigue, sore throat, and vision problems. We created additional variables for other symptoms written in by participants for each school year. These included cough, allergies, metallic taste, memory loss, eye symptoms (burning, itching, red, dry, or tearing), skin problems (eczema, rash, dry skin, itchy skin, or tingling skin), nasal symptoms (stuffy, itchy, burning, runny, or dry nose), throat symptoms (dry, scratchy, or sore), and voice symptoms (hoarse voice or laryngitis). Additionally, we grouped symptoms into upper respiratory symptoms (sinus problems, nasal symptoms, sneezing, throat symptoms, and/or voice symptoms), lower respiratory symptoms (breathing problem/asthma attack and/or cough), and mucous membrane irritation symptoms (eye symptoms, nasal symptoms, throat symptoms, and/or voice symptoms).

We grouped classroom and offices into six location categories (gym/cafeteria wing, administrative wing, media room wing, $\mathrm{k} / 1$ wing, $2 / 3$ wing, and $4 / 5$ wing). We assigned staff to a primary work wing based on the locations of their classrooms or offices. We assigned students to a classroom wing based on the locations of their primary classrooms/homerooms. If a student did not have a primary classroom/homeroom listed in the database, we assigned the student to a classroom wing based on his or her age (if it was available). Students 5 or 6 years old were assigned to the $\mathrm{k} / 1$ wing; students 8 years old to the $2 / 3$ wing; and students 10,11 , or 12 years old to the $4 / 5$ wing. We did not assign students aged 7 or 9 years old because their wing assignment would differ based on if they started kindergarten at 5 or 6 years old. Therefore in 2008/2009, we did not assign three 7 -year olds and four 9-year olds.

We used SAS version 9.2 (SAS Institute, Cary, NC) for data analyses. We calculated prevalence of symptoms reported by staff and parents for their children who attended Oak Ridge Elementary School during the 2008/2009 and/or 2007/2008 school year. We used the McNemar's test to determine if there was a statistical difference in symptom prevalence during the 2008/2009 and 2007/2008 school years in staff and students who worked or attended during the 2008/2009 and 2007/2008 school years and who had answered "yes" or wrote in a response to one of the six symptom questions on the first page of the health department questionnaire (Appendices A and 


\section{ApPendix M: NIOSH InteRIM Report, HETA 2009-0172 (CONTINUed)}

B). We used the likelihood ratio chi-square test to test for significance when we compared symptom prevalences during the 2008/2009 school year by classroom wing for staff and students. A probability level of $\mathrm{p}<0.05$ was considered statistically significant. A probability level of $\mathrm{p} \geq 0.05$ and $\mathrm{p}<0.10$ was considered marginally significant.

\section{RESULTS}

\section{Brief history of environmental findings and remediation at Oak Ridge Elementary School}

Prior to school opening in August 2005, library books were shrink-wrapped on homemade rolling press wood carts and moved from the library/media center in the old building to the pre-kindergarten classroom (room 613) in the $\mathrm{k} / 1$ wing (Figure 1) and stored for two months. In August 2005, when the 2/3 and 4/5 wings were completed, the books were moved to the temporary media center (classroom 500) in the 4/5 wing; at this time, the books and carts in the pre-k classroom were covered in a grayish green mold, and the room had an overwhelming moldy smell. A contractor cleaned the books before they were moved to classroom 500 and stored on wooden bookcases.

In August 2005, visible mold was also found on carpet in classrooms 427 and 625 (Figure 2), upholstered chairs in the records room (room 109) (Figure 3), and on picture frames in the storage room (room 111) (Figure 4). Later, after the school year began, mold started to grow back on some of the books in the temporary media center in classroom 500. Many books were thrown out while others were again cleaned. By the end of August 2005, visible mold had been identified in the $\mathrm{k} / 1$ wing (classroom 613, 625), 2/3 wing (classroom 427), 4/5 wing (classroom 500), and the administrative wing (rooms 109 and 111).

During this time frame, the HVAC system was being commissioned, and the school was having problems with high RH. Additionally, parents had begun notifying the school that their children were complaining of headaches and respiratory symptoms. On August 25, 2005, the Guilford County Department of Public Health received two complaints about mold on library books. The health department inspector who visited the school reported that the school was humid, and that this issue needed to be addressed; otherwise, the mold would likely come back. The inspector returned on August 31, 2005 and noted that the humidity issues had improved; however, staff reported headaches and irritation of the eyes, throat, and ears.

During September 2005, the school continued to adjust the HVAC system; mold and RH were still a concern. A consultant found that air filters in the heat pump (HP) units were very dusty and poorly fitting; they were replaced. The unit (HP 42) that served classroom 500 had some visible dry buildup on the coil front; a sample of the material grew mold (Pencillium). RH in the majority of rooms tested exceeded $60 \%$ (ranged from $61-72 \%$ ). Elevated mold counts in carpet samples resulted in the replacement of carpet in classroom 625 with new carpet and in the health center (room 101) with vinyl composition tile (VCT).

In September 2005, the North Carolina Division of Occupational Safety and Health received a complaint regarding 1) elevated carbon dioxide $\left(\mathrm{CO}_{2}\right)$ levels (2000 parts per million (ppm) and $\left.5000 \mathrm{ppm}\right)$ in classrooms 502 and 508 in the 4/5 wing, 2) exposure to mold in the school (including the media center which was closed due to mold on books, the health center, classroom 520, and around the gym), and 3) respiratory symptoms in employees. Additionally during this time, parents continued to report their children were having respiratory symptoms, headaches, and fatigue.

In response to the complaint to the state agency, the school hired an industrial hygiene consultant to test and monitor issues related to indoor air quality. He reported that a malfunctioning heat pump and damper were the cause of the elevated $\mathrm{CO}_{2}$ levels in classrooms 502 and 508. The heat pump was replaced, and the damper was fixed. The consultant also noted in the report that an outside wall in classroom 400 was water damaged due to an exterior leak during construction, and this was to be removed by a mold remediation firm. 


\section{Appendix M: NIOSH InTeRIM Report, HETA 2009-0172 (CONTINUED)}

In October 2005, the North Carolina Division of Occupational Safety and Health closed their case at the school on the grounds that the alleged hazardous conditions had been corrected or no longer existed. However during October 2005, the Guilford County Department of Public Health notified the school district that the health department continued to receive complaints from parents regarding indoor air quality issues such as mold and RH at Oak Ridge Elementary School. There were also concerns about the water supply and radon levels at the school which upon testing were found to be within normal range.

In November 2005, the school continued to have elevated $\mathrm{CO}_{2}$ levels in some classrooms. They included classroom 503, 1534 ppm (fully occupied); classroom 505, 1484 ppm (fully occupied); classroom 623, 1250 ppm (fully occupied), and classroom 625, 1370 ppm (fully occupied). Return air dampers were installed in some classrooms so more outdoor air could be delivered via the intake. However, during this same month, the water boiler and several heat pumps had "tripped out" for unknown reasons. Apparently, the heat pump fans may have been "off" during $\mathrm{CO}_{2}$ testing, and this may have resulted in the elevated $\mathrm{CO}_{2}$ levels. The $\mathrm{CO}_{2}$ set point for the four classrooms with elevated $\mathrm{CO}_{2}$ levels (HP 28 for classrooms 623 and 625 and HP 41 for classrooms 503 and 505 ) were set to $900 \mathrm{ppm}$. However, the school continued to have problems with elevated $\mathrm{CO}_{2}$ levels and high RH.

Mold continued to be found in the school. In the Testing Room (room 115) on February 2006, mold was identified from surface samples taken from the top of a dusty mobile cart and from a small canvas bag that sat on a dusty bookcase. The canvas bag was discarded. The industrial hygiene consultant recommended that the room and surfaces be professionally cleaned, which was done.

In May 2006, many teachers reported "stuffy" rooms, and left their windows open for end-of-grade testing to avoid symptoms in their students during the testing. In early June 2006, the construction company was notified (by the architect) that the $\mathrm{CO}_{2}$ controllers did not meet the specifications in the contract documents. Additionally, he notified them of a broken storm drainage line and problems with sewage lines (also identified in a February 2006 inspection).

In mid June 2006, a Trane MP581 programmable controller with temperature, $\mathrm{CO}_{2}$, and humidity sensors was installed at the school. A two day test run was done with the new unit controlling classroom 618 (HP-26) in the $\mathrm{k} / 1$ wing. This was compared to classroom 627 (HP-25) across the hall which did not have the new sequence installed. During the first day of testing, it was rainy with a temperature of 65 degrees Fahrenheit $\left({ }^{\circ} \mathrm{F}\right)$ with a $\mathrm{RH}$ of $90 \%$; during the second day, it was sunny with the outside temperature and $\mathrm{RH}$ ranging from $73-80{ }^{\circ} \mathrm{F}$ and $32-49 \%$, respectively. The Trane programmable unit sequence of operation called for the unit to be placed in dehumidification mode when the zone humidity reached $65 \% \mathrm{RH}$ and "off" when the zone humidity reached $55 \%$ RH. However, during the two days, the compressor did not turn off due to the zone humidity levels ranging between approximately $64 \%$ and $70 \%$ and never reaching the $55 \%$ level. The temperature stayed between approximately $71^{\circ} \mathrm{F}$ and $73^{\circ} \mathrm{F}$. The Trane consultants determined that since there was no type of reheat available, the only way to keep the room from over-cooling during the dehumidification mode was to switch the unit from cooling to heating. Once this occurred, the air entering the space was no longer dehumidified, and the humidity rapidly rose. However, once the discharge air temperature was low enough to dehumidify the air, the room again became over cooled, and the unit had to be changed back to the heating mode. The Trane consultants had concerns about the "wear and tear" on the equipment. During the test, the compressor never shut off because the room RH never reached 55\%. Also, the reversing valve changed from cooling to heating or from heating to cooling over 25 times during three hours of operation. During normal operation, the reversing valve should only change once or twice in a given day because it is usually heating or cooling, not switching back and forth between the two. The consultants thought that most of the humidity problems were associated with the introduction of outside air. They recommended that the outside air be "pre-treated" with dedicated units to allow the existing heat pumps to control the zone temperature.

The school continued to meet with Trane personnel, and they adjusted the "test" control system so it better 


\section{Appendix M: NIOSH InteRIM Report, HETA 2009-0172 (CONTINUed)}

handled humidity with minimal reversing of the unit from cooling to heating. In July 2006, the temperature and $\mathrm{RH}$ in classroom 520, a room with complaints, was monitored for five days. During the day, the temperature ranged from approximately $70-77^{\circ} \mathrm{F}$, and $\mathrm{RH}$ ranged from $50-60 \%$; however, during other times the temperature reached above $80^{\circ} \mathrm{F}$ and $65 \% \mathrm{RH}$.

On July 22, 2006, several roof leaks occurred in the school after a heavy rain. Water penetrated rooms in the $\mathrm{k} / 1$ wing $(603,606,608,610,616,619$, corridor between 603 and 608), 2/3 wing (401, 407, 418, 421, 427), 4/5 wing (500 and 505), the administrative wing $(104,106,108)$, and gym/cafeteria wing (201). In August 2006, visible mold was discovered on carpet in classrooms 406, 424, 427, and 623. The indoor RH was reported to be high during this time. The carpets in these rooms were cleaned and treated with an antimicrobial agent (or biocide). This same month, a leak occurred in a water line joint, which was subsequently fixed.

In February 2007, the North Carolina Department of Environmental and Natural Resources reported several ceiling leaks during a school inspection.

In October 2007, spore trap analysis was performed outside and inside the school. The outside total spore count was 14,302 spores $/ \mathrm{m}^{3}$; the top four outside fungal genera identified were Cladosporium, Ascospores, Basidiospores, and Aspergillus/Penicillium. Eleven indoor locations (416, 503, 505, 514, 517, 519, 521, 618, 625,627 , and the main office) were tested, and the total spore count ranged from 353 to $5,055 \mathrm{spores} / \mathrm{m}^{3}$. The five rooms with the highest total spore counts were classroom $521\left(7,680 \mathrm{spores} / \mathrm{m}^{3}\right)$, the main office $(5,055 \mathrm{spores} /$ $\left.\mathrm{m}^{3}\right)$, classroom $517\left(3,370\right.$ spores $\left./ \mathrm{m}^{3}\right)$, classroom $505\left(2,586\right.$ spores $\left./ \mathrm{m}^{3}\right)$, and classroom $503\left(2,429 \mathrm{spores} / \mathrm{m}^{3}\right)$. The top four outside fungal genera were also found in many of the classrooms but at lower levels. Alternaria counts were higher in the main office and in classrooms 503,505,514, 517, and 521 than levels found outside. Curvularia counts were higher in the main office and classrooms 503, 514, 517, and 521 than outdoor levels. Drechslera/Bipolaris counts were higher in the main office and classrooms 505, 517, and 521 than outdoor levels. Pithomyces was higher in the main office and classroom 521 than outdoors levels. Nigrospora was not found outside but was found in the main office and classrooms 517 and 521. Epicoccum counts were higher in classroom 521 than found outside. Myxomycete/Smut-like was not found outside but was found in the main office and classrooms 503, 505, 514, 517, 521, 625, and 627.

(NOTE: NIOSH indoor air scientists do not currently recommend routine air sampling for mold in indoor air quality evaluations because there are no established health-based standards for acceptable levels of biological agents and limited short-term air sampling may be misleading.)

Later in October 2007, consultants tested for $\mathrm{CO}_{2}$ and performed air, surface, and carpet samples for fungal materials. $\mathrm{CO}_{2}$ levels were above the level recommended by the American Society of Heating, Refrigeration, and Air-Conditioning Engineers (ASHRAE) in 10 (416, 503, 514, 517, 519, 520, 521, 618, 625, 627) of 13 rooms tested. Three (classrooms 416, 618, 625) of the 10 rooms with elevated $\mathrm{CO}_{2}$ had levels that exceeded the 1,500 parts per million (ppm) established by North Carolina schools. Spore trap analysis performed inside and outside the school revealed an outdoor total spore count of 2,083 spores $/ \mathrm{m}^{3}$ (outside the front of the school) and 2,983 spores $/ \mathrm{m}^{3}$ (outside classroom 405). The top four fungal genera identified in the sample collected outside the front of the school were Basidiospores, Cladosporium, Penicillum/Aspergillus, and Ascospores. Ten indoor locations (classrooms 405, 416, 503, 514, 517, 519, 521, 608, 625, and office lobby) were tested, and the total spore count ranged from 66 to 359 spores $/ \mathrm{m}^{3}$. The five rooms with the highest total spore counts were classroom 503 (359 spores $\left./ \mathrm{m}^{3}\right)$, classroom $517\left(346\right.$ spores $\left./ \mathrm{m}^{3}\right)$, classroom $521\left(293 \mathrm{spores} / \mathrm{m}^{3}\right)$, classroom $625\left(213 \mathrm{spores} / \mathrm{m}^{3}\right)$, and classroom 608 (212 spores $\left./ \mathrm{m}^{3}\right)$. The top four indoor fungal genera identified were Basidiospores, Cladosporium, Penicillum/Aspergillus, and Ascospores.

Surface samples were collected from the insulation in the supply air plenums of nine air handling units. Six of the nine samples had fungal growth of Cladosporium. Three of these air handlers (air handlers 37, 41, and 49) had trace to minimal fungal growth; two had low (air handler 26) to moderate (air handler 12) fungal growth, and 


\section{Appendix M: NIOSH InteRIM Report, HETA 2009-0172 (CONTINUed)}

one (air handler 14) had a high surface concentration of fungal growth. The consultants recommended that the insulation inside these six air handlers be cleaned or replaced. In December 2007, a cleaning company cleaned air handlers $12,14,26$, and 49.

Carpet dust samples from classrooms 423, 517, 519, 521, and the office lobby showed the concentration of viable fungal spores ranged from 132,600 cfu/gram in room 517 to 1,176,000 cfu/gram in classroom 423 . The consultants recommended that 1) carpets be cleaned with a commercial grade HEPA vacuum cleaner, 2) use of mats to capture particulate at the outdoor entry way to rooms or areas affected, and 3) possibly route students returning from the outside through the main entrance to the facility. Guilford County Schools reported they 1) delivered three new Pro-Team HEPA filtered back-pack vacuum cleaners and two new Windsor HEPA-filtered upright vacuum cleaners, 2) secured doorway mats, and 3) addressed student reentry into the school through the main entrance.

In January 2008, an inspector from the North Carolina Department of Environmental and Natural Resources reported a "bad leak" in the Central Receiving Room ceiling during a school inspection.

In September 2008, consultants performed temperature, humidity, and $\mathrm{CO}_{2}$ measurements and carpet sampling for fungal spores and total volatile organic compound (VOC) sampling. Indoor temperature (range measured: 71.3-72. $4^{\circ} \mathrm{F}$ ) and humidity readings (range measured: 48.9-53.0\%) were within the ASHRAE recommended levels. $\mathrm{CO}_{2}$ measurements (range measured: 657-1189 ppm) were elevated in classroom 514 (1189 ppm) and classroom $519(1086 \mathrm{ppm})$. The consultants reported that the air handling unit serving classroom 519 might not have been functioning properly at the time of testing. Additionally, the consultant noted the window in classroom $521(651 \mathrm{ppm})$ may have been open during testing in that room. These rooms were retested, and the $\mathrm{CO}_{2}$ levels in classroom 519 (954 ppm) and classroom $521(898 \mathrm{ppm})$ were below the ASHRAE recommended limit. Classroom 514 had an elevated $\mathrm{CO}_{2}$ measurement of 1,158 ppm. Guilford County Schools reported making adjustments to the air handler for room 514 to drop the $\mathrm{CO}_{2}$ levels. In classroom 407, carpet sampling for fungal spores revealed 1,300,000 cfu/g; the top three fungal genera identified were Alternaria, Aspergillus, and Cladosporium. Air sampling for fungal spores (spore trap analysis) performed in two locations outside of the school building revealed a total spore count of $10,736 \mathrm{spores} / \mathrm{m}^{3}$ and $6,896 \mathrm{spores} / \mathrm{m}^{3}$. Eleven indoor locations (office and classrooms 405, 407, 414, 501, 514, 519, 520, 521, 618, and 627) were tested, and the total spore count ranged from $<78$ to 392 spores $/ \mathrm{m}^{3}$. The three rooms with the highest total spore counts were classroom 514 (392 spores $\left./ \mathrm{m}^{3}\right)$, classroom 618 (313 spores $\left./ \mathrm{m}^{3}\right)$, and classroom 521 with window open $\left(313 \mathrm{spores} / \mathrm{m}^{3}\right)$. Three air samples for total VOCs were taken and all were below $0.5 \mathrm{mg} / \mathrm{m}^{3}$. The specific locations of the VOC sampling were not mentioned in the report.

In October 2008, carpet in the main lobby, the secretary/treasurer's office, and classrooms 407, 501, 503 were cleaned and treated with Sporicidin. HVAC units 12 (front office), 48 (classroom 501), and 50 (classroom 407) were cleaned including contact vacuuming with a HEPA vacuum. Debri was captured with a negative pressure HEPA vacuum. Additionally, fan blowers and coils were HEPA vacuumed, and the HVACs were sanitized with Sporicidin. The rooms were wiped down and HEPA vacuumed following the cleaning process.

In December 2008, an off-white substance was observed coming from the ductwork in the main office area and one of the classrooms (room 618) after cleaning had been conducted in some air handling units at the school. There was concern that the white substance was from the coil cleaning agent or possibly the mineral content in the water. An industrial hygiene consultant observed an off-white powdery substance in the three air handler units inspected and informed a school official who contacted the cleaning contractor and was told that no white substances were used in the cleaning of the coils. The coil cleaner contained butyl cellosolve (also called ethylene glycol monobutyl ether or 2-butoxyethanol), a type of glycol ether, which is water soluble and often used as a solvent. The industrial hygiene consultant performed total particulate monitoring, air sampling for butyl cellosolve, and air and surface sampling for fungal materials in the office area and room 618. In the three areas (office lobby and rooms 113 and 618) where the white substance was observed coming out of the duct work, the 


\section{ApPendix M: NIOSH InTeRIM RepORt, HETA 2009-0172 (CONTINUED)}

total particulate concentrations ranged from $0.20-0.53 \mathrm{mg} / \mathrm{m}^{3}$ and did not differ significantly from the break room (room 123) measurement of $0.34 \mathrm{mg} / \mathrm{m}^{3}$. The break room had not had coil cleaning and was tested in order to compare results with the affected areas. All three air sampling results (office lobby and rooms 113 and 618) for butyl cellosolve were below the detection limit $(<0.72 \mathrm{ppm})$ for the analytical method; thus, they were below the NIOSH recommended exposure limit (REL) $(5 \mathrm{ppm})$ and the Occupational Safety and Health Administration (OSHA) permissible exposure limit (PEL) $(50 \mathrm{ppm})$ at the time of testing. Two outdoor fungal air samples (spore trap analysis) revealed a total spore count of 1,746 structures $/ \mathrm{m}^{3}$ and 653 structures $/ \mathrm{m}^{3}$ compared to total spore counts of 53 structures $/ \mathrm{m}^{3}$ in room 618 , and 93 structures $/ \mathrm{m}^{3}$ in the office lobby, and 653 structures $/ \mathrm{m}^{3}$ in room 113. The laboratory detected an Aspergillus conidiophore in the air sample taken in room 113. The consultants conducted surface sampling on horizontal surfaces in three locations (office conference room table, lobby log-in book, and plastic lid in classroom 618) where the white substance was observed. Very few miscellaneous spores were present; no mold growth occurred in the samples taken.

In early April 2009, an industrial hygiene consultant from the Occupational and Environmental Epidemiology Branch of the North Carolina Department of Health and Human Services and a building systems engineer for the public schools of North Carolina performed site visits to evaluate the indoor environment at the school. During the site visit, the industrial hygiene consultant reported a moldy odor in the northwest corner of the assistant principal's office (room 108) where carpet had been pulled up. He noted that the northwest wall was a common wall to the new and the old buildings. He also noted a history of recurrent water damage to the ceiling tiles above the ramp which connects the new and old buildings. Additionally, there had been evidence of termites swarming that area. In classroom 520 in the 4/5 wing, he reported a musty, earthy odor or an odor similar to citrus-based cleaning products when a carpet square was lifted. He also reported a microbial odor outside the bathrooms in the 4/5 hall and a history of roof leaks in the music room (room 407) and the Spanish room (room 501) which share a common wall located under an area where a north-south roof intersects an east-west roof. In classroom 427 , he reported a history of recurring problems with mold growth in the carpet. He recommended the school district 1) remove the carpet (using proper procedures) and replace with VCT (after measurement of concrete floor moisture content) in rooms 108, 520, and the music room; 2) identify and correct the sources of water movement through the exterior wall in room 108; 3) repair flashing on the parapet where the old and new building were joined; 4) investigate the source of roof leaks above the music and Spanish room; 5) encourage students to use the main doors to enter and exit the building to reduce the amount of soils, dust, debris and allergens brought into the building; 6) install barrier mats at entrances that are long enough that a person would take at least five full steps on the mat; 7) vacuum carpet daily; 8) identify and correct odor sources at the bathroom in the 4/5 hall; 9) investigate sources of moisture under the concrete slab; 10) integrate pest management techniques to control the termite infestation where the new and old buildings meet; and 11) analyze absentee data or other data to look for patterns where problems may exist.

During the same site visit, the building systems engineer for the public schools of North Carolina reported ceiling tile stains throughout the school especially, in the area where the old and new buildings were structurally joined. He noted in his report there was a tendency to replace stained ceiling tiles before repairing the leaks. He recommended fixing leaks before replacing the ceiling tiles. He noted that foam roof sections in the older section of the school were showing signs of deterioration and would need to be replaced especially if there were an increase in the number of leaks. He noted that flashing connections in some areas over the media center did not cover enough of the wall to prevent rain penetration, and some of the exterior bricks on the wall had deteriorated which may have contributed to the leaks in the assistant principal's office. He recommended that the metal cap flashing on the north side be extended down to cover the gaps between the existing flashing and the brick wall. The engineer also reported that surface water drainage toward the building kept the slab wet. He recommended reducing moisture migration toward the building slab by installing a French drain between the school and the high ground of the playground area to drain groundwater toward the streets in order to keep it away from the building. He recommended construction of swales around the building on the playground side and improvement of drainage around the courtyard surface areas to better drain water away from the buildings. He noted that some of the existing drop inlets might need to be lowered. He reported variations in $\mathrm{RH}$ in different areas and that 


\section{ApPendix M: NIOSH INTERIM RepORT, HETA 2009-0172 (CONTINUED)}

the classrooms closest to the playground generally appeared to have moisture-related issues. He recommended keeping the RH ideally between 30 and $60 \%$ with installation of room dehumidifiers in classrooms where the HVAC system could not maintain an RH of $60 \%$ or below. The school district reported that they tested the RH in the school, and that on average, the RH readings were at or below $60 \%$. Lastly, he reported a significant amount of visible mold under the carpet in the assistant principal's office. He noted that several classrooms had odors which where masked by carpet cleaning compounds. He recommended removal of carpet and replacement with floor tile in carpeted rooms were odors and/or moisture where detected.

Later in April 2009, another industrial hygiene consultant from Workplace Group performed bulk sampling of adhesive beneath the carpet in classroom 501; surface testing of the underside of carpet for fungal materials in classroom 520 and the assistant principal's office; and monitored temperature, humidity, and $\mathrm{CO}_{2}$ in classrooms 516 and 520. Bulk sampling of the adhesive beneath the carpet in classroom 501 was negative for fungal growth or spores. In the assistant principal's office and classroom 520, Aspergillus and Pencillium were detected on the carpet backing. Mold growth was rated from 1 to 4 with 4 denoting the highest concentration. The mold score was 3 in classroom 520 and 4 in the assistant principal's office. In classroom 516, the temperature ranged from 66.7-75. ${ }^{\circ} \mathrm{F}$ (average $70.8^{\circ} \mathrm{F}$, median $70.2^{\circ} \mathrm{F}$ ); the RH ranged from $22.7-30.9 \%$ (average: $25.9 \%$, median: $26.1 \%$ ); and $\mathrm{CO}_{2}$ from 350-750 ppm (average: $500 \mathrm{ppm}$, median: $460 \mathrm{ppm}$ ). In room 520, the temperature ranged from $67.4^{\circ} \mathrm{F}-75.3$ (average: $73.0^{\circ} \mathrm{F}$, median: $73.5^{\circ} \mathrm{F}$ ); the RH from 20.6-51.0\% (average: $33.3 \%$, median $33.0 \%$ ); and $\mathrm{CO}_{2}$ from 330-1710 ppm (average: $700 \mathrm{ppm}$, median: $540 \mathrm{ppm}$ ), exceeding the recommended limit of 1,100 ppm at one point during testing.

In mid April 2009, floor consultants performed moisture testing of interior floor slabs in the assistant principal's office and classrooms 427, 516, 518, 520, and 618. During this same time frame, a consultant engineer performed a site visit to review floor and wall conditions. In his report, he noted that interior floor slabs were being tested for moisture by a flooring company, and the surface preparation was suspect, as evidenced by adhesive residue and curing compounds under the testing chamber. He mentioned that the school was reported to have had high levels of humidity for an extended period of time when the new addition was opened in 2005, and adjustments had been made to the HVAC system. Monitors for temperature and humidity were removed, and relative humidity was no longer monitored on the interior of the school. Surface water draining from the elevated ballfields was allowed to pond in areas adjacent to the concrete walkways instead of being directed across the gravel roadway. He also noted significant roof leaks on the flat roof over the utility/storage room at the southeast corner of the school just west of the Gym structure. He reported over 18 ceiling tiles were damaged. In the main hallway where the old and the new buildings interface, he reported damaged ceiling tiles from water intrusion which appeared to be from a roof leak.

In mid April, the carpet in the assistant principal' office was replaced with VCT.

During the second week in May 2009, a senior environmental health specialist from Guilford County Department of Public Health toured the site and noted a water stained ceiling tile in room 619. She noted in her report that a previous site visit by state and local agencies on April 3, 2009 outlined a variety of issues and recommendations.

Also during the second week of May 2009, an industrial hygiene consultant from Workplace Hygiene conducted carpet, surface, and air sampling for fungal materials (in rooms 115, 206, 420, 421, 422, 423, 425, 427, 500, 503, $514,515,516,610,616,618,627)$, and VOC air sampling. The indoor fungal air samples ranged from no spores detected in classroom 627 to 940 spores per cubic meter in classroom 420. The six rooms with the highest total spores were classroom $420\left(940\right.$ spores $\left./ \mathrm{m}^{3}\right)$, classroom $206\left(705 \mathrm{spores} / \mathrm{m}^{3}\right)$, classroom $427\left(549 \mathrm{spores} / \mathrm{m}^{3}\right)$, classroom 514 (549 spores $\left./ \mathrm{m}^{3}\right)$, classroom 115 (470 spores $\left./ \mathrm{m}^{3}\right)$, and classroom 425 (470 spores $\left./ \mathrm{m}^{3}\right)$. The outdoor samples (outside of $2 / 3$ and $\mathrm{k} / 1$ wings) ranged from 18,103 to 28,134 spores $/ \mathrm{m}^{3}$; the top three outside fungi species identified were Ascospores, Basidiospores, and Cladosporium. The outdoor fungi were higher than the indoor fungi except for Myxomycete/Smut-like which was higher in room 420. Surface sampling was conducted beneath the carpeting in the same 17 rooms, in a paper tray in room 115, and on a wall that experienced 


\section{ApPendix M: NIOSH InTeRIM RepORt, HETA 2009-0172 (CONTINUED)}

water damage in room 500. Because the carpet was glued to the floor, only one location was selected from each room. With the exception of the $\mathrm{k} / 1$ wing and room 206, the samples were collected near the exterior doors of each classroom because the consultants thought this area would likely have the moisture issues. The carpet in the rooms in the $\mathrm{k} / 1$ wing did not extend to exterior doors and room 206 did not have an exterior door. Spores were not detected on the carpet backing in rooms $425,503,515$, and 616. In the other areas sampled with the exception of room 421, the spore concentrations were characterized as "trace to light." The carpet backing in room 421 had a surface concentration of mold spores identified as Aspergillus/Penicillium characterized as "loaded" and concentrations of basidiospores and Cladosporium characterized as "trace" to "light".

Carpet sampling results ranged from 30,000 cfu/gram in room 115 to $>2,000,000 \mathrm{cfu} / \mathrm{gram}$ in classroom 516 . Six of the 16 results exceeded 1,000,000 cfu/gram (room 206, >1,333,333; classroom 514, >1,600,000; classroom 515, >1,600,000; classroom 516, >2,000,000; classroom 618, >1,000,000; classroom 627, 1,460,000 ). Total VOC air sampling ranged from $0.18 \mathrm{mg} / \mathrm{m}^{3}$ for a sample collected outdoors to $1.6 \mathrm{mg} / \mathrm{m}^{3}$ in classroom 425 . The consultants noted in their report that the Green Building Council established a recommended concentration limit for VOCs of $0.5 \mathrm{mg} / \mathrm{m}^{3}$ for new buildings, and Washington State established $0.5 \mathrm{mg} / \mathrm{m}^{3}$ in buildings. Six (420, $423,425,500,501,514)$ of the 20 areas sampled exceeded the $0.5 \mathrm{mg} / \mathrm{m}^{3}$ limit. The consultant reported cleaning activities were going on during the sampling, and this may have affected the results. The cleaning products used were not listed. The industrial hygiene consultant recommended the school district 1) test for aldehydes to determine whether their presence contributed to reports of bloody noses, 2) test (by smoke or pressure) the drain lines to check for leaks 3) inspect and clean the air handlers (if necessary), and replace any contaminated insulations (if found), and 4) forward the consultant's report to the county health department.

Later in May 2009, an engineer consultant assessed the school and found "slight humidity in the administration office", however, thought the air quality in the school seemed acceptable. He evaluated the mechanical systems in the $2 / 3$ and $4 / 5$ wings and thought the system was well designed. However, in an outdoor air unit, he found standing water in the condensate drain pan and noted the filters for these units needed to be replaced. The engineer relieved the pressure on the unit and drained the pan completely. He noted that the units were designed to be free of standing water and recommended that drainage of these units be looked at to determine the problem. Another contractor checked the drain pans and found that the drain piping did not appear to allow complete drainage in the drain pan. However, he did not note carryover of moisture to the fan compartment or ductwork and did not see any dirt or other issue that would cause a mold issue in the unit or drain pan. The two consultants agreed that an adjustment in the trays should solve the problem. A school staff member was reported to start the work immediately.

The engineer consultant also found that the $\mathrm{CO}_{2}$ ventilation system had been disabled, sensors had been "jumped out", and the outdoor air units had been set at $100 \%$ which appeared to be done to get as much air into the building as possible. However, outdoor air did not appear to be getting into the building. $\mathrm{CO}_{2}$ readings inside the building (that was unoccupied for three days) were around $500 \mathrm{ppm}$. Additionally, the building was noted to be in a neutral pressure condition but with the $\mathrm{CO}_{2}$ system disabled it apparently should have been in a positive pressure condition. The consultant noted that the HVAC system serving the school was a nice design but was not operating correctly. He recommended the system be re-commissioned and the outdoor air system be checked to see if it was sized correctly. A third consultant noted that that the $\mathrm{CO}_{2}$ sensors were part of a previous attempt to control fresh air intake and were not part of the current ventilation control. However, when the system was most recently balanced, the dampers were bypassed in the open position to allow the scheduled fresh air to be delivered from the new air handling unit to the classroom units to ensure the right amount of air reached each unit. The prior summer, when the new system was installed, fans and dampers were set up and a balance contractor verified flow to each unit and all were apparently $95 \%$ or better for air scheduled to go to the units. The school planned to have the ventilation air calculations double checked to determine if any further re-commissioning was necessary. The engineer consultant found a lot of construction dust in the return ductwork and recommended the duct system be cleaned. He noted the ductwork on the other side of the filter was clean, and the filters were being maintained. 


\section{Appendix M: NIOSH InTERIM RepORT, HETA 2009-0172 (CONTINUED)}

During May 2009, the carpet in classrooms 407, 501, 508, 518, and 625 was replaced with VCT. During the last weekend in May 2009, a carpet cleaning company cleaned all the remaining carpet in the school with an antimicrobial agent.

During the last week in May 2009, the Guilford County Department of Public Health distributed questionnaires for staff and parents at Oak Ridge Elementary School. In mid June 2009, the medical director of the health department and his colleagues toured the school. In a media briefing on the following day (June 16, 2009), he reported that when they toured the school a month earlier they could find no mold or dampness; however, the day before, they did observe moisture in a wall and a small patch of mildew behind a banner in the gymnasium

The Guilford County Department of Public Health reported in a document dated June 25, 2009 that approximately 800 questionnaire surveys were distributed and about half were returned within the week's deadline (in Appendix C). The document stated that the summary of the responses showed that the "driving" symptoms were headache and fatigue that occurred within the school and resolved when fresh air was obtained by opening windows and doors to the classrooms, by going outside, or by going home. The document noted that nosebleeds were also common, and other symptoms were tabulated, but their frequencies were not as elevated; nor was there a specific pattern evident.

In mid July 2009, NIOSH and the Turner Group performed a three day site visit at Oak Ridge Elementary School. On the last day during a breakfast meeting, NIOSH staff and the Turner Group met with representatives from Guilford County Schools. The Turner Group summarized their preliminary findings in a document dated July 21, 2009 (Appendix D). Their findings and recommendations included: 1) finding: dirt floor crawl space under the media center as possible source of mold / recommendation: install flexible vapor barrier and depressurize air space under the vapor barrier in the crawl space under the media center; 2) finding: inadequate flashing at junction between the parapet wall and gym wall above room 206 causing water intrusion leading to mold growth in the carpet / recommendation: install through-wall-flashing at edge of parapet to connect through brick to drainage layer behind; 3) finding: discolored drywall on exterior wall and repaired flashing at wall in classroom 400 (once a leak source with occupant reports of mold found under the cove base) / recommendation: repair roof junction joints to provide adequate flashing; 4) finding: dehumidification system operation suspect, evidence by high humidity $(70 \%+)$ in the $4 / 5$ wing / recommendation: provide retro commissioning of HVAC system with respect to verify proper system capacity and sequence of operation; 5) finding: possible heat pump refrigerant leaks indicated by $64^{\circ} \mathrm{F}$ discharge at HP serving classroom 520 and humidity levels monitored at $70-80 \%$ in room, or quantity of outside air being handled exceeded coil capacity / recommendation: provide retro commissioning of HVAC system with respect to verifying proper system capacity and sequence of operation; 6) finding: classroom wings not sealed at soffit, allowing excess air leakage in and out (depending on mechanical system operation) / recommendation: sealing of soffit to prevent air infiltration and ensure means for exhaust/relief; 7) finding: heat pump design conducive to growing mold due to proximity of filter and coiling coil / recommendation: provide new filter boxes upstream to create separation between coil and filter; 8) finding: concerns about odors from dry traps, particularly at heat pump drains, since air distributed to occupants / recommendation: review maintenance tasks and schedules, on-going maintenance issue; 9) finding: bathrooms not at negative pressure consistently with respect to hall / recommendation: balance air flows as part of retro commissioning process, possibly move some supply air to hall; 10) finding: mechanical room in office area under significant negative pressure / recommendation: provide duct joint sealant on all return air and possibly supply air and verify air flows on retro commissioning; 11) finding: unknown issues regarding storm runoff at the end of the $2 / 3$ and $4 / 5$ wings with possibility of water getting under slab if it pools against the exterior walls / recommendation: verify adequate site drainage, clean site drainage system as necessary, and provide means to limit runoff sand and other surface material; 12) finding: original design of classroom wings modulated between 3.5 cubic feet per minute per person and 7.5 cubic feet per minute per person from $1000 \mathrm{ppm} \mathrm{CO} 2$ to $1800 \mathrm{ppm}$; when dehumidification systems installed, carbon dioxide control eliminated and all areas receive 7.5cubic feet per minute per person / recommendation: provide balancing of outdoor air system and marking of dampers as part of retro 


\section{ApPendIX M: NIOSH INTERIM RePORT, HETA 2009-0172 (CONTINUED)}

commissioning project; 13) finding: numerous open bubbles on the foam roof with compromise of the aluminum paint / recommendation: obtain roofing contractors to estimate the repair/replace of the foam roof; 14) finding: possible leak from sewer vent and/or roof flashing of vent thru roof in classroom 500 / recommendation: replace or repair flashing; 15) finding: building not observed during the occupied mode and some operational conditions were not observed / recommendation: provide monitoring of conditions during occupancy to evaluate operation under occupancy (after retro commissioning of systems).

The Guilford County Schools continued to consult with the Turner Group to address issues found during the initial site visit in July 2009 and other issues found during further testing.

In August 2009, Oak Ridge Elementary School remained closed after the summer break for remediation. Students attended three alternate sites. Kindergartners and first-graders attended Colfax Elementary school; second and third graders attended Oak Ridge Military Academy; and fourth and fifth graders attended Northwest Middle School.

Some additional findings identified by the Turner Group (Appendices E and F) included visible mold on some small portions of gypsum board removed at floor level, water damaged walls in the clerestory area above the media center that were suspected to have supported mold growth in the past. The walls were removed or cleaned. Dried leaves and other dried organic material found trapped in the building construction in the media center were removed. Some of the clerestory windows were resealed and existing roof scuppers were lowered. An old ventilation system in the clerestory space that was no longer in use was removed and the openings sealed because it was defeating the building thermal envelope. Blower door tests and infrared imaging indicated that the soffits in the classroom wings were a source of many air leaks. Other heat and air leak areas identified on the thermal imaging included doors, windows, and the base of the building wall. The Turner Group consultants' reviewed photos that showed some plumbing waste line vents in the classroom wings were open to the space above the ceilings and should have been connected to the waste line vents. This was corrected. Additionally, some of the waste line vents through the roof were less than 10 feet from the roof mounted air inlets. They were apparently relocated to meet the minimum 10 feet distance between roof vents and air inlets specified by construction drawings. Slab testing results reported in the Turner Group's final report noted that the concrete flooring in some rooms $(106,110,400,425,502,514,608,610,614,616,617,618$, and 627) had more moisture (over 5.0 pounds per 1000 square feet per 24 hours) than other rooms. They also noted that some classrooms $(505,516,608$, and 614) had rusted sill plates possibly due to water damage. In addition to the recommendations in their preliminary report, the Turner Group also recommended 1) new floor maintenance systems and schedules since the school no longer had textile floor covers but either hard vinyl surfaces or TerraCotta; 2) limit noise by fitting desks, chairs, and other objects with felt pads on their feet, or fitted with rolling castor wheels designed for hard floors; 3 ) avoid the use of textile floor coverings of any size except track-off mats; 4) use track-off mats at all main entries and do not use classroom fire-exits as normal entry door; 5) set occupied temperature set point of $70^{\circ} \mathrm{F}$ heating and $74^{\circ} \mathrm{F}$ cooling in accordance with the recommendations made by the commissioning agent; 6) operate the school in an occupied cycle and an unoccupied cycle; 7) continue additional monitoring of space conditions throughout the remaining heating season of this year, and the upcoming cooling season, to verify the HVAC system and controls are operating properly; 8) additional evaluation and possible corrective work to further limit heat loss and air leaks from doors, windows, and base of the walls.

Appendix G summarizes the remediation activities completed at Oak Ridge Elementary School since the NIOSH site visit in July 2009.

\section{NIOSH walkthrough July 2009}

We noted a strong musty, moldy odor in the basement (Figure 5) and crawl space under the media center. Lime was observed on the crawl space dirt floor. We also noted a musty smell in the corridor around the media center, in particular near the school museum. Surface drainage from playgrounds drained towards the classroom wings; 
it appeared water could puddle near concrete walkways adjacent to classroom wings especially near the $2 / 3$ and 4/5 wings (Figures 6, 7, and 8). A storm drain outside the media center was blocked with standing water in it (Figure 9). The foam roof had multiple locations where the surface had been compromised; there were signs of softness and bubbling of the roof membrane (Figures 10 and 11). There did not appear to be adequate flashing at the junction between the parapet wall (above room 206) and the gym (Figure 12). Room 206 had stained carpet, wavy appearing baseboards, and walls which appeared newly painted. Inside the school, we noted moisture (with the infrared camera) in the gym's exterior concrete block wall in an area where a leak had been repaired. We also noted pressure airflow issues between rooms and spaces. Multiple classrooms were under negative pressure instead of positive pressure in regard to the corridor. Multiple restrooms had positive pressure instead of negative pressure. During the day, the plenum above the classroom ceilings was generally under positive pressure in regard to the exterior of the building. However at night when the dehumidifier (which served the classroom wings) was turned off, this sometimes resulted in the plenum being under negative pressure in regard to the outside of the building. This allowed outside humid air to enter the plenum through the soffits in the classroom wings. From the mezzanine area where the dehumidifier was located, we observed dust on classroom ceilings and ducts (Figures 13 and 14). Some of the coils for the HVAC air handlers had residue that may have been mold contamination (Figure 15).

On the last day of the site visit, we communicated these findings with the concurrence of the Turner Group at a breakfast meeting with officials from Guilford County Schools. The following week, we emailed school district officials a brief summary of the findings discussed during the breakfast meeting (Appendix H). Additionally in July 2009, we had a teleconference with school district officials in which we notified them that some Oak Ridge Elementary School employees interviewed over the telephone reported building-related symptoms suggestive of hypersensitivity pneumonitis. We also noted that there appeared to be a high prevalence of respiratory symptoms at the school based on the summary of the questionnaire survey results that Guilford County Department of Public Health provided to us (Appendices I and J); we had not yet done our own statistical analysis of the de-identified questionnaire data. In October 2009, we sent an email to parents who requested a summary of our findings from the site visit (Appendix K).

\section{Environmental results}

Water activity in floor materials, relative humidity, and temperature

Water activity on floor materials from the 23 areas ( 21 rooms) sampled ranged from 0.52 to 0.81 with a median of 0.69 on the date of investigation. Average water activity on the floor in the administration wing (0.76), media center wing (0.73), and 4/5 wing (0.69) were above the school median (0.688) while average water activity in the $\mathrm{k} / 1$ wing was lowest (0.57) (Table 2). RH in the building ranged from 34 to $69 \%$. Average RH was higher in the administrative wing (59\%) than the other wings of the building and lowest in $\mathrm{k} / 1$ wing $(37 \%)$, and 2/3 wing (39\%). Air temperature did not show a wide range within the building (range $=70.5-74.1^{\circ} \mathrm{F}$ ).

\section{Microbial concentration in floor dust samples}

From the 22 samples analyzed, we recovered 20 fungal species, yeasts, and unidentifiable non-sporulating fungi (Figure 16). Identified hydrophilic (water-loving) fungi from the dust samples included Alternaria alternata, Epicoccum nigrum, Trichoderma konigii, Aspergillus fumigatus, Mucor plumbeus, Rhizopus stolonifer, and yeasts [WHO 2009; Hung et al. 2005; Flannigan and Miller 2001]. Identified mesophilic fungi included Cladosporium cladosporioides, Aureobasidium pullulans, Fusarium oxysporum, Aspergillus ochraceus, Aspergillus niger, Aspergillus versicolor, Paecilomyces varioti, Penicillium aurantiogriseum [WHO 2009; Hung et al. 2005; Flannigan and Miller 2001]. Among the species recovered, Cladosporium cladosporioides occurred most frequently in dust samples (recovered from all 22 samples $=100 \%$ occurrence). Epicoccum nigrum and Alternaria alternata (water-loving fungi) were recovered from $86 \%$ and $64 \%$ of samples, respectively. Bipolaris australiensis [WHO 2009; Hung et al. 2005; Flannigan and Miller 2001] which was not identified as either hydrophilic or mesophilic was recovered from $64 \%$ of samples. 


\section{APPENDIX M: NIOSH INTERIM REPORT, HETA 2009-0172 (CONTINUED)}

The building overall geometric mean (GM) of total culturable fungi was 6,000 colony-forming units per gram (g) dust (Table 3). Overall building GM concentration of hydrophilic fungi was 1,200 cfu/g. Analysis of the fractions of hydrophilic and mesophilic fungi showed that on average, $83 \%$ of the total culturable fungi were either mesophilic (61\%) or hydrophilic (22\%). Among fungi identified from more than $40 \%$ of samples, Cladosporium cladosporioides ( $\mathrm{GM}=2,852 \mathrm{cfu} / \mathrm{g}$ ), Epicoccum nigrum (GM=1,130 cfu $/ \mathrm{g}$ ), and Aureobasidium pullulans $(\mathrm{GM}=733 \mathrm{cfu} / \mathrm{g})$ were recovered with higher concentration than others. The overall building $\mathrm{GM}$ of gram-positive bacteria $(396,000 \mathrm{cfu} / \mathrm{g})$ was higher than that of gram-negative bacteria $(8,500 \mathrm{cfu} / \mathrm{g})$. None of the 22 samples had Actinomycetes (fungus-like bacteria).

Levels of total fungi, gram-positive, and gram-negative bacteria by building wing are summarized in Table 4. The gym-cafeteria wing $(\mathrm{N}=1,24,000 \mathrm{cfu} / \mathrm{g})$ and 2/3 wing $(\mathrm{N}=4,23,500 \mathrm{cfu} / \mathrm{g})$ had the highest level of total culturable fungi in floor dust, approximately eight-times higher than those of the media center $(\mathrm{N}=3,2,900 \mathrm{cfu} / \mathrm{g})$ and $4 / 5$ wing $(\mathrm{N}=6,2,900 \mathrm{cfu} / \mathrm{g})$. We observed a similar pattern for the levels of hydrophilic fungi in dust. The percentages of hydrophilic fungi in total fungal concentration were similar among wings and ranged between $20 \%$ (administration wing) and 29\% ( $\mathrm{k} / 1 \mathrm{wing}$ ). Except for the $\mathrm{k} / 1 \mathrm{wing}$, we observed a similar pattern in gramnegative bacteria concentration by building wing as those observed with culturable fungi. The level of gramnegative bacteria was more than an order of magnitude higher in the $2 / 3 \mathrm{wing}(52,000 \mathrm{cfu} / \mathrm{g})$ than in the media center $(2,500 \mathrm{cfu} / \mathrm{g})$ and $4 / 5 \mathrm{wing}(3,900 \mathrm{cfu} / \mathrm{g})$. However, the levels of gram-positive bacteria were highest in $\mathrm{k} / 1$ wing $(855,600 \mathrm{cfu} / \mathrm{g})$, followed by $2 / 3$ wing $(660,100 \mathrm{cfu} / \mathrm{g})$, and the media center $(631,600 \mathrm{cfu} / \mathrm{g})$.

\section{Comparison of NIOSH measurements with historical measurements of fungi in floor dust}

We identified 10 rooms which had both NIOSH and previous consultants' measurements: two rooms in the administration area, one in the gym area, two in the $2 / 3$ wing, three in the $4 / 5$ wing, and two in the k/1wing. Eight of the 10 rooms had measurements in May 2009 and July 2009 (Table 5). Room 100 (lobby in the administration area) had measurements in October 2007 and July 2009 (Table 6). Room 407 had measurements in September and October 2008 and July 2009. Based on the comparisons, we found that the fungal levels measured during the 2009 NIOSH investigation were 4 to 1,000-fold lower than those measured previously by consultants in all rooms except for room 115 in the administration wing which showed similar levels. Measurements from three rooms in the 4/5 wing showed dramatically decreased (90 to 1000-fold lower) fungal levels in the NIOSH investigation compared to those measured about two months before (May 2009).

\section{Health results}

\section{Telephone interviews with three staff members}

\section{Staff member $A$}

During the early part of the 2008/2009 school year, this staff member, with no prior history of respiratory illness, developed headaches, scratchy throat, laryngitis, sinus infection, and deep cough. He/She noted that the carpet sometimes felt damp, and the room smelled like "a wet dog", or musty especially when it rained, and when the power was off, and there was no ventilation. He/She was diagnosed with bronchitis and then pneumonia. While he/she had pneumonia, he/she had profound fatigue, night sweats but no fever or chills. He/She did not respond to antibiotics and was eventually given steroid shots. He/She was restricted from work for a short period of time, improved, and then got sick again when he/she went back to work. He/She improved until the carpet was pulled up, at which time he/she had the same progression of symptoms, diagnoses, antibiotics with no effect, and finally prednisone, which made him/her feel better. The ventilation ducts were cleaned; however, during this time, he/she developed wheezing and was treated with an inhaler (corticosteroid with long acting bronchodilator $\beta_{2}$ agonist) and oral steroids. Additionally he/she reported that fish in one of the classrooms died, and the class was relocated for a few days because of the strong disinfectant smell. In the summer of 2009, while out of the school, he/she had no wheezing, cough, fatigue, headaches, or medication requirement.

\section{Staff member B}

Since the opening of the new school building in 2005, this staff member developed recurrent sinus infections. He/ 


\section{Appendix M: NIOSH InteRIM Report, HETA 2009-0172 (CONTINUed)}

She had post-nasal drip, difficulty breathing, chest tightness, a metallic taste in his/her mouth, lungs that "hurt", and clogged ears. Additionally, he/she described three episodes of "dizziness, lightheadedness and feeling" that he/she might "pass out" in the school building during the 2008/2009 school year. Other symptoms included a deep cough during exercise and some dyspnea on exertion as if he/she "couldn't take a deep breath" when he/ she walked. He/She noted his/her cough more outside of the building than inside, and it was sometimes dry and other times productive. Other than cough, he/she reported no symptoms outside of the school building. His/Her symptoms got progressively worse over the course of the work week. During holiday breaks and weekends, the symptoms improved, but he/she would get sick upon return to the building. During May and June 2009, his/her symptoms got much worse after the carpet was torn up at the school.

The media room made him/her nauseous, and he/she felt like he/she was getting the flu and had excessive fatigue. Sometimes he/she had night sweats and felt feverish and occasionally felt chilly. During the last two months of school during the 2008/2009 school year, he/she noted difficulty concentrating, remembering, and recalling. He/ She also had lots of wheezing and also reported sneezing attacks, itchy burning eyes, and headaches. By the end of the week, he/she thought he/she was getting sick, but he/she felt better on Saturdays. He/She normally is an active person but during the second half of the 2008/2009 school year, he/she gave up exercise because of excessive fatigue, but his/her fatigue went away within two weeks of the end of the 2008/2009 school year.

He/She reported no allergies on testing, except to cats, which was apparently minimal, and he/she does not have a cat. Medications have not helped his/her symptoms, and he/she has had particular difficulties the last couple months of the 2008/2009 school year with alternating antibiotics and steroids. However, within a couple of weeks of school being out this past summer, he/she has been healthy again, with no need for medications.

$\mathrm{He} / \mathrm{She}$ noted that the 4/5 wing has had leaks from the bathroom pipes. When it rained, the ceiling tiles got stained and were changed. The gym has had mold on the walls, and the physical education teacher's room had carpet separating from a damp wall. The administration offices smelt musty, like an old basement. A dehumidifier was placed in the administration area; however, it did not seem to serve all the offices. In the corridor near the library, there were two termite swarms.

\section{Staff member $C$}

During the 2008/2009 school year, this staff member with no prior history of respiratory illness developed respiratory and sinus infections, itchy/watery eye, rashes, decreased appetite, fatigue, and difficulty concentration and focusing. He/she did not note any cough, wheezing, fever, or chills but sometimes "gasped for air". Since the end of the 2008/2009 school year, his/her symptoms have improved, and he/she feels less fatigued and more alert.

$\mathrm{He} / \mathrm{She}$ noted many ceiling leaks at the school and water in fluorescent light covers in a classroom wing. He/She noted that stained ceiling tiles were constantly being replaced at the school, and the gym had issues with mold growth and condensation on the walls.

\section{De-identified questionnaire data (from Guilford County Department of Public Health)}

\section{Participation and Demographics}

Fifty-two of 88 (59\%) staff members participated in the questionnaire. All 52 participants worked at Oak Ridge Elementary School during the 2008/2009 school year; forty-five of the 52 participants also worked at the school during the 2007/2008 school year. Two of the staff participants who worked at the school during both school years stopped the questionnaire after the first page as directed by the questionnaire instructions; they had answered "no" to experiencing any of the following symptoms while in the school building during the 2008/2009 school year: 1) headaches, 2) dry eyes, 3) unusual sneezing episodes, 4) difficulty breathing/asthma attacks, 5) nosebleeds, or 6) other symptoms. Therefore, 2 (4\%) of 45 staff participants who worked at the school during both school years did not provide information on symptoms in the school building during the 2007/2008 school year, health conditions, medication, smoking status, or home exposures. 


\section{ApPendix M: NIOSH InteRIM Report, HETA 2009-0172 (CONTINUed)}

Seven hundred and thirty-eight (738) students attended Oak Ridge Elementary School in 2008/2009 and 734 in 2007/2008. Parents of 388 students completed the questionnaire. We excluded data from one questionnaire because the student did not attend the school during the 2008/2009 or 2007/2008 school year. Of the 387 students whose parents' who filled out the survey, 385 attended during the 2008/2009 school year; 308 attended during the 2007/2008 school year; and 306 attended during both schools years.

Of the 387 students, 122 did not have the symptoms (identified on the first page of the questionnaire) while in the school building during the 2008/2009 school year and were instructed to stop the questionnaire at that point; however, 11 continued on with the questionnaire. Parents for 77 (25\%) of 308 students who attended Oak Ridge Elementary School during the 2007/2008 school year stopped the questionnaire after the first page and did provide information on symptoms in the school building during the 2007/2008 school year, health conditions, medication, environmental tobacco exposure at home, and other home exposures. Parents for 34 (9\%) of 385 students who only attended Oak Ridge Elementary School during the 2008/2009 school year stopped the questionnaire after the first page and did provide any information on health conditions, medications, environmental tobacco exposure at home, and other home exposures. Therefore, $276(71 \%)$ of the 387 completed the entire questionnaire

Of the 48 staff who reported their 2008/2009 primary classroom or office, 40 (83\%) worked primarily in classroom wings and eight (17\%) in other wings (Table 7). Of the 385 students who attended the school during the $2008 / 2009$ school year, we were able to determine the primary classroom/homeroom wing for 376 students; $111(30 \%)$ were located in the k/1 wing, $136(36 \%)$ in the 2/3 wing, and $129(34 \%)$ in the 4/5 wing (Table 7).

Five of $50(10 \%)$ staff members reported smoking cigarettes, pipes, or cigars or having a smoker in their household. Nineteen of $276(7 \%)$ parents reported a smoker in the household.

Six of $50(12 \%)$ staff members reported one or more of: a history of water leaks in their homes; water stains on the wall and ceiling; or mold or mildew problems. Of the participating student households, 23 of $276(8 \%)$ had one or more of water leaks; water stains on the wall or ceiling, or mold or mildew.

\section{Six staff who reported daily breathing problems/asthma attacks during the 2008/2009 school year on the health} department questionnaire

As an indication of the presence of chronic lower respiratory problems possibly associated with the school building we have summarized the reports from the questionnaire of six staff members who reported having "difficulty breathing/ asthma attacks" daily while at work in the school building during the 2008/2009 school year. None of them smoked or had other smokers in their households. Two of the six staff members reported having asthma and one reported allergic rhinitis. We do not have systematic information on dates of diagnosis of these conditions, but one person reported an onset of 2007/2008 or earlier and the other reported an onset in 2008/2009. Only the two staff members with asthma reported being on prescription breathing medication.

These six staff members worked in the 2/3, 4/5 and administrative wings, and apart from their own work areas, also reported symptoms associated with being in the staff lounge and media center. Four of the six staff members reported that their symptoms went away after leaving the building with time frames for improvement ranging from hours to several days.

All of these employees reported a constellation of symptoms related to being in the building as well as breathing difficulties. As a group, their symptoms included chest-tightness, shortness of breath, coughing, difficulty breathing on awakening, fatigue, sore throat, headache, eye problems, nasal and sinus problems, skin problems, nosebleeds, nausea, vomiting, and a metallic taste in the mouth.

As an indication of severity of health problems, the health department questionnaire had asked about seeking medical attention. Five of these six staff members had visited a doctor multiple times (from two to more than 30 times) during the 2008/2009 school year for the health problems reported on the questionnaire. 


\section{Health conditions}

For staff, 34\% reported seasonal allergies; $8 \%$ asthma; and $8 \%$ allergic rhinitis. For students, $48 \%$ had seasonal allergies; $16 \%$ asthma; and $12 \%$ allergic rhinitis (Table 8 ).

Building-related symptoms during the 2008/2009 school year

Lower respiratory symptoms

Thirty-eight percent (38\%) of staff and 19\% of students experienced at least one lower respiratory symptom while at school; $31 \%$ of staff and $13 \%$ of students had difficulty breathing/asthma attacks (Table 9). Of the 16 staff who reported difficulty breathing/asthma attacks, 38\% (6/16) reported them daily, 56\% (9/16) sometimes, and 6\% (1/16) rarely. Of the 42 (out of 49) students with available frequency data, 5\% (2/42) had difficulty breathing/ asthma attacks daily, 79\% (33/42) sometimes, and 17\% (7/42) rarely. Fifteen percent of staff and 7\% of students had a cough at school.

\section{Upper respiratory symptoms}

Fifty-two percent $(52 \%)$ of staff and $28 \%$ of students had at least one upper respiratory symptom while at school. Unusual sneezing was the most common symptom reported with $31 \%$ of staff and $23 \%$ of students experiencing it.

Twenty-five percent of staff and 5\% of students had sinus problems; $27 \%$ of staff and $5 \%$ of students had voice or throat symptoms, and $10 \%$ of staff and $5 \%$ of students had nasal symptoms.

Mucous membrane irritation

Eighty-seven percent (87\%) of staff and 32\% of students had at least one mucous membrane symptom; $75 \%$ of staff and $28 \%$ of students had eye symptoms at school.

$\underline{\text { Headache }}$

Headache was the most common symptom in participating staff (92\%) and students (49\%) (Table 9). Of the 47 (out of 48) staff for which we had headache frequency data, 55\% (26/47) reported headaches daily at school, 43\% $(20 / 47)$ sometimes, and 2\% (1/47) rarely. Of the 182 (out of 187) students with available headache frequency data, 25\% (45/182) had headaches daily at school, 57\% (104/182) sometimes, and 18\% (33/182) rarely.

\section{Fatigue}

Forty-four percent (44\%) of staff and $6 \%$ of students had fatigue while at school.

\section{Nosebleed}

Twenty-five percent (25\%) of staff and $20 \%$ of students had nosebleeds. For the 10 out of 13 staff who reported nosebleed frequency, 10\% (1/10) reported them daily, 70\% (7/10) sometimes, and 20\% (2/10) rarely. For students, we had frequency data for 72 out of 77 students with nosebleeds; $11 \%$ (8/72) had them daily, 60\% (43/72) sometimes, and 29\% (21/72) rarely.

\section{Work-related symptoms}

Seventy-four percent (74\%) (37/50) of staff and 53\% (145/274) of students at Oak Ridge Elementary School during the 2008/2009 school year had symptoms/problems that went away after leaving the building.

\section{Symptoms by classroom wing}

The health department questionnaire asked the participants to provide a room number or description of areas in the school where they experienced more symptoms or discomfort. Of the 52 staff members who worked at the school during the 2008/2009 school year, 27 staff members wrote in one more rooms or areas that caused them to experience more symptoms or discomfort. Of these 27 staff members, 12 reported the media room, 12 reported areas in the administrative wing, 10 reported areas in the 4/5 wing, eight reported areas in the $2 / 3$ wing, four reported areas in the $\mathrm{k} / 1$ wing, and three reported areas in the gym-cafeteria wing. 


\section{Appendix M: NIOSH InteRIM RepORt, HETA 2009-0172 (CONTINUED)}

Of the 385 students who attended the school during the 2008/2009 school year, parents for 86 of these students wrote in one or more rooms or areas where their child experienced more symptoms or discomfort in the school. Fifty-four of the 86 parents reported areas in the 4/5 wing, 43 reported areas in the 2/3 wing, 18 reported areas in the $\mathrm{k} / 1$ wing, and two reported the gym. The music room was mentioned the most with 16 write-ins.

Table 10 shows the prevalence of reported symptoms by primary work wing of staff during the 2008/2009 school year. Upper respiratory symptoms were significantly $(\mathrm{p}<0.05)$ (Figure 17) more prevalent among staff who worked in the $2 / 3$ or $4 / 5$ wings compared to staff who worked in the $\mathrm{k} / 1$ wing. Lower respiratory symptoms were more prevalent (marginally significant, $\mathrm{p}<0.10$ ) among staff who worked in the $2 / 3$ or $4 / 5$ wings compared to staff who worked in the $\mathrm{k} / 1$ wing.

Table 11 shows the prevalence of reported symptoms by primary homeroom wing of students during the 2008/2009 school year. Headaches and nosebleeds were significantly more prevalent among students whose primary/homeroom classroom were in the $2 / 3$ or $4 / 5$ wing compared to students whose primary/homeroom classroom were in the $\mathrm{k} / 1$ wing. Dry eyes, unusual sneezing, breathing difficulties/asthma attacks, and upper respiratory symptoms were more prevalent (marginally significant, $\mathrm{p}<0.10$ ) among students whose primary/ homeroom classroom were in the $2 / 3$ or $4 / 5$ wing compared to students whose primary/homeroom classroom were in the $\mathrm{k} / 1$ wing (Figure 18).

Symptoms reported during the 2008/2009 and 2007/2008 school years

Staff (Figure 19) and students (Figure 20) who reported symptoms in the 2008/2009 school year and who worked or attended Oak Ridge Elementary School during 2007/2008 and 2008/2009 school year had headache, dry eyes, unusual sneezing, breathing difficulties/asthma attacks, nosebleeds, and upper and lower respiratory symptoms more frequently during the 2008/2009 school year than during the 2007/2008 school year; the results were statistically significant $(\mathrm{p}<0.05)$.

\section{DISCUSSION}

Oak Ridge Elementary School has had a long history of widespread dampness with mold growth since re-opening in August 2005 after renovations and the addition of three classroom wings. Visible mold was found recurrently on books, carpet, upholstered furniture, picture frames, and walls. The significance of visible mold is that it reflects chronic dampness and exposure hazards for staff and students. The significance of musty odors is that mold is actively growing. Mold cannot grow without moisture and a source of food, which is usually carbon in paper, wood, building materials, adhesives, and dirt. The dampness in Oak Ridge Elementary School had several sources. High relative humidity $(\mathrm{RH})$ reflects dampness and can support mold growth. Consultants reported measurements above $70 \%$, in contrast to recommended ranges of $30-60 \%$ for indoor light work. Ongoing problems with the building's heating, ventilation, and air-conditioning (HVAC) system and air flow issues allowed the incursion of outside, unconditioned air into the school through areas such as the crawl space under the media center, clerestory above the media center, and soffits in the classroom wings. There was potential for the formation of condensation. Additionally, the air flow and ventilation issues at the school may have allowed the transport of mold spores from reservoirs such as the crawl space and basement under the media center where a strong musty odor was detected.

Inadequate flashing was also a source of dampness at the school. During the July 2009 site visit, NIOSH and the Turner Group found inadequate flashing at the junction between the parapet wall above room 206 and the gym. The carpet in room 206 was stained and had wavy baseboards likely from water damage. A few months earlier, an engineer performing a site visit at the school reported inadequate flashing connections over areas of the media center resulting in deterioration of exterior bricks which likely allowed penetration of rain water and contributed to leaks in the assistant principal's office. During the same site visit, the consultant also reported finding a significant amount of visible mold under the carpet in this office. In 2005, an exterior wall in classroom 400 was water damaged due to an exterior leak during construction. In July 2009, the Turner Group reported that the drywall in this room was discolored, and that there had been occupant reports of mold found under the cove base. 


\section{$\overline{\text { ApPendIX M: NIOSH INTERIM RePORT, HETA 2009-0172 (CONTINUED) }}$}

Although the wall flashing had been repaired, the Turner Group recommended repair of the roof junction joints to provide adequate flashing.

Various consultants over the years have reported ceiling and roof leaks. Following a heavy rain in July 2006, roof leaks occurred in all the classroom wings as well as in the administrative wing and one of the offices near the gym. In February 2007, an official of the North Carolina Department of Environmental and Natural Resources reported several ceiling leaks. The following year during a similar inspection, a bad leak was reported in the ceiling over the central receiving room. In April 2009, an engineer consultant reported significant roof leaks over the utility/storage room near the gym. Also during April 2009, more than one consultant noted stained ceiling tiles throughout the school especially where the old and new buildings were structurally joined. There is also a history of roof leaks where the north-south roof intersects with the east-west roof above the music and Spanish rooms; these two rooms share a common wall. Previous consultants and building occupants reported a history of replacing ceiling tiles before repairing the leaks. During the NIOSH site visit, we observed no stained ceiling tiles; however, we did find defects in the flat roof over the old section of the school, suggesting the likelihood of water leaks.

Various consultants reported landscaping issues around the school that resulted in the drainage of water toward the school building allowing pooling of water adjacent to the school. Slab testing results reported in the Turner Group's final report noted that the concrete flooring in some rooms had more moisture (over 5.0 pounds per 1000 square feet per 24 hours) than other rooms. The Turner consultants also noted that some classrooms had rusted sill plates possibly due to water damage.

The school tried various ways to remediate mold, ventilation issues, humidity, and leakage through the building envelope from roof and flashing problems. However, additional indoor environmental quality issues may have resulted from use of cleaning agents such as biocides in the HVAC system and on the carpet. The school had tried to keep the carpets clean and vacuumed them with HEPA filters; however, the carpeting had been subjected to repeated moisture infusion from steam cleaning and chemical treatments with biocides intended to control mold growth. Carpet can act like a sponge to absorb and often hold these fluids and odors (as was noted by some consultants). Carpeted areas are frequently associated with increased building-related symptoms. They may release volatile organic compounds (VOCs), contain dust mites that can cause allergic symptoms, and are reservoirs for accumulated dusts. Dusts may contain fungi and bacteria as was the case at Oak Ridge Elementary School reflecting dampness effects on microbial proliferation.

Air, swab and vacuum sampling documented the presence of various microorganisms within the school over the years. Any indoor environment will have various microorganisms in such samples, and usually the indoor fungi reflect what has come in with outdoor air. Repeated air sampling for fungi often results in highly variable levels over the same day or at different locations in the same room. At present, no environmental standards exist with which to predict health risk. For that reason, NIOSH scientists do not recommend the common consultant practice of conducting air sampling, nor have we found air sampling helpful when there are reservoirs of hidden mold within walls or under carpets. Although counts of fungi in the air, whether spores or colony forming units, are difficult to interpret, sometimes sampling is helpful. If the qualitative or quantitative distribution of fungal types indoor differs from outdoors, an indoor source of fungal growth may exist. The consultant sampling documented some indoor fungal species that were higher in indoors samples than outdoor samples or did not occur in outdoor samples. This suggests that there were indoor sources of mold growth, as was evident visually by inspection and by smelling moldy odors. Water-loving fungi reflect indoor sources related to chronic dampness.

NIOSH collected floor dust samples and found that mesophilic and hydrophilic (water-loving) fungi together accounted for more than $80 \%$ of the total fungal concentration. According to the American Industrial Hygiene Association guidelines [Hung et al. 2005], samples dominated by the presence of water-loving fungi reflects chronic dampness, while the predominance of a species mix of mesophilic fungi indicates alternating wet and dry 


\section{$\overline{\text { ApPendix M: NIOSH INTERIM REPORT, HETA 2009-0172 (CONTINUED) }}$}

cycles.

Our results showed that the $2 / 3$ wing had the highest average fungal (both total and hydrophilic fungi) concentrations in floor dust while the $4 / 5$ wing and the media center had the lowest. We also found a high concentration of gram-negative bacteria in rooms of the $2 / 3$ wing. These findings suggest that the $2 / 3$ wing may have had underlying ongoing dampness issues because high levels of gram-negative bacteria and hydrophilic fungi which require high moisture contents in floor materials for proliferation. The rooms in the $\mathrm{k} / 1 \mathrm{wing}$ had the lowest average water activity on floor materials compared to the other wings at the school. This finding may imply that the carpets or tiles in this $\mathrm{k} / 1$ wing were relatively drier than those in other wings.

The concentrations of fungi in floor dust from the rooms of the school building collected during the NIOSH survey were consistently and substantially lower than environmental consultants reported before the NIOSH investigation. During the last weekend in May 2009, the school had cleaned all the carpets in the school with an antimicrobial (biocide) agent. Additionally in May 2009, the carpet in room 407 had been replaced with vinyl composite tile. The cleaning activity and replacement of carpet with tiles appeared to decrease the levels of culturable fungi in the floor carpet dust. However, if there are any unresolved dampness problems, the cleaning activity or replacement itself will not prevent recurrence of microbial growth in the future.

Upon review of environmental reports of activities at the school, we determined that the biocide Sporicidin (The Sporicidin Company, Rockville, MD) had been applied in the HVAC system and on carpets. Biocides, including fungicides and disinfectants, are chemical agents that destroy or harm living organisms [EPA 1997; Macher 1999; Prezant, Weekes, and Miller 2008]. Any biocide must be registered for use by the Environmental Protection Agency (EPA) under the Federal Insecticide, Fungicide, and Rodenticide Act. This EPA biocide registration is granted only for specific biocide uses; thus, only biocides specifically approved for use in a HVAC system can be applied in a HVAC system [EPA 1997]. Sporicidin Disinfectant Solution and Sporicidin Brand Disinfectant Spray are approved by the EPA for air duct cleaning (EPA Registration \# 8383-3 and 8383-4, respectively). The product label (Appendix L) indicates that Sporicidin must be only applied to hard, non-porous surfaces of air ducts. This product is not approved for use in internally-insulated air duct systems. According to the EPA, biocides registered for use in HVAC systems have low toxicity levels and the lowest level of efficacy [Lantz, 2009]; however, the EPA notes that risk assessment for use in HVAC systems has not been completed for Sporicidin. The product label (Appendix L) indicates that this product is approved for use on carpets; however, formal risk assessment for Sporicidin use on carpets has not been done. There is potential for exposure to biocide residues in treated carpet especially since the product label claims that this product has residual anti-fungal properties lasting for over six months.

An historical environmental sampling report stated that the coil cleaner used in December 2008 contained butyl cellosolve (2-butoxyethanol). The Agency for Toxic Substances and Disease Registry stated that exposure to butyl cellosolve may result in irritation of the nose and eyes, headache, a metallic taste, and vomiting. High exposure levels may cause breathing problems [ATSDR 1999]. Air sampling results for butyl cellosolve that occurred after the coil cleaning had been completed were below the NIOSH REL and OSHA PEL; however, we have no information on air levels of butyl cellosolve during the cleaning.

Use of biocides in HVAC systems is controversial. Although biocide use may help reduce mold and bacterial growth, all biocides have some level of toxicity and unnecessary exposures should be avoided. Biocide exposure may have contributed to some symptoms and the fish kill in a classroom fish tank. The growth of mold and bacteria is due to excess moisture in indoor environments; thus, application of biocide as a protective action is unnecessary if moisture is properly controlled. Applying biocides directly into HVAC ducts may result in dissemination of these chemicals throughout the building and into occupied office areas resulting in possible occupant exposures and health effects. Since some mold exposures can result in respiratory health effects even when they are not viable (alive), mold exposures can continue even with biocide treatment further detracting from biocide use in HVAC ducts [Macher 1999; NYCDHMH 2008; Prezant, Weekes, and Miller, 2008]. 


\section{Appendix M: NIOSH InteRIM Report, HETA 2009-0172 (CONTINUED)}

The New York City Guidelines and the American Conference of Governmental Industrial Hygienists do not recommend biocide use in HVAC systems as a routine cleaning practice due to both the questionable effectiveness of some of these chemicals to prevent microbiological growth and also due to uncertainties regarding health outcomes from occupant biocide exposures [Macher 1999; NYCDHMH 2008]. Biocides should not be applied in occupied buildings or in an operating HVAC system [Sesline 1994]. Given the uncertainty created by a lack of research on the safety of biocide use in HVAC system ducts, any biocide use in HVAC systems or ductwork, even EPA approved biocides such as Sporicidin, should be minimized, carefully monitored in terms of occupant health and air quality, and guided by professional judgment [Prezant, Weekes, and Miller 2008].

Excessive indoor dampness is a public health problem. In 2004, the Institute of Medicine of the National Academies (IOM) conducted an extensive review of past scientific studies on the health effects of damp buildings. Based on its review of the research that has been conducted, the IOM found sufficient evidence that indoor dampness or mold is associated with asthma symptoms (in sensitized asthmatic persons), cough, wheeze, and nasal and throat symptoms. In addition, the IOM found sufficient evidence that exposure to mold or bacteria in damp indoor environments is associated with hypersensitivity pneumonitis. It found limited or suggestive evidence that exposure to indoor dampness is associated with the development of shortness of breath and asthma. Since the IOM review, NIOSH research has documented several damp office buildings with increased risk of new-onset asthma.

In 2009, the World Health Organization (WHO) published guidelines for dampness and mold. The WHO Executive Summary states that excess moisture on almost all indoor materials leads to growth of microbes, such as mold and bacteria, which subsequently emit spores, cells, fragments and VOCs into indoor air. Additionally, dampness initiates chemical or biological degradation of materials, which also pollutes indoor air. In the chapter on health effects, the authors of the WHO guidelines concluded that there is sufficient epidemiological evidence of an association between indoor dampness-related factors and asthma development, asthma exacerbation, current asthma, respiratory infections, upper respiratory tract symptoms, cough, wheeze, and shortness of breath. There is now limited or suggestive evidence for bronchitis and allergic rhinitis being related to dampness-related exposures. There is clinical evidence that exposure to mold and other dampness-related microbial agents increase the risk for hypersensitivity pneumonitis, chronic rhinosinusitis, and allergic fungal sinusitis. Importantly, the WHO guidelines note that although atopy and allergy increases susceptibility to dampness-related health effects, such health effects are also found in non-atopic building occupants.

The WHO guidelines relevant to occupational environments are as follows:

- Persistent dampness and microbial growth on interior surfaces and in building structures should be avoided or minimized, as they may lead to adverse health effects.

- Indicators of dampness and microbial growth include the presence of condensation on surfaces or in structures, visible mold, perceived moldy odor and a history of water damage, leakage or penetration. Thorough inspection and, if necessary, appropriate measurements can be used to confirm indoor moisture and microbial growth.

- As the relations between dampness, microbial exposure and health effects cannot be quantified precisely, no quantitative health-based guideline values or thresholds can be recommended for acceptable levels of contamination with microorganisms. Instead, it is recommended that dampness and mold-related problems be prevented. When they occur, they should be remediated because they increase the risk of hazardous exposure to microbes and chemicals.

- Well-designed, well-constructed, well-maintained building envelopes are critical to the prevention and control of excess moisture and microbial growth, as they prevent thermal bridges and the entry of liquid or vapor-phase water. Management of moisture requires proper control of temperatures and ventilation to 


\section{ApPendix M: NIOSH InTeRIM RepORt, HETA 2009-0172 (CONTINUED)}

avoid excess humidity, condensation on surfaces and excess moisture in materials. Ventilation should be distributed effectively throughout spaces, and stagnant air zones should be avoided.

- Building owners are responsible for providing a healthy workplace or living environment free of excess moisture and mold, by ensuring proper building construction and maintenance. The occupants are responsible for managing the use of water, heating, ventilation and appliances in a manner that does not lead to dampness and mold growth.

As documented in this report and in other consultant reports, Oak Ridge Elementary School has had ongoing problems with their HVAC system since re-opening in August 2005. However, during the remediation process, the school district worked with ventilation experts, and the HVAC system was recently retro-commissioned. The report can be found on the Guilford County School's website (http://www.gcsnc.com/oakridge/turner.html).

At Oak Ridge Elementary School there was evidence of building-related symptoms. Staff during the 2008/2009 school year had high rates of lower respiratory symptoms, mucous membrane irritation, and headaches. Even if we adjust for the response rate of 59\%, these symptoms rates are still high. Staff and students who attended the school during the 2007/2008 and 2008/2009 school years had more upper and lower respiratory symptoms as well as headaches, dry eyes, and nosebleeds during the 2008/2009 school year than the 2007/2008 school year, suggesting the problems at the school were getting worse.

From our limited telephone interviews and from the health department questionnaire responses, we saw evidence of building-related lower respiratory problems in building occupants, and we suspect at least two employees had occupational hypersensitivity pneumonitis. Hypersensitivity pneumonitis is a serious lung disease in which an individual's immune system responds to repeated exposure to organic dusts (materials from living things such as plants, animals, bacteria, or fungi) or other sensitizing agents [Patel et al. 2001]. Symptoms can include cough, shortness of breath, muscle aches, fever, chills, and fatigue. In work-related hypersensitivity pneumonitis cases, the first sign that the illness is due to exposures at work may be that the worker's symptoms and medical tests (e.g., pulmonary function tests) improve during periods of time away from work and then worsen on return to work. Continued exposure can lead to lung scarring and permanent shortness of breath. The two staff members with symptoms suggestive of hypersensitivity pneumonitis both reported that their symptoms (e.g., fatigue, fever, chills, cough, and shortness of breath ) resolved with time away from the school and reoccurred upon return to the school building. Physicians rarely consider a diagnosis of hypersensitivity pneumonitis, particularly in nonindustrial workers.

Hypersensitivity pneumonitis is under-recognized, and a single case related to a building should be considered a sentinel event requiring investigation and remediation [Weltermann et al. 1998]. Hypersensitivity pneumonitis has been documented in workers in buildings with contaminated air-conditioners, ductwork, and HVAC filters [Bernstein et al. 1983; Scully et al. 1979; Fink et al. 1976; Banaszak et al. 1970; Arnow et al. 1978]. Hypersensitivity pneumonitis has also occurred in workers who worked in water-damaged buildings with roof leaks, plumbing leaks, poorly draining condensation pans, or high indoor relative humidity [Seuri et al. 2000; Thörn et al. 1996 Weltermann et al. 1998; Hoffman et al. 1993].

Once sources of moisture have been identified, mold and moisture-damaged materials should be promptly cleaned or removed with appropriate containment to minimize exposure to building occupants; and that necessary repairs be made to prevent further water entry into the building.

Accordingly, we think the decision to close Oak Ridge Elementary School during the remediation to address the underlying issues of water intrusion, humidity, and ventilation was a prudent one. We have found that remediation similar to what we recommended at Oak Ridge Elementary School is easier to do when a building is not occupied and that health effects associated with dampness commonly are made worse when remediation occurs in occupied buildings. 


\section{Appendix M: NIOSH InTeRIM Report, HETA 2009-0172 (CONTINUED)}

After remediation, employees and management often wish to know if the building is then "safe". Building consultants often recommend and perform "clearance" air sampling after work has been completed in an attempt to demonstrate that the building is safe for occupants. However, there is no scientific basis for the use of air sampling for this purpose. Once remediation is completed (moldy and damaged materials removed; musty odors no longer evident), the best evidence that the building is safe may be that employees no longer experience building-related symptoms. In large populations of workers, using employee health questionnaires may be helpful to collect information on building-related symptoms, particularly among persons new to the building after remediation (i.e., those without "sensitizing" historical exposures during a period of water damage). Unfortunately, even if most employees experience improvement in their symptoms, and new employees remain free of building-related symptoms, some employees with allergic conditions may not notice an improvement. Because their immune systems may continue to react to very small amounts of substances to which they are allergic, such individuals may have to avoid the building even after an otherwise successful remediation. An individualized management plan (such as assigning an affected employee to a different work location, perhaps at a remote site) is sometimes required, depending upon medical findings and recommendations of the individual's physician.

Our evaluation was built on the many efforts of the school administration, public health department, and consultants since 2005. Nevertheless, some limitations existed in the evaluation of this school. The health department questionnaire contained some open-ended questions in which the participants were asked to answer in their own words. These types of answers are often time consuming and difficult to categorize for statistical analysis. Additionally, the questionnaire asked for the presence of five specific symptoms but did not ask about cough, wheeze, chest tightness, itchy or runny nose, or other symptoms. Some participants wrote in these and other symptoms on the questionnaire. The questionnaire did ask about improvement of symptoms after leaving the school building; however, we could not determine which specific symptoms improved away from the school building. Additionally, the response rate (that is, the number of questionnaires that were returned to the health department) was low, and the results may not be representative of the whole school population. Parents filled out the questionnaires for their children instead of relying on self-report of the students. This type of surrogate or proxy reporting is often used for elementary-aged children, but may be less accurate than staff information. Lastly, the questionnaire did not ask about physician-diagnosed asthma and did not contain other standardized symptom questions. Accordingly, we were unable to compare results to national or state data to see if the school staff had elevated symptom rates. Within the school, staff in all areas appeared to have high rates of buildingrelated symptoms.

Nevertheless, the questionnaire was a systemic attempt to collect data on which to base policy and prioritize interventions. Priority had to be given to the entire school because of the prevalence of reported symptoms and the environmental findings. Additionally, the school building-relatedness of the symptoms reported by the staff and parents supported building-related exposures. Also, the case reports supported illness severe enough to have required medical consultation and treatment. Most worrisome were symptoms suggestive of hypersensitivity pneumonitis. This along with the high rates of asthma/breathing difficulties reported by staff and students during the 2008/2009 school year warranted public health action, as taken by the school, to relocate the staff and students during remediation.

Oak Ridge Elementary School reopened on February 22, 2010. NIOSH has been coordinating with officials from Guilford County Schools to administer a computerized health survey questionnaire to the employees at the school who wish to participate during March 2-4, 2010. Much of the questionnaire will address health symptoms experienced the four weeks prior to the re-opening of the school. We see this cross-sectional survey as part of surveillance to document the extent of resolution of a public health problem likely associated with dampness in the building before remediation. No environmental measurements are known to predict health outcomes, so assessing health is the only option. This assessment of health symptoms can serve as a baseline to help assess whether health is maintained after employees reoccupy the school. If there are no concerns, there may not be a reason to resurvey the employees. However, if concerns arise, such a survey would be invaluable. 
Appendix M: NIOSH INTERIM Report, HETA 2009-0172 (CONTINUED)

\section{RECOMMENDATIONS}

Although we realize many of the recommendations have been acted on (Appendix G) since NIOSH's site visit. For completeness, we have listed all our recommendations.

1. Improve surface drainage (landscaping) around the school to prevent drainage of water (e.g, rainwater and melting snow) toward the concrete walkways with puddling of water adjacent to the school.

2. Ensure proper functioning of storm drains. Ensure that the storm drains and pipes remain clear of leaves, grass cuttings, and other material.

3. Remove carpet and replace with hard floors (after testing slab for moisture content).

4. Install a vapor barrier and depressurize the basement and crawl space under the media center.

5. Repair the roof.

6. Repair inadequate flashing identified by NIOSH and the Turner Group.

7. Seal classroom wing soffits.

8. Recommission the HVAC system.

9. Maintain the HVAC system by following the manufacturer's recommended maintenance schedules including replacement of air filters, checking to make sure thermostats are in working order, and checking/cleaning ventilation system dampers in rooms to ensure proper functioning.

10. Continue to assess the school for water intrusion and damage and high relative humidity and correct these upon discovery. Prevent worker exposures and contamination of unaffected sections of the building during remediation.

11. Do not use biocides as standard procedures.

12. Implement a systematic way for faculty and staff to report problems in their work areas. Facilitate the confidential reporting of dampness problems and building-related health effects by employees by designating a contact person such as the school nurse. A locked drop box could also be used to report problems.

13. Use occupant health questionnaires to collect information on the building-related symptoms at re-opening and after occupancy, if needed

14. Use a dampness/mold check-sheet during routine inspections of the school. An example can be found in Appendix M.

15. Consider implementing the EPA's Indoor Air Quality Tools for Schools Program. Information can be found on the EPA website at http://www.epa.gov/iaq/schools/ 


\section{REFERENCES}

Agency for Toxic Substances and Disease Registry (ATSDR) [1999]. ToxFAQs for 2-Butoxyethanol and 2-Butoxyethanol Acetate [online]. [date assessed: 2010 Jan 13]. Available at http://www.atsdr.cdc.gov/tfacts118. $\underline{\text { html. }}$.

Arnow PM, Fink JN, Schlueter DP, Barboriak JJ, Mallison G, Said SI, Martin S, Unger GF, Scanlon G, Kurup VP [1978]. Early detection of hypersensitivity pneumonitis in office workers. Am J Med 64:236-242.

Banaszak EF, Thiede WH, Fink JN [1970]. Hypersensitivity pneumonitis due to contamination of an air conditioner. N Engl J Med 283:271-276.

Bernstein RS, Sorenson WG, Barabrant D, Reaux C, Treitman RD [1983]. Exposures to respirable, airborne Penicillium from a contaminated ventilation system: Clinical, environmental and epidemiological aspects. Am Ind Hyg Assoc J 44:161-169.

Environmental Protection Agency (EPA) [1997]. Should you have the air ducts in your home cleaned? [online]. [date assessed: 2010 Jan 7]. Available from URL:www.epa.gov/iaq/pdfs/airducts.pdf.

Environmental Protection Agency (EPA). Use of disinfectants and sanitizers in heating, ventilation, air conditioning, and refrigeration systems [online]. 2002. [date assessed: 2010 Jan 7]. Available from URL: $w w w$. epa.gov/oppad001/hvac.htm.

Flannigan B, Miller JD [2001]. Microbial growth in indoor environments. In: Flannigan B, Samson RA, Miller JD, eds. Microorganisms in home and indoor work environments: diversity, health impacts, investigation and control. $1^{\text {st }}$ ed. New York: Taylor \& Francis, Inc., pp.35-67.

Fink JN, Banaszak EF, Baroriak JJ, Hensley GT, Kurup VP, Scanlon GT, Schlueter DP, Sosman AJ, Thiede WH, Unger GF [1976]. Interstitial lung disease due to contamination of forced air systems. Ann Intern Med 84:406-413.

Hoffman RE, Wood RC, Kreiss K [1993]. Building-related asthma in Denver office workers. Am J Public Health 83:89-93.

Hung LL, Miller JD, Dillon HK, eds. [2005]. Field guide for the determination of biological contaminants in environmental samples. $2^{\text {nd }}$ ed. Fairfax, VA: American Industrial Hygiene Association, page 29-38.

Institute of Medicine (IOM) [2004]. Damp Indoor Spaces and Health. Washington, DC: National Academy of Sciences.

Lantz T (Lantz.Tracy@epamail.epa.gov) [2009]. Biocide use in HVAC. Private email message to Rena Saito (hve5@cdc.gov), December 8.

Macher J. ed. [1999]. Bioaerosols assessment and control. Cincinnati, OH: American Conference of Governmental Industrial Hygienists, Ch. 16.

New York City Department of Health and Mental Hygiene (NYCDHMH) [2008]. Guidelines on assessment and remediation of fungi in indoor environments [online]. [date assessed: 2010 Jan 7]. Available from URL: $\underline{w w w . n y c .}$ gov/html/doh/downloads/pdflepi/epi-mold-guidelines.pdf.

Patel A, Ryu J, Reed C [2001]. Hypersensitivity pneumonitis: current concepts and future questions. J Allergy Clin Immunol 108:661-670. 


\section{ApPENDIX M: NIOSH INTERIM REPORT, HETA 2009-0172 (CONTINUED)}

Prezant B, Weekes DM, Miller JD, ed. [2008]. Recognition, evaluation, and control of indoor mold. Fairfax, VA: American Industrial Hygiene Association, p. 207.

Scully RF, Galdabini JJ, McNeely BU [1979]. Case records of the Massachusetts General Hospital: Case 47-1979; Presentation of a case. N Engl J Med 301:1168-1174.

SeslineD, Ames RG, Howd RA [1994]. Irritative and systemic symptoms following exposure to Microban disinfectant through a school ventilation system. Arch Environ Health 49:439-444.

Seuri M, Husman K, Kinnunen H, Reiman M, Kreus R, Kuronen P, Lehtomäki K, Paananen M. [2000].

An outbreak of respiratory diseases among workers at a water-damaged building - a case report. Indoor Air 10:138-145.

Thörn A, Lewné M, Belin L [1996]. Allergic alveolitis in a school environment. Scan J Work Environ Health $22: 311-4$.

Weltermann BM, Hodgson M, Storey E, DeGraff AC, Bracker A, Groseclose S, Cole SR, Cartter M, Phillips D [1998]. Hypersensitivity pneumonitis: a sentinel event investigation in a wet building. Am J Ind Med 34:499-505.

World Health Organization (WHO) [2009]. WHO guidelines for indoor air quality: dampness and mould. WHO Regional Office for Europe. 
ApPendix M: NIOSH INTERIM RepORT, HETA 2009-0172 (CONTINUED)

Table 1. Microbial agents ${ }^{*}$ in floor samples ${ }^{\dagger}$ collected by NIOSH during July 2009 site visit

\begin{tabular}{|c|c|c|c|c|}
\hline \multirow[t]{2}{*}{ Room } & \multicolumn{2}{|l|}{ Fungi } & \multicolumn{2}{|l|}{ Bacteria } \\
\hline & Species & $\mathrm{cfu} / \mathrm{g}$ & & $\mathrm{cfu} / \mathrm{g}$ \\
\hline $100^{\mathrm{a}}$ & $\begin{array}{l}\text { Alternaria alternata } \\
\text { Cladosporium cladosporioides } \\
\text { Epicoccum nigrum } \\
\text { Fusarium oxysporum } \\
\text { Total fungi }\end{array}$ & $\begin{array}{l}2,000 \\
4,900 \\
2,000 \\
980 \\
9,800\end{array}$ & $\begin{array}{l}\text { Gram positive } \\
\text { Gram negative, non-fermentative } \\
\text { Broad spectrum }\end{array}$ & $\begin{array}{l}610,000 \\
7,300 \\
380,000\end{array}$ \\
\hline $113^{\mathrm{a}}$ & $\begin{array}{l}\text { Aureobasidium pullulans } \\
\text { Biopolaris australiensis } \\
\text { Cladosporium cladosporioides } \\
\text { Mucor plumbeus } \\
\text { Nonsporulating fungi } \\
\text { Penicillium lividium } \\
\text { Trichoderma koningii } \\
\text { Total fungi }\end{array}$ & $\begin{array}{l}640 \\
91 \\
1,500 \\
91 \\
180 \\
180 \\
91 \\
2,800\end{array}$ & $\begin{array}{l}\text { Gram positive } \\
\text { Gram negative, non-fermentative } \\
\text { Broad spectrum }\end{array}$ & $\begin{array}{l}280,000 \\
5,600 \\
450,000\end{array}$ \\
\hline $115^{\mathrm{a}}$ & $\begin{array}{l}\text { Aspergillus versicolor } \\
\text { Aureobasidium pullulans } \\
\text { Biopolaris australiensis } \\
\text { Cladosporium cladosporioides } \\
\text { Epicoccum nigrum } \\
\text { Nonsporulating fungi } \\
\text { Penicillium lividium } \\
\text { Total fungi }\end{array}$ & $\begin{array}{l}960 \\
960 \\
1,900 \\
13,000 \\
2,900 \\
1,900 \\
960 \\
23,000\end{array}$ & $\begin{array}{l}\text { Gram positive } \\
\text { Gram negative, non-fermentative } \\
\text { Broad spectrum }\end{array}$ & $\begin{array}{l}67,000 \\
6,100 \\
95,000\end{array}$ \\
\hline $116^{\mathrm{a}}$ & $\begin{array}{l}\text { Cladosporium cladosporioides } \\
\text { Epicoccum nigrum } \\
\text { Nonsporulating fungi } \\
\text { Penicillium aurantiogriseum } \\
\text { Total fungi }\end{array}$ & $\begin{array}{l}1,600 \\
540 \\
270 \\
89 \\
2,500\end{array}$ & $\begin{array}{l}\text { Gram positive } \\
\text { Gram negative, non-fermentative } \\
\text { Broad spectrum }\end{array}$ & $\begin{array}{l}450,000 \\
4,600 \\
63,000\end{array}$ \\
\hline $206^{\mathrm{a}}$ & $\begin{array}{l}\text { Alternaria alternata } \\
\text { Aspergillus glaucus } \\
\text { Aspergillus niger } \\
\text { Aspergillus versicolor } \\
\text { Biopolaris australiensis } \\
\text { Cladosporium cladosporioides } \\
\text { Epicoccum nigrum } \\
\text { Fusarium oxysporum } \\
\text { Rhizopus stolonifer } \\
\text { Total fungi }\end{array}$ & $\begin{array}{l}1,000 \\
2,000 \\
1,000 \\
1,000 \\
1,000 \\
14,000 \\
2,000 \\
1,000 \\
1,000 \\
24,000\end{array}$ & $\begin{array}{l}\text { Gram positive } \\
\text { Gram negative, non-fermentative } \\
\text { Broad spectrum }\end{array}$ & $\begin{array}{l}170,000 \\
7,500 \\
210,000\end{array}$ \\
\hline $407^{b}$ & $\begin{array}{l}\text { Alternaria alternata } \\
\text { Aspergillus glaucus } \\
\text { Aspergillus niger } \\
\text { Aspergillus ochraceus } \\
\text { Cladosporium cladosporioides }\end{array}$ & $\begin{array}{l}1,900 \\
2,800 \\
940 \\
940 \\
7,500\end{array}$ & $\begin{array}{l}\text { Gram positive } \\
\text { Gram negative, non-fermentative } \\
\text { Broad spectrum }\end{array}$ & $\begin{array}{l}94,000 \\
6,300 \\
1,200,000\end{array}$ \\
\hline
\end{tabular}




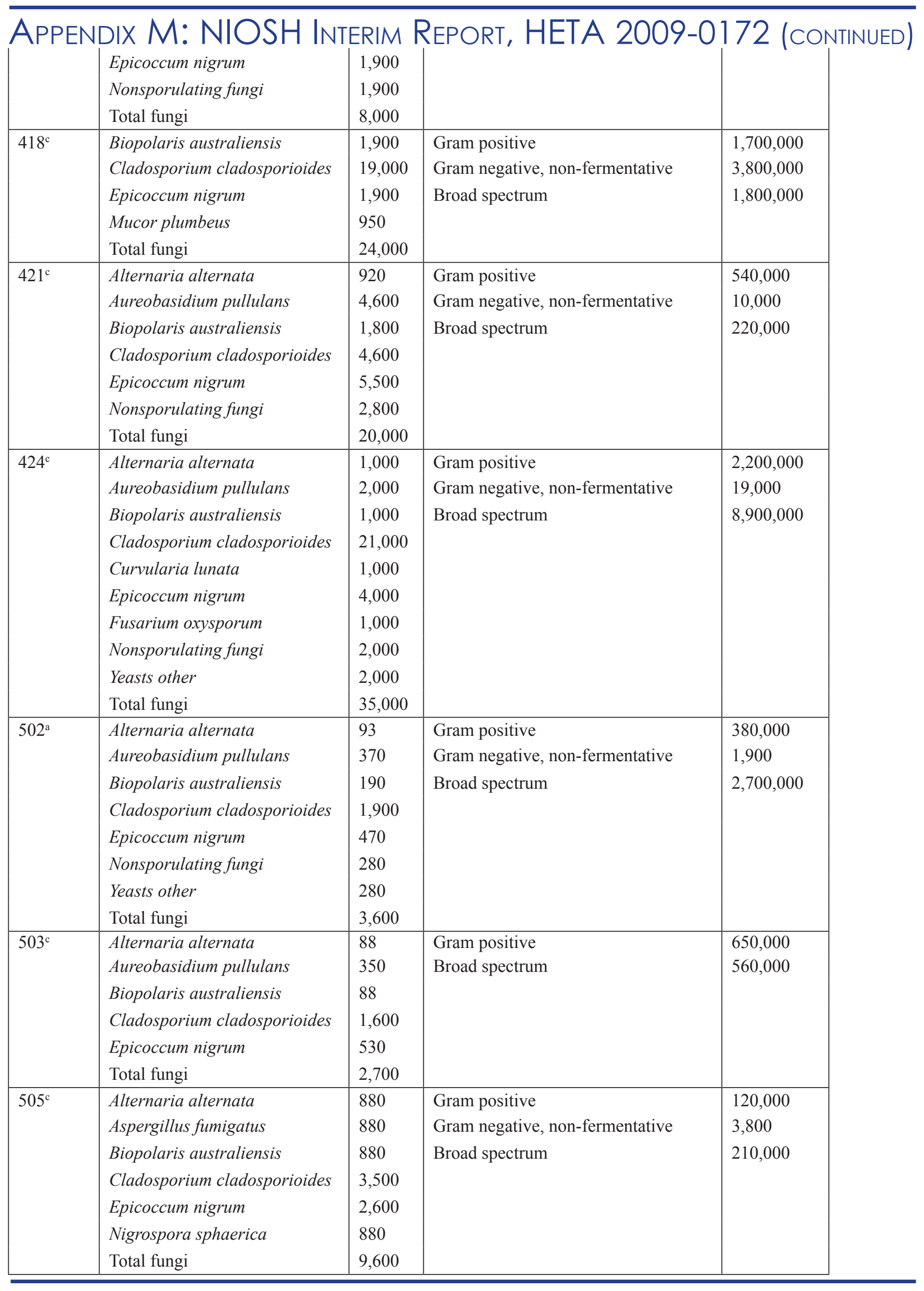




\begin{tabular}{|c|c|c|c|c|}
\hline $515^{\mathrm{c}}$ & $\begin{array}{l}\text { Alternaria alternata } \\
\text { Aspergillus niger } \\
\text { Biopolaris australiensis } \\
\text { Cladosporium cladosporioides } \\
\text { Epicoccum nigrum } \\
\text { Fusarium oxysporum } \\
\text { Nigrospora sphaerica } \\
\text { Total fungi }\end{array}$ & $\begin{array}{l}88 \\
270 \\
180 \\
710 \\
180 \\
88 \\
88 \\
1,600\end{array}$ & $\begin{array}{l}\text { Gram positive } \\
\text { Gram negative, non-fermentative } \\
\text { Broad spectrum }\end{array}$ & $\begin{array}{l}360,000 \\
3,800 \\
380,000\end{array}$ \\
\hline $516^{c}$ & $\begin{array}{l}\text { Alternaria alternata } \\
\text { Aspergillus niger } \\
\text { Cladosporium cladosporioides } \\
\text { Nonsporulating fungi } \\
\text { Total fungi }\end{array}$ & $\begin{array}{l}200 \\
300 \\
1,600 \\
400 \\
2,500\end{array}$ & $\begin{array}{l}\text { Gram positive } \\
\text { Gram negative, non-fermentative } \\
\text { Broad spectrum }\end{array}$ & $\begin{array}{l}1,000,000 \\
8,700 \\
360,000\end{array}$ \\
\hline $520^{b}$ & $\begin{array}{l}\text { Aspergillus versicolor } \\
\text { Cladosporium cladosporioides } \\
\text { Curvularia lunata } \\
\text { Epicoccum nigrum } \\
\text { Nigrospora sphaerica } \\
\text { Nonsporulating fungi } \\
\text { Total fungi }\end{array}$ & $\begin{array}{l}390 \\
490 \\
98 \\
290 \\
98 \\
200 \\
1,600\end{array}$ & $\begin{array}{l}\text { Gram positive } \\
\text { Gram negative, non-fermentative } \\
\text { Broad spectrum }\end{array}$ & $\begin{array}{l}5,900 \\
2,600 \\
19,000\end{array}$ \\
\hline $610^{c}$ & $\begin{array}{l}\text { Alternaria alternata } \\
\text { Aureobasidium pullulans } \\
\text { Biopolaris australiensis } \\
\text { Cladosporium cladosporioides } \\
\text { Epicoccum nigrum } \\
\text { Nonsporulating fungi } \\
\text { Trichoderma koningii } \\
\text { Total fungi }\end{array}$ & $\begin{array}{l}960 \\
960 \\
960 \\
5,800 \\
2,900 \\
1,900 \\
960 \\
14,000\end{array}$ & $\begin{array}{l}\text { Gram positive } \\
\text { Gram negative, non-fermentative } \\
\text { Broad spectrum }\end{array}$ & $\begin{array}{l}290,000 \\
2,000 \\
4,000,000\end{array}$ \\
\hline $612^{c}$ & $\begin{array}{l}\text { Biopolaris australiensis } \\
\text { Cladosporium cladosporioides } \\
\text { Epicoccum nigrum } \\
\text { Mucor plumbeus } \\
\text { Total fungi }\end{array}$ & $\begin{array}{l}980 \\
4,900 \\
2,000 \\
980 \\
8,800\end{array}$ & $\begin{array}{l}\text { Gram positive } \\
\text { Gram negative, non-fermentative } \\
\text { Broad spectrum }\end{array}$ & $\begin{array}{l}12,000,000 \\
5,200 \\
810,000\end{array}$ \\
\hline $618^{c}$ & $\begin{array}{l}\text { Alternaria alternata } \\
\text { Aspergillus fumigatus } \\
\text { Aureobasidium pullulans } \\
\text { Biopolaris australiensis } \\
\text { Cladosporium cladosporioides } \\
\text { Epicoccum nigrum } \\
\text { Nonsporulating fungi } \\
\text { Trichoderma koningii } \\
\text { Total fungi }\end{array}$ & $\begin{array}{l}93 \\
93 \\
190 \\
93 \\
1,300 \\
470 \\
93 \\
93 \\
2,400\end{array}$ & $\begin{array}{l}\text { Gram positive } \\
\text { Gram negative, non-fermentative } \\
\text { Broad spectrum }\end{array}$ & $\begin{array}{l}550,000 \\
170,000 \\
46,000,000\end{array}$ \\
\hline $623^{c}$ & $\begin{array}{l}\text { Aureobasidium pullulans } \\
\text { Biopolaris australiensis } \\
\text { Cladosporium cladosporioides } \\
\text { Epicoccum nigrum }\end{array}$ & $\begin{array}{l}460 \\
92 \\
1,500 \\
460\end{array}$ & $\begin{array}{l}\text { Gram positive } \\
\text { Gram negative, non-fermentative } \\
\text { Broad spectrum }\end{array}$ & $\begin{array}{l}280,000 \\
32,000 \\
220,000\end{array}$ \\
\hline
\end{tabular}




\begin{tabular}{|c|c|c|c|c|}
\hline APPEN & $\begin{array}{l}\text { DIX M: NIOSH IN } \\
\text { Total fungi }\end{array}$ & $\begin{array}{l}\text { ERIM } \\
2,500\end{array}$ & EPORT, HETA 200 & $721 \mathrm{c}$ \\
\hline MR-1 $\mathrm{A}^{\mathrm{d}}$ & $\begin{array}{l}\text { Alternaria alternata } \\
\text { Aspergillus niger } \\
\text { Aspergillus ochraceus } \\
\text { Aspergillus versicolor } \\
\text { Cladosporium cladosporioides } \\
\text { Fusarium oxysporum } \\
\text { Paecilomyces variotii } \\
\text { Penicillium crustosum } \\
\text { Total fungi }\end{array}$ & $\begin{array}{l}86 \\
86 \\
170 \\
340 \\
170 \\
170 \\
86 \\
260 \\
1,400\end{array}$ & $\begin{array}{l}\text { Gram positive } \\
\text { Gram negative, non-fermentative } \\
\text { Broad spectrum }\end{array}$ & $\begin{array}{l}6,000 \\
86 \\
26,000\end{array}$ \\
\hline MR-1Bd & $\begin{array}{l}\text { Aspergillus niger } \\
\text { Cladosporium cladosporioides } \\
\text { Epicoccum nigrum } \\
\text { Trichoderma koningii } \\
\text { Total fungi }\end{array}$ & $\begin{array}{l}93 \\
370 \\
93 \\
93 \\
650\end{array}$ & $\begin{array}{l}\text { Gram positive } \\
\text { Gram negative, non-fermentative } \\
\text { Broad spectrum }\end{array}$ & $\begin{array}{l}21,000,000 \\
1,600 \\
350,000\end{array}$ \\
\hline $\mathrm{MR}^{2 \mathrm{e}}$ & $\begin{array}{l}\text { Alternaria alternata } \\
\text { Aspergillus versicolor } \\
\text { Cladosporium cladosporioides } \\
\text { Epicoccum nigrum } \\
\text { Fusarium oxysporum } \\
\text { Nonsporulating fungi } \\
\text { Trichoderma koningii } \\
\text { Total fungi }\end{array}$ & $\begin{array}{l}1,700 \\
860 \\
17,000 \\
5,200 \\
860 \\
860 \\
860 \\
28,000\end{array}$ & $\begin{array}{l}\text { Gram positive } \\
\text { Gram negative, non-fermentative } \\
\text { Broad spectrum }\end{array}$ & $\begin{array}{l}2,000,000 \\
110,000 \\
4,100,000\end{array}$ \\
\hline
\end{tabular}

"Total fungi values have been rounded by the reporting laboratory; ${ }^{\dagger}$ room 108 was excluded because we were unable to collect enough dust for microbial analyses. Note: MR-1A, MR-1B = library - samples collected from edge; MR-2= librarysamples collected from rugs; ${ }^{\mathrm{a}}$ carpet; $\quad{ }^{\mathrm{b}}$ tile; ${ }^{\mathrm{c}}$ carpet with partial tile floor; ${ }^{\mathrm{d}}$ wood floor; ${ }^{\mathrm{e}}$ rug 
ApPendix M: NIOSH InTERIM RePORT, HETA 2009-0172 (CONTINUED)

Table 2. Arithmetic mean and standard deviation of water activity on the floor surface, and relative humidity and temperature in air by wing at Oak Ridge Elementary School

\begin{tabular}{|c|c|c|c|c|}
\hline Building wings & $\begin{array}{c}\text { Number of } \\
\text { samples }\end{array}$ & Water activity & $\begin{array}{c}\text { Relative humidity } \\
(\%)\end{array}$ & Temperature $\left({ }^{\circ} \mathrm{F}\right)$ \\
\hline Gym-cafeteria & 1 & $0.60(-)$ & $53.0(-)$ & $73.0(-)$ \\
\hline Admin & 5 & $0.76(0.09)$ & $59.0(9.4)$ & $71.6(0.7)$ \\
\hline Media center & 3 & $0.73(0.03)$ & $55.0(1.7)$ & $72.1(0.2)$ \\
\hline $\mathrm{k} / 1$ & 4 & $0.57(0.07)$ & $36.8(2.2)$ & $72.4(1.4)$ \\
\hline $2 / 3$ & 4 & $0.66(0.10)$ & $38.8(5.7)$ & $72.1(0.4)$ \\
\hline $4 / 5$ & 6 & $0.69(0.06)$ & $53.8(3.6)$ & $72.8(0.7)$ \\
\hline
\end{tabular}

Table 3. Overall building $(\mathrm{N}=22)$ concentration $(\mathrm{cfu} / \mathrm{g})$ of culturable fungi and bacteria

\begin{tabular}{|c|c|c|c|c|c|}
\hline & Total fungi & $\begin{array}{c}\text { Hydrophilic } \\
\text { fungi }\end{array}$ & $\begin{array}{c}\text { Mesophilic or } \\
\text { hydrophilic } \\
\text { fungi }\end{array}$ & $\begin{array}{c}\text { Gram negative } \\
\text { bacteria }\end{array}$ & $\begin{array}{c}\text { Gram positive } \\
\text { bacteria }\end{array}$ \\
\hline Minimum & 650 & 86 & 650 & 86 & 5,900 \\
\hline Maximum & 35,000 & 7,800 & 31,000 & $5,600,000$ & $21,000,000$ \\
\hline $\begin{array}{c}\text { Geometric } \\
\text { mean }\end{array}$ & 6,000 & 1,200 & 5,200 & 8,500 & 396,000 \\
\hline $\begin{array}{c}\text { Geometric } \\
\text { STD* }\end{array}$ & 3.3 & 4.2 & 3.3 & 8.1 & 7.0 \\
\hline
\end{tabular}

"STD: standard deviation

Table 4. Geometric mean (Geometric Standard Deviation ${ }^{\dagger}$ ) of culturable fungi and bacteria (cfu/g*) by wing

\begin{tabular}{|c|c|c|c|c|c|}
\hline Wings & $\mathrm{N}$ & $\begin{array}{c}\text { Total } \\
\text { fungi }\end{array}$ & $\begin{array}{c}\text { Hydrophilic } \\
\text { fungi }\end{array}$ & $\begin{array}{c}\text { Gram negative } \\
\text { bacteria }\end{array}$ & $\begin{array}{c}\text { Gram positive } \\
\text { bacteria }\end{array}$ \\
\hline $\begin{array}{c}\text { Gym- } \\
\text { cafeteria }\end{array}$ & 1 & $24,000(-)$ & $4,000(-)$ & $7,700(-)$ & $170,000(-)$ \\
\hline Admin & 4 & $6,300(2.9)$ & $1,000(4.3)$ & $5,900(1.2)$ & $267,900(2.7)$ \\
\hline Media center & 3 & $2,900(7.3)$ & $500(11.1)$ & $2,500(37.7)$ & $631,600(66.7)$ \\
\hline $\mathrm{k} / 1$ & 4 & $5,200(2.4)$ & $1,500(3.0)$ & $15,800(7.1)$ & $855,600(6.0)$ \\
\hline $2 / 3$ & 4 & $23,500(1.3)$ & $4,700(1.5)$ & $52,000(23.4)$ & $660,100(4.2)$ \\
\hline $4 / 5$ & 6 & $2,900(2.0)$ & $600(3.1)$ & $3,900(1.7)$ & $199,500(6.5)$ \\
\hline
\end{tabular}

"Geometric standard deviation; ${ }^{\dagger}$ colony forming unit per gram 
ApPendix M: NIOSH INTERIM RePORT, HETA 2009-0172 (CONTINUED)

Table 5. Comparison of results from historical environmental consultants' reports from May 2009 with $\mathrm{NIOSH}$ results for fungal concentration in floor dust

\begin{tabular}{|c|c|c|}
\hline Room number & $\begin{array}{l}05 / 08 / 2009 \\
\text { (Consultant results) cfu/g }\end{array}$ & $\begin{array}{l}\text { July } 2009 \\
\text { (NIOSH Results) cfu/g }\end{array}$ \\
\hline $\begin{array}{l}115 \\
\text { in admin wing }\end{array}$ & Total $(30,000)$ & $\begin{array}{l}\text { Aspergillus versicolor }(960) \\
\text { Aspergillus pullulans }(960) \\
\text { Biopolaris australiensis }(1,900) \\
\text { Cladosporium cladosporioides }(13,000) \\
\text { Epicoccum nigrum }(2,900) \\
\text { Nonsporulating fungi }(1,900) \\
\text { Penicillium lividium }(960) \\
\text { Total } \mathbf{( 2 3 , 0 0 0 )}\end{array}$ \\
\hline $\begin{array}{l}206 \\
\text { in gym-cafeteria wing }\end{array}$ & $\begin{array}{l}\text { Alternaria } \\
\text { Aspergillus } \\
\text { Epicoccum } \\
\text { Eurotium } \\
\text { Nonsporulating fungi } \\
\text { Penicillium } \\
\text { Yeast } \\
\\
\text { Total (>1.3 million)* }\end{array}$ & $\begin{array}{l}\text { Alternaria alternata }(1,000) \\
\text { Aspergillus glaucus }(2,000) \\
\text { Aspergillus niger }(1,000) \\
\text { Aspergillus versicolor }(1,000) \\
\text { Biopolaris australiensis }(1,000 \\
\text { Cladosporium cladosporioides }(14,000) \\
\text { Epicoccum nigrum }(2,000) \\
\text { Fusarium oxysporum }(1,000) \\
\text { Rhizopus stolonifer }(1,000) \\
\text { Total }(\mathbf{2 4 , 0 0 0 )}\end{array}$ \\
\hline $\begin{array}{l}421 \\
\text { in } 2 / 3 \text { wing }\end{array}$ & $\begin{array}{l}\text { Alternaria }(5,000) \\
\text { Aspergillus }(65,000) \\
\text { Bipolaris }(5,000) \\
\text { Cladosporium }(75,000) \\
\text { Curvularia }(5,000) \\
\text { Epicoccum }(60,000) \\
\text { Exserohilium }(5,000) \\
\text { Nonsporulating fungi }(70,000) \\
\text { Yeast }(40,000) \\
\text { Total }(\mathbf{3 3 0 , 0 0 0 )}\end{array}$ & $\begin{array}{l}\text { Alternaria alternata }(920) \\
\text { Aspergillus pullulans }(4,600) \\
\text { Biopolaris australiensis }(1,800) \\
\text { Cladosporium cladosporioides }(4,600) \\
\text { Epicoccum nigrum }(5,500) \\
\text { Nonsporulating fungi }(2,800)\end{array}$ \\
\hline $\begin{array}{l}503 \\
\text { in } 4 / 5 \text { wing }\end{array}$ & $\begin{array}{l}\text { Acremonium }(8,000) \\
\text { Alternaria }(40,000) \\
\text { Bipolaris }(4,000) \\
\text { Cladosporium }(36,000) \\
\text { Epicoccum }(48,000) \\
\text { Nonsporulating fungi }(60,000) \\
\text { Phoma }(12,000) \\
\text { Trichoderma }(12,000) \\
\text { Yeast }(24,000) \\
\text { Total }(\mathbf{2 4 4 , 0 0 0 )}\end{array}$ & $\begin{array}{l}\text { Alternaria alternata }(88) \\
\text { Aspergillus pullulans }(350) \\
\text { Biopolaris australiensis }(88) \\
\text { Cladosporium cladosporioides }(1,600) \\
\text { Epicoccum nigrum }(530)\end{array}$ \\
\hline $\begin{array}{l}515 \\
\text { in } 4 / 5 \text { wing }\end{array}$ & $\begin{array}{l}\text { Alternaria } \\
\text { Aspergillus } \\
\text { Aureobasidium } \\
\text { Bipolaris }\end{array}$ & $\begin{array}{l}\text { Alternaria alternata }(88) \\
\text { Aspergillus niger }(270) \\
\text { Biopolaris australiensis (180) } \\
\text { Cladosporium cladosporioides (710) }\end{array}$ \\
\hline
\end{tabular}




\begin{tabular}{|c|c|c|}
\hline APPEN & $\begin{array}{l}\text { SH INTERIM REP } \\
\text { Chrysosporium } \\
\text { Cladosporium } \\
\text { Curvularia } \\
\text { Epicoccum } \\
\text { Nonsporulating } \\
\text { Penicillium } \\
\text { Stemphylium } \\
\text { Trichoderma } \\
\text { Yeast } \\
\text { Total (>1.6 million) }\end{array}$ & $\begin{array}{l}\text { HETA 2009-0172 (C } \\
\text { Epicoccum nigrum (180) } \\
\text { Fusarium oxysporum (88) } \\
\text { Nigrospora sphaerica (88) }\end{array}$ \\
\hline $\begin{array}{l}516 \\
\text { in } 4 / 5 \text { wing }\end{array}$ & $\begin{array}{l}\text { Alternaria } \\
\text { Aspergillus } \\
\text { Bipolaris } \\
\text { Chrysosporium } \\
\text { Cladosporium } \\
\text { Epicoccum } \\
\text { Eurotium } \\
\text { Fusarium } \\
\text { Geotrichum } \\
\text { Mucor } \\
\text { Nonsporulating fungi } \\
\text { Penicillium } \\
\text { Yeast } \\
\text { Total (>2 million)* }\end{array}$ & $\begin{array}{l}\text { Alternaria alternata }(200) \\
\text { Aspergillus niger }(300) \\
\text { Cladosporium cladosporioides }(1,600) \\
\text { Nonsporulating fungi }(400)\end{array}$ \\
\hline $\begin{array}{l}610 \\
\text { in } \mathrm{k} / 1 \mathrm{wing}\end{array}$ & $\begin{array}{l}\text { Alternaria }(13,333) \\
\text { Bipolaris }(6,667) \\
\text { Cladosporium }(6,667) \\
\text { Epicoccum }(13,333) \\
\text { Nonsporulating }(13,333) \\
\text { Yeast }(13,333) \\
\text { Total }(\mathbf{8 6 , 6 6 7 )}\end{array}$ & $\begin{array}{l}\text { Alternaria alternata }(960) \\
\text { Aspergillus pullulans }(960) \\
\text { Biopolaris australiensis }(960) \\
\text { Cladosporium cladosporioides }(5,800) \\
\text { Epicoccum nigrum }(2,900) \\
\text { Nonsporulating fungi }(1,900) \\
\text { Trichoderma koningii }(960) \\
\text { Total }(\mathbf{1 4 , 0 0 0 )}\end{array}$ \\
\hline $\begin{array}{l}618 \\
\text { in } \mathrm{k} / 1 \mathrm{wing}\end{array}$ & $\begin{array}{l}\text { Alternaria } \\
\text { Aspergillus } \\
\text { Cladosporium } \\
\text { Epicoccum } \\
\text { Geotrichun } \\
\text { Mucor } \\
\text { Nonsporulating } \\
\text { Penicillium } \\
\text { Trichoderma } \\
\text { Yeast } \\
\text { Total (>1.0 million)* }\end{array}$ & $\begin{array}{l}\text { Alternaria alternata }(93) \\
\text { Aspergillus fumigatus }(93) \\
\text { Aspergillus pullulans }(190) \\
\text { Biopolaris australiensis }(93) \\
\text { Cladosporium cladosporioides }(1,300) \\
\text { Epicoccum nigrum }(470) \\
\text { Nonsporulating fungi }(93) \\
\text { Trichoderma koningii }(93) \\
\text { Total } \mathbf{( 2 , 4 0 0 )}\end{array}$ \\
\hline
\end{tabular}

*Too numerous to count - no individual spore type counts made 


\section{Appendix M: NIOSH Interim Report, HETA 2009-0172 (CONTINUEd)}

Table 6. Comparison of results from historical environmental consultants' reports from October 2007 and September and October 2008 with NIOSH results for fungal concentration in floor dust

\begin{tabular}{|c|c|c|c|c|}
\hline $\begin{array}{l}\text { Room } \\
\text { number }\end{array}$ & $\begin{array}{l}\text { 10/29/2007 } \\
\text { (Consultant) }\end{array}$ & $\begin{array}{l}\text { 09/24/2008 } \\
\text { (Consultant) cfu/g }\end{array}$ & $\begin{array}{l}10 / 27 / 2008 \\
\text { (Consultant) cfu/g }\end{array}$ & $\begin{array}{l}\text { July } 2009 \\
\text { (NIOSH Results) cfu/g }\end{array}$ \\
\hline $8 \frac{\text { ลे }}{8}$ & $\begin{array}{l}\text { Acremonium }(380,000) \\
\text { Cladosporium }(290,000) \\
\text { Curvularia lunata }(48,000) \\
\text { Epicoccum nigrum }(95,000) \\
\text { Fusarium solani }(95,000) \\
\text { Total }(\mathbf{9 0 8 , 0 0 0 )}\end{array}$ & & & $\begin{array}{l}\text { Alternaria alternata }(2,000) \\
\text { Cladosporium cladosporioides }(4,900) \\
\text { Epicoccum nigrum }(2,000) \\
\text { Fusarium oxysporum }(980) \\
\text { Total }(\mathbf{9 , 8 0 0 )}\end{array}$ \\
\hline 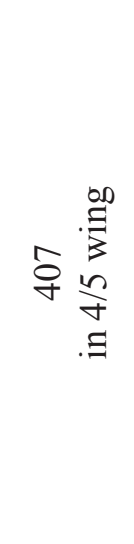 & & $\begin{array}{l}\text { Alternaria }(200,000) \\
\text { Aspergillus }(320,000) \\
\text { Cladosporium }(40,000) \\
\text { Bipolaris }(40,000) \\
\text { Epicoccum }(20,000) \\
\text { Penicillium }(20,000) \\
\text { Phoma }(600,000) \\
\text { Wallemia }(60,000) \\
\text { Total }(\mathbf{1 , 3 0 0 , 0 0 0 )}\end{array}$ & $\begin{array}{l}\text { Alternaria }(1,081) \\
\text { Aspergillus }(17,838) \\
\text { Aureobasidium }(1,081) \\
\text { Cladosporium }(4,865) \\
\text { Bipolaris }(1,622) \\
\text { Epicoccum }(6,486) \\
\text { Wallemia }(541) \\
\text { Yeast }(1,081) \\
\text { Nonsporulating fungi } \\
(541) \\
\text { Total }(\mathbf{3 5 , 1 3 5 )}\end{array}$ & $\begin{array}{l}\text { Alternaria alternata }(1,900) \\
\text { Aspergillus glaucus }(2,800) \\
\text { Aspergillus niger }(940) \\
\text { Aspergillus ochraceus }(940) \\
\text { Cladosporium cladosporioides }(7,500) \\
\text { Epicoccum nigrum }(1,900) \\
\text { Nonsporulating fungi }(1,9001)\end{array}$ \\
\hline
\end{tabular}


$\overline{\text { Appendix M: NIOSH InterIM RepORt, HETA 2009-0172 (CONTINUED) }}$

Table 7. Primary work area or homeroom wing of staff and students participating in the health department questionnaire

\begin{tabular}{|l|l|l|}
\hline & \multicolumn{1}{|c|}{ Staff } & \multicolumn{1}{c|}{ Student } \\
\hline Wings & $\begin{array}{l}2008-2009 \\
\text { school year } \\
\mathrm{n}=48^{*}\end{array}$ & $\begin{array}{l}\text { 2008-2009 } \\
\text { school year } \\
\mathrm{n}=376^{\dagger}\end{array}$ \\
\hline $\mathrm{k} / 1$ & $18(38 \%)$ & $111(30 \%)$ \\
\hline $2 / 3$ & $13(27 \%)$ & $136(36 \%)$ \\
\hline $4 / 5$ & $9(19 \%)$ & $129(34 \%)$ \\
\hline $\begin{array}{l}\text { Non-classroom } \\
\text { wings }\end{array}$ & $8(17 \%)$ & - \\
\hline
\end{tabular}

*four of the 52 participants did not provide their primary work location;

${ }^{\dagger}$ we could not classify the primary classroom wing for nine of the 385 participants;

${ }^{\ddagger}$ gym/cafeteria, administration, or media center wings.

Table 8. Prevalence of health conditions reported by staff and students

\begin{tabular}{|l|l|l|}
\hline & $\begin{array}{l}\text { Staff } \\
\mathrm{n}=50^{*}\end{array}$ & $\begin{array}{l}\text { Students } \\
\mathrm{n}=276^{\dagger}\end{array}$ \\
\hline $\begin{array}{l}\text { Seasonal } \\
\text { allergies }\end{array}$ & $17(34 \%)$ & $132(48 \%)$ \\
\hline Asthma & $4(8 \%)$ & $44(16 \%)$ \\
\hline $\begin{array}{l}\text { Allergic } \\
\text { rhinitis }\end{array}$ & $4(8 \%)$ & $33(12 \%)$ \\
\hline $\begin{array}{l}\text { Chronic } \\
\text { respiratory } \\
\text { disease }\end{array}$ & 0 & $4(1 \%)$ \\
\hline $\begin{array}{l}\text { Suppressed } \\
\text { immune } \\
\text { system }\end{array}$ & $1(2 \%)$ & $2(0.7 \%)$ \\
\hline Cystic fibrosis & $1(2 \%)$ & 0 \\
\hline $\begin{array}{l}\text { Radiation or } \\
\text { chemotherapy }\end{array}$ & 0 & 0 \\
\hline
\end{tabular}

"data was missing for two of the of the 52 staff participants; ${ }^{\dagger}$ data was missing for 111 of the 387 students whose parents participated in the questionnaire 
APPENDIX M: NIOSH INTERIM REPORT, HETA 2009-0172 (CONTINUED)

Table 9. Prevalence of symptoms* reported to occur while in the school building by school staff and students during the 2008/2009 school year

\begin{tabular}{|l|c|c|}
\hline & $\begin{array}{c}\text { Staff } \\
\mathrm{n}=52\end{array}$ & $\begin{array}{c}\text { Students } \\
\mathrm{n}=385\end{array}$ \\
\hline Headache $^{\dagger}$ & $48(92 \%)$ & $187(49 \%)$ \\
\hline Eye symptoms & $39(75 \%)$ & $106(28 \%)$ \\
\hline Fatigue & $23(44 \%)$ & $23(6 \%)$ \\
\hline Unusual sneezing & $16(31 \%)$ & $88(23 \%)$ \\
\hline $\begin{array}{l}\text { Breathing difficulty/ } \\
\text { asthma }\end{array}$ & $16(31 \%)$ & $49(13 \%)$ \\
\hline Sinus problems & $13(25 \%)$ & $18(5 \%)$ \\
\hline Nosebleed & $13(25 \%)$ & $77(20 \%)$ \\
\hline Throat symptoms & $12(23 \%)$ & $19(5 \%)$ \\
\hline Dizziness & $11(21 \%)$ & $8(2 \%)$ \\
\hline Skin problems & $10(19 \%)$ & $34(9 \%)$ \\
\hline Cough & $8(15 \%)$ & $28(7 \%)$ \\
\hline Nausea & $7(13 \%)$ & $16(4 \%)$ \\
\hline Memory loss & $7(13 \%)$ & $1(0.3 \%)$ \\
\hline Nasal symptoms & $5(10 \%)$ & $20(5 \%)$ \\
\hline Voice symptoms & $5(10 \%)$ & 0 \\
\hline Vision problems & $4(8 \%)$ & $6(2 \%)$ \\
\hline Metallic taste & $4(8 \%)$ & 0 \\
\hline Insomnia & $3(6 \%)$ & $3(0.8 \%)$ \\
\hline Allergies & $1(2 \%)$ & $9(2 \%)$ \\
\hline $\begin{array}{l}\text { Throat or voice } \\
\text { symptoms }\end{array}$ & $14(27 \%)$ & $19(5 \%)$ \\
\hline $\begin{array}{l}\text { Lower respiratory } \\
\text { symptoms }\end{array}$ & $20(38 \%)$ & $72(19 \%)$ \\
\hline $\begin{array}{l}\text { Upper respiratory } \\
\text { symptoms }\end{array}$ & $27(52 \%)$ & $106(28 \%)$ \\
\hline $\begin{array}{l}\text { Mucous membrane } \\
\text { irritation }\end{array}$ & $45(87 \%)$ & $12 \%)$ \\
\hline
\end{tabular}

includes write-in responses; ${ }^{\dagger}$ symptoms specifically asked about in the questionnaire. 
APPENDIX M: NIOSH INTERIM REPORT, HETA 2009-0172 (CONTINUED)

Table 10. Prevalence of symptoms by primary work wing of $48^{*}$ staff members during the 2008/2009

school year

\begin{tabular}{|l|c|l|l|l|}
\hline & $\mathrm{k} / 1$ wing & $4 / 5$ wing & $2 / 3$ wing & $\begin{array}{l}\text { Non- } \\
\text { classroom } \\
\text { wings } \\
\mathrm{n}=8\end{array}$ \\
\hline Headache $^{\dagger}$ & $18(100 \%)$ & $8(89 \%)$ & $12(92 \%)$ & $7(88 \%)$ \\
\hline Eye symptoms & $15(83 \%)$ & $8(89 \%)$ & $9(69 \%)$ & $4(50 \%)$ \\
\hline Fatigue & $9(50 \%)$ & $6(67 \%)$ & $5(38 \%)$ & $1(13 \%)$ \\
\hline Throat symptoms & $5(28 \%)$ & 0 & $6(46 \%)$ & 0 \\
\hline $\begin{array}{l}\text { Breathing difficulty/ } \\
\text { asthma }\end{array}$ & $4(22 \%)$ & $4(44 \%)$ & $5(38 \%)$ & $3(38 \%)$ \\
\hline Unusual sneezing & $4(22 \%)$ & $6(67 \%)$ & $3(23 \%)$ & $3(38 \%)$ \\
\hline Nosebleed & $4(22 \%)$ & $2(22 \%)$ & $3(23 \%)$ & $2(25 \%)$ \\
\hline Sinus problems & $2(11 \%)$ & $2(22 \%)$ & $6(46 \%)$ & $3(38 \%)$ \\
\hline Nausea & $2(11 \%)$ & $1(11 \%)$ & $3(23 \%)$ & $1(13 \%)$ \\
\hline Cough & $2(11 \%)$ & $2(22 \%)$ & $3(23 \%)$ & $1(13 \%)$ \\
\hline Skin problems & $1(6 \%)$ & $3(33 \%)$ & $4(31 \%)$ & $1(13 \%)$ \\
\hline Dizziness & $6(33 \%)$ & $1(11 \%)$ & $3(23 \%)$ & $1(13 \%)$ \\
\hline Memory loss & $1(6 \%)$ & $1(11 \%)$ & $3(23 \%)$ & $1(13 \%)$ \\
\hline Nasal symptoms & 0 & 0 & $3(23 \%)$ & $1(13 \%)$ \\
\hline Metallic taste & 0 & $1(11 \%)$ & $2(15 \%)$ & $1(13 \%)$ \\
\hline Voice symptoms & $1(6 \%)$ & $2(22 \%)$ & $2(15 \%)$ & 0 \\
\hline Insomnia & 0 & $1(11 \%)$ & $2(15 \%)$ & 0 \\
\hline Vision problems & $1(6 \%)$ & $2(22 \%)$ & 0 & 0 \\
\hline Allergies & 0 & 0 & 0 & $1(13 \%)$ \\
\hline $\begin{array}{l}\text { Lower respiratory } \\
\text { symptoms }\end{array}$ & $5(28 \%)$ & $5(56 \%)$ & $7(54 \%)$ & $3(38 \%)$ \\
\hline $\begin{array}{l}\text { Upper respiratory } \\
\text { symptoms }\end{array}$ & $7(39 \%)$ & $7(78 \%)$ & $9(69 \%)$ & $3(38 \%)$ \\
\hline $\begin{array}{l}\text { Mucous membrane } \\
\text { irritation }\end{array}$ & $16(89 \%)$ & $9(100 \%)$ & $13(100 \%)$ & $4(50 \%)$ \\
\hline
\end{tabular}

"four of the 52 participants did not provide their primary work location; ${ }^{\dagger}$ symptoms specifically asked about in the questionnaire; ${ }^{\ddagger} \mathrm{gym} /$ cafeteria, administration, or media center wings. 
ApPendix M: NIOSH InTERIM Report, HETA 2009-0172 (CONTINUED)

Table 11. Prevalence of symptoms by primary homeroom wing of $376^{*}$

students during the 2008/2009 school year

\begin{tabular}{|c|c|c|c|}
\hline & $\begin{array}{l}\mathrm{k} / 1 \text { wing } \\
\mathrm{n}=111\end{array}$ & $\begin{array}{l}2 / 3 \text { wing } \\
n=136\end{array}$ & $\begin{array}{l}\text { 4/5 wing } \\
n=129\end{array}$ \\
\hline Headache $^{\dagger}$ & $40(36 \%)$ & $72(53 \%)$ & $72(56 \%)$ \\
\hline Eye symptoms & $22(20 \%)$ & $41(30 \%)$ & $41(32 \%)$ \\
\hline Fatigue & $8(7 \%)$ & $5(4 \%)$ & $10(8 \%)$ \\
\hline Throat symptoms & $4(4 \%)$ & $6(4 \%)$ & $7(5 \%)$ \\
\hline $\begin{array}{l}\text { Breathing difficulty/ } \\
\text { asthma }^{\dagger}\end{array}$ & $9(8 \%)$ & $18(13 \%)$ & $20(16 \%)$ \\
\hline Usual sneezing $^{\dagger}$ & $19(17 \%)$ & $31(23 \%)$ & $36(28 \%)$ \\
\hline Nosebleed $^{\dagger}$ & $15(14 \%)$ & $35(26 \%)$ & $25(19 \%)$ \\
\hline Sinus problems & $6(5 \%)$ & $7(5 \%)$ & $5(4 \%)$ \\
\hline Nausea & $2(2 \%)$ & $7(5 \%)$ & $7(5 \%)$ \\
\hline Cough & $9(8 \%)$ & $12(9 \%)$ & $5(4 \%)$ \\
\hline Skin problems & $10(9 \%)$ & $11(8 \%)$ & $12(9 \%)$ \\
\hline Dizziness & $1(1 \%)$ & $6(4 \%)$ & $1(0.8 \%)$ \\
\hline Memory loss & 0 & 0 & $1(0.8 \%)$ \\
\hline Nasal symptoms & $5(5 \%)$ & $10(7 \%)$ & $4(3 \%)$ \\
\hline Metallic taste & 0 & 0 & 0 \\
\hline Voice symptoms & 0 & 0 & 0 \\
\hline Insomnia & 0 & $2(1 \%)$ & $1(0.8 \%)$ \\
\hline Vision problems & $1(1 \%)$ & $2(1 \%)$ & $3(2 \%)$ \\
\hline Allergies & $2(2 \%)$ & $6(4 \%)$ & $1(0.8 \%)$ \\
\hline $\begin{array}{l}\text { Throat or voice } \\
\text { symptoms }\end{array}$ & $4(4 \%)$ & $6(4 \%)$ & $7(5 \%)$ \\
\hline $\begin{array}{l}\text { Lower respiratory } \\
\text { symptoms }\end{array}$ & $17(15 \%)$ & $26(19 \%)$ & $25(19 \%)$ \\
\hline $\begin{array}{l}\text { Upper respiratory } \\
\text { symptoms }\end{array}$ & $24(22 \%)$ & $39(29 \%)$ & $40(31 \%)$ \\
\hline $\begin{array}{l}\text { Mucous membrane } \\
\text { irritation }\end{array}$ & $27(24 \%)$ & $45(33 \%)$ & $48(37 \%)$ \\
\hline
\end{tabular}

"we could not classify the primary classroom wing for nine of the 385 participants;

${ }^{\dagger}$ symptoms specifically asked about in the questionnaire. 
ApPendix M: NIOSH INTERIM RePORT, HETA 2009-0172 (CONTINUED) Figure1. Diagram of Oak Ridge Elementary School

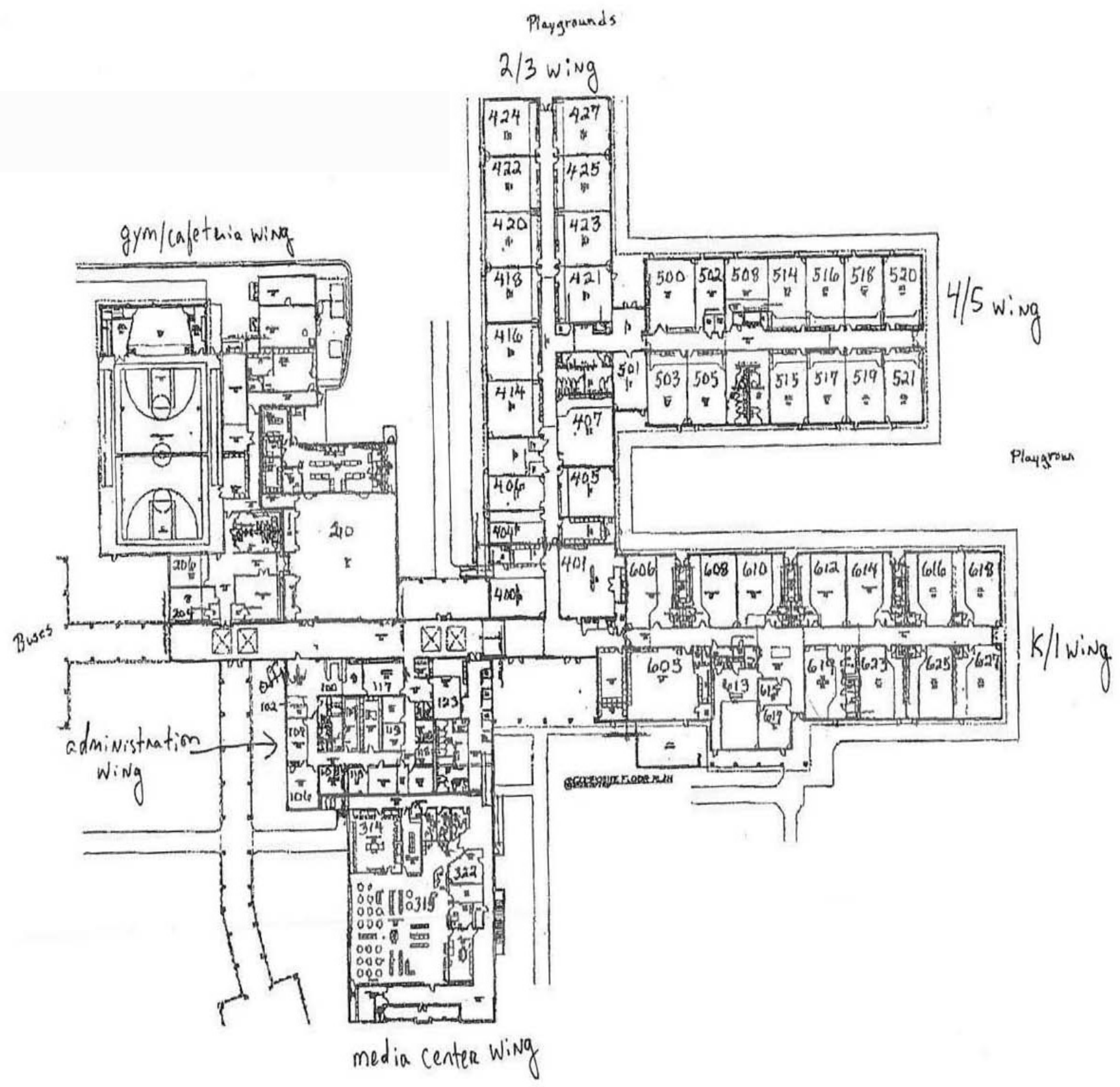




\section{Appendix M: NIOSH Interim Report, HETA 2009-0172 (CONTINUEd)}

Figure 2. Visible mold on carpet in classroom 625, August 10, 2005 (courtesy of building occupant)

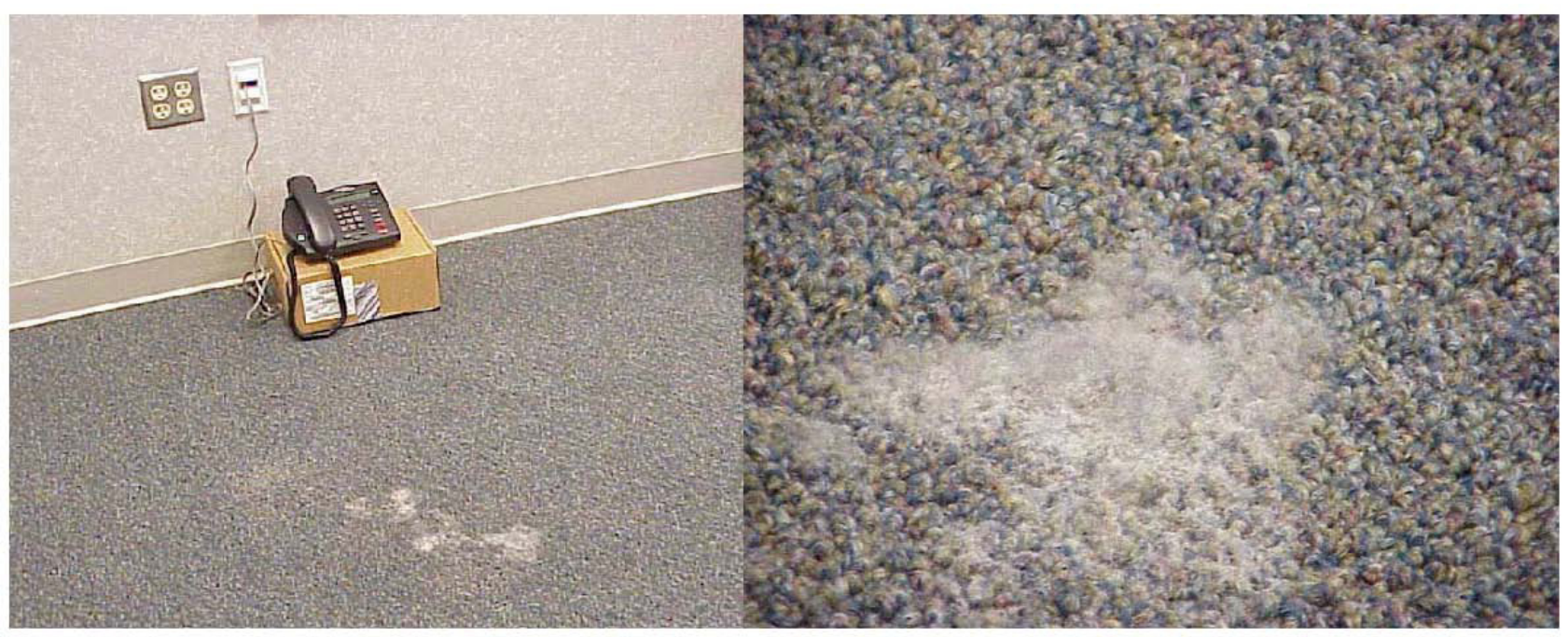

Figure 3. Visible mold on upholstered chairs in the records room (room 109), August 10, 2005 (courtesy of building occupant)

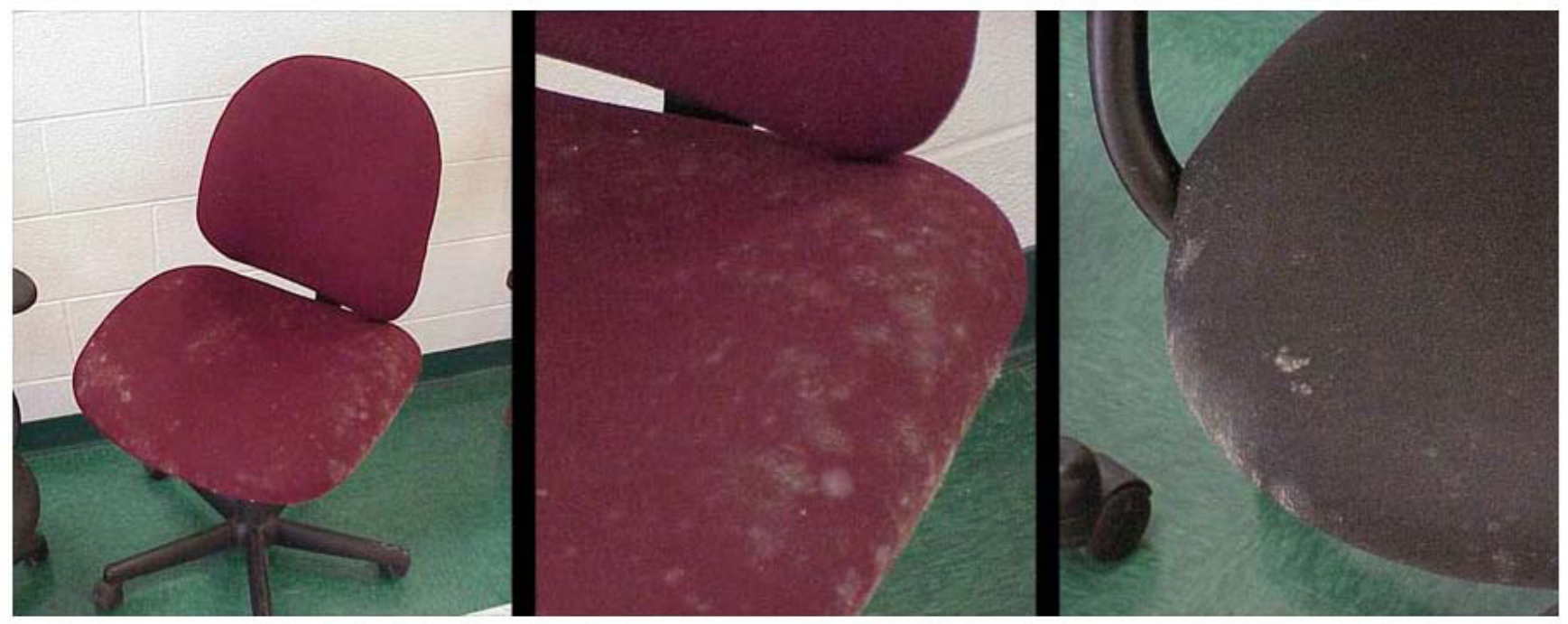




\section{Appendix M: NIOSH Interim Report, HETA 2009-0172 (CONTINUED)}

Figure 4. Visible mold on picture frames in the storage room (room 111), August 10, 2005 (courtesy of building occupant)
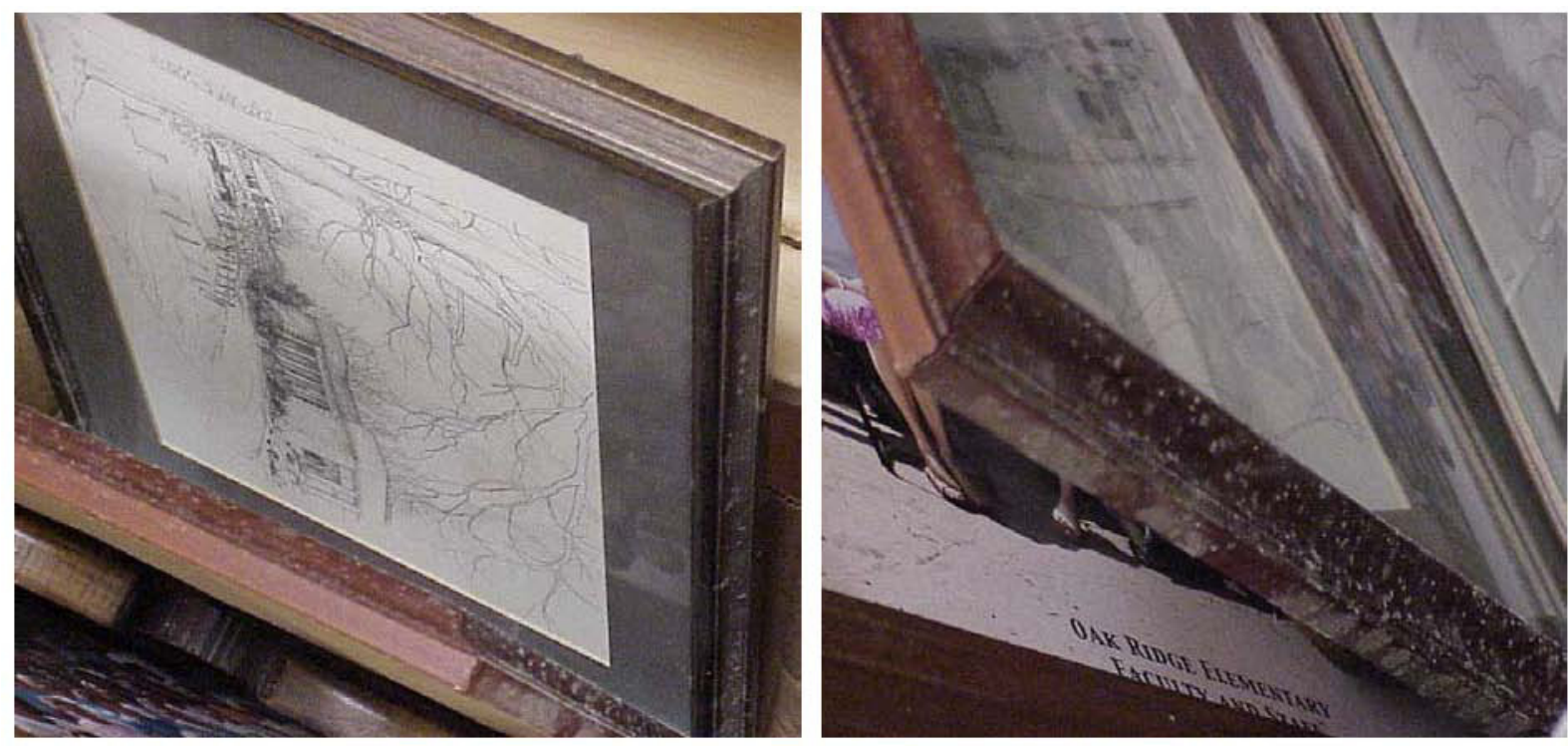

Figure 5. Basement below the media center wing, July 14, 2009

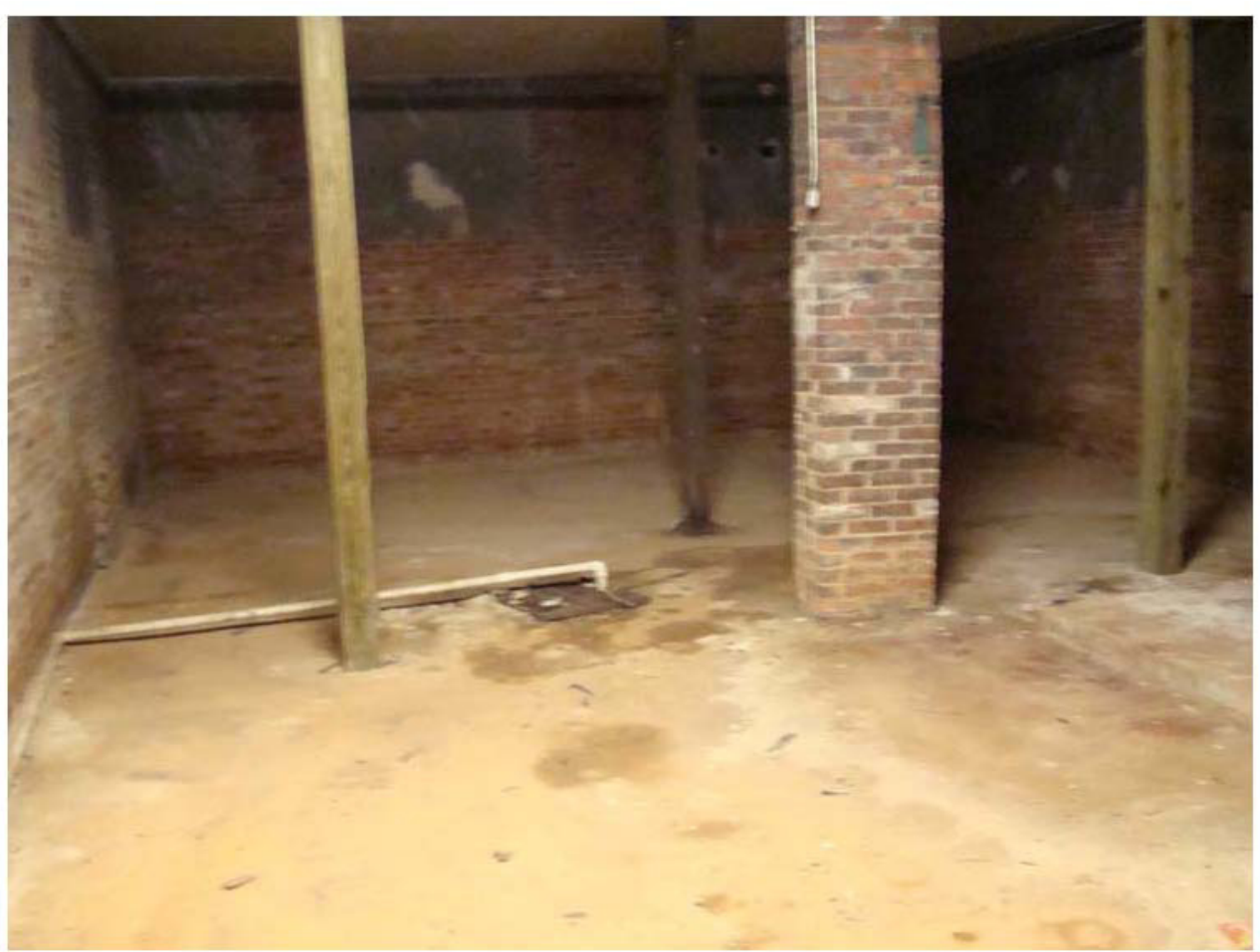




\section{Appendix M: NIOSH Interim Report, HETA 2009-0172 (CONTINUEd)}

Figure 6. Downward sloping ground towards classroom wings, July 14, 2009

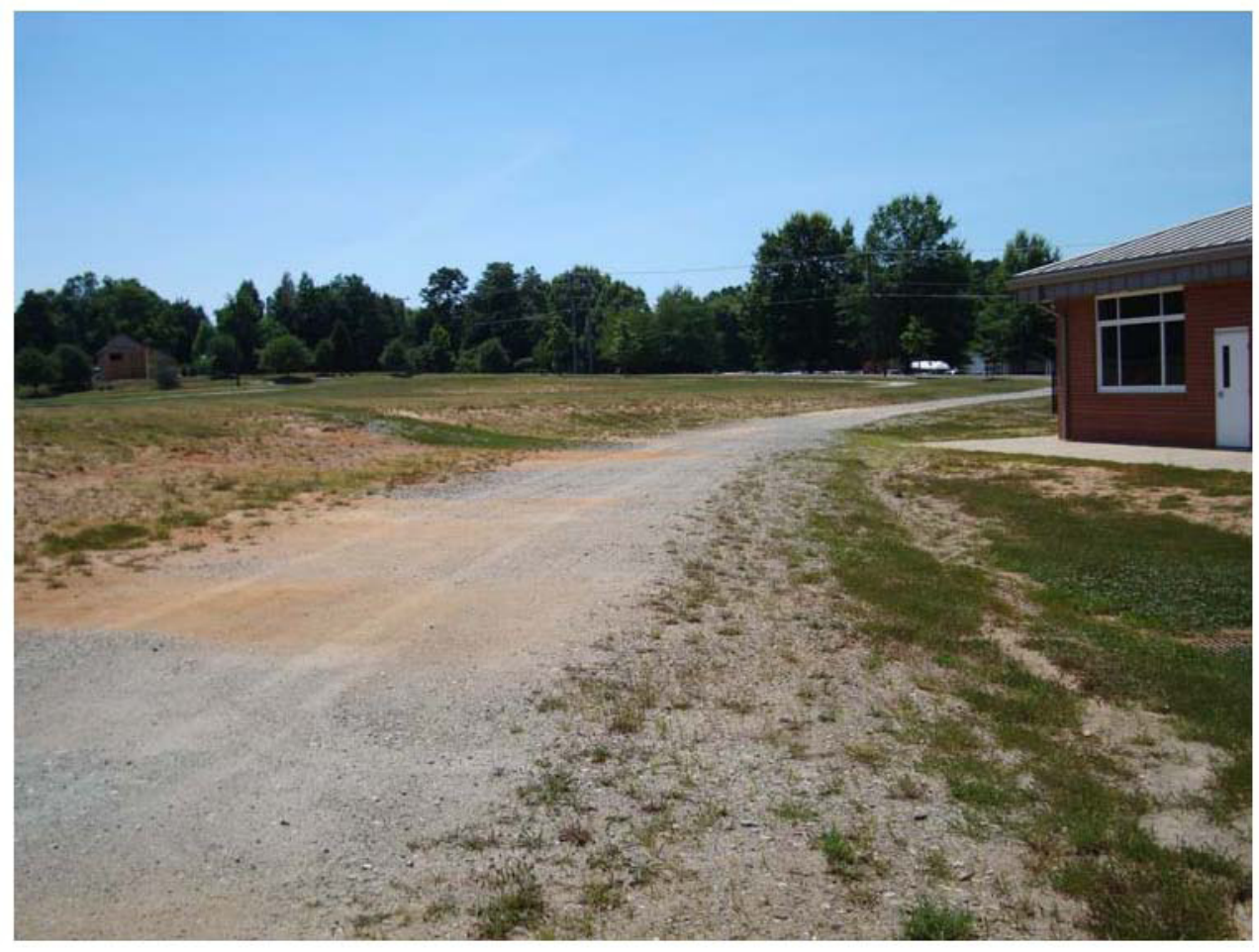

Figure 7. Downward sloping ground towards classroom wings, July 14, 2009

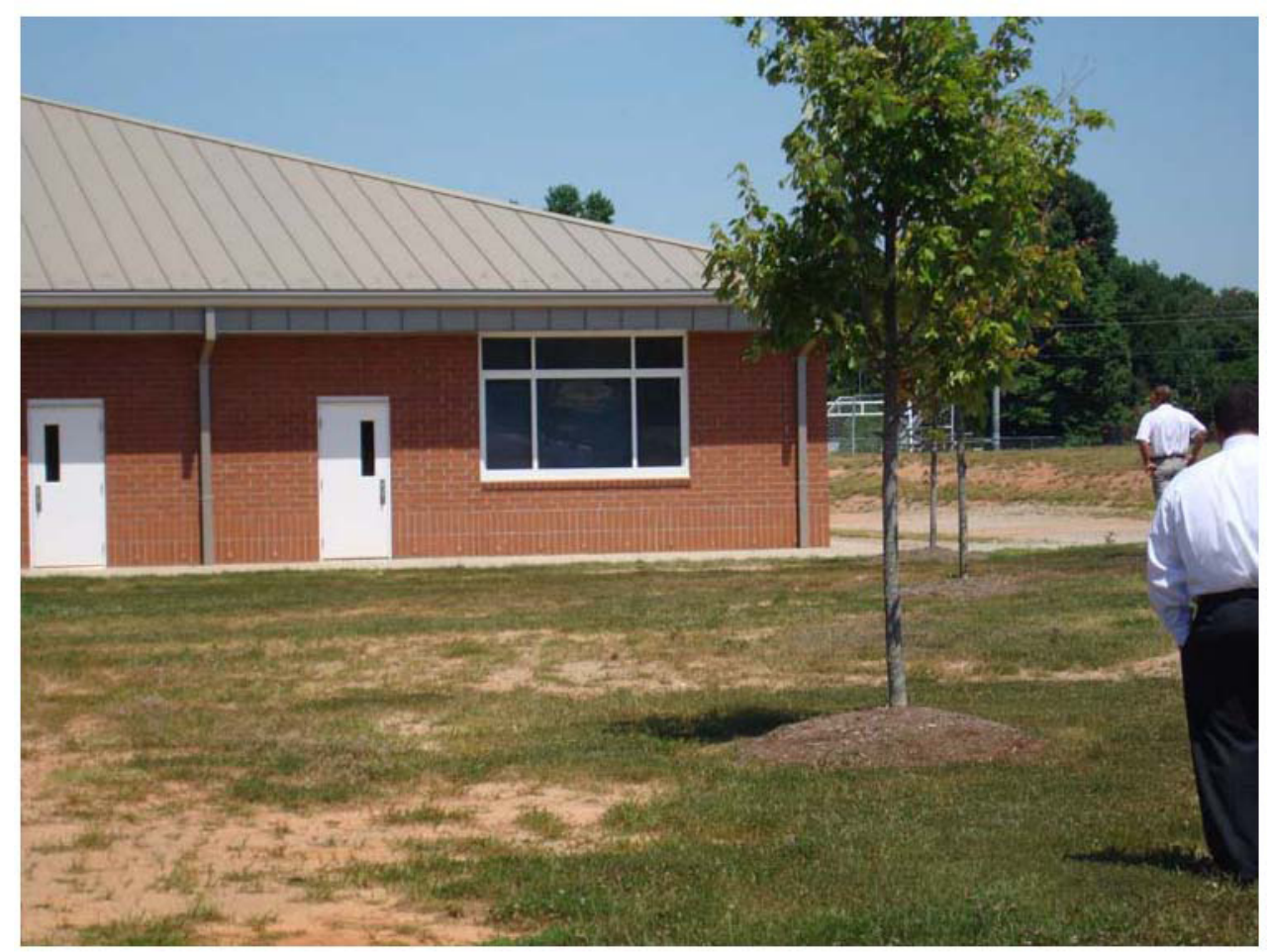




\section{Appendix M: NIOSH Interim Report, HETA 2009-0172 (CONTINUEd)}

Figure 8. Downward sloping ground towards classroom wings, July 14, 2009

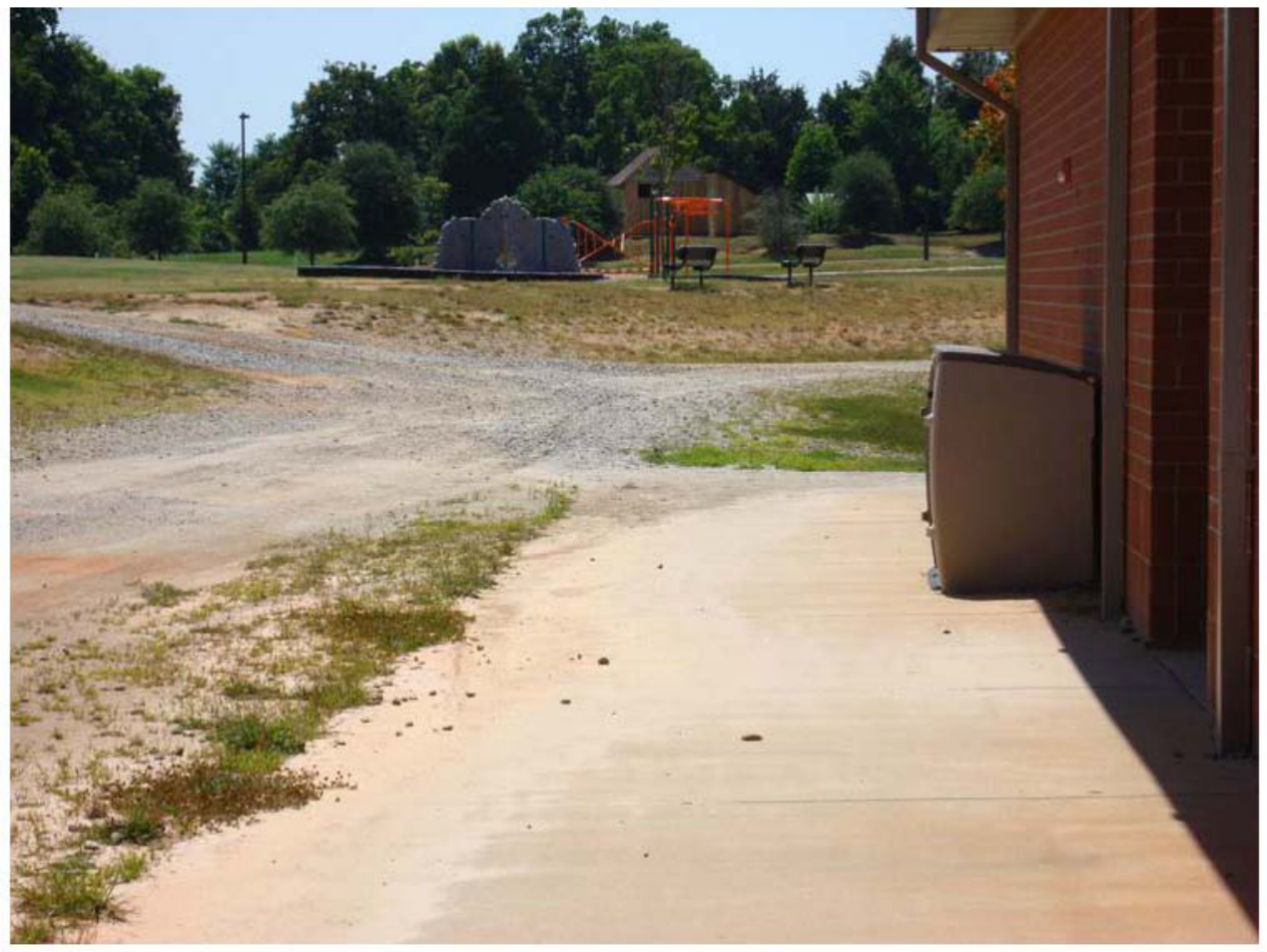

Figure 9. Blocked storm drain outside of the media center, July 14, 2009

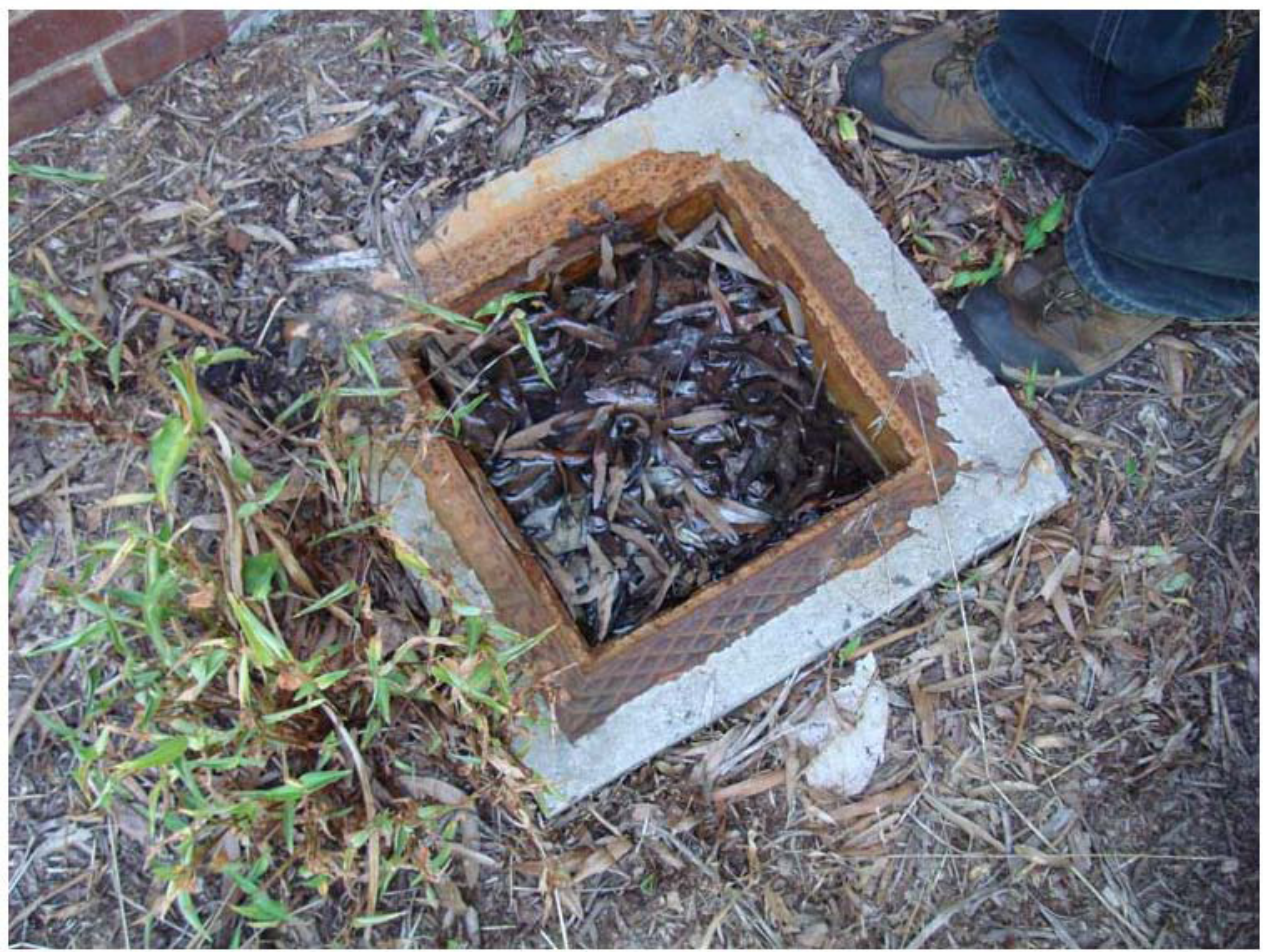




\section{Appendix M: NIOSH InterIM Report, HETA 2009-0172 (CONTINUED)}

Figure 10. Foam roof adjacent to gym, July 14, 2009

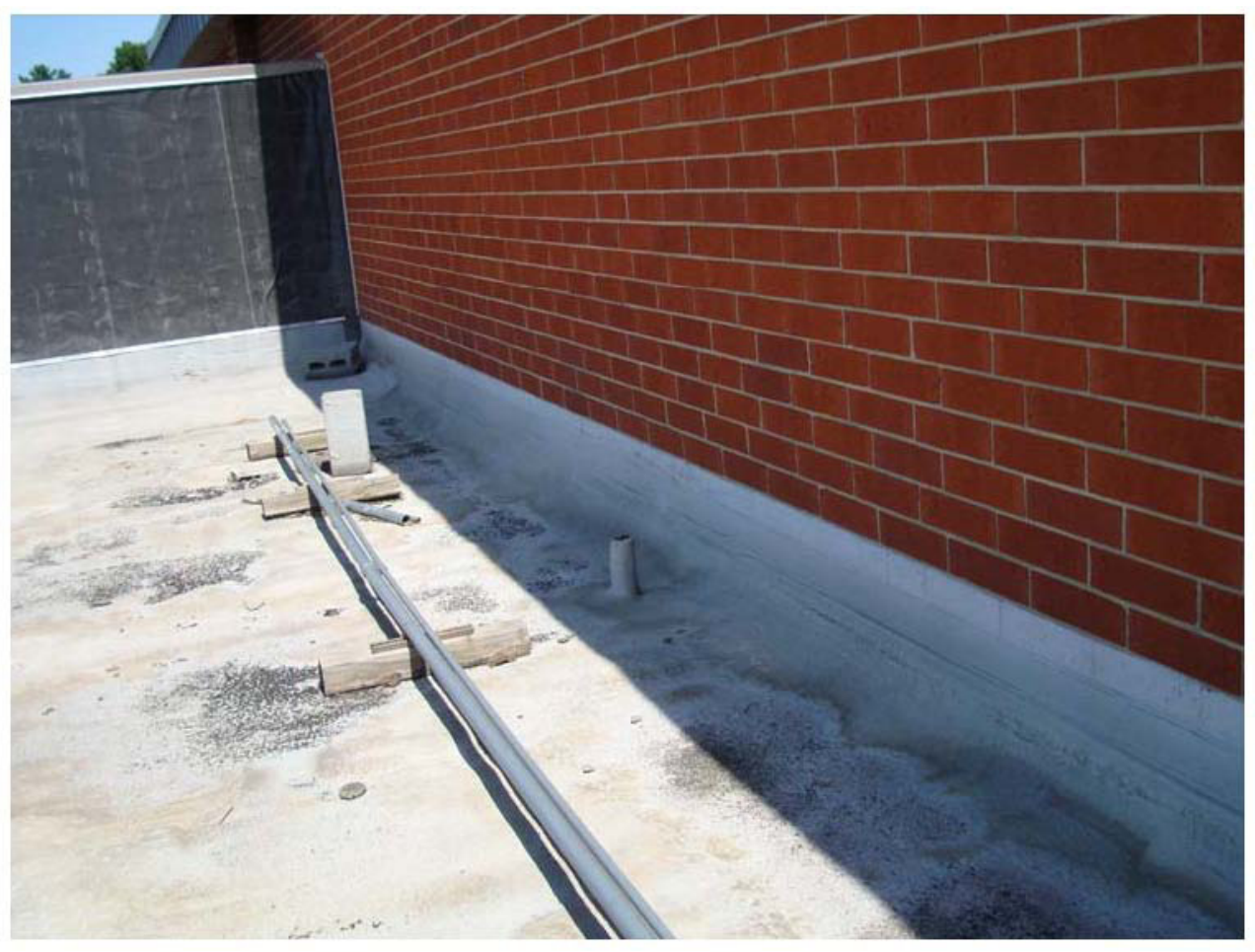

Figure 11. Foam roof over media center wing, July 14, 2009

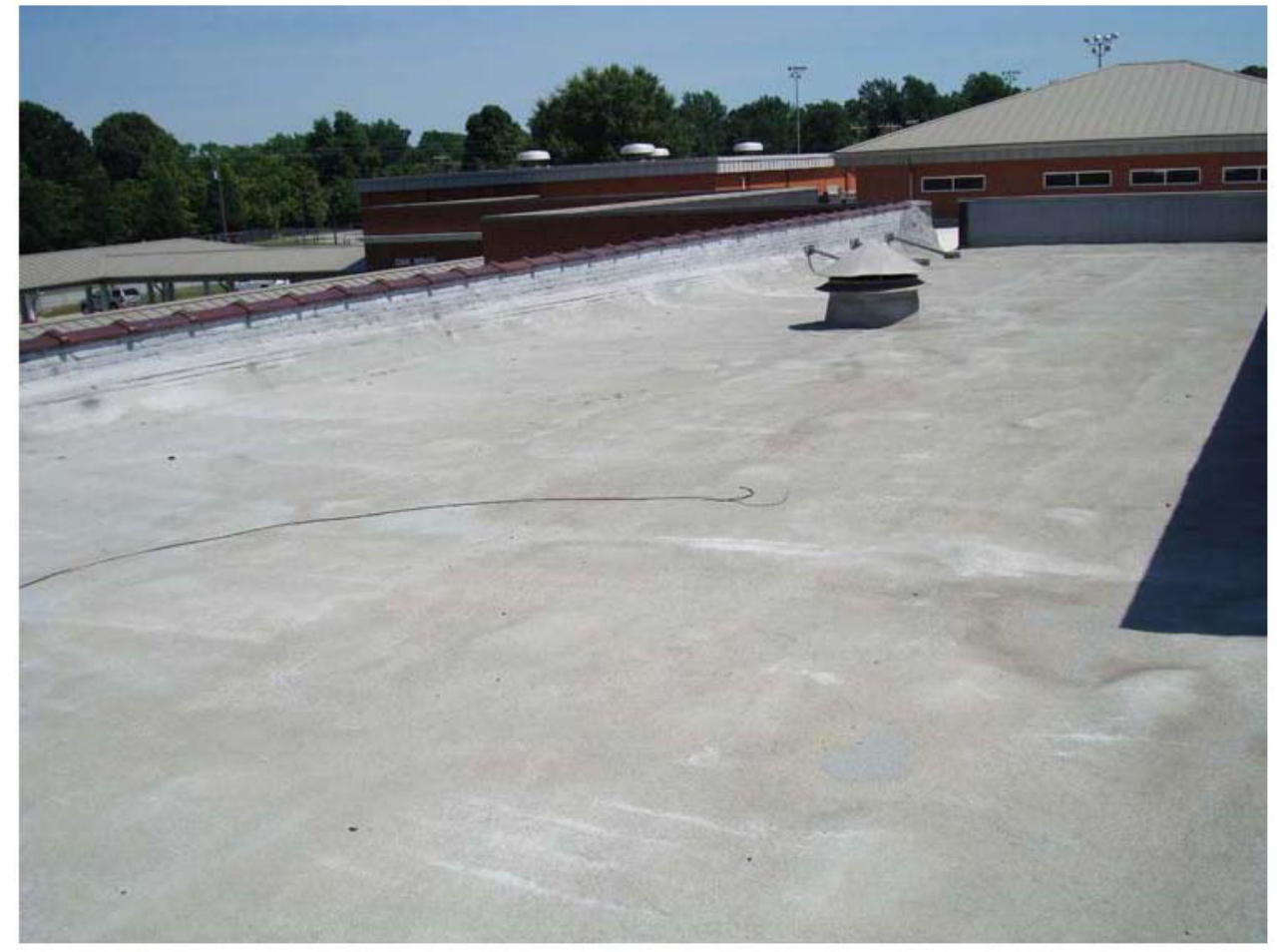




\section{$\overline{\text { Appendix M: NIOSH INTERIM RePORT, HETA 2009-0172 (CONTINUED) }}$}

Figure 12. Inadequate flashing that appeared to be mounted superficially at the junction between the parapet wall (above room 206) and the gym, July 14, 2009

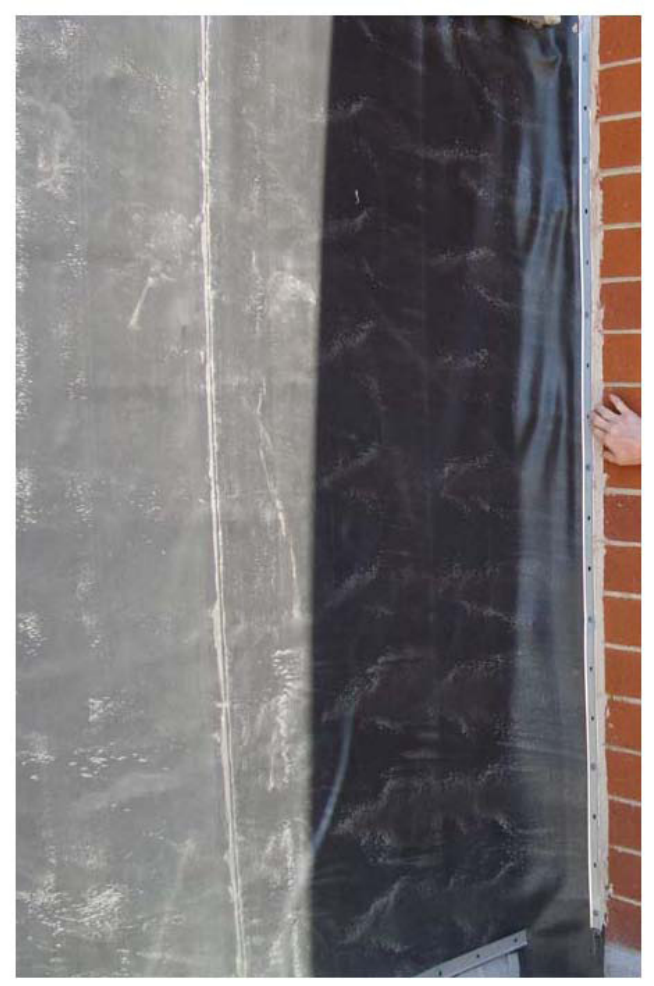

Figure 13. Dust on classroom ceilings and ducts in plenum, July 16, 2009

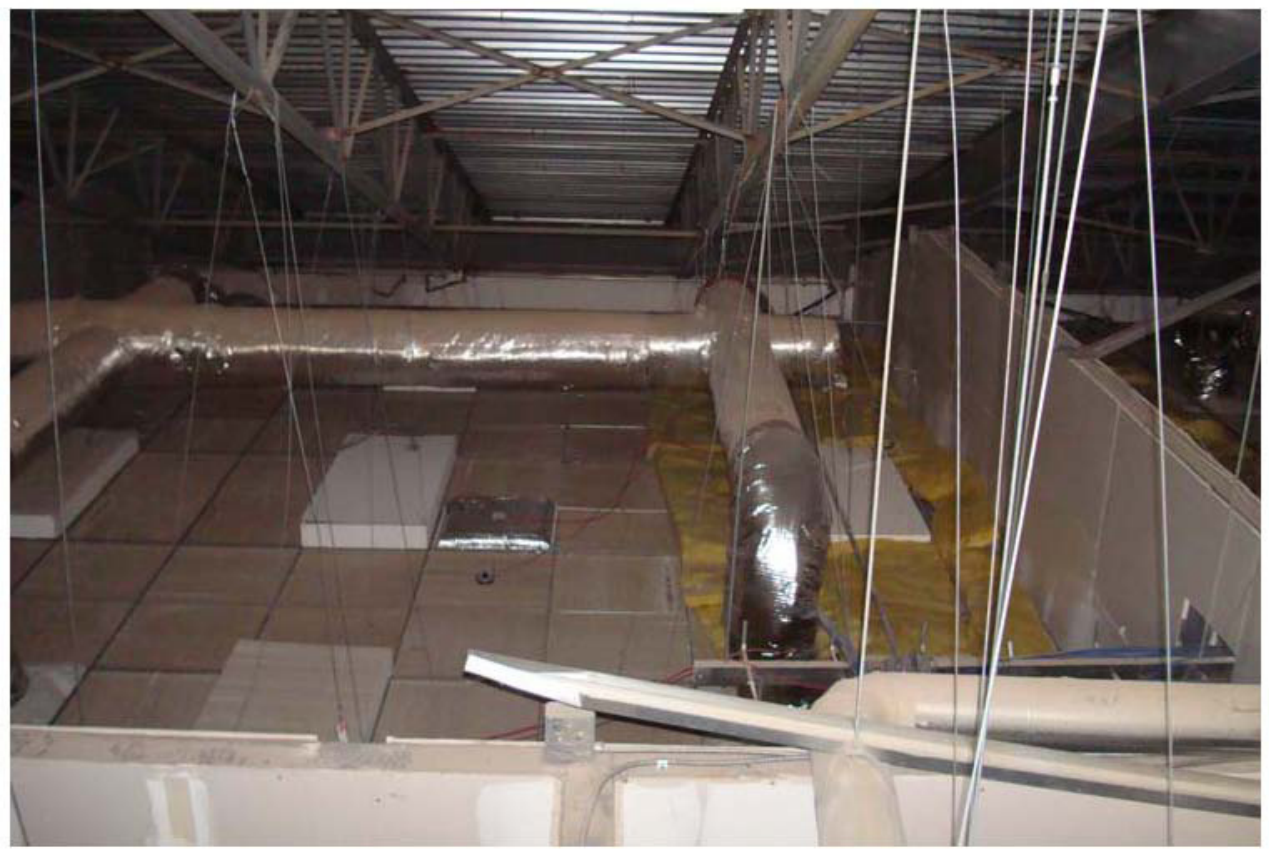


Appendix M: NIOSH Interim Report, HETA $2009-0172$ (CONTINUed)

Figure 14. Dust on classroom ceilings and ducts in plenum, July 16, 2009

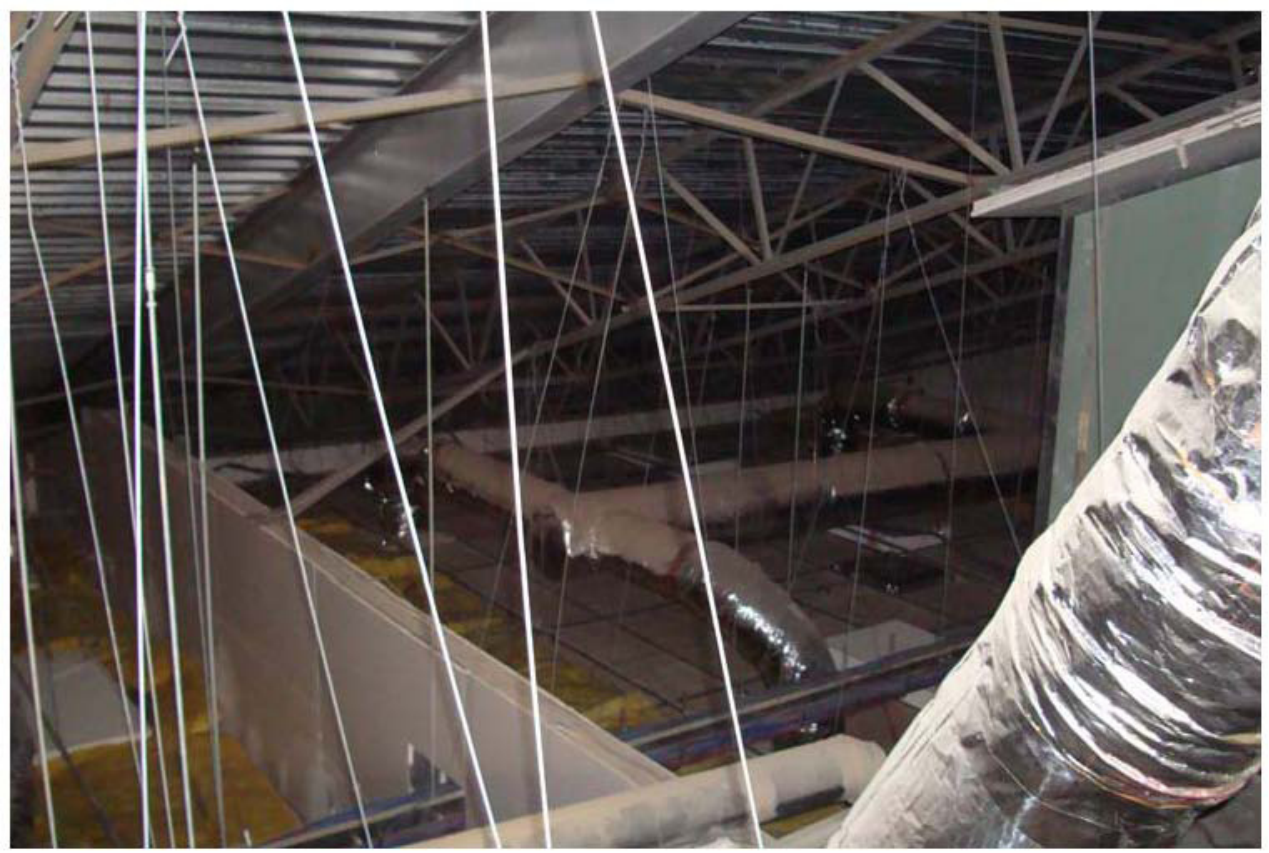

Figure 15. Coils for HVAC handlers showing dust and possible mold contamination , July 16, 2009
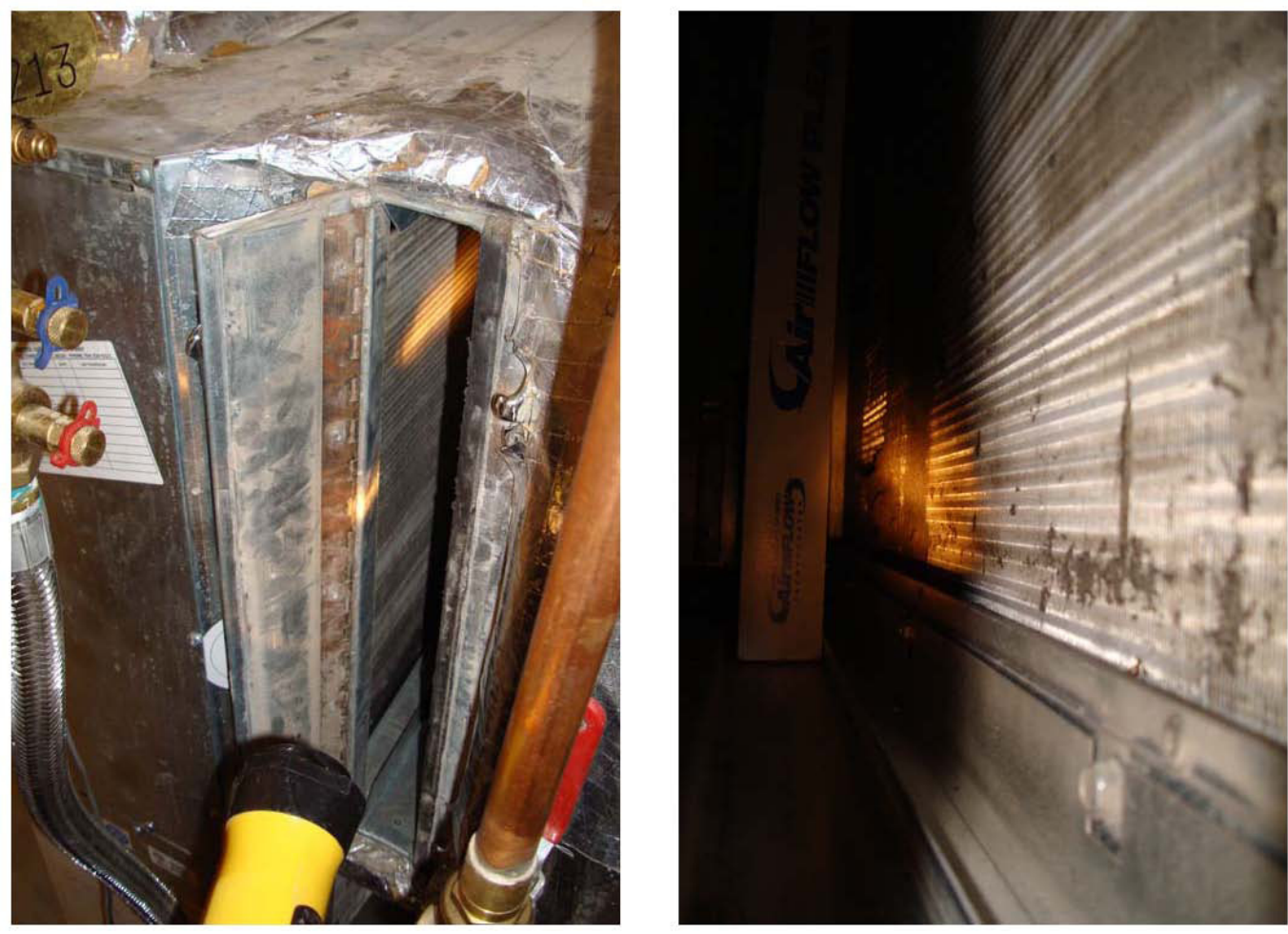


\section{Appendix M: NIOSH INTERIM Report, HETA 2009-0172 (CONTINUED)}

Figure 16. Percent frequency of occurrence in samples for individual fungal species

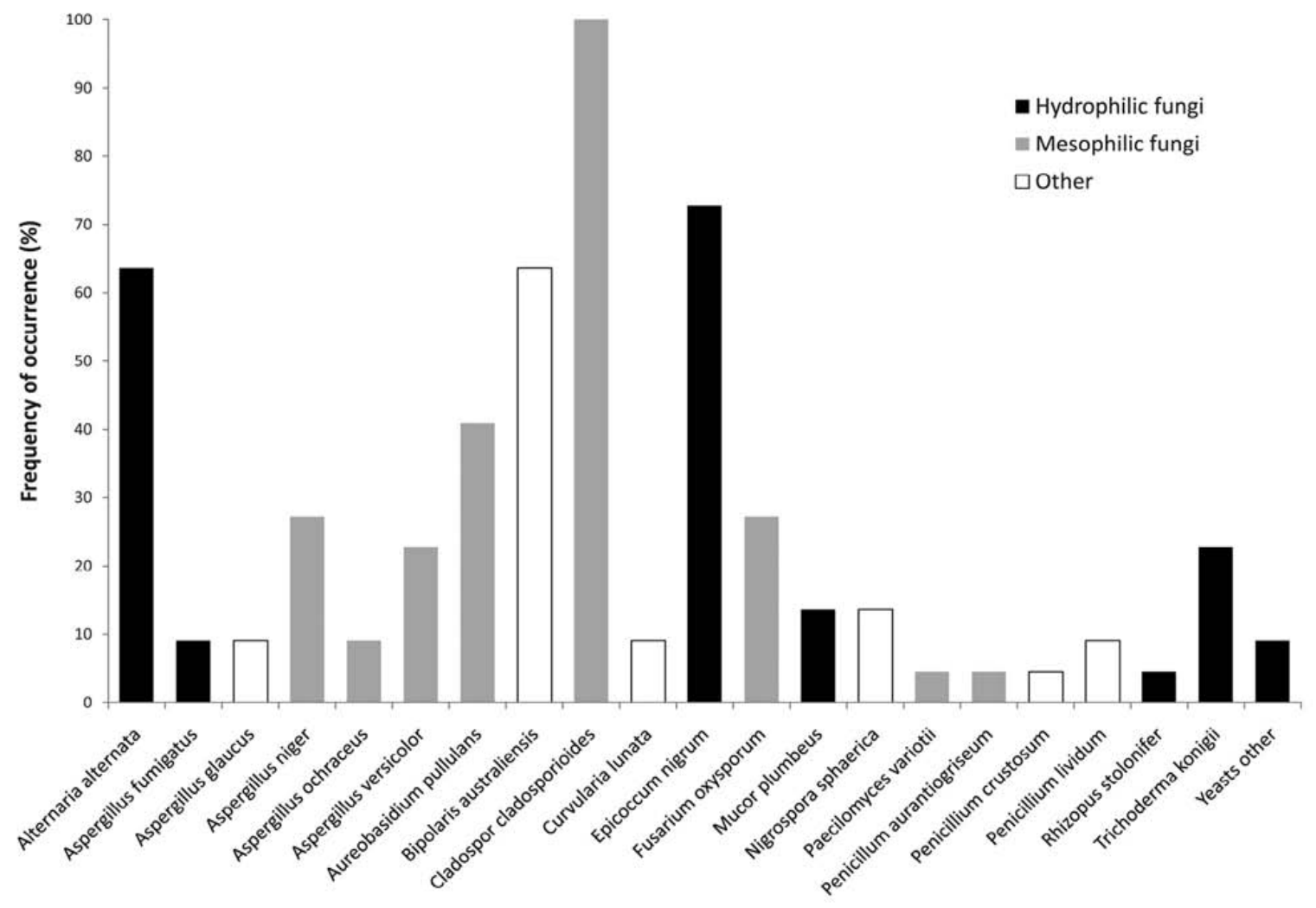


Figure 17. Comparison of symptom prevelances in 2008/2009 school year by classroom wing in 40 staff members ${ }^{1}$

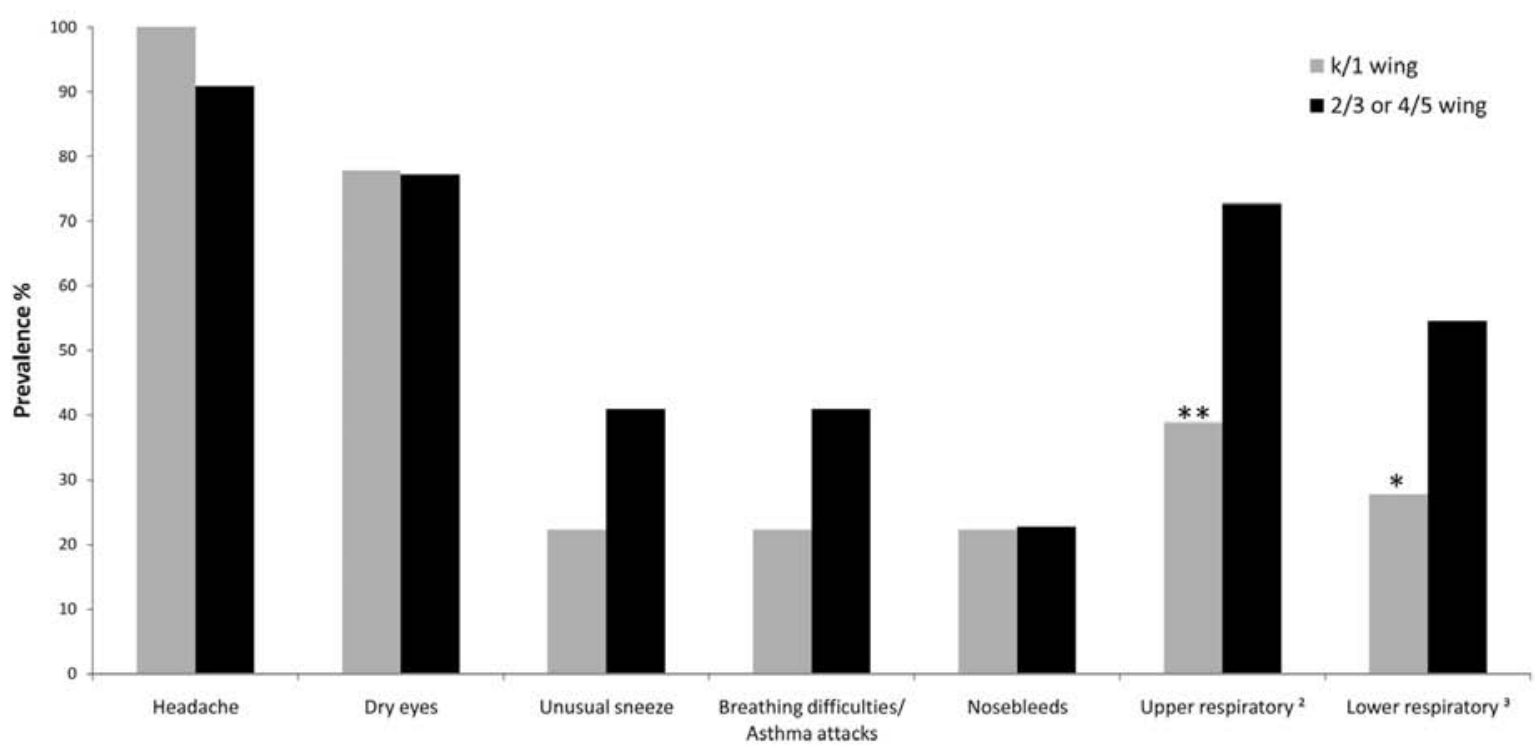

Likelihood ratio chi-square test different at " $" p<0.05$ and $" p<0.10$

${ }^{1}$ We did not have primary classroom wing information for four of the 52 staff members. Of the $48 \mathrm{staff}$ members with work area information, 40 worked primarily in classroom wings.
${ }^{2}$ upper respiratory symptoms include one more of the following: sinus problems, nasal symptoms, sneezing, throat symptoms, and/or voice symptoms.

${ }^{3}$ lower respiratory symptoms include cough and/or breathing problem/asthma attack. 
Figure 18. Comparison of symptom prevalences in 2008/2009 school year by classroom wing in 376 students ${ }^{1}$

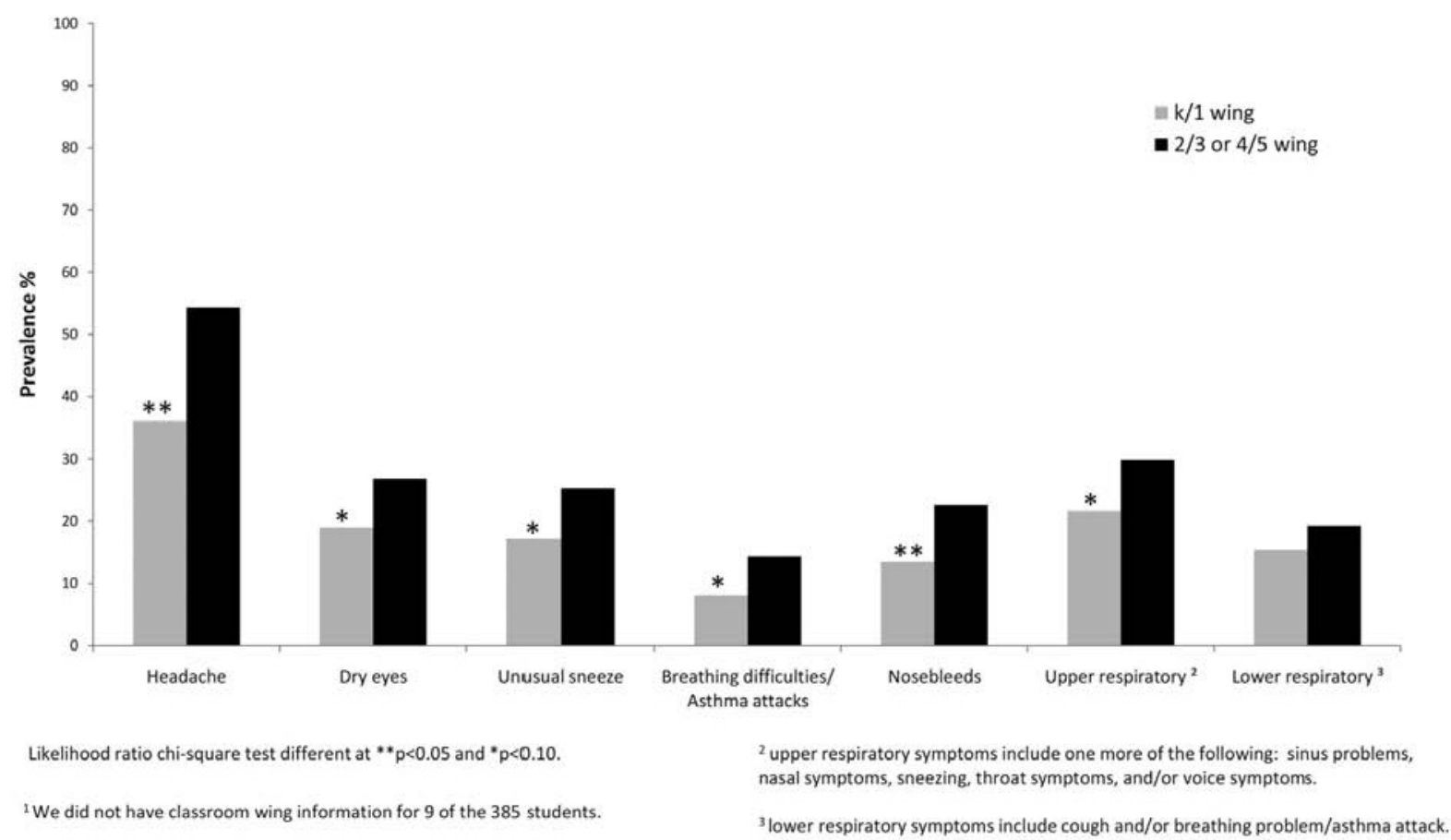


Figure 19. Comparison of symptom prevalences in 2007/2008 and 2008/2009 school years in 43 staff members ${ }^{1}$ who had symptoms during the 2008/2009 school year and worked at this school both years

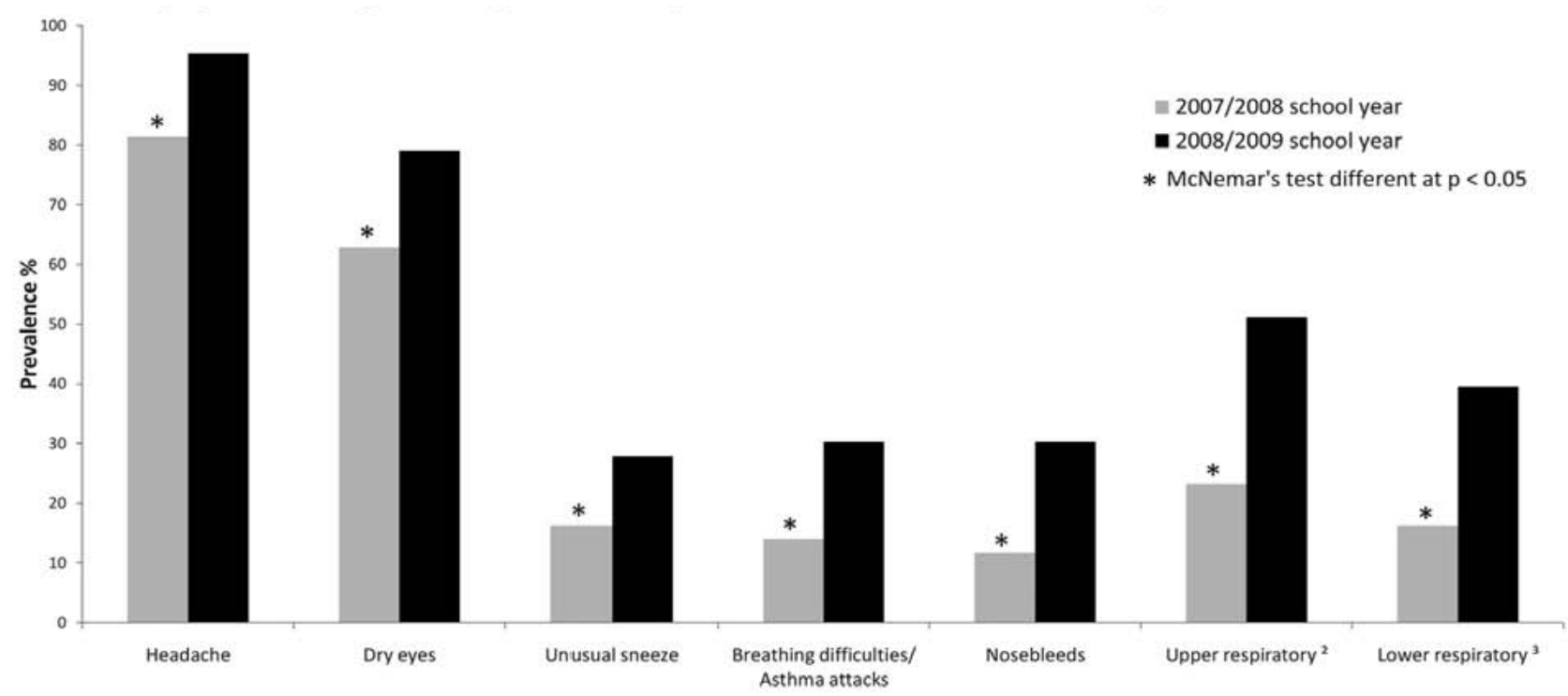

${ }^{1}$ Staff members who worked at Oak Ridge Elementary School both years and answered "yes" or wrote in a response to one of the six symptom questions on the first page of the health department questionnaire (Appendix A).

${ }^{2}$ upper respiratory symptoms include one more of the following: sinus problems, nasal symptoms, sneezing, throat symptoms, and/or voice symptoms.

${ }^{3}$ lower respiratory symptoms include cough and/or breathing problem/asthma attack. 


\section{APPENDIX M: NIOSH INTERIM RePORT, HETA 2009-0172 (CONTINUED)}

Figure 20. Comparison of symptom prevalences in 2007/2008 and 2008/2009 school years in 221 students ${ }^{1}$ who had symptoms during the 2008/2009 school year and attended this school both years

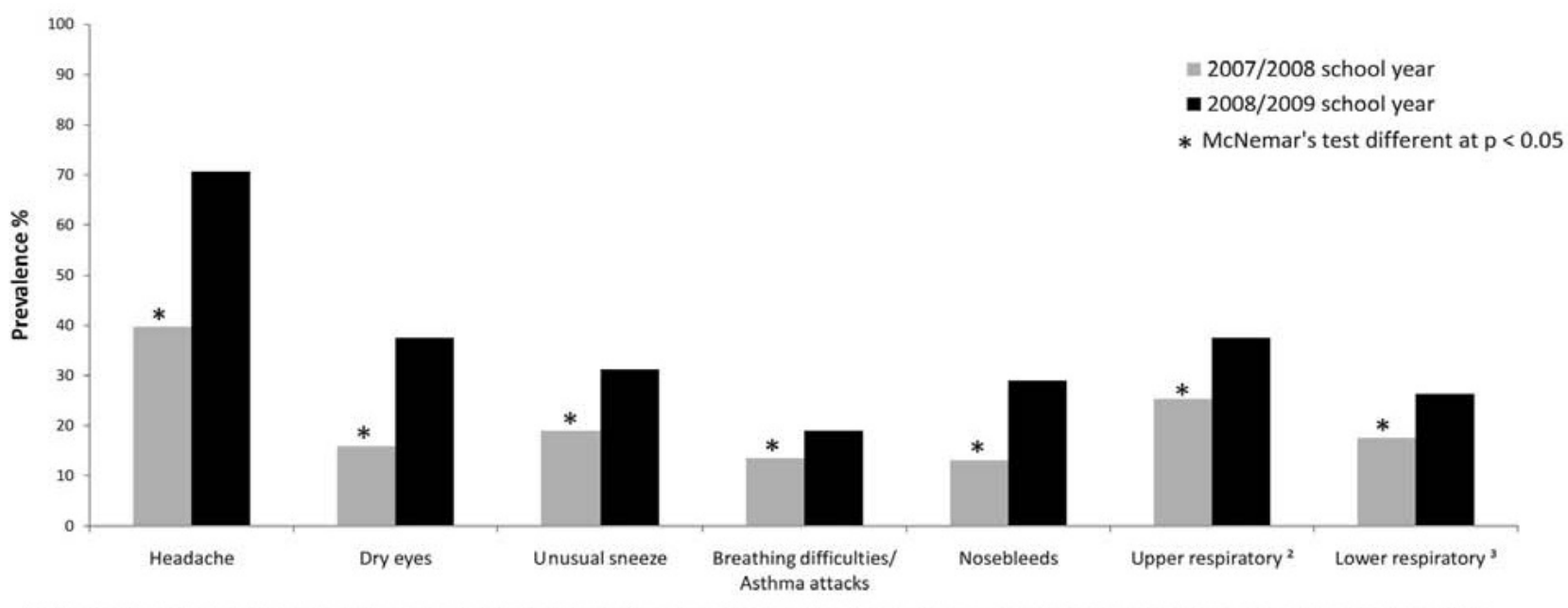

${ }^{1}$ Students who worked at Oak Ridge Elementary School both years and answered "yes" or wrote in a response to one of the six symptom questions on the first page of the health department questionnaire (Appendix A).

${ }^{2}$ upper respiratory symptoms include one more of the following: sinus problems, nasal symptoms, sneezing, throat symptoms, and/or voice symptoms.

${ }^{3}$ lower respiratory symptoms include cough and/or breathing problem/asthma attack. 
APPENDIX M: NIOSH INTERIM RePORT, HETA 2009-0172 (CONTINUEd)

Appendices

Appendix A: Guilford County Department of Public Health faculty/staff questionnaire

Appendix B: Guilford County Department of Public Health parent questionnaire

Appendix C: Guilford County Department of Public Health statement dated 25 June 2009

Appendix D: The H.S. Turner Group preliminary report dated 21 July 2009

Appendix E: The H.S. Turner Group final report dated 8 January 2010

Appendix F: The H.S. Turner Group final report pictures

Appendix G: Oak Ridge Elementary School-IAQ project progress update

Appendix H: NIOSH email dated 22 July: HETA 2009-0172 - Requested summary of findings discussed during the breakfast meeting on July 16, 2009.

Appendix I: Guilford County Department of Public Health report on the faculty/staff questionnaire results at Oak Ridge Elementary School

Appendix J: Guilford County Department of Public Health report on parent questionnaire results at Oak Ridge Elementary School

Appendix K: NIOSH email to dated 19 October 2009: HETA 2009-0172 - Oak Ridge

Elementary School

Appendix L: Sporicidin disinfectant solution label

Appendix M: NIOSH draft mold \& dampness assessment sheet 


\section{Appendix N: EMC Engineers, InC. Seasonal HVAC Retro-}

COMMISSIONING REPORT DATED MAY 2010

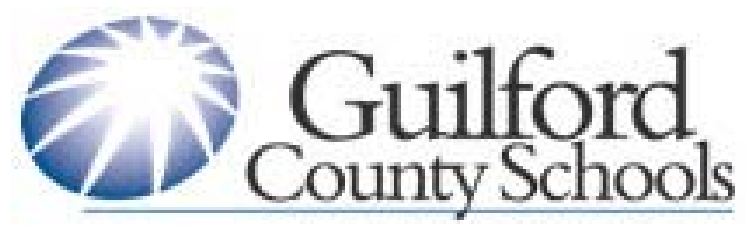

Seasonal Inspection Report

\section{Retro-Commissioning of HVAC Systems}

Oak Ridge Elementary

Oak Ridge, NC

Prepared for:

Guilford County Schools

EMC Project No. 1C409021.000

May 2010

Prepared by:

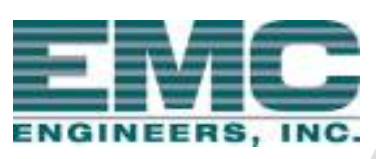

E M C Engineers, Inc.

706 Hillsborough Street, Suite 102

Raleigh, NC 27603 


\section{Appendix N: EMC Engineers, InC. SeAsonal HVAC Retro- COMMISSIONING Report DAted MAY 2010 (CONTINUED) INTENTIONAL BLANK PAGE}

Page $\mathbf{2}$ of $\mathbf{2 2}$ 


\section{Appendix N: EMC Engineers, InC. SeAsonal HVAC Retro- COMMISSIONING REPORT DATED MAY 2010 (CONTINUED)}

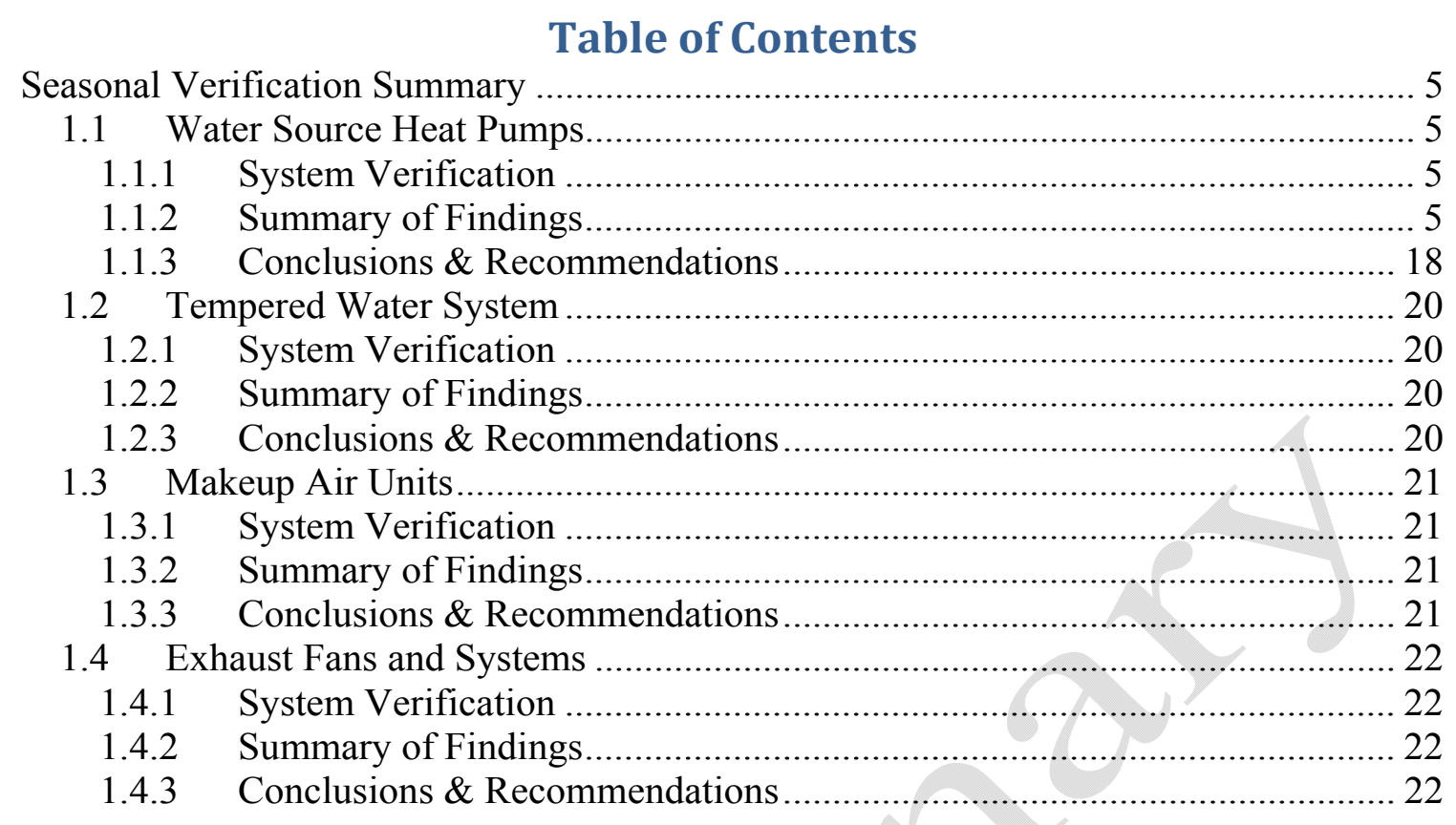

\section{List of Tables}

Table 1 - Outdoor conditions for testing period................................................................. 4

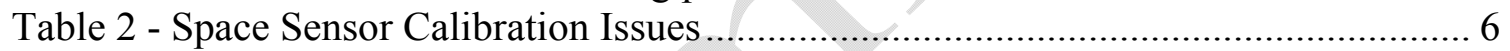

Table 3 - Units with multiple trouble calls after Feb 22, 2010 ....................................... 17

\section{Table of Figures}

Figure 1 - Rocks found at double doors at east-end of 400 hallway.................................. 7

Figure 2 - Rock found at double doors at north-end of 600 hallway .................................. 8

Figure 3 - Bricks found at double doors at middle of east-side of 600 hallway ................. 8

Figure 4 - Rock found at door on Linville Road side of main hallway .............................. 9

Figure 5 - Microphone stand base found outside classroom 407 outside door................... 9

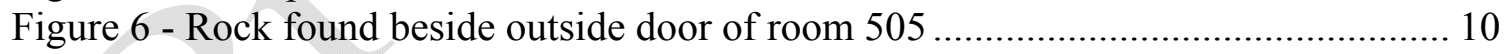

Figure 7 - Frame of outside door to classroom 521 damaged ......................................... 10

Figure 8-Outside door of classroom 521 dragging on threshold................................. 11

Figure 9 - Classroom 521 door showing $1 / 2$ " gap when door not physically pulled shut . 11

Figure 10 - Outdoor Air Humidity reading 122.9\% RH.............................................. 12

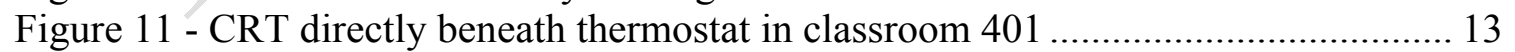

Figure 12 - HP-24 heat output @ 100\%, but discharge air temp. @ 44.2 deg F.............. 14

Figure 13 - HP-9 low pressure safety cutout .................................................................. 15

Figure 14 - Typical Outdoor Air Unit graphic screen (occupied operation) ..................... 21 


\section{Appendix N: EMC Engineers, InC. SeAsonal HVAC Retro- COMMISSIONING REPORT DATED MAY 2010 (CONTINUED)}

\section{Executive Summary}

EMC was selected to perform retro-commissioning functional testing at Oak Ridge Elementary School, a part of the Guilford County Schools System (GCS). Initial functional testing in the unoccupied school concluded in December, 2009 and the school was re-occupied by students and staff beginning February 22, 2010.

As part of its contract, EMC performed seasonal testing during the cooling season in order to assess the operation of systems at that time. Evaluation of building systems with the school fully furnished and occupied, while in the cooling mode was performed in order to identify any shortcomings of the systems or performance issues related to equipment or system operation in real-time. This report is a result of that seasonal testing, which was performed the week of May 3, 2010.

EMC performed cooling season tests on the following systems:

- Building automation system

- Verification of building automation system operator workstation graphics

- Heat Pumps, water pumps, piping, and associated equipment

- Make Up Air Units and associated glycol heat pumps

- Hot Water, pumps, piping, condensate, cooling towers, heat exchangers and associated equipment

- Exhaust Fans

Overall, EMC found the building systems to be operating well within the guidelines and recommendations established in the Turner Building Sciences report and the EMC Retro Commissioning Report. With the exception of a minor setpoint adjustment for the Office and Media Center areas, no adjustment of control parameters was found to be necessary during the course of seasonal testing. Weather conditions during seasonal testing were generally as follows:

Outdoor temperature and humidity conditions for the testing period were as follows:

\begin{tabular}{|l|c|c|c|c|c|}
\hline & $\mathbf{5 / 3}$ & $\mathbf{5 / 4}$ & $\mathbf{5 / 5}$ & $\mathbf{5 / 6}$ & $\mathbf{5 / 7}$ \\
\hline $\begin{array}{l}\text { High } \\
\text { Temperature }\end{array}$ & 76 & 83 & 85 & 88 & 87 \\
\hline $\begin{array}{l}\text { Low } \\
\text { Temperature }\end{array}$ & 71 & 65 & 59 & 63 & 61 \\
\hline $\begin{array}{l}\text { Humidity } \\
\text { High (\%RH) }\end{array}$ & $84 \%$ & $87 \%$ & $90 \%$ & $84 \%$ & $60 \%$ \\
\hline $\begin{array}{l}\text { Humidity } \\
\text { Low (\%RH) }\end{array}$ & $64 \%$ & $31 \%$ & $31 \%$ & $34 \%$ & $32 \%$ \\
\hline
\end{tabular}

Table 1 - Outdoor conditions for testing period 


\section{ApPendix N: EMC Engineers, InC. SeAsonal HVAC Retro- COMMISSIONING REPORT DATED MAY 2010 (CONTINUED)}

\section{Seasonal Verification Summary}

\subsection{Water Source Heat Pumps}

\subsubsection{System Verification}

EMC verified operation of the water source heat pumps in cooling mode to determine proper operation of the units and the units' ability to satisfy design conditions for the spaces they serve.

EMC performed testing as follows:

- Heat Pump operation in occupied cooling mode, including testing for overshoot after occupied transition

- Occupancy scheduling currently in place in the BAS

- Setpoint offset and fan control in unoccupied cooling mode

- Adherence to global space temperature setpoint ranges and occupancy schedules set forth in the January 2010 Turner Building Sciences report

- Verification of space temperature sensor calibrations

EMC also verified whether each heat pump's space temperature sensor was being blocked by classroom furnishings or being influenced by sources of heat or cold, which would impact the unit's ability to properly control space temperature.

\subsubsection{Summary of Findings}

- The BAS was found to control all heat pumps on the following schedules:

- Office and Media Center areas

- Occupied 5 a.m. - 10 p.m.

- Unoccupied 10 p.m. - 5 a.m.

- Classroom wings and Gymnasium / Cafeteria

- Occupied 6 a.m. - 10 p.m.

- Unoccupied 10 p.m. -6 a.m.

Data closets

- Occupied continuously

Observation of space temperatures immediately following transition from unoccupied to occupied operation revealed that the heat pumps would overshoot the occupied setpoint by up to $2 \frac{1 / 2}{2}$ degrees either side of the cooling setpoint. Classroom heat pumps took anywhere from 30 minutes to 1 hour to resolve the overshoot condition and begin maintaining space temperatures within the controller's deadband range. During the time when overshoot was occurring, there were instances of teachers arriving to prepare for class while room temperatures were either too warm or too cold. This resulted in unnecessary trouble calls to GCS Maintenance. Also, discussions with GCS operations staff indicated that there 


\section{Appendix N: EMC Engineers, InC. SeAsonal HVAC Retro- COMMISSIONING REPORT DATED MAY 2010 (CONTINUED)}

should typically not be a need to occupy the school after 9 p.m. As a result, the BAS occupancy schedule should be adjusted. Recommendations are shown in section 1.1.3.

- Setpoint control was found to be from the BAS for all heat pumps, with setpoints as follows:

- Office and Media Center

- Occupied Cooling at $74 \mathrm{deg} \mathrm{F}$

- Unoccupied Cooling at 79 deg F

- Classroom wings and Gymnasium / Cafeteria

- Occupied Cooling at $72 \mathrm{deg} \mathrm{F}$

- Unoccupied Cooling at $77 \operatorname{deg} \mathrm{F}$

A review of trouble calls generated since re-occupying the school revealed that a significant number of hot calls originated from personnel working in the office and media center. Testing of the heat pumps' ability to control these spaces to a setpoint consistent with the classroom wings was conducted and found to be satisfactory. Lowering cooling setpoints, while remaining within the guidelines of the aforementioned Turner report is recommended. Recommendations are shown in section 1.1.3.

- Heat pump space temperature sensor calibrations were found to generally be within 1 deg $\mathrm{F}(+/-.5 \operatorname{deg} \mathrm{F})$ with the following exceptions:

$\begin{array}{ccccc}\text { HP } & \text { Room } & \text { Tracer Temp. } & \text { Measured Temp. } & \text { Cal. Error } \\ 40 & 514 & 71.2 & 69.7 & -1.5 \text { deg F } \\ 47 & 422 & 72.2 & 71.0 & -1.1 \text { deg F } \\ 1 & \text { Central Receiving } & 74.4 & 76 & +1.6 \text { deg F } \\ 6 & \text { Gym Hallway } & 72.8 & 75.1 & +2.3 \text { deg F } \\ 7 & \text { Kitchen } & 74.9 & 73.1 & -1.8 \text { deg F } \\ 37 & \text { Rm 517 } & 72.8 & 73.6 & +0.8 \text { deg F } \\ 39 & \text { Rm 515 } & 71.9 & 72.7 & +0.8 \text { deg F } \\ 53 & \text { Rm 406 } & 72.1 & 70.4 & -1.7 \text { deg F } \\ 55 & \text { Rm 401 } & 71.4 & 69.9 & -1.5 \text { deg F } \\ 58 & \text { Office Hallway } & 74 & 75.9 & +1.9 \text { deg F }\end{array}$

Table 2 - Space Sensor Calibration Issues

Where calibration error in the list above is expressed in a negative (-) number, the space is generally colder than setpoint by that amount. Where this value is a positive $(+)$ number, the space is generally warmer than setpoint by that amount.

Observation of the position of outside doors and windows was made while conducting seasonal testing in order to verify compliance with EMC's earlier recommendations. The following was observed:

Page $\mathbf{6}$ of 22 


\section{Appendix N: EMC Engineers, InC. Seasonal HVAC Retro- COMmissioning Report dAted MAy 2010 (continued)}

- On 5/6/2010 at approximately 10:30 a.m., classroom 517 was observed while occupied and found to have the outside door propped open and the operable windows open. Outside conditions at the time were approximately 80 degrees and $74 \%$ relative humidity. The teacher in this classroom, Mrs. Jones informed EMC that she had been instructed by the principal, Ann Kraft to open the door and windows.

- All hallway doors leading to the outside were closed, however a number of these doors had a rock or some other heavy object located by the door opening. Discussion with various Oak Ridge school personnel indicated that these were used to prop the doors open at times. The following photographs were taken of doors where these objects were found:

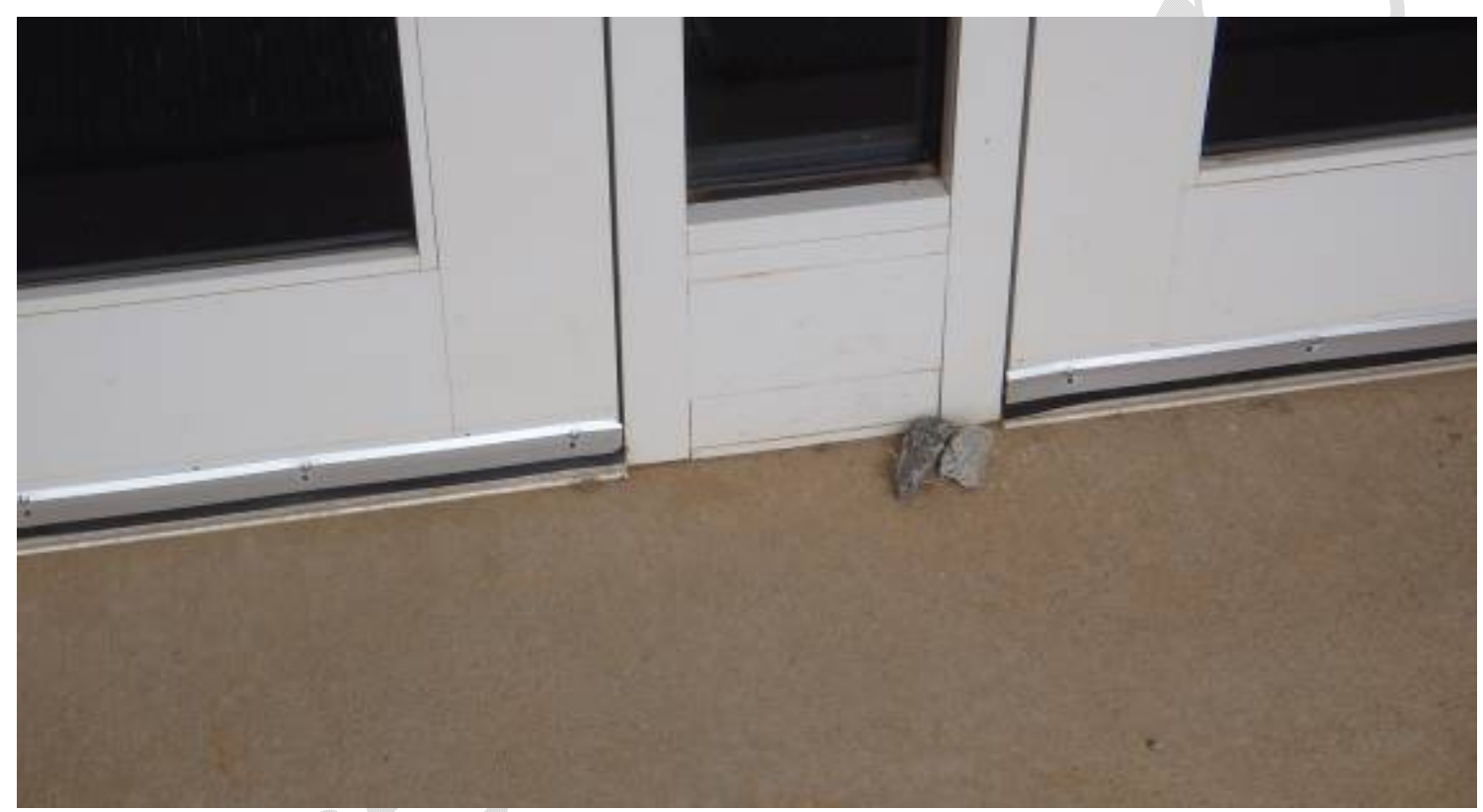

Figure 1 - Rocks found at double doors at east-end of 400 hallway 


\section{Appendix N: EMC Engineers, InC. SeAsonal HVAC Retro- COMMISSIONING REPORT DATED MAY 2010 (CONTINUED)}

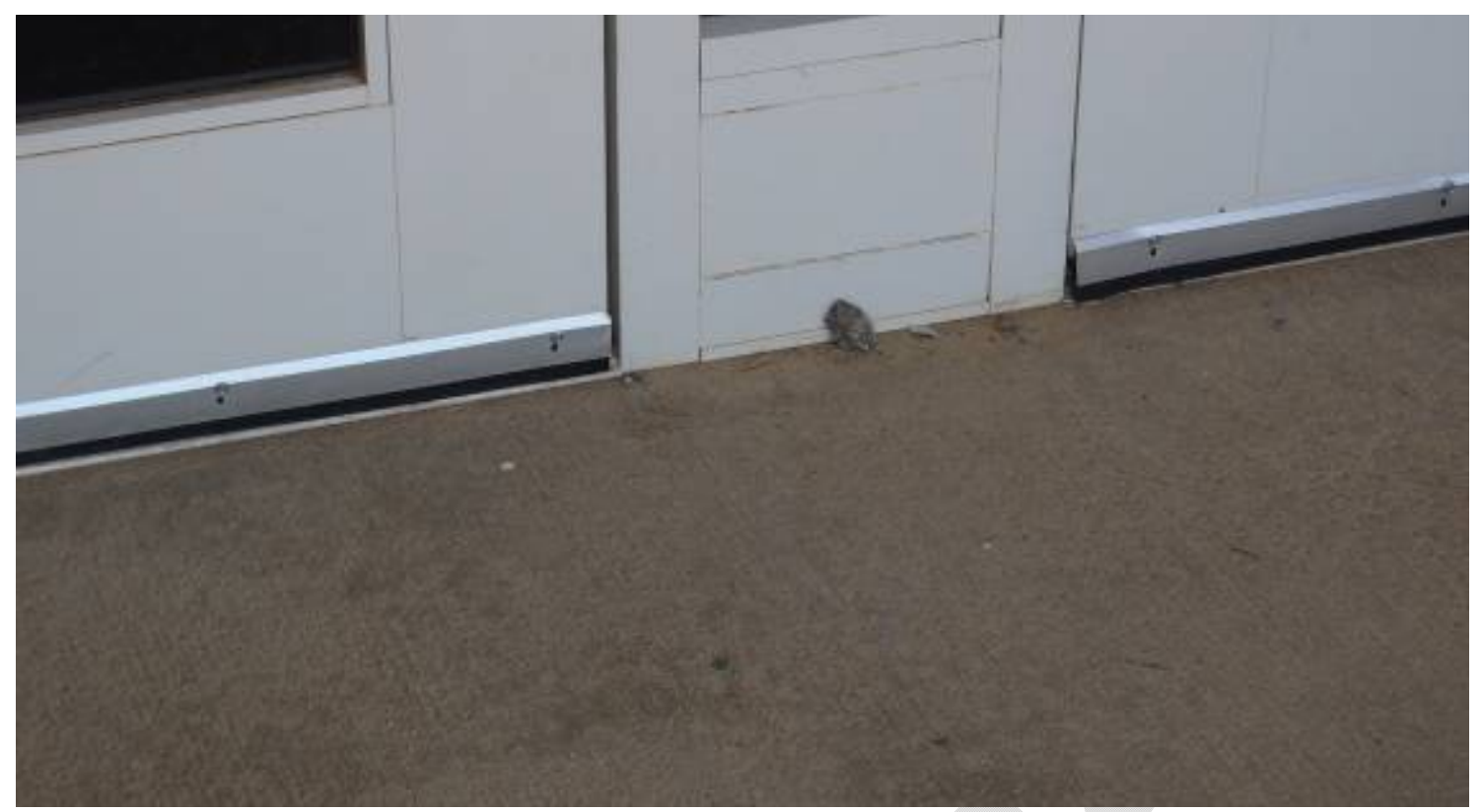

Figure 2 - Rock found at double doors at north-end of 600 hallway

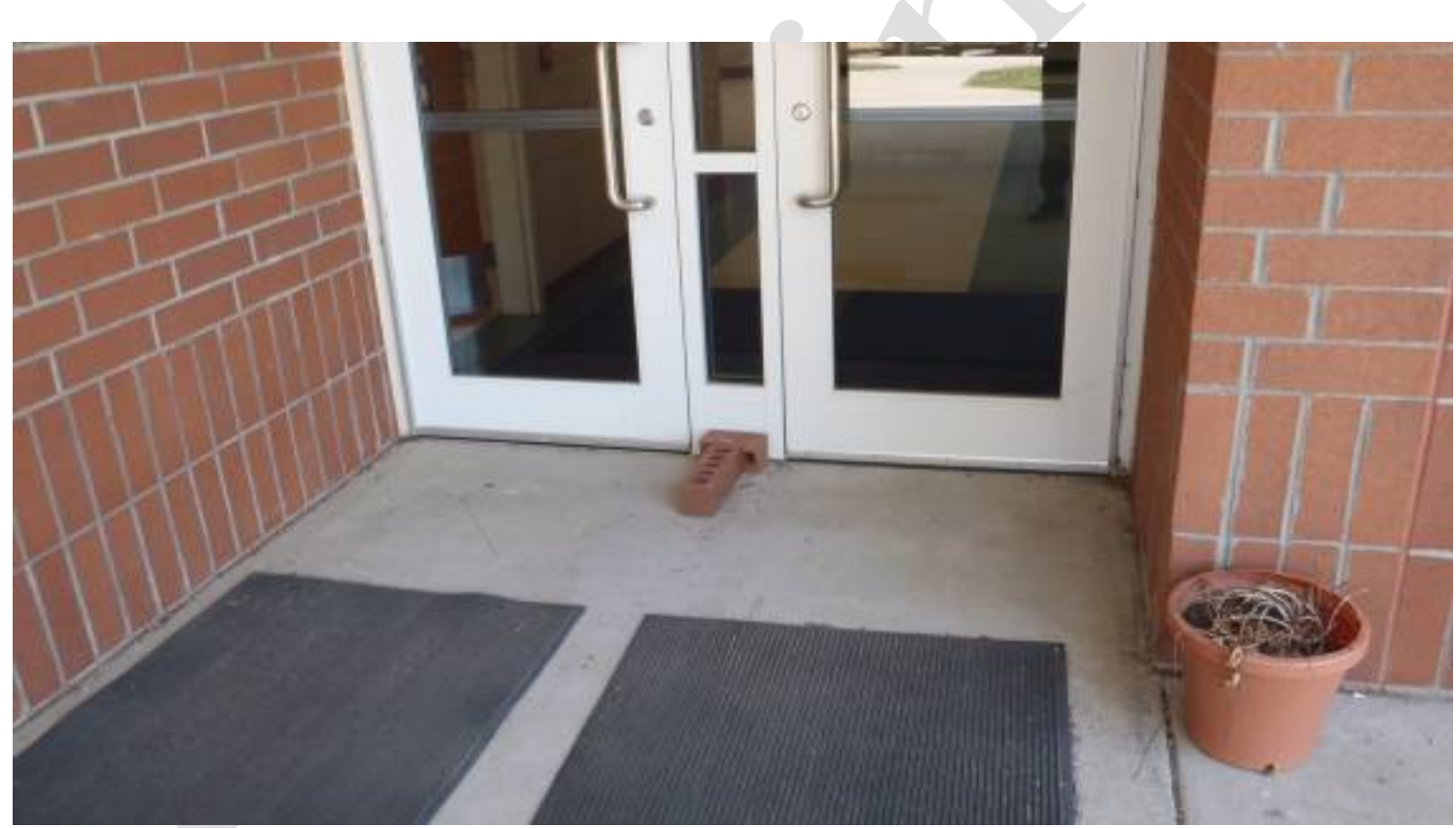

Figure 3 - Bricks found at double doors at middle of east-side of 600 hallway 


\section{Appendix N: EMC Engineers, InC. SeAsonal HVAC Retro- COMMISSIONING REPORT DATED MAY 2010 (CONTINUED)}

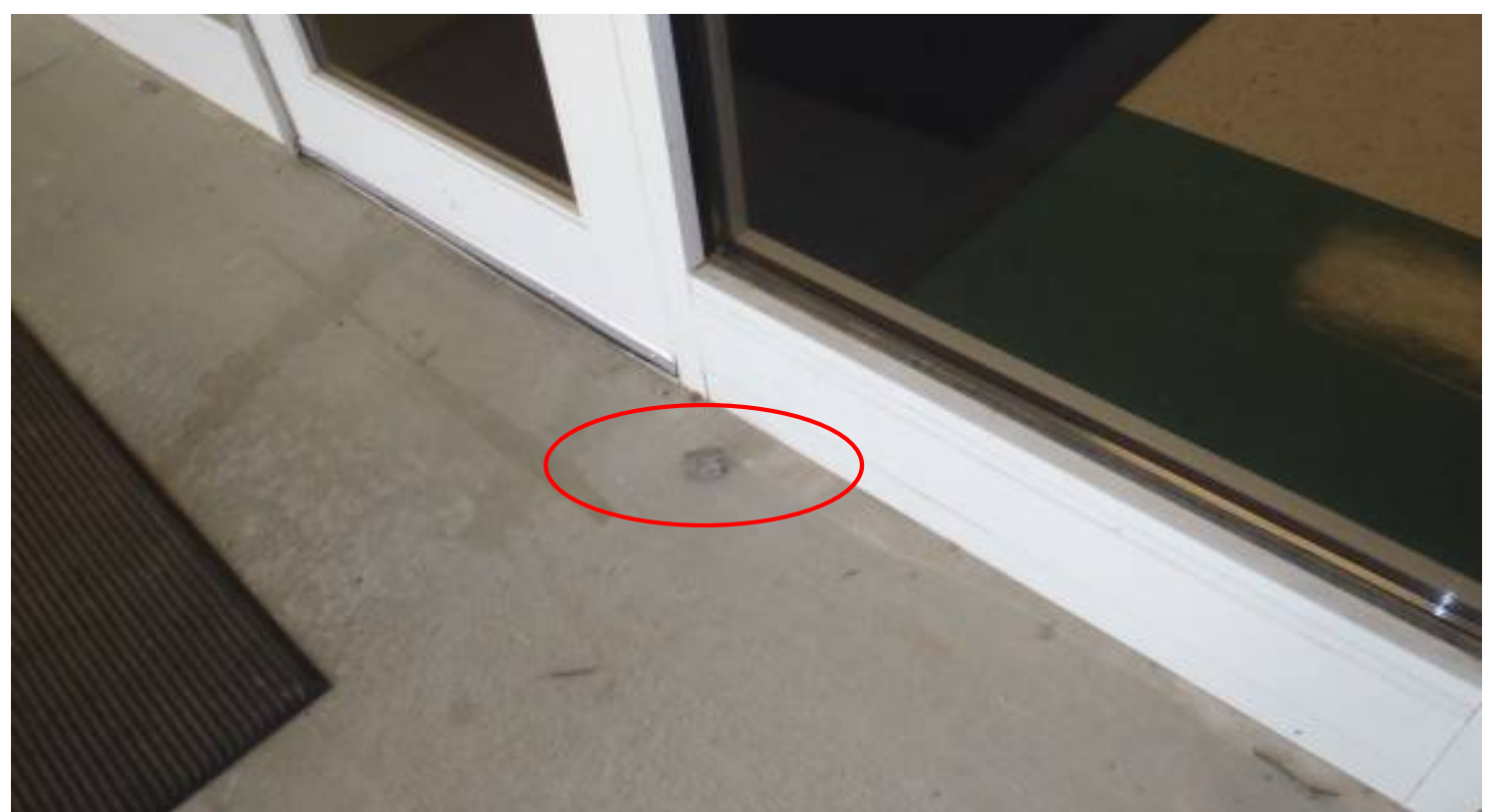

Figure 4 - Rock found at door on Linville Road side of main hallway

- Other classroom outside doors were found to have either a rock or another heavy object located beside them, which have also been used to prop these doors open. These are shown in figures 5 and 6.

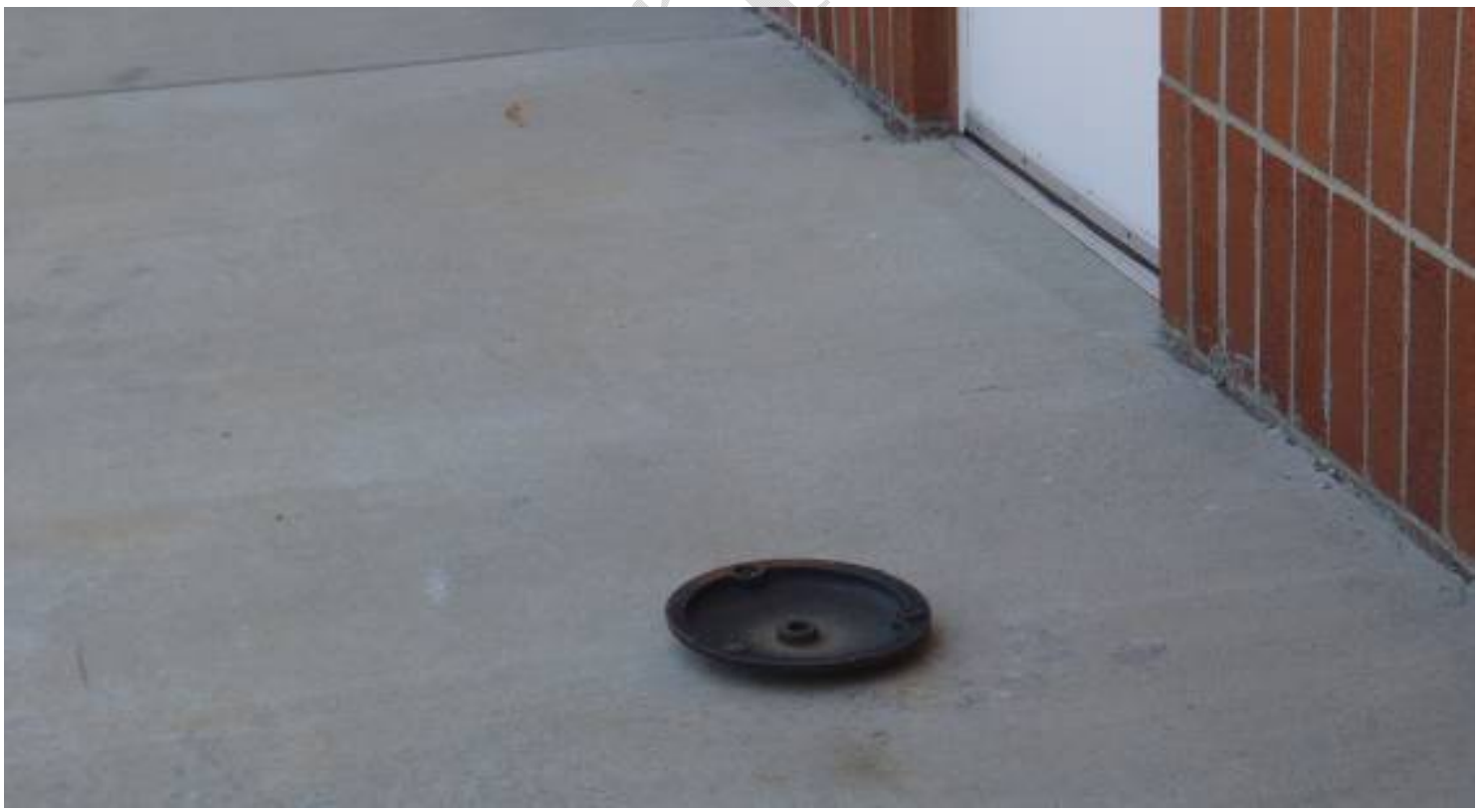

Figure 5 - Microphone stand base found outside classroom 407 outside door 


\section{Appendix N: EMC Engineers, InC. SeAsonal HVAC Retro- COMMISSIONING REPORT DATED MAY 2010 (CONTINUED)}

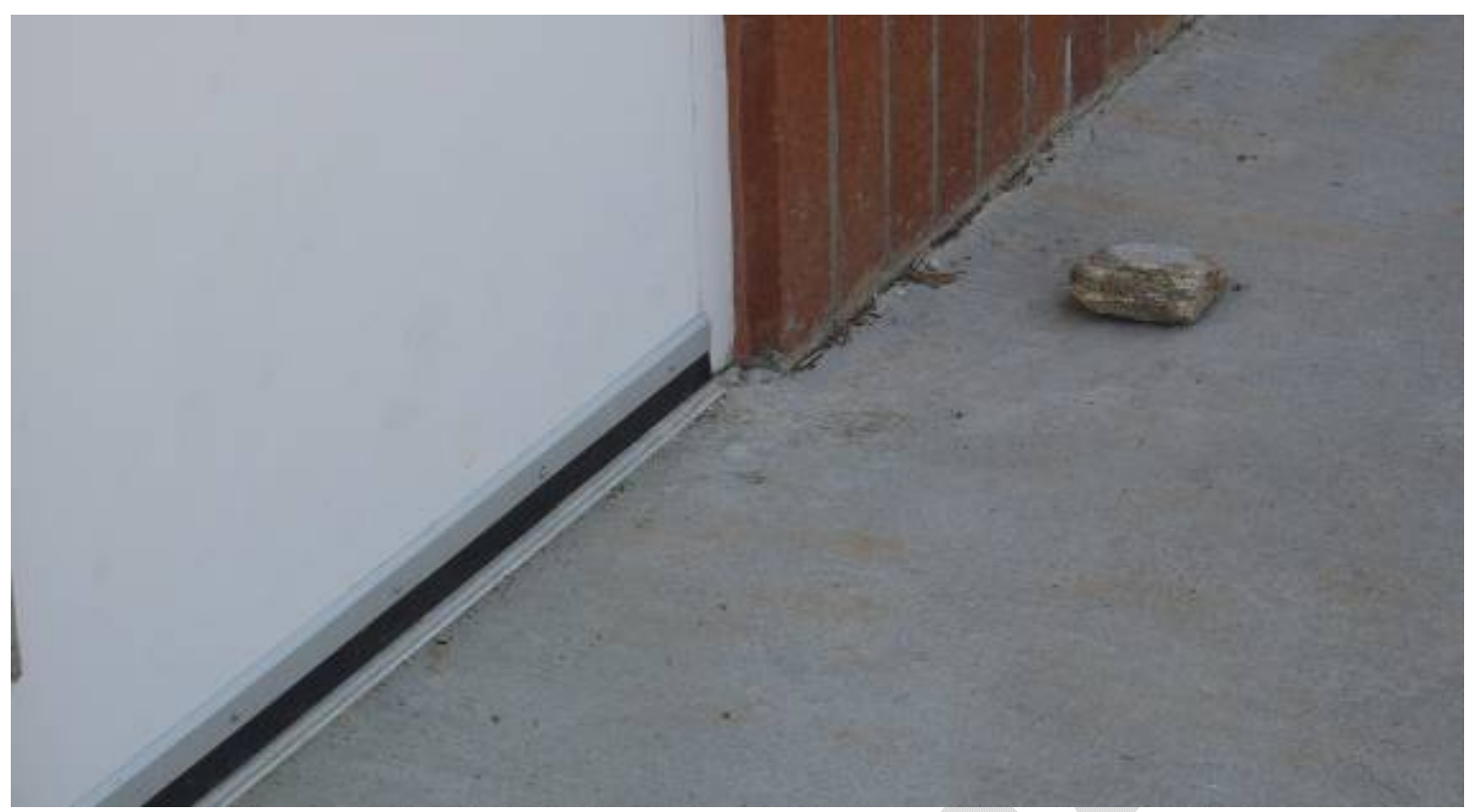

Figure 6 - Rock found beside outside door of room 505

- Figure 7 shows the outside door to classroom 521, and the door frame which has been badly damaged. The resulting misalignment of the door now requires deliberate action to pull the door shut to the point of latching. Figure 8 shows the door sweep and the spot the door is dragging on as a result of the door misalignment. There is a large open area when the door is not pulled shut shown in figure 9, which not only allows conditioned air to escape the room, but also has the potential to allow unauthorized access to the school if the door is not latched.

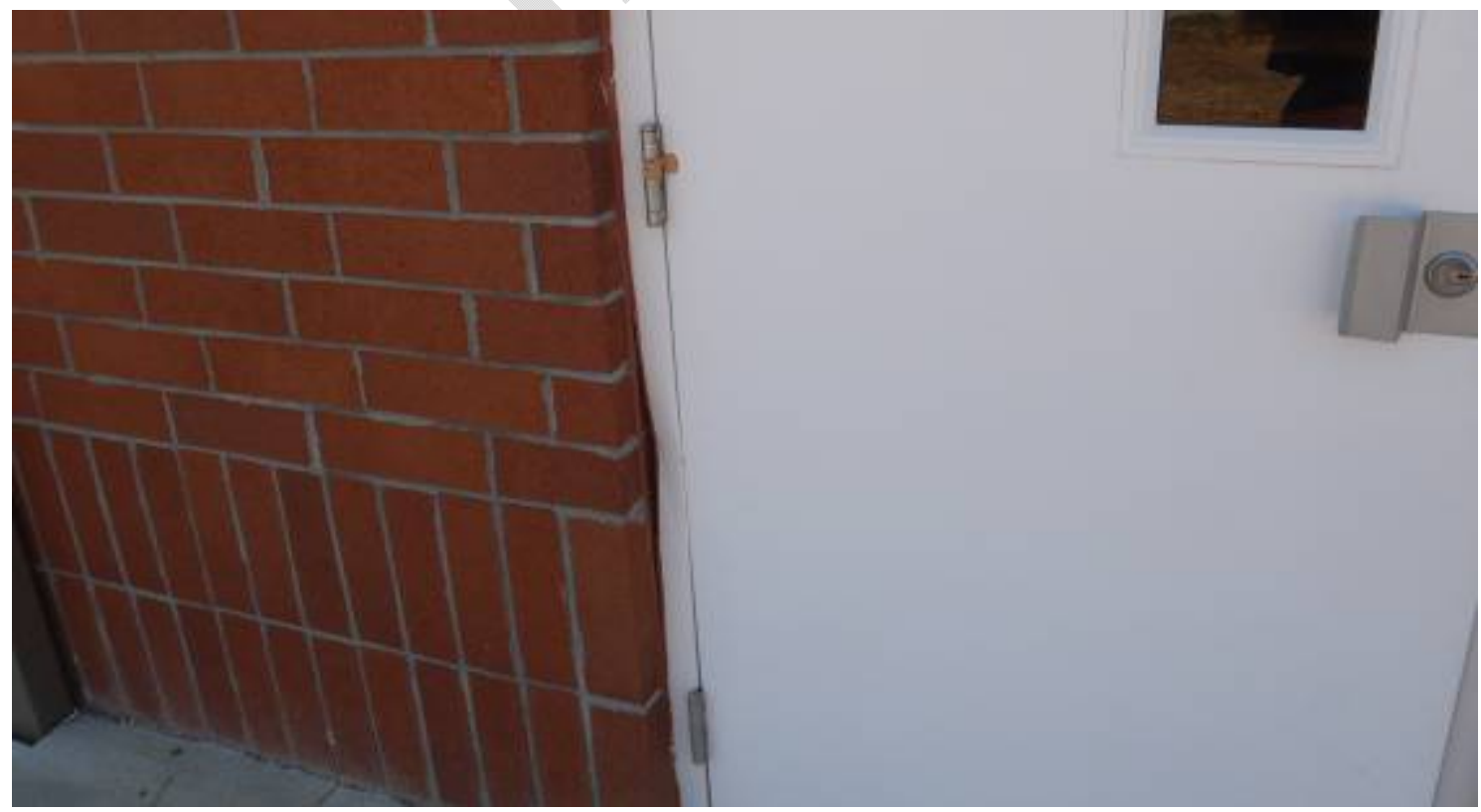

Figure 7 - Frame of outside door to classroom 521damaged

Page 10 of 22 


\section{Appendix N: EMC Engineers, InC. SeAsonal HVAC Retro- COMMISSIONING REPORT DATED MAY 2010 (CONTINUED)}

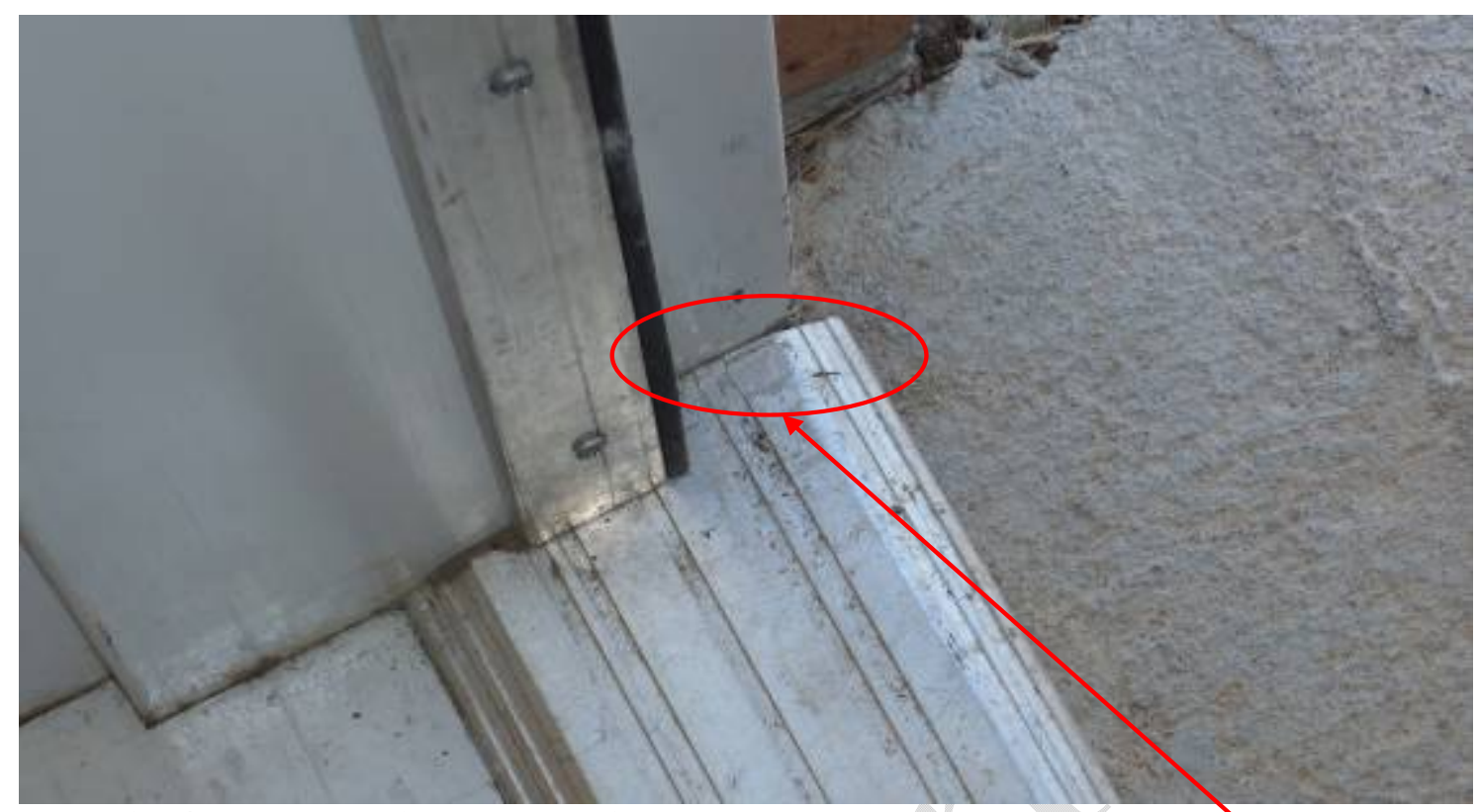

Figure 8 - Outside door of classroom 521 dragging on threshold

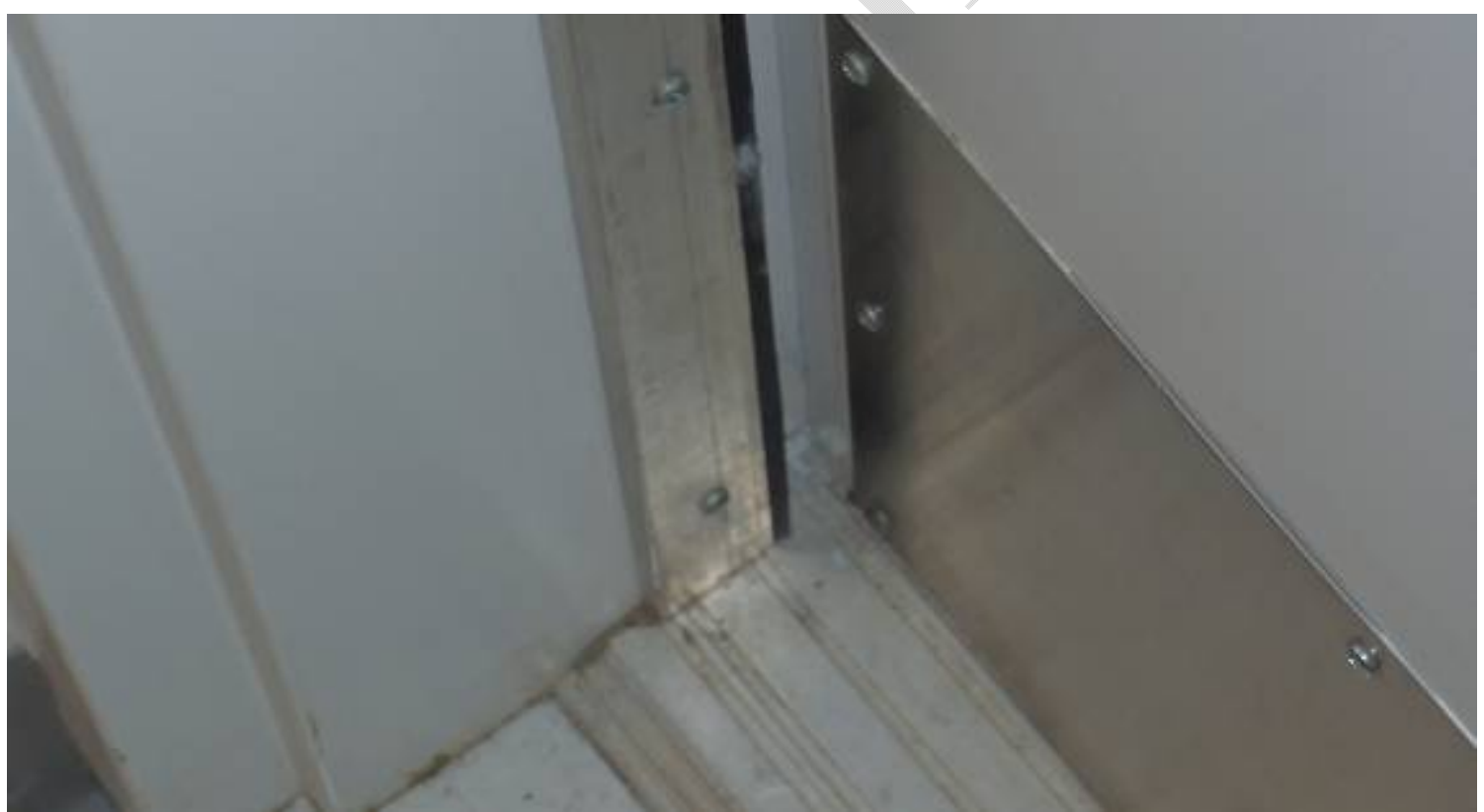

Figure 9 - Classroom 521 door showing 1/2" gap when door not physically pulled shut 


\section{Appendix N: EMC Engineers, InC. Seasonal HVAC Retro- COMMISSIONING RePORT DATED MAY 2010 (CONTINUED)}

- The outdoor Air Humidity sensor connected to the BAS was found to be reading incorrectly. Using a calibrated test instrument, outdoor humidity at this sensor was found to be $74 \%$, while the BAS reading showed $122.9 \%$

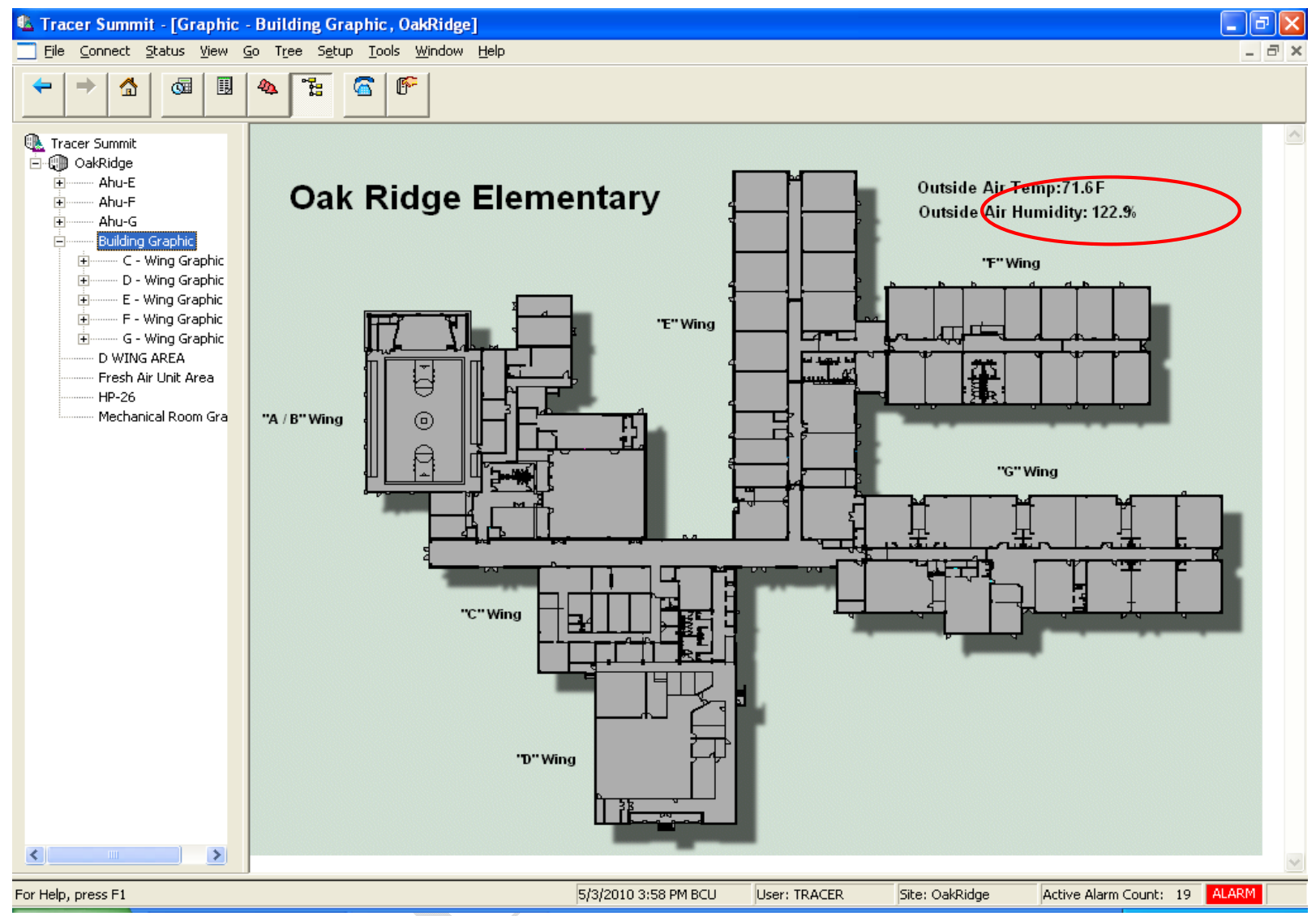

Figure 10 - Outdoor Air Humidity reading 122.9\% RH 


\section{Appendix N: EMC Engineers, InC. SeAsonal HVAC Retro- COMMISSIONING REPORT DATED MAY 2010 (CONTINUED)}

- Classroom 501, located on the BAS graphic screen does not have a link to HP48.

- A computer monitor (CRT) was found to have been placed directly beneath the thermostat of HP-55, in classroom 401 (Figure 11). The heat generated by the CRT was directly influencing the thermostat and keeping the room much colder than the BAS setpoint.

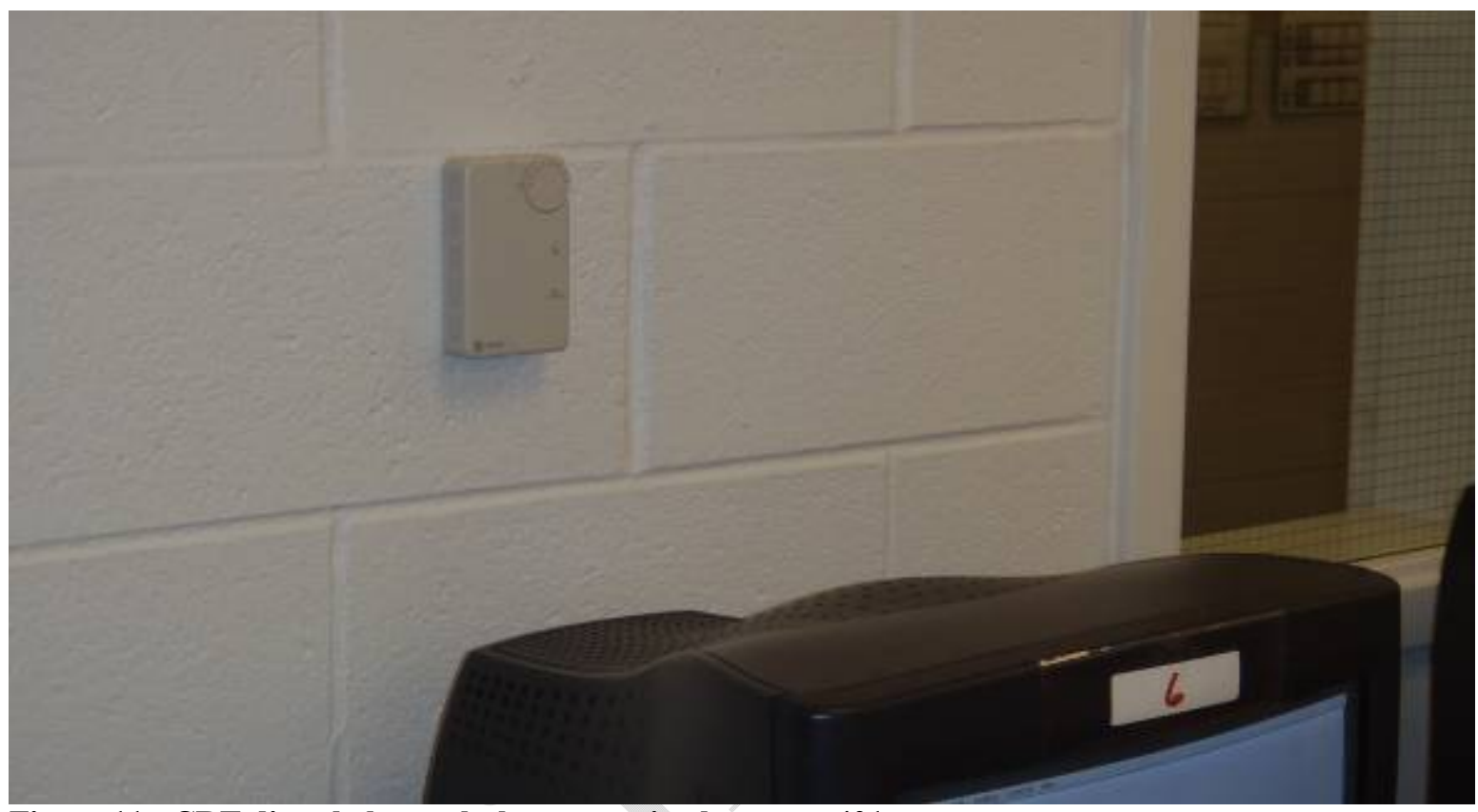

Figure 11 - CRT directly beneath thermostat in classroom 401

- HP-34 cooling setpoint was found to be at 74 deg F.

- The space temperature sensor installed in classroom 501 was found to be wired to an unknown point. EMC was unable to determine the termination point for this sensor due to no access above hard ceiling in the hallway. Classroom 501is served by HP-48, which also serves classroom 421 where the thermostat is located, and control of space temperatures of both classrooms is acceptable and consistent.

- Air filters for heat pumps showed expected amounts of build-up consistent with the approximately 90 days of use they have experienced.

- No issues with BAS communication to all heat pumps were present.

- Principal Ann Kraft demonstrated the unscientific method of using a facial tissue taped to the end of a yard stick to assess air flow in office spaces. This method is unreliable and cannot account for supply or return diffuser placement, air movement caused by sources outside the installed air conditioning system, or drafts which may be induced or naturally occurring. Since these air currents cannot be seen, this "tissue test" is unreliable. 


\section{Appendix N: EMC Engineers, InC. SeAsonal HVAC Retro- COMMISSIONING REPORT DATED MAY 2010 (CONTINUED)}

- HP-24 was found to constantly energize its reversing valve, thereby causing the unit to continually produce cold air. Temporarily altering the setpoints caused the heat pump controller to change to heating mode, however discharge air temperature remained at levels consistent with cooling operation. The reversing valve was tested and found to be operating properly, however the heat pump controller is constantly energizing the reversing valve.

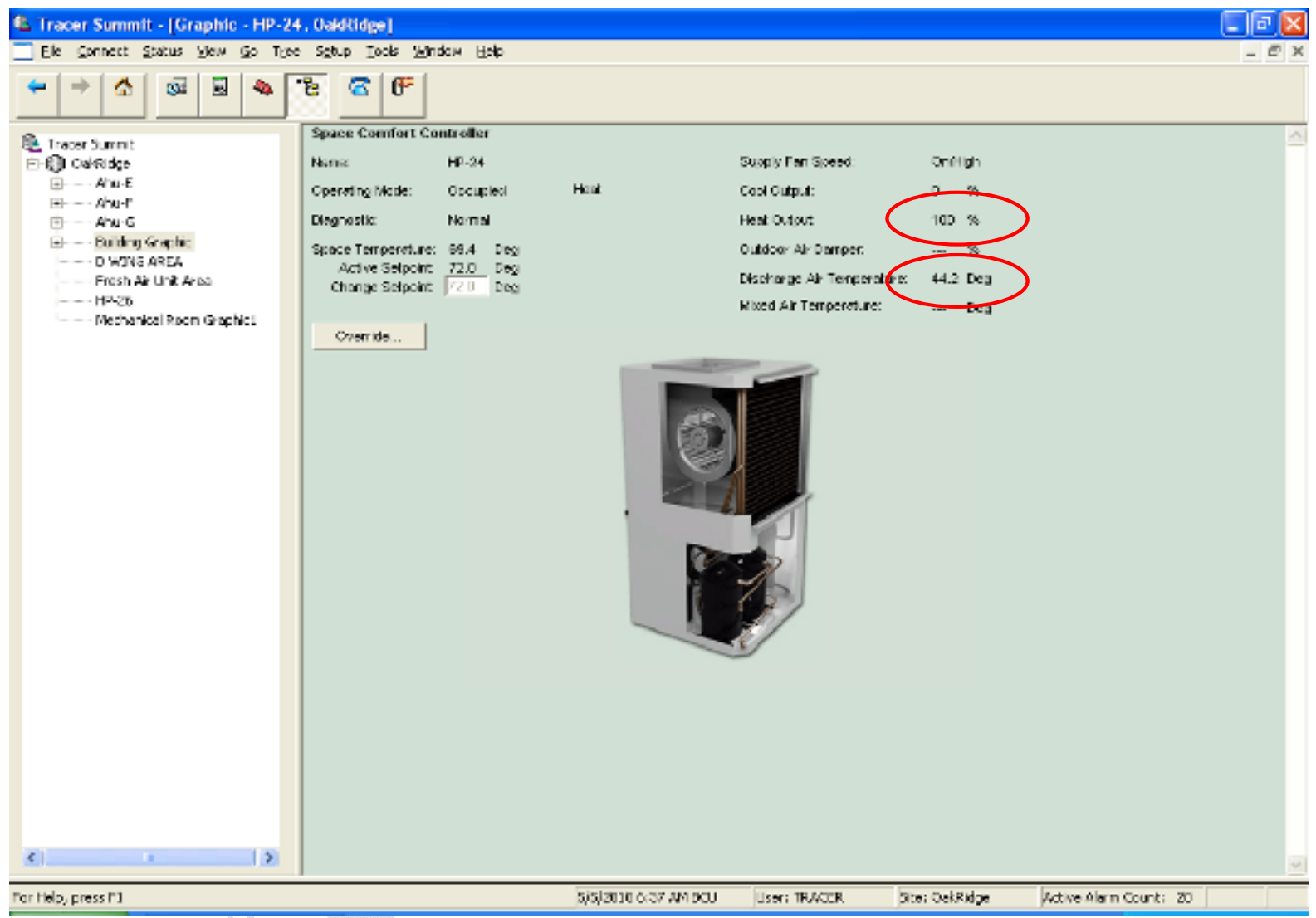

Figure 12 - HP-24 heat output @100\%, but discharge air temp.@44.2 deg F.

- Review of Tracer Summit alarm log revealed three instances during the week of May 3, 2010 where the Building Control Unit (BCU) communications ceased. No indication in alarm log of how long communications were down and no obvious reason why communications ceased. There was no impact to heat pumps operation or school environment conditions, however the Tracer Summit alarm log should be periodically reviewed to determine if future communication losses become more prevalent. 


\section{Appendix N: EMC Engineers, InC. SeAsonal HVAC Retro- COMMISSIONING REPORT DATED MAY 2010 (CONTINUED)}

- HP-9 circuit \#1 was found to be off on the low refrigerant pressure switch. Subsequent investigation by GCS personnel revealed that this circuit's refrigerant charge had escaped.

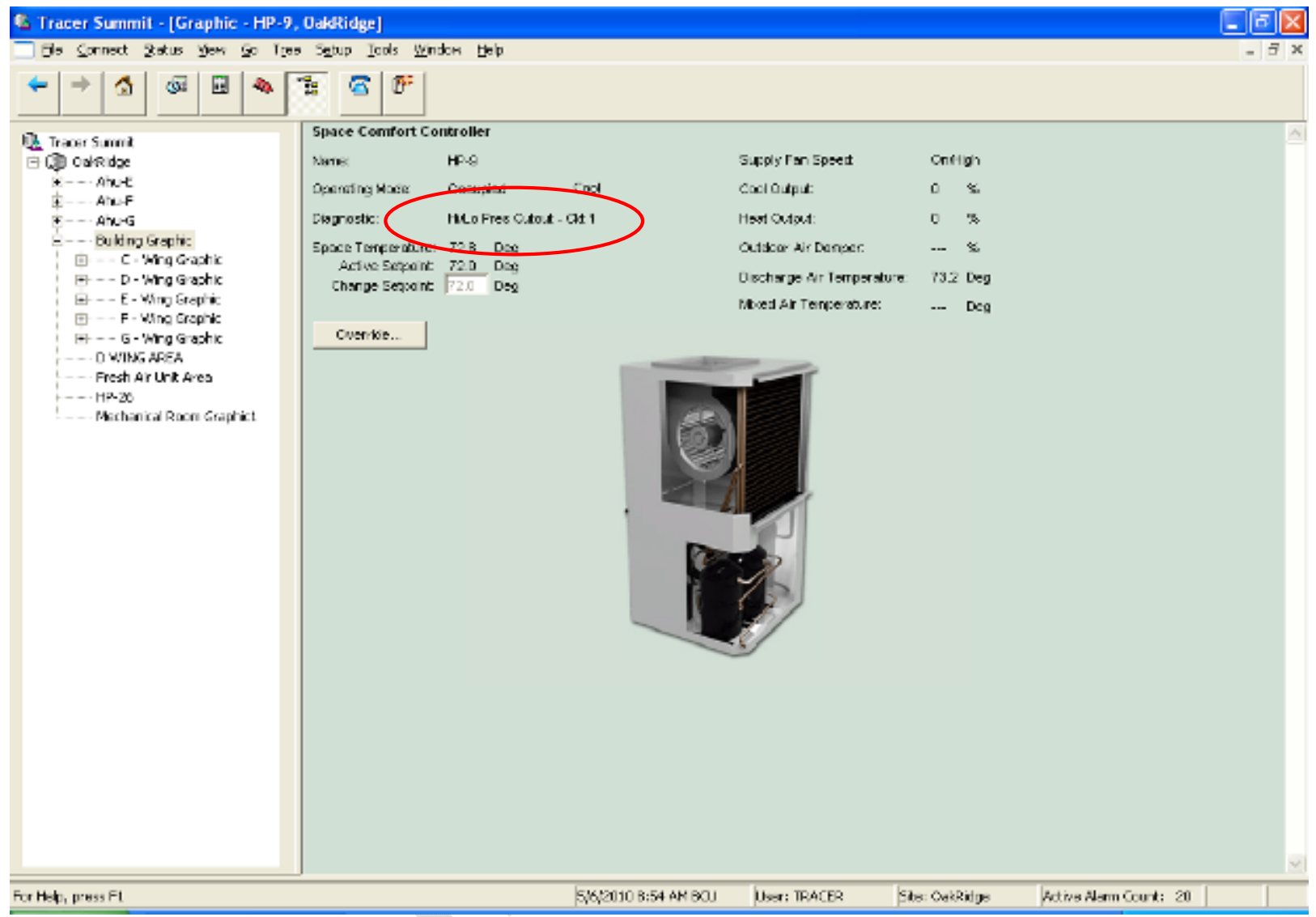

Figure 13 - HP-9 low pressure safety cutout

- The controller for HP-26 has been replaced with the same type of controller used for all other similar heat pumps in the school. This was a recommendation from EMC's original report. The operation of this heat pump is now consistent with the others.

- HP-12's supply fan motor stopped working while EMC was conducting seasonal testing. GCS Maintenance personnel responded to affect repairs.

- The rigid copper piping associated with the water source heat pumps' building loop, located in the mezzanines above the classroom wings lacks sufficient support in numerous locations. Unnecessary strain is being placed on soldered pipe joints, which will lead to premature failure of the joints and a potential for catastrophic flooding. 


\section{Appendix N: EMC Engineers, InC. SeAsonal HVAC Retro- COMMISSIONING REPORT DATED MAY 2010 (CONTINUED)}

- Space temperatures of a group of 9 heat pumps with multiple hot or cold calls since re-occupying the school on Feb 22, 2010 was trended. The following snapshot in Table 3 was captured on 5/6/2010:

\begin{tabular}{|c|c|c|c|c|c|c|c|c|c|}
\hline $\begin{array}{r}\text { HP-14 } \\
\text { Space Temperature: Active }\end{array}$ & HP-22 & HP-23 & HP-32 & HP-37 & HP-40 & HP-47 & HP-50 & HP-55 & HP-24 \\
\hline \multicolumn{10}{|l|}{ 5/6/2010 11:20:00 AM } \\
\hline 72.0 & 71.6 & 71.6 & 72.5 & 71.3 & 72.0 & 72.0 & 72.6 & 71.8 & 69.1 \\
\hline \multicolumn{10}{|l|}{ 5/6/2010 11:15:00 AM } \\
\hline 72.0 & 71.3 & 71.3 & 72.5 & 71.3 & 72.2 & 72.0 & 72.5 & 71.8 & 69.1 \\
\hline \multicolumn{10}{|l|}{ 5/6/2010 11:10:00 AM } \\
\hline 72.0 & 70.9 & 70.9 & 72.5 & 71.4 & 72.5 & 72.1 & 72.5 & 71.7 & 69.1 \\
\hline \multicolumn{10}{|l|}{ 5/6/2010 11:05:00 AM } \\
\hline 72.1 & 70.8 & 70.8 & 72.4 & 71.5 & 72.8 & 72.1 & 72.3 & 71.8 & 69.2 \\
\hline \multicolumn{10}{|l|}{ 5/6/2010 11:00:00 AM } \\
\hline \multirow{2}{*}{\multicolumn{10}{|c|}{ 5/6/2010 10:55:00 AM }} \\
\hline & & & & & & & & & \\
\hline 72.0 & 71.1 & 71.1 & 72.3 & 72.1 & 72.5 & 72.2 & 72.1 & 71.6 & 69.3 \\
\hline \multicolumn{10}{|l|}{ 5/6/2010 10:50:00 AM } \\
\hline 72.0 & 71.4 & 71.4 & 72.2 & 72.2 & 72.5 & 72.2 & 72.3 & 71.6 & 69.3 \\
\hline \multicolumn{10}{|l|}{ 5/6/2010 10:45:00 AM } \\
\hline 72.0 & 71.7 & 71.8 & 72.5 & 72.3 & 72.4 & 72.1 & 72.5 & 71.4 & 69.2 \\
\hline \multicolumn{10}{|l|}{ 5/6/2010 10:40:00 AM } \\
\hline 72.1 & 71.8 & 71.8 & 72.5 & 72.1 & 72.4 & 72.3 & 72.5 & 71.6 & 69.2 \\
\hline \multicolumn{10}{|l|}{ 5/6/2010 10:35:00 AM } \\
\hline 72.1 & 72.0 & 72.0 & 72.5 & 72.1 & 72.4 & 72.3 & 72.3 & 71.6 & 69.2 \\
\hline \multicolumn{10}{|l|}{ 5/6/2010 10:30:00 AM } \\
\hline 72.1 & 72.2 & 72.2 & 72.5 & 72.1 & 72.4 & 72.4 & 72.3 & 71.7 & 69.2 \\
\hline \multicolumn{10}{|l|}{ 5/6/2010 10:25:00 AM } \\
\hline \multirow{2}{*}{\multicolumn{10}{|c|}{ 5/6/2010 10:20:00 AM }} \\
\hline & & & & & & & & & \\
\hline 72.0 & 72.7 & 72.8 & 72.5 & 72.4 & 72.4 & 72.3 & 72.2 & 71.6 & 69.4 \\
\hline \multicolumn{9}{|l|}{ 5/6/2010 10:15:00 AM } & \\
\hline 72.0 & 72.8 & 72.8 & 72.5 & 72.4 & 72.4 & 72.4 & 72.1 & 71.7 & 69.3 \\
\hline \multicolumn{10}{|l|}{ 5/6/2010 10:10:00 AM } \\
\hline 72.0 & 72.8 & 72.8 & 72.4 & 72.4 & 72.4 & 72.4 & 72.1 & 71.6 & 69.3 \\
\hline \multicolumn{10}{|l|}{ 5/6/2010 10:05:00 AM } \\
\hline 71.9 & 72.9 & 72.9 & 72.3 & 72.6 & 72.4 & 72.8 & 71.8 & 71.7 & 69.2 \\
\hline \multicolumn{10}{|l|}{ 5/6/2010 10:00:00 AM } \\
\hline 5/6/2010 9:55:00 AM & 72.7 & 72.7 & 72.4 & 72.6 & 72.4 & 72.7 & 71.8 & 71.6 & 69.2 \\
\hline 71.9 & 72.5 & 72.5 & 72.4 & 72.8 & 72.4 & 72.6 & 71.8 & 71.6 & 69.3 \\
\hline 5/6/2010 9:50:00 AM & & & & & & & & & \\
\hline 71.9 & 72.1 & 72.1 & 72.2 & 72.8 & 72.4 & 72.5 & 71.8 & 71.7 & 69.3 \\
\hline 5/6/2010 9:45:00 AM & & & & & & & & & \\
\hline 72.0 & 71.8 & 71.8 & 72.5 & 72.9 & 72.4 & 72.5 & 71.8 & 71.9 & 69.5 \\
\hline $\begin{array}{c}\text { 5/6/2010 9:40:00 AM } \\
72.0\end{array}$ & & & & & & & & & \\
\hline 5/6/2010 9:35:00 AM & 71.5 & 71.5 & 72.4 & 73.0 & 72.5 & 72.5 & 71.9 & 71.7 & 69.4 \\
\hline 5/6/2010 9:30:00 AM & 71.2 & 70.9 & 72.6 & 73.2 & 72.4 & 72.6 & 71.8 & 71.8 & 69.5 \\
\hline 72.1 & 70.9 & 71.1 & 72.5 & 73.1 & 72.3 & 72.6 & 71.9 & 71.7 & 69.5 \\
\hline 5/6/2010 9:25:00 AM & & & & & & & & & \\
\hline 72.1 & 71.1 & 71.1 & 72.5 & 73.1 & 72.3 & 72.6 & 72.0 & 71.6 & 69.4 \\
\hline 5/6/2010 9:20:00 AM & & & & & & & & & \\
\hline 72.1 & 71.1 & 71.4 & 72.6 & 73.0 & 72.3 & 72.6 & 72.1 & 71.6 & 69.4 \\
\hline 5/6/2010 9:15:00 AM & & & & & & & & & \\
\hline 72.1 & 71.4 & 71.4 & 72.5 & 72.9 & 72.4 & 72.6 & 72.1 & 71.6 & 69.3 \\
\hline 5/6/2010 9:10:00 AM & & & & & & & & & \\
\hline 5/6/2010 9:05:00 AM & 71.7 & 71.7 & 72.4 & 72.8 & 72.3 & 72.5 & 72.3 & 71.9 & 69.4 \\
\hline 72.0 & 71.9 & 71.9 & 72.5 & 72.8 & 72.4 & 72.5 & 72.4 & 71.9 & 69.2 \\
\hline 5/6/2010 9:00:00 AM & & & & & & & & & \\
\hline 5/6/2010 8:55:00 AM & 72.0 & 72.0 & 72.5 & 72.8 & 72.4 & 72.6 & 72.5 & 71.8 & 69.3 \\
\hline
\end{tabular}

Page 16 of 22 


\section{Appendix N: EMC Engineers, InC. SeAsonal HVAC Retro- COMMISSIONING REPORT DATED MAY 2010 (CONTINUED)}

\begin{tabular}{|c|c|c|c|c|c|c|c|c|c|}
\hline \multirow{2}{*}{\multicolumn{10}{|c|}{ 5/6/2010 8:45:00 AM }} \\
\hline & & & & & & & & & \\
\hline 72.0 & 72.8 & 72.8 & 72.4 & 72.8 & 72.6 & 72.6 & 72.3 & 71.9 & 69.3 \\
\hline \multicolumn{10}{|l|}{ 5/6/2010 8:40:00 AM } \\
\hline 72.1 & 73.0 & 73.0 & 72.3 & 72.8 & 72.7 & 72.6 & 72.3 & 71.8 & 69.2 \\
\hline \multicolumn{10}{|l|}{ 5/6/2010 8:35:00 AM } \\
\hline 72.2 & 73.0 & 73.0 & 72.2 & 72.8 & 72.8 & 72.6 & 72.2 & 71.9 & 69.2 \\
\hline \multicolumn{10}{|l|}{ 5/6/2010 8:30:00 AM } \\
\hline 72.1 & 72.7 & 72.7 & 72.1 & 72.8 & 72.7 & 72.6 & 72.1 & 71.8 & 69.2 \\
\hline \multicolumn{10}{|l|}{ 5/6/2010 8:25:00 AM } \\
\hline 72.1 & 72.7 & 72.5 & 72.0 & 72.8 & 72.8 & 72.7 & 72.0 & 72.0 & 69.3 \\
\hline \multicolumn{10}{|l|}{ 5/6/2010 8:20:00 AM } \\
\hline 72.0 & 72.5 & 72.1 & 71.9 & 72.8 & 72.8 & 72.6 & 72.1 & 71.8 & 69.3 \\
\hline \multicolumn{10}{|l|}{ 5/6/2010 8:15:00 AM } \\
\hline \multirow{2}{*}{\multicolumn{10}{|c|}{ 5/6/2010 8:10:00 AM }} \\
\hline & & & & & & & & & \\
\hline 71.8 & 71.4 & 71.3 & 71.9 & 72.8 & 72.6 & 72.6 & 72.1 & 71.9 & 69.3 \\
\hline \multicolumn{10}{|l|}{ 5/6/2010 8:05:00 AM } \\
\hline 71.9 & 71.4 & 71.3 & 71.8 & 72.7 & 72.6 & 72.6 & 72.1 & 72.0 & 69.4 \\
\hline \multicolumn{10}{|l|}{ 5/6/2010 8:00:00 AM } \\
\hline 71.9 & 71.3 & 71.3 & 71.8 & 72.5 & 72.4 & 72.4 & 72.3 & 72.0 & 69.5 \\
\hline \multicolumn{10}{|l|}{ 5/6/2010 7:55:00 AM } \\
\hline 72.0 & 71.6 & 71.6 & 71.7 & 72.4 & 72.4 & 72.5 & 72.5 & 72.0 & 69.6 \\
\hline \multicolumn{10}{|l|}{ 5/6/2010 7:50:00 AM } \\
\hline 72.0 & 71.8 & 71.8 & 71.7 & 72.3 & 72.2 & 72.3 & 72.5 & 71.9 & 69.5 \\
\hline \multicolumn{10}{|l|}{ 5/6/2010 7:45:00 AM } \\
\hline 72.1 & 72.0 & 72.0 & 71.7 & 72.1 & 72.1 & 72.4 & 72.4 & 71.9 & 69.5 \\
\hline \multicolumn{10}{|l|}{ 5/6/2010 7:40:00 AM } \\
\hline \multirow{2}{*}{\multicolumn{10}{|c|}{ 5/6/2010 7:35:00 AM }} \\
\hline & & & & & & & & & \\
\hline 72.1 & 72.8 & 72.7 & 71.7 & 72.0 & 72.0 & 72.3 & 72.4 & 71.8 & 69.5 \\
\hline \multicolumn{10}{|l|}{ 5/6/2010 7:30:00 AM } \\
\hline 72.1 & 72.7 & 72.5 & 71.7 & 72.0 & 72.0 & 72.2 & 72.3 & 71.8 & 69.6 \\
\hline \multicolumn{10}{|l|}{ 5/6/2010 7:25:00 AM } \\
\hline 72.2 & 72.5 & 72.3 & 71.7 & 72.0 & 72.1 & 72.3 & 72.4 & 72.0 & 69.6 \\
\hline \multicolumn{10}{|l|}{ 5/6/2010 7:20:00 AM } \\
\hline 72.1 & 72.3 & 71.9 & 71.6 & 72.1 & 72.0 & 72.2 & 72.3 & 72.0 & 69.5 \\
\hline
\end{tabular}

Table 3 - Units with multiple trouble calls after Feb 22, 2010 


\section{Appendix N: EMC Engineers, InC. Seasonal HVAC Retro- COMMISSIONING RePORT DATED MAY 2010 (CONTINUED)}

\subsubsection{Conclusions \& Recommendations}

- To aid in having space temperatures settled within the controller's deadband by the time teachers and staff arrive in the mornings, the revised recommended schedule is as follows:

- Office and Media Center areas

- Occupied 4 a.m. - 9 p.m.

- Unoccupied 9 p.m. - 4 a.m.

- Classroom wings and Gymnasium / Cafeteria

- Occupied 5 a.m. - 9 p.m.

- Unoccupied 9 p.m. - 5 a.m.

- Data closets

- Occupied continuously

Note: As of 5/7/2010 the above schedule changes have been implemented.

- To provide acceptable space temperatures and alleviate unnecessary trouble calls to GCS Maintenance, the revised recommended temperature setpoints are as follows:

- Office and Media Center

- Occupied Cooling - 72 deg F

- Unoccupied Cooling - 77 deg F

- Classroom wings and Gymnasium / Cafeteria

- No changes

- Data closets

- No changes

(all unoccupied offsets should remain at $+/-5$ degrees)

Note: As of 5/7/2010 the above setpoint changes have been implemented.

- EMC recommends that regular air filter inspections continue to be performed on all units, including the makeup air units, at least every 3 months per manufacturer's recommendations, with filter changes conducted when visual inspection indicates necessity.

- Air flow assessments should be made by trained personnel with the proper calibrated equipment in order to prevent erroneous trouble calls stemming from inaccurate observations or assumptions.

- Thermostats shown in the list in section Table 2 above should be calibrated to within factory tolerances (+/- .5 deg F). If calibration is not possible, the thermostat should be replaced and the new thermostat calibration verified with a calibrated test instrument. 


\section{Appendix N: EMC Engineers, InC. SeAsonal HVAC Retro- COMMISSIONING REPORT DATED MAY 2010 (CONTINUED)}

- In order to aid in maintaining acceptable indoor conditions, all outside doors as well as all operable windows should continue to remain closed at all times. Additionally, custodial staff should regularly clear the areas around all exterior doors of rocks or objects that may deliberately or inadvertently impede the full closing of those doors.

- The outside door frame for room 521 should be replaced or repaired. If repair is made, the door alignment should be corrected as well.

- The outdoor air humidity transmitter for the BAS should be calibrated properly. The scaling of the device input in the BAS should also be verified as correct for the device.

- A link to HP-48 should be added to the F-wing graphic in the BAS to facilitate easy access to the heat pump controller from the E-wing graphic.

- HP-24 controller should be investigated to determine why the reversing valve is always energized, and repair or replace controller as determined necessary.

- HP-34 setpoints should be set the same as other classroom heat pumps.

- Continue following unit manufacturer's recommendations on frequency of air filter changes. Observe filters for increased build-up during periods of high traffic (i.e. move-in and move-out at beginning and end of school year) and adjust filter change periodicity as determined to be necessary.

- Review Trane Tracer Summit alarm log weekly, paying particular attention for BCU communication failures. If these failures start appearing more frequently than weekly, BCU replacement should be considered.

- Investigate HP-9 to determine the cause of refrigerant leak out of circuit \#1 and repair as necessary. Once repairs are made, HP-9 performance should be reviewed daily for 1 week to determine effectiveness of repairs.

- Install pipe supports every 6 feet, where missing on all building heat pump loop piping. These supports should be properly anchored to building structural components vice sheetrock anchors. Supports should be straight and plumb and their length should be such that the full weight of the copper piping is supported by the hangers. Threaded rod with saddle brackets sized to hold up to 2 " copper pipe, with dielectric insulator material should be used. 


\section{Appendix N: EMC Engineers, InC. Seasonal HVAC Retro- COMMISSIONING RePORT DATED MAY 2010 (CONTINUED)}

\subsection{Tempered Water System}

\subsubsection{System Verification}

EMC verified the operation of the Cooling Tower, Heat Exchanger, Building Loop Pumps, and Tower Pumps for proper cooling season operation. All digital commands, such as pump start/stop commands were visually verified for proper equipment reaction.

EMC worked to verify that the sequence of operations was fully and correctly implemented, and that the transient changes in operation of the units were still made apparent on the Graphics for the BAS.

\subsubsection{Summary of Findings}

No issues were discovered with respect to the operation of the cooling tower, heat exchanger or pumps in cooling season operation.

\subsubsection{Conclusions \& Recommendations}

This tempered water system is operating as designed. No recommendations at this time. 


\section{Appendix N: EMC Engineers, InC. Seasonal HVAC Retro- COMMISSIONING RePORT DATED May 2010 (continued)}

\subsection{Makeup Air Units}

\subsubsection{System Verification}

EMC verified that during occupied periods supply air temperature was generally at or near $70 \operatorname{deg} \mathrm{F}$, and supply air humidity was generally between 45 and 50\% RH. During unoccupied periods, an offset of 5 degrees was observed when units were activated upon a call for operation of one or more associated heat pumps in that building wing. Supply humidity levels during unoccupied periods were observed as remaining unchanged. Reheat water temperatures generally ranged from $95-100$ deg F and glycol supply temperatures were observed to be between $40-45$ deg F.

The outdoor air units followed the occupancy schedule of the classroom wings and stopped off when the wing went into unoccupied, unless there was at least one heat pump calling for cooling, in which case the outdoor unit would start or continue to run.

\subsubsection{Summary of Findings}

No issues were discovered with respect to the operation of the outdoor air units.

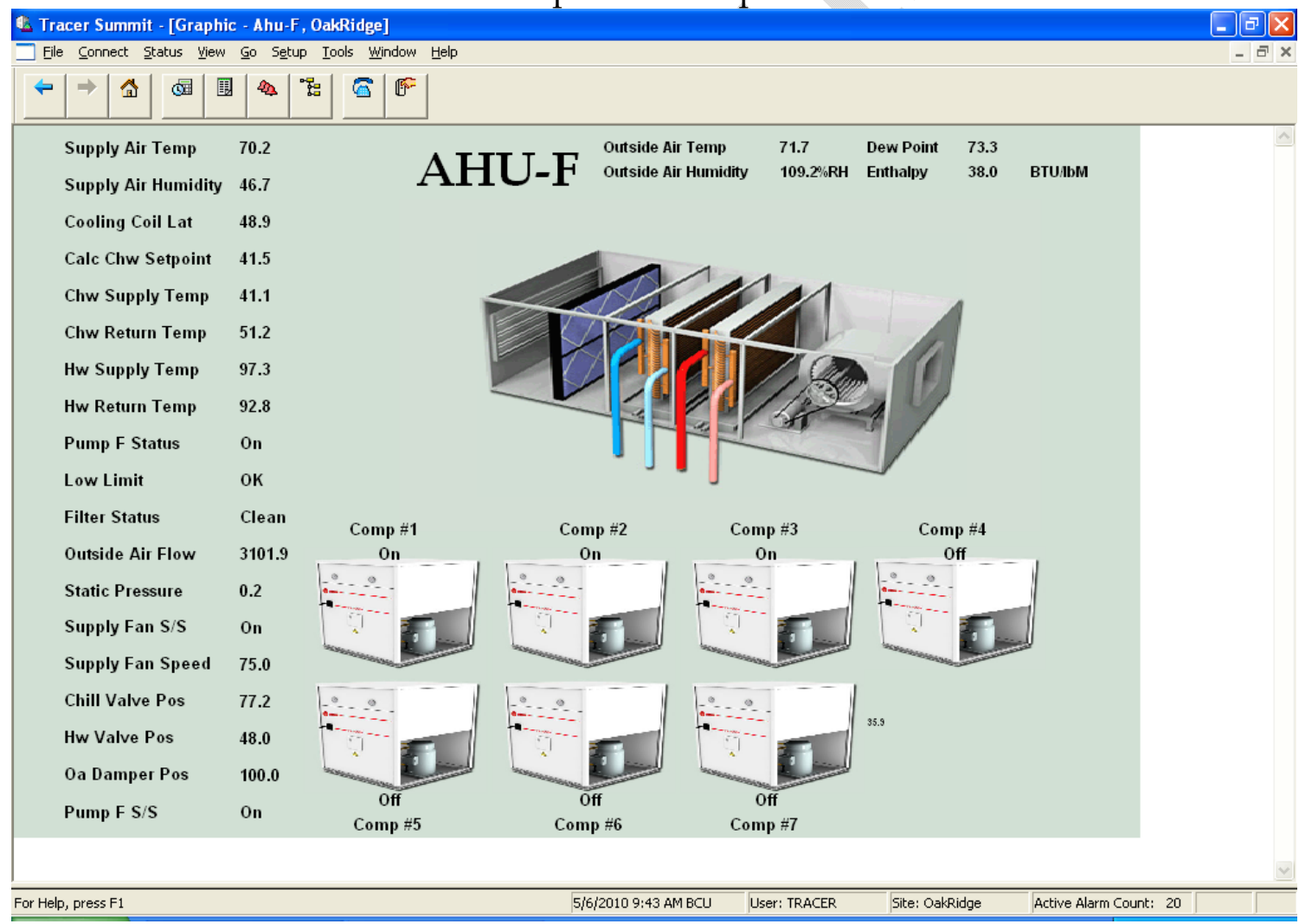

Figure 14 - Typical Outdoor Air Unit graphic screen (occupied operation)

\subsubsection{Conclusions \& Recommendations}

The units are operating as designed. No recommendations at this time. 


\section{Appendix N: EMC Engineers, InC. Seasonal HVAC Retro- COMMISSIONING RePORT DATED MAY 2010 (CONtINUEd)}

\subsection{Exhaust Fans and Systems}

\subsubsection{System Verification}

EMC verified operation of the building exhaust fans. Fans controlled by heat pumps were operational, as were thermostatically-controlled fans. No issues related to exhaust fans were discovered during seasonal inspection.

\subsubsection{Summary of Findings}

No issues were discovered with respect to the operation of the exhaust fans.

\subsubsection{Conclusions \& Recommendations}

The exhaust fans are operating correctly. No recommendations at this time. 


\section{Appendix O: Elementary SChOOL IAQ PROJECt PROGRESS UPDATE \\ Oak Ridge Elementary School-IAQ Project \\ As of $01 / 12 / 10$ \\ Progress Update}

\begin{tabular}{|c|c|c|c|c|c|c|}
\hline \multirow[b]{2}{*}{ \# } & \multirow[b]{2}{*}{ ACTIVITY } & \multicolumn{3}{|c|}{ \% Complete } & \multirow{2}{*}{$\begin{array}{l}\text { SUBSTANTIAL } \\
\text { COMPLETION DATE }\end{array}$} & \multirow[b]{2}{*}{ STATUS } \\
\hline & & $\mathbf{P}$ & \begin{tabular}{|l|l}
$\mathbf{D}$ \\
\end{tabular} & $\mathbf{C}$ & & \\
\hline 1 & $\begin{array}{l}\text { Remove and clean building contents, move to new } \\
\text { sites and storage. }\end{array}$ & 100 & 100 & 100 & $8 / 14 / 2009$ & Complete \\
\hline 2 & Sheetrock repaired from initial NIOSH and Turner visit & 100 & 100 & 100 & $8 / 17 / 2009$ & Complete \\
\hline 3 & Removal of Carpeting & 100 & 100 & 100 & $9 / 4 / 2009$ & Complete \\
\hline 4 & Installation of VCT/moisture testing & 100 & 100 & 100 & $9 / 11 / 2009$ & Complete \\
\hline 5 & $\begin{array}{l}\text { Installation of new filter boxes upstream from water } \\
\text { source heat pump \& clean coils }\end{array}$ & 100 & 100 & 100 & $12 / 6 / 2009$ & Complete \\
\hline 6 & $\begin{array}{l}\text { Removal of old ceiling tiles above suspended ceiling } \\
\text { media center hall and sealing around windows }\end{array}$ & 100 & 100 & 100 & $11 / 9 / 2009$ & Complete \\
\hline 7 & $\begin{array}{l}\text { Sheetrock replacement/repair resulting from } \\
\text { exploratory and base cove work }\end{array}$ & 100 & 100 & 100 & $10 / 19 / 2009$ & Complete \\
\hline 8 & $\begin{array}{l}\text { Vapor barrier and depressurization of crawl space } \\
\text { under the media center. }\end{array}$ & 100 & 100 & 100 & $10 / 23 / 2009$ & Complete \\
\hline 9 & Roof flashing and roof repairs & 100 & 100 & 100 & $12 / 30 / 2009$ & Complete \\
\hline 10 & Sealing of classroom wings/soffits/exhaust & 100 & 100 & 100 & $12 / 13 / 2009$ & Complete \\
\hline 11 & Site drainage review/modifications & 100 & 100 & 0 & TBD & $\begin{array}{l}\text { Pending due to weather } \\
\text { conditions/ wet grounds- } \\
\text { Turner Consulting has } \\
\text { verbally informed us that it is } \\
\text { not necessary to complete } \\
\text { this work in order to occupy } \\
\text { the building. }\end{array}$ \\
\hline 12 & Retro-commissioning of HVAC system & 100 & 100 & 100 & $12 / 23 / 2009$ & Complete \\
\hline 13 & Building Cleaning & 100 & 100 & 100 & $12 / 18 / 2009$ & $\begin{array}{l}\text { Complete- Note: Cleaning } \\
\text { efforts will be ongoing as the } \\
\text { move-in occurs. }\end{array}$ \\
\hline 14 & Moisture in gym exterior walls & 100 & 100 & 100 & $10 / 30 / 2009$ & Complete \\
\hline 15 & Turner Consulting- Site Review/Data Review & NA & NA & NA & $1 / 5 / 2010$ & $\begin{array}{l}\text { Complete- Turner on-site } \\
12 / 15 \text { thru } 12 / 18\end{array}$ \\
\hline 16 & Turner Consulting Report & NA & NA & NA & $1 / 12 / 2010$ & Complete \\
\hline
\end{tabular}

\begin{tabular}{|l|} 
Phase: \\
P - Planning \\
D - Design/Protocol Development \\
C - Construction
\end{tabular}

Notes:

1. In addition, to the Indoor Air Quality Work (IAQ) related work currently underway staff is also coordinating with the County building department/fire marshal's office and any other necessary Authorities Having Jurisdiction (AHJ's) to resolve any code related issues discovered as a result of ongoing building evaluations. Based on recent discussions with the County building department and the fire marshal's office we do not believe that any of the work currently being proposed (Ex. add an exterior door and reconfigure some interior doors in the media center) would impact re-occupancy of ORE in a Jan.-Feb. timeframe.

2. This is intended to provide a snapshot of the work that has been identified to date in working with Turner Consulting. Additional work resulting from ongoing evaluations, pending NIOSH report and any unforseen conditions may be added and/or modify line items noted above, as applicable 


\section{$\overline{\text { ApPENDIX O: ElEMENTARY SCHOOL IAQ PROJECT PROGRESS UPDATE }}$}

(CONTINUED)

\section{\#3}

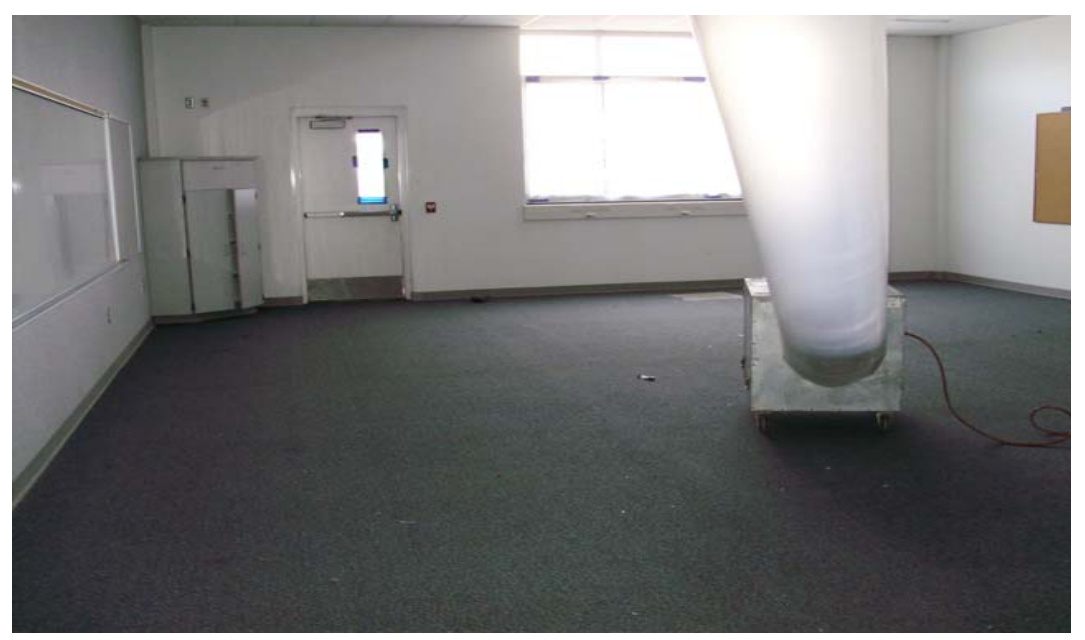

Removal of CARPETING

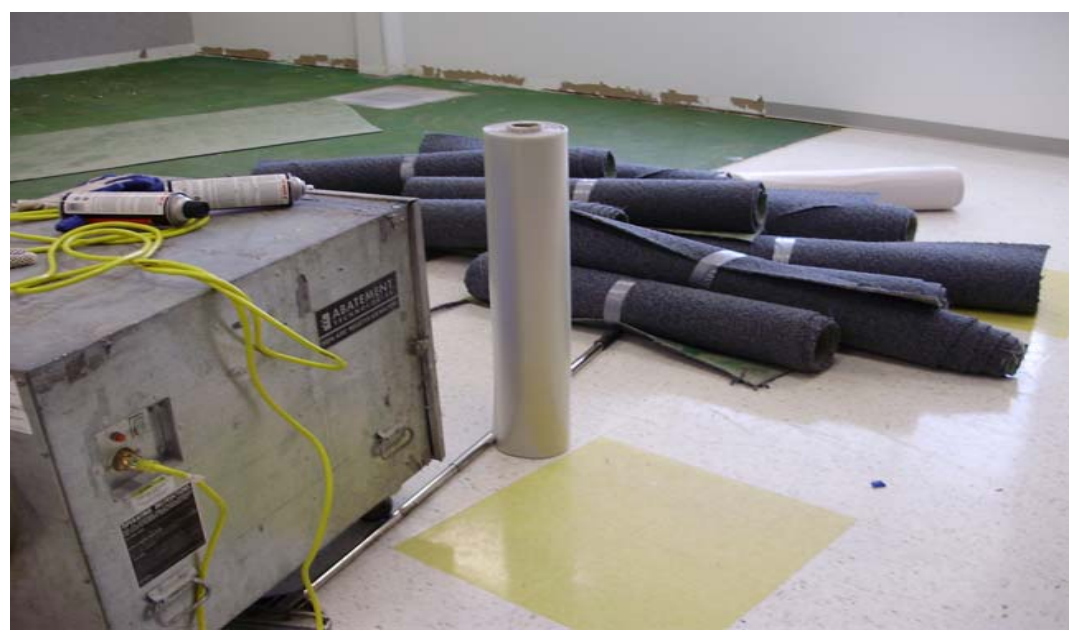




\section{$\overline{\text { Appendix O: ElEMENTARY SCHOOL IAQ PROJECT PROGRESS UPDATE }}$}

(CONTINUED)

\section{$\# 4$}

Moisture Testing

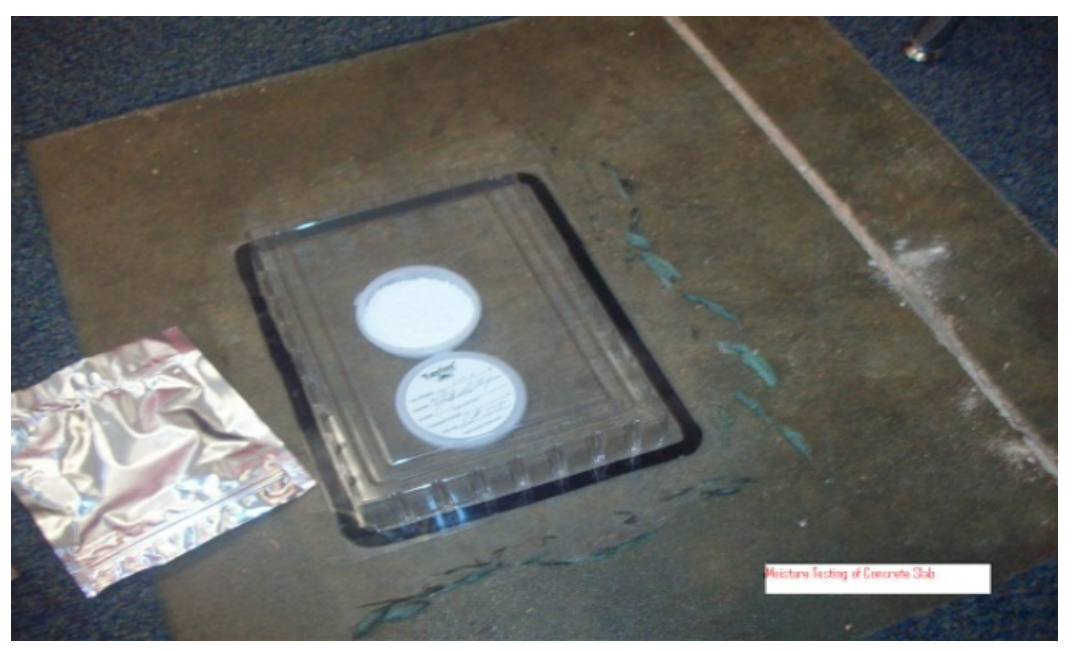

INSTALLATION OF VCT

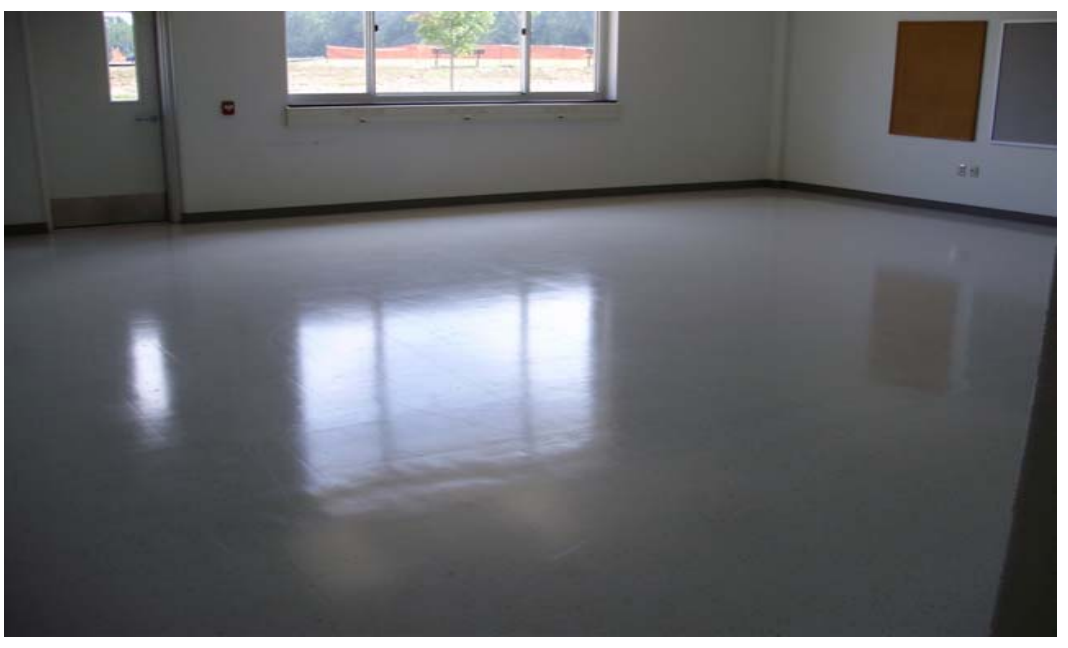




\section{$\overline{\text { ApPENDIX O: ElEMENTARY SCHOOL IAQ PROJECT PROGRESS UPDATE }}$}

(CONTINUED)

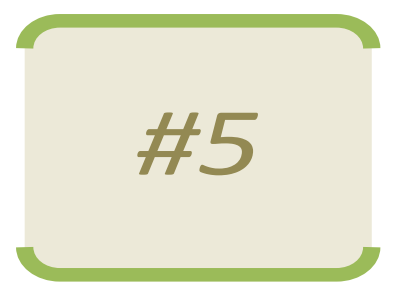

Removal of Air Filters at HeAt

PUMP

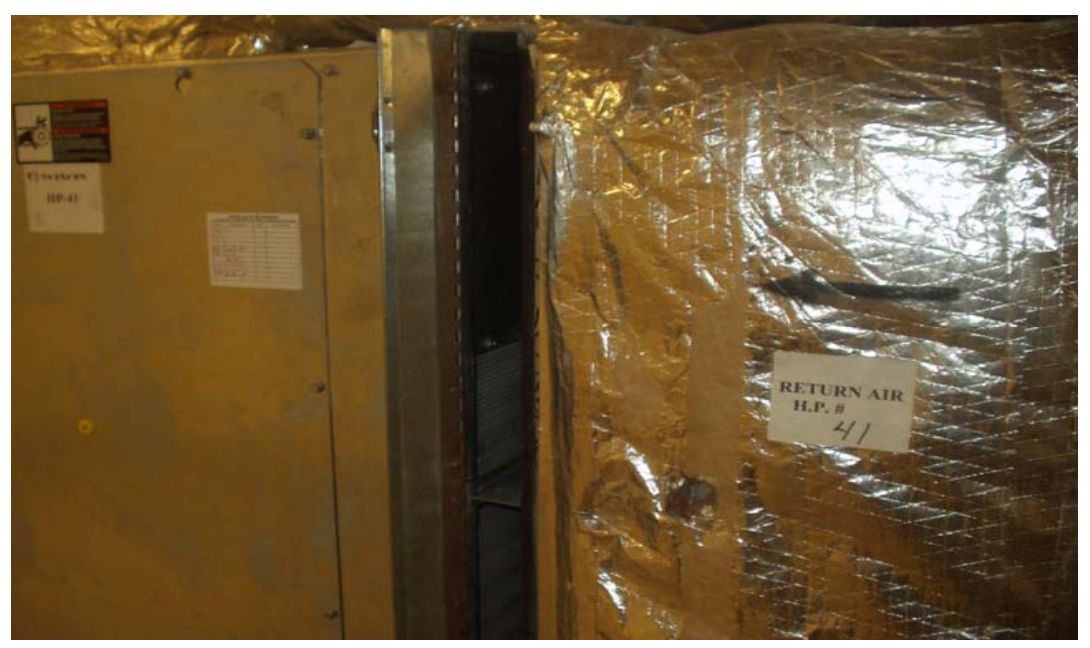

INSTALLATION OF NEW Filter

BOXES IN ClASSROOM

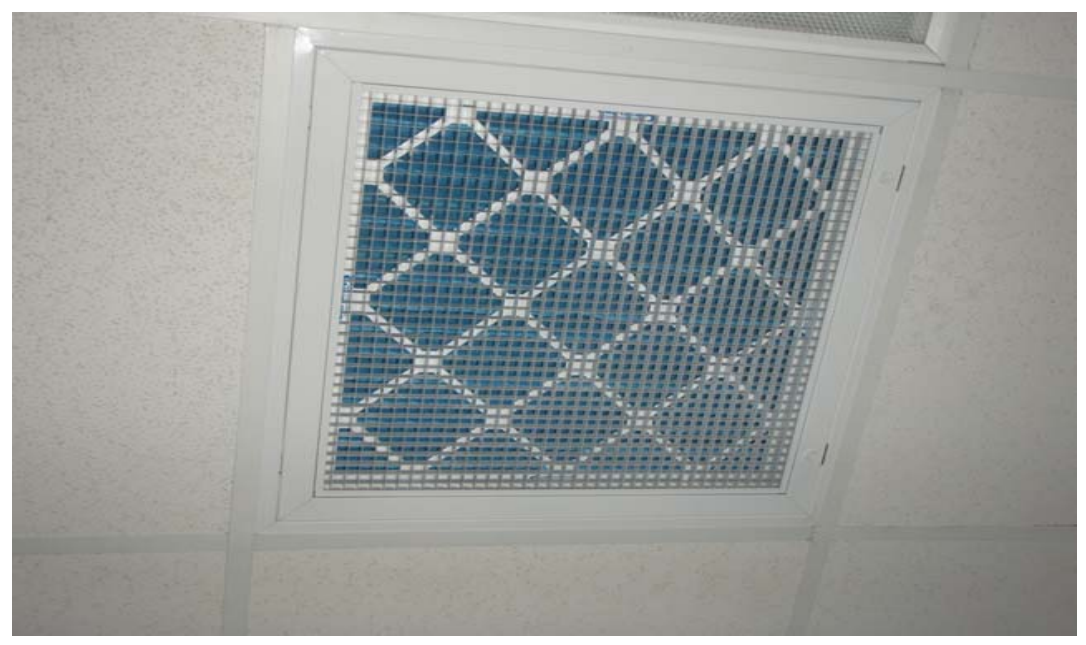




\section{$\overline{\text { Appendix O: Elementary SCHOOL IAQ PROJECt PROGRESS UPDATE }}$
(CONTINUEd)}

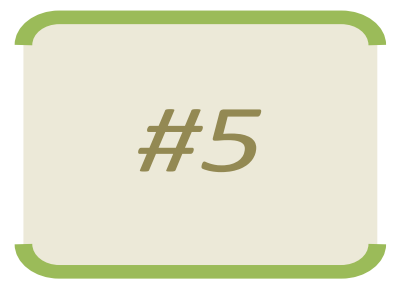

DiRTY COILS

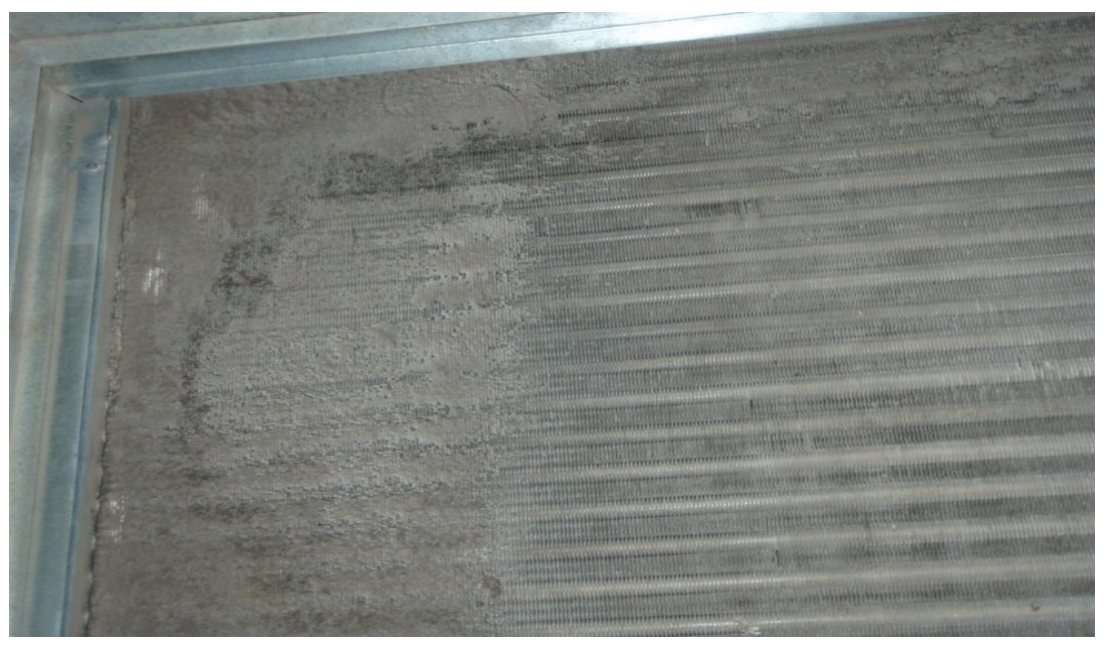

Cleaned Coils

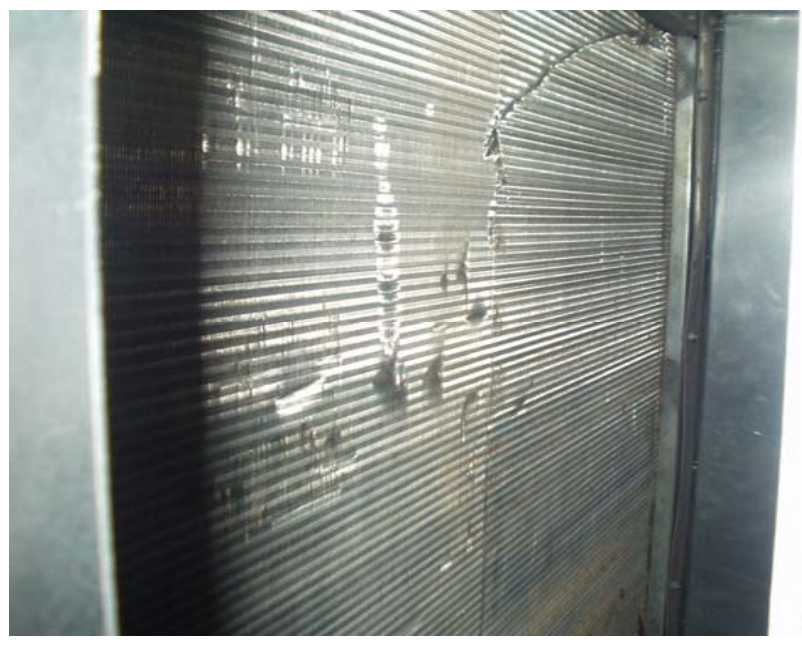




\section{APPENDIX O: EleMENTARY SCHOOL IAQ PROJECT PROGRESS UPDATE}

(CONTINUED)

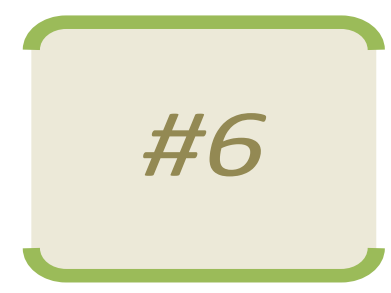

Removal of Old Ceiling Tiles

Above Suspended Ceiling Media

Center Hall

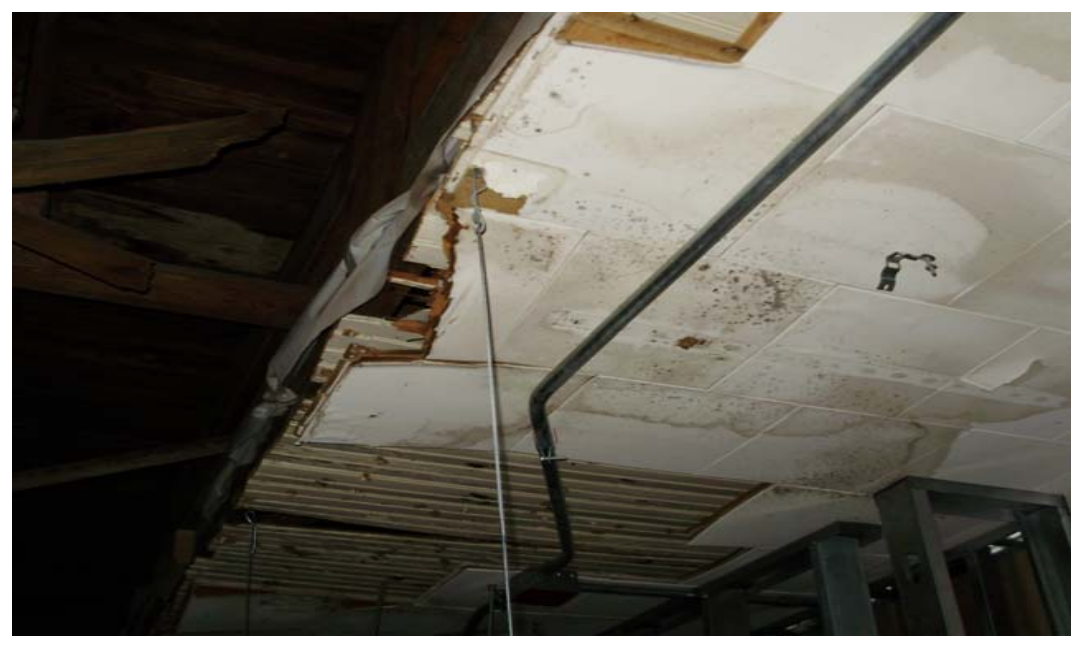

Ceiling Tiles Removed

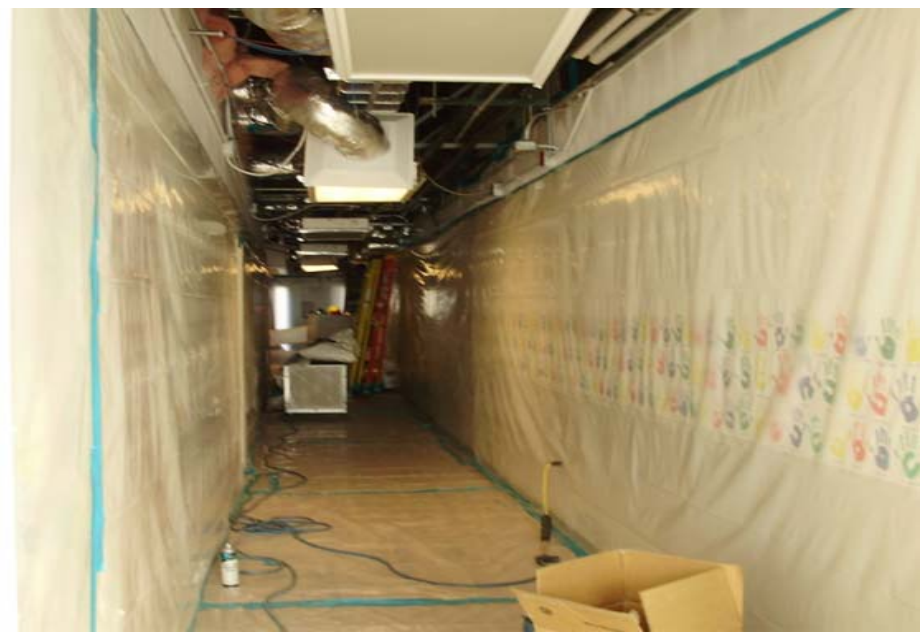




\section{ApPendix O: EleMENTARY SCHOOL IAQ PROJECT PROGRESS UPDATE}

(CONTINUED)

\section{\#6}

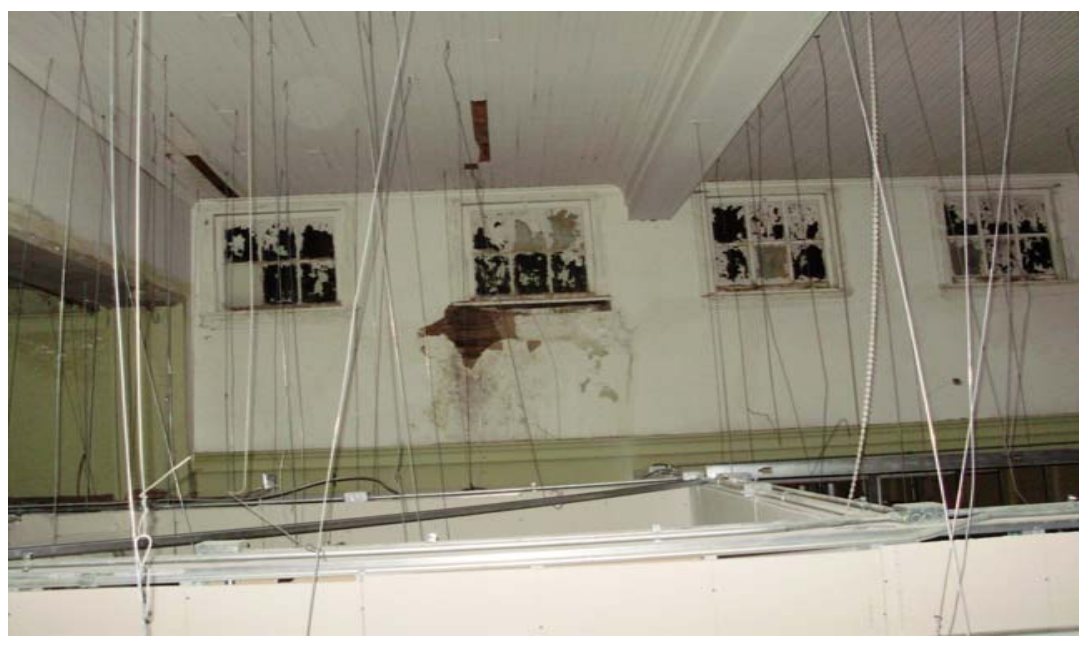

Cleaned and Sealed Windows Above Media Center

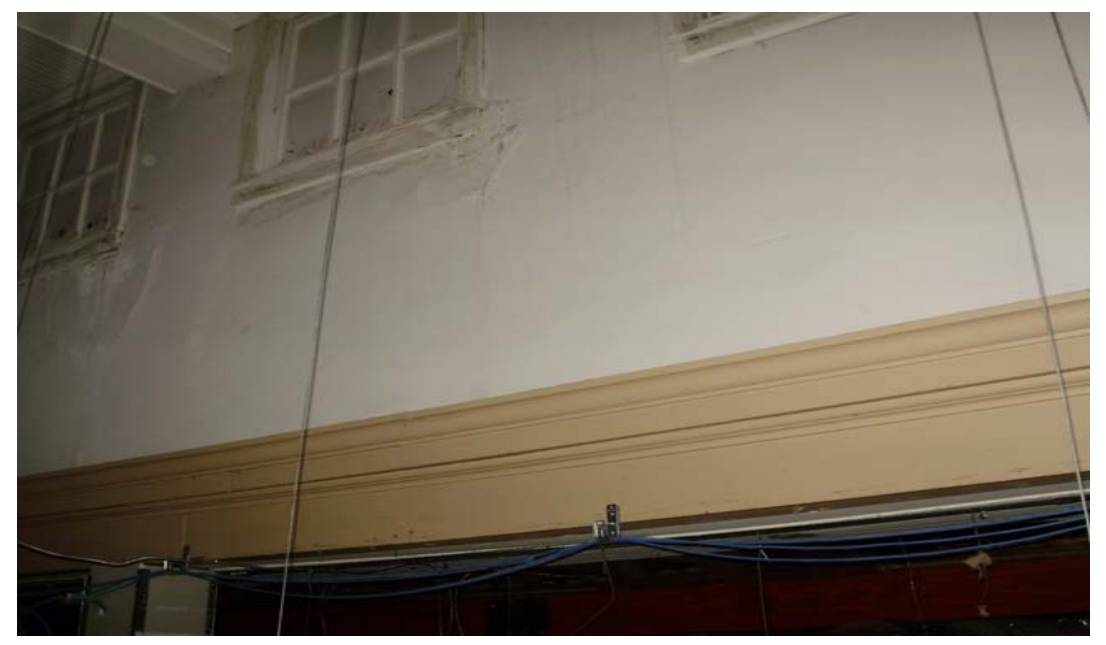




\section{APPENDIX O: ElEMENTARY SCHOOL IAQ PROJECT PROGRESS UPDATE}

(CONTINUED)

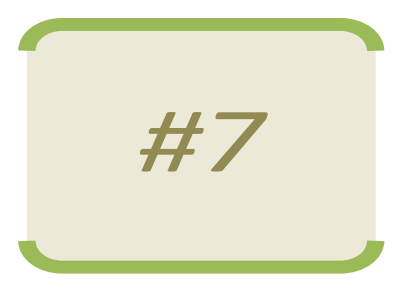

FINDINGS FROM EXPLORATORY AND BASE COVE WORK

SHEETROCK REPLACEMENT AND REPAIR

REPAIRED WALL

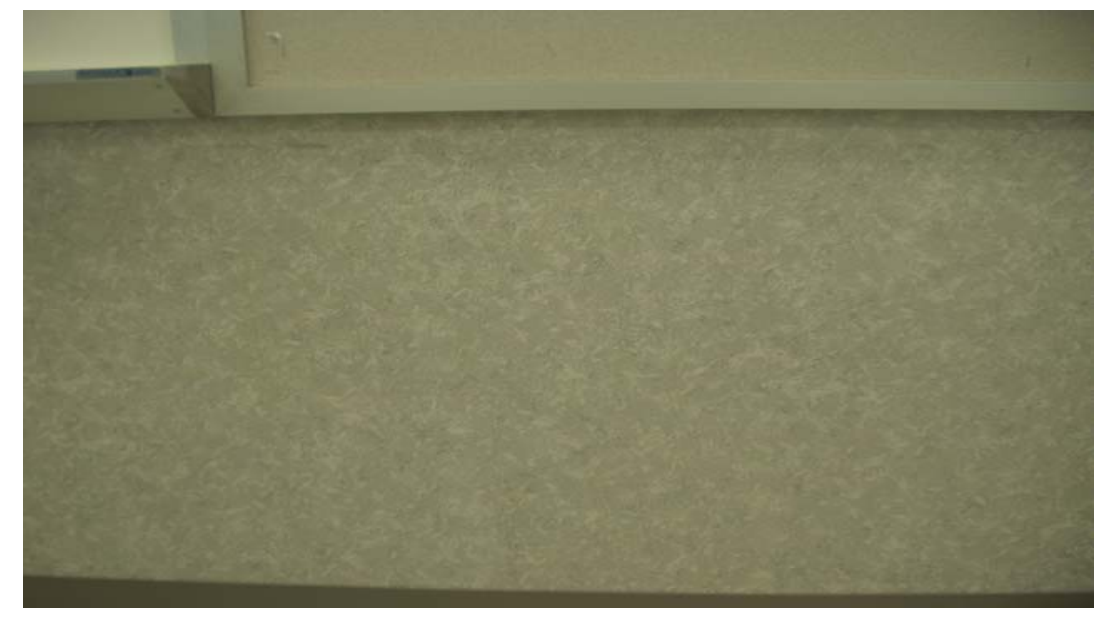

Health Hazard Evaluation Report 2009-0172-3124
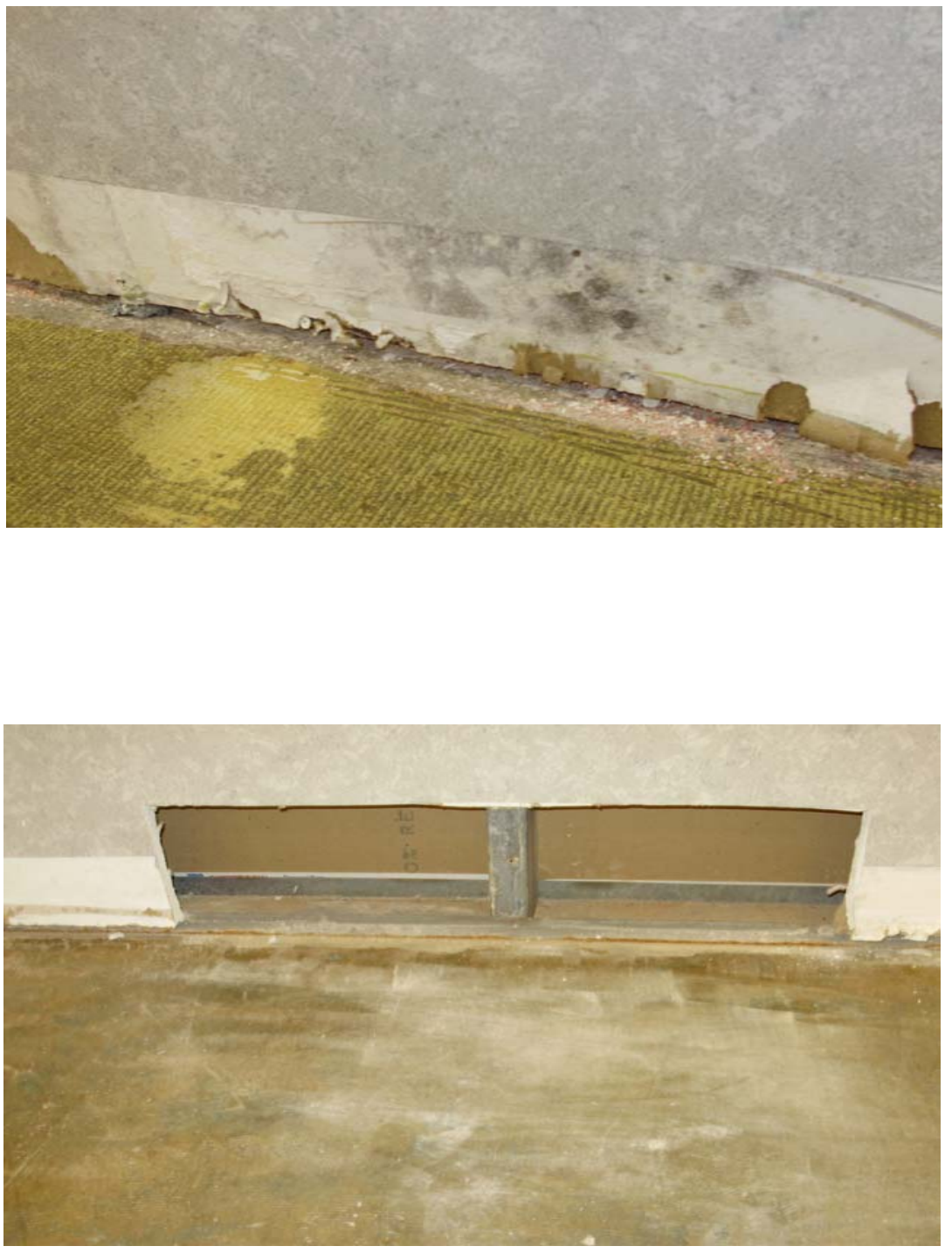


\section{$\overline{\text { ApPendix O: EleMENTARY SCHOOL IAQ PROJeCt PROGRESS UPDATE }}$}

(CONTINUED)

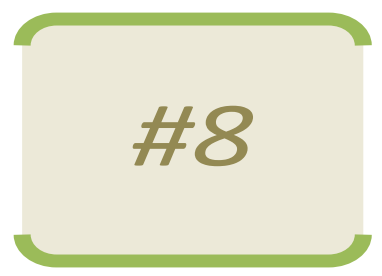

Molded Floor System

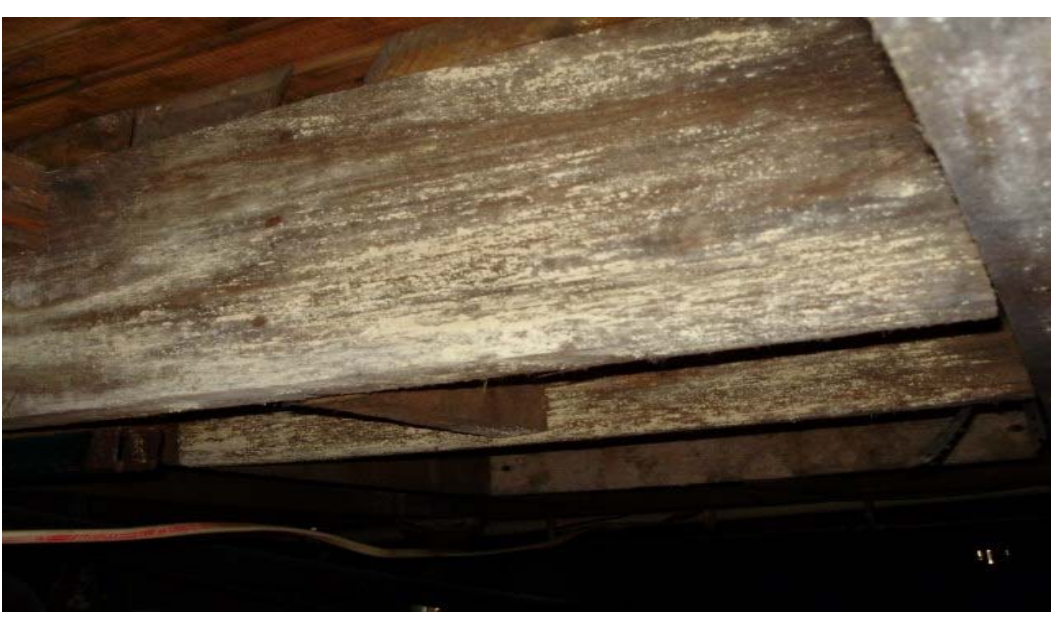

Cleaned Floor System AND INSTALLED VAPOR BARRIER

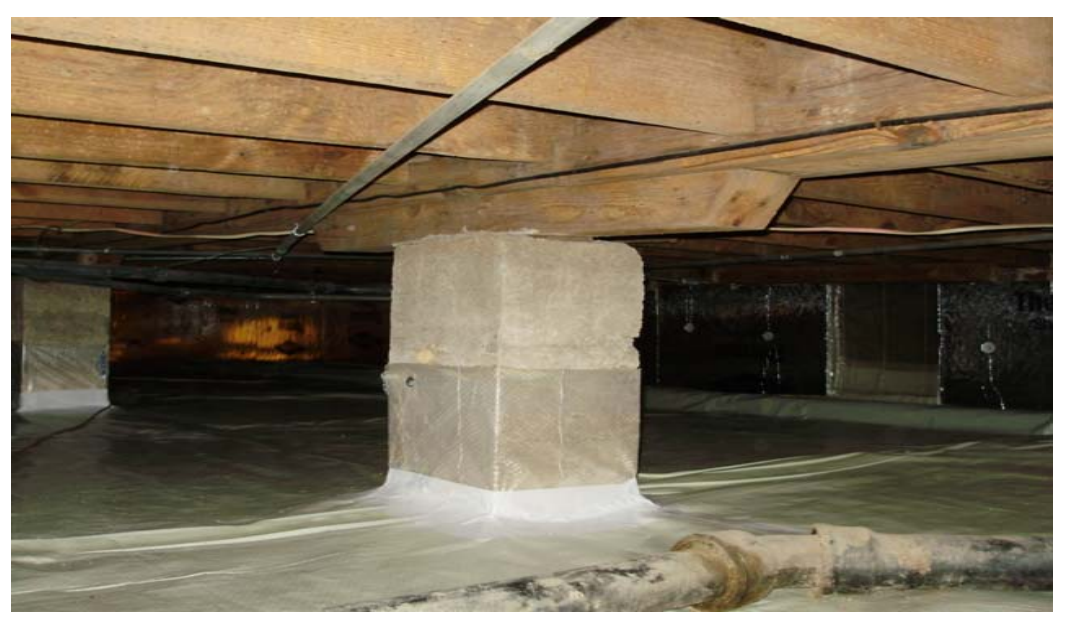

DEHUMIDIFIER INSTALLED

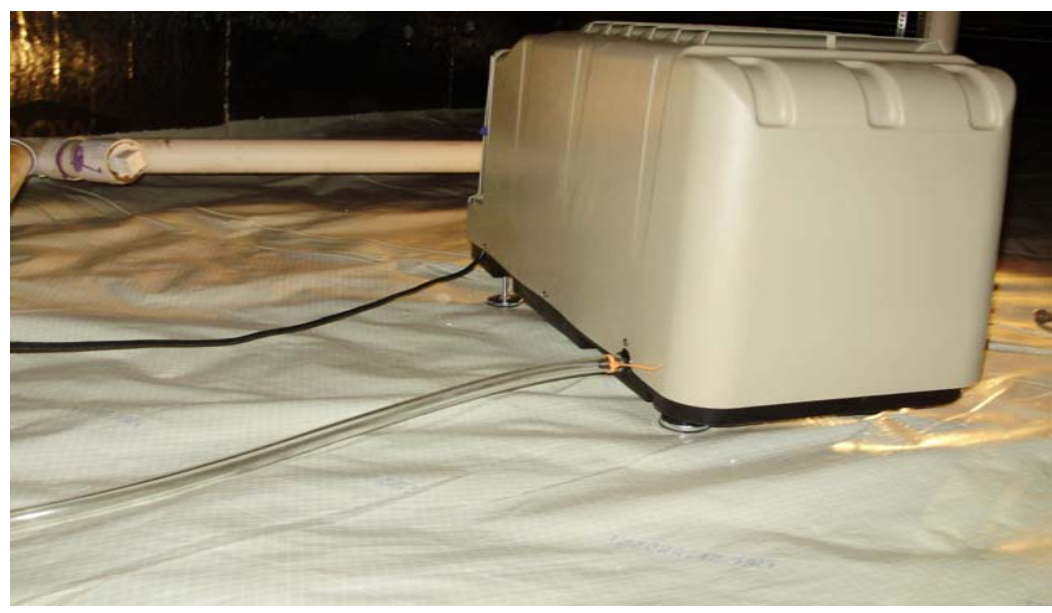




\section{APPENDIX O: ElEMENTARY SCHOOL IAQ PROJECT PROGRESS UPDATE}

(CONTINUED)

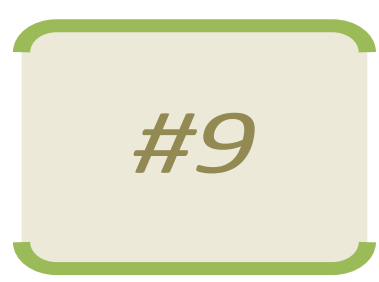

Problematic Roof Flashing

ROOF Flashing REPAIRS IN

PROGRESS

Completed RoOf Flashing

REPAIR
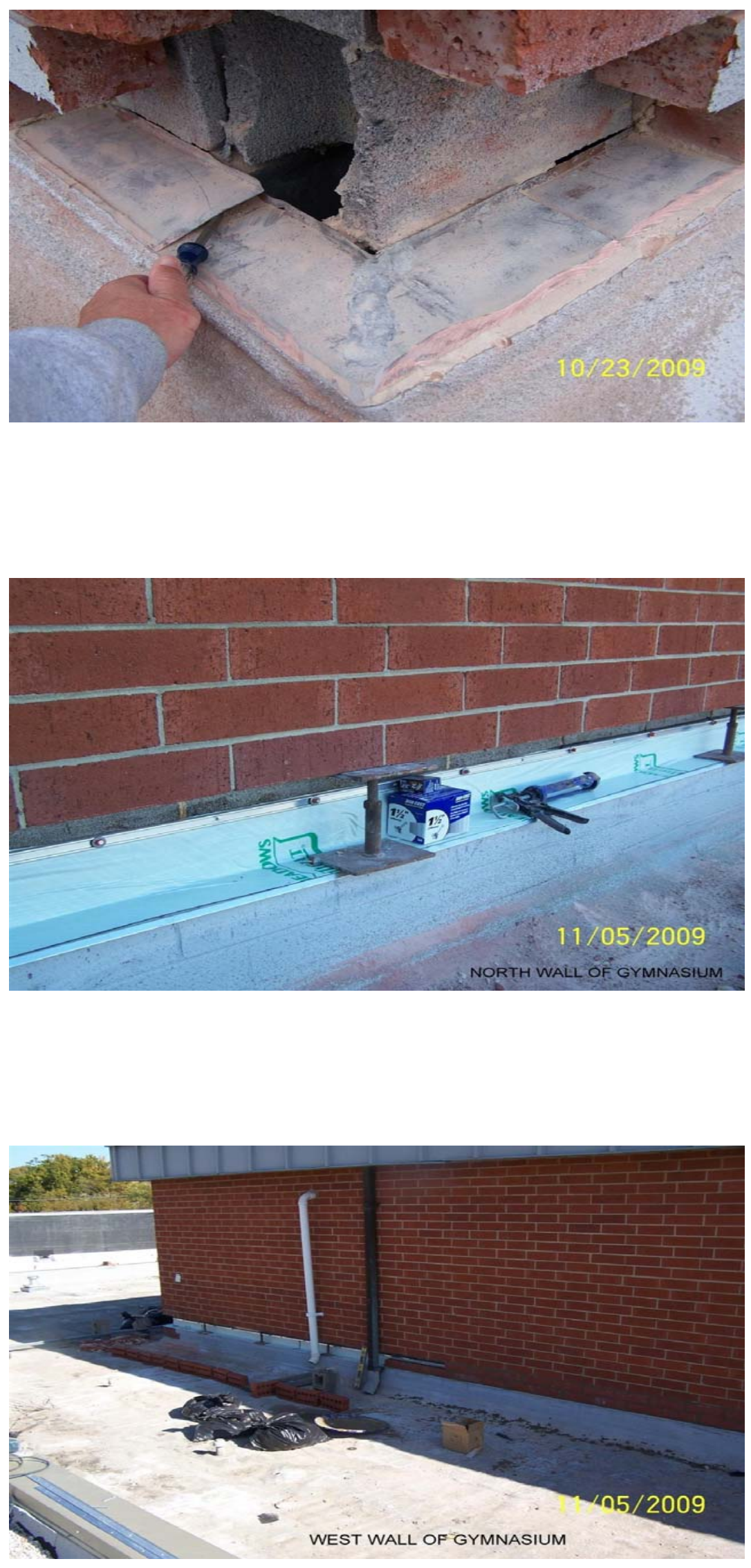

Health Hazard Evaluation Report 2009-0172-3124 


\section{$\overline{\text { APPENDIX O: ElEMENTARY SCHOOL IAQ PROJECT PROGRESS UPDATE }}$}

(CONTINUED)
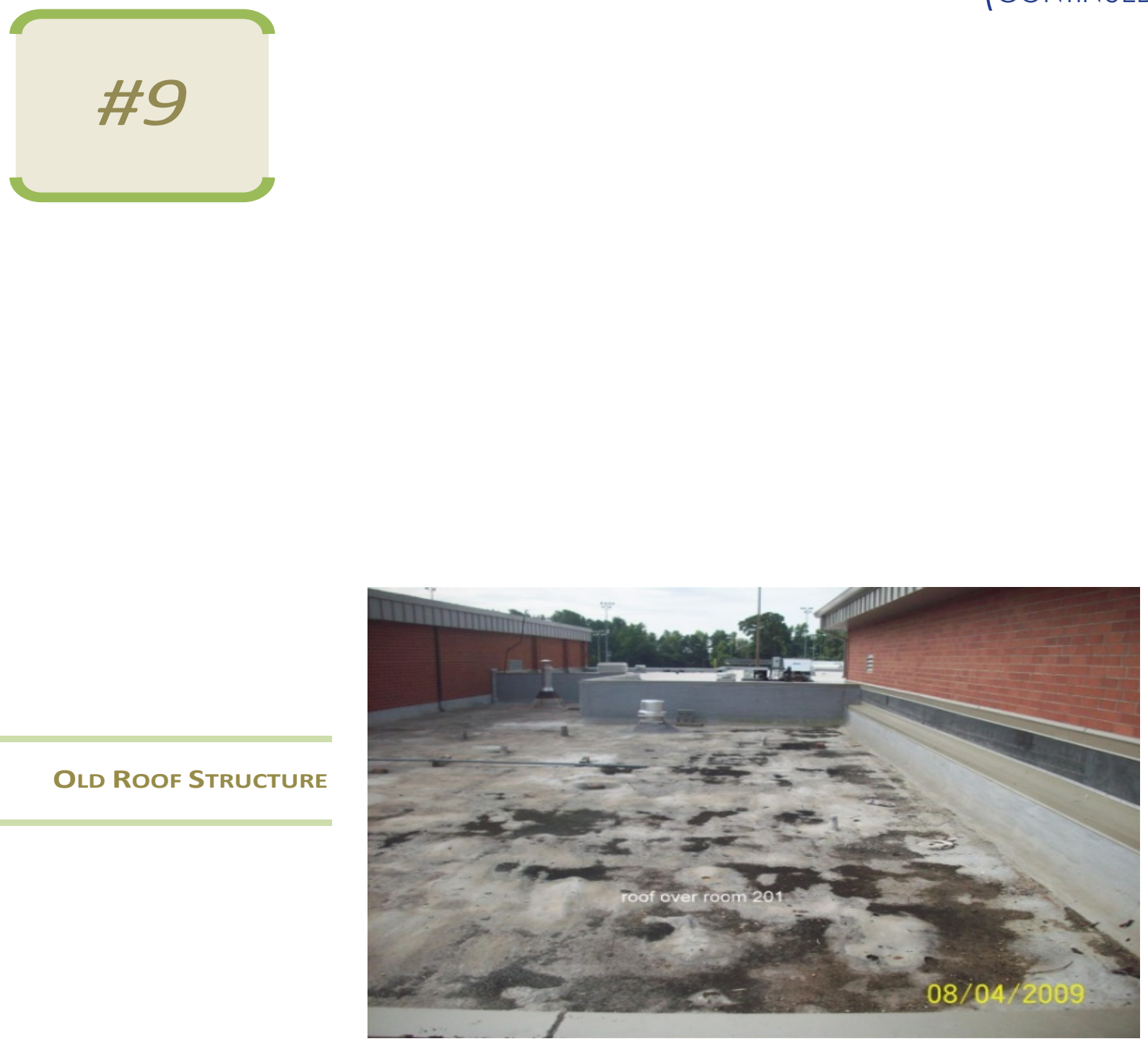

NeW Roof Structure

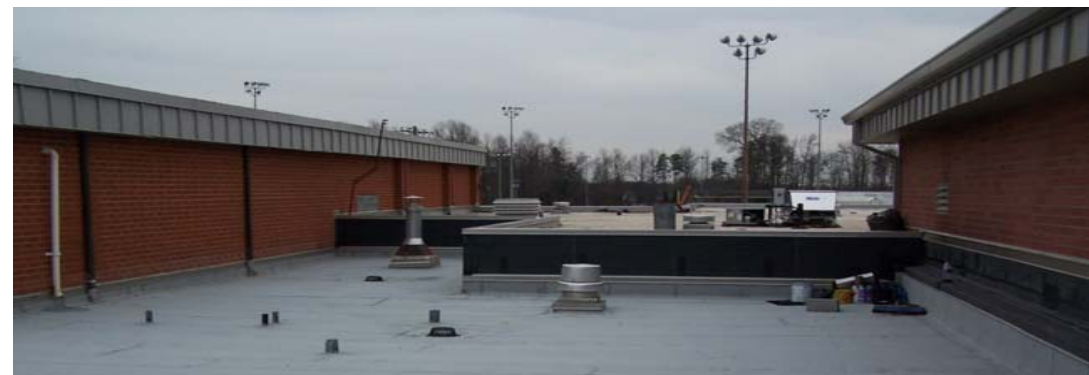

$12 / 15 / 2009$ 


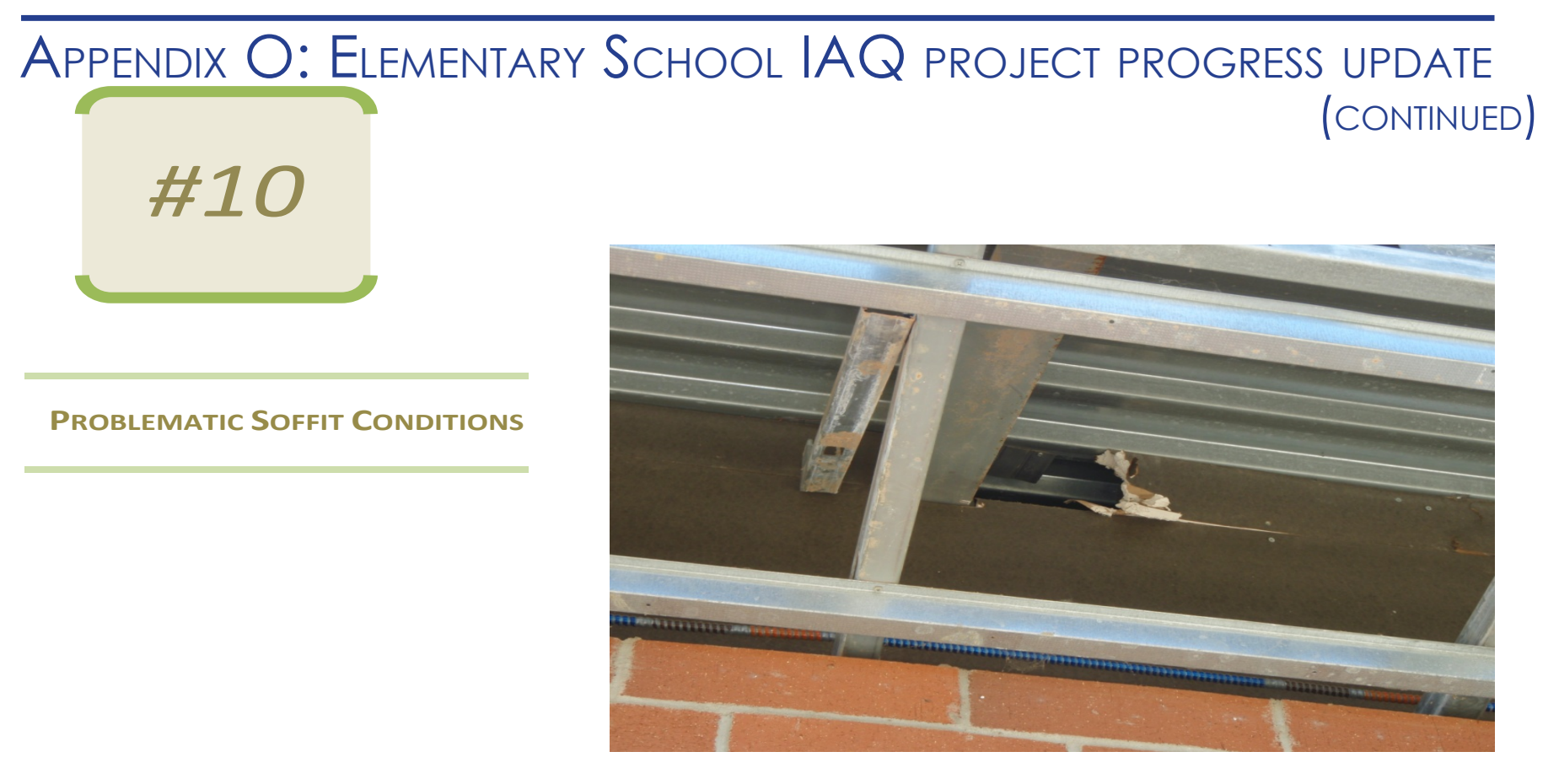

SOFFIt REPAIR AND FIRE CAULKED SEAMS

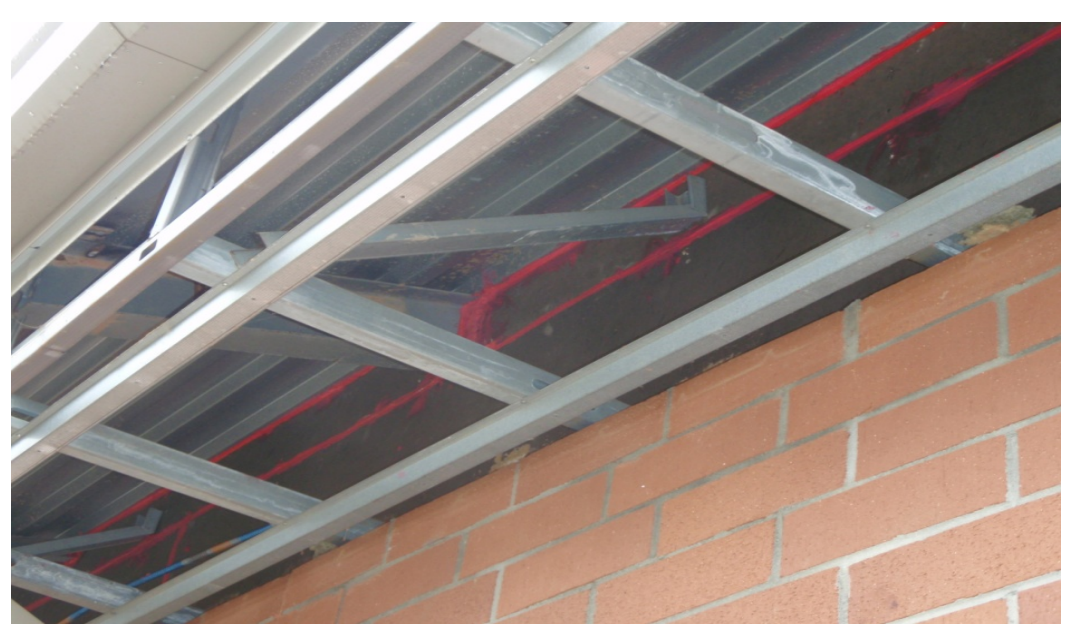

COMPLETED SOFFIT REPAIR WITH

FOAM INSULATION

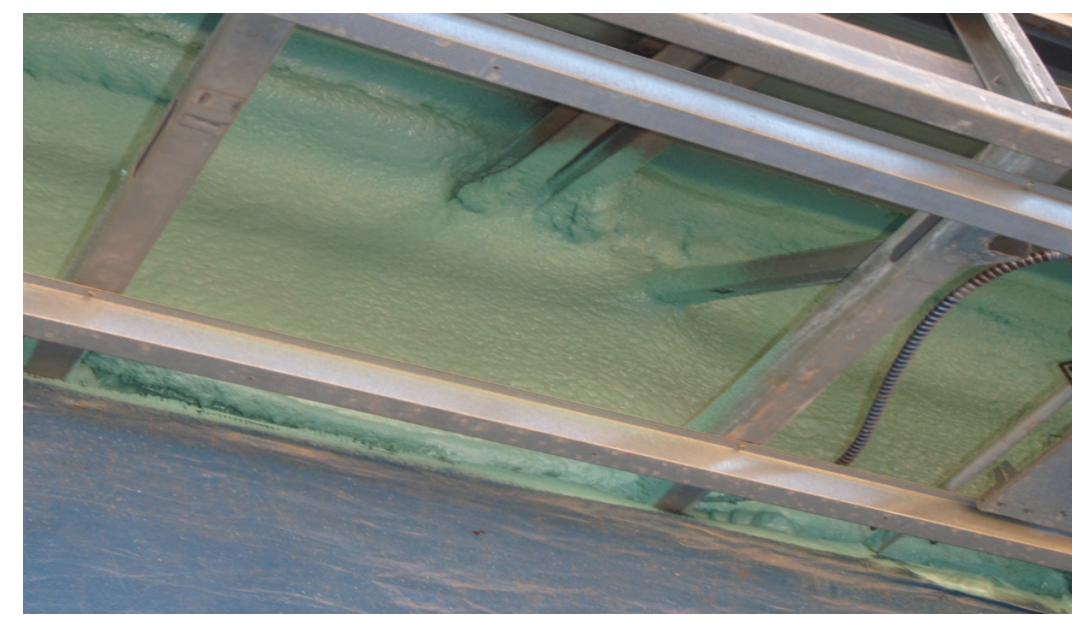




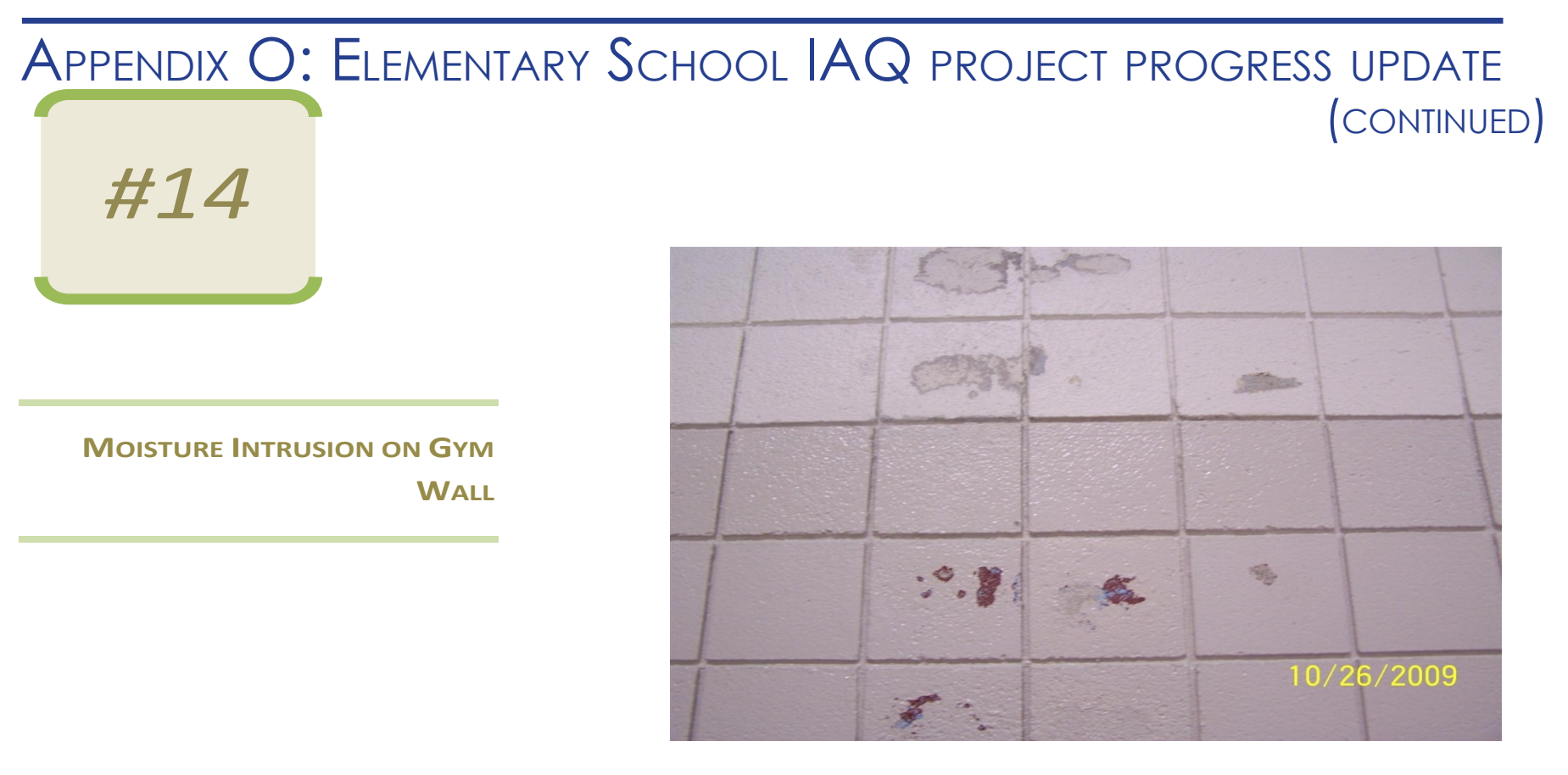

Damaged Drain Causing Moisture INTRUSION

DRAIN REPAIRED GyM WALL REPAINTED
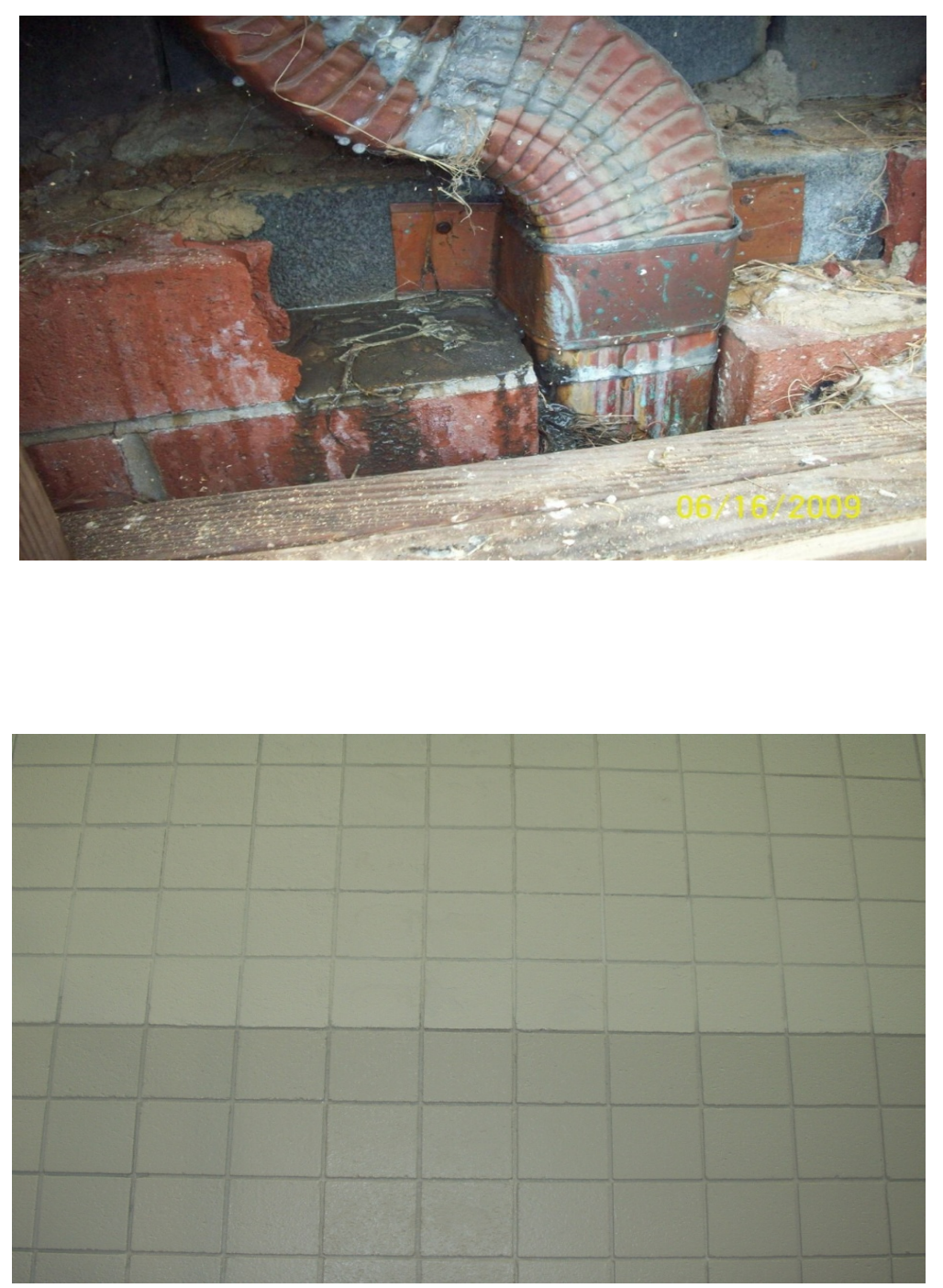


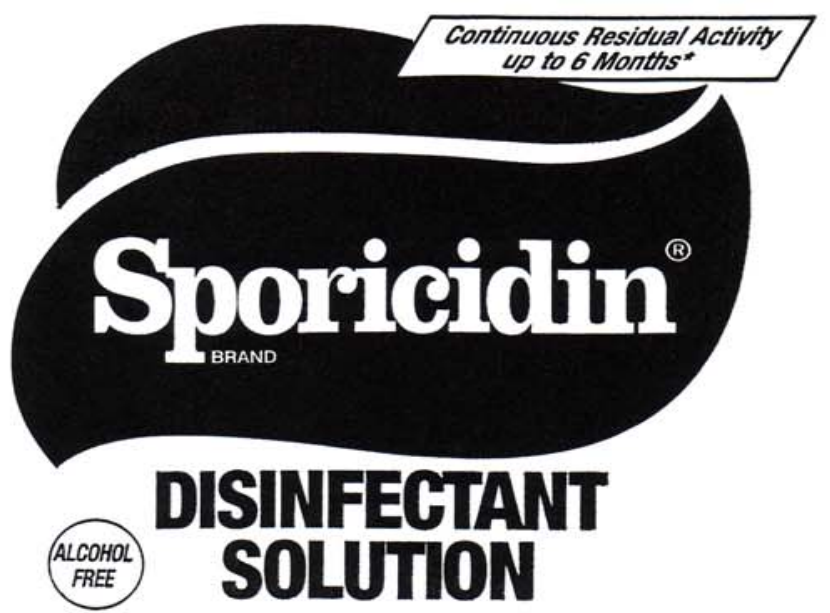

CLEANS • DISINFECTS • DEODORIZES

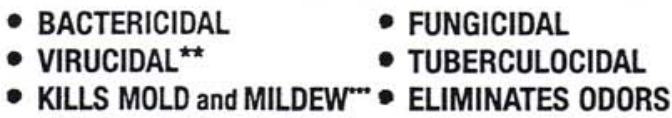
Active Ingredients Phenol ....................... $1.56 \%$ EPA Reg. No. 8383-3 Inert Ingredients ...........98.38\% EPA Est. No. 047179-IL-001 Total.................................100\% Net Contents: 1 U.S. Gal. (3.79 Itr.)

Keep Out of Reach of Children CAUTION

See additional precautions on back panel

\section{ACCEPTED}

FES 202003

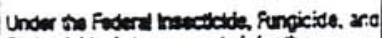
fodentiction Act as ananded, for the

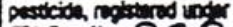
Ex $\operatorname{Rag}$ No. $_{2} 383$ -

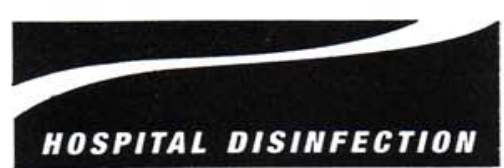

HOSPITAL DISINFECTION
PRESOAK DISINFECT

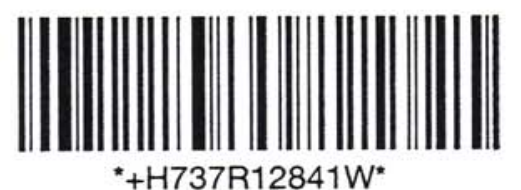

* +H737R12841W*
An effective cleaner, disinfectant and active deodorant for hospitals, clinics, medical and veterinary offices, laboratories, industrial clean rooms, homes, nursing homes, ambulances, hotels, restaurants, schools, airplanes, trains, boats, autos, buses, health spas and toilets. DISINFECTANT AND DEODORIZER FOR HARD, NON-POROUS SURFACES in medical treatment and patient rooms, operating rooms, emergency rooms, recovery rooms and batthrooms. Use on medical and dental equipment such as beds, surgical carts, counter tops, manikins, telephones, furniture, wheelchairs, walkers, sinks, floors, walls, light switches, linen hampers, shower stalls, toilet seats, trash containers and damp and musty areas, including animal enclosures. Reduces the hazards of cross-contamination.

ACTIVE DEODORIZER": Provides continuous residual bacteriostatic activity against odor-causing organisms for up to 6 months, if not removed from surfaces including plastics, latex, vinyl, glass, wood, metal, porcelain, tile and paint.

- Kills Trichophyton mentagrophytes (Nirus that causes Athlete's Foot) on bathroom, shower and pool surfaces - Penetrates cracks - Non-staining $\bullet$ Contains no harmful abrasives - Self-sanitizes surfaces against odor-causing bacteria. SPORICIDIN Brand DISINFECTANT SOLUTION is germicidal when used as directed for disinfection but differs in composition from other Sporicidin products. PASSES AOAC EFFICACY STANDARDS FOR INSTITUTIONAL AND HOSPITAL DISINFECTION

- Kills HIV-1 (AIDS virus) in one minute. (See Sporicidin Bulletin No. 303)

- Kills Staphylococcus aureus, Salmonella choleraesuis. Pseudomonas aeruginosa, Methicillin resistant Staphylococcus aureus (MRSA), Vancomycin resistan Enterococcus faecium (NRE) and Trichophyton mentagrophytes (Athlete's Foo fungus) in three minutes.

- Kills vegetative organisms including Streptococcus pyogenes, Streptococcus salivarius, Escherichia coli, "*Herpes simplex types $1 / F$ and $2 / G$ (oral, ocular, and genital), influenza A; (Japan 305/57 Asian strain). Pellicularia filamentosa, Penicillium variable. Vaccinia, Canine parvovirus, Cytomegalovirus, Coronavirus and Polio type 1 viruses and Mycobacterium tubercuCoronavirus and Polio type 1 viruses and $M y c d$
losis in ten minutes at $20^{\circ} \mathrm{C} / 68^{\circ} \mathrm{F}$ or above.

DIRECTIONS FOR USE: it is a violation of U.S. Federal Law to use this product in a manner inconsistent with its labeling.
Sporicidinin

Rockville, MD 20852
PRECLEAN surfaces using Sporicidin Brand Disinfectant Solution to remove soil and filth. Wipe dry with paper towel, cloth or sponge.

TO DISINFECT AND DEODORIZE: Thoroughly wet pre-cleaned surface with disinfectant. Use contact times as stated above at room temperature $\left(20^{\circ} \mathrm{C} / 68^{\circ} \mathrm{F}\right.$ or above) to kill listed organisms. NOTE: See Sporicidin Bulletin No. 303 for HIV-1 (AIDS virus) instructions.

**TO KILL, CLEAN AND PREVENT MOLD AND MILDEW: Thoroughly wet precleaned contaminated area with sprayer or saturated Sporicidin towelette. Repeat application if new growth appears. Effective against Stachybotrys chartarum, Chaetomium globosum and Aspergillus niger.

TO CLEAN AIR DUCTS: Apply to hard, nonporous, surfaces.

TO CLEAN, DEODORIZE AND DECONTAMINATE CARPETS: For manual cleaning, spray product onto carpet and allow to dry. For machine cleaning, apply product in accordance with the machine manufacturer's guidelines

FOR FOGGING CLEAN ROOMS: See Sporicidin Bulletin No. 301.

PRECAUTIONARY STATEMENTS: Hazard to Humans and Domestic Animals.

CAUTION: Causes moderate eye irritation. Avoid contact with eyes or clothing. Wash thoroughly with soap and water after handling.

FIRST AID: If in eyes, hold eye open and rinse slowly and gently with water for 15 20 minutes. Remove contact lenses, if present, after the first 5 minutes, then continue rinsing eye. Call a poison control center or doctor for treatment advice. Have product container or label with you when calling a poison control center or doctor, or going for treatment.

STORAGE AND DISPOSAL: Store at room temperature. Discard empty container in regular trash.

NOTE: This product is not to be used as a terminal sterilant/high level disinfectant on any surface or instrument that (1) is introduced directly into the human body.
either into or in contact with the bloodstream or normally sterilc areas of the body, or (2) contacts intact mucous membranes but which does not ord:narily penetrate the blood barrier or otherwise enter normally sterile areas of the body. This product may be used to preclean or decontaminate critical or semi-critical medical devices prior to sterilization or high level disinfection.

2316 


\section{Appendix Q: NIOSH Draft Mold \& Dampness Assessment Sheet}
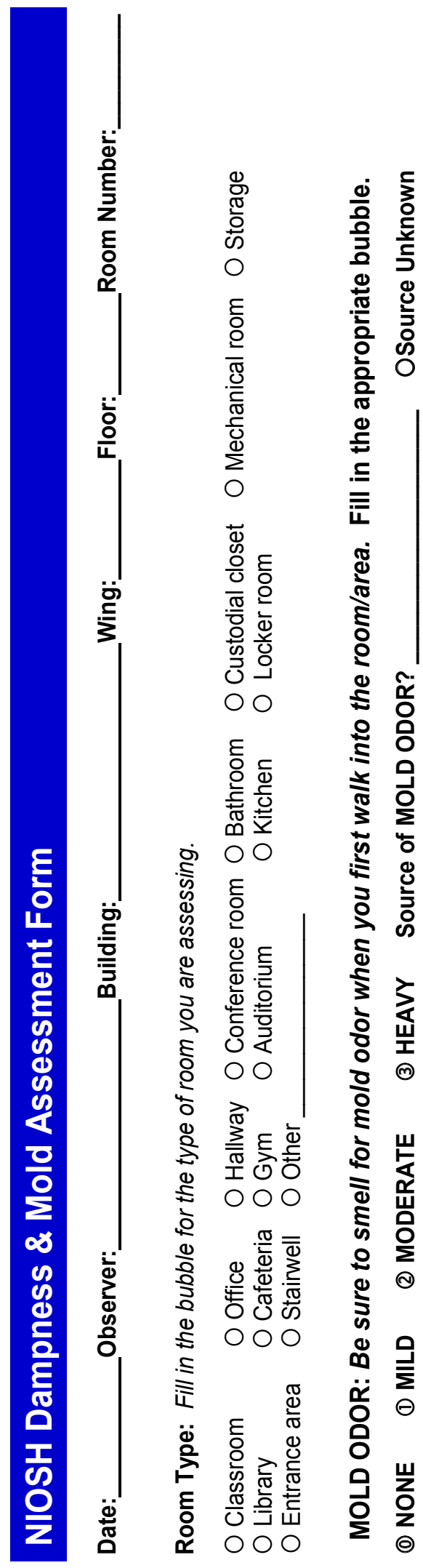

总

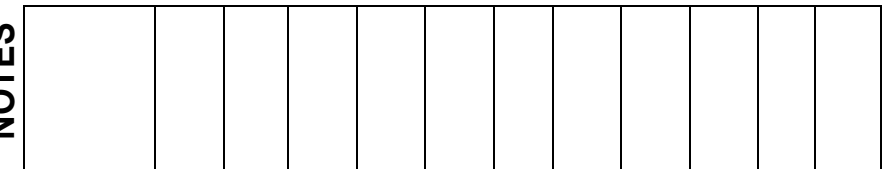

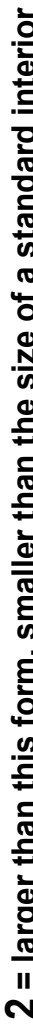

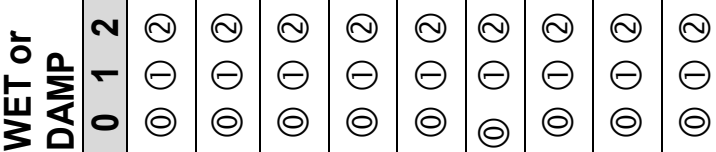

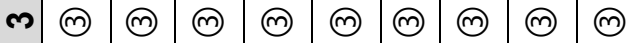

芫

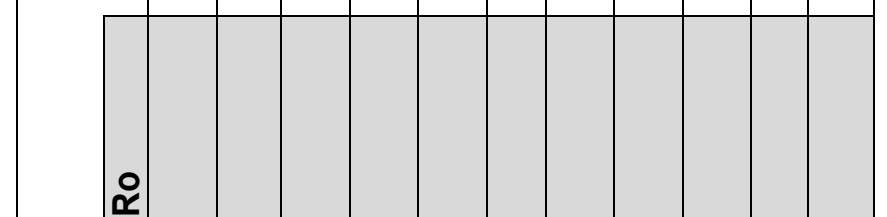

$\stackrel{ }{=} 0$

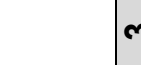

m

के

岁过。

(m)

ปั

ఏ

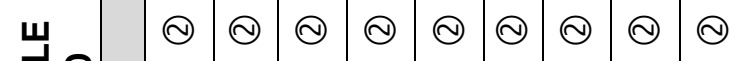

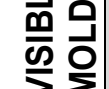

$\ominus \ominus$

$\ominus \Theta$

$\ominus \ominus \ominus \ominus \ominus$

$\omega$

$\infty \odot$

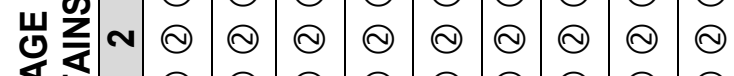

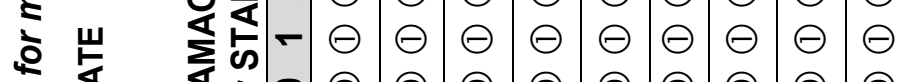

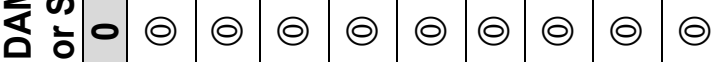

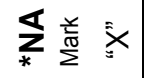

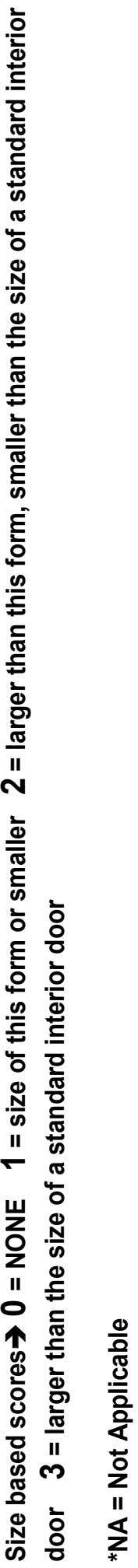




\section{Appendix Q: NIOSH Draft Mold \& Dampness Assessment SheEt}

(CONTINUED)

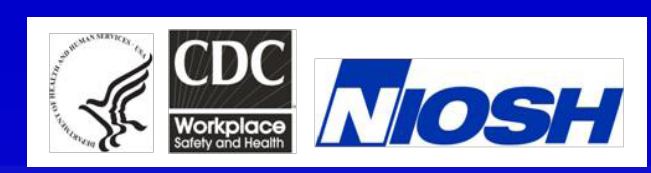

PILOT Study

National Institute for Occupational Safety and Health (NIOSH)

Centers for Disease Control and Prevention (CDC)

Department of Health and Human Services

NIOSH Contact: Michelle Martin 304-285-5734 or moldsheet\#1@cdc.gov

\section{Dampness and Mold Assessment Tool Instructions Packet}

$\mathrm{NIOSH}$ has developed an observational assessment tool for dampness and mold in buildings.

- GOAL: To provide valuable information for motivating remediation, prioritizing intervention, and evaluating remediation effectiveness.

Through past research, $\mathrm{NIOSH}$ has shown in studies of a community college and a health care facility involving 15 buildings that the dampness and mold scores, obtained by using a similar assessment tool, were associated with respiratory health outcomes in the building occupants (Park et al. 2004, Indoor Air; 14:425-433; Cox-Ganser et al. 2009, Indoor Air 19(4):280-290).

- The tool consists of:

1. A form which is used to assess signs of dampness, water damage, mold growth, and musty odors in rooms and areas throughout a building.

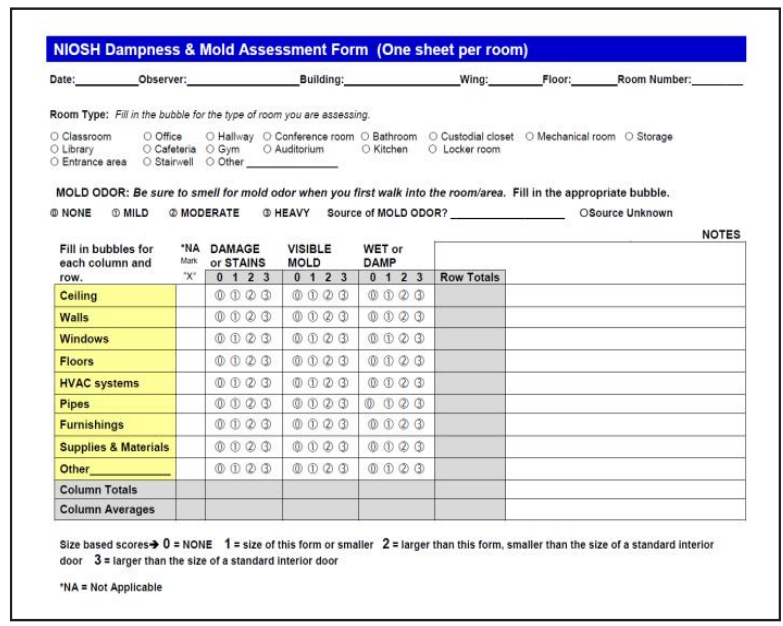

2. A Microsoft Access database application to enter data collected from hard copy assessment forms for electronic record keeping and reports.

Through a National Occupational Research Agenda project and a Public Health Project, NIOSH has partnered with the Maine Indoor Air Quality Council (MIAQC), the University of Connecticut Health Center, and school districts in both Maine and Connecticut to pilot the use of the observational assessment tool. $\mathrm{NIOSH}$ has developed a training presentation and a hard copy package, and we have successfully trained school district facility managers in the use of the tool. In working together with school business managers, facility managers, and custodians, the form has been used in small and large school districts of different building types. With their feedback, we have made changes to make the tool more useful.

The Dampness and Mold Assessment Tool is free to the public. We encourage using the tool to assist with proactive environmental efforts in buildings and request feedback on the tool's utility. Please send all feedback to: mold\#1@cdc.gov. 


\title{
Purpose of the Dampness and Mold Assessment Tool
}

\author{
NIOSH PILOT Study
}

NIOSH Contact: Michelle Martin 3042855734 or moldsheet\#1@cdc.gov

\section{The purpose of this tool is to:}

- Identify and record areas of dampness or mold throughout your building.

- Trigger early repair and remediation to avoid potential health effects and more costly repair and remediation.

- Create awareness of potential problem areas.

- Track (monitor) past and present problem areas by repeating the use of this tool at the frequency which your individual facility determines.

\section{Assessment, identification, repair and remediation cycle:}

STEP 1. Use the Dampness and Mold Assessment Form in all rooms and areas of your building.

Facilities staff or trained professionals should use separate forms to assess each room and area of your building.

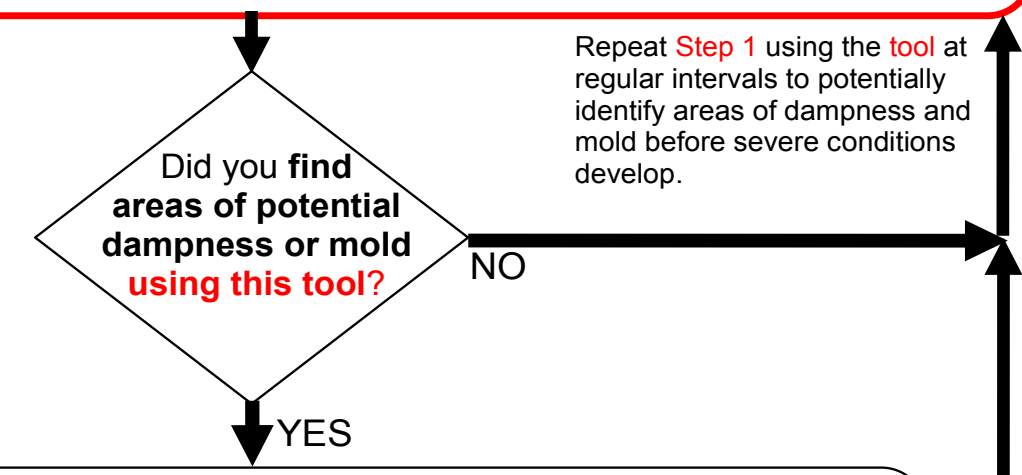

STEP 2. Find the sources of moisture identified in Step 1.

Facilities staff or trained professionals should conduct further inspection of the areas of dampness and mold to identify sources of water incursion, and repair and remediation needs.

\section{STEP 3. Perform repairs and remediation.}

Facilities staff or trained professionals should repair sources of moisture then remediate damaged areas following guidelines such as Mold Remediation in Schools and Commercial Buildings (Environmental Protection Agency) http://www.epa.gov/iedmold1/pdfs/moldremediation.pdf or New York City Guidelines on Assessment and Remediation of Fungi in Indoor Environments (New York City Department of Health and Mental Hygiene) http://www.nyc.gov/html/doh/downloads/pdf/epi/epi-mold-guidelines.pdf

Should assessments end after remediation? No! Go back to Step 1. 


\title{
General Instructions (assessing rooms with the form)
}

\author{
NIOSH PILOT Study
}

NIOSH Contact: Michelle Martin 3042855734 or moldsheet\#1@cdc.gov

Individuals who are experiencing respiratory health symptoms, and have concerns that exposures to dampness and mold are related to their symptoms, are cautioned about using the form in potential problem areas.

1) When completing the assessment form, first fill-in the date, observer, building, wing, floor, room number, and room type that you are inspecting in the top area of the sheet. Use one sheet per room.

2) Fill in any observation of MOLD ODOR. Be sure to smell for mold odor when you first walk into each room. When listing a source, refer to the first (yellow shaded) column on the left of the page. Determine whether a smell is mild, moderate, or heavy (strong).

3) Typical room components within a room are listed in the first (yellow shaded) column and should be systematically assessed in the order given. If a room has other components you will assess than those listed, write the name of the component on the line provided next to the "Other" row.

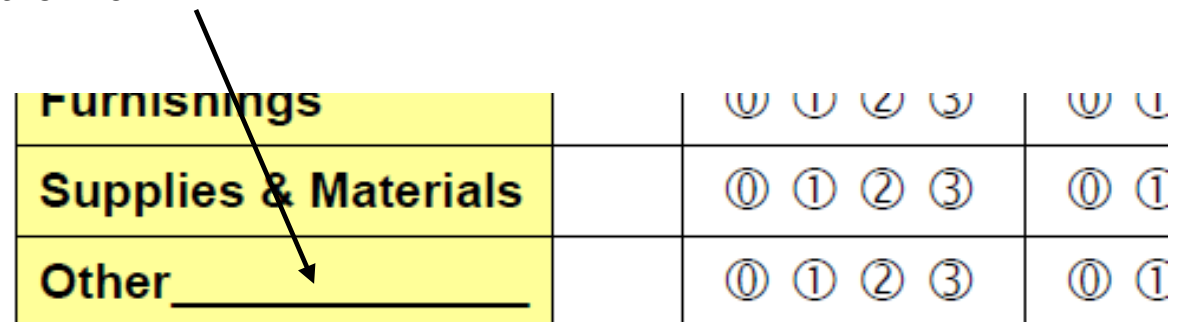

4) An "X" should be placed in the next column next to any room component that is not applicable (NA) or does not exist in the room you are assessing.

5) In the next three columns, for each room component, score the combined size of the following types of observed areas: damage or stains, visible mold, and wetness or dampness. Base scores solely on size (see next page).

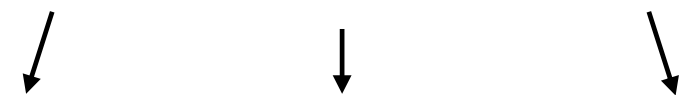

\begin{tabular}{|c|c|c|c|}
\hline $\begin{array}{l}{ }^{*} \mathrm{NA} \\
\text { Mark }\end{array}$ & $\begin{array}{l}\text { DAMAGE } \\
\text { or STAINS }\end{array}$ & $\begin{array}{l}\text { VISIBLE } \\
\text { MOLD }\end{array}$ & $\begin{array}{l}\text { WET or } \\
\text { DAMP }\end{array}$ \\
\hline$" \mathrm{X} "$ & $\begin{array}{llll}0 & 1 & 2 & 3 \\
\end{array}$ & $\begin{array}{llll}0 & 1 & 2 & 3 \\
\end{array}$ & $\begin{array}{llll}0 & 1 & 2 & 3 \\
\end{array}$ \\
\hline & (0) (1) (2) (3) & (0) (1) (2) (3) & (1) (1) (2) (3) \\
\hline & (a) (1) (2) (3) & (1) (1) (2) (3) & (1) (1) (2) (3) \\
\hline
\end{tabular}




\section{Appendix Q: NIOSH Draft Mold \& Dampness Assessment Sheet}

\section{General Instructions (assessing rooms with the form)}

\section{NIOSH PILOT Study}

NIOSH Contact: Michelle Martin 3042855734 or moldsheet\#1@cdc.gov

6) Provide additional information in the "Notes" column.

7) Do not complete the gray areas for "column totals", "column averages", or "row totals". Save this for later.

\section{Determining Size-based Scores}

Scoring is based on four sizes:

$0=$ NONE

$1=$ the combined size of damage/stain, visible mold, or wet/damp areas that are approximately the size of the actual "Dampness and Mold Assessment Form" or smaller.

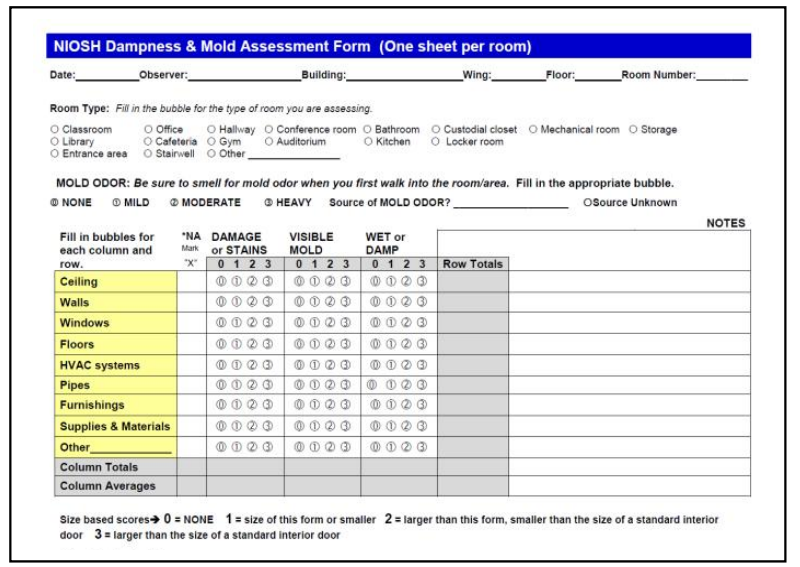

Form size $=8^{1 / 2}$ inches $X 11$ inches

$2=$ the combined size of damage/stain, visible mold, or wet/damp areas that are between the size of the "Dampness and Mold Assessment Form" and the size of a standard interior door.

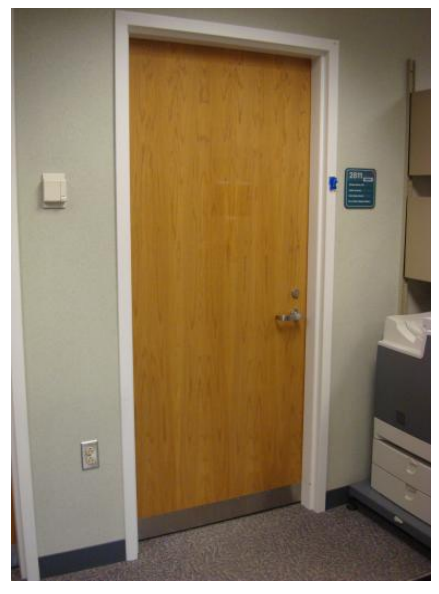

Door size $=32$ inches $X 80$ inches

$3=$ the combined size of the damage/stain, visible mold, or wet/damp areas that are larger than the size of an interior door. 


\section{Special Notes}

NIOSH PILOT Study

NIOSH Contact: Michelle Martin 3042855734 or moldsheet\#1@cdc.gov

Is it a stain or is it mold? Most of the time this is difficult to determine. If you are not certain what you see is mold:

1) mark as "None" in the "Visible Mold" column

2) score the size of the stain in the "Damage or Stains" column

3) write a note in the "Notes" column

If you observe severe areas of damage or visible mold:

1) score according to size for both "Damage or Stains" and "Visible Mold"

2) write a note in the "Notes" column on how severe you observe the area to be

Photographs can be useful for documenting conditions. You may consider taking a photograph of an area that appears to be severe and referring to the photograph in the "Notes" column.

Is it currently wet or damp? It may be beneficial to touch an area to determine whether it is moist. The purpose of this question is to identify how current a leak may be.

- We recommend that you wash your hands after touching damp areas. Areas of obvious moisture or mold DO NOT need to be touched.

If you observe areas of moisture on fixtures, such as condensation on pipes under toilets and sinks, include your observations by filling in the appropriate bubble for the size of the affected area in the "Wet or Damp" column and make a note in the "NOTES" section.

If you notice a stain on an item that appears unrelated to sources of water incursion within the room, include this in the "NOTES" section as "incidental".

- After being in a building for a lengthy period of time, it may become difficult to determine if there is a mold odor present in a room. To prevent this problem, we recommend that you take periodic breaks outside of the building. If you smell something other than mold odor, include this in the "NOTES" column. Always try to detect the smell of mold immediately as you enter each room.

- Remember the scoring is based on size, not on density or darkness of the stain or mold. 


\section{Appendix Q: NIOSH Draft MOld \& Dampness Assessment Sheet}

\section{Example 1}

NIOSH PILOT Study

NIOSH Contact: Michelle Martin 3042855734 or moldsheet\#1@cdc.gov

\section{Example 1: Assessing the CEILING}

Picture of stained ceiling tiles. These two stains were the only stains observed on the entire ceiling in this room. If there were other areas of damage/stain, visible mold, or damp/wet conditions, you would also combine the size of those areas for one score for the entire ceiling.

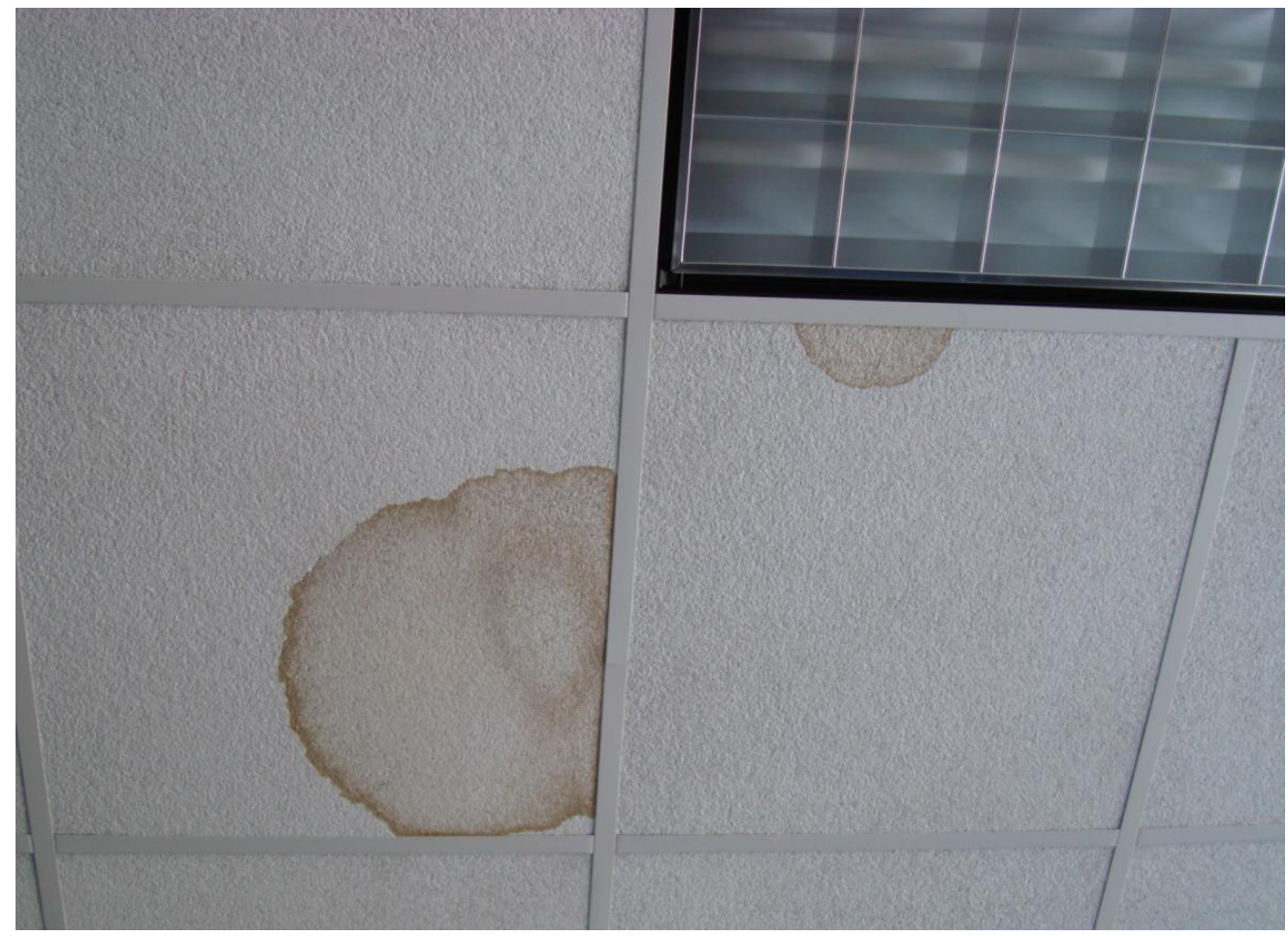

\section{1) DAMAGE or STAIN}

The damage to this ceiling area appears to be somewhat extensive. A score for this would be a 2 because both stains together are bigger than the size of the assessment form but smaller than a standard interior door.

\section{2) VISIBLE MOLD}

It is hard to determine if there is mold in this example, so you might score this as a 0 but note (in the "Notes" column) that there may be mold growth.

\section{3) WET or DAMP}

Both areas feel damp by touch and both together appear to be larger than the size of the assessment form but smaller than the size of a standard interior door. This example would have a score of 2 .

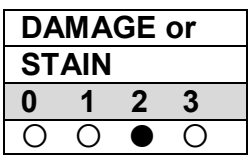

\begin{tabular}{|llll|}
\hline \multicolumn{3}{|l|}{ MOLD } \\
\hline AREA & \\
\hline $\mathbf{0}$ & $\mathbf{1}$ & $\mathbf{2}$ & $\mathbf{3}$ \\
\hline $\mathbf{0}$ & $\mathrm{O}$ & $\bigcirc$ & $\bigcirc$ \\
\hline
\end{tabular}

\begin{tabular}{|llll|}
\hline \multicolumn{3}{|l|}{ WET or } \\
\hline DAMP \\
\hline $\mathbf{0}$ & $\mathbf{1}$ & $\mathbf{2}$ & $\mathbf{3}$ \\
\hline $\mathrm{O}$ & $\mathrm{O}$ & $\mathbf{0}$ & $\mathrm{O}$ \\
\hline
\end{tabular}




\section{Example 2}

\section{NIOSH PILOT Study}

NIOSH Contact: Michelle Martin 3042855734 or moldsheet\#1@cdc.gov

\section{Example 2: Assessing the WALLS}

Picture of wall and floor. This area of wall damage and mold is the only problem area observed on all the walls in this room. If there were other areas of damage/stain, visible mold, or wet/damp conditions, you would also combine the size of those areas for one score for all the walls in the room.

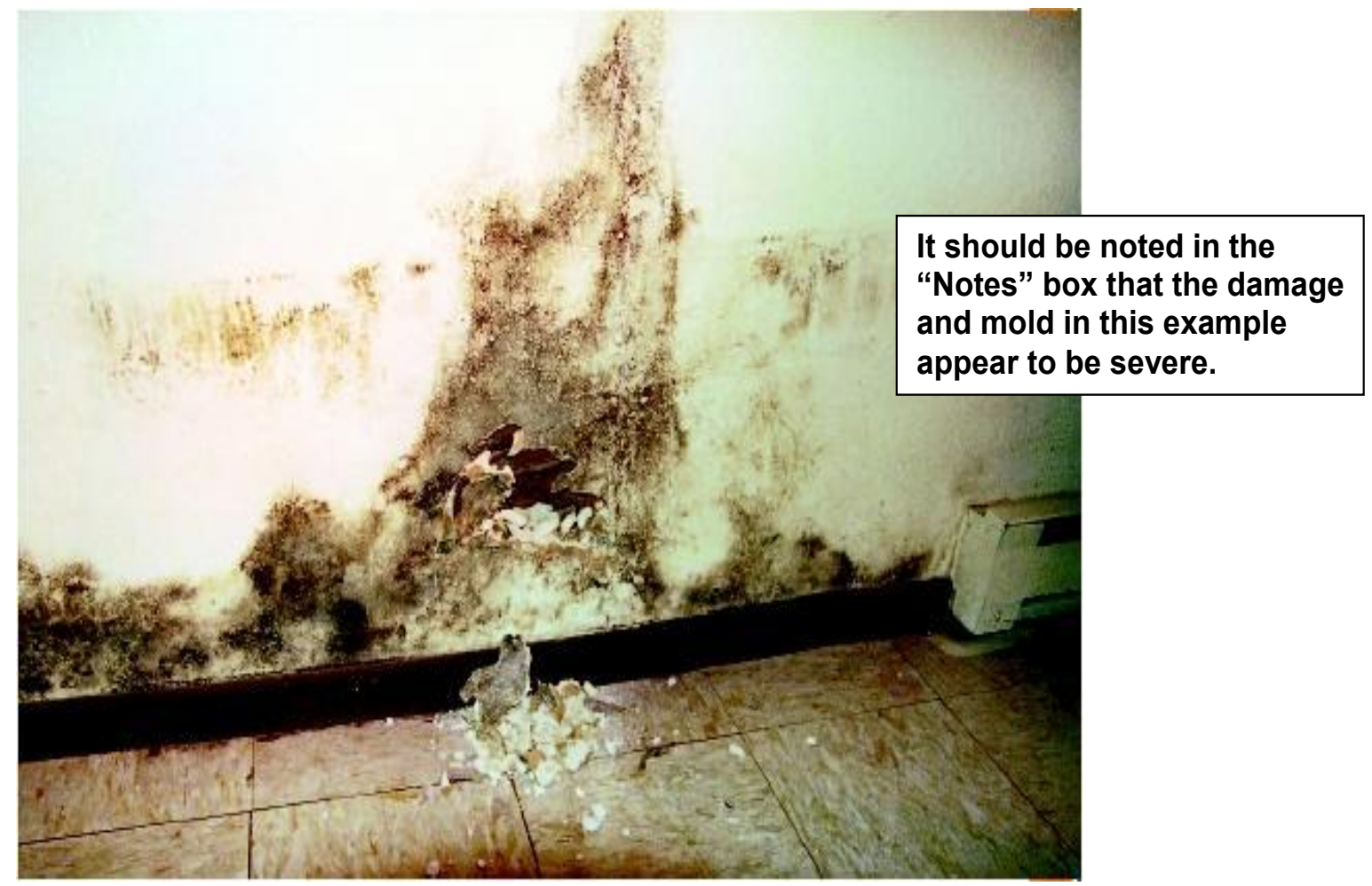

\section{1) DAMAGE or STAIN}

There appears to be a large area of visible mold and damage to the wall in this example. The score would be 2 because the area is bigger than the size of the assessment form and smaller than a standard interior door.

\section{2) VISIBLE MOLD}

The area of visible mold appears to be significant. The score would be 2 because the area is bigger than the size of the assessment form and smaller than a standard interior door.

\begin{tabular}{|llll|}
\hline \multicolumn{4}{|l|}{ DAMAGE or } \\
\hline STAIN \\
\hline $\mathbf{0}$ & $\mathbf{1}$ & $\mathbf{2}$ & $\mathbf{3}$ \\
\hline $\mathrm{O}$ & $\mathrm{O}$ & $\mathbf{0}$ & $\mathrm{O}$ \\
\hline
\end{tabular}

\begin{tabular}{|llll|}
\hline \multicolumn{3}{|l|}{ WET or } \\
\hline DAMP \\
\hline $\mathbf{0}$ & $\mathbf{1}$ & $\mathbf{2}$ & $\mathbf{3}$ \\
\hline & 0 & 0 & 0 \\
\hline
\end{tabular}




\section{How to Calculate Total or Average Scores per Room}

\section{NIOSH PILOT Study NIOSH Contact: Michelle Martin 3042855734 or moldsheet\#1@cdc.gov}

There are two ways you can choose to score your results using hardcopies. Method I is calculating total scores per room and Method II is calculating average scores per room. When the Microsoft Access software for this tool becomes available, it will automatically provide results and summary reports based on both methods when data is entered.

\section{Scoring Method I - Total Room Score}

After completing the assessment form:

a) add circled numbers in each column and enter in "Column Totals"

b) add circled numbers in each row and enter in "Row Totals"

c) Add the 3 column totals for the Total Room Score (example: $8+4+6=18$ )

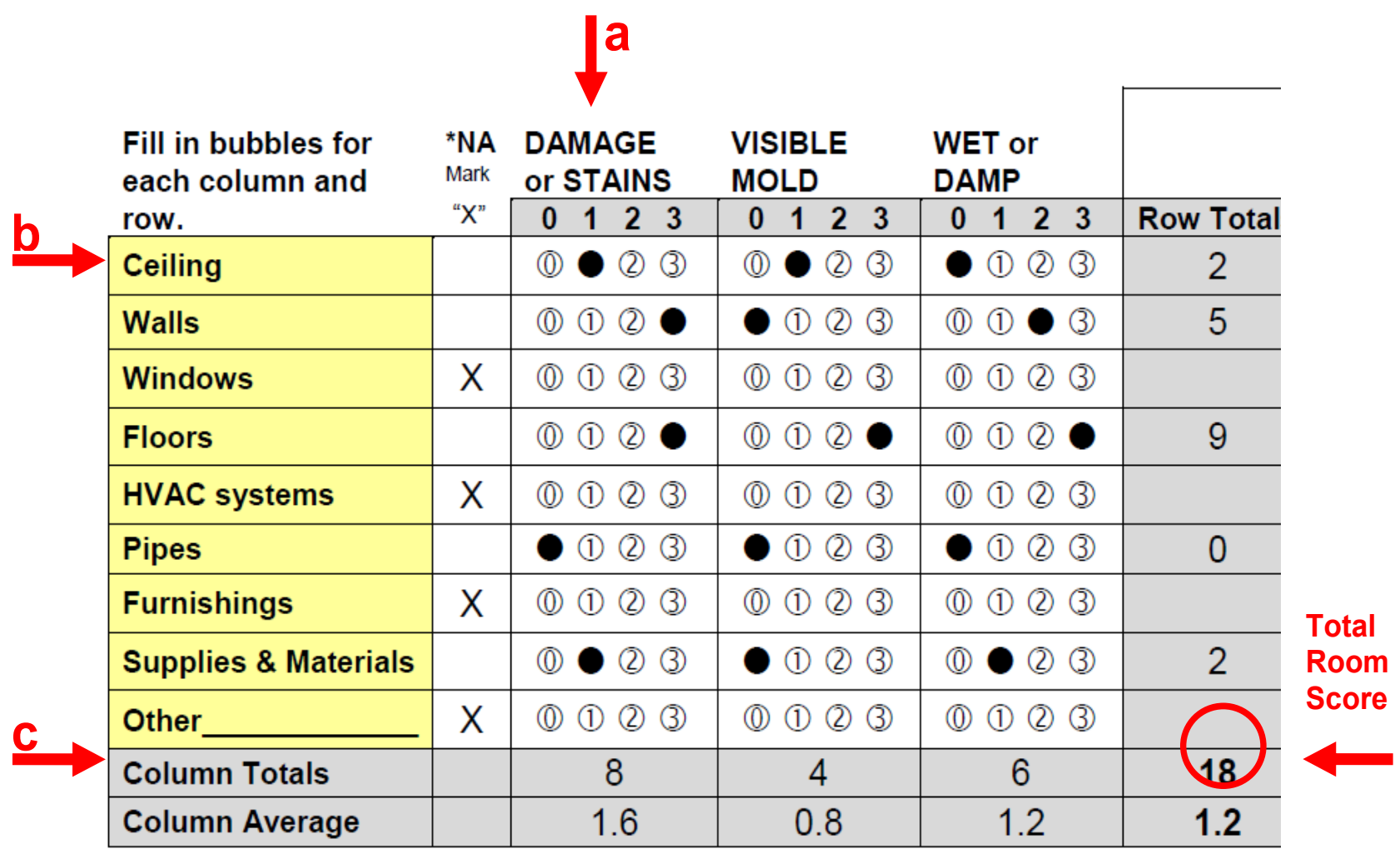




\section{How to Calculate Total or Average Scores per Room}

\section{NIOSH PILOT Study N NIOSH Contact: Michelle Martin 3042855734 or moldsheet\#1@cdc.gov}

\section{Scoring Method II - Average Room Score}

After completing the assessment form:

a) Complete Scoring Method I for total room score.

b) In each column, divide the column total by the number of scores in the bubbles above and enter that number in the column average (example: $8 \div 5=1.6$ )

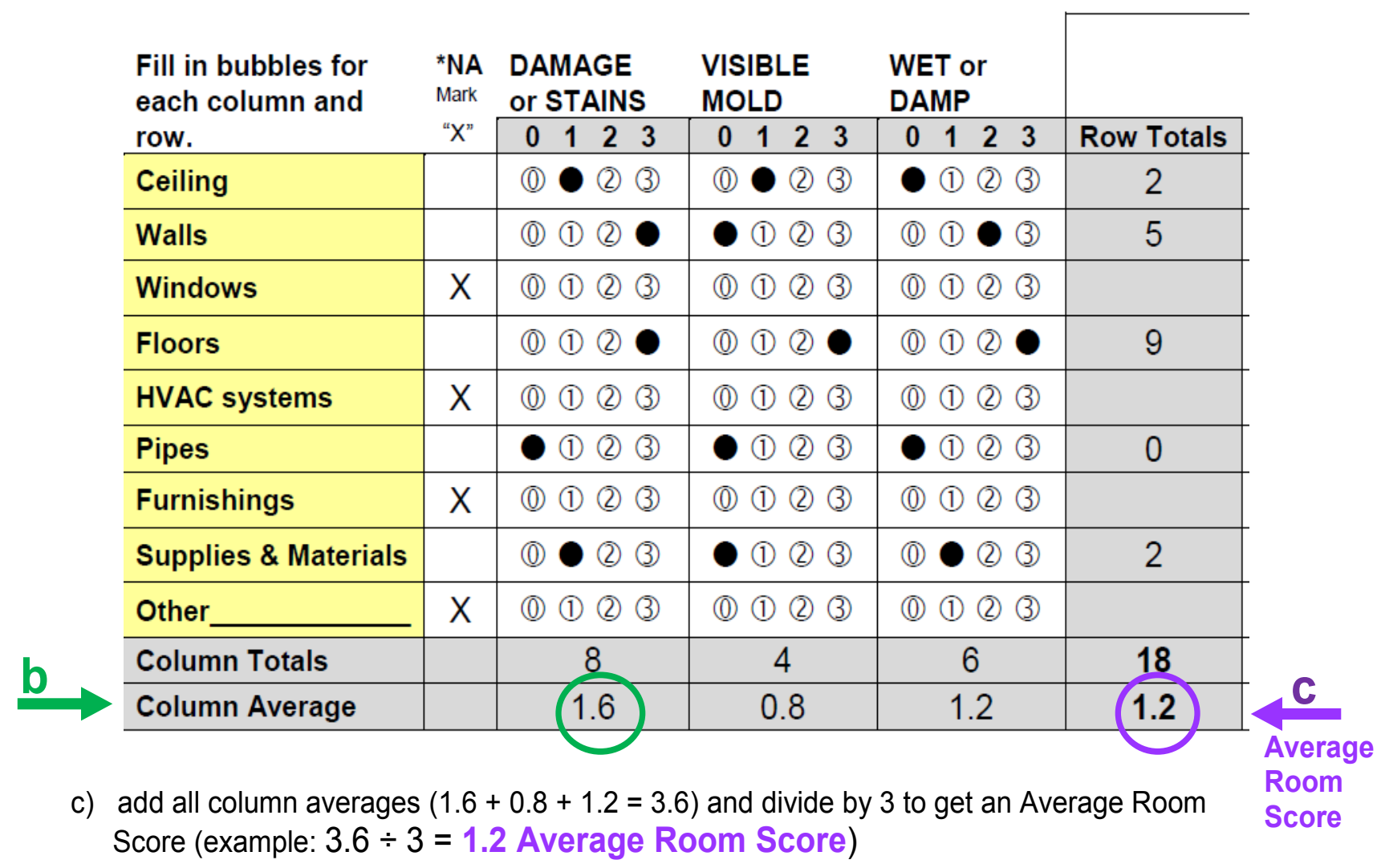

Overall totals are used for record keeping and for comparison. Use these totals to determine whether the areas observed were overall better or worse than the previous assessment taken. 
岕号

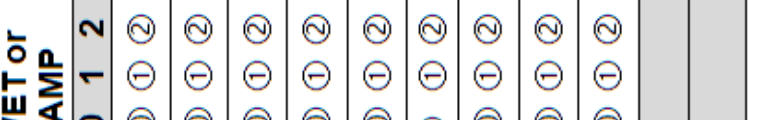

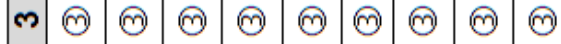

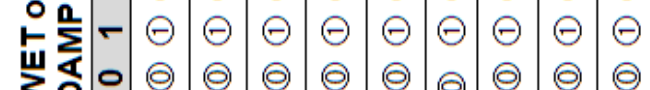
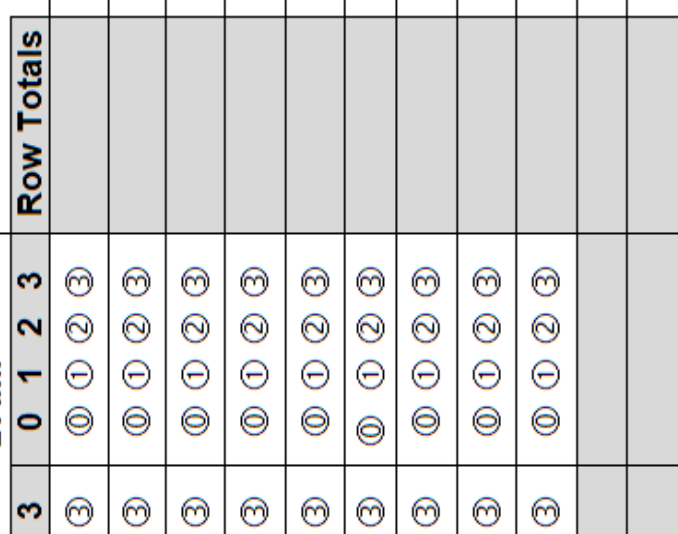

山

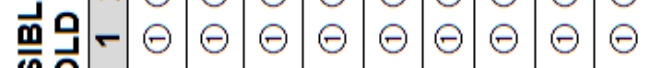

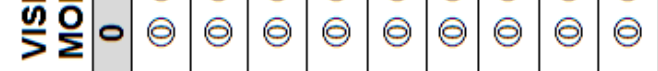

$\sum_{\substack{a \\ 0}}^{\mathbb{a}}$

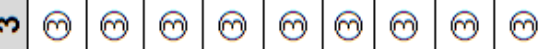

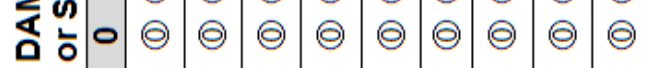

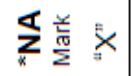

ติ

(2)

के

$\ddot{\ddot{\mathbf{x}}} \sum_{\Theta}$

훈 듬

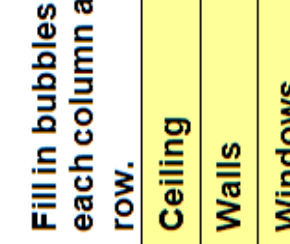

을

○े

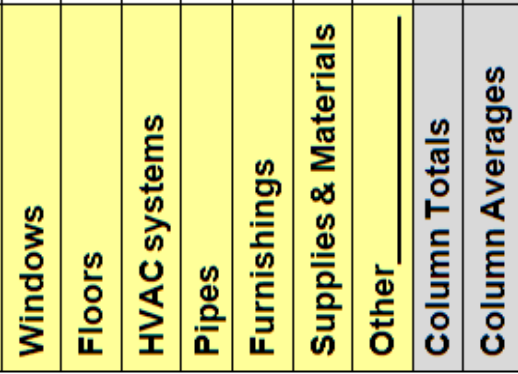

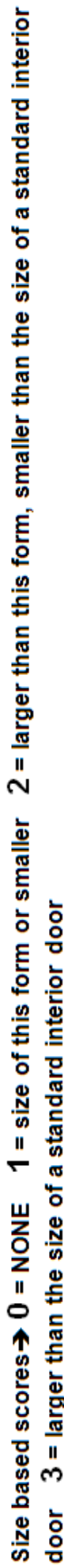

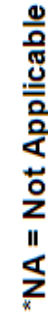


Appendix Q: NIOSH Draft Mold \& Dampness Assessment Sheet (CONTINUED)

\title{
CDC/NIOSH PILOT
}

\author{
NIOSH Contact: \\ Michelle Martin 304-285-5734 or \\ moldsheet\#1@cdc.gov
}


ACKNOWLEDGEMENTS AND

AVAILABILITY OF REPORT

The Respiratory Disease Hazard Evaluation and Technical Assistance Program (RDHETAP) of NIOSH conducts field investigations of possible health hazards in the workplace. These investigations are conducted under the authority of Section 20(a) (6) of the Occupational Safety and Health (OSH) Act of 1970, 29 U.S.C. 669(a)(6), or Section 501(a)(11) of the Federal Mine Safety and Health Act of 1977, 30 U.S.C. 951(a)(11), which authorizes the Secretary of Health and Human Services, following a written request from any employers or authorized representative of employees, to determine whether any substance normally found in the place of employment has potentially toxic effects in such concentrations as used or found.

RDHETAP also provides, upon request, technical and consultative assistance to federal, state, and local agencies; labor; industry; and other groups or individuals to control occupational health hazards and to prevent related trauma and disease.

The findings and conclusions in this report are those of the authors and do not necessarily represent the views of NIOSH. Mention of any company or product does not constitute endorsement by NIOSH. In addition, citations to websites external to NIOSH do not constitute NIOSH endorsement of the sponsoring organizations or their programs or products. Furthermore, NIOSH is not responsible for the content of these websites. All Web addresses referenced in this document were accessible as of the publication date.

This report was prepared by Rachel Bailey, Ju-Hyeong Park, Rena Saito, Kathleen Kreiss, and Jean Cox-Ganser of the Division of Respiratory Disease Studies. Field assistance was provided by Michael Beaty, David Spainhour, Eva Suarthana, and Sandra White. Database management was provided by Nicole Edwards and Brian Tift. Analytical support was provided by Nicole Edwards, Kathy Fedan, and Brian Tift. Desktop publishing was performed by Tia McClelland.

Copies of this report have been sent to representatives at the school district, elementary school, local parent teacher association, county health department, and OSHA Regional Office. This report is 


\section{ACKNOWLEDGEMENTS AND AVAILABILITY OF REPORT (CONTINUED)}

not copyrighted and may be freely reproduced. The report may be viewed and printed from the following internet address: $\underline{\mathrm{http}: / /}$ www.cdc.gov/niosh/hhe. Copies may be purchased from the National Technical Information Service at 5825 Port Royal Road, Springfield, Virginia 22161. Information regarding the NTIS stock number may be obtained from the NIOSH Publications Office at the Cincinnati address. 
Below is a recommended citation for this report:

NIOSH [2011]. Health Hazard Evaluation Report: Evaluation of dampness-

associated respiratory symptoms with relocation of staff during remediation of an elementary school, North Carolina. Cincinnati, OH: U.S. Department of Health and Human Services, Public Health Service, Centers for Disease Control and

Prevention, National Institutute for Occupational Safety and Health, NIOSH HETA No. 2009-0172-3124.

National Institute for Occupational Safety and Health

Delivering on the Nation's promise: Safety and health at work for all people through research and prevention.

To receive NIOS

occupational sat

contact NIOSH at:

1-800-35-NIOSt (1-800-356-4674)

Fax: 1-513-533-8573

E-mail: pubstaft@cdc.gov

or visit the NIOSH web site at:

http://www.cdc.gov/niosh/hhe 